
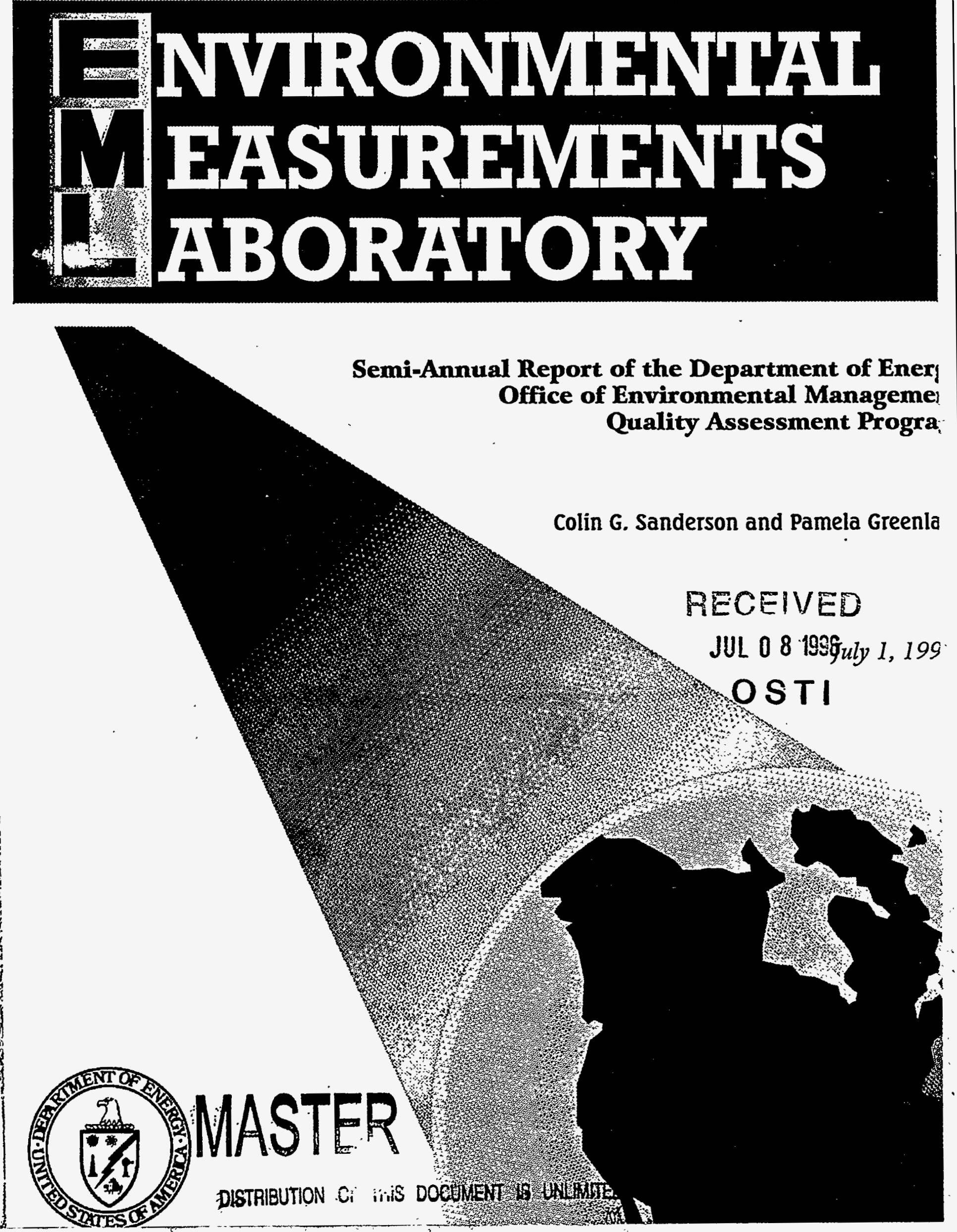


\title{
SEMI-ANNUAL REPORT OF THE DEPARTMENT OF ENERGY, OFFICE OF ENVIRONMENTAL MANAGEMENT, QUALITY ASSESSMENT PROGRAM
}

\author{
Colin G. Sanderson and Pamela Greenlaw \\ Environmental Measurements Laboratory \\ U. S. Department of Energy \\ New York, NY 10014-4811
}

July 1, 1996

\begin{abstract}
DISCLAIMER
"This report was prepared as an account of work sponsored by an agency of the United States Government. Neither the United States Government nor any agency thereof, nor any of their employees, makes any warranty, express or implied, or assumes any legal liability or responsibility for the accuracy, completeness, or usefulness of any information, apparatus, product, or process disclosed, or represents that its use would not infringe privately owned rights. Reference.herein to any specific commercial product, process, or service by trade name, trademark, manufacturer, or otherwise, does not necessarily constitute or imply its endorsement, recommendation, or favoring by the United States Government or any agency thereof. The views and opinions of authors expressed herein do not necessarily state or reflect those of the United States Government or any agency thereof."
\end{abstract}

This report has been reproduced directly from the best available copy.

Available from the National Technical Information Service, U.S. Department of Commerce, 5285 Port Royal Road, Springfield, Virginia 22161. 


\begin{abstract}
This report presents the results from the analysis of the 44th set of environmental quality assessment samples (QAP XLIV) that were received on or before June 3, 1996.
\end{abstract}




\section{INTRODUCTION}

This Quality Assessment Program (QAP) is designed to test the quality of the environmental measurements being reported to the Department of Energy by its contractors. Since 1976, real or synthetic environmental samples that have been prepared and thoroughly analyzed at the Environmental Measurements Laboratory (EML) have been distributed at first quarterly and then semi-annually to these contractors. Their results, which are returned to EML within 90 days, are compiled with EML's results and are reported back to the participating contractors 30 days later. A summary of the reported results is available to the participants 2 days after the reporting deadline via a modem-telephone connection to the EML computer.

This is the 48th report of this program. Preceding reports in this series are:

HASL-317
HASL-319
HASL-323
HASL-331
EML-336
EML-337
EML-340
EML-343
EML-346
EML-350
EML-351
EML-354
EML-358
EML-364
EML-368
EML-377
EML-387
EML-388
EML-393
EML-402
EML-414
EML-417
EML-426
PNL-5079
EML-431

(February 1, 1977)

(May 2, 1977)

(August 1, 1977)

(November 1, 1977)

(January 1, 1978)

(February 1, 1978)

(May 1, 1978)

(August 1, 1978)

(November 1, 1978)

(February 1, 1979)

(February 1, 1979)

(May 1, 1979)

(August 1, 1979)

(November 1, 1979)

(February 1, 1980)

(August 1, 1980)

(February 1, 1981)

(February 1, 1981)

(August 3, 1981)

(February 1, 1982)

(April 1, 1983)

(September 1, 1983)

(March 1, 1984)

(April 1, 1984)

(September 1, 1984) $\begin{array}{ll}\text { EML-432 } & \text { (November 1, 1984) } \\ \text { EML-438 } & \text { (March 1, 1985) } \\ \text { EML-439 } & \text { (March 1, 1985) } \\ \text { EML-448 } & \text { (October 1, 1985) } \\ \text { EML-453 } & \text { (March 1, 1986) } \\ \text { EML-454 } & \text { (March 1, 1986) } \\ \text { EML-477 } & \text { (October 1, 1986) } \\ \text { EML-478 } & \text { (March 1, 1987) } \\ \text { EML-498 } & \text { (September 1, 1987) } \\ \text { EML-518 } & \text { (January 2, 1989) } \\ \text { EML-525* } & \text { (August 1, 1989) } \\ \text { EML-526 } & \text { (January 2, 1990) } \\ \text { EML-530 } & \text { (July 2, 1990) } \\ \text { EML-535 } & \text { (January 1, 1991) } \\ \text { EML-539 } & \text { (July 1, 1991) } \\ \text { EML-543 } & \text { (January 2, 1992) } \\ \text { EML-546 } & \text { (July 1, 1992) } \\ \text { EML-551 } & \text { (January 4, 1993) } \\ \text { EML-556 } & \text { (July 1, 1993) } \\ \text { EML-559 } & \text { (January 5, 1994) } \\ \text { EML-561 } & \text { (July 1, 1994) } \\ \text { EML-565 } & \text { (January 5, 1995) } \\ \text { EML-569 } & \text { (July 3, 1995) } \\ \text { EML-576 } & \text { (February 1, 1996, Revised) }\end{array}$

"Please note this is a corrected report number. 


\section{DISCLAIMER}

Portions of this document may be illegible in electronic image products. Images are produced from the best available original document. 


\section{RESULTS}

The results from the analysis of QAP-XLIV samples received on or before June 3,1996 are listed according to the TABLE OF CONTENTS. The data for the different kinds of samples are given in the following units:

\section{Nuclear Species}

$\begin{array}{ll}\text { Air Filters } & \mathrm{Bq} \text { filter } \\ \text { Soil } & \mathrm{Bq} \mathrm{kg}^{-1} \\ \text { Tissue } & \mathrm{Bq} \mathrm{kg}^{-1} \\ \text { Vegetation } & \mathrm{Bq} \mathrm{kg}^{-1} \\ \text { Water } & \mathrm{Bq} \mathrm{L}^{-1}\end{array}$

The values for elemental uranium are reported in $\mu \mathrm{g}$ filter ${ }^{-1}, \mathrm{~g}$, or $\mathrm{mL}$. Some programs require the use of $\mathrm{pCi}$ as reporting units, the conversion can be found on page 3 .

The 'EML value' listed in the tables to which the contractors' results are compared is the mean of replicate determinations for each nuclide. The EML uncertainty is the standard error of the mean. All other uncertainties are as reported by the participants.

The control limits were established from percentiles of historic data distributions (1982-1992). The evaluation of this historic data and the development of the control limits is presented in DOE report EML-564.

Participants' analytical performance is evaluated based on the historical analytical capabilities for individual analyte/matrix pairs. The criteria for acceptable performance, " $A$ ", has been chosen to be between the $15^{\text {th }}$ and $85^{\text {th }}$ percentile of the cumulative normalized distribution, which can be viewed as the middle $70 \%$ of all historic measurements. The acceptable with warning criteria, "W", is between the $5^{\text {th }}$ and $15^{\text {th }}$ percentile and between the $85^{\text {th }}$ and $95^{\text {th }}$ percentile. In other words, the middle $90 \%$ of all reported values are acceptable, while the outer $5^{\text {th }}-15^{\text {th }}(10 \%)$ and $85^{\text {th }}-95^{\text {th }}$ percentiles $(10 \%)$ are in the warning area. The not acceptable criteria, " $N "$ ", is established at less than the $5^{\text {th }}$ percentile and greater than the $95^{\text {th }}$ percentile, that is, the outer $10 \%$ of the historical data. These control limits for all $48 \mathrm{i} / \mathrm{j}$ pairs are listed in the Table of Control Limits \& Performance Criteria (p. 4).

QAP is an external assessment of environmental radiological analyses. If your laboratory is performing other types of analyses (screening, high-level radiological), this evaluation system may not be appropriate, and you should continue to use an evaluation system appropriate to your data objectives. 


\section{TABLE OF CONTENTS}

Summary Figure of QAP-XLIV Evaluations $\ldots \ldots \ldots \ldots \ldots \ldots \ldots \ldots \ldots \ldots \ldots \ldots \ldots \ldots \ldots$

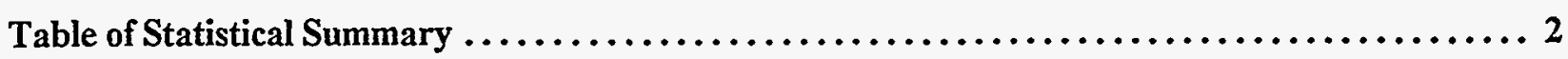

Table of Control Limits and Performance Criteria $\ldots \ldots \ldots \ldots \ldots \ldots \ldots \ldots \ldots \ldots \ldots \ldots$

Summary of Matrix Evaluations by Laboratory $\ldots \ldots \ldots \ldots \ldots \ldots \ldots \ldots \ldots \ldots \ldots \ldots \ldots$

Summary of Laboratory Evaluations by Matrix $\ldots \ldots \ldots \ldots \ldots \ldots \ldots \ldots \ldots \ldots \ldots \ldots \ldots \ldots$

Summary of Matrix Evaluations by Nuclide $\ldots \ldots \ldots \ldots \ldots \ldots \ldots \ldots \ldots \ldots \ldots \ldots \ldots \ldots, 43$

Results Ordered by Laboratory

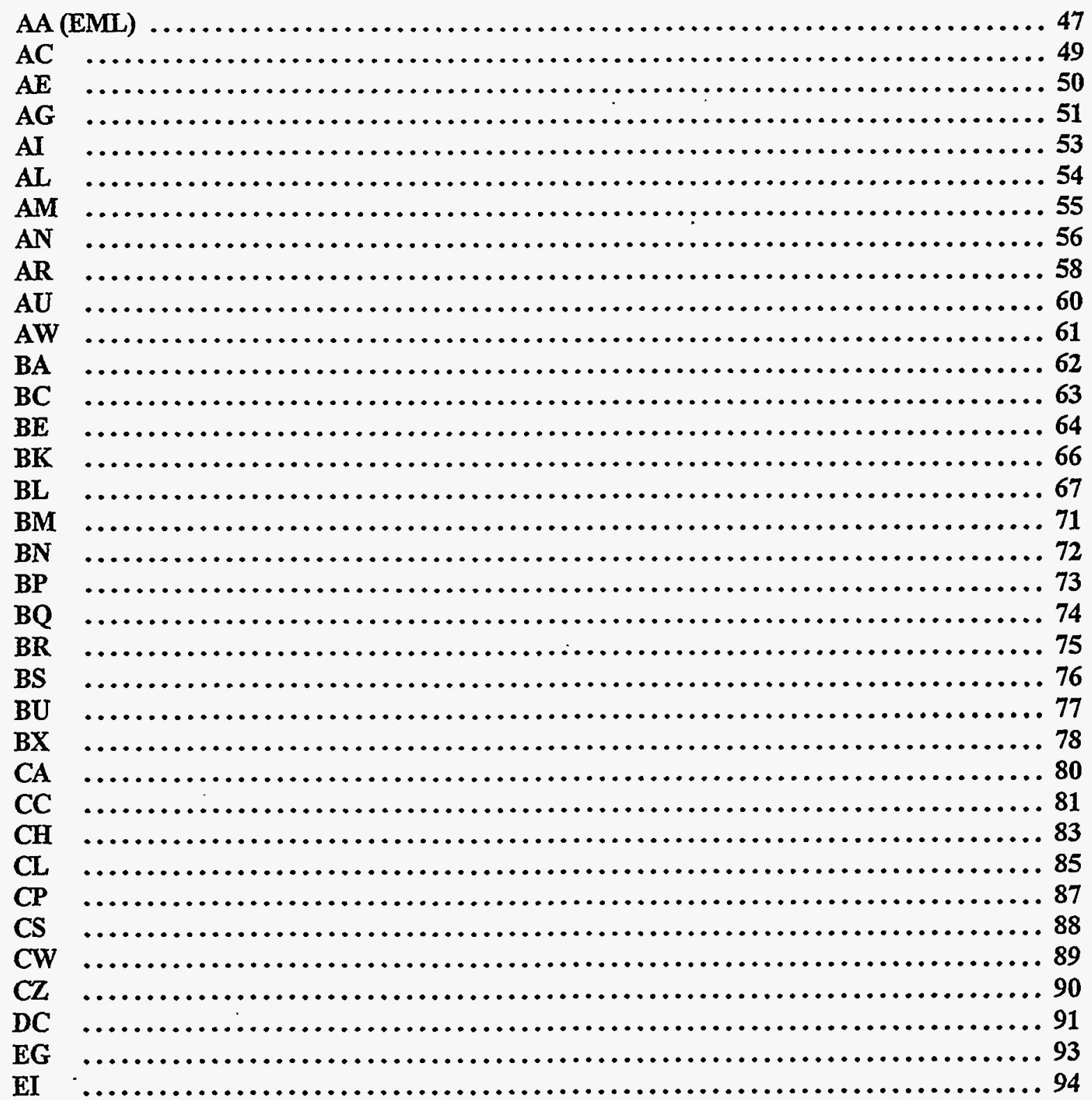




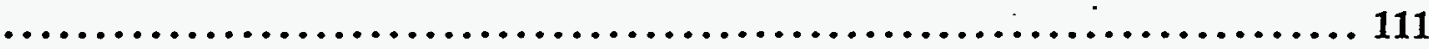

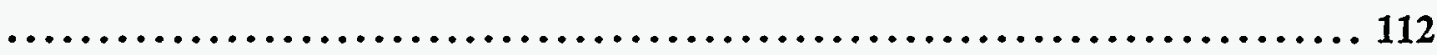

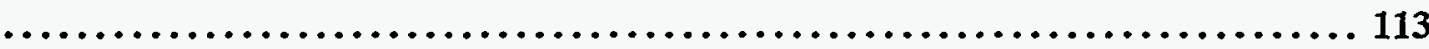

IL

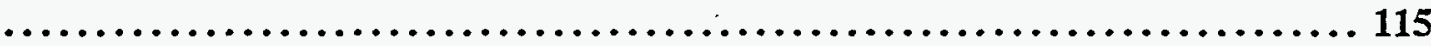



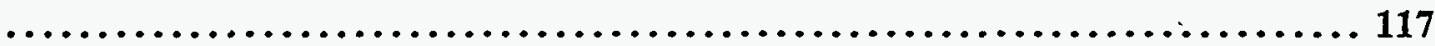



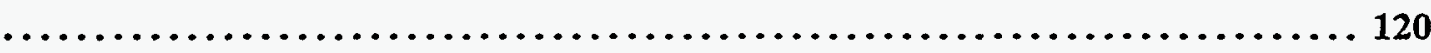

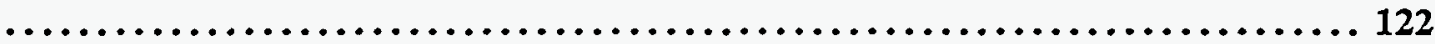

$\mathbf{J P}$

KA

LA

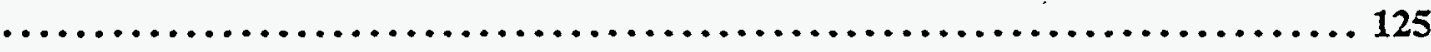

LB

LH

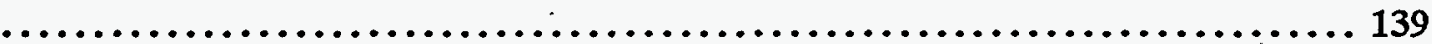

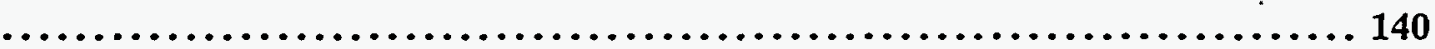

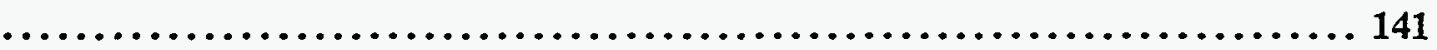

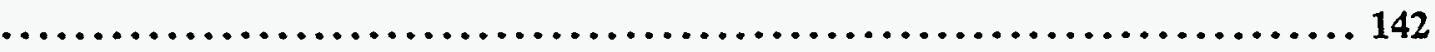

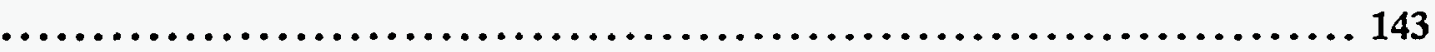

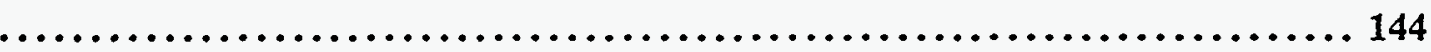

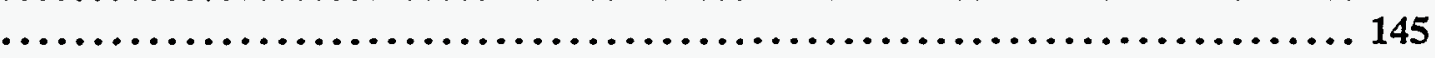



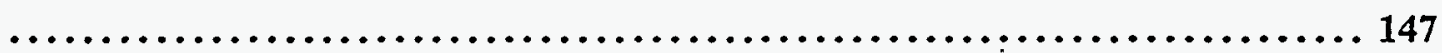

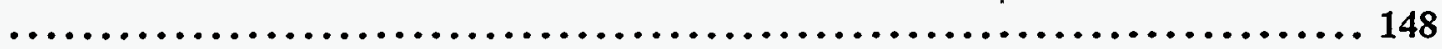



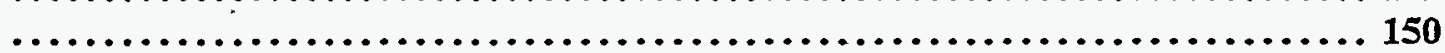

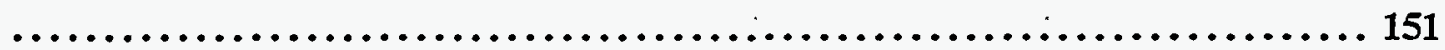

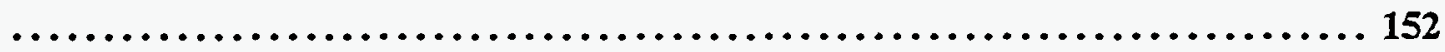

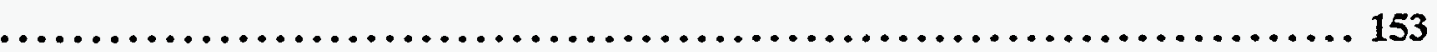

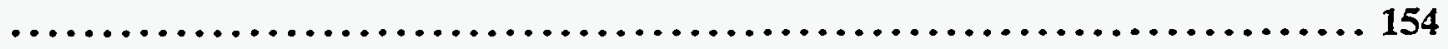

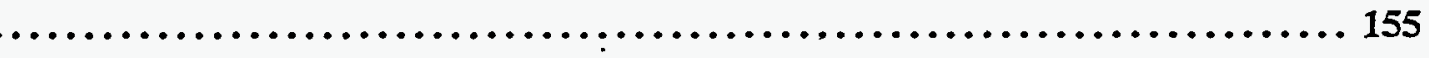




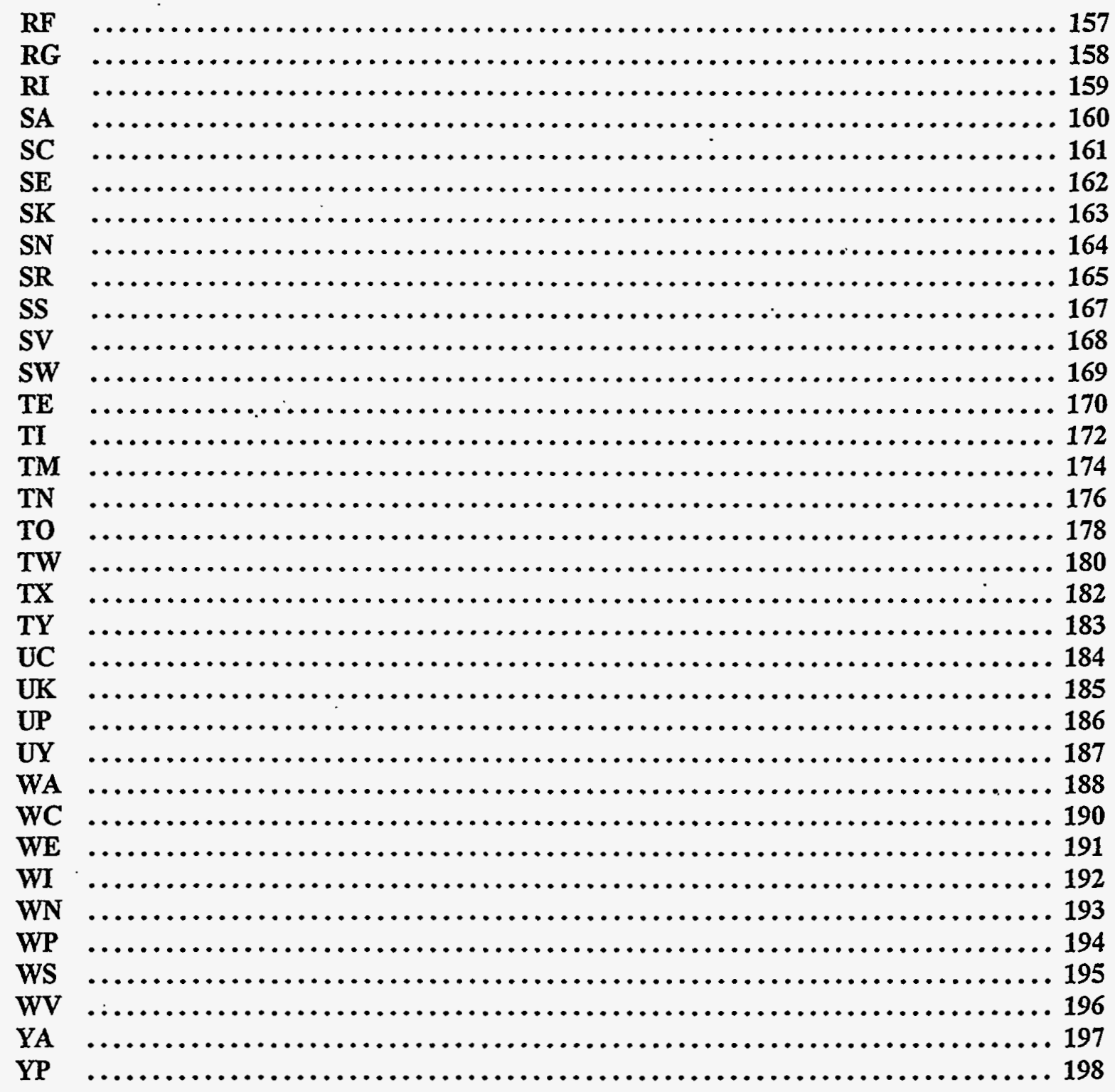

Results Ordered by Matrix/Nuclide

Air

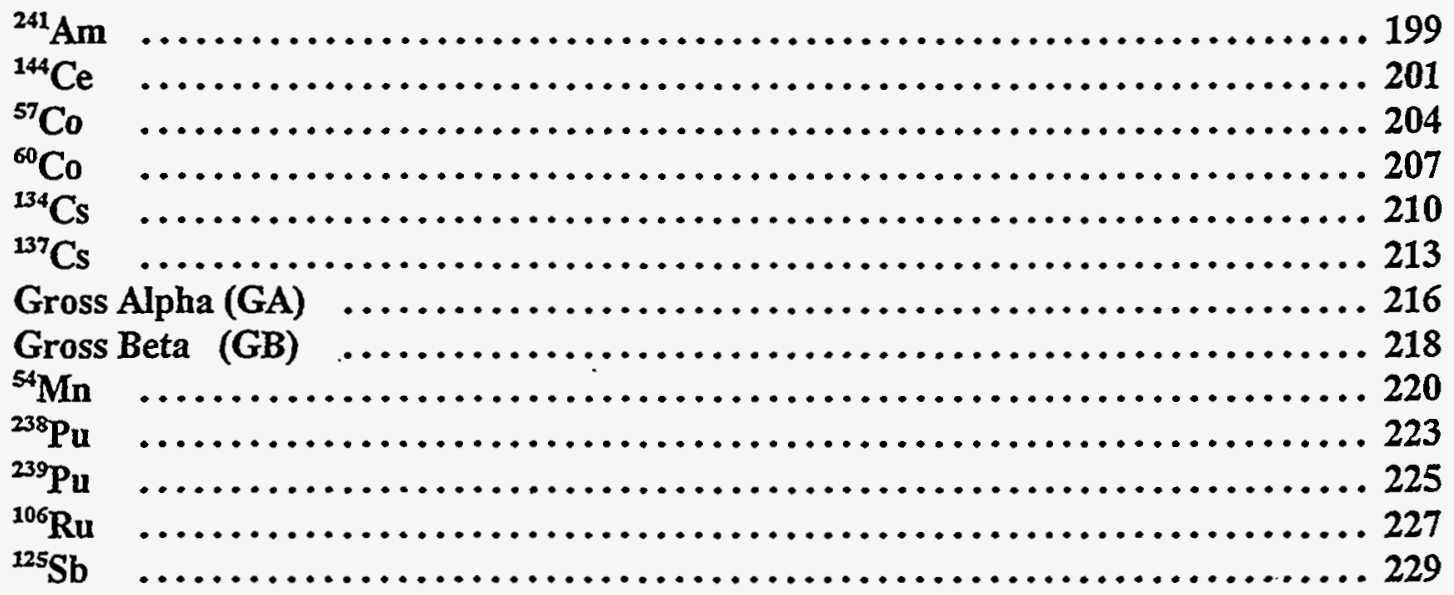




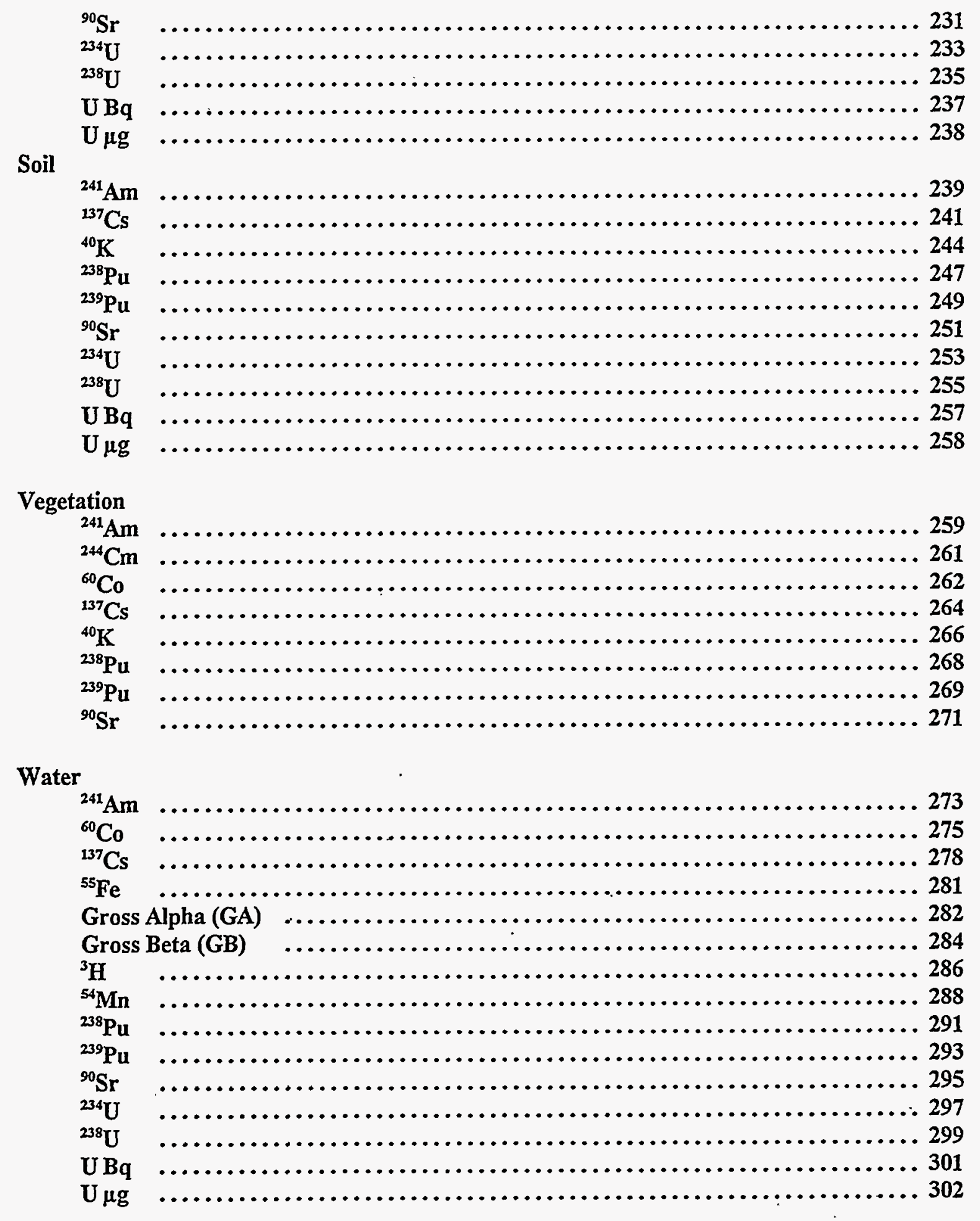

List of Labcodes of Participating Laboratories for EML QAP XLIII

Laboratories Reporting Data

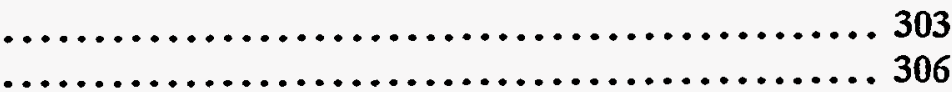

Laboratories Not Reporting Data 


\section{QAP 44 Summary of Evaluations of 3262 Reported Analyses}

Air Filter:

1208 Analyses

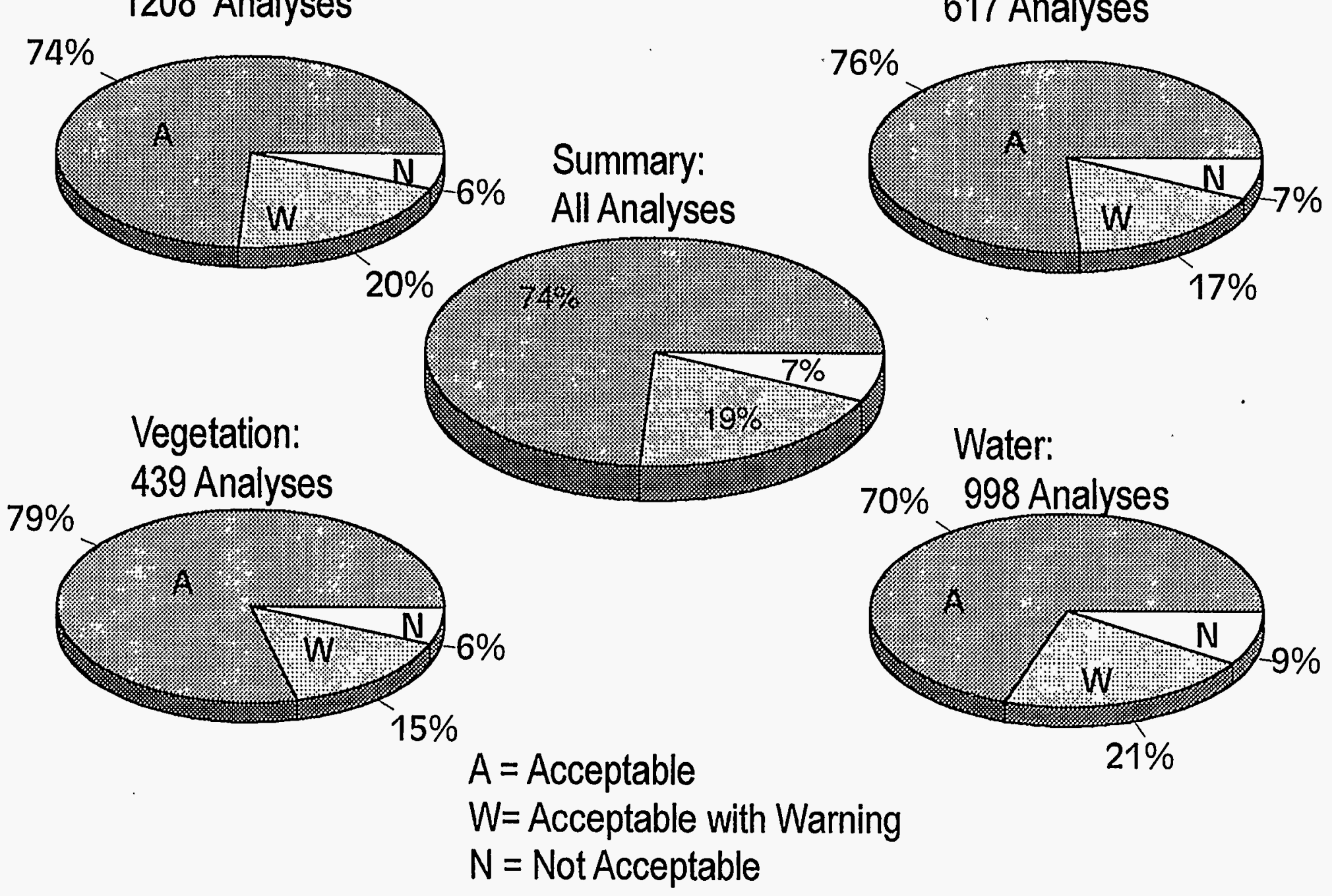


QAP44 Statistical Summary

Reported Values EML Value

No. of

Reported

Nualide

EMI Value

EMLFrror

Mean

Median

Std. Dev

Values

Matrix: Al

$\begin{array}{lr}\text { AM241 } & 0.189 \\ \text { CE144 } & 33.300 \\ \text { CO 57 } & 8.900 \\ \text { CO 60 } & 29.500 \\ \text { CS134 } & 14.700 \\ \text { CS137 } & 6.640 \\ \text { GA 1 } & 1.620 \\ \text { GB 2 } & 1.770 \\ \text { MN 54 } & 3.440 \\ \text { PU238 } & 0.096 \\ \text { PU239 } & 0.093 \\ \text { RU106 } & 11.600 \\ \text { SB125 } & 9.780 \\ \text { SR 90 } & 1.060 \\ \text { U 234 } & 0.052 \\ \text { U 238 } & 0.053 \\ \text { U BO } & 0.107 \\ \text { U UG } & 4.310\end{array}$

0.007
3.300
0.900
2.900
1.460
0.700
0.150
0.150
0.380
0.002
0.003
1.440
1.030
0.037
0.002
0.002
0.003
0.100

1.09

0.78

1.04

0.22

0.81

0.89

0.75

0.79

0.89

0.91

0.91

1.16

1.08

0.92

0.99

1.04

0.94

0.94

0.98

1.21

1.16

1.25

0.91

0.89

1.17

1.03

0.90

1.00

1.05

0.95

0.94

0.95

1.17

1.13

1.12

$1.09 \quad 1.02$

0.14

0.14

0.08

0.09 .

0.11

0.18

0.20

0.12

0.12

0.12

0.16

0.15

0.26

0.24

0.19

0.30

0.19

60

80

85

84

87

88

59

57

81

61

61

76

86

47

41

39

13

25

Matrix: So

$\begin{array}{lr}\text { AM241 } & 3.690 \\ \text { CS137 } & 359.000 \\ \text { K 40 } & 465.000 \\ \text { PU238 } & 43.000 \\ \text { PU239 } & 9.230 \\ \text { SR 90 } & 1340.000 \\ \text { U 234 } & 34.200 \\ \text { U 238 } & 35.900 \\ \text { U BQ } & 71.700 \\ \text { U UG } & 2.900\end{array}$

0.454

10.000

30.000

2.440

0.346

113.000

3.520

3.630

4.150

0.290

$\begin{array}{ll}1.08 & 1.00 \\ 1.06 & 1.06 \\ 1.06 & 1.04 \\ 0.98 & 1.00 \\ 1.03 & 1.01 \\ 0.88 & 0.89 \\ 1.01 & 1.05 \\ 1.02 & 1.00 \\ 0.95 & 0.98 \\ 0.96 & 0.97\end{array}$

0.33

0.11

0.13

0.12

0.13

0.13

0.16

0.20

0.18

0.21

60
95
86
67
70
44
46
58
17
33

Matrix: VE

$\begin{array}{lr}\text { AM241 } & 5.600 \\ \text { CM244 } & 4.440 \\ \text { CO 60 } & 59.700 \\ \text { CS137 } & 944.000 \\ \text { K 40 } & 1030.000 \\ \text { PU238 } & 0.820 \\ \text { PU239 } & 9.820 \\ \text { SR 90 } & 1300.000\end{array}$

0.184

0.202

0.963

16.200

33.000

0.104

1.220

52.400

$\begin{array}{ll}1.16 & 1.10 \\ 1.01 & 1.01 \\ 1.04 & 1.04 \\ 1.14 & 1.14 \\ 1.09 & 1.10 \\ 1.09 & 1.15 \\ 0.96 & 0.95 \\ 0.95 & 0.96\end{array}$

0.27

0.20

0.13

0.12

0.15

0.24

0.16

0.17

54
35
76
69
72
7
55
44

Statistical summary of "A" and "W" reported values 


\section{QAP44 Statistical Summary}

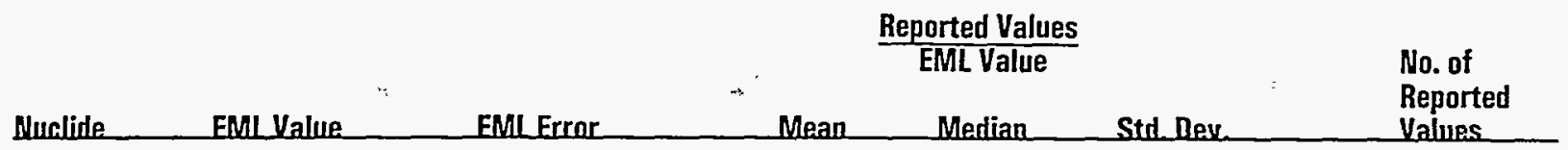

\section{Matrix: WA}

$\begin{array}{lr}\text { AM241 } & 0.766 \\ \text { CO 60 } & 32.800 \\ \text { CS137 } & 38.300 \\ \text { FE 55 } & 83.000 \\ \text { GA 1 } & 1850.000 \\ \text { GB 2 } & 744.000 \\ \text { H 3 } & 251.000 \\ \text { MN 54 } & 38.400 \\ \text { PU238 } & 0.982 \\ \text { PU239 } & 0.772 \\ \text { SR 90 } & 1.450 \\ \text { U 234 } & 0.274 \\ \text { U 238 } & 0.275 \\ \text { U BO } & 0.559 \\ \text { U UG } & 0.022\end{array}$

0.013
0.580
0.881
3.440
185.000
74.000
11.400
1.160
0.074
0.056
0.034
0.019
0.024
0.047
0.003

$\begin{array}{ll}1.07 & 1.05 \\ 1.03 & 1.02 \\ 1.10 & 1.10 \\ 1.03 & 0.98 \\ 1.01 & 1.00 \\ 1.06 & 1.08 \\ 0.95 & 0.88 \\ 1.09 & 1.09 \\ 0.96 & 0.97 \\ 0.98 & 0.99 \\ 1.09 & 1.06 \\ 1.10 & 1.06 \\ 1.08 & 1.09 \\ 1.05 & 1.07 \\ 1.02 & 1.04\end{array}$

0.17
0.06
0.07
0.23
0.12
0.19
0.22
0.07
0.08
0.11
0.19
0.11
0.10
0.23
0.10

69

97

96

13

63

43

$74.000 \quad 11.400$
38.400

0.982

0.772

0.274

0.275

0.022

0.003

Units for matrices: $\mathrm{Al}=\mathrm{Bq} / \mathrm{filter} \mathrm{SO}=\mathrm{Bq} / \mathrm{kg} V \mathrm{~V}=\mathrm{Bq} / \mathrm{kg} W A=B \mathrm{~g} / \mathrm{L}$. Values for elemental uranium in $\mu \mathrm{g} /$ filter, $\mathrm{g}$ or $\mathrm{mL}$.

Conversion from $\mathrm{Bq}$ to $\mathrm{pCi}$ :

$$
1 \mathrm{~Bq}=27 \mathrm{pCi}
$$

Example: Convert $3 \mathrm{~Bq}$ to $\mathrm{pCi}$

$$
3 \mathrm{~Bq} \times 27 \mathrm{pCi} / \mathrm{Bq}=81 \mathrm{pCi}
$$


QAP44 Control Limits* by Matrix

Nuclide

Lower limit

Lower Middle Limit

Upper Middle Limit

Upper Limit

Matrix: Al

$\begin{array}{lllll}\text { AM241 } & 0.62 & 0.82 & 1.25 & 1.93 \\ \text { CE144 } & 0.61 & 0.69 & 1.09 & 1.31 \\ \text { CO 57 } & 0.63 & 0.72 & 1.08 & 1.29 \\ \text { CO 60 } & 0.74 & 0.83 & 1.10 & 1.25 \\ \text { CS134 } & 0.70 & 0.80 & 1.08 & 1.21 \\ \text { CS137 } & 0.72 & 0.84 & 1.13 & 1.32 \\ \text { GA 1 } & 0.82 & 0.91 & 1.30 & 1.58 \\ \text { GB 2 } & 0.75 & 0.89 & 1.61 & 1.94 \\ \text { MN 54 } & 0.76 & 0.85 & 1.12 & 1.33 \\ \text { PU238 } & 0.61 & 0.82 & 1.15 & 1.55 \\ \text { PU239 } & 0.67 & 0.86 & 1.16 & 1.58 \\ \text { RU106 } & 0.54 & 0.64 & 1.16 & 1.59 \\ \text { SB125 } & 0.35 & 0.66 & 1.12 & 1.40 \\ \text { SR 90 } & 0.62 & 0.83 & 1.45 & 2.26 \\ \text { U 234 } & 0.78 & 0.90 & 1.37 & 1.93 \\ \text { U 238 } & 0.80 & 0.89 & 1.37 & 2.63 \\ \text { U BO } & 0.79 & 0.87 & 1.66 & 2.88 \\ \text { U UG } & 0.54 & 0.84 & 1.26 & 1.73\end{array}$

Matrix: SO

$\begin{array}{ll}\text { AM241 } & 0.52 \\ \text { CS137 } & 0.74 \\ \text { K 40 } & 0.70 \\ \text { PU238 } & 0.22 \\ \text { PU239 } & 0.62 \\ \text { SR 90 } & 0.58 \\ \text { U 234 } & 0.39 \\ \text { U 238 } & 0.35 \\ \text { U BO } & 0.27 \\ \text { U UG } & 0.30\end{array}$

0.52

$\begin{array}{ll}0.52 & 0.75 \\ 0.74 & 0.88\end{array}$

0.84

0.62

0.84

0.76

0.64

0.64

0.42

0.51

1.49

1.23

1.23

1.19

1.28

1.66

1.06

1.09

1.05

1.10

Matrix: VE

$\begin{array}{ll}\text { AM241 } & 0.58 \\ \text { CM244 } & 0.40 \\ \text { CO 60 } & 0.64 \\ \text { CS137 } & 0.75 \\ \text { K 40 } & 0.45 \\ \text { PU238 } & 0.56 \\ \text { PU239 } & 0.60 \\ \text { SR 90 } & 0.50\end{array}$

0.81

0.84

0.82

0.92

0.81

0.82

0.81

0.71
1.60

1.55

1.23

1.25

1.20

1.63

1.36

1.11
2.86

1.87

1.49

1.48

1.51

2.76

1.98

1.37

Matrix: WA

$\begin{array}{lllll}\text { AM241 } & 0.66 & 0.84 & 1.20 & 1.56 \\ \text { CD 60 } & 0.87 & 0.95 & 1.11 & 1.17 \\ \text { CS137 } & 0.90 & 0.97 & 1.15 & 1.25 \\ \text { FE 55 } & 0.27 & 0.80 & 1.28 & 1.62\end{array}$

* Control limits are reported as: the ratio of Reported Value vs. EML Value 


\section{QAP44 Control Limits* by Matrix}

\begin{tabular}{lcccc} 
Nuclide & Lower Limit & Lower Middle Limit & Upper Middle Limit & Upper Limit \\
\hline & & 2 & & \\
GA 1 & 0.55 & 0.80 & 1.22 & 1.31 \\
GB 2 & 0.75 & 0.93 & 1.52 & 1.65 \\
H 3 & 0.69 & 0.84 & 1.20 & 1.91 \\
MN 54 & 0.88 & 0.95 & 1.13 & 1.21 \\
PU238 & 0.68 & 0.91 & 1.14 & 1.33 \\
PU239 & 0.62 & 0.78 & 1.16 & 1.65 \\
SR 90 & 0.73 & 0.87 & 1.24 & 1.47 \\
U 234 & 0.77 & 0.90 & 1.19 & 1.40 \\
U 238 & 0.78 & 0.90 & 1.14 & 1.45 \\
U BD & 0.40 & 0.82 & 1.21 & 1.35 \\
U UG & 0.73 & 0.89 & &
\end{tabular}

Control limits are established from historical QAP data.

Where historical data are insufficient, limits of $\pm 20 \%$ and $\pm 50 \%$ are applied.

The following are recommended performance criteria for analysis of environmental levels of analytes:

Acceptable: Lower Middle Limit $\leq A \leq$ Upper Middle Limit

Acceptable with Warning: Lower Limit $\leq W<$ Lower Middle Limit $\quad$ or Upper Middle Limit $\quad<W \leq$ Upper Limit

Not Acceptable: $\mathbf{N}<$ Lower Limit or $\mathbf{N}>$ Upper Limit

"Control limits are reported as: the ratio of Reported Value vs. EML Value 


\section{QAP44 Summary of Matrix Evaluations by Laboratory}

\begin{tabular}{|c|c|c|c|c|c|c|c|}
\hline \multicolumn{4}{|c|}{ Evaluation Summary } & & \multicolumn{3}{|c|}{ Evaluation Percentages } \\
\hline Matrix & $A$ & $w$ & $N$ & Analuses & $\%$ & $\% w$ & $\% N$ \\
\hline
\end{tabular}

Lab: AA Environmental Measurements Laboratory

\begin{tabular}{|c|c|c|c|c|c|c|c|}
\hline $\begin{array}{l}\mathrm{Al} \\
\mathrm{Al} \\
\text { SO } \\
\mathrm{VE} \\
\text { WA }\end{array}$ & $\begin{array}{r}1 \\
17 \\
10 \\
8 \\
15\end{array}$ & $\begin{array}{l}0 \\
0 \\
0 \\
0 \\
0\end{array}$ & $\begin{array}{l}0 \\
0 \\
0 \\
0 \\
0\end{array}$ & $\begin{array}{r}1 \\
17 \\
10 \\
8 \\
15\end{array}$ & $\begin{array}{l}100 \\
100 \\
100 \\
100 \\
100\end{array}$ & $\begin{array}{l}0 \\
0 \\
0 \\
0 \\
0\end{array}$ & $\begin{array}{l}0 \\
0 \\
0 \\
0 \\
0\end{array}$ \\
\hline Totals: & 51 & 0 & 0 & 51 & $100 \%$ & 0\% & $0 \%$ \\
\hline
\end{tabular}

Lab: AC Analytical Chemistry Laboratory. Argonne National Lab

\begin{tabular}{|c|c|c|c|c|c|c|c|}
\hline $\begin{array}{l}\text { Al } \\
\text { SO } \\
\text { WA }\end{array}$ & $\begin{array}{l}9 \\
5 \\
6\end{array}$ & $\begin{array}{l}3 \\
0 \\
1\end{array}$ & $\begin{array}{l}0 \\
0 \\
1\end{array}$ & $\begin{array}{r}12 \\
5 \\
8\end{array}$ & $\begin{array}{r}75 \\
100 \\
75\end{array}$ & $\begin{array}{r}25 \\
0 \\
13\end{array}$ & $\begin{array}{r}0 \\
0 \\
13\end{array}$ \\
\hline Totals: & 20 & 4 & 1 & 25 & $80 \%$ & $16 \%$ & $4 \%$ \\
\hline
\end{tabular}

Lab: AE Analytical Resources, inc., Seattle

\begin{tabular}{|c|c|c|c|c|c|c|c|}
\hline $\begin{array}{l}\text { So } \\
\text { WA }\end{array}$ & $\begin{array}{l}6 \\
9\end{array}$ & $\begin{array}{l}1 \\
1\end{array}$ & $\begin{array}{l}0 \\
0 \\
0\end{array}$ & $\begin{array}{r}7 \\
10\end{array}$ & $\begin{array}{l}86 \\
90\end{array}$ & $\begin{array}{l}14 \\
10\end{array}$ & $\begin{array}{l}0 \\
0\end{array}$ \\
\hline Totals: & 15 & 2 & 0 & 17 & $88 \%$ & $12 \%$ & $0 \%$ \\
\hline
\end{tabular}

Lah: AG Paragon Analytics, Inc, Fort Collins, CO

\begin{tabular}{|c|c|c|c|c|c|c|c|}
\hline $\begin{array}{l}\text { Al } \\
\text { SO } \\
\text { VE } \\
\text { WA }\end{array}$ & $\begin{array}{r}13 \\
6 \\
7 \\
10\end{array}$ & $\begin{array}{l}3 \\
2 \\
0 \\
1\end{array}$ & $\begin{array}{l}0 \\
0 \\
0 \\
2\end{array}$ & $\begin{array}{r}16 \\
8 \\
7 \\
13\end{array}$ & $\begin{array}{r}81 \\
75 \\
100 \\
77\end{array}$ & $\begin{array}{r}19 \\
25 \\
0 \\
8\end{array}$ & $\begin{array}{r}0 \\
0 \\
0 \\
15\end{array}$ \\
\hline Totals: & 36 & 6 & 2 & 44 & $82 \%$ & $14 \%$ & $5 \%$ \\
\hline
\end{tabular}

Lab: Al Nuclear Technalogy Services, Inc., Roswell, GA

$\begin{array}{llllrrrr}\text { Al } & 6 & 3 & 3 & 12 & 50 & 25 & 25 \\ \text { SO } & 2 & 3 & 1 & 6 & 33 & 50 & 17 \\ \text { VE } & 0 & 2 & 1 & 3 & 0 & 67 & 33 \\ \text { WA } & 5 & 3 & 1 & 9 & 56 & 33 & 11\end{array}$




\section{QAP44 Summary of Matrix Evaluations by Laboratory}

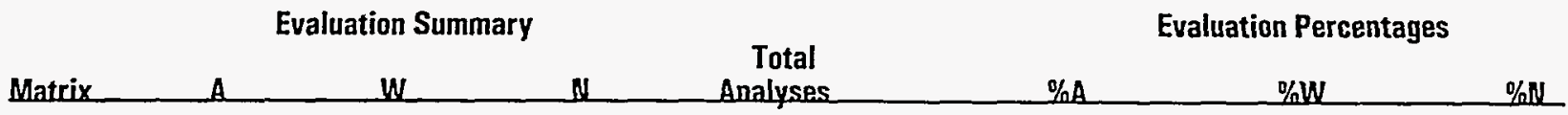

Lab: Al Nuclear Technology Services, Inc., Roswell, GA

$\begin{array}{lllllllll} & & & & & & & & \\ \text { Totals: } & 13 & 11 & 6 & 30 & 33 \% & & 37 \% & 20 \%\end{array}$

Lab:- AL Ames Laboratory, Ames, IA

\begin{tabular}{|c|c|c|c|c|c|c|c|}
\hline $\begin{array}{l}\text { Al } \\
\text { SD } \\
\text { VE } \\
\text { WA }\end{array}$ & $\begin{array}{l}2 \\
1 \\
2 \\
3\end{array}$ & $\begin{array}{l}0 \\
0 \\
0 \\
0\end{array}$ & $\begin{array}{l}4 \\
0 \\
0 \\
0\end{array}$ & $\begin{array}{l}6 \\
1 \\
2 \\
3\end{array}$ & $\begin{array}{r}33 \\
100 \\
100 \\
100\end{array}$ & $\begin{array}{l}0 \\
0 \\
0 \\
0\end{array}$ & $\begin{array}{r}67 \\
0 \\
0 \\
0\end{array}$ \\
\hline Totals: & 8 & 0 & 4 & 12 & $67 \%$ & $0 \%$ & $33 \%$ \\
\hline
\end{tabular}

Lah: AM American Radiation Services, Inc., Baton Rouge

\begin{tabular}{lrrrrrrrr} 
Al & 5 & 5 & 1 & 11 & & 45 & 45 & 9 \\
SO & 3 & 2 & 0 & 5 & 60 & 40 & 0 \\
WA & 4 & 2 & 1 & 7 & & 57 & 29 & 14 \\
\cline { 2 - 7 } Totals: & 12 & 9 & 2 & 23 &. & $52 \%$. & $39 \%$ & $9 \%$
\end{tabular} .

Lab: AN Argonne National Laboratory

\begin{tabular}{|c|c|c|c|c|c|c|c|}
\hline $\begin{array}{l}A l \\
S O \\
V E \\
W A\end{array}$ & $\begin{array}{r}15 \\
7 \\
7 \\
11\end{array}$ & $\begin{array}{l}1 \\
1 \\
1 \\
1\end{array}$ & $\begin{array}{l}0 \\
0 \\
0 \\
0\end{array}$ & $\begin{array}{r}16 \\
8 \\
8 \\
12\end{array}$ & $\begin{array}{l}94 \\
88 \\
88 \\
92\end{array}$ & $\begin{array}{r}6 \\
13 \\
13 \\
8\end{array}$ & $\begin{array}{l}0 \\
0 \\
0 \\
0\end{array}$ \\
\hline Totals: & 40 & 4 & 0 & 44 & $91 \%$ & $\mathbf{9} \%$ & $0 \%$ \\
\hline
\end{tabular}

Lab: AR Accu-Labs Research Inc., Golden, CO

\begin{tabular}{|c|c|c|c|c|c|c|c|}
\hline $\begin{array}{l}A I \\
\text { SO } \\
\text { VE } \\
W A\end{array}$ & $\begin{array}{r}16 \\
7 \\
3 \\
6\end{array}$ & $\begin{array}{l}2 \\
3 \\
4 \\
6\end{array}$ & $\begin{array}{l}0 \\
0 \\
1 \\
2\end{array}$ & $\begin{array}{r}18 \\
10 \\
8 \\
14\end{array}$ & $\begin{array}{l}89 \\
70 \\
38 \\
43\end{array}$ & $\begin{array}{l}11 \\
30 \\
50 \\
43\end{array}$ & $\begin{array}{r}0 \\
0 \\
13 \\
14\end{array}$ \\
\hline Totals: & 32 & 15 & 3 & 50 & $64 \%$ & $30 \%$ & $6 \%$ \\
\hline
\end{tabular}




\section{QAP44 Summary of Matrix Evaluations by Laboratory}

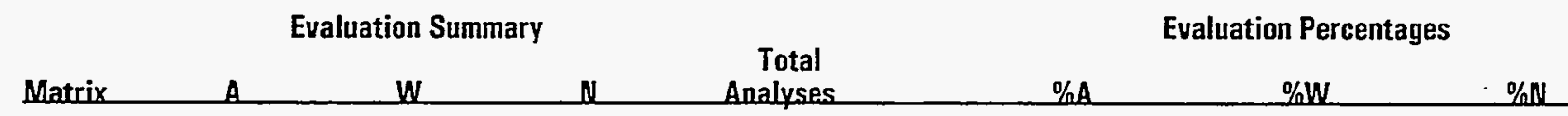

Lab: AU ORISE EESD/ESSAP, Oak Ridge

\begin{tabular}{|c|c|c|c|c|c|c|c|}
\hline $\begin{array}{l}\mathrm{Al} \\
\mathrm{SO} \\
\mathrm{VE} \\
\mathrm{WA}\end{array}$ & $\begin{array}{l}1 \\
2 \\
2 \\
2\end{array}$ & $\begin{array}{l}1 \\
0 \\
1 \\
4\end{array}$ & $\begin{array}{l}4 \\
4 \\
2 \\
3\end{array}$ & $\begin{array}{l}6 \\
6 \\
5 \\
9\end{array}$ & $\begin{array}{l}17 \\
33 \\
40 \\
22\end{array}$ & $\begin{array}{r}17 \\
0 \\
20 \\
44\end{array}$ & $\begin{array}{l}67 \\
67 \\
40 \\
33\end{array}$ \\
\hline Totals: & 7 & 6 & 13 & 26 & $27 \%$ & $23 \%$ & $50 \%$ \\
\hline
\end{tabular}

Lab: AW Argonne National Laboratory, Idaho Falls

\begin{tabular}{|c|c|c|c|c|c|c|c|}
\hline $\begin{array}{l}\text { Al } \\
\text { WA }\end{array}$ & $\begin{array}{l}9 \\
5\end{array}$ & $\begin{array}{l}0 \\
1\end{array}$ & $\begin{array}{l}0 \\
1\end{array}$ & $\begin{array}{l}9 \\
7\end{array}$ & $\begin{array}{r}100 \\
71\end{array}$ & $\begin{array}{r}0 \\
14\end{array}$ & $\begin{array}{r}0 \\
14\end{array}$ \\
\hline Totals: & 14 & 1 & 1 & 16 & $88 \%$ & $6 \%$ & $6 \%$ \\
\hline
\end{tabular}

Lab: BA Bettis Atomic Power Lab, West Mifflin, PA

\begin{tabular}{|c|c|c|c|c|c|c|c|}
\hline $\begin{array}{l}\text { Al } \\
\text { SO } \\
\text { VE } \\
\text { WA }\end{array}$ & $\begin{array}{l}8 \\
1 \\
4 \\
5\end{array}$ & $\begin{array}{l}3 \\
0 \\
0 \\
2\end{array}$ & $\begin{array}{l}0 \\
0 \\
0 \\
0\end{array}$ & $\begin{array}{r}11 \\
1 \\
4 \\
7\end{array}$ & $\begin{array}{r}73 \\
100 \\
100 \\
71\end{array}$ & $\begin{array}{r}27 \\
0 \\
0 \\
29\end{array}$ & $\begin{array}{l}0 \\
0 \\
0 \\
0\end{array}$ \\
\hline Totals: & 18 & 5 & 0 & 23 & $78 \%$ & $22 \%$ & $0 \%$ \\
\hline
\end{tabular}

Lab: BC Babcock \& Wilcox MC \#42, Lynchburg, VA

\begin{tabular}{|c|c|c|c|c|c|c|c|}
\hline $\begin{array}{l}\text { Al } \\
\text { SD } \\
\text { VE } \\
\text { WA }\end{array}$ & $\begin{array}{r}12 \\
2 \\
2 \\
4\end{array}$ & $\begin{array}{l}1 \\
3 \\
1 \\
4\end{array}$ & $\begin{array}{l}0 \\
0 \\
0 \\
0\end{array}$ & $\begin{array}{r}13 \\
5 \\
3 \\
8\end{array}$ & $\begin{array}{l}92 \\
40 \\
67 \\
50\end{array}$ & $\begin{array}{r}8 \\
60 \\
33 \\
50\end{array}$ & $\begin{array}{l}0 \\
0 \\
0 \\
0\end{array}$ \\
\hline Totals: & 20 & 9 & 0 & 29 & $69 \%$ & $31 \%$ & $0 \%$ \\
\hline
\end{tabular}

Lab: BE RUST Geotech, Grand Junction, CO

$\begin{array}{lrrrrrrr}\text { Al } & 10 & 7 & 0 & 17 & 59 & 41 & 0 \\ \text { SO } & 6 & 3 & 0 & 9 & 67 & 33 & 0 \\ \text { VE } & 5 & 2 & 0 & 7 & 71 & 29 & 0 \\ \text { WA } & 7 & 4 & 2 & 13 & 54 & 31 & 15\end{array}$




\section{QAP44 Summary of Matrix Evaluations by Laboratory}

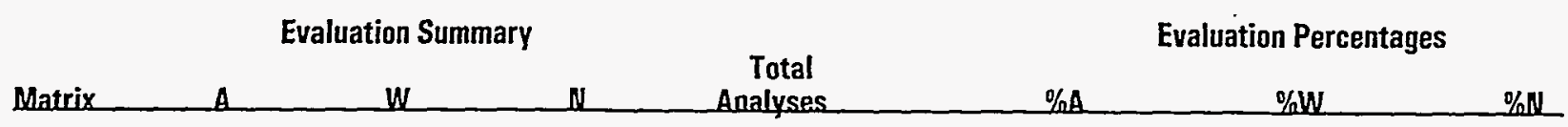

Lah: BE RUST Geatech, Grand Junction, CO

$\begin{array}{lllll}\text { Totals: } & 28 & 16 & 2 & 46\end{array}$

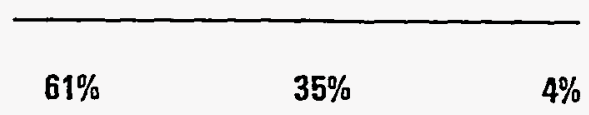

Lab: BK Brookhaven National Laboratory, Unton, NY

$\begin{array}{lllllllll}\text { WA } & 1 & 3 & 0 & 4 & 25 & 75 & 0 \\ \text { Totals: } & 1 & 3 & 0 & 4 & 25 \% & 75 \% & 0 \%\end{array}$

Lab: BL Barringer Labaratories Inc., Golden, CO

\begin{tabular}{|c|c|c|c|c|c|c|c|}
\hline $\begin{array}{l}\text { AI } \\
\text { SO } \\
\text { VE } \\
\text { WA }\end{array}$ & $\begin{array}{r}43 \\
9 \\
23 \\
38\end{array}$ & $\begin{array}{r}9 \\
10 \\
1 \\
3\end{array}$ & $\begin{array}{l}0 \\
3 \\
0 \\
6\end{array}$ & $\begin{array}{l}52 \\
22 \\
24 \\
47\end{array}$ & $\begin{array}{l}83 \\
41 \\
96 \\
81\end{array}$ & $\begin{array}{r}17 \\
45 \\
4 \\
6\end{array}$ & $\begin{array}{r}0 \\
14 \\
0 \\
13\end{array}$ \\
\hline tals: & 113 & 23 & 9 & 145 & $78 \%$ & $16 \%$ & $6 \%$ \\
\hline
\end{tabular}

Lab: BM Battelle Memorial Institute, Columbus, $\mathrm{OH}$

\begin{tabular}{|c|c|c|c|c|c|c|c|}
\hline $\begin{array}{l}\text { Al } \\
\text { SO } \\
\text { VE } \\
\text { WA }\end{array}$ & $\begin{array}{l}9 \\
0 \\
2 \\
6\end{array}$ & $\begin{array}{l}5 \\
2 \\
2 \\
2\end{array}$ & $\begin{array}{l}0 \\
2 \\
1 \\
0\end{array}$ & $\begin{array}{r}14 \\
4 \\
5 \\
8\end{array}$ & $\begin{array}{r}64 \\
0 \\
40 \\
75\end{array}$ & $\begin{array}{l}36 \\
50 \\
40 \\
25\end{array}$ & $\begin{array}{r}0 \\
50 \\
20 \\
0\end{array}$ \\
\hline Totals: & 17 & 11 & 3 & 31 & $55 \%$ & $35 \%$ & $10 \%$ \\
\hline
\end{tabular}

Lab: BN Broakhaven National Laboratory, Upton, NY

\begin{tabular}{|c|c|c|c|c|c|c|c|}
\hline $\begin{array}{l}\text { Al } \\
\text { SO } \\
\text { VE } \\
\text { WA }\end{array}$ & $\begin{array}{l}8 \\
2 \\
2 \\
7\end{array}$ & $\begin{array}{l}2 \\
0 \\
1 \\
0\end{array}$ & $\begin{array}{l}0 \\
0 \\
0 \\
0\end{array}$ & $\begin{array}{r}10 \\
2 \\
3 \\
7\end{array}$ & $\begin{array}{r}80 \\
100 \\
67 \\
100\end{array}$ & $\begin{array}{r}20 \\
0 \\
33 \\
0\end{array}$ & $\begin{array}{l}0 \\
0 \\
0 \\
0\end{array}$ \\
\hline & 19 & 3 & 0 & 22 & $86 \%$ & $14 \%$ & $0 \%$ \\
\hline
\end{tabular}


DAP44 Summary of Matrix Evaluations by Laboratory

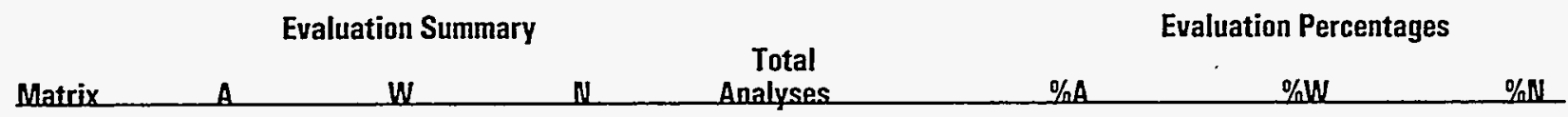

Lab: BP Battelle Pacific Northwest Laboratory

$\begin{array}{lrrrrrrr}\text { Al } & 10 & 1 & 0 & 11 & 91 & 9 & 0 \\ \text { SO } & 7 & 0 & 0 & 7 & 100 & 33 & 0 \\ \text { VE } & 1 & 1 & 1 & 3 & 44 & 44 & 11 \\ \text { WA } & 4 & 4 & 1 & 9 & 73 \% & 20 \% & 7 \%\end{array}$

Lab: BQ Becquerel Laboratories Inc. Mississauga, Ontario, Canada

\begin{tabular}{|c|c|c|c|c|c|c|c|}
\hline $\begin{array}{l}\text { Al } \\
\text { SO } \\
\text { VE } \\
\text { WA }\end{array}$ & $\begin{array}{l}7 \\
2 \\
1 \\
3\end{array}$ & $\begin{array}{l}2 \\
0 \\
1 \\
1\end{array}$ & $\begin{array}{l}0 \\
1 \\
1 \\
0\end{array}$ & $\begin{array}{l}9 \\
3 \\
3 \\
4\end{array}$ & $\begin{array}{l}78 \\
67 \\
33 \\
75\end{array}$ & $\begin{array}{r}22 \\
0 \\
33 \\
25\end{array}$ & $\begin{array}{r}0 \\
33 \\
33 \\
0\end{array}$ \\
\hline Totals: & 13 & 4 & 2 & 19 & $68 \%$ & $21 \%$ & $11 \%$ \\
\hline
\end{tabular}

Lah: BR US Army Research Laboratory, Aberdeen Proving Ground

\begin{tabular}{|c|c|c|c|c|c|c|c|}
\hline $\begin{array}{l}\text { Al } \\
\text { SO } \\
\text { VE } \\
\text { WA }\end{array}$ & $\begin{array}{l}6 \\
1 \\
0 \\
1\end{array}$ & $\begin{array}{l}2 \\
1 \\
1 \\
2\end{array}$ & $\begin{array}{l}0 \\
0 \\
1 \\
0\end{array}$ & $\begin{array}{r}8 \\
2 \\
2 \\
-\quad 3\end{array}$ & $\begin{array}{r}75 \\
50 \\
0 \\
33\end{array}$ & $\begin{array}{l}25 \\
50 \\
50 \\
67\end{array}$ & $\begin{array}{r}0 \\
0 \\
50 \\
0\end{array}$ \\
\hline Totals: & 8 & 6 & 1 & 15 & $53 \%$ & $40 \%$ & $7 \%$ \\
\hline
\end{tabular}

Lab: BS B\&W Nuclear Envir. Services, Leechburg, PA

\begin{tabular}{|c|c|c|c|c|c|c|c|}
\hline $\begin{array}{l}\text { Al } \\
\text { SO } \\
\text { VE } \\
\text { WA }\end{array}$ & $\begin{array}{l}7 \\
3 \\
0 \\
5\end{array}$ & $\begin{array}{l}4 \\
0 \\
3 \\
1\end{array}$ & $\begin{array}{l}0 \\
1 \\
1 \\
0\end{array}$ & $\begin{array}{r}11 \\
4 \\
4 \\
6\end{array}$ & $\begin{array}{r}64 \\
75 \\
0 \\
83\end{array}$ & $\begin{array}{r}36 \\
0 \\
75 \\
17\end{array}$ & $\begin{array}{r}0 \\
25 \\
25 \\
0\end{array}$ \\
\hline Totals: & 15 & 8 & 2 & 25 & $60 \%$ & $32 \%$ & $8 \%$ \\
\hline
\end{tabular}

Lab: BU Autoridad Requlatoria, Buenos Aires, Argentina

\begin{tabular}{lrrrrrrr} 
Al & 11 & 0 & 2 & 13 & 85 & 0 & 15 \\
SO & 5 & 0 & 1 & 6 & 83 & 80 & 0 \\
VE & 4 & 0 & 1 & 5 & 78 & 17 & 20 \\
WA & 7 & 1 & 1 & 9 & & 11 \\
\hline
\end{tabular}




\section{QAP44 Summary of Matrix Evaluations by Laboratory}

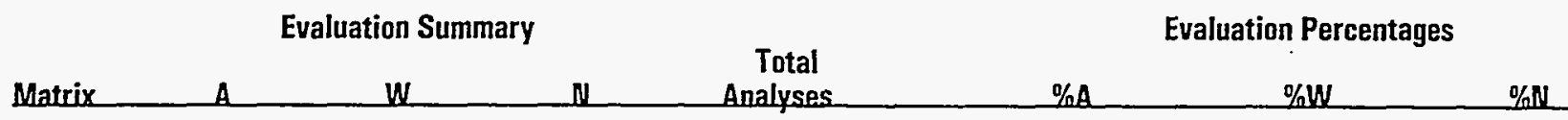

Lab: BU Autoridad Regulatoria, Buenos Aires, Argentina

$\begin{array}{llllllll} & & & & & & & \\ \text { Totals: } & 27 & 1 & 5 & 33 & 82 \% & 3 \% & 15 \%\end{array}$

Lab: BX B\&W Nuclear Envir. Services, Lynchburg. VA

\begin{tabular}{|c|c|c|c|c|c|c|c|}
\hline $\begin{array}{l}\text { AI } \\
\text { SO } \\
\text { VE } \\
\text { WA }\end{array}$ & $\begin{array}{r}14 \\
4 \\
3 \\
9\end{array}$ & $\begin{array}{l}2 \\
3 \\
3 \\
4\end{array}$ & $\begin{array}{l}0 \\
1 \\
0 \\
0\end{array}$ & $\begin{array}{r}16 \\
8 \\
6 \\
13\end{array}$ & $\begin{array}{l}88 \\
50 \\
50 \\
69\end{array}$ & $\begin{array}{l}13 \\
38 \\
50 \\
31\end{array}$ & $\begin{array}{r}0 \\
13 \\
0 \\
0\end{array}$ \\
\hline Totals: & 30 & 12 & 1 & 43 & $70 \%$ & $28 \%$ & $2 \%$ \\
\hline
\end{tabular}

Lab: CA Atomic Energy Control Board, Ottawa, Canada

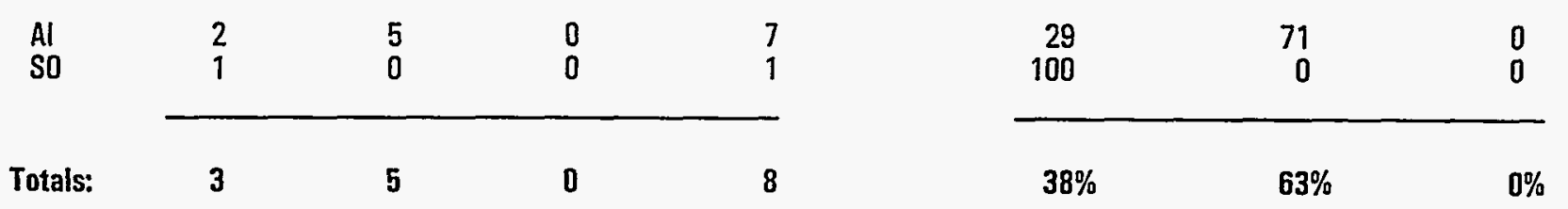

Lab: CC Compuchem Environmental Corp., RTP, NC

\begin{tabular}{lrrrrrrr} 
Al & 9 & 5 & 0 & 14 & 64 & 36 & 0 \\
SO & 7 & 2 & 0 & 9 & 78 & 22 & 0 \\
VE & 4 & 3 & 0 & 7 & 57 & 43 & 0 \\
WA & 10 & 0 & 1 & 11 & 91 & 0 & 9 \\
\cline { 2 - 8 } & & & & & & & \\
Totals: & 30 & 10 & 1 & 41 & $73 \%$ & $24 \%$ & $2 \%$
\end{tabular}

Lab: CH California State Dept. Health Serv, Sanitation \& Radiation Laboratory

\begin{tabular}{|c|c|c|c|c|c|c|c|}
\hline $\begin{array}{l}\text { Al } \\
\text { SO } \\
\text { VE } \\
\text { WA }\end{array}$ & $\begin{array}{r}15 \\
9 \\
6 \\
10\end{array}$ & $\begin{array}{l}3 \\
1 \\
1 \\
3\end{array}$ & $\begin{array}{l}0 \\
0 \\
0 \\
1\end{array}$ & $\begin{array}{r}18 \\
10 \\
7 \\
14\end{array}$ & $\begin{array}{l}83 \\
90 \\
86 \\
71\end{array}$ & $\begin{array}{l}17 \\
10 \\
14 \\
21\end{array}$ & $\begin{array}{l}0 \\
0 \\
0 \\
7\end{array}$ \\
\hline tals: & 40 & 8 & 1 & 49 & $82 \%$ & $16 \%$ & $2 \%$ \\
\hline
\end{tabular}




\section{QAP44 Summary of Matrix Evaluations by Laboratory}

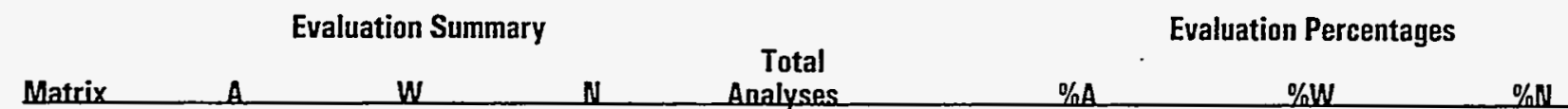

Lab: CL Core Laboratories, Casper, WY

\begin{tabular}{|c|c|c|c|c|c|c|c|}
\hline $\begin{array}{l}\text { Al } \\
\text { SO } \\
\text { VE } \\
\text { WA }\end{array}$ & $\begin{array}{r}11 \\
4 \\
6 \\
7\end{array}$ & $\begin{array}{l}3 \\
4 \\
0 \\
4\end{array}$ & $\begin{array}{l}1 \\
1 \\
1 \\
0\end{array}$ & $\begin{array}{r}15 \\
9 \\
7 \\
11\end{array}$ & $\begin{array}{l}73 \\
44 \\
86 \\
64\end{array}$ & $\begin{array}{r}20 \\
44 \\
0 \\
36\end{array}$ & $\begin{array}{r}7 \\
11 \\
14 \\
0\end{array}$ \\
\hline Totals: & 28 & 11 & 3 & 42 & $67 \%$ & $26 \%$ & $7 \%$ \\
\hline
\end{tabular}

Lab: CP Controls for Environmental Polliution, Santa Fe

\begin{tabular}{|c|c|c|c|c|c|c|c|}
\hline $\begin{array}{l}\mathrm{Al} \\
\mathrm{SO} \\
\mathrm{VE} \\
\mathrm{WA}\end{array}$ & $\begin{array}{l}9 \\
3 \\
3 \\
5\end{array}$ & $\begin{array}{l}1 \\
0 \\
1 \\
0\end{array}$ & $\begin{array}{l}1 \\
0 \\
0 \\
1\end{array}$ & $\begin{array}{r}11 \\
3 \\
4 \\
6\end{array}$ & $\begin{array}{r}82 \\
100 \\
75 \\
83\end{array}$ & $\begin{array}{r}9 \\
0 \\
25 \\
0\end{array}$ & $\begin{array}{r}9 \\
0 \\
0 \\
17\end{array}$ \\
\hline Totals: & 20 & 2 & 2 & 24 & $83 \%$ & $8 \%$ & $8 \%$ \\
\hline
\end{tabular}

Lab: CS Rockwell International Carp., Canoga Park, CA

\begin{tabular}{|c|c|c|c|c|c|c|c|}
\hline $\begin{array}{l}\text { Al } \\
\text { WA }\end{array}$ & $\begin{array}{l}1 \\
1\end{array}$ & $\begin{array}{l}2 \\
0\end{array}$ & $\begin{array}{l}7 \\
0\end{array}$ & $\begin{array}{r}10 \\
1\end{array}$ & $\begin{array}{r}10 \\
100\end{array}$ & $\begin{array}{r}20 \\
0\end{array}$ & $\begin{array}{r}70 \\
0\end{array}$ \\
\hline Totals: & 2 & 2 & 7 & 11 & $18 \%$ & $18 \%$ & $64 \%$ \\
\hline
\end{tabular}

Lab: CW Carlsbad Environmental Moritoring Research Center, NM

\begin{tabular}{llllllll} 
Al & 5 & 0 & 0 & 5 & 100 & 0 & 0 \\
SO & 5 & 0 & 0 & 5 & 100 & 0 & 0 \\
VE & 3 & 0 & 0 & 3 & 100 & 0 & 0 \\
WA & 5 & 0 & 0 & 5 & 100 & 0 & 0 \\
\cline { 2 - 7 } & & & 0 & 0 & 18 & $100 \%$ & $0 \%$
\end{tabular}

Lab: CZ ACZ Labaratories, Inc., Steamboat Springs, CO

\begin{tabular}{llllllll} 
WA & 1 & 0 & 0 & 1 & 100 & 0 & 0 \\
\cline { 2 - 5 } & 1 & 0 & 0 & 1 & $100 \%$ & $0 \%$ & $0 \%$
\end{tabular}




\section{QAP44 Summary of Matrix Evaluations by Laboratory}

Evaluation Summary

$\begin{array}{lrrrr}\text { Matrix } & \text { A } & \text { W } & \text { Total } \\ \text { Analyses }\end{array}$

Total

Evaluation Percentages

yses

\%W

$\% \mathbb{N}$

$\begin{array}{lllll}\text { Totals: } & 42 & 14 & 3 & 59\end{array}$

\begin{tabular}{llr}
59 & 35 & 6 \\
74 & 26 & 0 \\
86 & 14 & 0 \\
75 & 13 & 13 \\
\hline $71 \%$ & $24 \%$ & $5 \%$
\end{tabular}

Lab: EG LITCO/INEL, Scoville

\begin{tabular}{|c|c|c|c|c|c|c|c|}
\hline $\begin{array}{l}\text { Al } \\
\text { SO } \\
\text { VE } \\
W A\end{array}$ & $\begin{array}{l}9 \\
5 \\
4 \\
6\end{array}$ & $\begin{array}{l}1 \\
2 \\
0 \\
5\end{array}$ & $\begin{array}{l}0 \\
1 \\
0 \\
0\end{array}$ & $\begin{array}{r}10 \\
8 \\
4 \\
11\end{array}$ & $\begin{array}{r}90 \\
63 \\
100 \\
55\end{array}$ & $\begin{array}{r}10 \\
25 \\
0 \\
45\end{array}$ & $\begin{array}{r}0 \\
13 \\
0 \\
0\end{array}$ \\
\hline Totals: & 24 & 8 & 1 & 33 & $73 \%$ & $24 \%$ & $3 \%$ \\
\hline
\end{tabular}

Lab: El Eichrom Industries, Inc., Argonne

\begin{tabular}{|c|c|c|c|c|c|c|c|}
\hline $\begin{array}{l}A I \\
S O \\
V E \\
W A\end{array}$ & $\begin{array}{l}6 \\
1 \\
3 \\
2\end{array}$ & $\begin{array}{l}0 \\
2 \\
0 \\
5\end{array}$ & $\begin{array}{l}0 \\
1 \\
1 \\
0\end{array}$ & $\begin{array}{l}6 \\
4 \\
4 \\
7\end{array}$ & $\begin{array}{r}100 \\
25 \\
75 \\
29\end{array}$ & $\begin{array}{r}0 \\
50 \\
0 \\
71\end{array}$ & $\begin{array}{r}0 \\
25 \\
25 \\
0\end{array}$ \\
\hline Totals: & 12 & 7 & 2 & 21 & $57 \%$ & $33 \%$ & $10 \%$ \\
\hline
\end{tabular}

Lab: EL Energy Laborataries, Inc, Casper, WY

\begin{tabular}{|c|c|c|c|c|c|c|c|}
\hline $\begin{array}{l}\text { Al } \\
\text { SO } \\
\text { VE } \\
W A\end{array}$ & $\begin{array}{l}8 \\
2 \\
2 \\
4\end{array}$ & $\begin{array}{l}5 \\
3 \\
1 \\
4\end{array}$ & $\begin{array}{l}0 \\
1 \\
1 \\
1\end{array}$ & $\begin{array}{r}13 \\
6 \\
4 \\
9\end{array}$ & $\begin{array}{l}62 \\
33 \\
50 \\
44\end{array}$ & $\begin{array}{l}38 \\
50 \\
25 \\
44\end{array}$ & $\begin{array}{r}0 \\
17 \\
25 \\
11\end{array}$ \\
\hline Totals: & 16 & 13 & 3 & 32 & $50 \%$ & $41 \%$ & $9 \%$ \\
\hline
\end{tabular}

Lab: EP US EPA, Las Vegas

\begin{tabular}{lrrrrrrr} 
Al & 10 & 0 & 0 & 10 & 100 & 0 & 0 \\
SO & 2 & 0 & 1 & 3 & 67 & 0 & 33 \\
VE & 1 & 0 & 1 & 2 & 50 & 0 & 50 \\
WA & 6 & 3 & 0 & 9 & 67 & 33 & 0 \\
\hline
\end{tabular}




\section{QAP44 Summary of Matrix Evaluations by Laboratory}

\begin{tabular}{|c|c|c|c|c|c|c|}
\hline \multicolumn{3}{|c|}{ Evaluation Summary } & & \multicolumn{3}{|c|}{ Evaluation Percentages } \\
\hline Matrix & $A$ & $\mathbf{w}$ & $\begin{array}{c}\text { lotal } \\
\text { Analuses }\end{array}$ & $\% A$ & $\% W$ & $\% N$ \\
\hline
\end{tabular}

Lab: EP US EPA, Las Vegas

$\begin{array}{llllllll} & & & & & & & \\ \text { Totals: } & 19 & 3 & 2 & 24 & 79 \% & 13 \% & 8 \%\end{array}$

Lab: ES Environmental Sci. \& Engr., Inc., Gainesville, FL

\begin{tabular}{|c|c|c|c|c|c|c|c|}
\hline $\begin{array}{l}\text { AI } \\
\text { SO } \\
\text { VE } \\
\text { WA }\end{array}$ & $\begin{array}{r}14 \\
11 \\
6 \\
11\end{array}$ & $\begin{array}{l}2 \\
1 \\
1 \\
4\end{array}$ & $\begin{array}{l}2 \\
1 \\
0 \\
1\end{array}$ & $\begin{array}{r}18 \\
13 \\
7 \\
16\end{array}$ & $\begin{array}{l}78 \\
85 \\
86 \\
69\end{array}$ & $\begin{array}{r}11 \\
8 \\
14 \\
25\end{array}$ & $\begin{array}{r}11 \\
8 \\
0 \\
6\end{array}$ \\
\hline Totals: & 42 & 8 & 4 & 54 & $78 \%$ & $15 \%$ & $7 \%$ \\
\hline
\end{tabular}

Lab: FG FGL Environmental, Santa Paula, CA

\begin{tabular}{|c|c|c|c|c|c|c|c|}
\hline $\begin{array}{l}\text { Al } \\
\text { SO } \\
\text { WA }\end{array}$ & $\begin{array}{l}9 \\
2 \\
8\end{array}$ & $\begin{array}{l}2 \\
2 \\
1\end{array}$ & $\begin{array}{l}0 \\
0 \\
2\end{array}$ & $\begin{array}{r}11 \\
4 \\
11\end{array}$ & $\begin{array}{l}82 \\
50 \\
73\end{array}$ & $\begin{array}{r}18 \\
50 \\
9\end{array}$ & $\begin{array}{r}0 \\
0 \\
18\end{array}$ \\
\hline Totals: & 19 & 5 & 2 & 26 & $73 \%$ & $19 \%$ & $8 \%$ \\
\hline
\end{tabular}

Lab: FL Florida Dept of Health \& Rehab. Serv, Orlando

\begin{tabular}{|c|c|c|c|c|c|c|c|}
\hline $\begin{array}{l}\text { Al } \\
\text { SO } \\
\text { VE } \\
\text { WA }\end{array}$ & $\begin{array}{r}13 \\
6 \\
4 \\
5\end{array}$ & $\begin{array}{l}0 \\
0 \\
0 \\
4\end{array}$ & $\begin{array}{l}0 \\
0 \\
0 \\
0\end{array}$ & $\begin{array}{r}13 \\
6 \\
4 \\
9\end{array}$ & $\begin{array}{r}100 \\
100 \\
100 \\
56\end{array}$ & $\begin{array}{r}0 \\
0 \\
0 \\
44\end{array}$ & $\begin{array}{l}0 \\
0 \\
0 \\
0\end{array}$ \\
\hline Totals: & 28 & 4 & 0 & 32 & $88 \%$ & $13 \%$ & $0 \%$ \\
\hline
\end{tabular}

Lab: FM Florida Mobile Emergency Radiological Laboratory, Orlando

\begin{tabular}{|c|c|c|c|c|c|c|c|}
\hline $\begin{array}{l}\text { AI } \\
\text { WA }\end{array}$ & $\begin{array}{l}9 \\
3\end{array}$ & $\begin{array}{l}0 \\
1\end{array}$ & $\begin{array}{l}0 \\
0\end{array}$ & $\begin{array}{l}9 \\
4\end{array}$ & $\begin{array}{r}100 \\
75\end{array}$ & $\begin{array}{r}0 \\
25\end{array}$ & $\begin{array}{l}0 \\
0\end{array}$ \\
\hline Totals: & 12 & 1 & 0 & 13 & $92 \%$ & $8 \%$ & 0\% \\
\hline
\end{tabular}




\section{QAP44 Summary of Matrix Evaluations by Laboratory}

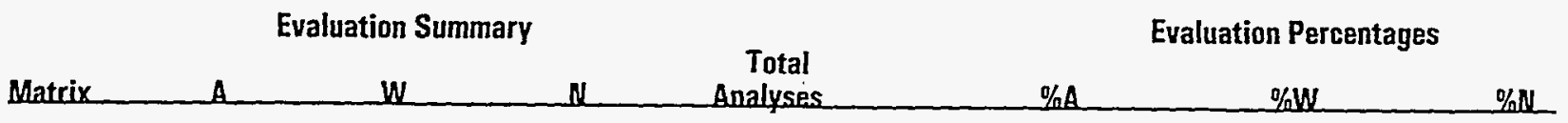

Lab: FN Fermi Lab, Batavia, IL

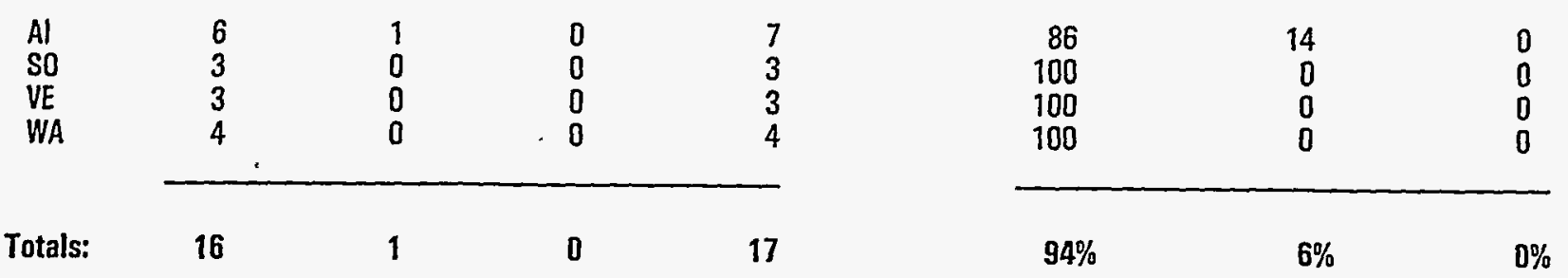

Lab: FS Florida State University, Tallahassee

\begin{tabular}{lllllllll} 
So & 5 & 2 & 0 & 7 & & 71 & 29 & 0 \\
\cline { 2 - 3 } Totals: & 5 & 2 & 0 & 7 & & $71 \%$ & $29 \%$ & $0 \%$
\end{tabular}

Lab: GA Lockheed Martin, Pikton, OH

\begin{tabular}{|c|c|c|c|c|c|c|c|}
\hline $\begin{array}{l}\text { AI } \\
\text { SO } \\
\text { VE } \\
\text { WA }\end{array}$ & $\begin{array}{r}12 \\
7 \\
5 \\
10\end{array}$ & $\begin{array}{l}3 \\
1 \\
1 \\
0\end{array}$ & $\begin{array}{l}0 \\
0 \\
0 \\
0\end{array}$ & $\begin{array}{r}15 \\
8 \\
6 \\
10\end{array}$ & $\begin{array}{r}80 \\
88 \\
83 \\
100\end{array}$ & $\begin{array}{r}20 \\
13 \\
17 \\
0\end{array}$ & $\begin{array}{l}0 \\
0 \\
0 \\
0\end{array}$ \\
\hline tals: & 34 & 5 & 0 & 39 & $87 \%$ & $13 \%$ & $0 \%$ \\
\hline
\end{tabular}

Lab: GE Environmental Physics, Inc., Charleston, SC

\begin{tabular}{lrrrrrrr} 
AI & 16 & 1 & 0 & 17 & 94 & 6 & 0 \\
SO & 8 & 1 & 0 & 9 & 89 & 11 & 0 \\
VE & 7 & 0 & 0 & 7 & 100 & 0 & 0 \\
WA & 5 & 8 & 0 & 13 & 38 & 62 & 0 \\
\cline { 2 - 7 } & Totals: & 36 & 10 & 0 & 46 & $78 \%$ & $22 \%$
\end{tabular}

Lah: GS USGSINWOL, Arvada, CO

\begin{tabular}{llllllll}
\hline WA & 0 & 1 & 0 & 1 & 0 & 100 & 0 \\
\cline { 2 - 6 } & 0 & 1 & 0 & 1 & $0 \%$ & $100 \%$ & $0 \%$
\end{tabular}


QAP44 Summary of Matrix Evaluations by Laboratory

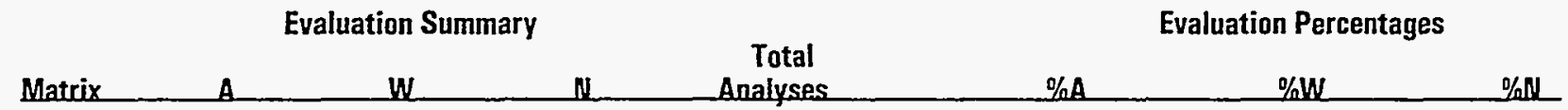

Lab: HA NUS Laboratory, Pittsburgh

\begin{tabular}{|c|c|c|c|c|c|c|c|}
\hline $\begin{array}{l}\text { SO } \\
\text { WA }\end{array}$ & $\begin{array}{l}4 \\
9\end{array}$ & $\begin{array}{l}3 \\
4\end{array}$ & $\begin{array}{l}0 \\
0\end{array}$ & $\begin{array}{r}7 \\
13\end{array}$ & $\begin{array}{l}57 \\
69\end{array}$ & $\begin{array}{l}43 \\
31\end{array}$ & $\begin{array}{l}0 \\
0\end{array}$ \\
\hline Totals: & 13 & 7 & 0 & 20 & $65 \%$ & $35 \%$ & $0 \%$ \\
\hline
\end{tabular}

Lab: HC Lawrence Livermore Laboratory, California

\begin{tabular}{|c|c|c|c|c|c|c|c|}
\hline $\begin{array}{l}\text { Al } \\
\text { WA }\end{array}$ & $\begin{array}{l}2 \\
2\end{array}$ & $\begin{array}{l}0 \\
1\end{array}$ & $\begin{array}{l}0 \\
0\end{array}$ & $\begin{array}{r}-2 \\
3\end{array}$ & $\begin{array}{r}100 \\
67\end{array}$ & $\begin{array}{r}0 \\
33\end{array}$ & $\begin{array}{l}0 \\
0\end{array}$ \\
\hline Totals: & 4 & 1 & 0 & 5 & $80 \%$ & $20 \%$ & $0 \%$ \\
\hline
\end{tabular}

Lab: HL Heritage Laboratories, Inc., Romeoville, IL

\begin{tabular}{llllllll} 
S0 & 4 & 3 & 0 & 7 & 57 & 43 & 0 \\
WA & 6 & 2 & 0 & 8 & 75 & 25 & 0 \\
\cline { 2 - 5 } & 10 & 5 & 0 & 15 & $67 \%$ & $33 \%$
\end{tabular}

Lab: ID DPRA - IRD/CNEN, Rio de Janeiro, Brazil

\begin{tabular}{|c|c|c|c|c|c|c|c|}
\hline $\begin{array}{l}\text { Al } \\
\text { SO } \\
\text { VE } \\
\text { WA }\end{array}$ & $\begin{array}{l}8 \\
7 \\
5 \\
5\end{array}$ & $\begin{array}{l}2 \\
0 \\
1 \\
2\end{array}$ & $\begin{array}{l}0 \\
0 \\
0 \\
1\end{array}$ & $\begin{array}{r}10 \\
7 \\
6 \\
8\end{array}$ & $\begin{array}{r}80 \\
100 \\
83 \\
63\end{array}$ & $\begin{array}{r}20 \\
0 \\
17 \\
25\end{array}$ & $\begin{array}{r}0 \\
0 \\
0 \\
13\end{array}$ \\
\hline & 25 & 5 & 1 & 31 & $81 \%$ & $16 \%$ & $3 \%$ \\
\hline
\end{tabular}

Lab: IE IEA, Inc., Morrisville, NC

\begin{tabular}{|c|c|c|c|c|c|c|c|}
\hline $\begin{array}{l}\text { Al } \\
\text { SO } \\
\text { VE } \\
\text { WA }\end{array}$ & $\begin{array}{r}12 \\
9 \\
7 \\
10\end{array}$ & $\begin{array}{l}3 \\
0 \\
0 \\
1\end{array}$ & $\begin{array}{l}0 \\
0 \\
0 \\
2\end{array}$ & $\begin{array}{r}15 \\
9 \\
7 \\
13\end{array}$ & $\begin{array}{r}80 \\
100 \\
100 \\
77\end{array}$ & $\begin{array}{r}20 \\
0 \\
0 \\
8\end{array}$ & $\begin{array}{r}0 \\
0 \\
0 \\
15\end{array}$ \\
\hline als: & 38 & 4 & 2 & 44 & $86 \%$ & $9 \%$ & $5 \%$ \\
\hline
\end{tabular}


QAP44 Summary of Matrix Evaluations by Laboratory

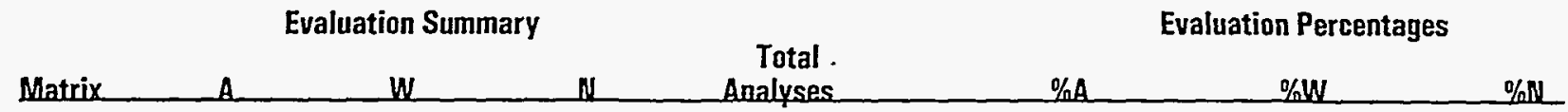

Lah: IL ISU Environmental Monitoring Program, Pocatello, ID

\begin{tabular}{|c|c|c|c|c|c|c|c|}
\hline $\begin{array}{l}\text { Al } \\
\text { SO } \\
\text { VE } \\
\text { VE } \\
\text { WA }\end{array}$ & $\begin{array}{l}1 \\
0 \\
0 \\
0 \\
1\end{array}$ & $\begin{array}{l}1 \\
0 \\
0 \\
0 \\
2\end{array}$ & $\begin{array}{l}0 \\
2 \\
2 \\
1 \\
2\end{array}$ & $\begin{array}{l}2 \\
2 \\
2 \\
1 \\
5\end{array}$ & $\begin{array}{r}50 \\
0 \\
0 \\
0 \\
20\end{array}$ & $\begin{array}{r}50 \\
0 \\
0 \\
0 \\
40\end{array}$ & $\begin{array}{r}0 \\
100 \\
100 \\
100 \\
40\end{array}$ \\
\hline Totals: & 2 & 3 & 7 & 12 & $17 \%$ & $25 \%$ & $\mathbf{5 8} \%$ \\
\hline
\end{tabular}

Lab: IN WINCO, Idaho Falls

\begin{tabular}{|c|c|c|c|c|c|c|c|}
\hline $\begin{array}{l}\text { Al } \\
\text { SO } \\
\text { VE } \\
W A\end{array}$ & $\begin{array}{l}4 \\
4 \\
2 \\
5\end{array}$ & $\begin{array}{l}3 \\
2 \\
1 \\
2\end{array}$ & $\begin{array}{l}1 \\
0 \\
0 \\
0\end{array}$ & $\begin{array}{l}8 \\
6 \\
3 \\
7\end{array}$ & $\begin{array}{l}50 \\
67 \\
67 \\
71\end{array}$ & $\begin{array}{l}38 \\
33 \\
33 \\
29\end{array}$ & $\begin{array}{r}13 \\
0 \\
0 \\
0\end{array}$ \\
\hline Totals: & 15 & 8 & 1 & 24 & $63 \%$ & $33 \%$ & $4 \%$ \\
\hline
\end{tabular}

Lab: IR Idaho National Engineering Laboratory

\begin{tabular}{|c|c|c|c|c|c|c|c|}
\hline $\begin{array}{l}\text { Al } \\
\text { WA }\end{array}$ & $\begin{array}{l}1 \\
0\end{array}$ & $\begin{array}{l}0 \\
0\end{array}$ & $\begin{array}{l}0 \\
1\end{array}$ & $\begin{array}{l}1 \\
1\end{array}$ & $\begin{array}{r}100 \\
0\end{array}$ & $\begin{array}{l}0 \\
0\end{array}$ & $\begin{array}{r}0 \\
100\end{array}$ \\
\hline Totals: & 1 & 0 & 1 & 2 & $50 \%$ & $0 \%$ & $50 \%$ \\
\hline
\end{tabular}

Lab: IS Quanterra-St. Louis

\begin{tabular}{|c|c|c|c|c|c|c|c|}
\hline $\begin{array}{l}A I \\
\text { SD } \\
\text { VE } \\
\text { WA }\end{array}$ & $\begin{array}{l}5 \\
7 \\
6 \\
8\end{array}$ & $\begin{array}{l}7 \\
1 \\
0 \\
3\end{array}$ & $\begin{array}{l}2 \\
1 \\
0 \\
1\end{array}$ & $\begin{array}{r}14 \\
9 \\
6 \\
12\end{array}$ & $\begin{array}{r}36 \\
78 \\
100 \\
67\end{array}$ & $\begin{array}{r}50 \\
11 \\
0 \\
25\end{array}$ & $\begin{array}{r}14 \\
11 \\
0 \\
8\end{array}$ \\
\hline Totals: & 26 & 11 & 4 & 41 & $63 \%$ & $27 \%$ & $10 \%$ \\
\hline
\end{tabular}

Lab: IT Quanterra-Richland Laboratory

$\begin{array}{llllrlll}\text { AI } & 8 & 6 & 1 & 15 & 53 & 40 & 7 \\ \text { SO } & 6 & 1 & 0 & 7 & 86 & 14 & 0 \\ \text { VE } & 4 & 3 & 0 & 7 & 57 & 43 & 0 \\ \text { WA } & 5 & 5 & 1 & 11 & 45 & 45 & 9\end{array}$




\section{QAP44 Summary of Matrix Evaluations by Laboratory}

\begin{tabular}{|c|c|c|c|c|c|c|c|}
\hline \multirow[b]{2}{*}{ Matrix } & \multicolumn{3}{|c|}{ Evaluation Summary } & \multirow{2}{*}{$\begin{array}{c}\text { Total } \\
\text { Analyses }\end{array}$} & \multicolumn{3}{|c|}{ Evaluation Percentages } \\
\hline & A & $\mathbf{w}$ & $\mathbb{N}$ & & $\%$ & $\% w$ & \% \\
\hline Lab: IT & \multicolumn{7}{|c|}{ Quanterra-Richland Laboratory } \\
\hline Totals: & 23 & 15 & 2 & 40 & $57 \%$ & $38 \%$ & $5 \%$ \\
\hline Lab: JP & \multicolumn{7}{|c|}{ Japan Chemical Analysis Center } \\
\hline $\begin{array}{l}\text { Al } \\
\text { SO } \\
\text { VE } \\
\text { WA }\end{array}$ & $\begin{array}{r}13 \\
8 \\
7 \\
12\end{array}$ & $\begin{array}{l}2 \\
0 \\
0 \\
0\end{array}$ & $\begin{array}{l}0 \\
0 \\
0 \\
0\end{array}$ & $\begin{array}{r}15 \\
8 \\
7 \\
12\end{array}$ & $\begin{array}{r}87 \\
100 \\
100 \\
100\end{array}$ & $\begin{array}{r}13 \\
0 \\
0 \\
0\end{array}$ & $\begin{array}{l}0 \\
0 \\
0 \\
0\end{array}$ \\
\hline Totals: & 40 & 2 & 0 & 42 & $95 \%$ & $5 \%$ & $\mathbf{0} \%$ \\
\hline
\end{tabular}

Lab: KA Knolls Atomic Power Lab_ Schenectady

\begin{tabular}{|c|c|c|c|c|c|c|c|}
\hline $\begin{array}{l}\text { Al } \\
\text { SO } \\
\text { WA }\end{array}$ & $\begin{array}{r}1 \\
5 \\
10\end{array}$ & $\begin{array}{l}1 \\
0 \\
1\end{array}$ & $\begin{array}{l}0 \\
0 \\
0\end{array}$ & $\begin{array}{r}2 \\
5 \\
11\end{array}$ & $\begin{array}{r}50 \\
100 \\
91\end{array}$ & $\begin{array}{r}50 \\
0 \\
9\end{array}$ & $\begin{array}{l}0 \\
0 \\
0\end{array}$ \\
\hline Totals: & 16 & 2 & 0 & 18 & $89 \%$ & $11 \%$ & D\% \\
\hline
\end{tabular}

Lab: LA Los Alamos National Laboratory, NM

\begin{tabular}{|c|c|c|c|c|c|c|c|}
\hline $\begin{array}{l}\text { Al } \\
\text { SO } \\
\text { VE } \\
\text { WA }\end{array}$ & $\begin{array}{l}31 \\
21 \\
17 \\
19\end{array}$ & $\begin{array}{r}14 \\
0 \\
1 \\
5\end{array}$ & $\begin{array}{l}0 \\
0 \\
0 \\
9\end{array}$ & $\begin{array}{l}45 \\
21 \\
18 \\
33\end{array}$ & $\begin{array}{r}69 \\
100 \\
94 \\
58\end{array}$ & $\begin{array}{r}31 \\
0 \\
6 \\
15\end{array}$ & $\begin{array}{r}0 \\
0 \\
0 \\
27\end{array}$ \\
\hline Totals: & 88 & 20 & 9 & 117 & $75 \%$ & $17 \%$ & $8 \%$ \\
\hline
\end{tabular}

Lab: LB Lawrence Berkelev Lab UCB

\begin{tabular}{|c|c|c|c|c|c|c|c|}
\hline $\begin{array}{l}\text { Al } \\
\text { SO } \\
\text { VE } \\
\text { WA }\end{array}$ & $\begin{array}{l}7 \\
2 \\
3 \\
1\end{array}$ & $\begin{array}{l}3 \\
1 \\
0 \\
2\end{array}$ & $\begin{array}{l}0 \\
0 \\
1 \\
1\end{array}$ & $\begin{array}{r}10 \\
3 \\
4 \\
4\end{array}$ & $\begin{array}{l}70 \\
67 \\
75 \\
25\end{array}$ & $\begin{array}{r}30 \\
33 \\
0 \\
50\end{array}$ & $\begin{array}{r}0 \\
0 \\
25 \\
25\end{array}$ \\
\hline Totals: & 13 & 6 & 2 & 21 & $62 \%$ & $29 \%$ & $10 \%$ \\
\hline
\end{tabular}




\section{QAP44 Summary of Matrix Evaluations by Laboratory}

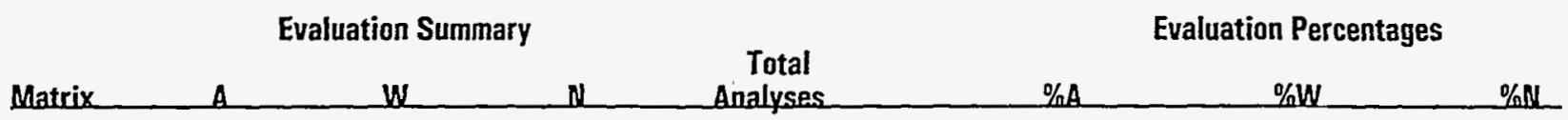

Lab: LH Lockheed Analytical Laboratory, Las Vegas

\begin{tabular}{|c|c|c|c|c|c|c|c|}
\hline $\begin{array}{l}\text { Al } \\
\text { SO } \\
\text { VE } \\
\text { WA }\end{array}$ & $\begin{array}{r}7 \\
8 \\
6 \\
12\end{array}$ & $\begin{array}{l}8 \\
0 \\
1 \\
0\end{array}$ & $\begin{array}{l}1 \\
0 \\
0 \\
1\end{array}$ & $\begin{array}{r}16 \\
8 \\
7 \\
13\end{array}$ & $\begin{array}{r}44 \\
100 \\
86 \\
92\end{array}$ & $\begin{array}{r}50 \\
0 \\
14 \\
0\end{array}$ & $\begin{array}{l}6 \\
0 \\
0 \\
8\end{array}$ \\
\hline Totals: & 33 & 9 & 2 & 44 & $75 \%$ & $20 \%$ & $5 \%$ \\
\hline
\end{tabular}

Lab: LL Lawrence Livermore National Lab, CA

\begin{tabular}{|c|c|c|c|c|c|c|c|}
\hline $\begin{array}{l}\text { Al } \\
\text { SO } \\
\text { VE } \\
\text { WA }\end{array}$ & $\begin{array}{r}11 \\
4 \\
4 \\
5\end{array}$ & $\begin{array}{l}1 \\
0 \\
0 \\
2\end{array}$ & $\begin{array}{l}2 \\
0 \\
0 \\
3\end{array}$ & $\begin{array}{r}14 \\
4 \\
4 \\
10\end{array}$ & $\begin{array}{r}79 \\
100 \\
100 \\
50\end{array}$ & $\begin{array}{r}7 \\
0 \\
0 \\
20\end{array}$ & $\begin{array}{r}14 \\
0 \\
0 \\
30\end{array}$ \\
\hline Totals: & 24 & 3 & 5 & 32 & $75 \%$ & $\mathbf{9} \%$ & $16 \%$ \\
\hline
\end{tabular}

Lab: LM Los Alamos National Lab, Mercury, NV

\begin{tabular}{|c|c|c|c|c|c|c|c|}
\hline $\begin{array}{l}\text { Al } \\
\text { SO } \\
\text { WA }\end{array}$ & $\begin{array}{l}2 \\
0 \\
1\end{array}$ & $\begin{array}{l}3 \\
0 \\
0\end{array}$ & $\begin{array}{l}5 \\
2 \\
3\end{array}$ & $\begin{array}{r}10 \\
2 \\
4\end{array}$ & $\begin{array}{r}20 \\
0 \\
25\end{array}$ & $\begin{array}{r}30 \\
0 \\
0\end{array}$ & $\begin{array}{r}50 \\
100 \\
75\end{array}$ \\
\hline Totals: & 3 & 3 & 10 & 16 & $19 \%$ & $19 \%$ & $63 \%$ \\
\hline
\end{tabular}

Lab: LV UNLV, Dept of Health Physics

\begin{tabular}{|c|c|c|c|c|c|c|c|}
\hline $\begin{array}{l}\text { AI } \\
\text { SO } \\
\text { VE. } \\
\text { WA }\end{array}$ & $\begin{array}{l}7 \\
2 \\
2 \\
2\end{array}$ & $\begin{array}{l}2 \\
1 \\
2 \\
2\end{array}$ & $\begin{array}{l}0 \\
0 \\
0 \\
0\end{array}$ & $\begin{array}{l}9 \\
3 \\
4 \\
4\end{array}$ & $\begin{array}{l}78 \\
67 \\
50 \\
50\end{array}$ & $\begin{array}{l}22 \\
33 \\
50 \\
50\end{array}$ & $\begin{array}{l}0 \\
0 \\
0 \\
0\end{array}$ \\
\hline Totals: & 13 & 7 & D & 20 & $65 \%$ & $35 \%$ & $0 \%$ \\
\hline
\end{tabular}

Lab: LW Lawrence Livermore National Lab, CA

$\begin{array}{lllllrrr}\text { SO } & 3 & 1 & 0 & 4 & 75 & 25 & 0 \\ \text { VE } & 4 & 0 & 0 & 4 & 100 & 0 & 0 \\ \text { WA } & 5 & 1 & 0 & 6 & 83 & 17 & 0\end{array}$




\section{QAP44 Summary of Matrix Evaluations by Laboratory}

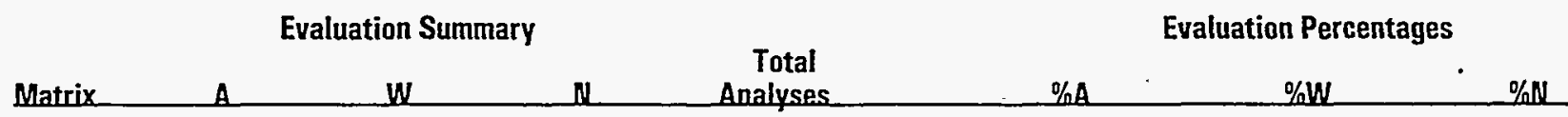

Lab: LW Lawrence Livermore National Lab, CA

$\begin{array}{lllll}\text { Totals: } & 12 & 2 & 0 & 14\end{array}$

$86 \% \quad 14 \%$

$8 \%$

Lab: MA ORNL Health Sciences Research Div.

\begin{tabular}{|c|c|c|c|c|c|c|c|}
\hline $\begin{array}{l}\text { SO } \\
\text { VE }\end{array}$ & $\begin{array}{l}2 \\
3\end{array}$ & $\begin{array}{l}1 \\
1\end{array}$ & $\begin{array}{l}0 \\
0\end{array}$ & $\begin{array}{l}3 \\
4\end{array}$ & $\begin{array}{l}67 \\
75\end{array}$ & $\begin{array}{l}33 \\
25\end{array}$ & $\begin{array}{l}0 \\
0\end{array}$ \\
\hline Totals: & 5 & 2 & 0 & 7 & $71 \%$ & $29 \%$ & $0 \%$ \\
\hline
\end{tabular}

Lab: ME Radiation Control Program, Jamaica Plain, MA

\begin{tabular}{|c|c|c|c|c|c|c|c|}
\hline $\begin{array}{l}\text { Al } \\
\text { SO } \\
\text { VE } \\
\text { WA }\end{array}$ & $\begin{array}{l}7 \\
1 \\
2 \\
1\end{array}$ & $\begin{array}{l}0 \\
1 \\
1 \\
2\end{array}$ & $\begin{array}{l}1 \\
0 \\
0 \\
0\end{array}$ & $\begin{array}{l}8 \\
2 \\
3 \\
3\end{array}$ & $\begin{array}{l}88 \\
50 \\
67 \\
33\end{array}$ & $\begin{array}{r}0 \\
50 \\
33 \\
67\end{array}$ & $\begin{array}{r}13 \\
0 \\
0 \\
0\end{array}$ \\
\hline Totals: & 11 & 4 & 1 & 16 & $69 \%$ & $25 \%$ & $6 \%$ \\
\hline
\end{tabular}

Lab: MI Massachusetts Institute of Technology

\begin{tabular}{|c|c|c|c|c|c|c|c|}
\hline $\begin{array}{l}\mathrm{Al} \\
\text { WA }\end{array}$ & $\begin{array}{l}2 \\
4\end{array}$ & $\begin{array}{l}3 \\
6\end{array}$ & $\begin{array}{l}0 \\
2\end{array}$ & $\begin{array}{r}5 \\
12\end{array}$ & $\begin{array}{l}40 \\
33\end{array}$ & $\begin{array}{l}60 \\
50\end{array}$ & $\begin{array}{r}0 \\
17\end{array}$ \\
\hline Totals: & 6 & 9 & 2 & 17 & $35 \%$ & $53 \%$ & $12 \%$ \\
\hline
\end{tabular}

Lab: ML EG\&G Mound Applied Technologies; Miamisburg, $\mathrm{OH}$

\begin{tabular}{|c|c|c|c|c|c|c|c|}
\hline $\begin{array}{l}\text { Al } \\
\text { SO } \\
\text { VE } \\
\text { WA }\end{array}$ & $\begin{array}{r}11 \\
6 \\
4 \\
7\end{array}$ & $\begin{array}{l}1 \\
0 \\
0 \\
1\end{array}$ & $\begin{array}{l}0 \\
0 \\
0 \\
0\end{array}$ & $\begin{array}{r}12 \\
6 \\
4 \\
8\end{array}$ & $\begin{array}{r}92 \\
100 \\
100 \\
88\end{array}$ & $\begin{array}{r}8 \\
0 \\
0 \\
13\end{array}$ & $\begin{array}{l}0 \\
0 \\
0 \\
0\end{array}$ \\
\hline Totals: & 28 & 2 & 0 & 30 & $93 \%$ & $7 \%$ & $0 \%$ \\
\hline
\end{tabular}

Lab: MS Manufacturing Sciences Corporation, Qak Ridge

\begin{tabular}{llllllll} 
Al & 6 & 1 & 0 & 7 & 86 & 14 & 0 \\
\hline
\end{tabular}




\section{QAP44 Summary of Matrix Evaluations by Laboratory}

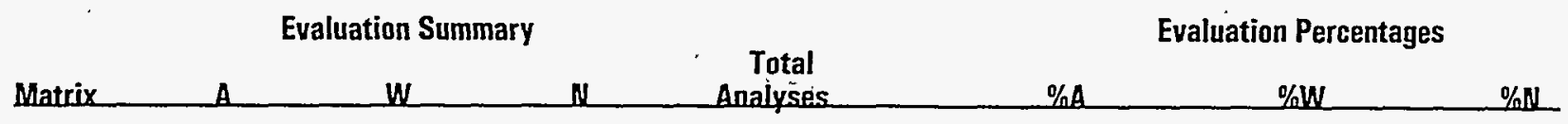

Lab: MS Manufacturing Sciences Corporation, Oak Ridge

\begin{tabular}{|c|c|c|c|c|c|c|c|}
\hline $\begin{array}{l}\text { SO } \\
\text { WA }\end{array}$ & $\begin{array}{l}2 \\
3\end{array}$ & $\begin{array}{l}0 \\
1\end{array}$ & $\begin{array}{l}0 \\
0\end{array}$ & $\begin{array}{l}2 \\
4\end{array}$ & $\begin{array}{r}100 \\
75\end{array}$ & $\begin{array}{r}0 \\
25\end{array}$ & $\begin{array}{l}0 \\
0\end{array}$ \\
\hline Totals: & 11 & 2 & 0 & 13 & $85 \%$ & $15 \%$ & $0 \%$ \\
\hline
\end{tabular}

Lab: NA US EPA NAREL, Montgomery, AL

\begin{tabular}{|c|c|c|c|c|c|c|c|}
\hline $\begin{array}{l}\text { AI } \\
\text { SO } \\
\text { VE } \\
\text { WA }\end{array}$ & $\begin{array}{r}12 \\
7 \\
7 \\
5\end{array}$ & $\begin{array}{l}0 \\
0 \\
0 \\
2\end{array}$ & $\begin{array}{l}0 \\
0 \\
0 \\
1\end{array}$ & $\begin{array}{r}12 \\
7 \\
7 \\
8\end{array}$ & $\begin{array}{r}100 \\
100 \\
100 \\
63\end{array}$ & $\begin{array}{r}0 \\
0 \\
0 \\
25\end{array}$ & $\begin{array}{r}0 \\
0 \\
0 \\
13\end{array}$ \\
\hline Totals: & 31 & 2 & 1 & 34 & $91 \%$ & $6 \%$ & $3 \%$ \\
\hline
\end{tabular}

Lab: NC Nuclear Services North Carolina State University, Raleigh

\begin{tabular}{|c|c|c|c|c|c|c|c|}
\hline $\begin{array}{l}\text { Al } \\
\text { SO } \\
\text { VE } \\
\text { WA }\end{array}$ & $\begin{array}{l}7 \\
1 \\
2 \\
3\end{array}$ & $\begin{array}{l}0 \\
0 \\
0 \\
0\end{array}$ & $\begin{array}{l}0 \\
0 \\
0 \\
0\end{array}$ & $\begin{array}{l}7 \\
1 \\
2 \\
3\end{array}$ & $\begin{array}{l}100 \\
100 \\
100 \\
100\end{array}$ & $\begin{array}{l}0 \\
0 \\
0 \\
0\end{array}$ & $\begin{array}{l}0 \\
0 \\
0 \\
0\end{array}$ \\
\hline Tatals: & 13 & 0 & 0 & 13 & $100 \%$ & $0 \%$ & $0 \%$ \\
\hline
\end{tabular}

Lab: NL FERMCD, Cincinnati, OH

\begin{tabular}{|c|c|c|c|c|c|c|c|}
\hline $\begin{array}{l}\text { Al } \\
\text { SO } \\
\text { WA }\end{array}$ & $\begin{array}{r}12 \\
5 \\
6\end{array}$ & $\begin{array}{l}0 \\
1 \\
2\end{array}$ & $\begin{array}{l}1 \\
0 \\
0\end{array}$ & $\begin{array}{r}13 \\
6 \\
8\end{array}$ & $\begin{array}{l}92 \\
83 \\
75\end{array}$ & $\begin{array}{r}0 \\
17 \\
25\end{array}$ & $\begin{array}{l}8 \\
0 \\
0\end{array}$ \\
\hline Totals: & 23 & 3 & 1 & 27 & $85 \%$ & $11 \%$ & $4 \%$ \\
\hline
\end{tabular}

Lab: NM Environmental Evaluation Group, Carlshad, NM

\begin{tabular}{|c|c|c|c|c|c|c|c|}
\hline $\begin{array}{l}\text { Al } \\
\text { SO } \\
\text { WA }\end{array}$ & $\begin{array}{l}7 \\
4 \\
8\end{array}$ & $\begin{array}{l}0 \\
0 \\
1\end{array}$ & $\begin{array}{l}2 \\
1 \\
0\end{array}$ & $\begin{array}{l}9 \\
5 \\
9\end{array}$ & $\begin{array}{l}78 \\
80 \\
89\end{array}$ & $\begin{array}{r}0 \\
0 \\
11\end{array}$ & $\begin{array}{r}22 \\
20 \\
0\end{array}$ \\
\hline Totals: & 19 & 1 & 3 & 23 & $83 \%$ & $4 \%$ & $13 \%$ \\
\hline
\end{tabular}




\section{QAP44 Summary of Matrix Evaluations by Laboratory}

Evaluation Summary

Matrix

W

Total Analyses
Evaluation Percentages

\%W $\% \mathrm{~N}$

Lab: NR Naval Reactors Facility Chemistry, Scoville, ID

\begin{tabular}{|c|c|c|c|c|c|c|c|}
\hline $\begin{array}{l}\text { SO } \\
\text { VE }\end{array}$ & $\begin{array}{l}1 \\
1\end{array}$ & $\begin{array}{l}1 \\
2\end{array}$ & $\begin{array}{l}0 \\
0\end{array}$ & $\begin{array}{l}2 \\
3\end{array}$ & $\begin{array}{l}50 \\
33\end{array}$ & $\begin{array}{l}50 \\
67\end{array}$ & $\begin{array}{l}0 \\
0\end{array}$ \\
\hline Totals: & 2 & 3 & 0 & 5 & $40 \%$ & $60 \%$ & $0 \%$ \\
\hline
\end{tabular}

Lab: OB OBG Laboratories, East Syracuse, NY

\begin{tabular}{|c|c|c|c|c|c|c|c|}
\hline $\begin{array}{l}\text { Al } \\
\text { SO } \\
\text { WA }\end{array}$ & $\begin{array}{l}2 \\
0 \\
1\end{array}$ & $\begin{array}{l}0 \\
2 \\
1\end{array}$ & $\begin{array}{l}0 \\
0 \\
0\end{array}$ & $\begin{array}{l}2 \\
2 \\
2\end{array}$ & $\begin{array}{r}100 \\
0 \\
50\end{array}$ & $\begin{array}{r}0 \\
100 \\
50\end{array}$ & $\begin{array}{l}0 \\
0 \\
0\end{array}$ \\
\hline tals: & 3 & 3 & 0 & 6 & $50 \%$ & $50 \%$ & $0 \%$ \\
\hline
\end{tabular}

Lab: OD ORNL, Radiobioassay Lab

\begin{tabular}{|c|c|c|c|c|c|c|c|}
\hline $\begin{array}{l}\text { Al } \\
\text { WA }\end{array}$ & $\begin{array}{r}10 \\
4\end{array}$ & $\begin{array}{l}1 \\
2\end{array}$ & $\begin{array}{l}0 \\
0\end{array}$ & $\begin{array}{r}11 \\
6\end{array}$ & $\begin{array}{l}91 \\
67\end{array}$ & $\begin{array}{r}9 \\
33\end{array}$ & $\begin{array}{l}0 \\
0\end{array}$ \\
\hline Totals: & 14 & 3 & 0 & 17 & $82 \%$ & $18 \%$ & $0 \%$ \\
\hline
\end{tabular}

Lab: OL ORNL Environmental Sciences Div.

\begin{tabular}{|c|c|c|c|c|c|c|c|}
\hline $\begin{array}{l}\text { Al } \\
\text { SO } \\
\text { VE } \\
\text { WA }\end{array}$ & $\begin{array}{l}7 \\
2 \\
3 \\
3\end{array}$ & $\begin{array}{l}1 \\
0 \\
0 \\
0\end{array}$ & $\begin{array}{l}0 \\
0 \\
0 \\
0\end{array}$ & $\begin{array}{l}8 \\
2 \\
3 \\
3\end{array}$ & $\begin{array}{r}88 \\
100 \\
100 \\
100\end{array}$ & $\begin{array}{r}13 \\
0 \\
0 \\
0\end{array}$ & $\begin{array}{l}0 \\
0 \\
0 \\
0\end{array}$ \\
\hline Totals: & 15 & 1 & 0 & 16 & $94 \%$ & $6 \%$ & $0 \%$ \\
\hline
\end{tabular}

Lab: OR Oak Ridge National Lab

\begin{tabular}{|c|c|c|c|c|c|c|c|}
\hline $\begin{array}{l}\text { Al } \\
\text { SO } \\
\text { VE } \\
\text { WA }\end{array}$ & $\begin{array}{r}12 \\
6 \\
6 \\
8\end{array}$ & $\begin{array}{l}1 \\
0 \\
1 \\
1\end{array}$ & $\begin{array}{l}0 \\
1 \\
0 \\
0\end{array}$ & $\begin{array}{r}13 \\
7 \\
7 \\
9\end{array}$ & $\begin{array}{l}92 \\
86 \\
86 \\
89\end{array}$ & $\begin{array}{r}8 \\
0 \\
14 \\
11\end{array}$ & $\begin{array}{r}0 \\
14 \\
0 \\
0\end{array}$ \\
\hline tals: & 32 & 3 & 1 & 36 & $89 \%$ & $8 \%$ & $3 \%$ \\
\hline
\end{tabular}


QAP44 Summary of Matrix Evaluations by Laboratory

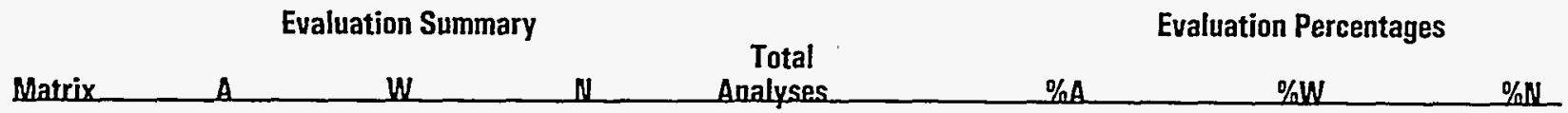

Lab: OS Oregon Health Division Radiation Controls Section, Portland

$\begin{array}{llllllll}\text { So } & 1 & 0 & 0 & 1 & 100 & 0 & 0 \\ \text { Totals: } & 1 & 0 & 0 & 1 & 100 \% & 0 \% & 0 \%\end{array}$

Lab: OT ORNL Radioactive Material Analysis Lab

\begin{tabular}{|c|c|c|c|c|c|c|c|}
\hline $\begin{array}{l}\text { Al } \\
\text { SO } \\
\text { VE } \\
\text { WA }\end{array}$ & $\begin{array}{l}9 \\
4 \\
4 \\
6\end{array}$ & $\begin{array}{l}2 \\
1 \\
0 \\
4\end{array}$ & $\begin{array}{l}4 \\
0 \\
1 \\
1\end{array}$ & $\begin{array}{r}15 \\
5 \\
5 \\
11\end{array}$ & $\begin{array}{l}60 \\
80 \\
80 \\
55\end{array}$ & $\begin{array}{r}13 \\
20 \\
0 \\
36\end{array}$ & $\begin{array}{r}27 \\
0 \\
20 \\
9\end{array}$ \\
\hline als: & 23 & 7 & 6 & 36 & $64 \%$ & $19 \%$ & $17 \%$ \\
\hline
\end{tabular}

Lab: PA Mason \& Hanger-Silas Mason Co., Inc., Battelle Pantex, Amarillo, TX

\begin{tabular}{|c|c|c|c|c|c|c|c|}
\hline $\begin{array}{l}\text { Al } \\
\text { SO } \\
\text { VE } \\
\text { WA }\end{array}$ & $\begin{array}{l}3 \\
3 \\
0 \\
5\end{array}$ & $\begin{array}{l}0 \\
0 \\
1 \\
0\end{array}$ & $\begin{array}{l}2 \\
0 \\
0 \\
1\end{array}$ & $\begin{array}{l}5 \\
3 \\
1 \\
6\end{array}$ & $\begin{array}{r}60 \\
100 \\
0 \\
83\end{array}$ & $\begin{array}{r}0 \\
0 \\
100 \\
0\end{array}$ & $\begin{array}{r}40 \\
0 \\
0 \\
17\end{array}$ \\
\hline Totals: & 11 & 1 & 3 & 15 & $73 \%$ & $7 \%$ & $20 \%$ \\
\hline
\end{tabular}

Lab: PB Mason \& Hanger-Silas Mason Co. Inc. Battelle Pantex, Amarillo, TX

\begin{tabular}{|c|c|c|c|c|c|c|c|}
\hline $\begin{array}{l}\text { SO } \\
\text { VE } \\
\text { WA }\end{array}$ & $\begin{array}{l}3 \\
1 \\
5\end{array}$ & $\begin{array}{l}0 \\
0 \\
0\end{array}$ & $\begin{array}{l}0 \\
0 \\
1\end{array}$ & $\begin{array}{l}3 \\
1 \\
6\end{array}$ & $\begin{array}{r}100 \\
100 \\
83\end{array}$ & $\begin{array}{l}0 \\
0 \\
0\end{array}$ & $\begin{array}{r}0 \\
0 \\
17\end{array}$ \\
\hline Totals: & $\mathbf{9}$ & 0 & 1 & 10 & $90 \%$ & $0 \%$ & $10 \%$ \\
\hline
\end{tabular}

Lab: PI Lockheed Martin Specialty Components, Largo, FL

\begin{tabular}{llllllll} 
Al & 2 & 0 & 0 & 2 & 100 & 0 & 0 \\
SO & 2 & 0 & 0 & 2 & 100 & 0 & 0 \\
WA & 5 & 0 & 0 & 5 & 100 & 0 & 0 \\
\cline { 2 - 8 } & & 9 & 0 & 0 & 9 & $100 \%$ & $0 \%$
\end{tabular}




\section{QAP44 Summary of Matrix Evaluations by Laboratory}

\begin{tabular}{|c|c|c|c|c|c|c|}
\hline \multicolumn{3}{|c|}{ Evaluation Summary } & & \multicolumn{3}{|c|}{ Evaluation Percentages } \\
\hline Matrix & $A$ & $\mathbf{w}$ & $\begin{array}{l}\text { Iotat } \\
\text { Analuses }\end{array}$ & $\% A$ & $\% w$ & $\% N$ \\
\hline
\end{tabular}

Lab: RA V. G. Khlopin Radium Institute, St. Petersburg, Russia

\begin{tabular}{|c|c|c|c|c|c|c|c|}
\hline $\begin{array}{l}\text { Al } \\
\text { SO } \\
\text { VE }\end{array}$ & $\begin{array}{l}7 \\
7 \\
5\end{array}$ & $\begin{array}{l}3 \\
0 \\
1\end{array}$ & $\begin{array}{l}1 \\
2 \\
1\end{array}$ & $\begin{array}{r}11 \\
9 \\
7\end{array}$ & $\begin{array}{l}64 \\
78 . \\
71\end{array}$ & $\begin{array}{r}27 \\
0 \\
14\end{array}$ & $\begin{array}{r}9 \\
22 \\
14\end{array}$ \\
\hline Totals: & 19 & 4 & 4 & 27 & $70 \%$ & $15 \%$ & $15 \%$ \\
\hline
\end{tabular}

Lab: RE Bechtel Nevada, Mercury, NV

\begin{tabular}{|c|c|c|c|c|c|c|c|}
\hline $\begin{array}{l}\text { Al } \\
\text { SO } \\
\text { VE } \\
\text { WA }\end{array}$ & $\begin{array}{r}10 \\
6 \\
6 \\
11\end{array}$ & $\begin{array}{l}4 \\
1 \\
0 \\
2\end{array}$ & $\begin{array}{l}2 \\
0 \\
0 \\
0\end{array}$ & $\begin{array}{r}16 \\
7 \\
6 \\
13\end{array}$ & $\begin{array}{r}63 \\
86 \\
100 \\
85\end{array}$ & $\begin{array}{r}25 \\
14 \\
0 \\
15\end{array}$ & $\begin{array}{r}13 \\
0 \\
0 \\
0\end{array}$ \\
\hline Totals: & 33 & 7 & 2 & 42 & $79 \%$ & $17 \%$ & $5 \%$ \\
\hline
\end{tabular}

Lab: RF EG\&G Rocky Flats Plant, Golden

\begin{tabular}{|c|c|c|c|c|c|c|c|}
\hline $\begin{array}{l}\text { Al } \\
\text { SO } \\
\text { VE } \\
\text { WA }\end{array}$ & $\begin{array}{l}0 \\
3 \\
1 \\
6\end{array}$ & $\begin{array}{l}0 \\
1 \\
0 \\
2\end{array}$ & $\begin{array}{l}2 \\
0 \\
0 \\
0\end{array}$ & $\begin{array}{l}2 \\
4 \\
1 \\
8\end{array}$ & $\begin{array}{r}0 \\
75 \\
100 \\
75\end{array}$ & $\begin{array}{r}0 \\
25 \\
0 \\
25\end{array}$ & $\begin{array}{r}100 \\
0 \\
0 \\
0\end{array}$ \\
\hline Totals: & 10 & 3 & 2 & 15 & $67 \%$ & $20 \%$ & $13 \%$ \\
\hline
\end{tabular}

Lab: RG EG\&G Rocky Flats Plant, Golden

\begin{tabular}{|c|c|c|c|c|c|c|c|}
\hline $\begin{array}{l}\text { SO } \\
\text { WA }\end{array}$ & $\begin{array}{l}6 \\
7\end{array}$ & $\begin{array}{l}0 \\
2\end{array}$ & $\begin{array}{l}0 \\
0\end{array}$ & $\begin{array}{l}6 \\
9\end{array}$ & $\begin{array}{r}100 \\
78\end{array}$ & $\begin{array}{r}0 \\
22\end{array}$ & $\begin{array}{l}0 \\
0\end{array}$ \\
\hline Totals: & 13 & 2 & 0 & 15 & $87 \%$ & $13 \%$ & $0 \%$ \\
\hline
\end{tabular}

Lab: BI Westinghouse Hanford Co. Analytical Labs

$\begin{array}{lrrrrrrr}\text { Al } & 5 & 3 & 4 & 12 & 42 & 25 & 33 \\ \text { SO } & 0 & 0 & 2 & 2 & 0 & 0 & 100 \\ \text { VE } & 3 & 1 & 2 & 6 & 50 & 17 & 33 \\ \text { WA } & 5 & 2 & 2 & 9 & 56 & 22 & 22\end{array}$




\section{QAP44 Summary of Matrix Evaluations by Laboratory}

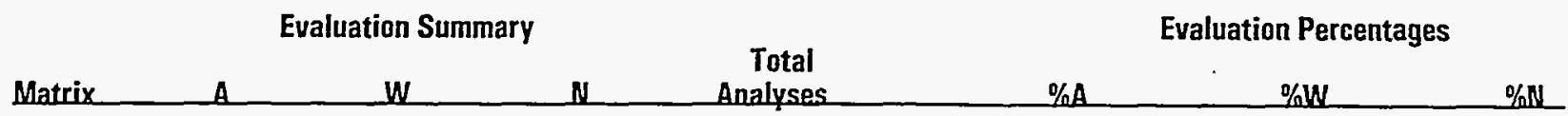

Lab: Rl Westinghouse Hanford Co. Analytical Labs

$\begin{array}{llllllll}\text { Totals: } & 13 & 6 & 10 & 29 & 45 \% & 21 \% & 34 \%\end{array}$

Lab: SA Sandia Labs Radioactive Sample Diaq. Prog. NM

\begin{tabular}{|c|c|c|c|c|c|c|c|}
\hline $\begin{array}{l}\text { Al } \\
\text { SO } \\
\text { WA }\end{array}$ & $\begin{array}{l}0 \\
2 \\
5\end{array}$ & $\begin{array}{l}7 \\
0 \\
0\end{array}$ & $\begin{array}{l}0 \\
0 \\
0\end{array}$ & $\begin{array}{l}7 \\
2 \\
5\end{array}$ & $\begin{array}{r}0 \\
100 \\
100\end{array}$ & $\begin{array}{r}100 \\
0 \\
0\end{array}$ & $\begin{array}{l}0 \\
0 \\
0\end{array}$ \\
\hline Totals: & 7 & 7 & 0 & 14 & $50 \%$ & $50 \%$ & $0 \%$ \\
\hline
\end{tabular}

Lab: SC S-Cubed Division Maxwell Labs, La Jolla, CA

\begin{tabular}{|c|c|c|c|c|c|c|c|}
\hline $\begin{array}{l}\text { Al } \\
\text { SO } \\
\text { WA }\end{array}$ & $\begin{array}{l}2 \\
5 \\
9\end{array}$ & $\begin{array}{l}0 \\
1 \\
2\end{array}$ & $\begin{array}{l}0 \\
1 \\
2\end{array}$ & $\begin{array}{r}2 \\
7 \\
13\end{array}$ & $\begin{array}{r}100 \\
71 \\
69\end{array}$ & $\begin{array}{r}0 \\
14 \\
15\end{array}$ & $\begin{array}{r}0 \\
14 \\
15\end{array}$ \\
\hline Totals: & 16 & 3 & 3 & 22 & $73 \%$ & $14 \%$ & $14 \%$ \\
\hline
\end{tabular}

Lab: SE Shealy Environmental Services inc., Cayce, SC

\begin{tabular}{|c|c|c|c|c|c|c|c|}
\hline $\begin{array}{l}\text { Al } \\
\text { SO } \\
\text { VE } \\
\text { WA }\end{array}$ & $\begin{array}{l}8 \\
1 \\
5 \\
5\end{array}$ & $\begin{array}{l}1 \\
4 \\
2 \\
5\end{array}$ & $\begin{array}{l}0 \\
1 \\
0 \\
1\end{array}$ & $\begin{array}{r}9 \\
6 \\
7 \\
11\end{array}$ & $\begin{array}{l}89 \\
17 \\
71 \\
45\end{array}$ & $\begin{array}{l}11 \\
67 \\
29 \\
45\end{array}$ & $\begin{array}{r}0 \\
17 \\
0 \\
9\end{array}$ \\
\hline Totals: & 19 & 12 & 2 & 33 & $58 \%$ & $36 \%$ & $6 \%$ \\
\hline
\end{tabular}

Lab: SK Savannah River Plant

\begin{tabular}{|c|c|c|c|c|c|c|c|}
\hline $\begin{array}{l}\mathrm{Al} \\
\text { SO } \\
\mathrm{VE} \\
\text { WA }\end{array}$ & $\begin{array}{l}3 \\
2 \\
3 \\
4\end{array}$ & $\begin{array}{l}4 \\
0 \\
0 \\
0\end{array}$ & $\begin{array}{l}1 \\
0 \\
0 \\
0\end{array}$ & $\begin{array}{l}8 \\
2 \\
3 \\
4\end{array}$ & $\begin{array}{r}38 \\
100 \\
100 \\
100\end{array}$ & $\begin{array}{r}50 \\
0 \\
0 \\
0\end{array}$ & $\begin{array}{r}13 \\
0 \\
0 \\
0\end{array}$ \\
\hline Totals: & 12 & 4 & 1 & 17 & $71 \%$ & $24 \%$ & $6 \%$ \\
\hline
\end{tabular}




\section{QAP44 Summary of Matrix Evaluations by Laboratory}

\begin{tabular}{|c|c|c|c|c|c|}
\hline \multirow[b]{2}{*}{ Matrix } & \multicolumn{2}{|c|}{ Evaluation Summary } & \multicolumn{3}{|c|}{ Evaluation Percentages } \\
\hline & $\mathbf{w}$ & Analuses & $\% A$ & $\% W$ & $\% \mathrm{~N}$ \\
\hline
\end{tabular}

Lab: SN Sanford Cohen Associates, Inc., Montgomery, AL

\begin{tabular}{|c|c|c|c|c|c|c|c|}
\hline $\begin{array}{l}\text { Al } \\
\text { SO } \\
\text { VE } \\
\text { WA }\end{array}$ & $\begin{array}{l}4 \\
4 \\
3 \\
6\end{array}$ & $\begin{array}{l}0 \\
1 \\
1 \\
1\end{array}$ & $\begin{array}{l}0 \\
1 \\
0 \\
0\end{array}$ & $\begin{array}{l}4 \\
6 \\
4 \\
7\end{array}$ & $\begin{array}{r}100 \\
67 \\
75 \\
86\end{array}$ & $\begin{array}{r}0 \\
17 \\
25 \\
14\end{array}$ & $\begin{array}{r}0 \\
17 \\
0 \\
0\end{array}$ \\
\hline Totals: & 17 & 3 & 1 & 21 & $81 \%$ & $14 \%$ & $5 \%$ \\
\hline
\end{tabular}

Lab: SR Savannah River Plant

\begin{tabular}{|c|c|c|c|c|c|c|c|}
\hline $\begin{array}{l}\text { Al } \\
\text { SO } \\
\text { VE } \\
\text { WA }\end{array}$ & $\begin{array}{r}13 \\
4 \\
2 \\
10\end{array}$ & $\begin{array}{l}2 \\
0 \\
1 \\
2\end{array}$ & $\begin{array}{l}1 \\
1 \\
2 \\
0\end{array}$ & $\begin{array}{r}16 \\
5 \\
5 \\
12\end{array}$ & $\begin{array}{l}81 \\
80 \\
40 \\
83\end{array}$ & $\begin{array}{r}13 \\
0 \\
20 \\
17\end{array}$ & $\begin{array}{r}6 \\
20 \\
40 \\
0\end{array}$ \\
\hline Totals: & 29 & 5 & 4 & 38 & $76 \%$ & $13 \%$ & $11 \%$ \\
\hline
\end{tabular}

Lab: SS Savannah River Tech Center

\begin{tabular}{|c|c|c|c|c|c|c|c|}
\hline $\begin{array}{l}\text { Al } \\
\text { SO } \\
\text { VE } \\
\text { WA }\end{array}$ & $\begin{array}{l}5 \\
1 \\
3 \\
3\end{array}$ & $\begin{array}{l}3 \\
1 \\
0 \\
0\end{array}$ & $\begin{array}{l}0 \\
0 \\
0 \\
0\end{array}$ & $\begin{array}{l}8 \\
2 \\
3 \\
3\end{array}$ & $\begin{array}{r}63 \\
50 \\
100 \\
100\end{array}$ & $\begin{array}{r}38 \\
50 \\
0 \\
0\end{array}$ & $\begin{array}{l}0 \\
0 \\
0 \\
0\end{array}$ \\
\hline Totals: & 12 & 4 & B & 16 & $75 \%$ & $25 \%$ & $0 \%$ \\
\hline
\end{tabular}

Lab: SV Savannah Lab \& Environmental Services, Inc., Tampa, FL

\begin{tabular}{|c|c|c|c|c|c|c|c|}
\hline WA & 5 & 0 & 0 & 5 & 100 & 0 & 0 \\
\hline Totals: & 5 & 0 & 0 & 5 & $100 \%$ & $0 \%$ & $0 \%$ \\
\hline
\end{tabular}

Lab: SW Southwest Research Institute, San Antonio, TX

$\begin{array}{llllrrrr}\text { Al } & 6 & 5 & 2 & 13 & 46 & 38 & 15 \\ \text { SO } & 1 & 2 & 1 & 4 & 25 & 50 & 25 \\ \text { VE } & 5 & 2 & 0 & 7 & 71 & 29 & 0 \\ \text { WA } & 5 & 0 & 2 & 7 & 71 & 0 & 29\end{array}$


QAP44 Summary of Matrix Evaluations by Laboratory

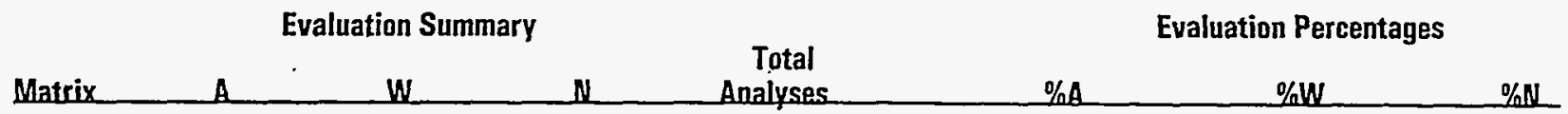

Lab: SW Southwest Research Institute, San Antonio, TX

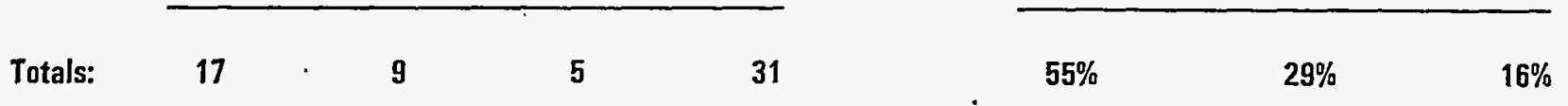

Lab: TE Teledyne isotopes Midwest Lab, Northbrook, IL

\begin{tabular}{|c|c|c|c|c|c|c|c|}
\hline $\begin{array}{l}\text { Al } \\
\text { SO } \\
\text { VE } \\
\text { WA }\end{array}$ & $\begin{array}{r}12 \\
6 \\
7 \\
9\end{array}$ & $\begin{array}{l}3 \\
1 \\
0 \\
3\end{array}$ & $\begin{array}{l}0 \\
0 \\
0 \\
0\end{array}$ & $\begin{array}{r}15 \\
7 \\
7 \\
12\end{array}$ & $\begin{array}{r}80 \\
86 \\
100 \\
75\end{array}$ & $\begin{array}{r}20 \\
14 \\
0 \\
25\end{array}$ & $\begin{array}{l}0 \\
0 \\
0 \\
0\end{array}$ \\
\hline Totals: & 34 & 7 & 0 & 41 & $83 \%$ & $17 \%$ & $0 \%$ \\
\hline
\end{tabular}

Lab: II Teledyne Brown Engineering Environmental Services, Westwood, NJ

\begin{tabular}{|c|c|c|c|c|c|c|c|}
\hline $\begin{array}{l}\text { Al } \\
\text { SO } \\
\text { VE } \\
\text { WA }\end{array}$ & $\begin{array}{r}14 \\
9 \\
4 \\
9\end{array}$ & $\begin{array}{l}2 \\
0 \\
3 \\
1\end{array}$ & $\begin{array}{l}1 \\
0 \\
0 \\
4\end{array}$ & $\begin{array}{r}17 \\
9 \\
7 \\
14\end{array}$ & $\begin{array}{r}82 \\
100 \\
57 \\
64\end{array}$ & $\begin{array}{r}12 \\
0 \\
43 \\
7\end{array}$ & $\begin{array}{r}6 \\
0 \\
0 \\
29\end{array}$ \\
\hline Totals: & 36 & 6 & 5 & 47 & $77 \%$ & $13 \%$ & $11 \%$ \\
\hline
\end{tabular}

Lah: TM TMA/Eberline-Albuquerque Lab, NM

\begin{tabular}{lrrrrrrrr} 
Al & 14 & 2 & 1 & 17 & 82 & 12 & 6 \\
SO & 8 & 1 & 0 & 9 & 89 & 11 & 0 \\
VE & 6 & 1 & 0 & 7 & 86 & 14 & 0 \\
WA & 10 & 3 & 0 & 13 & 77 & 23 & 0 \\
\cline { 2 - 8 } & Totals: & 38 & 7 & 1 & 46 & $83 \%$ & $15 \%$ & $2 \%$
\end{tabular}

Lab: TN TMAINORCAL, Richmond, CA

\begin{tabular}{|c|c|c|c|c|c|c|c|}
\hline $\begin{array}{l}\text { Al } \\
\text { SO } \\
\text { VE } \\
\text { WA }\end{array}$ & $\begin{array}{r}16 \\
8 \\
6 \\
10\end{array}$ & $\begin{array}{l}1 \\
1 \\
1 \\
4\end{array}$ & $\begin{array}{l}0 \\
0 \\
0 \\
1\end{array}$ & $\begin{array}{r}17 \\
9 \\
7 \\
15\end{array}$ & $\begin{array}{l}94 \\
89 \\
86 \\
67\end{array}$ & $\begin{array}{r}6 \\
11 \\
14 \\
27\end{array}$ & $\begin{array}{l}0 \\
0 \\
0 \\
7\end{array}$ \\
\hline Totals: & 40 & 7 & 1 & 48 & $83 \%$ & $15 \%$ & $2 \%$ \\
\hline
\end{tabular}




\section{QAP44 Summary of Matrix Evaluations by Laboratory}

Evaluation Summary

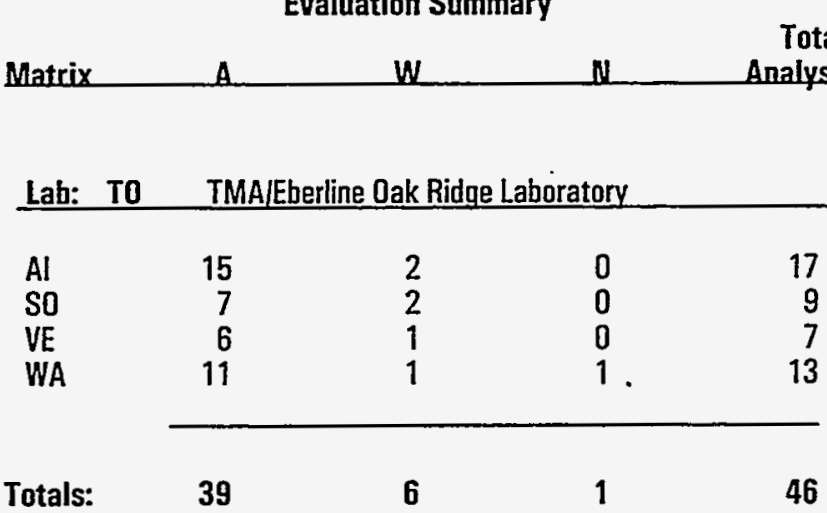

Evaluation Percentages

Total

$\% w$

$\%$ N

$\% A$

Lab: TW Taiwan Radiation Monitoring Center

\begin{tabular}{|c|c|c|c|c|c|c|c|}
\hline $\begin{array}{l}\text { Al } \\
\text { SO } \\
\text { VE } \\
\text { WA }\end{array}$ & $\begin{array}{r}13 \\
7 \\
7 \\
6\end{array}$ & $\begin{array}{l}1 \\
1 \\
0 \\
4\end{array}$ & $\begin{array}{l}2 \\
0 \\
0 \\
2\end{array}$ & $\begin{array}{r}16 \\
8 \\
7 \\
12\end{array}$ & $\begin{array}{r}81 \\
88 \\
100 \\
50\end{array}$ & $\begin{array}{r}6 \\
13 \\
0 \\
33\end{array}$ & $\begin{array}{r}13 \\
0 \\
0 \\
17\end{array}$ \\
\hline Totals: & 33 & 6 & 4 & 43 & $77 \%$ & $14 \%$ & $9 \%$ \\
\hline
\end{tabular}

Lab: TX Texas Dept. of Health/Lahoratories, Austin

\begin{tabular}{|c|c|c|c|c|c|c|c|}
\hline $\begin{array}{l}\text { Al } \\
\text { SO } \\
\text { VE } \\
\text { WA }\end{array}$ & $\begin{array}{l}9 \\
5 \\
4 \\
2\end{array}$ & $\begin{array}{l}4 \\
3 \\
2 \\
5\end{array}$ & $\begin{array}{l}0 \\
0 \\
0 \\
3\end{array}$ & $\begin{array}{r}13 \\
8 \\
6 \\
10\end{array}$ & $\begin{array}{l}69 \\
63 \\
67 \\
20\end{array}$ & $\begin{array}{l}31 \\
38 \\
33 \\
50\end{array}$ & $\begin{array}{r}0 \\
0 \\
0 \\
30\end{array}$ \\
\hline Totals: & 20 & 14 & 3 & 37 & $54 \%$ & $38 \%$ & $8 \%$ \\
\hline
\end{tabular}

Lab: TY Scientific Production Association, Russia

\begin{tabular}{|c|c|c|c|c|c|c|c|}
\hline $\begin{array}{l}\text { Al } \\
\text { SO } \\
\text { VE }\end{array}$ & $\begin{array}{l}2 \\
2 \\
4\end{array}$ & $\begin{array}{l}2 \\
0 \\
1\end{array}$ & $\begin{array}{l}4 \\
1 \\
0\end{array}$ & $\begin{array}{l}8 \\
3 \\
5\end{array}$ & $\begin{array}{l}25 \\
67 \\
80\end{array}$ & $\begin{array}{r}25 \\
0 \\
20\end{array}$ & $\begin{array}{r}50 \\
33 \\
0\end{array}$ \\
\hline Totals: & 8 & 3 & 5 & 16 & $50 \%$ & $19 \%$ & $31 \%$ \\
\hline
\end{tabular}

Lab: UC Lockheed Martin, Paducah, KY

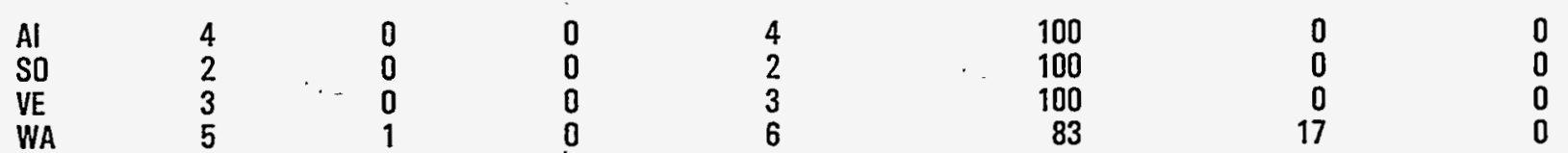


QAP44 Summary of Matrix Evaluations by Laboratory

Evaluation Summary

Total

Evaluation Percentages

Matrix W Anàlyses $\% w$ $\%$ H

Lab: UC Lockheed Martin, Paducah, KY

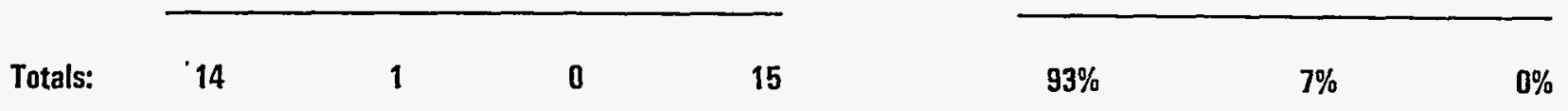

Lab: UK Lockheed Martin Energy Systems, Oak Ridge

\begin{tabular}{|c|c|c|c|c|c|c|c|}
\hline $\begin{array}{l}\text { Al } \\
\text { SO } \\
\text { WA }\end{array}$ & $\begin{array}{l}5 \\
4 \\
6\end{array}$ & $\begin{array}{l}5 \\
1 \\
0\end{array}$ & $\begin{array}{l}0 \\
1 \\
1\end{array}$ & $\begin{array}{r}10 \\
6 \\
7\end{array}$ & $\begin{array}{l}50 \\
67 \\
86\end{array}$ & $\begin{array}{r}50 \\
17 \\
0\end{array}$ & $\begin{array}{r}0 \\
17 \\
14\end{array}$ \\
\hline Totals: & 15 & 6 & 2 & 23 & $65 \%$ & $26 \%$ & $9 \%$ \\
\hline
\end{tabular}

Lab: UP Lockheed Martin Energy Systems, Y-12 Plant, Oak Ridge

\begin{tabular}{|c|c|c|c|c|c|c|c|}
\hline $\begin{array}{l}\mathrm{Al} \\
\text { SO } \\
W A\end{array}$ & $\begin{array}{r}6 \\
6 \\
11\end{array}$ & $\begin{array}{r}10 \\
1 \\
2\end{array}$ & $\begin{array}{l}1 \\
0 \\
0\end{array}$ & $\begin{array}{r}17 \\
7 \\
13\end{array}$ & $\begin{array}{l}35 \\
86 \\
85\end{array}$ & $\begin{array}{l}59 \\
14 \\
15\end{array}$ & $\begin{array}{l}6 \\
0 \\
0\end{array}$ \\
\hline Totals: & 23 & 13 & 1 & 37 & $62 \%$ & $35 \%$ & 3 \\
\hline
\end{tabular}

Lab: UY Lockheed Martin Energy Systems, Y-12 Plant, Oak Ridge

\begin{tabular}{|c|c|c|c|c|c|c|c|}
\hline $\begin{array}{l}\text { Al } \\
\text { SO } \\
\text { WA }\end{array}$ & $\begin{array}{r}13 \\
7 \\
11\end{array}$ & $\begin{array}{l}3 \\
1 \\
0\end{array}$ & $\begin{array}{l}0 \\
0 \\
2\end{array}$ & $\begin{array}{r}16 \\
8 \\
13\end{array}$ & $\begin{array}{l}81 \\
88 \\
85\end{array}$ & $\begin{array}{r}19 \\
13 \\
0\end{array}$ & $\begin{array}{r}0 \\
0 \\
15\end{array}$ \\
\hline Totals: & 31 & 4 & 2 & 37 & $84 \%$ & $11 \%$ & $5 \%$ \\
\hline
\end{tabular}

Lab: WA Environmental Radiation Lab, Off. of Public Health Labs. Seattle

\begin{tabular}{|c|c|c|c|c|c|c|c|}
\hline $\begin{array}{l}A I \\
\text { SO } \\
\text { VE } \\
W A\end{array}$ & $\begin{array}{r}14 \\
6 \\
4 \\
10\end{array}$ & $\begin{array}{l}3 \\
2 \\
1 \\
1\end{array}$ & $\begin{array}{l}0 \\
1 \\
1 \\
2\end{array}$ & $\begin{array}{r}17 \\
9 \\
6 \\
13\end{array}$ & $\begin{array}{l}82 \\
67 \\
67 \\
77\end{array}$ & $\begin{array}{r}18 \\
22 \\
17 \\
8\end{array}$ & $\begin{array}{r}0 \\
11 \\
17 \\
15\end{array}$ \\
\hline & 34 & 7 & 4 & 45 & $76 \%$ & $16 \%$ & $9 \%$ \\
\hline
\end{tabular}




\section{QAP44 Summary of Matrix Evaluations by Laboratory}

Evaluation Summary

Matrix A

W N Total Analyses $\% \mathrm{~A}$ \%W $\%$ N

Lab: WC Westinghouse Hanford Co.

\begin{tabular}{lrrrr} 
Al & 9 & 6 & 1 & 16 \\
SO & 2 & 0 & 0 & 2 \\
VE & 3 & 0 & 0 & 3 \\
WA & 4 & 7 & 1 & 12 \\
\cline { 2 - 4 } & & & & \\
Totals: & 18 & 13 & 2 & 33
\end{tabular}

Lab: WE Westinghouse Electric Corp., Madison, PA

\begin{tabular}{|c|c|c|c|c|c|c|c|}
\hline $\begin{array}{l}\text { AI } \\
\text { SO } \\
\text { VE } \\
\text { WA }\end{array}$ & $\begin{array}{l}3 \\
4 \\
3 \\
3\end{array}$ & $\begin{array}{l}3 \\
1 \\
0 \\
4\end{array}$ & $\begin{array}{l}7 \\
2 \\
2 \\
1\end{array}$ & $\begin{array}{r}13 \\
7 \\
5 \\
8\end{array}$ & $\begin{array}{l}23 \\
57 \\
60 \\
38\end{array}$ & $\begin{array}{r}23 \\
14 \\
0 \\
50\end{array}$ & $\begin{array}{l}54 \\
29 \\
40 \\
13\end{array}$ \\
\hline Totals: & 13 & 8 & 12 & 33 & $39 \%$ & $24 \%$ & $36 \%$ \\
\hline
\end{tabular}

Lab: WI WIPP Site, Westinghouse Electric Corp.

\begin{tabular}{|c|c|c|c|c|c|c|c|}
\hline $\begin{array}{l}\mathrm{Al} \\
\text { WA }\end{array}$ & $\begin{array}{l}7 \\
1\end{array}$ & $\begin{array}{l}0 \\
2\end{array}$ & $\begin{array}{l}0 \\
0\end{array}$ & $\begin{array}{l}7 \\
3\end{array}$ & $\begin{array}{r}100 \\
33\end{array}$ & $\begin{array}{r}0 \\
67\end{array}$ & $\begin{array}{l}0 \\
0\end{array}$ \\
\hline Totals: & 8 & 2 & 0 & 10 & $80 \%$ & $20 \%$ & $0 \%$ \\
\hline
\end{tabular}

Lab: WN WINCO, Idaho Chemical Processing Plant, Scoville

\begin{tabular}{|c|c|c|c|c|c|c|c|}
\hline $\begin{array}{l}\text { Al } \\
\text { SO } \\
\text { VE } \\
\text { WA }\end{array}$ & $\begin{array}{r}11 \\
2 \\
4 \\
8\end{array}$ & $\begin{array}{l}0 \\
0 \\
0 \\
2\end{array}$ & $\begin{array}{l}0 \\
0 \\
0 \\
1\end{array}$ & $\begin{array}{r}11 \\
2 \\
4 \\
11\end{array}$ & $\begin{array}{r}100 \\
100 \\
100 \\
73\end{array}$ & $\begin{array}{r}0 \\
0 \\
0 \\
18\end{array}$ & $\begin{array}{l}0 \\
0 \\
0 \\
9\end{array}$ \\
\hline Is: & 25 & 2 & 1 & 28 & $89 \%$ & $7 \%$ & $4 \%$ \\
\hline
\end{tabular}

Lab: WP Washington Public Power Supply System, Richland

$\begin{array}{lllllrrr}\text { Al } & 9 & 0 & 0 & 9 & 100 & 0 & 0 \\ \text { SO } & 3 & 0 & 0 & 3 & 100 & 0 & 0 \\ \text { VE } & 2 & 2 & 0 & 4 & 50 & 50 & 0 \\ \text { WA } & 5 & 0 & 0 & 5 & 100 & 0 & 0\end{array}$




\section{QAP44 Summary of Matrix Evaluations by Laboratory}

\begin{tabular}{|c|c|c|c|c|c|c|c|}
\hline \multirow[b]{2}{*}{ Matrix } & \multicolumn{3}{|c|}{ Evaluation Summary } & & \multicolumn{3}{|c|}{ Evaluation Percentages } \\
\hline & A & $w$ & in & Analyses & $\% A$ & $\% w$ & \\
\hline
\end{tabular}

Lab: WP Washington Public Power Supply System, Richland

$\begin{array}{lllllllll}\text { Totals: } & 19 & 2 & 0 & 21 & 90 \% & 10 \% & 0 \%\end{array}$

Lab: WS Weldon Springs Site, St Charles, MO

\begin{tabular}{cccccccc} 
So & 4 & 0 & 0 & 4 & 100 & 0 & 0 \\
\cline { 2 - 6 } & 4 & 0 & 0 & 4 & $100 \%$ & $0 \%$ & $0 \%$
\end{tabular}

Lab: WV West Valley Nuclear Services Co, Inc, NY

\begin{tabular}{|c|c|c|c|c|c|c|c|}
\hline $\begin{array}{l}\mathrm{Al} \\
\text { WA }\end{array}$ & $\begin{array}{l}2 \\
6\end{array}$ & $\begin{array}{l}0 \\
1\end{array}$ & $\begin{array}{l}0 \\
0\end{array}$ & $\frac{2}{7}$ & $\begin{array}{r}100 \\
86\end{array}$ & $\begin{array}{r}0 \\
14\end{array}$ & $\begin{array}{l}0 \\
0\end{array}$ \\
\hline Totals: & 8 & 1 & 0 & 9 & $89 \%$ & $11 \%$ & $0 \%$ \\
\hline
\end{tabular}

Lah: YA Yankee Atomic Electric Company, Westboro, MA

\begin{tabular}{|c|c|c|c|c|c|c|c|}
\hline $\begin{array}{l}\mathrm{Al} \\
\text { SO } \\
\text { VE } \\
\text { WA }\end{array}$ & $\begin{array}{l}5 \\
5 \\
6 \\
8\end{array}$ & $\begin{array}{l}0 \\
0 \\
0 \\
1\end{array}$ & $\begin{array}{l}0 \\
0 \\
0 \\
0\end{array}$ & $\begin{array}{l}5 \\
5 \\
6 \\
9\end{array}$ & $\begin{array}{r}100 \\
100 \\
100 \\
89\end{array}$ & $\begin{array}{r}0 \\
0 \\
0 \\
11\end{array}$ & $\begin{array}{l}0 \\
0 \\
0 \\
0\end{array}$ \\
\hline Totals: & 24 & 1 & D & 25 & $96 \%$ & $4 \%$ & 0\% \\
\hline
\end{tabular}

Lab: YP US Army Proving Ground, Yuma, AZ

\begin{tabular}{|c|c|c|c|c|c|c|c|}
\hline $\begin{array}{l}\text { Al } \\
\text { SO } \\
\text { WA }\end{array}$ & $\begin{array}{l}1 \\
0 \\
1\end{array}$ & $\begin{array}{l}0 \\
1 \\
0\end{array}$ & $\begin{array}{l}0 \\
0 \\
0\end{array}$ & $\begin{array}{l}1 \\
1 \\
1\end{array}$ & $\begin{array}{r}100 \\
0 \\
100\end{array}$ & $\begin{array}{r}0 \\
100 \\
0\end{array}$ & $\begin{array}{l}0 \\
0 \\
0\end{array}$ \\
\hline Totals: & 2 & 1 & 0 & 3 & $67 \%$ & $33 \%$ & $0 \%$ \\
\hline
\end{tabular}


QAP44 Summary of Laboratory Evaluations by Matrix

Matrix: Al

Evaluation Summary

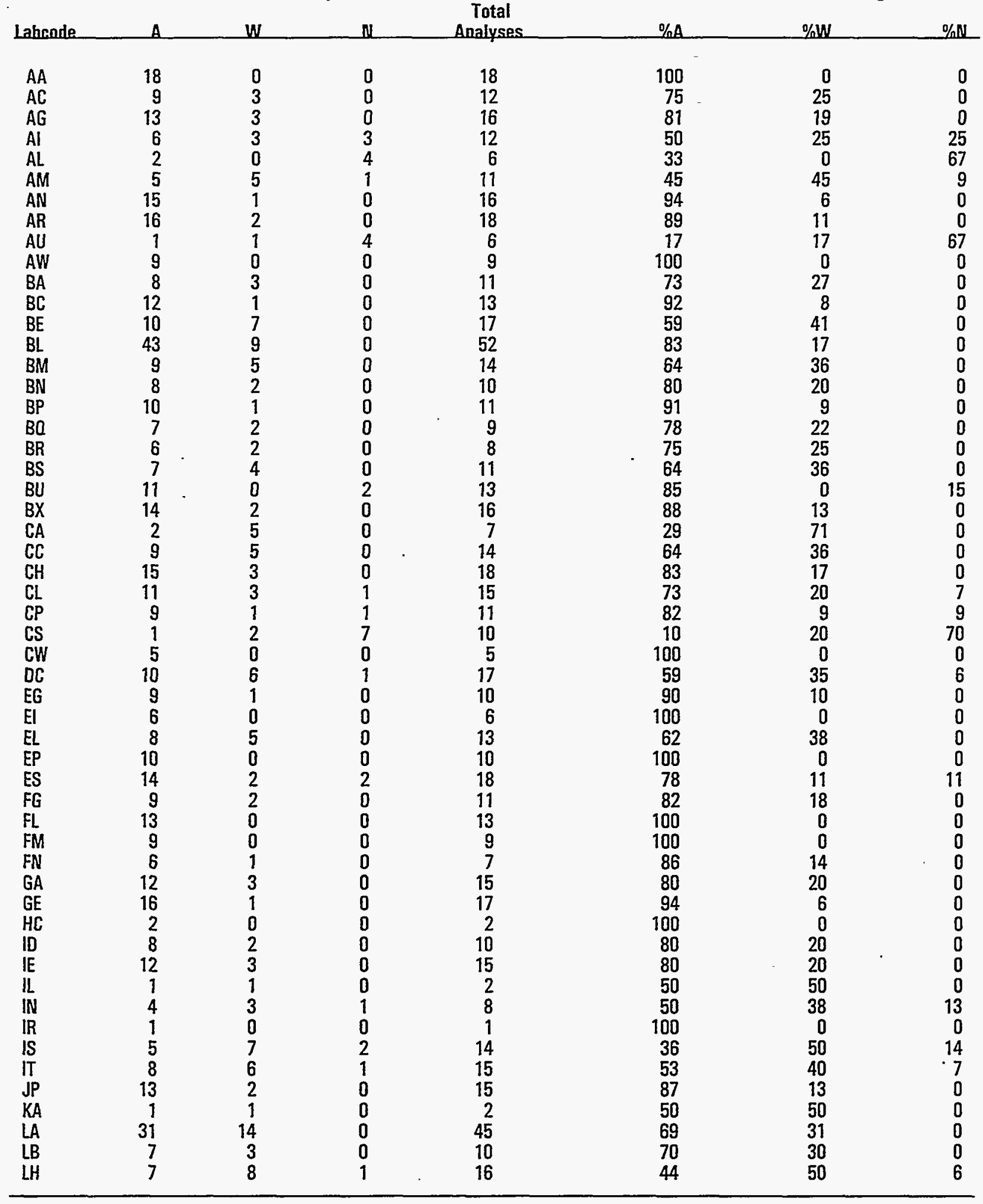

Evaluation Percentages Total
Analyse $\% \mathrm{~W} \quad \% \mathrm{~N}$ 
QAP44 Summary of Laboratory Evaluations by Matrix

Matrix: Al

Evaluation Summary

\begin{tabular}{|c|c|c|c|c|c|c|c|}
\hline Lahcade & A & $\mathbf{W}$ & $N$ & Analyses & $\% \mathrm{~A}$ & $\% W$ & $\% N$ \\
\hline LH & 7 & 8 & 1 & 16 & 44 & 50 & 6 \\
\hline $\mathrm{LL}$ & 11 & 1 & 2 & 14 & 79 & 7 & 14 \\
\hline LM & 2 & 3 & 5 & 10 & 20 & 30 & 50 \\
\hline LV & $\overline{7}$ & 2 & 0 & 9 & 78 & 22 & 0 \\
\hline ME & 7 & 0 & 1 & 8 & 88 & 0 & 13 \\
\hline MI & 2 & 3 & 0 & 5 & 40 & 60 & 0 \\
\hline$M L$ & 11 & 1 & 0 & 12 & 92 & 8 & 0 \\
\hline MS & 6 & 1 & 0 & 7 & 86 & 14 & 0 \\
\hline NA & 12 & 0 & 0 & 12 & 100 & 0 & 0 \\
\hline NC & 7 & 0 & 0 & 7 & 100 & 0 & 0 \\
\hline NL & 12 & 0 & 1 & 13 & 92 & 0 & 8 \\
\hline NM & 7 & 0 & 2 & 9 & 78 & 0 & 22 \\
\hline $\mathrm{OB}$ & 2 & 0 & 0 & 2 & 100 & 0 & 0 \\
\hline OD & 10 & 1 & 0 & 11 & 91 & 9 & 0 \\
\hline $\mathrm{OL}$ & 7 & 1 & 0 & 8 & 88 & 13 & 0 \\
\hline $\mathrm{OR}$ & 12 & 1 & 0 & 13 & 92 & 8 & 0 \\
\hline OT & 9 & 2 & 4 & 15 & 60 & 13 & 27 \\
\hline PA & 3 & 0 & 2 & 5 & 60 & 0 & 40 \\
\hline $\mathrm{Pl}$ & 2 & 0 & $\overline{0}$ & 2 & 100 & 0 & 0 \\
\hline RA & 7 & 3 & 1 & 11 & 64 & 27 & 9 \\
\hline $\mathrm{RE}$ & 10 & 4 & 2 & 16 & 63 & 25 & 13 \\
\hline $\mathrm{RF}^{\circ}$ & 0 & 0 & 2 & 2 & 0 & 0 & 100 \\
\hline $\mathbf{R I}$ & 5 & 3 & 4 & 12 & 42 & 25 & 33 \\
\hline SA & 0 & 7 & 0 & 7 & 0 & 100 & 0 \\
\hline SC & 2 & 0 & 0 & 2 & 100 & 0 & 0 \\
\hline SE & 8 & 1 & 0 & $\overrightarrow{9}$ & 89 & 11 & 0 \\
\hline SK & 3 & 4 & 1 & 8 & 38 & 50 & 13 \\
\hline SN & 4 & 0 & 0 & 4 & 100 & 0 & 0 \\
\hline SR & 13 & 2 & 1 & 16 & 81 & 13 & 6 \\
\hline SS & 5 & 3 & 0 & 8 & 63 & 38 & 0 \\
\hline SW & 6 & 5 & 2 & 13 & 46 & 38 & 15 \\
\hline TE & 12 & 3 & 0 & 15 & 80 & 20 & 0 \\
\hline $\mathrm{TI}$ & 14 & 2 & 1 & 17 & 82 & 12 & 6 \\
\hline TM & 14 & 2 & 1 & 17 & 82 & 12 & 6 \\
\hline $\mathrm{TN}$ & 16 & 1 & 0 & 17 & 94 & 6 & 0 \\
\hline TO & 15 & 2 & 0 & 17 & 88 & 12 & 0 \\
\hline TW & 13 & 1 & 2 & 16 & 81 & 6 & 13 \\
\hline$T X$ & 9 & 4 & 0 & 13 & 69 & 31 & 0 \\
\hline TY & 2 & 2 & 4 & 8 & 25 & 25 & 50 \\
\hline UC & 4 & 0 & 0 & 4 & 100 & 0 & 0 \\
\hline UK & 5 & 5 & 0 & 10 & 50 & 50 & 0 \\
\hline UP & 6 & 10 & 1 & 17 & 35 & 59 & 6 \\
\hline UY & 13 & 3 & 0 & 16 & 81 & 19 & 0 \\
\hline WA & 14 & 3 & 0 & 17 & 82 & 18 & 0 \\
\hline WC & 9 & 6 & 1 & 16 & 56 & 38 & 6 \\
\hline WE & 3 & 3 & 7 & 13 & 23 & 23 & 54 \\
\hline WI & 7 & 0 & 0 & 7 & 100 & 0 & 0 \\
\hline WN & 11 & 0 & 0 & 11 & 100 & 0 & 0 \\
\hline WP & 9 & 0 & 0 & 9 & 100 & 0 & 0 \\
\hline WV & 2 & 0 & 0 & 2 & 100 & 0 & 0 \\
\hline YA & 5 & 0 & 0 & 5 & 100 & 0 & 0 \\
\hline YP & 1 & 0 & 0 & 1 & 100 & 0 & 0 \\
\hline
\end{tabular}




\section{QAP44 Summary of Laboratory Evaluations by Matrix}

Matrix: Al

\begin{tabular}{|c|c|c|c|c|c|c|c|}
\hline \multirow[b]{2}{*}{ Lahcade } & \multicolumn{3}{|c|}{ Evaluation Summary } & \multirow{2}{*}{$\begin{array}{c}\text { Total } \\
\text { Analyses }\end{array}$} & \multicolumn{3}{|c|}{ Evaluation Percentages } \\
\hline & A & $w$ & $\mathbf{N}$ & & $\% A$ & $\% W$ & $\%$ N \\
\hline $\begin{array}{l}\text { Totals } \\
106\end{array}$ & 000 & 241 & & $17 n 0$ & $70 \%$ & סחר & $6 \%$ \\
\hline
\end{tabular}


QAP44 Summary of Laboratory Evaluations by Matrix

Matrix: SO

Evaluation Summary

\begin{tabular}{|c|c|c|c|c|c|c|c|}
\hline Laheade. & A & $w$ & $\mathbf{N}$ & Analyses & $\% A$ & $\% W$ & $\% N$ \\
\hline$A A$ & 10 & 0 & 0 & 10 & 100 & 0 & 0 \\
\hline$A C$ & 5 & 0 & 0 & 5 & 100 & 0 & 0 \\
\hline $\mathrm{AE}$ & 6 & 1 & 0 & 7 & 86 & 14 & 0 \\
\hline$A G$ & 6 & 2 & 0 & 8 & 75 & 25 & 0 \\
\hline $\mathrm{Al}$ & 2 & 3 & 1 & 6 & 33 & 50 & 17 \\
\hline AL & 1 & 0 & 0 & 1 & 100 & 0 & 0 \\
\hline AM & 3 & 2 & 0 & 5 & 60 & 40 & 0 \\
\hline AN & 7 & 1 & 0 & 8 & 88 & 13 & 0 \\
\hline AR & 7 & 3 & 0 & 10 & 70 & 30 & 0 \\
\hline AU & 2 & 0 & 4 & 6 & 33 & 0 & 67 \\
\hline BA & 1 & 0 & 0 & 1 & 100 & 0 & 0 \\
\hline$\cdot B C$ & 2 & 3 & 0 & 5 & 40 & 60 & 0 \\
\hline $\mathrm{BE}$ & 6 & 3 & 0 & 9 & 67 & 33 & 0 \\
\hline $\mathrm{BL}$ & 9 & 10 & 3 & 22 & 41 & 45 & 14 \\
\hline$B M$ & 0 & 2 & 2 & 4 & 0 & 50 & 50 \\
\hline $\mathrm{BN}$ & 2 & 0 & 0 & 2 & 100 & 0 & 0 \\
\hline $\mathrm{BP}$ & 7 & 0 & 0 & 7 & 100 & 0 & 0 \\
\hline $\mathrm{BO}$ & 2 & 0 & 1 & 3 & 67 & 0 & 33 \\
\hline$B R$ & 1 & 1 & 0 & 2 & 50 & 50 & 0 \\
\hline BS & 3 & 0 & 1 & 4 & 75 & 0 & 25 \\
\hline $\mathrm{BU}$ & 5 & 0 & 1 & 6 & 83 & 0 & 17 \\
\hline $\begin{array}{l}B X \\
C A\end{array}$ & 4 & 3 & 1 & $\begin{array}{l}8 \\
1\end{array}$ & 50 & 38 & 13 \\
\hline $\begin{array}{l}\text { CA } \\
\text { CC }\end{array}$ & $\begin{array}{l}1 \\
7\end{array}$ & $\begin{array}{l}0 \\
2\end{array}$ & 0 & $\begin{array}{l}1 \\
9\end{array}$ & $\begin{array}{r}100 \\
78\end{array}$ & 0 & $\begin{array}{l}0 \\
0\end{array}$ \\
\hline $\mathrm{CH}$ & 9 & $\overline{1}$ & 0 & 10 & $\begin{array}{l}78 \\
90\end{array}$ & $\begin{array}{l}22 \\
10\end{array}$ & $\begin{array}{l}0 \\
0\end{array}$ \\
\hline CL & 4 & 4 & 1 & 9 & 44 & 44 & 11 \\
\hline$C P$ & 3 & 0 & 0 & 3 & 100 & 0 & 0 \\
\hline $\mathrm{CW}$ & 5 & 0 & 0 & 5 & 100 & 0 & 0 \\
\hline$D C$ & 14 & 5 & 0 & 19 & 74 & 26 & 0 \\
\hline EG & 5 & 2 & 1 & 8 & 63 & 25 & 13 \\
\hline EI & 1 & 2 & 1 & 4 & 25 & 50 & 25 \\
\hline EL & 2 & 3 & $i$ & 6 & 33 & 50 & 17 \\
\hline EP & 2 & 0 & 1 & 3 & 67 & 0 & 33 \\
\hline ES & 11 & 1 & 1 & 13 & 85 & 8 & 8 \\
\hline FG & 2 & 2 & 0 & 4 & 50 & 50 & 0 \\
\hline $\mathrm{FL}$ & 6 & 0 & 0 & 6 & 100 & 0 & 0 \\
\hline FN & 3 & 0 & 0 & 3 & 100 & 0 & 0 \\
\hline FS & 5 & 2 & 0 & 7 & 71 & 29 & 0 \\
\hline GA & 7 & 1 & 0 & 8 & 88 & 13 & 0 \\
\hline GE & 8 & 1 & 0 & 9 & 89 & 11 & 0 \\
\hline HA & 4 & 3 & 0 & 7 & 57 & 43 & 0 \\
\hline HL & 4 & 3 & 0 & 7 & 57 & 43 & 0 \\
\hline ID & 7 & 0 & 0 & 7 & 100 & 0 & 0 \\
\hline IE & 9 & 0 & 0 & 9 & 100 & 0 & 0 \\
\hline IL. & 0 & 0 & 2 & 2 & 0 & 0 & 100 \\
\hline IN & 4 & 2 & 0 & 6 & 67 & 33 & 0 \\
\hline IS & 7 & 1 & 1 & 9 & 78 & 11 & 11 \\
\hline IT & 6 & 1 & 0 & 7 & 86 & 14 & 0 \\
\hline$J P$ & 8 & 0 & 0 & 8 & 100 & 0 & 0 \\
\hline KA & 5 & 0 & 0 & 5 & 100 & 0 & 0 \\
\hline LA & 21 & 0 & 0 & 21 & 100 & 0 & 0 \\
\hline LB & 2 & 1 & 0 & 3 & 67 & 33 & 0 \\
\hline LH & 8 & 0 & 0 & 8 & 100 & 0 & 0 \\
\hline $\mathrm{LL}$ & 4 & 0 & 0 & 4 & 100 & 0 & 0 \\
\hline
\end{tabular}

Evaluation Percentages

Total

0

17

0

0

0

0

50

0

0

13

0

11

0
0
0

13

17

33

0

0

0

0

0

0

0

0 
QAP44 Summary of Laboratory Evaluations by Matrix

Matrix: SO

Evaluation Summary

Total

Evaluation Percentages

\begin{tabular}{|c|c|c|c|c|c|c|c|}
\hline Lahende. & A & $w$ & $\mathrm{~N}$ & $\begin{array}{c}\text { lotal } \\
\text { Analyses }\end{array}$ & $\% A$ & $\% \mathrm{~W}$ & ONN \\
\hline LM & 0 & 0 & 2 & 2 & 0 & 0 & 100 \\
\hline LV & 2 & 1 & 0 & 3 & 67 & 33 & 0 \\
\hline$L W$ & 3 & 1 & 0 & 4 & 75 & 25 & 0 \\
\hline$M A$ & 2 & 1 & 0 & 3 & 67 & 33 & 0 \\
\hline $\mathrm{ME}$ & $\overline{1}$ & 1 & 0 & 2 & 50 & 50 & 0 \\
\hline$M L$ & 6 & 0 & 0 & 6 & 100 & 0 & 0 \\
\hline MS & 2 & 0 & 0 & 2 & 100 & 0 & 0 \\
\hline NA & $\overrightarrow{7}$ & 0 & 0 & 7 & 100 & 0 & 0 \\
\hline NC & 1 & 0 & 0 & 1 & 100 & 0 & 0 \\
\hline $\mathrm{NL}$ & 5 & 1 & 0 & 6 & 83 & 17 & 0 \\
\hline NM & 4 & 0 & 1 & 5 & 80 & 0 & 20 \\
\hline NR & 1 & 1 & 0 & 2 & 50 & 50 & 0 \\
\hline $\mathrm{OB}$ & 0 & 2 & 0 & 2 & 0 & 100 & 0 \\
\hline OL & 2 & 0 & 0 & 2 & 100 & 0 & 0 \\
\hline $\mathrm{DR}$ & 6 & 0 & 1 & $\overline{7}$ & 86 & 0 & 14 \\
\hline OS & 1 & 0 & 0 & 1 & 100 & 0 & 0 \\
\hline OT & 4 & 1 & 0 & 5 & 80 & 20 & 0 \\
\hline $\mathrm{PA}$ & 3 & 0 & 0 & 3 & 100 & 0 & 0 \\
\hline PB & 3 & 0 & 0 & 3 & 100 & 0 & 0 \\
\hline $\mathrm{PI}$ & 2 & 0 & 0 & 2 & 100 & 0 & 0 \\
\hline RA & $\overline{7}$ & 0 & 2 & 9 & 78 & 0 & 22 \\
\hline $\mathrm{RE}$ & 6 & 1 & 0 & 7 & 86 & 14 & 0 \\
\hline $\mathrm{RF}$ & 3 & 1 & 0 & 4 & 75 & 25 & 0 \\
\hline RG & 6 & 0 & 0 & 6 & 100 & 0 & 0 \\
\hline Rl & 0 & 0 & 2 & 2 & 0 & 0 & 100 \\
\hline SA & 2 & 0 & 0 & 2 & 100 & 0 & 0 \\
\hline SC & 5 & 1 & 1 & 7 & 71 & 14 & 14 \\
\hline SE & 1 & 4 & 1 & 6 & 17 & 67 & 17 \\
\hline SK & 2 & 0 & 0 & 2 & 100 & 0 & 0 \\
\hline SN & 4 & 1 & 1 & 6 & 67 & 17 & 17 \\
\hline SR & 4 & 0 & 1 & 5 & 80 & 0 & 20 \\
\hline SS & $i$ & 1 & 0 & 2 & 50 & 50 & 0 \\
\hline SW & 1 & 2 & 1 & 4 & 25 & 50 & 25 \\
\hline TE & 6 & 1 & 0 & 7 & 86 & 14 & 0 \\
\hline $\mathrm{TI}$ & 9 & 0 & 0 & 9 & 100 & 0 & 0 \\
\hline$T M$ & 8 & 1 & 0 & 9 & 89 & 11 & 0 \\
\hline $\mathrm{TN}$ & 8 & 1 & 0 & g & 89 & 11 & 0 \\
\hline TO & 7 & 2 & 0 & 9 & 78 & 22 & 0 \\
\hline TW & 7 & 1 & 0 & 8 & 88 & 13 & 0 \\
\hline TX & 5 & 3 & 0 & 8 & 63 & 38 & 0 \\
\hline TY & 2 & 0 & 1 & 3 & 67 & 0 & 33 \\
\hline UC & 2 & 0 & 0 & 2 & 100 & 0 & 0 \\
\hline UK & 4 & 1 & 1 & 6 & 67 & 17 & 17 \\
\hline UP & 6 & 1 & 0 & 7 & 86 & 14 & 0 \\
\hline UY & 7 & 1 & 0 & 8 & 88 & 13 & 0 \\
\hline WA & 6 & 2 & 1 & 9 & 67 & 22 & 11 \\
\hline WC & 2 & 0 & 0 & 2 & 100 & 0 & 0 \\
\hline WE & 4 & 1 & 2 & 7 & 57 & 14 & 29 \\
\hline WN & 2 & 0 & 0 & 2 & 100 & 0 & 0 \\
\hline WP & 3 & 0 & 0 & 3 & 100 & 0 & 0 \\
\hline WS & 4 & 0 & 0 & 4 & 100 & 0 & 0 \\
\hline YA & 5 & 0 & 0 & 5 & 100 & 0 & 0 \\
\hline YP & 0 & 1 & 0 & 1 & 0 & 100 & 0 \\
\hline
\end{tabular}




\section{QAP44 Summary of Laboratory Evaluations by Matrix}

Matrix: SO

\begin{tabular}{|c|c|c|c|c|c|c|c|}
\hline \multirow[b]{2}{*}{ Lahonde } & \multicolumn{3}{|c|}{ Evaluation Summary } & \multirow[b]{2}{*}{$\begin{array}{c}\text { Total } \\
\text { Analyses }\end{array}$} & \multicolumn{3}{|c|}{ Evaluation Percentages } \\
\hline & A & $w$ & N & & $\% A$ & $\% w$ & $\%$ \\
\hline $\begin{array}{l}\text { Totals } \\
107 \text { Labs: }\end{array}$ & 469 & 107 & 41 & 617 & $76 \%$ & $17 \%$ & $7 \%$ \\
\hline
\end{tabular}


QAP44 Summary of Laboratory Evaluations by Matrix

Matrix: VE

Evaluation Sụmmary

\begin{tabular}{|c|c|c|c|c|c|c|c|}
\hline Lahonde & A & $W$ & $\mathrm{~N}$ & Analyses & $\% A$ & $\% W$ & $\% \mathbf{N}$ \\
\hline$A A$ & 8 & 0 & 0 & 8 & 100 & 0 & 0 \\
\hline$A G$ & 7 & 0 & 0 & 7 & 100 & 0 & 0 \\
\hline Al & 0 & 2 & 1 & 3 & 0 & 67 & 33 \\
\hline $\mathrm{AL}$ & 2 & 0 & 0 & 2 & 100 & 0 & 0 \\
\hline AN & 7 & 1 & 0 & 8 & 88 & 13 & 0 \\
\hline$A R$ & 3 & 4 & 1 & 8 & 38 & 50 & 13 \\
\hline $\mathrm{AU}$ & 2 & 1 & 2 & 5 & 40 & 20 & 40 \\
\hline$B A$ & 4 & 0 & 0 & 4 & 100 & 0 & 0 \\
\hline$B C$ & 2 & 1 & 0 & 3 & 67 & 33 & 0 \\
\hline $\mathrm{BE}$ & 5 & 2 & 0 & 7 & 71 & 29 & 0 \\
\hline$B L$ & 23 & 1 & 0 & 24 & 96 & 4 & 0 \\
\hline$B M$ & 2 & 2 & 1 & 5 & 40 & 40 & 20 \\
\hline $\mathrm{BN}$ & 2 & 1 & 0 & 3 & 67 & 33 & 0 \\
\hline $\mathrm{BP}$ & 1 & $i$ & 1 & 3 & 33 & 33 & 33 \\
\hline $\mathrm{BO}$ & 1 & 1 & 1 & 3 & 33 & 33 & 33 \\
\hline $\mathrm{BR}$ & .0 & 1 & $i$ & 2 & 0 & 50 & 50 \\
\hline BS & 0 & 3 & 1 & 4 & 0 & 75 & 25 \\
\hline BU & 4 & 0 & $i$ & 5 & 80 & 0 & 20 \\
\hline $\mathrm{BX}$ & 3 & 3 & 0 & 6 & 50 & 50 & 0 \\
\hline CC & 4 & 3 & 0 & 7 & 57 & 43 & 0 \\
\hline $\mathrm{CH}$ & 6 & 1 & 0 & 7 & 86 & 14 & 0 \\
\hline$C L$ & 6 & 0 & 1 & 7 & 86 & 0 & 14 \\
\hline $\mathrm{CP}$ & 3 & 1 & 0 & 4 & 75 & 25 & 0 \\
\hline CW & 3 & 0 & 0 & 3 & 100 & 0 & 0 \\
\hline DC & 6 & 1 & 0 & 7 & 86 & 14 & 0 \\
\hline$E G$ & 4 & 0 & 0 & 4 & 100 & 0 & 0 \\
\hline EI & 3 & 0 & 1 & 4 & 75 & 0 & 25 \\
\hline EL & 2 & 1 & 1 & 4 & 50 & 25 & 25 \\
\hline EP & 1 & 0 & 1 & 2 & 50 & 0 & 50 \\
\hline ES & 6 & 1 & 0 & 7 & 86 & 14 & 0 \\
\hline $\mathrm{FL}$ & 4 & 0 & 0 & 4 & 100 & 0 & 0 \\
\hline FN & 3 & 0 & 0 & 3 & 100 & 0 & 0 \\
\hline GA & 5 & 1 & 0 & 6 & 83 & 17 & 0 \\
\hline $\mathrm{GE}$ & 7 & 0 & 0 & 7 & 100 & 0 & 0 \\
\hline ID & 5 & 1 & 0 & 6 & 83 & 17 & 0 \\
\hline IE & 7 & 0 & 0 & 7 & 100 & 0 & 0 \\
\hline II & 0 & 0 & 1 & 1 & 0 & 0 & 100 \\
\hline IL & 0 & 0 & 2 & 2 & 0 & 0 & 100 \\
\hline IN & 2 & 1 & 0 & 3 & 67 & 33 & 0 \\
\hline is & 6 & 0 & 0 & 6 & 100 & 0 & 0 \\
\hline IT & 4 & 3 & 0 & 7 & 57 & 43 & 0 \\
\hline $\mathrm{JP}$ & 7 & 0 & 0 & 7 & 100 & 0 & 0 \\
\hline LA & 17 & 1 & 0 & 18 & 94 & 6 & 0 \\
\hline LB & 3 & 0 & 1 & 4 & 75 & 0 & 25 \\
\hline LH & 6 & 1 & 0 & 7 & 86 & 14 & 0 \\
\hline LL & 4 & 0 & 0 & 4 & 100 & 0 & 0 \\
\hline LV & 2 & 2 & 0 & 4 & 50 & 50 & 0 \\
\hline$L W$ & 4 & 0 & 0 & 4 & 100 & 0 & 0 \\
\hline MA & 3 & 1 & 0 & 4 & 75 & 25 & 0 \\
\hline $\mathrm{ME}$ & 2 & 1 & 0 & 3 & 67 & 33 & 0 \\
\hline$M L$ & 4 & 0 & 0 & 4 & 100 & 0 & 0 \\
\hline NA & 7 & 0 & 0 & 7 & 100 & 0 & 0 \\
\hline NC & 2 & 0 & 0 & 2 & 100 & 0 & 0 \\
\hline NR & $\overline{1}$ & 2 & 0 & 3 & 33 & 67 & 0 \\
\hline
\end{tabular}

Evaluation Percentages

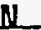


DAP44 Summary of Laboratory Evaluations by Matrix

Matrix: VE

\begin{tabular}{|c|c|c|c|c|c|c|c|}
\hline \multirow[b]{2}{*}{ Lahcorle } & \multicolumn{3}{|c|}{ Evaluation Summary } & \multirow{2}{*}{$\begin{array}{c}\text { Total } \\
\text { Analyses }\end{array}$} & \multicolumn{3}{|c|}{ Evaluation Percentages } \\
\hline & $A$ & $\mathbf{w}$ & N & & $\% \mathrm{~A}$ & $\% w$ & $\% N$ \\
\hline $\begin{array}{l}O L \\
O R \\
O T \\
O T \\
P A \\
P B \\
R A \\
R E \\
R E \\
R F \\
R I \\
S E \\
S K \\
S K \\
S N \\
S R \\
S S \\
S W \\
T E \\
T I \\
T M \\
T N \\
T D \\
T D \\
T W \\
T X \\
T Y \\
U C \\
W C \\
W A \\
W C \\
W E \\
W N \\
W P \\
\text { W } \\
Y A\end{array}$ & $\begin{array}{l}3 \\
6 \\
4 \\
0 \\
1 \\
5 \\
5 \\
6 \\
1 \\
3 \\
5 \\
3 \\
3 \\
2 \\
3 \\
5 \\
5 \\
7 \\
4 \\
6 \\
6 \\
6 \\
7 \\
7 \\
4 \\
4 \\
3 \\
4 \\
3 \\
3\end{array}$ & $\begin{array}{l}0 \\
1 \\
0 \\
1 \\
0 \\
1 \\
0 \\
0 \\
1 \\
2 \\
0 \\
1 \\
1 \\
0 \\
2 \\
0 \\
3 \\
1 \\
1 \\
1 \\
0 \\
2 \\
1 \\
0 \\
1 \\
0 \\
0 \\
0 \\
2 \\
0\end{array}$ & $\begin{array}{l}0 \\
0 \\
1 \\
0 \\
0 \\
0 \\
1 \\
0 \\
0 \\
0 \\
2 \\
0 \\
0 \\
0 \\
0 \\
0 \\
2 \\
0 \\
0 \\
0 \\
0 \\
0 \\
0 \\
0 \\
0 \\
0 \\
0 \\
0 \\
0 \\
0 \\
0 \\
0\end{array}$ & $\begin{array}{l}3 \\
7 \\
5 \\
5 \\
1 \\
1 \\
7 \\
6 \\
7 \\
7 \\
6 \\
7 \\
3 \\
4 \\
4 \\
5 \\
3 \\
7 \\
7 \\
7 \\
7 \\
7 \\
7 \\
7 \\
7 \\
7 \\
6 \\
5 \\
5 \\
3 \\
6 \\
3\end{array}$ & $\begin{array}{r}100 \\
86 \\
80 \\
0 \\
100 \\
71 \\
100 \\
100 \\
50 \\
71 \\
100 \\
75 \\
40 \\
100 \\
71 \\
100 \\
57 \\
86 \\
86 \\
86 \\
100 \\
67 \\
80 \\
100 \\
67 \\
100 \\
60 \\
100 \\
50 \\
100\end{array}$ & $\begin{array}{r}0 \\
14 \\
0 \\
100 \\
0 \\
14 \\
0 \\
0 \\
17 \\
29 \\
0 \\
25 \\
20 \\
0 \\
29 \\
0 \\
43 \\
14 \\
14 \\
14 \\
0 \\
33 \\
20 \\
0 \\
17 \\
0 \\
0 \\
0 \\
50 \\
0\end{array}$ & $\begin{array}{r}0 \\
0 \\
20 \\
0 \\
0 \\
14 \\
0 \\
0 \\
33 \\
0 \\
0 \\
0 \\
40 \\
0 \\
0 \\
0 \\
0 \\
0 \\
0 \\
0 \\
0 \\
0 \\
0 \\
0 \\
17 \\
0 \\
40 \\
0 \\
0 \\
0\end{array}$ \\
\hline $\begin{array}{l}\text { Totals } \\
84 \text { Labs: }\end{array}$ & 344 & 68 & 27 & 439 & $78 \%$ & $15 \%$ & $6 \%$ \\
\hline
\end{tabular}


QAP44 Summary of Laboratory Evaluations by Matrix

Matrix: WA

\begin{tabular}{|c|c|c|c|c|c|c|c|}
\hline \multirow[b]{2}{*}{ Lahende. } & \multicolumn{3}{|c|}{ Evaluation Summary } & \multirow{2}{*}{$\begin{array}{c}\text { Total } \\
\text { Analyses }\end{array}$} & \multicolumn{3}{|c|}{ Evaluation Percentages } \\
\hline & A. & $w$ & $\mathbf{N}$ & & $\%$ & $\% W$ & $\% \mathrm{~N}$ \\
\hline AA & 15 & 0 & 0 & 15 & 100 & 0 & 0 \\
\hline$A C$ & 6 & 1 & 1 & 8 & 75 & 13 & 13 \\
\hline $\mathrm{AE}$ & 9 & 1 & 0 & 10 & 90 & 10 & 0 \\
\hline$\ddot{A G}$ & 10 & 1 & 2 & 13 & 77 & 8 & 15 \\
\hline Al & 5 & 3 & 1 & 9 & 56 & 33 & 11 \\
\hline AL: & 3 & 0 & 0 & 3 & 100 & 0 & 0 \\
\hline$A M$ & 4 & 2 & 1 & 7 & 57 & 29 & 14 \\
\hline AN & 11 & 1 & 0 & 12 & 92 & 8 & 0 \\
\hline AR & 6 & 6 & 2 & 14 & 43 & 43 & 14 \\
\hline$A U$ & 2 & 4 & 3 & 9 & 22 & 44 & 33 \\
\hline AW & 5 & 1 & 1 & 7 & 71 & 14 & 14 \\
\hline BA & 5 & 2 & 0 & 7 & 71 & 29 & 0 \\
\hline $\mathrm{BC}$ & 4 & 4 & 0 & 8 & 50 & 50 & 0 \\
\hline BE & 7 & 4 & 2 & 13 & 54 & 31 & 15 \\
\hline $\mathrm{BK}$ & 1 & 3 & 0 & 4 & 25 & 75 & 0 \\
\hline BL & 38 & 3 & 6 & 47 & 81 & 6 & 13 \\
\hline BM & 6 & 2 & 0 & 8 & 75 & 25 & 0 \\
\hline BN & 7 & 0 & 0 & 7 & 100 & 0 & 0 \\
\hline $\mathrm{BP}$ & 4 & 4 & 1 & 9 & 44 & 44 & 11 \\
\hline BQ & 3 & 1 & 0 & 4 & 75 & 25 & 0 \\
\hline $\mathrm{BR}$ & 1 & 2 & 0 & 3 & 33 & 67 & 0 \\
\hline BS & 5 & 1 & 0 & 6 & 83 & 17 & 0 \\
\hline $\mathrm{BU}$ & 7 & 1 & 1 & 9 & 78 & 11 & 11 \\
\hline BX & 9 & 4 & 0 & 13 & 69 & 31 & 0 \\
\hline CC & 10 & 0 & 1 & 11 & 91 & 0 & 9 \\
\hline $\mathrm{CH}$ & 10 & 3 & 1 & 14 & 71 & 21 & 7 \\
\hline $\mathrm{CL}$ & 7 & 4 & 0 & 11 & 64 & 36 & 0 \\
\hline $\mathrm{CP}$ & 5 & 0 & 1 & 6 & 83 & 0 & 17 \\
\hline CS & 1 & 0 & 0 & 1 & 100 & 0 & 0 \\
\hline CW & 5 & 0 & 0 & 5 & 100 & 0 & 0 \\
\hline CZ & 1 & 0 & 0 & 1 & 100 & 0 & 0 \\
\hline$D C$ & 12 & 2 & 2 & 16 & 75 & 13 & 13 \\
\hline EG & 6 & 5 & 0 & 11 & 55 & 45 & 0 \\
\hline $\mathrm{EI}$ & 2 & 5 & 0 & 7 & 29 & 71 & 0 \\
\hline $\mathrm{EL}$ & 4 & 4 & 1 & 9 & 44 & 44 & 11 \\
\hline EP & 6 & 3 & 0 & 9 & 67 & 33 & 0 \\
\hline ES & 11 & 4 & 1 & 16 & 69 & 25 & 6 \\
\hline FG & 8 & $i$ & 2 & 11 & 73 & 9 & 18 \\
\hline $\mathrm{FL}$ & 5 & 4 & 0 & 9 & 56 & 44 & 0 \\
\hline$F M$ & 3 & $i$ & 0 & 4 & 75 & 25 & 0 \\
\hline FN & 4 & 0 & 0 & 4 & 100 & 0 & 0 \\
\hline $\mathrm{GA}$ & 10 & 0 & 0 & 10 & 100 & 0 & 0 \\
\hline GE & 5 & 8 & 0 & 13 & 38 & 62 & 0 \\
\hline GS & 0 & 1 & 0 & 1 & 0 & 100 & 0 \\
\hline HA & 9 & 4 & 0 & 13 & 69 & 31 & 0 \\
\hline $\mathrm{HC}$ & 2 & 1 & 0 & 3 & 67 & 33 & 0 \\
\hline HL & 6 & 2 & 0 & 8 & 75 & 25 & 0 \\
\hline 10 & 5 & 2 & 1 & 8 & 63 & 25 & 13 \\
\hline IE & 10 & 1 & 2 & 13 & 77 & 8 & 15 \\
\hline II & 1 & 2 & 2 & 5 & 20 & 40 & 40 \\
\hline IN & 5 & 2 & 0 & 7 & 71 & 29 & 0 \\
\hline $\mathbb{R}$ & 0 & 0 & 1 & 1 & 0 & 0 & 100 \\
\hline IS & 8 & 3 & 1 & 12 & 67 & 25 & 8 \\
\hline IT & 5 & 5 & $i$ & 11 & 45 & 45 & 9 \\
\hline
\end{tabular}


QAP44 Summary of Laboratory Evaluations by Matrix

Matrix: WA

Evaluation Summary

\begin{tabular}{|c|c|c|c|c|c|c|c|}
\hline \multirow[b]{2}{*}{ Lahonde } & \multicolumn{3}{|c|}{ Evaluation Summary } & & \multicolumn{2}{|c|}{ Evaluation Percentages } \\
\hline & A & $w$ & $\mathbf{N}$ & Analyses. & $\% A$ & $\% W$ & $\%$ \\
\hline $\mathrm{JP}$ & 12 & 0 & 0 & 12 & 100 & 0 & 0 \\
\hline KA & 10 & 1 & 0 & 11 & 91 & 9 & 0 \\
\hline LA & 19 & 5 & 9 & 33 & 58 & 15 & 27 \\
\hline LB & 1 & 2 & 1 & 4 & 25 & 50 & 25 \\
\hline $\mathrm{LH}$ & 12 & 0 & 1 & 13 & 92 & 0 & 8 \\
\hline$\ddot{L L}$ & 5 & 2 & 3 & 10 & 50 & 20 & 30 \\
\hline$L M$ & 1 & $\overline{0}$ & 3 & 4 & 25 & 0 & 75 \\
\hline LV & 2 & 2 & 0 & 4 & 50 & 50 & 0 \\
\hline$L W$ & 5 & 1 & 0 & 6 & 83 & 17 & 0 \\
\hline$M E$ & 1 & 2 & 0 & 3 & 33 & 67 & 0 \\
\hline$M I$ & 4 & 6 & 2 & 12 & 33 & 50 & 17 \\
\hline$M L$ & 7 & 1 & 0 & 8 & 88 & 13 & 0 \\
\hline MS & 3 & 1 & 0 & 4 & 75 & 25 & 0 \\
\hline NA & 5 & 2 & 1 & 8 & 63 & 25 & 13 \\
\hline $\mathrm{NC}$ & 3 & 0 & 0 & 3 & 100 & 0 & 0 \\
\hline NL & 6 & 2 & 0 & 8 & 75 & 25 & 0 \\
\hline NM & 8 & 1 & 0 & 9 & 89 & 11 & 0 \\
\hline $\mathrm{OB}$ & 1 & $i$ & 0 & 2 & 50 & 50 & 0 \\
\hline$O D$ & 4 & 2 & 0 & 6 & 67 & 33 & 0 \\
\hline $\mathrm{OL}$ & 3 & 0 & 0 & 3 & 100 & 0 & 0 \\
\hline $\mathrm{OR}$ & 8 & 1 & 0 & 9 & 89 & 11 & 0 \\
\hline OT & 6 & 4 & 1 & 11 & 55 & 36 & 9 \\
\hline PA & 5 & 0 & 1 & 6 & 83 & 0 & 17 \\
\hline $\mathrm{PB}$ & 5 & 0 & 1 & 6 & 83 & 0 & 17 \\
\hline $\mathrm{PI}$ & 5 & 0 & 0 & 5 & 100 & 0 & $\ddot{0}$ \\
\hline RE & 11 & 2 & 0 & 13 & 85 & 15 & 0 \\
\hline $\mathrm{RF}$ & 6 & 2 & 0 & 8 & 75 & 25 & 0 \\
\hline $\mathrm{RG}$ & 7 & 2 & 0 & 9 & 78 & 22 & 0 \\
\hline $\mathrm{RI}$ & 5 & 2 & 2 & 9 & 56 & 22 & 22 \\
\hline SA & 5 & 0 & 0 & 5 & 100 & 0 & 0 \\
\hline SC & 9 & 2 & 2 & 13 & 69 & 15 & 15 \\
\hline SE & 5 & 5 & 1 & 11 & 45 & 45 & 9 \\
\hline SK & 4 & 0 & 0 & 4 & 100 & 0 & 0 \\
\hline SN & 6 & 1 & 0 & 7 & 86 & 14 & 0 \\
\hline SR & 10 & 2 & 0 & 12 & 83 & 17 & 0 \\
\hline SS & 3 & 0 & 0 & 3 & 100 & 0 & 0 \\
\hline SV & 5 & 0 & 0 & 5 & 100 & 0 & 0 \\
\hline SW & 5 & 0 & 2 & 7 & 71 & 0 & 29 \\
\hline $\mathrm{TE}$ & 9 & 3 & 0 & 12 & 75 & 25 & 0 \\
\hline TI & 9 & 1 & 4 & 14 & 64 & 7 & 29 \\
\hline $\mathrm{TM}$ & 10 & 3 & 0 & 13 & 77 & 23 & 0 \\
\hline TN & 10 & 4 & 1 & 15 & 67 & 27 & 7 \\
\hline TO & 11 & $i$ & 1 & 13 & 85 & 8 & 8 \\
\hline TW & 6 & 4 & 2 & 12 & 50 & 33 & 17 \\
\hline TX & 2 & 5 & 3 & 10 & 20 & 50 & 30 \\
\hline UC & $\overline{5}$ & 1 & 0 & 6 & 83 & 17 & 0 \\
\hline UK & 6 & 0 & 1 & 7 & 86 & 0 & 14 \\
\hline UP & 11 & 2 & 0 & 13 & 85 & 15 & 0 \\
\hline UY & 11 & 0 & 2 & 13 & 85 & 0 & 15 \\
\hline WA & 10 & 1 & 2 & 13 & 77 & 8 & 15 \\
\hline WC & 4 & 7 & 1 & 12 & 33 & 58 & 8 \\
\hline WE & 3 & 4 & 1 & 8 & 38 & 50 & 13 \\
\hline WI & 1 & 2 & 0 & 3 & 33 & 67 & 0 \\
\hline WN & 8 & 2 & 1 & 11 & 73 & 18 & 9 \\
\hline
\end{tabular}

Evaluation Percentages 


\section{QAP44 Summary of Laboratory Evaluations by Matrix}

Matrix: WA

\begin{tabular}{|c|c|c|c|c|c|c|c|}
\hline \multirow[b]{2}{*}{ Lahcade } & \multicolumn{3}{|c|}{ Evaluation Summary } & \multirow{2}{*}{$\begin{array}{c}\text { Total } \\
\text { Analyses }\end{array}$} & \multicolumn{3}{|c|}{ Evaluation Percentages } \\
\hline & A & $\mathbf{w}$ & $n$ & & $\%$ & \%w & $\% N$ \\
\hline $\begin{array}{l}W P \\
W V \\
Y A \\
Y P\end{array}$ & $\begin{array}{l}5 \\
6 \\
8 \\
1\end{array}$ & $\begin{array}{l}0 \\
1 \\
1 \\
0\end{array}$ & $\begin{array}{l}0 \\
0 \\
0 \\
0\end{array}$ & $\begin{array}{l}5 \\
7 \\
9 \\
1\end{array}$ & $\begin{array}{r}100 \\
86 \\
89 \\
100\end{array}$ & $\begin{array}{r}0 \\
14 \\
11 \\
0\end{array}$ & $\begin{array}{l}0 \\
0 \\
0 \\
0\end{array}$ \\
\hline $\begin{array}{l}\text { Totals } \\
112 \text { Labs: }\end{array}$ & 697 & 214 & 87 & 998 & $70 \%$ & $21 \%$ & $\mathbf{9 \%}$ \\
\hline
\end{tabular}


QAP44 Summary of Matrix Evaluations by Radionuclide

Matrix: AI

\begin{tabular}{|c|c|c|c|c|c|c|c|}
\hline \multirow{2}{*}{$\begin{array}{l}\text { Radio. } \\
\text { Nuclide }\end{array}$} & \multicolumn{3}{|c|}{ Evaluation Summary } & \multirow[b]{2}{*}{$\begin{array}{c}\text { Total } \\
\text { Analyses }\end{array}$} & \multicolumn{3}{|c|}{ Evaluation Percentages } \\
\hline & A & $w$ & $\mathbf{N}$ & & $\% A$ & $\% \mathrm{~W}$ & $\% \mathrm{~N}$ \\
\hline $\begin{array}{l}\text { AM241 } \\
\text { CE144 } \\
\text { CD 57 } \\
\text { CO 60 } \\
\text { CS134 } \\
\text { CS137 } \\
\text { GA 1 } \\
\text { GB 2 } \\
\text { MN 54 } \\
\text { PU238 } \\
\text { PU239 } \\
\text { RU106 } \\
\text { SB125 } \\
\text { SR 90 } \\
\text { U 234 } \\
\text { U 238 } \\
\text { U BO } \\
\text { U UG }\end{array}$ & $\begin{array}{l}45 \\
59 \\
61 \\
67 \\
73 \\
59 \\
46 \\
49 \\
51 \\
55 \\
52 \\
67 \\
74 \\
36 \\
30 \\
34 \\
11 \\
20\end{array}$ & $\begin{array}{r}15 \\
21 \\
24 \\
17 \\
14 \\
29 \\
13 \\
8 \\
30 \\
6 \\
9 \\
9 \\
12 \\
11 \\
11 \\
5 \\
2 \\
5\end{array}$ & $\begin{array}{r}2 \\
11 \\
5 \\
9 \\
4 \\
5 \\
5 \\
5 \\
9 \\
1 \\
2 \\
0 \\
1 \\
4 \\
5 \\
5 \\
3 \\
2\end{array}$ & $\begin{array}{l}62 \\
91 \\
90 \\
93 \\
91 \\
93 \\
64 \\
62 \\
90 \\
62 \\
63 \\
76 \\
87 \\
51 \\
46 \\
44 \\
16 \\
27\end{array}$ & $\begin{array}{l}73 \\
65 \\
68 \\
72 \\
80 \\
63 \\
72 \\
79 \\
57 \\
89 \\
83 \\
88 \\
85 \\
71 \\
65 \\
77 \\
69 \\
74\end{array}$ & $\begin{array}{l}24 \\
23 \\
27 \\
18 \\
15 \\
31 \\
20 \\
13 \\
33 \\
10 \\
14 \\
12 \\
14 \\
22 \\
24 \\
11 \\
13 \\
19\end{array}$ & $\begin{array}{r}3 \\
12 \\
6 \\
10 \\
4 \\
5 \\
8 \\
8 \\
10 \\
2 \\
3 \\
0 \\
1 \\
8 \\
11 \\
11 \\
19 \\
7\end{array}$ \\
\hline Totals: & 889 & 241 & 78 & 1208 & $74 \%$ & $20 \%$ & $6 \%$ \\
\hline
\end{tabular}




\section{QAP44 Summary of Matrix Evaluations by Radionuclide}

Matrix: SO

\begin{tabular}{|c|c|c|c|c|c|c|c|}
\hline \multirow[b]{2}{*}{$\begin{array}{l}\text { Radio- } \\
\text { Nuclide }\end{array}$} & \multicolumn{3}{|c|}{ Evaluation Summary } & \multirow[b]{2}{*}{$\begin{array}{c}\text { Total } \\
\text { Analyses }\end{array}$} & \multicolumn{3}{|c|}{ Evaluation Percentages } \\
\hline & A & $w$ & $\boldsymbol{N}$ & & $\% A$ & $\% w$ & $\% N$ \\
\hline $\begin{array}{l}\text { AM241 } \\
\text { CS137 } \\
\text { K } 40 \\
\text { PU238 } \\
\text { PU239 } \\
\text { SR 90 } \\
\text { U } 234 \\
\text { U } 238 \\
\text { U BO } \\
\text { U UG }\end{array}$ & $\begin{array}{l}42 \\
86 \\
76 \\
66 \\
60 \\
33 \\
25 \\
41 \\
14 \\
26\end{array}$ & $\begin{array}{r}18 \\
9 \\
10 \\
1 \\
10 \\
11 \\
21 \\
17 \\
3 \\
7\end{array}$ & $\begin{array}{r}2 \\
4 \\
6 \\
2 \\
3 \\
11 \\
7 \\
2 \\
2 \\
2\end{array}$ & $\begin{array}{l}62 \\
99 \\
92 \\
69 \\
73 \\
55 \\
53 \\
60 \\
19 \\
35\end{array}$ & $\begin{array}{l}68 \\
87 \\
83 \\
96 \\
82 \\
60 \\
47 \\
68 \\
74 \\
74\end{array}$ & $\begin{array}{r}29 \\
9 \\
11 \\
1 \\
14 \\
20 \\
40 \\
28 \\
16 \\
20\end{array}$ & $\begin{array}{r}3 \\
4 \\
7 \\
3 \\
4 \\
20 \\
13 \\
3 \\
11 . \\
6\end{array}$ \\
\hline Totals: & 469 & 107 & 41 & 617 & $76 \%$ & $17 \%$ & $7 \%$ \\
\hline
\end{tabular}


QAP44 Summary of Matrix Evaluations by Radionuclide

Matrix: VE

\begin{tabular}{|c|c|c|c|c|c|c|c|}
\hline \multirow[b]{2}{*}{$\begin{array}{l}\text { Radio- } \\
\text { Mualide }\end{array}$} & \multicolumn{3}{|c|}{ Evaluation Summary } & \multirow[b]{2}{*}{$\begin{array}{c}\text { Total } \\
\text { Analyses }\end{array}$} & \multicolumn{3}{|c|}{ Evaluation Percentages } \\
\hline & A & $w$ & N & & $\% A$ & $\% W$ & $\% \mathrm{~N}$ \\
\hline $\begin{array}{l}\text { AM241 } \\
\text { CM244 } \\
\text { CO 60 } \\
\text { CS137 } \\
\text { K 40 } \\
\text { PU238 } \\
\text { PU239 } \\
\text { SR } 90\end{array}$ & $\begin{array}{r}48 \\
27 \\
68 \\
55 \\
58 \\
6 \\
49 \\
33\end{array}$ & $\begin{array}{r}6 \\
8 \\
8 \\
14 \\
14 \\
1 \\
6 \\
11\end{array}$ & $\begin{array}{l}0 \\
0 \\
3 \\
9 \\
3 \\
1 \\
4 \\
7\end{array}$ & $\begin{array}{r}54 \\
35 \\
79 \\
78 \\
75 \\
8 \\
59 \\
51\end{array}$ & $\begin{array}{l}89 \\
77 \\
86 \\
71 \\
77 \\
75 \\
83 \\
65\end{array}$ & $\begin{array}{l}11 \\
23 \\
10 \\
18 \\
19 \\
13 \\
10 \\
22\end{array}$ & $\begin{array}{r}0 \\
0 \\
4 \\
12 \\
4 \\
13 \\
7 \\
14\end{array}$ \\
\hline Totals: & 344 & 68 & 27 & 439 & $78 \%$ & $15 \%$ & $6 \%$ \\
\hline
\end{tabular}


QAP44 Summary of Matrix Evaluations by Radionuclide

Matrix: WA

\begin{tabular}{|c|c|c|c|c|c|c|c|}
\hline \multirow[b]{2}{*}{$\begin{array}{l}\text { Radio- } \\
\text { Nuclide }\end{array}$} & \multicolumn{3}{|c|}{ Evaluation Summary } & \multirow[b]{2}{*}{$\begin{array}{c}\text { Totál } \\
\text { Analyses }\end{array}$} & \multicolumn{3}{|c|}{ Evaluation Percentages } \\
\hline & $A$ & $w$ & $\mathbf{N}$ & & $\%$ & \%W & $\%$ \\
\hline $\begin{array}{l}\text { AM241 } \\
\text { CD 60 } \\
\text { CS137 } \\
\text { FE 55 } \\
\text { GA 1 } \\
\text { GB 2 } \\
\text { H } 3 \\
\text { MN 54 } \\
\text { PU238 } \\
\text { PU239 } \\
\text { SR 90 } \\
\text { U } 234 \\
\text { U } 238 \\
\text { U BO } \\
\text { U UG }\end{array}$ & $\begin{array}{r}53 \\
83 \\
74 \\
7 \\
58 \\
31 \\
49 \\
61 \\
54 \\
70 \\
44 \\
40 \\
40 \\
11 \\
22\end{array}$ & $\begin{array}{r}16 \\
14 \\
22 \\
6 \\
5 \\
12 \\
32 \\
25 \\
18 \\
8 \\
16 \\
14 \\
16 \\
9 \\
1\end{array}$ & $\begin{array}{r}5 \\
4 \\
5 \\
0 \\
4 \\
23 \\
2 \\
12 \\
1 \\
0 \\
5 \\
5 \\
4 \\
3 \\
14\end{array}$ & $\begin{array}{r}74 \\
101 \\
101 \\
13 \\
67 \\
66 \\
83 \\
98 \\
73 \\
78 \\
65 \\
59 \\
60 \\
23 \\
37\end{array}$ & $\begin{array}{l}72 \\
82 \\
73 \\
54 \\
87 \\
47 \\
59 \\
62 \\
74 \\
90 \\
68 \\
68 \\
67 \\
48 \\
59\end{array}$ & $\begin{array}{r}22 \\
14 \\
22 \\
46 \\
7 \\
18 \\
39 \\
26 \\
25 \\
10 \\
25 \\
24 \\
27 \\
39 \\
3\end{array}$ & $\begin{array}{r}7 \\
4 \\
5 \\
0 \\
6 \\
35 \\
2 \\
12 \\
1 \\
0 \\
8 \\
8 \\
7 \\
13 \\
38\end{array}$ \\
\hline Totals: & 697 & 214 & 87 & 998 & $70 \%$ & $21 \%$ & $\mathbf{9} \%$ \\
\hline
\end{tabular}




\section{OAP44 Results by Laboratory}

Lab: AA Environmental Measurements Laboratory

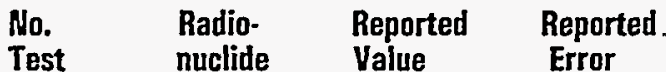

Matrix: Al

$\begin{array}{llrl}1 & \text { AM241 } & 0.189 & 0.007 \\ 1 & \text { CE144 } & 33.300 & 3.300 \\ 1 & \text { CO 57 } & 8.900 & 0.900 \\ 1 & \text { CO 60 } & 29.500 & 2.900 \\ 1 & \text { CS134 } & 14.700 & 1.460 \\ 1 & \text { CS137 } & 6.640 & 0.700 \\ 1 & \text { GA 1 } & 1.620 & 0.150 \\ 1 & \text { GB 2 } & 1.770 & 0.150 \\ 1 & \text { MN 54 } & 3.440 & 0.380 \\ 1 & \text { PU238 } & 0.096 & 0.002 \\ 1 & \text { PU239 } & 0.093 & 0.003 \\ 1 & \text { RU106 } & 11.600 & 1.440 \\ 1 & \text { SB125 } & 9.780 & 1.030 \\ 1 & \text { SR 90 } & 1.060 & 0.037 \\ 1 & \text { U 234 } & 0.052 & 0.002 \\ 1 & \text { U 238 } & 0.053 & 0.002 \\ 1 & \text { U B0 } & 0.107 & 0.003 \\ 1 & \text { U UG } & 4.310 & 0.100\end{array}$

Matrix: So

$\begin{array}{llrr}1 & \text { AMi241 } & 3.690 & 0.454 \\ 1 & \text { CS137 } & 359.000 & 10.000 \\ 1 & \text { K 40 } & 465.000 & 30.000 \\ 1 & \text { PU238 } & 43.000 & 2.440 \\ 1 & \text { PU239 } & 9.230 & 0.346 \\ 1 & \text { SR } 90 & 1340.000 & 113.000 \\ 1 & \text { U } 234 & 34.200 & 3.520 \\ 1 & \text { U } 238 & 35.900 & 3.630 \\ 1 & \text { U BO } & 71.700 & 4.150 \\ 1 & \text { U UG } & 2.900 & 0.290\end{array}$

Matrix: VE

$\begin{array}{rlrr}1 & \text { AM241 } & 5.600 & 0.184 \\ 1 & \text { CM244 } & 4.440 & 0.202 \\ 1 & \text { CO 60 } & 59.700 & 0.963 \\ \text { CS137 } & 944.000 & 16.200 \\ 1 & \text { K 40 } & 1030.000 & 33.000 \\ 1 & \text { PU238 } & 0.820 & 0.104 \\ 1 & \text { PU239 } & 9.820 & 1.220 \\ 1 & \text { SR 90 } & 1300.000 & 52.400\end{array}$

Matrix: WA

$\begin{array}{llll}1 & \text { AM241 } & 0.766 & 0.013\end{array}$

Units for matrices: $A \mathrm{l}=\mathrm{Bq} / \mathrm{filter} \mathrm{SO}=\mathrm{Bq} / \mathrm{kg} V \mathrm{E}=\mathrm{Bq} / \mathrm{kg} W \mathrm{~W}=\mathrm{Bq} / \mathrm{L}$. Values for elemental uranium are reported in $\mu \mathrm{g} / f i l t e r, \mathrm{~g}$, or $\mathrm{mL}$.

Evaluation: $A=A c c e p t a b l e, W=A c c e p t a b l e$ with Warning, $N=$ Not Acceptable

$\mathrm{pCi}=\mathrm{Bq} \times 27$ 


\section{OAP44 Results by Laboratory}

Lab: AA Environmental Measurements Laboratory

\begin{tabular}{llll}
$\begin{array}{l}\text { No. } \\
\text { Test }\end{array}$ & $\begin{array}{l}\text { Radio- } \\
\text { nuclide }\end{array}$ & $\begin{array}{l}\text { Reported } \\
\text { Value }\end{array}$ & $\begin{array}{l}\text { Reported } \\
\text { Error }\end{array}$ \\
\hline
\end{tabular}

Matrix: WA

$\begin{array}{llrr}1 & \text { CO 60 } & 32.800 & 0.580 \\ 1 & \text { CS137 } & 38.300 & 0.881 \\ 1 & \text { FE 55 } & 83.000 & 3.440 \\ 1 & \text { GA 1 } & 1850.000 & 185.000 \\ 1 & \text { GB 2 } & 744.000 & 74.000 \\ 1 & \text { H } 3 & 251.000 & 11.400 \\ 1 & \text { MN 54 } & 38.400 & 1.160 \\ 1 & \text { PU238 } & 0.982 & 0.074 \\ 1 & \text { PU239 } & 0.772 & 0.056 \\ 1 & \text { SR 90 } & 1.450 & 0.034 \\ 1 & \text { U 234 } & 0.274 & 0.019 \\ 1 & \text { U 238 } & 0.275 & 0.024 \\ 1 & \text { U BO } & 0.559 & 0.047 \\ 1 & \text { U UG } & 0.022 & 0.003\end{array}$

Units for matrices: $\mathrm{Al}=\mathrm{Bq} /$ filter $S O=B q / \mathrm{kg} V E=B q / \mathrm{kg} W A=B q \mid L$. Values for elemental uranium are reported in $\mu \mathrm{g} / \mathrm{filter}, \mathrm{g}$, or $\mathrm{mL}$. $\mathrm{pCi}=\mathrm{Bq} \times 27$ 
QAP44 Results by Laboratory

Lab: AC Analytical Chemistry Laboratory, Argonne National Lab

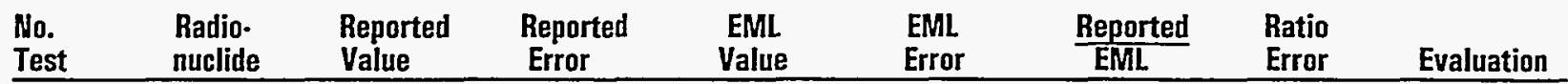

Matrix: Al

$\begin{array}{llrllllll}1 & \text { AM241 } & 0.174 & 0.005 & 0.189 & 0.007 & 0.920 & 0.04 & \text { A } \\ 1 & \text { CE144 } & 23.100 & 2.300 & 33.300 & 3.300 & 0.690 & 0.10 & \text { A } \\ 1 & \text { CO 57 } & 6.240 & 0.110 & 8.900 & 0.900 & 0.700 & 0.07 & \text { W } \\ 1 & \text { CO 60 } & 24.900 & 0.400 & 29.500 & 2.900 & 0.840 & 0.08 & \text { A } \\ 1 & \text { CS134 } & 13.500 & 0.300 & 14.700 & 1.460 & 0.920 & 0.09 & \text { A } \\ 1 & \text { CS137 } & 5.530 & 0.210 & 6.640 & 0.700 & 0.830 & 0.09 & \text { W } \\ 1 & \text { MN 54 } & 2.660 & 0.140 & 3.440 & 0.380 & 0.770 & 0.09 & \text { W } \\ 1 & \text { PU238 } & 0.098 & 0.004 & 0.096 & 0.002 & 1.020 & 0.05 & \text { A } \\ 1 & \text { PU239 } & 0.104 & 0.004 & 0.093 & 0.003 & 1.120 & 0.06 & \text { A } \\ 1 & \text { SB125 } & 9.530 & 0.800 & 9.780 & 1.030 & 0.970 & 0.13 & \text { A } \\ 1 & \text { U 234 } & 0.050 & 0.002 & 0.052 & 0.002 & 0.970 & 0.05 & \text { A } \\ 1 & \text { U 238 } & 0.048 & 0.002 & 0.053 & 0.002 & 0.900 & 0.05 & \text { A }\end{array}$

Matrix: So

$\begin{array}{llrrrrrrr}1 & \text { AM241 } & 4.550 & 0.440 & 3.690 & 0.454 & 1.230 & 0.19 & \text { A } \\ 1 & \text { CS137 } & 382.000 & 2.000 & 359.000 & 10.000 & 1.060 & 0.03 & \text { A } \\ 1 & \text { K 40 } & 472.000 & 12.000 & 465.000 & 30.000 & 1.020 & 0.07 & \text { A } \\ 1 & \text { PU238 } & 44.800 & 1.500 & 43.000 & 2.440 & 1.040 & 0.07 & \text { A } \\ 1 & \text { PU239 } & 9.250 & 0.460 & 9.230 & 0.346 & 1.000 & 0.06 & \text { A }\end{array}$

Matrix: WA

$\begin{array}{llrlrllll}1 & \text { AM241 } & 0.815 & 0.044 & 0.766 & 0.013 & 1.060 & 0.06 & \text { A } \\ 1 & \text { CO 60 } & 34.000 & 0.900 & 32.800 & 0.580 & 1.040 & 0.03 & \text { A } \\ 1 & \text { CS137 } & 40.200 & 0.800 & 38.300 & 0.881 & 1.050 & 0.03 & \text { A } \\ 1 & \text { MN 54 } & 50.300 & 1.200 & 38.400 & 1.160 & 1.310 & 0.05 & \text { N } \\ 1 & \text { PU238 } & 1.010 & 0.060 & 0.982 & 0.074 & 1.030 & 0.10 & \text { A } \\ 1 & \text { PU239 } & 0.813 & 0.048 & 0.772 & 0.056 & 1.050 & 0.10 & \text { A } \\ 1 & \text { U 234 } & 0.241 & 0.013 & 0.274 & 0.019 & 0.880 & 0.08 & \text { W } \\ 1 & \text { U 238 } & 0.246 & 0.014 & 0.275 & 0.024 & 0.900 & 0.09 & \text { A }\end{array}$

Units for matrices: $\mathrm{Al}=\mathrm{Bq}$ lifilter $\mathrm{SO}=\mathrm{Bq} / \mathrm{kg} V \mathrm{VE}=\mathrm{Bq} / \mathrm{kg} W \mathrm{WA}=\mathrm{Bq} \mid \mathrm{L}$. Values for elemental uranium are reported in $\mu \mathrm{g} / \mathrm{filter}, \mathrm{g}$, or $\mathrm{mL}$.

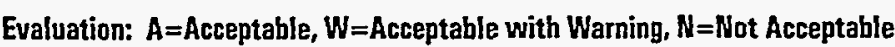

$\mathrm{pCi}=\mathrm{Bq} \times 27$ 


\section{QAP44 Results by Laboratory}

Lab: AE Analytical Resources, Inc., Seattle

No.

Radio-

Reported · Reported

EML

Error

EML

Value Error

Reported Ratio

EML Error

Evaluation

Matrix: So

$\begin{array}{llrr}1 & \text { AM241 } & 2.660 & 0.510 \\ 1 & \text { CS137 } & 405.000 & 25.700 \\ 1 & \text { K } 40 & 534.000 & 72.000 \\ 1 & \text { PU238 } & 42.800 & 1.620 \\ 1 & \text { PU239 } & 8.510 & 0.740 \\ 1 & \text { U 234 } & 32.300 & 2.180 \\ 1 & \text { U 238 } & 31.400 & 2.120\end{array}$

0.510

25.700

72.000

0.740

2.120

$\begin{array}{rr}3.690 & 0.454 \\ 359.000 & 10.000 \\ 465.000 & 30.000 \\ 43.000 & 2.440 \\ 9.230 & 0.346 \\ 34.200 & 3.520 \\ 35.900 & 3.630\end{array}$

0.720

1.130

1.150

1.000

0.920

0.940

0.880

0.16

0.08

0.17

0.07

0.09

0.12

0.11

W
$\mathbf{A}$
$\mathbf{A}$
$\mathbf{A}$
$\mathbf{A}$
$\mathbf{A}$
$\mathbf{A}$

Matrix: WA

$\begin{array}{llr}1 & \text { AM241 } & 0.665 \\ 1 & \text { CD 60 } & 29.500 \\ 1 & \text { CS137 } & 40.600 \\ 1 & H 3 & 212.000 \\ 1 & \text { MN 54 } & 38.400 \\ 1 & \text { PU238 } & 0.950 \\ 1 & \text { PU239 } & 0.750 \\ 1 & \text { SR 90 } & 1.500 \\ 1 & \text { U 234 } & 0.280 \\ 1 & \text { U 238 } & 0.280\end{array}$

0.090
2.010
3.380
13.800
3.050
0.070
0.060
0.340
0.030
0.030

0.766
32.800
38.300
251.000
38.400
0.982
0.772
1.450
0.274
0.275

0.013
0.580
0.881
11.400
1.160
0.074
0.056
0.034
0.019
0.024

0.870
0.900
1.060
0.850
1.000
0.970
0.970
1.030
1.020
1.020

0.12

0.06

0.09

0.07

0.09

0.10

0.11

0.24

0.13

0.14

A
W
A
A
A
A
A
A
A
A

Units for matrices: $A I=B q /$ filter $S O=B q / k g V E=B q / k g ~ W A=B q / L$. Values for elemental uranium are reported in $\mu g / f i l t e r, g$, or $\mathrm{mL}$. 
QAP44 Results by Laboratory

Lab: AG Paragon Analytics, Inc, Fort Collins, CO

\begin{tabular}{|c|c|c|c|c|c|c|c|}
\hline $\begin{array}{l}\text { No. } \\
\text { Test }\end{array}$ & $\begin{array}{l}\text { Radio. } \\
\text { nuclide }\end{array}$ & $\begin{array}{l}\text { Reported } \\
\text { Value }\end{array}$ & $\begin{array}{l}\text { Reported } \\
\text { Error }\end{array}$ & $\begin{array}{l}\text { EML } \\
\text { Value }\end{array}$ & $\begin{array}{l}\text { EML } \\
\text { Error }\end{array}$ & $\frac{\text { Reported }}{\text { EML }}$ & $\begin{array}{l}\text { Ratio } \\
\text { Error }\end{array}$ \\
\hline
\end{tabular}

Matrix: Al

$\begin{array}{lrrrrrrr}\text { AM241 } & 0.169 & 0.022 & 0.189 & 0.007 & 0.890 & 0.12 & \text { A } \\ \text { CE144 } & 25.300 & 1.800 & 33.300 & 3.300 & 0.760 & 0.09 & \text { A } \\ \text { CO 57 } & 7.440 & 0.490 & 8.900 & 0.900 & 0.840 & 0.10 & \text { A } \\ \text { CO 60 } & 26.500 & 1.800 & 29.500 & 2.900 & 0.900 & 0.11 & \text { A } \\ \text { CS134 } & 14.500 & 0.940 & 14.700 & 1.460 & 0.990 & 0.12 & \text { A } \\ \text { CS137 } & 6.090 & 0.440 & 6.640 & 0.700 & 0.920 & 0.12 & \text { A } \\ \text { GA 1 } & 1.850 & 0.082 & 1.620 & 0.150 & 1.140 & 0.12 & \text { A } \\ \text { GB 2 } & 1.730 & 0.066 & 1.770 & 0.150 & 0.980 & 0.09 & \text { A } \\ \text { MN 54 } & 2.890 & 0.200 & 3.440 & 0.380 & 0.840 & 0.11 & \text { W } \\ \text { PU238 } & 0.085 & 0.011 & 0.096 & 0.002 & 0.880 & 0.12 & \text { A } \\ \text { PU239 } & 0.086 & 0.011 & 0.093 & 0.003 & 0.930 & 0.12 & \text { A } \\ \text { RU106 } & 11.400 & 1.800 & 11.600 & 1.440 & 0.980 & 0.20 & \text { A } \\ \text { SB125 } & 9.780 & 0.710 & 9.780 & 1.030 & 1.000 & 0.13 & \text { A } \\ \text { SR 90 } & 0.870 & 0.160 & 1.060 & 0.037 & 0.820 & 0.15 & \text { W } \\ \text { U 234 } & 0.080 & 0.012 & 0.052 & 0.002 & 1.550 & 0.24 & \text { W } \\ \text { U 238 } & 0.068 & 0.010 & 0.053 & 0.002 & 1.280 & 0.19 & \text { A }\end{array}$

Matrix: So

$\begin{array}{lrrrrr}\text { AM241 } & 3.330 & 0.440 & 3.690 & 0.454 & 0.900 \\ \text { CS137 } & 452.000 & 29.000 & 359.000 & 10.000 & 1.260 \\ \text { K 40 } & 585.000 & 45.000 & 465.000 & 30.000 & 1.260 \\ \text { PU238 } & 41.000 & 4.800 & 43.000 & 2.440 & 0.950 \\ \text { PU239 } & 9.200 & 1.100 & 9.230 & 0.346 & 1.000 \\ \text { SR 90 } & 1040.000 & 140.000 & 1340.000 & 113.000 & 0.780 \\ \text { U 234 } & 31.400 & 3.700 & 34.200 & 3.520 & 0.920 \\ \text { U 238 } & 32.200 & 4.000 & 35.900 & 3.630 & 0.900\end{array}$

$\begin{array}{ll}0.16 & \text { A } \\ 0.09 & \text { W } \\ 0.13 & \text { W } \\ 0.12 & \text { A } \\ 0.13 & \text { A } \\ 0.12 & \text { A } \\ 0.14 & \text { A } \\ 0.14 & \text { A }\end{array}$

Matrix: VE

$\begin{array}{rlrr}1 & \text { AM241 } & 6.170 & 0.740 \\ 1 & \text { CM244 } & 3.950 & 0.370 \\ 1 & \text { CO 60 } & 61.800 & 4.100 \\ 1 & \text { CS137 } & 1180.000 & 75.000 \\ 1 & \text { K 40 } & 1220.000 & 84.000 \\ 1 & \text { PU239 } & 9.400 & 1.100 \\ 1 & \text { SR 90 } & 1220.000 & 220.000\end{array}$

.740
.370
.100
.000
.000
.100
.000

5.600
4.440
59.700
944.000
1030.000
9.820
1300.000

0.184
0.202
0.963
16.200
33.000
1.220
52.400

1.100
0.890
1.040
1.250
1.180
0.960
0.940

0.14
0.09
0.07
0.08
0.09
0.16
0.17

$\mathbf{A}$
$\mathbf{A}$
$\mathbf{A}$
$\mathbf{A}$
$\mathbf{A}$
$\mathbf{A}$
$\mathbf{A}$

Matrix: WA

\begin{tabular}{llrrrrrrr}
1 & AM241 & 0.788 & 0.097 & 0.766 & 0.013 & 1.030 & 0.13 & A \\
1 & CO 60 & 31.900 & 2.600 & 32.800 & 0.580 & 0.970 & 0.08 & A \\
1 & CS137 & 40.500 & 3.300 & 38.300 & 0.881 & 1.060 & 0.09 & $A$ \\
1 & GA 1 & 1850.000 & 233.000 & 1850.000 & 185.000 & 1.000 & 0.16 & A \\
1 & GB 2 & 474.000 & 59.000 & 744.000 & 74.000 & 0.640 & 0.10 & N \\
1 & H 3 & 195.000 & 29.000 & 251.000 & 11.400 & 0.780 & 0.12 & W \\
\hline
\end{tabular}

Units for matrices: $\mathrm{Al}=\mathrm{Bq} / \mathrm{filter} \mathrm{SO}=\mathrm{Bq} / \mathrm{kg} \mathrm{VE}=\mathrm{Bq} / \mathrm{kg} W \mathrm{~W}=\mathrm{Bq} / \mathrm{L}$. Values for elemental uranium are reported in $\mu \mathrm{g} / \mathrm{filter}, \mathrm{g}$, or $\mathrm{mL}$. 


\section{OAP44 Results by Laboratory}

Lab: AG Paragon Analytics, Inc, Fort Collins, CO

\begin{tabular}{llllllll} 
No. & Radio- & Reported & Reported & EML & EML & Reported & Ratio \\
Test & nuclide & Value & Error & Value & Error & EML & Error \\
\hline
\end{tabular}

Matrix: WA

$\begin{array}{lllllllll}1 & \text { MN 54 } & 40.000 & 3.400 & 38.400 & 1.160 & 1.040 & 0.09 & \\ 1 & \text { PU238 } & 0.940 & 0.120 & 0.982 & 0.074 & 0.960 & 0.14 & \text { A } \\ 1 & \text { PU239 } & 0.715 & 0.089 & 0.772 & 0.056 & 0.930 & 0.13 & \text { A } \\ 1 & \text { SR 90 } & 1.500 & 0.300 & 1.450 & 0.034 & 1.030 & 0.21 & \text { A } \\ 1 & \text { U 234 } & 0.293 & 0.037 & 0.274 & 0.019 & 1.070 & 0.15 & \text { A } \\ 1 & \text { U 238 } & 0.283 & 0.037 & 0.275 & 0.024 & 1.030 & 0.16 & \text { A } \\ 1 & \text { U BO } & 0.147 & 0.012 & 0.559 & 0.047 & 0.260 & 0.03 & \text { N }\end{array}$

Units for matrices: $\mathrm{Al}=\mathrm{Bq} / \mathrm{filter} \mathrm{SO}=\mathrm{Bq} / \mathrm{kg} \mathrm{VE}=\mathrm{Bq} / \mathrm{kg} \mathrm{WA}=\mathrm{Bq} / \mathrm{L}$. Values for elemental uranium are reported in $\mu \mathrm{g} / \mathrm{filter}, \mathrm{g}$, or $\mathrm{mL}$.

$\mathrm{pCi}=\mathrm{Bq} \times 27$ 


\section{QAP44 Results by Laboratory}

Lab: Al Nuclear Technology Services, Inc., Roswell, GA

\begin{tabular}{|c|c|c|c|c|c|c|}
\hline $\begin{array}{l}\text { Radio. } \\
\text { nuclide }\end{array}$ & $\begin{array}{l}\text { Reported } \\
\text { Value }\end{array}$ & $\begin{array}{l}\text { Repported } \\
\text { Error }\end{array}$ & $\begin{array}{l}\text { EML } \\
\text { Value }\end{array}$ & $\begin{array}{l}\text { EML } \\
\text { Error }\end{array}$ & $\frac{\text { Reported }}{\text { EML }}$ & $\begin{array}{l}\text { Ratio } \\
\text { Error }\end{array}$ \\
\hline
\end{tabular}

Matrix: Al

$\begin{array}{lrrrrrrr}\text { CE144 } & 6.910 & 0.360 & 33.300 & 3.300 & 0.210 & 0.02 & \text { N } \\ \text { CO 57 } & 5.850 & 0.055 & 8.900 & 0.900 & 0.660 & 0.07 & \text { W } \\ \text { CO 60 } & 19.200 & 0.110 & 29.500 & 2.900 & 0.650 & 0.06 & \text { N } \\ \text { CS134 } & 10.600 & 0.820 & 14.700 & 1.460 & 0.720 & 0.09 & \text { W } \\ \text { CS137 } & 4.830 & 0.085 & 6.640 & 0.700 & 0.730 & 0.08 & \text { W } \\ \text { MN54 } & 2.300 & 0.089 & 3.440 & 0.380 & 0.670 & 0.08 & \text { N } \\ \text { PU238 } & 0.082 & 0.009 & 0.096 & 0.002 & 0.850 & 0.10 & \text { A } \\ \text { PU239 } & 0.097 & 0.010 & 0.093 & 0.003 & 1.040 & 0.11 & \text { A } \\ \text { RU106 } & 8.770 & 0.710 & 11.600 & 1.440 & 0.760 & 0.11 & \text { A } \\ \text { SB125 } & 7.660 & 0.160 & 9.780 & 1.030 & 0.780 & 0.08 & \text { A } \\ \text { U 234 } & 0.054 & 0.003 & 0.052 & 0.002 & 1.040 & 0.07 & \text { A } \\ \text { U 238 } & 0.055 & 0.004 & 0.053 & 0.002 & 1.040 & 0.08 & \text { A }\end{array}$

Matrix: So

$\begin{array}{llrrrrrrr}1 & \text { CS137 } & 267.000 & 4.600 & 359.000 & 10.000 & 0.740 & 0.02 & W \\ 1 & \text { K 40 } & 308.000 & 35.900 & 465.000 & 30.000 & 0.660 & 0.09 & \mathbf{N} \\ 1 & \text { PU238 } & 42.300 & 2.500 & 43.000 & 2.440 & 0.980 & 0.08 & \text { A } \\ 1 & \text { PU239 } & 9.580 & 0.850 & 9.230 & 0.346 & 1.040 & 0.10 & \text { A } \\ 1 & \text { U 234 } & 40.500 & 11.000 & 34.200 & 3.520 & 1.180 & 0.34 & W \\ 1 & \text { U 238 } & 50.700 & 13.000 & 35.900 & 3.630 & 1.410 & 0.39 & \mathbf{W}\end{array}$

Matrix: VE

\begin{tabular}{|c|c|c|c|c|c|c|}
\hline $\begin{array}{l}\text { CO 60 } \\
\text { CS137 } \\
\text { PU239 }\end{array}$ & $\begin{array}{r}39.900 \\
700.000 \\
6.170\end{array}$ & $\begin{array}{l}2.400 \\
7.700 \\
1.200\end{array}$ & $\begin{array}{r}59.700 \\
944.000 \\
9.820\end{array}$ & $\begin{array}{r}0.963 \\
16.200 \\
1.220\end{array}$ & $\begin{array}{l}0.670 \\
0.740 \\
0.630\end{array}$ & $\begin{array}{l}0.04 \\
0.02 \\
0.15\end{array}$ \\
\hline
\end{tabular}

Matrix: WA

$\begin{array}{llr}1 & \text { AM241 } & 0.652 \\ 1 & \text { CO 60 } & 27.600 \\ 1 & \text { CS137 } & 36.100 \\ 1 & H 3 & 400.000 \\ 1 & \text { MN 54 } & 34.300 \\ 1 & \text { PU238 } & 1.020 \\ 1 & \text { PU239 } & 0.824 \\ 1 & \text { U 234 } & 0.290 \\ 1 & \text { U 238 } & 0.260\end{array}$

0.067
0.750
1.120
45.000
1.130
0.073
0.065
0.030
0.030

0.766
32.800
38.300
251.000
38.400
0.982
0.772
0.274
0.275

0.013
0.580
0.881
11.400
1.160
0.074
0.056
0.019
0.024

0.850
0.840
0.940
1.590
0.890
1.040
1.070
1.060
0.950

0.09
0.03
0.04
0.19
0.04
0.11
0.11
0.13
0.14

$\mathbf{A}$
$\mathbf{N}$
$\mathbf{W}$
$\mathbf{W}$
$\mathbf{W}$
$\mathbf{A}$
$\mathbf{A}$
$\mathbf{A}$
$\mathbf{A}$

Units for matrices: $\mathrm{Al}=\mathrm{Bq} / \mathrm{filter} \mathrm{SO}=\mathrm{Bq} / \mathrm{kg}$ VE=Bq/kg $W A=B q / L$. Values for elemental uranium are reported in $\mu \mathrm{g} /$ filter, $\mathrm{g}$, or $\mathrm{mL}$. $\mathrm{pCi}=\mathrm{Bq} \times 27$ 


\section{QAP44 Results by Laboratory}

Lab: AL Ames Laboratory, Ames, IA

\begin{tabular}{llllllll} 
No. & Radio- & $\begin{array}{l}\text { Reported } \\
\text { Value }\end{array}$ & $\begin{array}{l}\text { Reported } \\
\text { Error }\end{array}$ & $\begin{array}{c}\text { EML } \\
\text { Value }\end{array}$ & $\begin{array}{l}\text { EML } \\
\text { Error }\end{array}$ & $\frac{\text { Reported }}{\text { EML }}$ & $\begin{array}{c}\text { Ratio } \\
\text { Error }\end{array}$ \\
\hline
\end{tabular}

Matrix: Al

$\begin{array}{lllllllll}1 & \text { CE144 } & 44.400 & 1.060 & 33.300 & 3.300 & 1.330 & 0.14 & \text { N } \\ 1 & \text { CO 60 } & 43.700 & 0.360 & 29.500 & 2.900 & 1.480 & 0.15 & \text { N } \\ 1 & \text { CS134 } & 13.500 & 0.210 & 14.700 & 1.460 & 0.920 & 0.09 & \text { A } \\ 1 & \text { CS137 } & 10.500 & 0.230 & 6.640 & 0.700 & 1.580 & 0.17 & \text { N } \\ 1 & \text { MN 54 } & 3.200 & 0.120 & 3.440 & 0.380 & 0.930 & 0.11 & \text { A } \\ 1 & \text { SB125 } & 14.700 & 0.390 & 9.780 & 1.030 & 1.500 & 0.16 & N\end{array}$

Matrix: SO

$1 \quad \operatorname{CS} 137 \quad 319.000$

10.000

0.890

0.03

A

Matrix: VE

$1 \quad C 060 \quad 56.20$

$1 \quad$ CS137 980.000

$2.070 \quad 59.700$

944.000

0.963

0.940

1.040

0.04

0.02

A

Matrix: WA

$\begin{array}{ll}1 & \text { CO.60 } \\ 1 & \text { CS137 } \\ 1 & \text { MN } 54\end{array}$

31.400
41.100
40.000

0.650

0.640

32.800

38.300

38.400

$\begin{array}{ll}0.580 & 0.960 \\ 0.881 & 1.070 \\ 1.160 & 1.040\end{array}$

0.03

0.03

0.04

$N$
$N$
$A$
$N$
$A$
$N$




\section{QAP44 Results by Laboratory}

Lab: AM American Radiation Services, Inc., Baton Rouge

\begin{tabular}{|c|c|c|c|c|c|c|c|}
\hline $\begin{array}{l}\text { No. } \\
\text { Test }\end{array}$ & $\begin{array}{l}\text { Radio- } \\
\text { nuclide }\end{array}$ & $\begin{array}{l}\text { Reported } \\
\text { Value }\end{array}$ & $\begin{array}{l}\text { Reported } \\
\text { Error }\end{array}$ & $\begin{array}{c}\text { EML } \\
\text { Value }\end{array}$ & $\begin{array}{l}\text { EML } \\
\text { Error }\end{array}$ & $\frac{\text { Reported }}{\text { EML }}$ & $\begin{array}{l}\text { Ratio } \\
\text { Error }\end{array}$ \\
\hline
\end{tabular}

Matrix: AI

\begin{tabular}{|c|c|c|c|c|c|c|}
\hline $\begin{array}{l}\text { AM241 } \\
\text { CE144 } \\
\text { CO } 57 \\
\text { CO 60 } \\
\text { CS134 } \\
\text { CS137 } \\
\text { GA } 1 \\
\text { GB } 2 \\
\text { MN 54 } \\
\text { RU106 } \\
\text { SB125 }\end{array}$ & $\begin{array}{r}0.270 \\
25.600 \\
6.220 \\
24.500 \\
9.810 \\
5.630 \\
2.400 \\
2.540 \\
2.850 \\
8.910 \\
6.080\end{array}$ & $\begin{array}{l}0.050 \\
0.450 \\
0.070 \\
0.210 \\
0.130 \\
0.150 \\
0.050 \\
0.040 \\
0.240 \\
0.660 \\
0.230\end{array}$ & $\begin{array}{r}0.189 \\
33.300 \\
8.900 \\
29.500 \\
14.700 \\
6.640 \\
1.620 \\
1.770 \\
3.440 \\
11.600 \\
9.780\end{array}$ & $\begin{array}{l}0.007 \\
3.300 \\
0.900 \\
2.900 \\
1.460 \\
0.700 \\
0.150 \\
0.150 \\
0.380 \\
1.440 \\
1.030\end{array}$ & $\begin{array}{l}1.430 \\
0.770 \\
0.700 \\
0.830 \\
0.670 \\
0.850 \\
1.480 \\
1.440 \\
0.830 \\
0.770 \\
0.620\end{array}$ & $\begin{array}{l}0.27 \\
0.08 \\
0.07 \\
0.08 \\
0.07 \\
0.09 \\
0.14 \\
0.12 \\
0.12 \\
0.11 \\
0.07\end{array}$ \\
\hline
\end{tabular}

Matrix: $\quad$ So

$\begin{array}{ll}1 & \text { AM2 } 21 \\ 1 & \text { CS137 } \\ 1 & \text { K } 40 \\ 1 & \text { PU239 } \\ 1 & \text { U } 238 \\ & \\ \text { Watrix: } & \text { WA }\end{array}$

$\begin{array}{llrrrrrrr}1 & \text { AM241 } & 1.060 & 0.590 & 0.766 & 0.013 & 1.380 & 0.77 & \text { W } \\ 1 & \text { CO 60 } & 31.300 & 0.610 & 32.800 & 0.580 & 0.950 & 0.03 & \text { A } \\ 1 & \text { CS137 } & 40.700 & 1.060 & 38.300 & 0.881 & 1.060 & 0.04 & \text { A } \\ 1 & \text { GA 1 } & 709.000 & 15.900 & 1850.000 & 185.000 & 0.380 & 0.04 & \text { N } \\ 1 & \text { GB 2 } & 833.000 & 12.300 & 744.000 & 74.000 & 1.120 & 0.11 & \text { A } \\ 1 & \text { MN 54 } & 41.100 & 1.020 & 38.400 & 1.160 & 1.070 & 0.04 & \text { A } \\ 1 & \text { U BQ } & 0.360 & 0.070 & 0.559 & 0.047 & 0.640 & 0.14 & \text { W }\end{array}$

Units for matrices: $A|=B q| f i l t e r ~ S O=B q / k g$ VE=Bqlkg $W A=B q \mid L$. Values for elemental uranium are reported in $\mu g \mid f i l t e r, g$, or $\mathrm{mL}$. 


\section{QAP44 Results by Laboratory}

Lab: AN Argonne National Laboratory

\begin{tabular}{ccccccccc} 
No. & $\begin{array}{l}\text { Radio- } \\
\text { nuclide }\end{array}$ & $\begin{array}{l}\text { Reported } \\
\text { Value }\end{array}$ & $\begin{array}{c}\text { Reported } \\
\text { Error }\end{array}$ & $\begin{array}{c}\text { EML } \\
\text { Value }\end{array}$ & $\begin{array}{c}\text { EML } \\
\text { Error }\end{array}$ & $\frac{\text { Reported }}{\text { EML }}$ & $\begin{array}{l}\text { Ratio } \\
\text { Error }\end{array}$ & Evaluation \\
\hline
\end{tabular}

Matrix: Al

$\begin{array}{llrlrllll}1 & \text { AM241 } & 0.192 & 0.006 & 0.189 & 0.007 & 1.020 & 0.05 & \text { A } \\ 1 & \text { CE144 } & 23.100 & 0.700 & 33.300 & 3.300 & 0.690 & 0.07 & \text { A } \\ 1 & \text { CD 57 } & 6.620 & 0.200 & 8.900 & 0.900 & 0.740 & 0.08 & \text { A } \\ 1 & \text { CD 60 } & 26.100 & 0.800 & 29.500 & 2.900 & 0.890 & 0.09 & \text { A } \\ 1 & \text { CS134 } & 13.100 & 0.400 & 14.700 & 1.460 & 0.890 & 0.09 & \text { A } \\ 1 & \text { CS137 } & 5.850 & 0.180 & 6.640 & 0.700 & 0.880 & 0.10 & \text { A } \\ 1 & \text { GA 1 } & 1.980 & 0.200 & 1.620 & 0.150 & 1.220 & 0.17 & \text { A } \\ 1 & \text { GB 2 } & 2.250 & 0.230 & 1.770 & 0.150 & 1.270 & 0.17 & \text { A } \\ 1 & \text { MN 54 } & 2.890 & 0.140 & 3.440 & 0.380 & 0.840 & 0.10 & \text { W } \\ 1 & \text { PU238 } & 0.096 & 0.003 & 0.096 & 0.002 & 1.000 & 0.04 & \text { A } \\ 1 & \text { PU239 } & 0.100 & 0.003 & 0.093 & 0.003 & 1.080 & 0.05 & \text { A } \\ 1 & \text { RU106 } & 10.700 & 0.300 & 11.600 & 1.440 & 0.920 & 0.12 & \text { A } \\ 1 & \text { SB125 } & 8.760 & 0.260 & 9.780 & 1.030 & 0.900 & 0.10 & \text { A } \\ 1 & \text { SR 90 } & 1.120 & 0.030 & 1.060 & 0.037 & 1.060 & 0.05 & \text { A } \\ 1 & \text { U 234 } & 0.056 & 0.003 & 0.052 & 0.002 & 1.080 & 0.06 & \text { A } \\ 1 & \text { U 238 } & 0.060 & 0.003 & 0.053 & 0.002 & 1.130 & 0.06 & \text { A }\end{array}$

Matrix: So

\begin{tabular}{|c|c|c|c|c|c|c|}
\hline $\begin{array}{l}\text { AM241 } \\
\text { CS137 } \\
\text { K } 40 \\
\text { PU238 } \\
\text { PU239 } \\
\text { SR 90 } \\
\text { U } 234 \\
\text { U } 238\end{array}$ & $\begin{array}{r}3.460 \\
410.000 \\
519.000 \\
46.000 \\
10.300 \\
1310.000 \\
37.900 \\
37.800\end{array}$ & $\begin{array}{r}0.120 \\
12.000 \\
16.000 \\
1.400 \\
0.300 \\
39.000 \\
1.100 \\
1.100\end{array}$ & $\begin{array}{r}3.690 \\
359.000 \\
465.000 \\
43.000 \\
9.230 \\
1340.000 \\
34.200 \\
35.900\end{array}$ & $\begin{array}{r}0.454 \\
10.000 \\
30.000 \\
2.440 \\
0.346 \\
113.000 \\
3.520 \\
3.630\end{array}$ & $\begin{array}{l}0.940 \\
1.140 \\
1.120 \\
1.070 \\
1.120 \\
0.980 \\
1.110 \\
1.050\end{array}$ & $\begin{array}{l}0.12 \\
0.05 \\
0.08 \\
0.07 \\
0.05 \\
0.09 \\
0.12 \\
0.11\end{array}$ \\
\hline
\end{tabular}

Matrix: VE

$\begin{array}{rlrrrrrrr}1 & \text { AM241 } & 5.850 & 0.180 & 5.600 & 0.184 & 1.040 & 0.05 & \text { A } \\ 1 & \text { CM244 } & 4.400 & 0.130 & 4.440 & 0.202 & 0.990 & 0.05 & \text { A } \\ 1 & \text { CO 6D } & 64.400 & 1.900 & 59.700 & 0.963 & 1.080 & 0.04 & \text { A } \\ 1 & \text { CS137 } & 1130.000 & 34.000 & 944.000 & 16.200 & 1.200 & 0.04 & \text { A } \\ 1 & \text { K 40 } & 1170.000 & 35.000 & 1030.000 & 33.000 & 1.140 & 0.05 & \text { A } \\ 1 & \text { PU238 } & 0.701 & 0.028 & 0.820 & 0.104 & 0.860 & 0.11 & \mathbf{A} \\ 1 & \text { PU239 } & 9.450 & 0.280 & 9.820 & 1.220 & 0.960 & 0.12 & \mathbf{A} \\ 1 & \text { SR 90 } & 1450.000 & 44.000 & 1300.000 & 52.400 & 1.120 & 0.06 & \mathbf{W}\end{array}$

Matrix: WA

\begin{tabular}{rlrrrrrrr}
1 & AM241 & 0.804 & 0.024 & 0.766 & 0.013 & 1.050 & 0.04 & A \\
1 & CO 60 & 33.200 & 1.000 & 32.800 & 0.580 & 1.010 & 0.04 & A \\
1 & CS137 & 40.900 & 1.200 & 38.300 & 0.881 & 1.070 & 0.04 & $A$ \\
1 & GA 1 & 2040.000 & 200.000 & 1850.000 & 185.000 & 1.100 & 0.15 & A \\
1 & GB 2 & 570.000 & 57.000 & 744.000 & 74.000 & 0.770 & 0.11 & W \\
\hline
\end{tabular}

Units for matrices: $A \mathrm{l}=\mathrm{Bq} \mid$ filter $\mathrm{SO}=\mathrm{Bq} / \mathrm{kg} V E=B q / \mathrm{kg} W A=B q \mid \mathrm{L}$. Values for elemental uranium are reported in $\mu \mathrm{g} / \mathrm{filter}, \mathrm{g}$, or $\mathrm{mL}$.

Evaluation: $A=$ Acceptable, $W=$ Acceptable with Warning, $N=$ Not Acceptable

$\mathrm{pCi}=\mathrm{Bq} \times 27$ 


\section{QAP44 Results by Laboratory}

Lab: AN Argonne National Laboratory

\begin{tabular}{llllllll} 
No. & Radio- & $\begin{array}{l}\text { Reported } \\
\text { Value }\end{array}$ & $\begin{array}{c}\text { Reported } \\
\text { Error }\end{array}$ & $\begin{array}{c}\text { EML } \\
\text { Value }\end{array}$ & $\begin{array}{l}\text { EML } \\
\text { Error }\end{array}$ & $\begin{array}{l}\text { Reported } \\
\text { EML }\end{array}$ & $\begin{array}{l}\text { Ratio } \\
\text { Error }\end{array}$ \\
\hline
\end{tabular}

Matrix: WA

$\begin{array}{llrrrrrrr}1 & \text { H 3 } & 220.000 & 7.000 & 251.000 & 11.400 & 0.880 & 0.05 & \text { A } \\ 1 & \text { MN 54 } & 41.900 & 1.300 & 38.400 & 1.160 & 1.090 & 0.05 & \text { A } \\ 1 & \text { PU238 } & 0.920 & 0.028 & 0.982 & 0.074 & 0.940 & 0.08 & \text { A } \\ 1 & \text { PU239 } & 0.723 & 0.022 & 0.772 & 0.056 & 0.940 . & 0.07 & \text { A } \\ 1 & \text { SR 90 } & 1.580 & 0.050 & 1.450 & 0.034 & 1.090 & 0.04 & \text { A } \\ 1 & \text { U 234 } & 0.291 & 0.009 & 0.274 & 0.019 & 1.060 & 0.08 & \text { A } \\ 1 & \text { U 238 } & 0.299 & 0.009 & 0.275 & 0.024 & 1.090 & 0.10 & \text { A }\end{array}$

Units for matrices: $\mathrm{Al}=\mathrm{Bq} / \mathrm{filter} \mathrm{SO}=\mathrm{Bq} / \mathrm{kg} \mathrm{VE}=\mathrm{Bq} / \mathrm{kg} \mathrm{WA}=\mathrm{Bq} / \mathrm{L}$. Values for elemental uranium are reported in $\mu \mathrm{g} / \mathrm{filter}, \mathrm{g}$, or $\mathrm{mL}$. 


\section{OAP44 Results by Laboratory}

Lab: AR Accu-Labs Research Inc., Golden, CO

\begin{tabular}{|c|c|c|c|c|c|c|}
\hline $\begin{array}{l}\text { No. } \\
\text { Test }\end{array}$ & $\begin{array}{l}\text { Radio- } \\
\text { nuclide }\end{array}$ & $\begin{array}{l}\text { Reported } \\
\text { Value }\end{array}$ & $\begin{array}{l}\text { Reported } \\
\text { Error }\end{array}$ & $\begin{array}{c}\text { EML } \\
\text { Value }\end{array}$ & $\begin{array}{l}\text { EML } \\
\text { Error }\end{array}$ & tion \\
\hline
\end{tabular}

Matrix: Al

$\begin{array}{llrlrllll}1 & \text { AM241 } & 0.176 & 0.013 & 0.189 & 0.007 & 0.930 & 0.08 & \text { A } \\ 1 & \text { CE144 } & 32.200 & 2.100 & 33.300 & 3.300 & 0.970 & 0.12 & \text { A } \\ 1 & \text { CO 57 } & 7.110 & 0.300 & 8.900 & 0.900 & 0.800 & 0.09 & \text { A } \\ 1 & \text { CO 60 } & 27.100 & 0.900 & 29.500 & 2.900 & 0.920 & 0.10 & \text { A } \\ 1 & \text { CS134 } & 13.000 & 0.600 & 14.700 & 1.460 & 0.880 & 0.10 & \text { A } \\ 1 & \text { CS137 } & 6.000 & 0.300 & 6.640 & 0.700 & 0.900 & 0.11 & \text { A } \\ 1 & \text { GA 1 } & 1.540 & 0.030 & 1.620 & 0.150 & 0.950 & 0.09 & \text { A } \\ 1 & \text { GB 2 } & 1.500 & 0.030 & 1.770 & 0.150 & 0.850 & 0.07 & \text { W } \\ 1 & \text { MN 54 } & 3.000 & 0.180 & 3.440 & 0.380 & 0.870 & 0.11 & \text { A } \\ 1 & \text { PU238 } & 0.097 & 0.013 & 0.096 & 0.002 & 1.010 & 0.13 & \text { A } \\ 1 & \text { PU239 } & 0.091 & 0.013 & 0.093 & 0.003 & 0.980 & 0.14 & \text { A } \\ 1 & \text { RU106 } & 10.300 & 1.400 & 11.600 & 1.440 & 0.890 & 0.16 & \text { A } \\ 1 & \text { SB125 } & 10.100 & 0.900 & 9.780 & 1.030 & 1.030 & 0.14 & \text { A } \\ 1 & \text { SR 90 } & 0.730 & 0.192 & 1.060 & 0.037 & 0.690 & 0.18 & \text { W } \\ 1 & \text { U 234 } & 0.060 & 0.011 & 0.052 & 0.002 & 1.160 & 0.21 & \text { A } \\ 1 & \text { U 238 } & 0.055 & 0.011 & 0.053 & 0.002 & 1.040 & 0.20 & \text { A } \\ 1 & \text { U BQ } & 0.115 & 0.015 & 0.107 & 0.003 & 1.080 & 0.14 & \text { A } \\ 1 & \text { U UG } & 4.400 & 0.060 & 4.310 & 0.100 & 1.020 & 0.03 & \text { A }\end{array}$

Matrix: So

$\begin{array}{llrr}1 & \text { AM241 } & 2.320 & 0.310 \\ 1 & \text { CS137 } & 327.000 & 11.000 \\ 1 & \text { K 40 } & 369.000 & 22.000 \\ 1 & \text { PU238 } & 44.200 & 3.300 \\ 1 & \text { PU239 } & 11.300 & 1.700 \\ 1 & \text { SR 90 } & 1340.000 & 60.000 \\ 1 & \text { U 234 } & 37.800 & 3.300 \\ 1 & \text { U 238 } & 31.800 & 3.000 \\ 1 & \text { U BQ } & 69.600 & 4.500 \\ 1 & \text { U UG } & 2.340 & 0.030\end{array}$

$\begin{array}{rr}0.310 & 3.690 \\ 11.000 & 359.000 \\ 22.000 & 465.000 \\ 3.300 & 43.000 \\ 1.700 & 9.230 \\ 60.000 & 1340.000 \\ 3.300 & 34.200 \\ 3.000 & 35.900 \\ 4.500 & 71.700 \\ 0.030 & 2.900\end{array}$

0.454
10.000
30.000
2.440
0.346
113.000
3.520
3.630
4.150
0.290

$$
\begin{aligned}
& 0.630 \\
& 0.910 \\
& 0.790 \\
& 1.030 \\
& 1.220 \\
& 1.000 \\
& 1.110 \\
& 0.890 \\
& 0.970 \\
& 0.810
\end{aligned}
$$

0.11
0.04
0.07
0.10
0.19
0.10
0.15
0.12
0.08
0.08

W
A
W
A
A
A
W
A
A
A

Matrix: VE

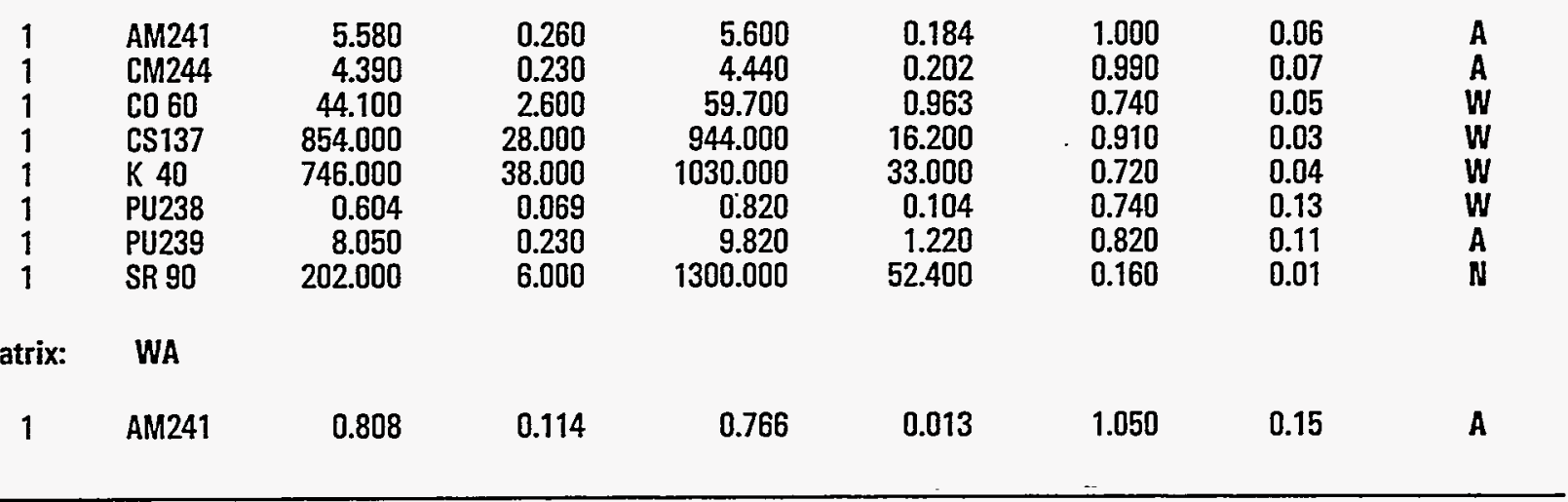

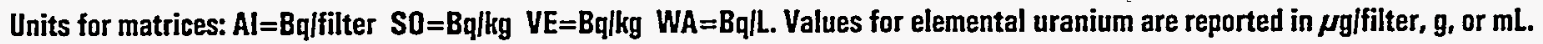
Evaluation: $A=$ Acceptable, $W=$ Acceptable with Warning, $N=$ Not Acceptable $\mathrm{pCi}=\mathrm{Bq} \times 27$ 


\section{QAP44 Results by Laboratory}

Lab: AR Accu-Labs Research Inc., Golden, CO

\begin{tabular}{llllllll} 
No. & Radio- & Reported & Reported & EML & EML & Reported & Ratio \\
Test & nuclide & Value & Error & Value & Error & $\frac{\text { EML }}{\text { EML }}$ & Evaluation \\
\hline
\end{tabular}

Matrix: WA

$\begin{array}{llrrrrrrr}1 & \text { CO 60 } & 36.900 & 2.600 & 32.800 & 0.580 & 1.130 & 0.08 & \text { W } \\ 1 & \text { CS137 } & 45.600 & 3.600 & 38.300 & 0.881 & 1.190 & 0.10 & \text { W } \\ 1 & \text { GA 1 } & 1820.000 & 200.000 & 1850.000 & 185.000 & 0.980 & 0.15 & \text { A } \\ 1 & \text { GB 2 } & 528.000 & 66.000 & 744.000 & 74.000 & 0.710 & 0.11 & \text { N } \\ 1 & \text { H 3 } & 224.000 & 9.000 & 251.000 & 11.400 & 0.890 & 0.05 & A \\ 1 & \text { MN 54 } & 44.100 & 3.600 & 38.400 & 1.160 & 1.150 & 0.10 & \text { W } \\ 1 & \text { PU238 } & 0.853 & 0.122 & 0.982 & 0.074 & 0.870 & 0.14 & \text { W } \\ 1 & \text { PU239 } & 0.839 & 0.120 & 0.772 & 0.056 & 1.090 & 0.17 & A \\ 1 & \text { SR 90 } & 1.520 & 0.190 & 1.450 & 0.034 & 1.050 & 0.13 & A \\ 1 & \text { U 234 } & 0.328 & 0.042 & 0.274 & 0.019 & 1.200 & 0.17 & \text { W } \\ 1 & \text { U 238 } & 0.320 & 0.040 & 0.275 & 0.024 & 1.160 & 0.18 & \text { W } \\ 1 & \text { U BO } & 0.648 & 0.058 & 0.559 & 0.047 & 1.160 & 0.14 & A \\ 1 & \text { U UG } & 25.700 & 1.100 & 0.022 & 0.003 & * * * & * * * * & \text { N }\end{array}$

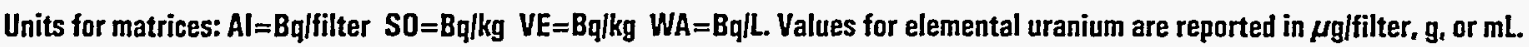




\section{QAP44 Results by Laboratory}

Lab: AU ORISE EESD/ESSAP, Oak Ridge

\begin{tabular}{lllllllll}
$\begin{array}{l}\text { No. } \\
\text { Test }\end{array}$ & $\begin{array}{l}\text { Radio- } \\
\text { nuclide }\end{array}$ & $\begin{array}{l}\text { Reported } \\
\text { Value }\end{array}$ & $\begin{array}{l}\text { Reported } \\
\text { Error }\end{array}$ & $\begin{array}{l}\text { EML } \\
\text { Value }\end{array}$ & $\begin{array}{l}\text { EML } \\
\text { Error }\end{array}$ & $\frac{\text { Reported }}{\text { EML }}$ & $\begin{array}{l}\text { Ratio } \\
\text { Error }\end{array}$ & Evaluation \\
\hline
\end{tabular}

Matrix: Al

$\begin{array}{lllllllll}1 & \text { GA 1 } & 2.120 & 0.040 & 1.620 & 0.150 & 1.310 & 0.12 & \text { W } \\ 1 & \text { GB 2 } & 2.090 & 0.040 & 1.770 & 0.150 & 1.180 & 0.10 & \mathbf{A} \\ 1 & \text { PU238 } & 0.290 & 0.070 & 0.096 & 0.002 & 3.020 & 0.73 & \mathbf{N} \\ 1 & \text { PU239 } & 0.340 & 0.080 & 0.093 & 0.003 & 3.670 & 0.87 & \mathbf{N} \\ 1 & \text { U 234 } & 0.180 & 0.040 & 0.052 & 0.002 & 3.480 & 0.78 & \mathbf{N} \\ 1 & \text { U 238 } & 0.160 & 0.030 & 0.053 & 0.002 & 3.000 & 0.58 & \mathbf{N}\end{array}$

Matrix: So

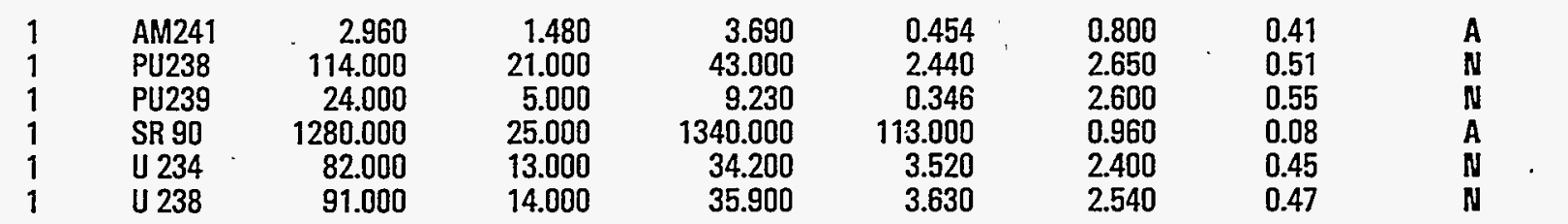

Matrix: VE

$\begin{array}{rrrrrrrrr}1 & \text { AM241 } & 5.700 & 1.000 & 5.600 & 0.184 & .1 .020 & 0.18 & \text { A } \\ 1 & \text { CM244 } & 3.300 & 0.700 & 4.440 & 0.202 & 0.740 & 0.16 & W \\ 1 & \text { PU238 } & 9.000 & 2.000 & 0.820 & 0.104 & * * * & 2.81 & \text { N } \\ 1 & \text { PU239 } & 29.000 & 6.000 & 9.820 & 1.220 & 2.950 & 0.71 & \text { N } \\ 1 & \text { SR 9D } & 1200.000 & 18.000 & 1300.000 & 52.400 & 0.920 & 0.04 & \text { A }\end{array}$

Matrix: WA

\begin{tabular}{|c|c|c|c|c|c|c|}
\hline AM241 & 0.790 & 0.100 & 0.766 & 0.013 & 1.030 & 0.13 \\
\hline GA 1 & 150.000 & 1.880 & 1850.000 & 185.000 & 0.080 & 0.01 \\
\hline GB 2 & 79.800 & 1.140 & 744.000 & 74.000 & 0.110 & 0.01 \\
\hline H 3 & 246.000 & 18.000 & 251.000 & 11.400 & 0.980 & 0.08 \\
\hline PU238 & 1.360 & 0.360 & 0.982 & 0.074 & 1.390 & 0.38 \\
\hline PU239 & 1.060 & 0.300 & 0.772 & 0.056 & 1.370 & 0.40 \\
\hline SR 90 & 2.300 & 0.370 & 1.450 & 0.034 & 1.590 & 0.26 \\
\hline U 234 & 0.370 & 0.100 & 0.274 & 0.019 & 1.350 . & 0.38 \\
\hline U 238 & 0.340 & 0.100 & 0.275 & 0.024 & 1.240 & 0.38 \\
\hline
\end{tabular}

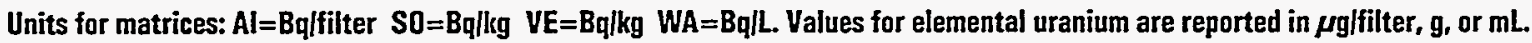

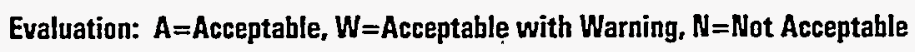
$\mathrm{pCi}=\mathrm{Bq} \times 27$ 
QAP44 Results by Laboratory

Lab: AW Argonne National Laboratory, Idaho Falls

\begin{tabular}{llllllll} 
No. & Radio- \\
Test & nuclide & $\begin{array}{l}\text { Reported } \\
\text { Value }\end{array}$ & $\begin{array}{l}\text { Reported } \\
\text { Error }\end{array}$ & $\begin{array}{c}\text { EML } \\
\text { Value }\end{array}$ & $\begin{array}{l}\text { EML } \\
\text { Error }\end{array}$ & $\frac{\text { Reported }}{\text { EML }}$ & $\begin{array}{l}\text { Ratio } \\
\text { Error }\end{array}$ \\
\hline
\end{tabular}

Matrix: Al

$\begin{array}{llrlrllll}1 & \text { AM241 } & 0.224 & 0.100 & 0.189 & 0.007 & 1.180 & 0.53 & \text { A } \\ 1 & \text { CE144 } & 33.400 & 5.010 & 33.300 & 3.300 & 1.000 & 0.18 & \text { A } \\ 1 & \text { CO 57 } & 9.390 & 1.410 & 8.900 & 0.900 & 1.050 & 0.19 & \text { A } \\ 1 & \text { CO 60 } & 30.200 & 4.530 & 29.500 & 2.900 & 1.020 & 0.18 & \text { A } \\ 1 & \text { CS134 } & 14.500 & 2.180 & 14.700 & 1.460 & 0.990 & 0.18 & \text { A } \\ 1 & \text { CS137 } & 6.950 & 1.040 & 6.640 & 0.700 & 1.050 & 0.19 & \text { A } \\ 1 & \text { MN 54 } & 3.500 & 0.530 & 3.440 & 0.380 & 1.020 & 0.19 & \text { A } \\ 1 & \text { RU106 } & 11.100 & 1.670 & 11.600 & 1.440 & 0.960 & 0.19 & \text { A } \\ 1 & \text { SB125 } & 10.200 & 1.530 & 9.780 & 1.030 & 1.040 & 0.19 & \text { A }\end{array}$

Matrix: WA

$\begin{array}{llrrrrrrr}1 & \text { CO 60 } & 30.600 & 4.600 & 32.800 & 0.580 & 0.930 & 0.14 & \text { W } \\ 1 & \text { CS137 } & 39.500 & 5.900 & 38.300 & 0.881 & 1.030 & 0.16 & \text { A } \\ 1 & \text { GA 1 } & 1700.000 & 170.000 & 1850.000 & 185.000 & 0.920 & 0.13 & \text { A } \\ 1 & \text { GB 2 } & 980.000 & 98.000 & 744.000 & 74.000 & 1.320 & 0.19 & \text { A } \\ 1 & \text { H 3 } & 220.000 & 22.000 & 251.000 & 11.400 & 0.880 & 0.10 & \text { A } \\ 1 & \text { MN 54 } & 39.100 & 5.900 & 38.400 & 1.160 & 1.020 & 0.16 & A \\ 1 & \text { U UG } & 0.016 & 0.002 & 0.022 & 0.003 & 0.720 & 0.11 & N\end{array}$

Units for matrices: $A \mathrm{l}=\mathrm{Bq} / \mathrm{filter} \mathrm{SD}=\mathrm{Bq} / \mathrm{kg} \mathrm{VE}=\mathrm{Bq} / \mathrm{kg} W \mathrm{~W}=\mathrm{Bq} / \mathrm{L}$. Values for elemental uranium are reported in $\mu \mathrm{g} / \mathrm{filter}, \mathrm{g}$, or $\mathrm{mL}$.

Evaluation: $A=A c c e p t a b l e, W=A c c e p t a b l e$ with Warning, $N=$ Not Acceptable

$\mathrm{pCi}=\mathrm{Bq} \times 27$ 


\section{QAP44 Results by Laboratory}

Lab: BA Bettis Atomic Power Lab, West Mifflin, PA

No.

Radio- Reported Reported EML

Value

EML

Reported Ratio

Test nuclide

Value

Error

Error EML

Error

Evaluation

Matrix: Al

$\begin{array}{rlrl}1 & \text { CE144 } & 30.400 & 2.600 \\ 1 & \text { CO 57 } & 8.460 & 0.620 \\ 1 & \text { CO 60 } & 31.200 & 0.920 \\ 1 & \text { CS134 } & 15.300 & 0.850 \\ 1 & \text { CS137 } & 7.120 & 0.680 \\ 1 & \text { MN 54 } & 4.170 & 0.600 \\ 1 & \text { PU238 } & 0.087 & 0.019 \\ 1 & \text { PU239 } & 0.102 & 0.020 \\ 1 & \text { RU106 } & 16.100 & 4.600 \\ 1 & \text { SB125 } & 11.600 & 0.990 \\ 1 & \text { SR 90 } & 1.010 & 0.120\end{array}$

33.300

8.900

29.500

14.700

6.640

3.440

0.096

0.093

11.600

9.780

1.060

Matrix: so

CS137

337.000

21.000

359.000

10.000

3.300
0.900
2.900
1.460
0.700
0.380
0.002
0.003
1.440
1.030
0.037

0.910

0.950

1.060

1.040

1.070

1.210

0.900

1.100

1.390

1.190

0.950

0.12

0.12

0.11

0.12

0.15

0.22

0.20

0.22

0.43

0.16

0.12

$A$
$A$
$A$
$A$
$A$
$W$
$A$
$A$
$W$
$W$
A

Matrix: VE

$\begin{array}{cc}1 & \text { CS137 } \\ 1 & \text { PU23 } \\ 1 & \text { PU239 } \\ 1 & \text { SR 90 } \\ & \\ \text { atrix: } & \text { WA }\end{array}$

$\begin{array}{ll}1 & \text { CO 60 } \\ 1 & \text { CS137 } \\ 1 & \text { MN 54 } \\ 1 & \text { PU238 } \\ 1 & \text { PU239 } \\ 1 & \text { U 234 } \\ 1 & \text { U 238 }\end{array}$

34.800
43.900
42.000
1.040
0.766
0.342
0.346

2.200
4.200
4.300
0.170
0.120
0.062
0.063

59.000
0.210
1.600
12.000

944.000
0.820
9.820
1300.000

16.200
0.104
1.220
52.400

1.010
1.260
1.010
0.870

0.06

0.30

0.21

1130.000

12.000

1300.000

32.800
38.300
38.400
0.982
0.772
0.274
0.275

0.580
0.881
1.160
0.074
0.056
0.019
0.024

1.060
1.150
1.090
1.060
0.990
1.250
1.260

0.07
0.11
0.12
0.19
0.17
0.24
0.26

$A$
$A$
$A$
$A$
$A$
$W$
$W$

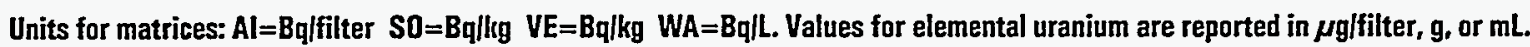




\section{QAP44 Results by Laboratory}

Lab: BC Babcock \& Wilcox MC \#42, Lynchburg, VA

\begin{tabular}{lllllllll} 
No. & $\begin{array}{l}\text { Radio- } \\
\text { nuclide }\end{array}$ & $\begin{array}{l}\text { Reported } \\
\text { Value }\end{array}$ & $\begin{array}{l}\text { Reported } \\
\text { Error }\end{array}$ & $\begin{array}{c}\text { EML } \\
\text { Value }\end{array}$ & $\begin{array}{c}\text { EML } \\
\text { Error }\end{array}$ & $\frac{\text { Reported }}{\text { EML }}$ & $\begin{array}{l}\text { Ratio } \\
\text { Error }\end{array}$ & Evaluation \\
\hline
\end{tabular}

Matrix: Al

$\begin{array}{llrlrllll}1 & \text { CE144 } & 23.100 & 0.670 & 33.300 & 3.300 & 0.690 & 0.07 & \text { A } \\ 1 & \text { CO 57 } & 6.770 & 0.196 & 8.900 & 0.900 & 0.760 & 0.08 & A \\ 1 & \text { CO 60 } & 25.300 & 0.304 & 29.500 & 2.900 & 0.860 & 0.08 & A \\ 1 & \text { CS134 } & 13.300 & 0.613 & 14.700 & 1.460 & 0.910 & 0.10 & A \\ 1 & \text { CS137 } & 5.850 & 0.322 & 6.640 & 0.700 & 0.880 & 0.11 & A \\ 1 & \text { GA 1 } & 1.690 & 0.058 & 1.620 & 0.150 & 1.040 & 0.10 & \text { A } \\ 1 & \text { GB 2 } & 3.050 & 0.083 & 1.770 & 0.150 & 1.720 & 0.15 & \text { W } \\ 1 & \text { MN 54 } & 2.990 & 0.156 & 3.440 & 0.380 & 0.870 & 0.11 & A \\ 1 & \text { RU106 } & 11.200 & 0.877 & 11.600 & 1.440 & 0.970 & 0.14 & A \\ 1 & \text { SB125 } & 9.030 & 0.478 & 9.780 & 1.030 & 0.920 & 0.11 & A \\ 1 & \text { SR 90 } & 1.220 & 0.244 & 1.060 & 0.037 & 1.150 . & 0.23 & A \\ 1 & \text { U 234 } & 0.064 & 0.004 & 0.052 & 0.002 & 1.230 & 0.09 & A \\ 2 & \text { U 238 } & 0.071 & 0.006 & 0.053 & 0.002 & 1.330 & 0.13 & A\end{array}$

Matrix: So

$\begin{array}{rlrrrrrrr}1 & \text { CS137 } & 411.000 & 35.300 & 359.000 & 10.000 & 1.150 & 0.10 & \text { A } \\ 1 & \text { K 40 } & 451.000 & 25.700 & 465.000 & 30.000 & 0.970 & 0.08 & \text { A } \\ 1 & \text { SR 90 } & 936.000 & 71.400 & 1340.000 & 113.000 & 0.700 & 0.08 & \text { W } \\ 1 & \text { U 234 } & 42.200 & 2.460 & 34.200 & 3.520 & 1.230 & 0.15 & \text { W } \\ 1 & \text { U 238 } & 42.600 & 2.210 & 35.900 & 3.630 & 1.190 & 0.14 & \text { W }\end{array}$

Matrix: VE

$\begin{array}{rrrrrrrrr}1 & \text { CO 60 } & 61.800 & 3.520 & 59.700 & 0.963 & 1.040 & 0.06 & \text { A } \\ 1 & \text { K 40 } & 1130.000 & 62.300 & 1030.000 & 33.000 & 1.100 & 0.07 & \text { A } \\ 1 & \text { SR 90 } & 707.000 & 42.200 & 1300.000 & 52.400 & 0.540 & 0.04 & \text { W }\end{array}$

Matrix: WA

$\begin{array}{llrrrrrrr}1 & \text { CO 60 } & 32.800 & 1.570 & 32.800 & 0.580 & 1.000 & 0.05 & \text { A } \\ 1 & \text { CS137 } & 43.700 & 3.100 & 38.300 & 0.881 & 1.140 & 0.09 & \text { A } \\ 1 & \text { GA 1 } & 2330.000 & 62.900 & 1850.000 & 185.000 & 1.260 & 0.13 & \text { W } \\ 1 & \text { GB 2 } & 951.000 & 28.600 & 744.000 & 74.000 & 1.280 & 0.13 & \text { A } \\ 1 & \text { MN 54 } & 43.300 & 2.510 & 38.400 & 1.160 & 1.130 & 0.07 & \text { A } \\ 1 & \text { SR 90 } & 2.180 & 0.518 & 1.450 & 0.034 & 1.500 & 0.36 & \text { W } \\ 1 & \text { U 234 } & 0.335 & 0.021 & 0.274 & 0.019 & 1.220 & 0.11 & \text { W } \\ 1 & \text { U 238 } & 0.336 & 0.026 & 0.275 & 0.024 & 1.220 & 0.14 & \text { W }\end{array}$

Units for matrices: $\mathrm{Al}=\mathrm{Bq} /$ filter $\mathrm{SO}=\mathrm{Bq} / \mathrm{kg}$ VE=Bq/kg $W \mathrm{~A}=\mathrm{Bq} / \mathrm{L}$. Values for elemental uranium are reported in $\mu \mathrm{g} /$ filter, $\mathrm{g}$, or $\mathrm{mL}$.

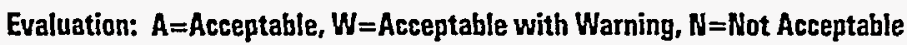

$\mathrm{pCi}=\mathrm{Bq} \times 27$ 


\section{QAP44 Results by Laboratory}

Lab: BE RUST Geotech, Grand Junction, CO

\begin{tabular}{lllllllll} 
No. & Radio- & Reported & Reported & EML & EML & Reported & Ratio & Evor \\
Test & nuclide & Value & Error & Value & Error & $\frac{\text { EML }}{\text { Error }}$ & Evaluation \\
\hline
\end{tabular}

Matrix: Al

\begin{tabular}{|c|c|c|c|c|c|c|}
\hline AM241 & 0.180 & 0.010 & 0.189 & 0.007 & 0.950 & 0.06 \\
\hline CE144 & 21.300 & 2.200 & 33.300 & 3.300 & 0.640 & 0.09 \\
\hline $\mathrm{CO} 57$ & 6.160 & 0.420 & 8.900 & 0.900 & 0.690 & 0.08 \\
\hline CO 60 & 23.600 & 1.610 & 29.500 & 2.900 & 0.800 & 0.10 \\
\hline CS134 & 11.600 & 0.820 & 14.700 & 1.460 & 0.790 & 0.10 \\
\hline CS137 & 5.480 & 0.390 & 6.640 & 0.700 & 0.830 & 0.11 \\
\hline GA 1 & 2.200 & 0.200 & 1.620 & 0.150 & 1.360 & 0.18 \\
\hline GB 2 & 1.800 & 0.100 & 1.770 & 0.150 & 1.020 & 0.10 \\
\hline MN 54 & 2.900 & 0.230 & 3.440 & 0.380 & 0.840 & 0.12 \\
\hline PU238 & 0.100 & 0.010 & 0.096 & 0.002 & 1.040 & 0.11 \\
\hline PU239 & 0.100 & 0.010 & 0.093 & 0.003 & 1.080 & 0.11 \\
\hline RU106 & 8.280 & 1.020 & 11.600 & 1.440 & 0.710 & 0.13 \\
\hline SB125 & 7.020 & 0.560 & 9.780 & 1.030 & 0.720 & 0.09 \\
\hline SR 90 & 1.060 & 0.090 & 1.060 & 0.037 & 1.000 & 0.09 \\
\hline U 234 & 0.052 & 0.010 & 0.052 & 0.002 & 1.010 & 0.20 \\
\hline U 238 & 0.055 & 0.010 & 0.053 & 0.002 & 1.030 & 0.19 \\
\hline U UG & 4.500 & 0.000 & 4.310 & 0.100 & 1.040 & 0.02 \\
\hline
\end{tabular}

Matrix: so

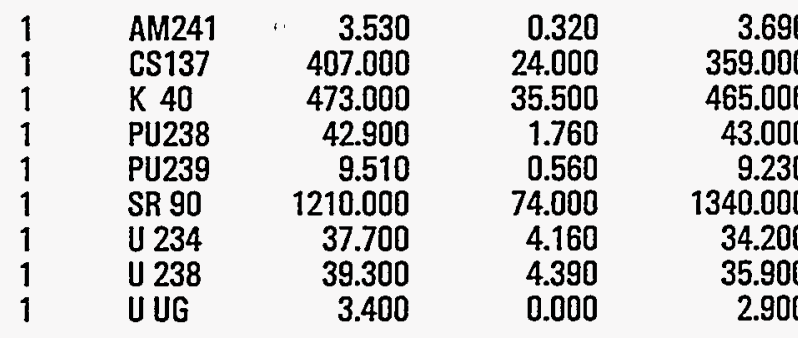

Matrix: VE

$\begin{array}{llrrrrrrr}1 & \text { AM241 } & 6.170 & 0.580 & 5.600 & 0.184 & 1.100 & 0.11 & \text { A } \\ 1 & \text { CM244 } & 3.670 & 0.410 & 4.440 & 0.202 & 0.830 & 0.10 & \text { W } \\ 1 & \text { C0 60 } & 55.400 & 4.000 & 59.700 & 0.963 & 0.930 & 0.07 & \text { A } \\ 1 & \text { CS137 } & 1190.000 & 59.800 & 944.000 & 16.200 & 1.260 & 0.07 & \text { W } \\ 1 & \text { K 40 } & 1080.000 & 73.000 & 1030.000 & 33.000 & 1.050 & 0.08 & \text { A } \\ 1 & \text { PU239 } & 9.290 & 1.050 & 9.820 & 1.220 & 0.950 & 0.16 & \text { A } \\ 1 & \text { SR 90 } & 1120.000 & 62.000 & 1300.000 & 52.400 & 0.860 & 0.06 & \text { A }\end{array}$

Matrix: WA

$\begin{array}{llrrrrrrr}1 & \text { AM241 } & 0.800 & 0.060 & 0.766 & 0.013 & 1.040 & 0.08 & A \\ 1 & \text { CO 60 } & 35.500 & 1.470 & 32.800 & 0.580 & 1.080 & 0.05 & A \\ 1 & \text { CS137 } & 46.000 & 1.850 & 38.300 & 0.881 & 1.200 & 0.06 & \text { W } \\ 1 & \text { GA 1 } & 1840.000 & 34.000 & 1850.000 & 185.000 & 1.000 & 0.10 & \text { A }\end{array}$

Units for matrices: $\mathrm{Al}=\mathrm{Bq} / \mathrm{filter} \mathrm{SO}=\mathrm{Bq} / \mathrm{kg}$ VE=Bq/kg $W A=B q / \mathrm{L}$. Values for elemental uranium are reported in $\mu \mathrm{g} / f i l t e r, \mathrm{~g}$, or $\mathrm{mL}$. Evaluation: A=Acceptable, $W=A c c e p t a b l e$ with Warning, $N=$ Not Acceptable $\mathrm{pCi}=\mathrm{Bq} \times 27$ 


\section{QAP44 Results by Laboratory}

Lab: BE RUST Geotech, Grand Junction, CO

\begin{tabular}{llllllll} 
No. & Radio \\
Test & nuclide & $\begin{array}{l}\text { Reported } \\
\text { Value }\end{array}$ & $\begin{array}{c}\text { Reported } \\
\text { Error }\end{array}$ & $\begin{array}{c}\text { EML } \\
\text { Value }\end{array}$ & $\begin{array}{l}\text { EML } \\
\text { Error }\end{array}$ & $\frac{\text { Reported }}{\text { EML }}$ & $\begin{array}{l}\text { Ratio } \\
\text { Error }\end{array}$ Evaluation \\
\hline
\end{tabular}

Matrix: WA

$\begin{array}{llrrrrrrr}1 & \text { GB 2 } & 578.000 & 6.800 & 744.000 & 74.000 & 0.780 & 0.08 & \text { W } \\ 1 & \text { H } 3 & 206.000 & 20.500 & 251.000 & 11.400 & 0.820 & 0.09 & \text { W } \\ 1 & \text { MN 54 } & 47.500 & 1.860 & 38.400 & 1.160 & 1.240 & 0.06 & \text { N } \\ 1 & \text { PU238 } & 0.960 & 0.090 & 0.982 & 0.074 & 0.980 & 0.12 & \text { A } \\ 1 & \text { PU239 } & 0.740 & 0.070 & 0.772 & 0.056 & 0.960 & 0.11 & \text { A } \\ 1 & \text { SR 90 } & 1.860 & 0.350 & 1.450 & 0.034 & 1.280 & 0.24 & W \\ 1 & \text { U 234 } & 0.284 & 0.054 & 0.274 & 0.019 & 1.040 & 0.21 & A \\ 1 & \text { U 238 } & 0.275 & 0.054 & 0.275 & 0.024 & 1.000 & 0.22 & \text { A } \\ 1 & \text { U UG } & 23.900 & 0.000 & 0.022 & 0.003 & * * * & * * * * & \text { N }\end{array}$

Units for matrices: $\mathrm{Al}=\mathrm{Bq} / \mathrm{filter} \mathrm{SO}=\mathrm{Bq} / \mathrm{kg} \mathrm{VE}=\mathrm{Bq} / \mathrm{kg} \mathrm{WA}=\mathrm{Bq} / \mathrm{L}$. Values for elemental uranium are reported in $\mu \mathrm{g} / \mathrm{filter}, \mathrm{g}$, or $\mathrm{mL}$. 


\section{QAP44 Results by Laboratory}

Lab: BK Brookhaven National Laboratory, Upton, NY

\begin{tabular}{llllllll} 
No. & Radio & Reported & Reported & EML & EML & Reported & Ratio \\
Test & nuclide & Value & Error & Value & Error & $\frac{1}{\text { EML }}$ & $\begin{array}{l}\text { Error } \\
\text { Evaluation }\end{array}$ \\
\hline
\end{tabular}

Matrix: WA

$\begin{array}{lllllllll}1 & \text { CO 60 } & 35.300 & 2.390 & 32.800 & 0.580 & 1.080 & 0.08 & \\ 1 & \text { CS137 } & 45.700 & 2.390 & 38.300 & 0.881 & 1.190 & 0.07 & \text { W } \\ 1 & \text { MN 54 } & 45.300 & 2.090 & 38.400 & 1.160 & 1.180 & 0.07 & W \\ 1 & \text { PU239 } & 0.550 & 0.060 & 0.772 & 0.056 & 0.710 & 0.09 & W\end{array}$

Units for matrices: $A \mid=B q /$ filter $S D=B q / k g ~ V E=B q / k g ~ W A=B q / L$. Values for elemental uranium are reported in $\mu g / f i l t e r, g$, or $\mathrm{mL}$. 


\section{QAP44 Results by Laboratory}

Lab: BL Barringer Laboratories Inc., Golden, CO

\begin{tabular}{lllllllll}
$\begin{array}{l}\text { No. } \\
\text { Test }\end{array}$ & $\begin{array}{l}\text { Radio- } \\
\text { nuclide }\end{array}$ & $\begin{array}{l}\text { Reported } \\
\text { Value }\end{array}$ & $\begin{array}{l}\text { Reported } \\
\text { Error }\end{array}$ & $\begin{array}{c}\text { EML } \\
\text { Value }\end{array}$ & $\begin{array}{c}\text { EML } \\
\text { Error }\end{array}$ & $\begin{array}{c}\text { Reported } \\
\text { EML }\end{array}$ & $\begin{array}{l}\text { Ratio } \\
\text { Error }\end{array}$ & Evaluation \\
\hline
\end{tabular}

Matrix: Al

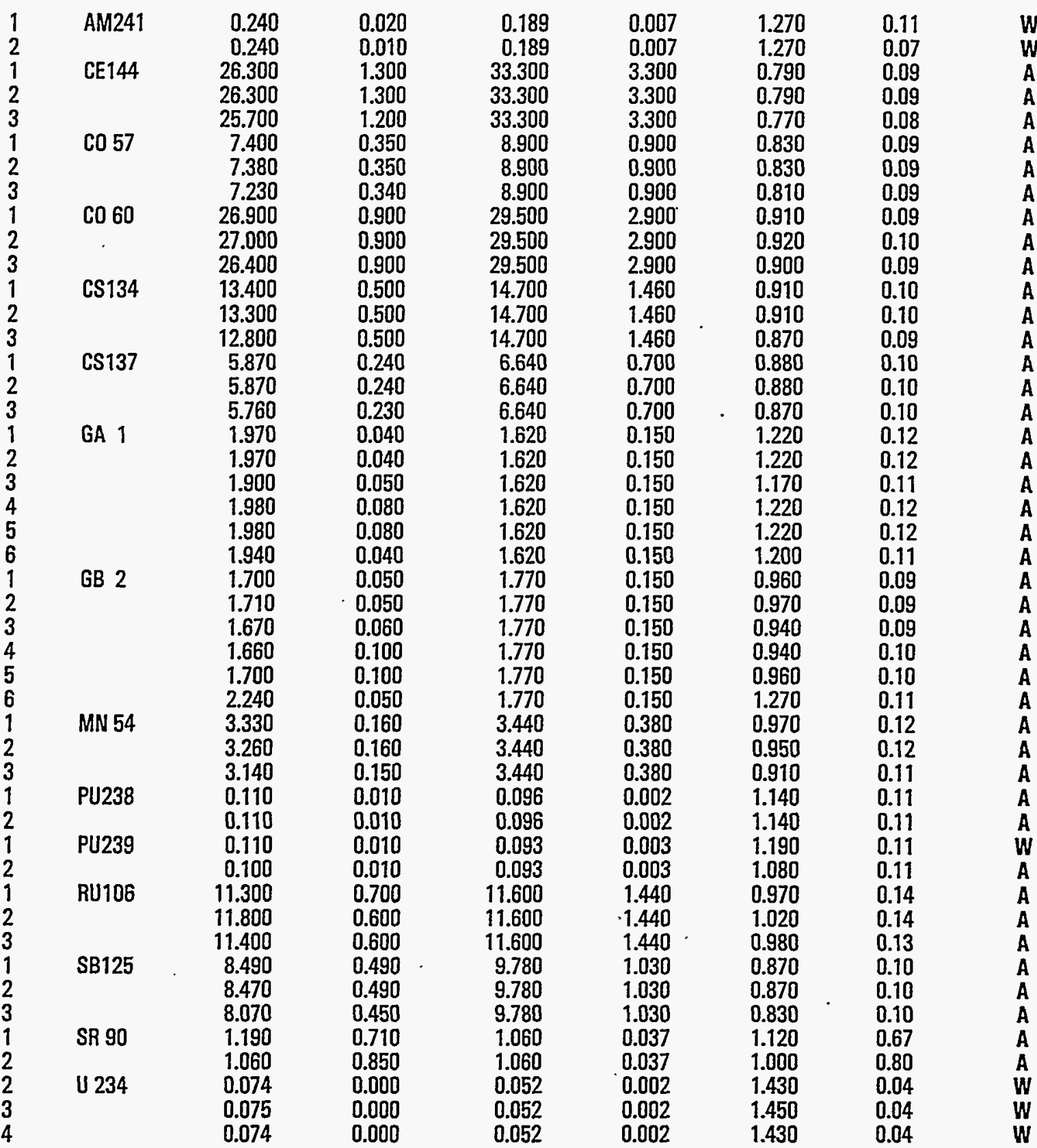

Units for matrices: $\mathrm{Al}=\mathrm{Bq} / \mathrm{filter} \mathrm{SO}=\mathrm{Bq} / \mathrm{kg}$ VE=Bq/kg $\mathrm{WA}=\mathrm{Bq} / \mathrm{L}$. Values for elemental uranium are reported in $\mu \mathrm{g} / \mathrm{filter}, \mathrm{g}$, or $\mathrm{mL}$.

Evaluation: $A=$ Acceptable, $W=$ Acceptable with Warning, $N=$ Not Acceptable $\mathrm{pCi}=\mathrm{Bq} \times 27$ 


\section{QAP44 Results by Laboratory}

Lab: BL Barringer Laboratories Inc., Golden, CO

\begin{tabular}{lllllllll} 
No. & $\begin{array}{l}\text { Radio- } \\
\text { nuclide }\end{array}$ & $\begin{array}{l}\text { Reported } \\
\text { Value }\end{array}$ & $\begin{array}{c}\text { Reported } \\
\text { Error }\end{array}$ & $\begin{array}{c}\text { EML } \\
\text { Value }\end{array}$ & $\begin{array}{c}\text { EML } \\
\text { Error }\end{array}$ & $\frac{\text { Reported }}{\text { EML }}$ & $\begin{array}{l}\text { Ratio } \\
\text { Error }\end{array}$ & Evaluation \\
\hline
\end{tabular}

Matrix: Al

$\begin{array}{lllllllll}2 & \text { U 238 } & 0.073 & 0.000 & 0.053 & 0.002 & 1.370 & 0.05 & A \\ 3 & & 0.072 & 0.000 & 0.053 & 0.002 & 1.350 & 0.05 & A \\ 1 & \text { U UG } & 6.080 & 0.110 & 4.310 & 0.100 & 1.410 & 0.04 & \text { W } \\ 2 & & 6.110 & 0.120 & 4.310 & 0.100 & 1.420 & 0.04 & W \\ 3 & & 6.080 & 0.110 & 4.310 & 0.100 & 1.410 & 0.04 & \text { W }\end{array}$

Matrix: SO

$\begin{array}{llrrrrrrr}1 & \text { AM241 } & 2.630 & 0.180 & 3.690 & 0.454 & 0.710 & 0.10 & \text { W } \\ 1 & \text { CS137 } & 372.000 & 8.000 & 359.000 & 10.000 & 1.040 & 0.04 & \text { A } \\ 2 & & 374.000 & 8.000 & 359.000 & 10.000 & 1.040 & 0.04 & \text { A } \\ 3 & & 375.000 & 8.000 & 359.000 & 10.000 & 1.040 & 0.04 & \text { A } \\ 1 & \text { K 40 } & 528.000 & 17.000 & 465.000 & 30.000 & 1.140 & 0.08 & \text { A } \\ 2 & & 519.000 & 18.000 & 465.000 & 30.000 & 1.120 & 0.08 & \text { A } \\ 3 & & 522.000 & 17.000 & 465.000 & 30.000 & 1.120 & 0.08 & \text { A } \\ 1 & \text { PU238 } & 45.000 & 0.900 & 43.000 & 2.440 & 1.050 & 0.06 & \text { A } \\ 2 & & 46.600 & 0.020 & 43.000 & 2.440 & 1.080 & 0.06 & \text { A } \\ 1 & \text { PU239 } & 10.100 & 0.430 & 9.230 & 0.346 & 1.090 & 0.06 & \text { A } \\ 2 & & 12.400 & 0.010 & 9.230 & 0.346 & 1.340 & 0.05 & \text { W } \\ 1 & \text { SR 90 } & 958.000 & 45.000 & 1340.000 & 113.000 & 0.720 & 0.07 & \text { W } \\ 2 & & 958.000 & 34.000 & 1340.000 & 113.000 & 0.720 & 0.07 & \text { W } \\ 1 & \text { U 234 } & 48.500 & 0.000 & 34.200 & 3.520 & 1.420 & 0.15 & \mathbf{N} \\ 2 & & 49.700 & 0.000 & 34.200 & 3.520 & 1.450 & 0.15 & \mathbf{N} \\ 3 & & 48.500 & 0.000 & 34.200 & 3.520 & 1.420 & 0.15 & \mathbf{N} \\ 1 & \text { U 238 } & 47.300 & 0.000 & 35.900 & 3.630 & 1.320 & 0.13 & \mathbf{W} \\ 2 & & 48.500 & 0.000 & 35.900 & 3.630 & 1.350 & 0.14 & \mathbf{W} \\ 3 & & 47.300 & 0.000 & 35.900 & 3.630 & 1.320 & 0.13 & \mathbf{W} \\ 1 & \text { U UG } & 3.970 & 0.090 & 2.900 & 0.290 & 1.370 & 0.14 & \mathbf{W} \\ 2 & & 4.070 & 0.090 & 2.900 & 0.290 & 1.400 & 0.14 & \mathbf{W} \\ 3 & & 3.970 & 0.090 & 2.900 & 0.290 & 1.370 & 0.14 & \mathbf{W}\end{array}$

Matrix: VE

$\begin{array}{rrrrrrrrr}1 & \text { AM241 } & 7.310 & 0.260 & 5.600 & 0.184 & 1.310 & 0.06 & \text { A } \\ 2 & & 8.260 & 0.290 & 5.600 & 0.184 & 1.480 & 0.07 & \text { A } \\ 2 & & 10.900 & 0.790 & 5.600 & 0.184 & 1.950 & 0.16 & \text { W } \\ 3 & & 8.540 & 0.900 & 5.600 & 0.184 & 1.530 & 0.17 & \text { A } \\ 1 & \text { CM244 } & 4.860 & 0.200 & 4.440 & 0.202 & 1.100 & 0.07 & A \\ 2 & & 4.600 & 0.610 & 4.440 & 0.202 & 1.040 & 0.15 & \text { A } \\ 2 & & 4.410 & 0.190 & 4.440 & 0.202 & 0.990 & 0.06 & A \\ 3 & & 4.770 & 0.660 & 4.440 & 0.202 & 1.070 & 0.16 & A \\ 1 & \text { C0 60 } & 64.000 & 2.300 & 59.700 & 0.963 & 1.070 & 0.04 & A \\ 2 & & 60.400 & 2.200 & 59.700 & 0.963 & 1.010 & 0.04 & A \\ 3 & & 62.900 & 2.400 & 59.700 & 0.963 & 1.050 & 0.04 & A \\ 1 & \text { CS137 } & 1030.000 & 22.000 & 944.000 & 16.200 & 1.090 & 0.03 & A \\ 2 & & 1020.000 & 21.000 & 944.000 & 16.200 & 1.080 & 0.03 & \text { A }\end{array}$

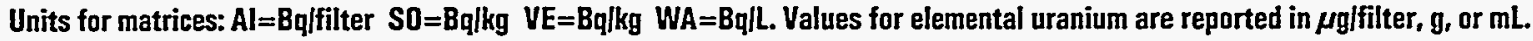

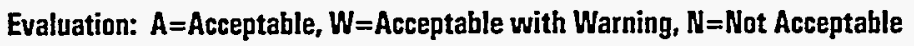
$\mathrm{pCi}=\mathrm{Bq} \times 27$ 


\section{QAP44 Results by Laboratory}

Lab: BL Barringer Laboratories Inc.; Golden, CO

\begin{tabular}{|c|c|c|c|c|c|c|c|c|}
\hline No. & $\begin{array}{l}\text { Radio- } \\
\text { nuclide }\end{array}$ & $\begin{array}{l}\text { Reported } \\
\text { Value }\end{array}$ & $\begin{array}{l}\text { Reported } \\
\text { Error }\end{array}$ & $\begin{array}{c}\text { EML } \\
\text { Value }\end{array}$ & $\begin{array}{l}\text { EML } \\
\text { Error }\end{array}$ & $\frac{\text { Reporte }}{\text { EML }}$ & $\begin{array}{l}\text { Ratio } \\
\text { Error }\end{array}$ & Evaluation \\
\hline
\end{tabular}

Matrix: VE

$\begin{array}{lrrrrrrr}\text { CS137 } & 1020.000 & 22.000 & 944.000 & 16.200 & 1.080 & 0.03 & \text { A } \\ \text { K 40 } & 1140.000 & 35.000 & 1030.000 & 33.000 & 1.110 & 0.05 & \text { A } \\ & 1130.000 & 35.000 & 1030.000 & 33.000 & 1.100 & 0.05 & \text { A } \\ & 1160.000 & 37.000 & 1030.000 & 33.000 & 1.130 & 0.05 & \text { A } \\ \text { PU238 } & 0.940 & 0.100 & 0.820 & 0.104 & 1.150 & 0.19 & \text { A } \\ & 0.990 & 0.340 & 0.820 & 0.104 & 1.210 & 0.44 & \text { A } \\ \text { PU239 } & 9.590 & 0.320 & 9.820 & 1.220 & 0.980 & 0.13 & \text { A } \\ & 9.020 & 0.020 & 9.820 & 1.220 & 0.920 & 0.11 & \text { A } \\ & 9.130 & 0.660 & 9.820 & 1.220 & 0.930 & 0.13 & \text { A } \\ \text { SR 90 } & 1390.000 & 38.000 & 1300.000 & 52.400 & 1.070 & 0.05 & \text { A } \\ & 1320.000 & 34.000 & 1300.000 & 52.400 & 1.020 & 0.05 & \text { A }\end{array}$

Matrix: WA

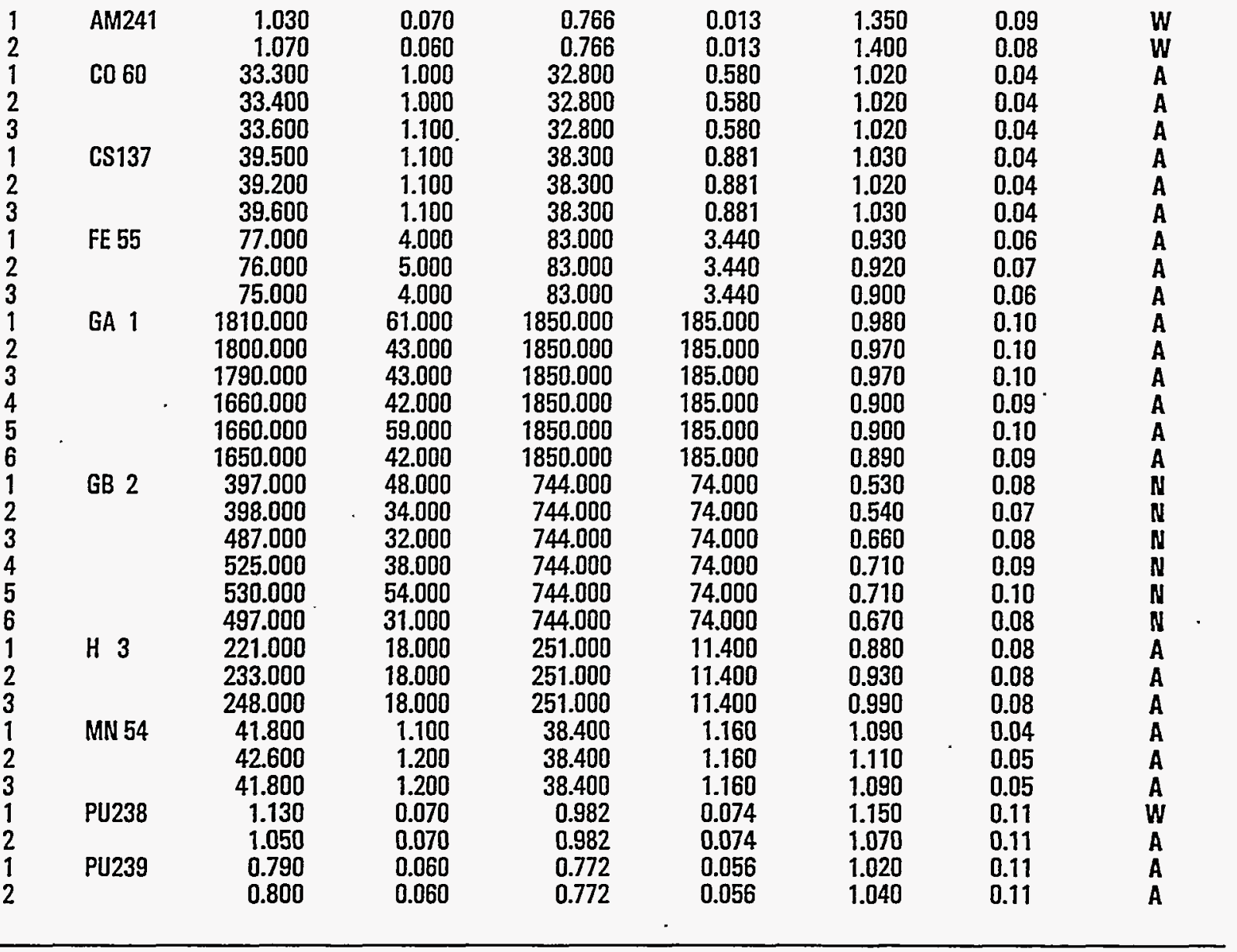

Units for matrices: $\mathrm{Al}=\mathrm{Bq} /$ filter $\mathrm{SO}=\mathrm{Bq} / \mathrm{kg}$ VE=Bq/kg $W A=B q / \mathrm{L}$. Values for elemental uranium are reported in $\mu \mathrm{g} /$ filter, $\mathrm{g}$, or $\mathrm{mL}$.

Evaluation: $A=$ Acceptable, $W=$ Acceptable with Warning, $N=$ Not Acceptable

$\mathrm{pCi}=\mathrm{Bq} \times 27$ 


\section{QAP44 Results by Laboratory}

Lab: BL Barringer Laboratories Inc., Golden, CO

\begin{tabular}{lllllllll} 
No. & Radio- & Reported & Reported & EML & EML & Reported & Ratio \\
Test & nuclide & Value & Error & Value & Error & EML & Error & Evaluation \\
\hline
\end{tabular}

Matrix: WA

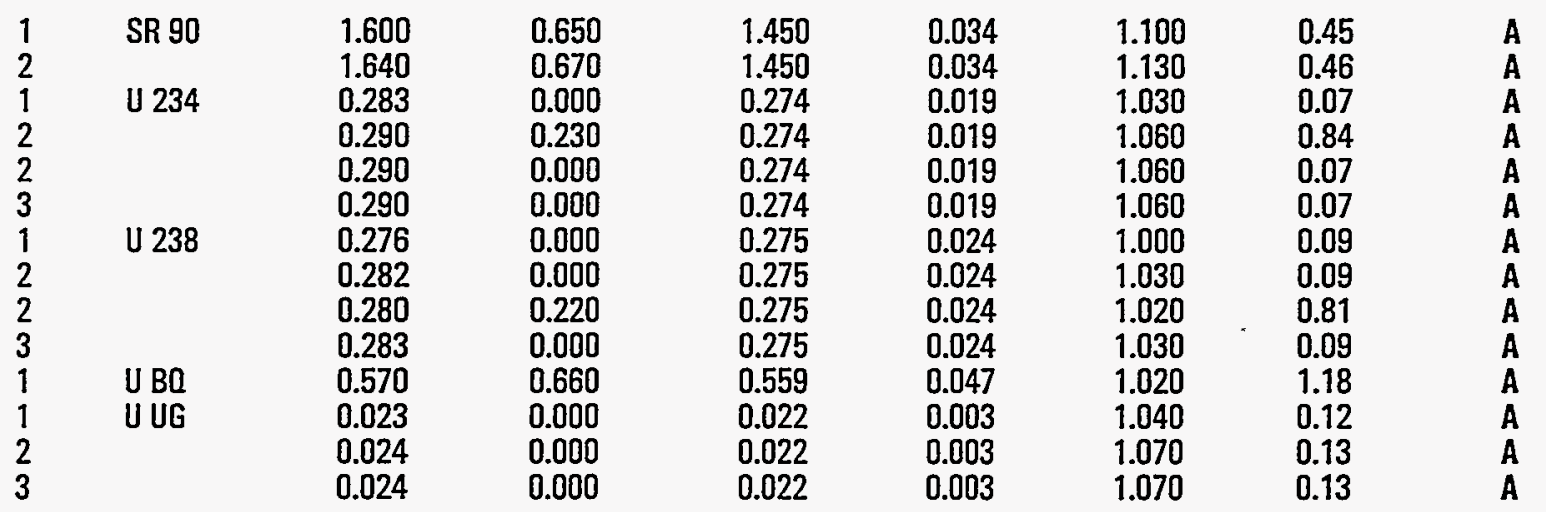

Units for matrices: $\mathrm{Al}=\mathrm{Bq} / \mathrm{filter} \mathrm{SO}=\mathrm{Bq} / \mathrm{kg}$ VE $=\mathrm{Bq} / \mathrm{kg} W \mathrm{~W}=\mathrm{Bq} / \mathrm{L}$. Values for elemental uranium are reported in $\mu \mathrm{g} /$ filter, $\mathrm{g}$, or $\mathrm{mL}$. 


\section{QAP44 Results by Laboratory}

Lab: BM Battelle Memorial Institute, Columbus, $\mathrm{OH}$

\begin{tabular}{|c|c|c|c|c|c|c|c|c|}
\hline $\begin{array}{l}\text { No. } \\
\text { Test }\end{array}$ & $\begin{array}{l}\text { Radio- } \\
\text { nuclide }\end{array}$ & $\begin{array}{l}\text { Reported } \\
\text { Value }\end{array}$ & $\begin{array}{l}\text { Reported } \\
\text { Error }\end{array}$ & $\begin{array}{c}\text { EML } \\
\text { Value }\end{array}$ & $\begin{array}{l}\text { EML } \\
\text { Error }\end{array}$ & $\frac{\text { Reporte }}{\text { EML }}$ & $\begin{array}{l}\text { Ratio } \\
\text { Error }\end{array}$ & Fualuation \\
\hline
\end{tabular}

Matrix: Al

$\begin{array}{lrrrrrrr}\text { AM241 } & 0.260 & 0.100 & 0.189 & 0.007 & 1.380 & 0.53 & \text { W } \\ \text { CE144 } & 28.000 & 2.000 & 33.300 & 3.300 & 0.840 & 0.10 & \text { A } \\ \text { CO 57 } & 8.010 & 0.560 & 8.900 & 0.900 & 0.900 & 0.11 & \text { A } \\ \text { CO 60 } & 26.300 & 1.800 & 29.500 & 2.900 & 0.890 & 0.11 & \text { A } \\ \text { CS134 } & 14.600 & 0.700 & 14.700 & 1.460 & 0.990 & 0.11 & \text { A } \\ \text { CS137 } & 6.390 & 0.380 & 6.640 & 0.700 & 0.960 & 0.12 & \text { A } \\ \text { MN 54 } & 3.260 & 0.230 & 3.440 & 0.380 & 0.950 & 0.12 & \text { A } \\ \text { PU238 } & 0.120 & 0.030 & 0.096 & 0.002 & 1.250 & 0.31 & \text { W } \\ \text { PU239 } & 0.120 & 0.030 & 0.093 & 0.003 & 1.290 & 0.33 & \text { W } \\ \text { RU106 } & 12.500 & 1.400 & 11.600 & 1.440 & 1.080 & 0.18 & \text { A } \\ \text { SB125 } & 9.500 & 0.700 & 9.780 & 1.030 & 0.970 & 0.13 & \text { A } \\ \text { SR 90 } & 0.820 & 0.090 & 1.060 & 0.037 & 0.770 & 0.09 & \text { W } \\ \text { U 234 } & 0.077 & 0.017 & 0.052 & 0.002 & 1.490 & 0.33 & \text { W } \\ \text { U 238 } & 0.066 & 0.017 & 0.053 & 0.002 & 1.240 & 0.32 & \text { A }\end{array}$

Matrix: So

$\begin{array}{rlrrrrrrr}1 & \text { CS137 } & 306.000 & 22.000 & 359.000 & 10.000 & 0.850 & 0.07 & W \\ 1 & \text { K 40 } & 380.000 & 60.000 & 465.000 & 30.000 & 0.820 & 0.14 & W \\ 1 & \text { SR 90 } & 506.000 & 60.000 & 1340.000 & 113.000 & 0.380 & 0.05 & \mathbf{N} \\ 1 & \text { U UG } & 68.000 & 12.000 & 2.900 & 0.290 & .^{* * *} & 4.76 & \mathbf{N}\end{array}$

Matrix: VE

\begin{tabular}{|c|c|c|c|c|c|c|}
\hline $\begin{array}{l}\text { AM241 } \\
\text { CO 60 } \\
\text { CS137 } \\
\text { K } 40 \\
\text { SR 90 }\end{array}$ & $\begin{array}{r}9.100 \\
51.000 \\
840.000 \\
870.000 \\
209.000\end{array}$ & $\begin{array}{r}4.500 \\
3.600 \\
60.000 \\
45.000 \\
20.000\end{array}$ & $\begin{array}{r}5.600 \\
59.700 \\
944.000 \\
1030.000 \\
1300.000\end{array}$ & $\begin{array}{r}0.184 \\
0.963 \\
16.200 \\
33.000 \\
52.400\end{array}$ & $\begin{array}{l}1.630 \\
0.850 \\
0.890 \\
0.850 \\
0.160\end{array}$ & $\begin{array}{l}0.81 \\
0.06 \\
0.07 \\
0.05 \\
0.02\end{array}$ \\
\hline
\end{tabular}

Matrix: WA

$\begin{array}{llrllllll}1 & \text { CO 60 } & 31.300 & 2.200 & 32.800 & 0.580 & 0.950 & 0.07 & \text { A } \\ 1 & \text { CS137 } & 43.500 & 3.100 & 38.300 & 0.881 & 1.140 & 0.09 & \text { A } \\ 1 & \text { MN 54 } & 42.000 & 3.000 & 38.400 & 1.160 & 1.090 & 0.08 & \text { A } \\ 1 & \text { PU238 } & 0.930 & 0.160 & 0.982 & 0.074 & 0.950 & 0.18 & \text { A } \\ 1 & \text { PU239 } & 0.710 & 0.120 & 0.772 & 0.056 & 0.920 & 0.17 & \text { A } \\ 1 & \text { SR 90 } & 1.170 & 0.230 & 1.450 & 0.034 & 0.810 & 0.16 & \text { W } \\ 1 & \text { U 234 } & 0.300 & 0.060 & 0.274 & 0.019 & 1.100 & 0.23 & \text { A } \\ 1 & \text { U 238 } & 0.320 & 0.060 & 0.275 & 0.024 & 1.160 & 0.24 & \text { W }\end{array}$

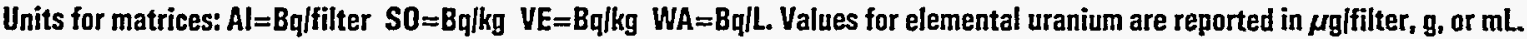

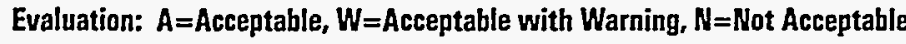
$\mathrm{pCi}=\mathrm{Bq} \times 27$ 


\section{QAP44 Results by Laboratory}

Lab: BN Brookhaven National Laboratory, Upton, NY

\begin{tabular}{lllllllll} 
No. & Radio- & Reported & Reported & EML & EML & Reported & Ratio \\
nest & nuclide & Value & Error & Value & Error & EML & Error & Evaluation \\
\hline
\end{tabular}

Matrix: Al

$\begin{array}{llrlrllll}1 & \text { CE144 } & 32.000 & 2.260 & 33.300 & 3.300 & 0.960 & 0.12 & \text { A } \\ 1 & \text { CO 57 } & 9.120 & 0.600 & 8.900 & 0.900 & 1.030 & 0.12 & \text { A } \\ 1 & \text { CO 60 } & 26.100 & 0.170 & 29.500 & 2.900 & 0.890 & 0.09 & \text { A } \\ 1 & \text { CS134 } & 13.000 & 0.020 & 14.700 & 1.460 & 0.880 & 0.09 & \text { A } \\ 1 & \text { CS137 } & 7.940 & 0.400 & 6.640 & 0.700 & 1.200 & 0.14 & \text { W } \\ 1 & \text { GA 1 } & 1.940 & 0.050 & 1.620 & 0.150 & 1.200 & 0.12 & \text { A } \\ 1 & \text { GB 2 } & 2.290 & 0.050 & 1.770 & 0.150 & 1.290 & 0.11 & \text { A } \\ 1 & \text { MN 54 } & 3.860 & 0.160 & 3.440 & 0.380 & 1.120 & 0.13 & A \\ 1 & \text { RU106 } & 12.000 & 0.350 & 11.600 & 1.440 & 1.030 & 0.13 & A \\ 1 & \text { SB125 } & 12.400 & 0.640 & 9.780 & 1.030 & 1.270 & 0.15 & \text { W }\end{array}$

Matrix: So

\begin{tabular}{|c|c|c|c|c|c|c|}
\hline $\begin{array}{l}\text { CS137 } \\
\text { K } 40\end{array}$ & $\begin{array}{l}354.000 \\
414.000\end{array}$ & $\begin{array}{r}19.300 \\
7.940\end{array}$ & $\begin{array}{l}359.000 \\
465.000\end{array}$ & $\begin{array}{l}10.000 \\
30.000\end{array}$ & $\begin{array}{l}0.990 \\
0.890\end{array}$ & $\begin{array}{l}0.06 \\
0.06\end{array}$ \\
\hline VE & & & & & & \\
\hline $\begin{array}{l}\text { CO } 60 \\
\text { CS137 } \\
K 40\end{array}$ & $\begin{array}{r}46.500 \\
949.000 \\
885.000\end{array}$ & $\begin{array}{r}0.940 \\
31.200 \\
21.600\end{array}$ & $\begin{array}{r}59.700 \\
944.000 \\
1030.000\end{array}$ & $\begin{array}{r}0.963 \\
16.200 \\
33.000\end{array}$ & $\begin{array}{l}0.780 \\
1.010 \\
0.860\end{array}$ & $\begin{array}{l}0.02 \\
0.04 \\
0.03\end{array}$ \\
\hline
\end{tabular}

Matrix: WA

$\begin{array}{llrrrrrrr}1 & \text { CO 60 } & 32.700 & 0.600 & 32.800 & 0.580 & 1.000 & 0.03 & \text { A } \\ 1 & \text { CS137 } & 42.500 & 0.410 & 38.300 & 0.881 & 1.110 & 0.03 & \text { A } \\ 1 & \text { GA 1 } & 2050.000 & 24.200 & 1850.000 & 185.000 & 1.110 & 0.11 & . \\ 1 & \text { GB 2 } & 924.000 & 13.700 & 744.000 & 74.000 & 1.240 & 0.13 & \text { A } \\ 1 & \text { H 3 } & 213.000 & 7.970 & 251.000 & 11.400 & 0.850 & 0.05 & \text { A } \\ 1 & \text { MN 54 } & 43.000 & 0.420 & 38.400 & 1.160 & 1.120 & 0.04 & \text { A } \\ 1 & \text { SR 90 } & 1.320 & 0.000 & 1.450 & 0.034 & 0.910 & 0.02 & \text { A }\end{array}$

Units for matrices: $A \mathrm{~A}=\mathrm{Bq} / \mathrm{filter} \mathrm{SO}=\mathrm{Bq} / \mathrm{kg}$ VE=Bq/kg $W A=B q[\mathrm{~L}$. Values for elemental uranium are reported in $\mu \mathrm{g} / \mathrm{filter}, \mathrm{g}$, or $\mathrm{mL}$. Evaluation: $A=A c c e p t a b l e, W=A c c e p t a b l e$ with Warning, $N=$ Not Acceptable $\mathrm{pCi}=\mathrm{Bq} \times 27$ 


\section{QAP44 Results by Laboratory}

Lab: BP Battelle Pacific Northwest Laboratory

\begin{tabular}{llllllll}
$\begin{array}{l}\text { No. } \\
\text { Test }\end{array}$ & $\begin{array}{l}\text { Radio. } \\
\text { nuclide }\end{array}$ & $\begin{array}{l}\text { Reported } \\
\text { Value }\end{array}$ & $\begin{array}{l}\text { Reported } \\
\text { Error }\end{array}$ & $\begin{array}{c}\text { EML } \\
\text { Value }\end{array}$ & $\begin{array}{c}\text { EML } \\
\text { Error }\end{array}$ & $\frac{\text { Reported }}{\text { EML }}$ & $\begin{array}{l}\text { Ratio } \\
\text { Error }\end{array}$ \\
\hline
\end{tabular}

Matrix: AI

$\begin{array}{lrrrrrrr}\text { AM241 } & 0.201 & 0.012 & 0.189 & 0.007 & 1.060 & 0.07 & \text { A } \\ \text { CE144 } & 26.200 & 7.000 & 33.300 & 3.300 & 0.790 & 0.22 & \text { A } \\ \text { CO 57 } & 5.800 & 1.200 & 8.900 & 0.900 & 0.650 & 0.15 & \text { W } \\ \text { CD 60 } & 24.500 & 1.700 & 29.500 & 2.900 & 0.830 & 0.10 & \text { A } \\ \text { CS134 } & 14.100 & 1.100 & 14.700 & 1.460 & 0.960 & 0.12 & \text { A } \\ \text { CS137 } & 7.400 & 1.400 & 6.640 & 0.700 & 1.110 & 0.24 & \text { A } \\ \text { MN 54 } & 3.100 & 1.400 & 3.440 & 0.380 & 0.900 & 0.42 & \text { A } \\ \text { PU238 } & 0.103 & 0.006 & 0.096 & 0.002 & 1.070 & 0.07 & \text { A } \\ \text { PU239 } & 0.099 & 0.006 & 0.093 & 0.003 & 1.070 & 0.07 & \\ \text { SB125 } & 8.900 & 2.900 & 9.780 & 1.030 & 0.910 & 0.31 & \text { A } \\ \text { SR 90 } & 1.010 & 0.080 & 1.060 & 0.037 & 0.950 & 0.08 & \text { A }\end{array}$

Matrix: SO

$\begin{array}{llrrrr}1 & \text { AM241 } & 3.440 & 0.320 & 3.690 & 0.454 \\ 1 & \text { CS137 } & 428.000 & 7.000 & 359.000 & 10.000 \\ 1 & \text { K 40 } & 542.000 & 14.000 & 465.000 & 30.000 \\ 1 & \text { PU238 } & 45.200 & 1.900 & 43.000 & 2.440 \\ 1 & \text { PU239 } & 10.100 & 0.300 & 9.230 & 0.346 \\ 1 & \text { SR 90 } & 1210.000 & 75.000 & 1340.000 & 113.000 \\ 1 & \text { U UG } & 3.040 & 0.000 & 2.900 & 0.29\end{array}$

Matrix: VE

$\begin{array}{rlrr}1 & C 060 & 69.800 & 2.200 \\ 1 & \text { CS137 } & 1850.000 & 43.000\end{array}$

K $40 \quad 1330.000 \quad 43.000$

Matrix: WA

1
1
1
1
1
1
1
1
1

$\begin{array}{lrrr}\text { AM241 } & 0.708 & 0.020 & 0.766 \\ \text { CO 60 } & 34.400 & 0.580 & 32.800 \\ \text { CS137 } & 45.100 & 1.100 & 38.300 \\ \text { H } 3 & 187.000 & 22.000 & 251.000 \\ \text { MN 54 } & 43.800 & 0.840 & 38.400 \\ \text { PU238 } & 0.931 & 0.063 & 0.982 \\ \text { PU239 } & 0.704 & 0.023 & 0.772 \\ \text { SR 90 } & 1.240 & 0.110 & 1.450 \\ \text { U UG } & 24.300 & 0.000 & 0.022\end{array}$

0.963
16.200
33.000

1.170

1.960

1.290
0.14

0.04

0.08

0.07

0.05

0.09

0.11

1.050

0.04

0.06

$\stackrel{A}{\mathbf{N}}$

0.013
0.580
0.881
11.400
1.160
0.074
0.056
0.034
0.003

0.920

1.050

1.180

0.750

1.140

0.950

0.910

0.860

****

$\begin{array}{ll}0.03 & A \\ 0.03 & A \\ 0.04 & W \\ 0.09 & W \\ 0.04 & W \\ 0.10 & \text { A } \\ 0.07 & A \\ 0.08 & \text { W } \\ \cdots \cdots & \text { N }\end{array}$

Units for matrices: $A|=B q|$ filter $S O=B q / \mathrm{kg} V E=B q / \mathrm{kg} W A=B q / L$. Values for elemental uranium are reported in $\mu \mathrm{g} /$ filter, $\mathrm{g}$, or $\mathrm{mL}$. 


\section{QAP44 Results by Laboratory}

Lab: BQ Becquerel Laboratories Inc., Mississauga, Ontario, Canada

\begin{tabular}{|c|c|c|c|c|c|c|c|c|}
\hline $\begin{array}{l}\text { No. } \\
\text { Test }\end{array}$ & $\begin{array}{l}\text { Radio- } \\
\text { nuclide }\end{array}$ & $\begin{array}{l}\text { Reported } \\
\text { Value } \\
\end{array}$ & $\begin{array}{l}\text { Reported } \\
\text { Error }\end{array}$ & $\begin{array}{c}\text { EML } \\
\text { Value } \\
\end{array}$ & $\begin{array}{l}\text { EML } \\
\text { Error } \\
\end{array}$ & $\frac{\text { Reported }}{\text { EML }}$ & $\begin{array}{l}\text { Ratio } \\
\text { Error } \\
\end{array}$ & Evalua \\
\hline Matrix: & Al & & & & & & & \\
\hline $\begin{array}{l}1 \\
1 \\
1 \\
1 \\
1 \\
1 \\
1 \\
1 \\
1\end{array}$ & $\begin{array}{l}\text { CE144 } \\
\text { CO } 57 \\
\text { CO 60 } \\
\text { CS134 } \\
\text { CS137 } \\
\text { MN } 54 \\
\text { RU106 } \\
\text { SB125 } \\
\text { U UG }\end{array}$ & $\begin{array}{r}30.000 \\
7.500 \\
24.300 \\
14.500 \\
6.800 \\
2.900 \\
12.000 \\
9.700 \\
4.300\end{array}$ & $\begin{array}{l}0.900 \\
0.100 \\
0.600 \\
0.500 \\
0.300 \\
0.300 \\
2.000 \\
0.500 \\
0.200\end{array}$ & $\begin{array}{r}33.300 \\
8.900 \\
29.500 \\
14.700 \\
6.640 \\
3.440 \\
11.600 \\
9.780 \\
4.310\end{array}$ & $\begin{array}{l}3.300 \\
0.900 \\
2.900 \\
1.460 \\
0.700 \\
0.380 \\
1.440 \\
1.030 \\
0.100\end{array}$ & $\begin{array}{l}0.900 \\
0.840 \\
0.820 \\
0.990 \\
1.020 \\
0.840 \\
1.030 \\
0.990 \\
1.000\end{array}$ & $\begin{array}{l}0.09 \\
0.09 \\
0.08 \\
0.10 \\
0.12 \\
0.13 \\
0.22 \\
0.12 \\
0.05\end{array}$ & $\begin{array}{l}\text { A } \\
\text { A } \\
\text { W } \\
\text { A } \\
\text { A } \\
\text { W } \\
\text { A } \\
\text { A }\end{array}$ \\
\hline
\end{tabular}

Matrix: $\quad$ SO

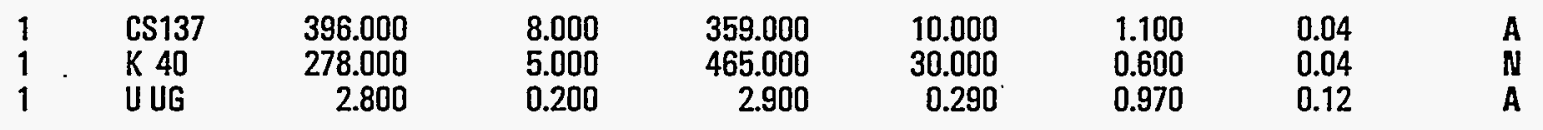

Matrix: VE

$\begin{array}{rlrrrrrrr}1 & \text { C0 60 } & 81.900 & 7.000 & 59.700 & 0.963 & 1.370 & 0.12 & \text { W } \\ 1 & \text { CS137 } & .1100 .000 & 14.000 & 944.000 & 16.200 & 1.170 & 0.02 & \text { A } \\ 1 & \text { K 40 } & 371.000 & 44.000 & 1030.000 & 33.000 & 0.360 & 0.04 & \text { N }\end{array}$

Matrix: WA

$\begin{array}{llrlrllll}1 & \text { CO 60 } & 35.000 & 2.000 & 32.800 & 0.580 & 1.070 & 0.06 & \\ 1 & \text { CS137 } & 42.000 & 1.000 & 38.300 & 0.881 & 1.100 & 0.04 & \text { A } \\ 1 & \text { MN 54 } & 44.000 & 2.000 & 38.400 & 1.160 & 1.150 & 0.06 & \text { W } \\ 1 & \text { U UG } & 0.026 & 0.001 & 0.022 & 0.003 & 1.180 & 0.14 & \text { A }\end{array}$

Units for matrices: $\mathrm{Al}=\mathrm{Bq} / \mathrm{filter} \mathrm{SO}=\mathrm{Bq} / \mathrm{kg}$ VE=Bqlkg $W A=B q / L$. Values for elemental uranium are reported in $\mu \mathrm{g} / \mathrm{filter}, \mathrm{g}$, or $\mathrm{mL}$.

Evaluation: A=Acceptable, $W=$ Acceptable with Warning, $N=$ Not Acceptable

$\mathrm{pCi}=\mathrm{Bq} \times 27$ 


\section{QAP44 Results by Laboratory}

Lab: BR US Army Research Laboratory, Aberdeen Proving Ground

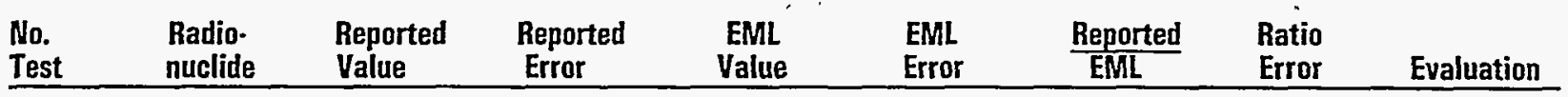

Matrix: Al

$\begin{array}{llrrrrrrr}1 & \text { CE144 } & 29.700 & 2.940 & 33.300 & 3.300 & 0.890 & 0.13 & \text { A } \\ 1 & \text { CO 57 } & 7.980 & 0.480 & 8.900 & 0.900 & 0.900 & 0.11 & \text { A } \\ 1 & \text { CO 60 } & 29.800 & 1.310 & 29.500 & 2.900 & 1.010 & 0.11 & \text { A } \\ 1 & \text { CS134 } & 14.300 & 0.880 & 14.700 & 1.460 & 0.970 & 0.11 & \text { A } \\ 1 & \text { CS137 } & 6.640 & 0.930 & 6.640 & 0.700 & 1.000 & 0.18 & \text { A } \\ 1 & \text { MN 54 } & 4.450 & 1.200 & 3.440 & 0.380 & 1.290 & 0.38 & \text { W } \\ 1 & \text { RU106 } & 13.500 & 3.460 & 11.600 & 1.440 & 1.160 & 0.33 & \text { A } \\ 1 & \text { SB125 } & 12.200 & 1.730 & 9.780 & 1.030 & 1.250 & 0.22 & \text { W }\end{array}$

Matrix: So

\begin{tabular}{|c|c|}
\hline $\begin{array}{l}\text { AM241 } \\
\text { CS137 }\end{array}$ & $\begin{array}{l}3.800 \\
9.000\end{array}$ \\
\hline
\end{tabular}

Matrix: VE

\begin{tabular}{|c|}
\hline $\begin{array}{l}\text { CO 60 } \\
\text { CS137 }\end{array}$ \\
\hline
\end{tabular}

Matrix: WA

$\begin{array}{lllllllll}1 & \text { CO 60 } & 33.000 & 1.400 & 32.800 & 0.580 & 1.010 & 0.05 & \text { A } \\ 1 & \text { CS137 } & 46.300 & 2.100 & 38.300 & 0.881 & 1.210 & 0.06 & \text { W } \\ 1 & \text { MN 54 } & 46.000 & 2.500 & 38.400 & 1.160 & 1.200 & 0.07 & \text { W }\end{array}$

Units for matrices: $A \mid=B q / f i l t e r ~ S 0=B q / k g \quad V E=B q / k g ~ W A=B q / L$. Values for elemental uranium are reported in $\mu q / f i l t e r, g$, or $\mathrm{mL}$. 


\section{QAP44 Results by Laboratory}

Lab: BS B\&W Nuclear Envir. Services, Leechburg, PA

\begin{tabular}{|c|c|c|c|c|c|c|c|c|}
\hline $\begin{array}{l}\text { No. } \\
\text { Test }\end{array}$ & $\begin{array}{l}\text { Radio- } \\
\text { nuclide }\end{array}$ & $\begin{array}{l}\text { Reported } \\
\text { Value }\end{array}$ & $\begin{array}{l}\text { Reported } \\
\text { Error }\end{array}$ & $\begin{array}{l}\text { EML } \\
\text { Valu }\end{array}$ & $\begin{array}{l}\text { EMI } \\
\text { Erro }\end{array}$ & $\frac{\text { Reportt }}{\text { EML }}$ & & valuation \\
\hline
\end{tabular}

Matrix: Al

$\begin{array}{llrlrllll}1 & \text { AM241 } & 0.280 & 0.040 & 0.189 & 0.007 & 1.480 & 0.22 & \text { W } \\ 1 & \text { CE144 } & 23.300 & 0.240 & 33.300 & 3.300 & 0.700 & 0.07 & \text { A } \\ 1 & \text { CO 57 } & 6.440 & 0.050 & 8.900 & 0.900 & 0.720 & 0.07 & \text { A } \\ 1 & \text { CO 60 } & 24.900 & 0.190 & 29.500 & 2.900 & 0.840 & 0.08 & \text { A } \\ 1 & \text { CS134 } & 13.100 & 0.150 & 14.700 & 1.460 & 0.890 & 0.09 & \text { A } \\ 1 & \text { CS137 } & 5.370 & 0.090 & 6.640 & 0.700 & 0.810 & 0.09 & \text { W } \\ 1 & \text { GA 1 } & 2.200 & 0.010 & 1.620 & 0.150 & 1.360 & 0.13 & W \\ 1 & \text { GB 2 } & 1.870 & 0.010 & 1.770 & 0.150 & 1.060 & 0.09 & \text { A } \\ 1 & \text { MN 54 } & 2.860 & 0.100 & 3.440 & 0.380 & 0.830 & 0.10 & \text { W } \\ 1 & \text { RU106 } & 7.700 & 0.640 & 11.600 & 1.440 & 0.660 & 0.10 & \text { A } \\ 1 & \text { SB125 } & 7.630 & 0.200 & 9.780 & 1.030 & 0.780 & 0.08 & \text { A }\end{array}$

Matrix: So

$\begin{array}{llrrrrr}1 & \text { AM241 } & 4.600 & 0.100 & 3.690 & 0.454 & 1.250 \\ 1 & \text { CS137 } & 374.000 & 0.200 & 359.000 & 10.000 & 1.040 \\ 1 & \text { K 40 } & 482.000 & 10.900 & 465.000 & 30.000 & 1.040 \\ 1 & \text { U BO } & 141.000 & 18.500 & 71.700 & 4.150 & 1.970\end{array}$

Matrix: VE

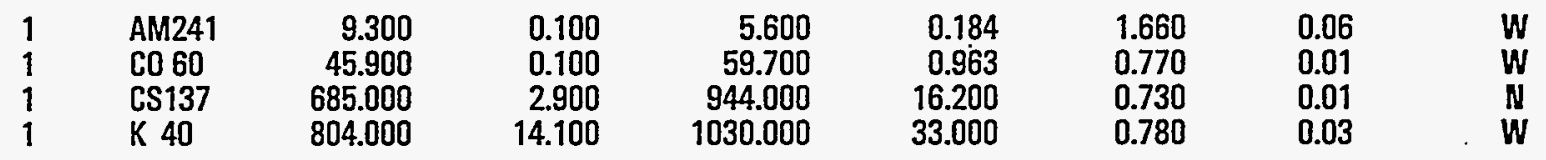

Matrix: WA

\begin{tabular}{|c|c|c|c|c|c|c|}
\hline $\begin{array}{l}\text { AM241 } \\
\text { CO } 60 \\
\text { CS137 } \\
\text { GA } 1 \\
\text { GB } 2 \\
\text { MN } 54\end{array}$ & $\begin{array}{r}0.870 \\
32.500 \\
41.100 \\
1590.000 \\
732.000 \\
43.700\end{array}$ & $\begin{array}{l}0.160 \\
0.480 \\
0.480 \\
7.900 \\
4.300 \\
0.510\end{array}$ & $\begin{array}{r}0.766 \\
32.800 \\
38.300 \\
1850.000 \\
744.000 \\
38.400\end{array}$ & $\begin{array}{r}0.013 \\
0.580 \\
0.881 \\
185.000 \\
74.000 \\
1.160\end{array}$ & $\begin{array}{l}1.140 \\
0.990 \\
1.070 \\
0.860 \\
0.980 \\
1.140\end{array}$ & $\begin{array}{l}0.21 \\
0.02 \\
0.03 \\
0.09 \\
0.10 \\
0.04\end{array}$ \\
\hline
\end{tabular}

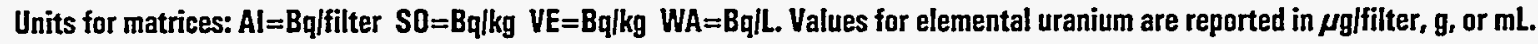

Evaluation: $A=A c c e p t a b l e, W=A c c e p t a b l e$ with Warning, $N=$ Not Acceptable

$\mathrm{pCi}=\mathrm{Bq} \times 27$ 


\section{QAP44 Results by Laboratory}

Lab: BU $^{\circ}$ Autoridad Regulatoria, Buenos Aires, Argentina

\begin{tabular}{|c|c|c|c|c|c|c|}
\hline Nost & $\begin{array}{l}\text { Radio- } \\
\text { nuclide }\end{array}$ & $\begin{array}{l}\text { Reported } \\
\text { Value }\end{array}$ & $\begin{array}{l}\text { Reported } \\
\text { Error }\end{array}$ & $\begin{array}{c}\text { EML } \\
\text { Value }\end{array}$ & $\begin{array}{l}\text { EMI } \\
\text { Erro }\end{array}$ & ion \\
\hline
\end{tabular}

Matrix: Al

$\begin{array}{lrrrrrrr}\text { AM241 } & 0.580 & 0.130 & 0.189 & 0.007 & 3.070 & 0.70 & \text { N } \\ \text { CE144 } & 33.000 & 6.000 & 33.300 & 3.300 & 0.990 & 0.21 & \text { A } \\ \text { C0 57 } & 8.900 & 0.900 & 8.900 & 0.900 & 1.000 & 0.14 & \text { A } \\ \text { CO 60 } & 29.000 & 3.000 & 29.500 & 2.900 & 0.980 & 0.14 & \text { A } \\ \text { CS134 } & 14.000 & 1.000 & 14.700 & 1.460 & 0.950 & 0.12 & \text { A } \\ \text { CS137 } & 6.900 & 0.700 & 6.640 & 0.700 & 1.040 & 0.15 & \text { A } \\ \text { GA 1 } & 1.600 & 0.100 & 1.620 & 0.150 & 0.990 & 0.11 & \text { A } \\ \text { MN 54 } & 3.400 & 0.300 & 3.440 & 0.380 & 0.990 & 0.14 & \text { A } \\ \text { PU238 } & 0.087 & 0.005 & 0.096 & 0.002 & 0.900 & 0.06 & \text { A } \\ \text { PU239 } & 0.089 & 0.005 & 0.093 & 0.003 & 0.960 & 0.06 & \text { A } \\ \text { RU106 } & 11.000 & 2.000 & 11.600 & 1.440 & 0.950 & 0.21 & \text { A } \\ \text { SB125 } & 10.000 & 1.000 & 9.780 & 1.030 & 1.020 & 0.15 & \text { A } \\ \text { UBO } & 5.800 & 0.400 & 0.107 & 0.003 & \because * * & 3.96 & \text { N }\end{array}$

Matrix: So

$\begin{array}{llrrrrrrr}1 & \text { AM241 } & 2.900 & 0.700 & 3.690 & 0.454 & 0.790 & 0.21 & \text { A } \\ 1 & \text { CS137 } & 360.000 & 30.000 & 359.000 & 10.000 & 1.000 & 0.09 & \text { A } \\ 1 & \text { K 40 } & .470 .000 & 80.000 & 465.000 & 30.000 & 1.010 & 0.18 & \text { A } \\ 1 & \text { PU238 } & 39.100 & 0.900 & 43.000 & 2.440 & 0.910 & 0.06 & \text { A } \\ 1 & \text { PU239 } & 9.400 & 0.300 & 9.230 & 0.346 & 1.020 & 0.05 & \text { A } \\ 1 & \text { U BO } & 7.900 & 0.800 & 71.700 & 4.150 & 0.110 & 0.01 & \text { N }\end{array}$

Matrix: VE

$\begin{array}{rlrrrrrrr}1 & \text { AM241 } & 7.400 & 1.800 & 5.600 & 0.184 & 1.320 & 0.32 & \text { A } \\ 1 & \text { CO 60 } & 72.000 & 7.000 & 59.700 & 0.963 & 1.210 & 0.12 & \text { A } \\ 1 & \text { CS137 } & 1000.000 & 90.000 & 944.000 & 16.200 & 1.060 & 0.10 & \text { A } \\ 1 & \text { K 40 } & 1100.000 & 200.000 & 1030.000 & 33.000 & 1.070 & 0.20 & \text { A } \\ 1 & \text { PU239 } & 3.000 & 0.300 & 9.820 & 1.220 & 0.310 & 0.05 & \mathbf{N}\end{array}$

Matrix: WA

$\begin{array}{llr}1 & \text { AM241 } & 0.870 \\ 1 & \text { CO 60 } & 31.000 \\ 1 & \text { CS137 } & 38.000 \\ 1 & \text { GA } 1 & 2040.000 \\ 1 & H 3 & 226.000 \\ 1 & \text { MN } 54 & 37.000 \\ 1 & \text { PU238 } & 0.740 \\ 1 & \text { PU239 } & 0.780 \\ 1 & \text { U BO } & 0.024\end{array}$

0.200
3.000
3.000
100.000
5.000
3.000
0.030
0.030
0.002

0.766
32.800
38.300
1850.000
251.000
38.400
0.982
0.772
0.559

0.013
0.580
0.881
185.000
11.400
1.160
0.074
0.056
0.047

1.140
0.950
0.990
1.100
0.900
0.960
0.750
1.010
0.040

0.26
0.09
0.08
0.12
0.05
0.08
0.06
0.08
0.01

$A$
$A$
$A$
$A$
$A$
$A$
$W$
$A$
N

Units for matrices: $A|=B q|$ filter $S 0=B q \mid k g V E=B q / k g ~ W A=B q l L$. Values for elemental uranium are reported in $\mu g / f i l t e r, g$, or $\mathrm{mL}$.

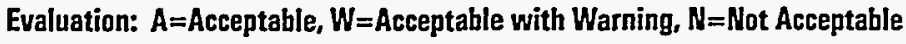
$\mathrm{pCi}=\mathrm{Bq} \times 27$ 


\section{QAP44 Results by Laboratory}

Lab: BX B\&W Nuclear Envir. Services, Lynchburg, VA

\begin{tabular}{lllllllll} 
No. & Radio- & Reported & Reported & EML & EML & Reported & Ratio & \\
Test & nuclide & Value & Error & Value & Error & $\frac{\text { EML }}{\text { Error }}$ & Evaluation \\
\hline
\end{tabular}

Matrix: Al

$\begin{array}{llrlrllll}1 & \text { AM241 } & 0.204 & 0.010 & 0.189 & 0.007 & 1.080 & 0.07 & \text { A } \\ 1 & \text { CE144 } & 23.300 & 0.676 & 33.300 & 3.300 & 0.700 & 0.07 & A \\ 1 & \text { CO 57 } & 6.660 & 0.200 & 8.900 & 0.900 & 0.750 & 0.08 & A \\ 1 & \text { CO 60 } & 25.100 & 0.301 & 29.500 & 2.900 & 0.850 & 0.08 & A \\ 1 & \text { CS134 } & 13.300 & 0.611 & 14.700 & 1.460 & 0.910 & 0.10 & A \\ 1 & \text { CS137 } & 5.920 & 0.320 & 6.640 & 0.700 & 0.890 & 0.11 & \text { A } \\ 1 & \text { GA 1 } & 1.430 & 0.053 & 1.620 & 0.150 & 0.880 & 0.09 & \text { W } \\ 1 & \text { GB 2 } & 2.690 & 0.077 & 1.770 & 0.150 & 1.520 & 0.14 & A \\ 1 & \text { MN 54 } & 3.040 & 0.164 & 3.440 & 0.380 & 0.880 & 0.11 & A \\ 1 & \text { PU238 } & 0.128 & 0.009 & 0.096 & 0.002 & 1.330 & 0.09 & \text { W } \\ 1 & \text { PU239 } & 0.097 & 0.007 & 0.093 & 0.003 & 1.050 & 0.09 & A \\ 1 & \text { RU106 } & 11.600 & 0.822 & 11.600 & 1.440 & 1.000 & 0.14 & A \\ 1 & \text { SB125 } & 8.950 & 0.466 & 9.780 & 1.030 & 0.920 & 0.11 & A \\ 1 & \text { SR 90 } & 1.270 & 0.245 & 1.060 & 0.037 & 1.200 & 0.24 & A \\ 1 & \text { U 234 } & 0.067 & 0.006 & 0.052 & 0.002 & 1.300 & 0.13 & A \\ 1 & \text { U 238 } & 0.063 & 0.008 & 0.053 & 0.002 & 1.180 & 0.16 & \text { A }\end{array}$

Matrix: So

$\begin{array}{llrrrrrrr}1 & \text { AM241 } & 2.020 & 0.051 & 3.690 & 0.454 & 0.550 & 0.07 & \text { W } \\ 1 & \text { CS137 } & 392.000 & 27.100 & 359.000 & 10.000 & 1.090 & 0.08 & A \\ 1 & \text { K 40 } & 437.000 & 27.100 & 465.000 & 30.000 & 0.940 & 0.08 & \text { A } \\ 1 & \text { PU238 } & 36.900 & 0.940 & 43.000 & 2.440 & 0.860 & 0.05 & \text { A } \\ 1 & \text { PU239 } & 7.220 & 0.185 & 9.230 & 0.346 & 0.780 & 0.04 & \text { W } \\ 1 & \text { SR 90 } & 618.000 & 42.200 & 1340.000 & 113.000 & 0.460 & 0.05 & \mathbf{N} \\ 1 & \text { U 234 } & 36.700 & 2.500 & 34.200 & 3.520 & 1.070 & 0.13 & \text { W } \\ 1 & \text { U 238 } & 32.900 & 2.090 & 35.900 & 3.630 & 0.920 & 0.11 & \text { A }\end{array}$

Matrix: VE

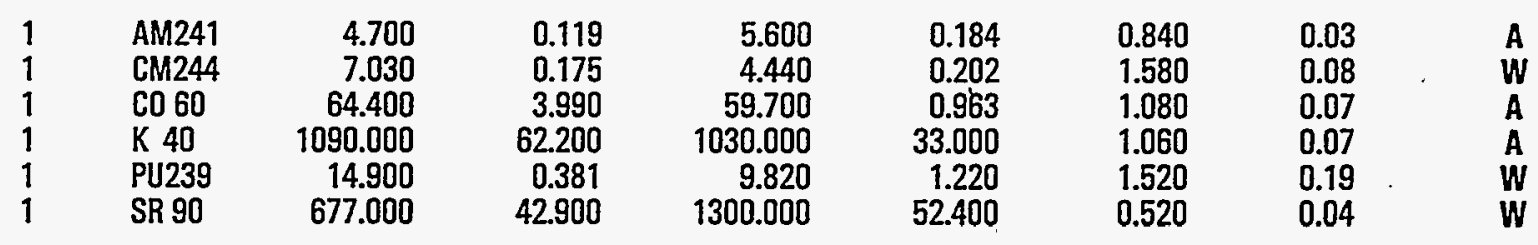

Matrix: WA

$\begin{array}{llrrrrrrr}1 & \text { AM241 } & 0.796 & 0.028 & 0.766 & 0.013 & 1.040 & 0.04 & \text { A } \\ 1 & \text { CO 60 } & 32.600 & 1.630 & 32.800 & 0.580 & 0.990 & 0.05 & \text { A } \\ 1 & \text { CS137 } & 42.600 & 2.850 & 38.300 & 0.881 & 1.110 & 0.08 & \text { A } \\ 1 & \text { FE 55 } & 57.000 & 4.660 & 83.000 & 3.440 & 0.690 & 0.06 & \text { W } \\ 1 & \text { GA 1 } & 2040.000 & 58.800 & 1850.000 & 185.000 & 1.100 & 0.12 & \text { A } \\ 1 & \text { GB 2 } & 866.000 & 27.300 & 744.000 & 74.000 & 1.160 & 0.12 & \text { A } \\ 1 & \text { H 3 } & 212.000 & 23.200 & 251.000 & 11.400 & 0.850 & 0.10 & \text { A }\end{array}$

Units for matrices: $A|=B q|$ filter $S O=B q \mid k g$ VE=Bq/kg $W A=B q / L$. Values for elemental uranium are reported in $\mu g / f i l t e r, g$, or $\mathrm{mL}$.

Evaluation: $A=A c c e p t a b l e, ~ W=A c c e p t a b l e$ with Warning, $N=$ Not Acceptable $\mathrm{pCi}=\mathrm{Bq} \times 27$ 


\section{QAP44 Results by Laboratory}

Lab: BX B\&W Nuclear Envir. Services, Lynchburg, VA

\begin{tabular}{|c|c|c|c|c|c|c|c|c|}
\hline $\begin{array}{l}\text { No. } \\
\text { Test }\end{array}$ & $\begin{array}{l}\text { Radio- } \\
\text { nuclide }\end{array}$ & $\begin{array}{l}\text { Reported } \\
\text { Value }\end{array}$ & $\begin{array}{l}\text { Reported } \\
\text { Error }\end{array}$ & $\begin{array}{c}\text { EML } \\
\text { Value }\end{array}$ & $\begin{array}{l}\text { EML } \\
\text { Error }\end{array}$ & $\frac{\text { Reported }}{E M L}$ & $\begin{array}{l}\text { Ratio } \\
\text { Error }\end{array}$ & Evaluation \\
\hline
\end{tabular}

\section{Matrix: WA}

$\begin{array}{rrrrrrrrr}1 & \text { MN 54 } & 41.800 & 2.300 & 38.400 & 1.160 & 1.090 & 0.07 & \text { A } \\ 1 & \text { PU238 } & 0.969 & 0.039 & 0.982 & 0.074 & 0.990 & 0.08 & \text { A } \\ 1 & \text { PU239 } & 0.703 & 0.031 & 0.772 & 0.056 & 0.910 & 0.08 & \text { A } \\ 1 & \text { SR 90 } & 2.000 & 0.485 & 1.450 & 0.034 & 1.380 & 0.34 & \text { W } \\ 1 & \text { U 234 } & .0 .334 & 0.021 & 0.274 & 0.019 & 1.220 & 0.11 & \text { W } \\ 1 & \text { U 238 } & 0.336 & 0.026 & 0.275 & 0.024 & 1.220 & 0.15 & \text { W }\end{array}$

Units for matrices: $\mathrm{Al}=\mathrm{Bq} /$ filter $\mathrm{SO}=\mathrm{Bq} / \mathrm{kg}$ VE=Bq/kg $W A=B q / \mathrm{L}$. Values for elemental uranium are reparted in $\mu \mathrm{g} / \mathrm{filter,} \mathrm{g}$, or $\mathrm{mL}$. $\mathrm{pCi}=\mathrm{Bq} \times 27$ 


\section{OAP44 Results by Laboratory}

Lab: CA Atomic Energy Control Board, Ottawa, Canada

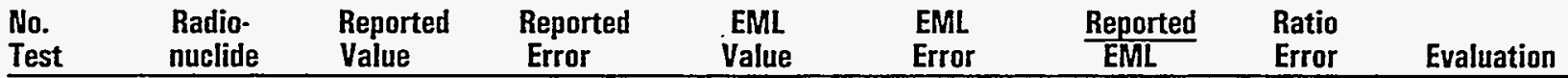

Matrix: Al

$\begin{array}{llrlrllll}1 & \text { CE144 } & 20.300 & 0.900 & 33.300 & 3.300 & 0.610 & 0.07 & \text { W } \\ 1 & \text { CO 57 } & 6.030 & 0.090 & 8.900 & 0.900 & 0.680 & 0.07 & \text { W } \\ 1 & \text { CO 60 } & 24.200 & 0.300 & 29.500 & 2.900 & 0.820 & 0.08 & \text { W } \\ 1 & \text { CS134 } & 13.200 & 0.100 & 14.700 & 1.460 & 0.900 & 0.09 & \text { A } \\ 1 & \text { CS137 } & 5.200 & 0.500 & 6.640 & 0.700 & 0.780 & 0.11 & \text { W } \\ 1 & \text { GA 1 } & 1.550 & 0.020 & 1.620 & 0.150 & 0.960 & 0.09 & \text { A } \\ 1 & \text { GB 2 } & 1.460 & 0.030 & 1.770 & 0.150 & 0.830 & 0.07 & \text { W }\end{array}$

Matrix: So

$3.000 \quad 0.200$

2.900

0.290

1.030

0.12

A

Units for matrices: $A I=B q / f i l t e r ~ S O=B q / k g ~ V E=B q / k g ~ W A=B q / L$. Values for elemental uranium are reported in $\mu \mathrm{g} /$ filter, $\mathrm{g}$, or $\mathrm{mL}$.

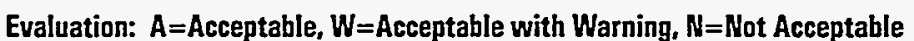
$\mathrm{pCi}=\mathrm{Bq} \times 27$ 


\section{QAP44 Results by Laboratory}

Lab: CC Compuchem Environmental Corp., RTP, NC

\begin{tabular}{llllllll} 
No. & $\begin{array}{l}\text { Radio- } \\
\text { nuclide }\end{array}$ & $\begin{array}{l}\text { Reported } \\
\text { Value }\end{array}$ & $\begin{array}{l}\text { Reported } \\
\text { Error }\end{array}$ & $\begin{array}{c}\text { EML } \\
\text { Value }\end{array}$ & $\begin{array}{l}\text { EML } \\
\text { Error }\end{array}$ & $\begin{array}{l}\text { Reported } \\
\text { EML }\end{array}$ & $\begin{array}{l}\text { Ratio } \\
\text { Error }\end{array}$ \\
\hline
\end{tabular}

Matrix: Al

$\begin{array}{llrlrllll}1 & \text { AM241 } & 0.180 & 0.000 & 0.189 & 0.007 & 0.950 & 0.03 & \text { A } \\ 1 & \text { CE144 } & 20.400 & 0.000 & 33.300 & 3.300 & 0.610 & 0.06 & \text { W } \\ 1 & \text { CD 57 } & 6.150 & 0.000 & 8.900 & 0.900 & 0.690 & 0.07 & \text { W } \\ 1 & \text { CD 60 } & 23.800 & 0.000 & 29.500 & 2.900 & 0.810 & 0.08 & \text { W } \\ 1 & \text { CS134 } & 12.700 & 0.000 & 14.700 & 1.460 & 0.860 & 0.09 & \mathbf{A} \\ 1 & \text { CS137 } & 5.570 & 0.000 & 6.640 & 0.700 & 0.840 & 0.09 & \text { A } \\ 1 & \text { MN 54 } & 2.750 & 0.000 & 3.440 & 0.380 & 0.800 & 0.09 & \text { W } \\ 1 & \text { PU238 } & 0.090 & 0.000 & 0.096 & 0.002 & 0.940 & 0.02 & \mathbf{A} \\ 1 & \text { PU239 } & 0.090 & 0.000 & 0.093 & 0.003 & 0.970 & 0.03 & \text { A } \\ 1 & \text { SB125 } & 5.690 & 0.000 & 9.780 & 1.030 & 0.580 & 0.06 & \text { W } \\ 1 & \text { SR 90 } & 0.970 & 0.000 & 1.060 & 0.037 & 0.920 & 0.03 & \text { A } \\ 1 & \text { U 234 } & 0.050 & 0.000 & 0.052 & 0.002 & 0.970 & 0.03 & \text { A } \\ 1 & \text { U 238 } & 0.060 & 0.000 & 0.053 & 0.002 & 1.130 & 0.04 & \text { A } \\ 1 & \text { U UG } & 4.560 & 0.000 & 4.310 & 0.100 & 1.060 & 0.02 & \text { A }\end{array}$

Matrix: So

$\begin{array}{llrlrrrrr}1 & \text { AM241 } & 2.510 & 0.000 & 3.690 & 0.454 & 0.680 & 0.08 & \text { W } \\ 1 & \text { CS137 } & .441 .000 & 0.000 & 359.000 & 10.000 & 1.230 & 0.03 & \text { A } \\ 1 & \text { K 40 } & 539.000 & 0.000 & 465.000 & 30.000 & 1.160 & 0.07 & \text { A } \\ 1 & \text { PU238 } & 45.500 & 0.000 & 43.000 & 2.440 & 1.060 & 0.06 & \text { A } \\ 1 & \text { PU239 } & 10.100 & 0.000 & 9.230 & 0.346 & 1.090 & .0 .04 & \text { A } \\ 1 & \text { SR 90 } & 994.000 & 0.000 & 1340.000 & 113.000 & 0.740 & 0.06 & \text { W } \\ 1 & \text { U 234 } & 35.800 & 0.000 & 34.200 & 3.520 & 1.050 & 0.11 & A \\ 1 & \text { U 238 } & 36.300 & 0.000 & 35.900 & 3.630 & 1.010 & 0.10 & A \\ 1 & \text { U UG } & 2.910 & 0.000 & 2.900 & 0.290 & 1.000 & 0.10 & \text { A }\end{array}$

Matrix: VE

\begin{tabular}{|c|c|c|c|c|c|c|}
\hline $\begin{array}{l}\text { AM241 } \\
\text { CM244 } \\
\text { CO 60 } \\
\text { CS137 } \\
\text { K } 40 \\
\text { PU239 } \\
\text { SR 90 }\end{array}$ & $\begin{array}{r}6.080 \\
4.710 \\
73.700 \\
1390.000 \\
1430.000 \\
10.000 \\
1320.000\end{array}$ & $\begin{array}{l}0.000 \\
0.000 \\
0.000 \\
0.000 \\
0.000 \\
0.000 \\
0.000\end{array}$ & $\begin{array}{r}5.600 \\
4.440 \\
59.700 \\
944.000 \\
1030.000 \\
9.820 \\
1300.000\end{array}$ & $\begin{array}{r}0.184 \\
0.202 \\
0.963 \\
16.200 \\
33.000 \\
1.220 \\
52.400\end{array}$ & $\begin{array}{l}1.090 \\
1.060 \\
1.240 \\
1.470 \\
1.390 \\
1.020 \\
1.020\end{array}$ & $\begin{array}{l}0.04 \\
0.05 \\
0.02 \\
0.03 \\
0.04 \\
0.13 \\
0.04\end{array}$ \\
\hline
\end{tabular}

Matrix: WA

\begin{tabular}{llrlrrrrr}
1 & AM241 & 0.810 & 0.000 & 0.766 & 0.013 & 1.060 & 0.02 & A \\
1 & CD 60 & 32.900 & 0.000 & 32.800 & 0.580 & 1.000 & 0.02 & A \\
1 & CS137 & 43.900 & 0.000 & 38.300 & 0.881 & 1.150 & 0.03 & A \\
1 & H 3 & 156.000 & 0.000 & 251.000 & 11.400 & 0.620 & 0.03 & N \\
1 & MN 54 & 43.400 & 0.000 & 38.400 & 1.160 & 1.130 & 0.03 & A \\
1 & PU238 & 1.000 & 0.000 & 0.982 & 0.074 & 1.020 & 0.08 & A \\
1 & PU239 & 0.760 & 0.000 & 0.772 & 0.056 & 0.980 & 0.07 & A \\
\hline
\end{tabular}

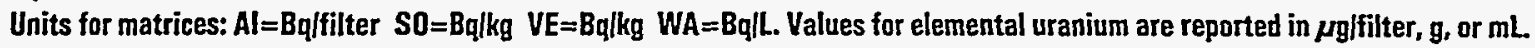

Evaluation: $A=A c c e p t a b l e, W=A c c e p t a b l e ~ w i t h ~ W a r n i n g, ~ N=$ Not Acceptable $\mathrm{PCi}=\mathrm{Bq} \times 27$ 


\section{QAP44 Results by Laboratory}

Lab: CC Compuchem Environmental Corp., RTP, NC

\begin{tabular}{|c|c|c|c|c|c|c|c|c|}
\hline $\begin{array}{l}\text { No. } \\
\text { Test }\end{array}$ & $\begin{array}{l}\text { Radio- } \\
\text { nuclide }\end{array}$ & $\begin{array}{l}\text { Reported } \\
\text { Value } \\
\end{array}$ & $\begin{array}{l}\text { Reported } \\
\text { Error }\end{array}$ & $\begin{array}{c}\text { EML } \\
\text { Value }\end{array}$ & $\begin{array}{l}\text { EML } \\
\text { Error }\end{array}$ & $\frac{\text { Reported }}{\text { EML }}$ & $\begin{array}{l}\text { Ratio } \\
\text { Error }\end{array}$ & Evalua \\
\hline Matrix: & WA & & & & & & & \\
\hline $\begin{array}{l}1 \\
1 \\
1 \\
1\end{array}$ & $\begin{array}{l}\text { SR 90 } \\
\text { U } 234 \\
\text { U } 238 \\
\text { U UG }\end{array}$ & $\begin{array}{l}1.500 \\
0.310 \\
0.300 \\
0.020\end{array}$ & $\begin{array}{l}0.000 \\
0.000 \\
0.000 \\
0.000\end{array}$ & $\begin{array}{l}1.450 \\
0.274 \\
0.275 \\
0.022\end{array}$ & $\begin{array}{l}0.034 \\
0.019 \\
0.024 \\
0.003\end{array}$ & $\begin{array}{l}1.030 \\
1.130 \\
1.090 \\
0.900\end{array}$ & $\begin{array}{l}0.02 \\
0.08 \\
0.10 \\
0.11\end{array}$ & $\begin{array}{l}A \\
A \\
A \\
A\end{array}$ \\
\hline
\end{tabular}

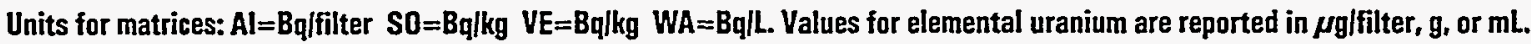
Evaluation: $A=A c c e p t a b l e, W=A c c e p t a b l e$ with Warning, $N=$ Not Acceptable $\mathrm{pCi}=\mathrm{Bq} \times 27$ 


\section{QAP44 Results by Laboratory}

Lab: CH California State Dept. Health Serv, Sanitation \& Radiation Laboratory

\begin{tabular}{llllllll} 
No. & Radio- & Reported & Reported & EML & EML & Reported & Ratio \\
Test & nuclide & Value & Error & Value & Error & $\frac{1}{\text { EML }}$ & $\begin{array}{l}\text { Error } \\
\text { Evaluation }\end{array}$ \\
\hline
\end{tabular}

Matrix: Al

$\begin{array}{lrrrrrrr}\text { AM241 } & 0.197 & 0.034 & 0.189 & 0.007 & 1.040 & 0.19 & \text { A } \\ \text { CE144 } & 21.100 & 1.710 & 33.300 & 3.300 & 0.630 & 0.08 & \text { W } \\ \text { CO 57 } & 6.370 & 1.700 & 8.900 & 0.900 & 0.720 & 0.20 & \text { A } \\ \text { CD 60 } & 25.000 & 0.970 & 29.500 & 2.900 & 0.850 & 0.09 & \text { A } \\ \text { CS134 } & 12.600 & 0.552 & 14.700 & 1.460 & 0.860 & 0.09 & \text { A } \\ \text { CS137 } & 5.560 & 0.552 & 6.640 & 0.700 & 0.840 & 0.12 & \text { A } \\ \text { GA 1 } & 2.090 & 0.086 & 1.620 & 0.150 & 1.290 & 0.13 & \text { A } \\ \text { GB 2 } & 1.840 & 0.126 & 1.770 & 0.150 & 1.040 & 0.11 & \text { A } \\ \text { MN 54 } & 2.880 & 0.504 & 3.440 & 0.380 & 0.840 & 0.17 & \text { W } \\ \text { PU238 } & 0.097 & 0.021 & 0.096 & 0.002 & 1.010 & 0.22 & \text { A } \\ \text { PU239 } & 0.099 & 0.022 & 0.093 & 0.003 & 1.060 & 0.23 & \text { A } \\ \text { RU106 } & 10.000 & 3.630 & 11.600 & 1.440 & 0.860 & 0.33 & \text { A } \\ \text { SB125 } & 7.850 & 1.140 & 9.780 & 1.030 & 0.800 & 0.14 & \text { A } \\ \text { SR 90 } & 0.860 & 0.154 & 1.060 & 0.037 & 0.810 & 0.15 & \text { W } \\ \text { U 234 } & 0.070 & 0.024 & 0.052 & 0.002 & 1.360 & 0.47 & \text { A } \\ \text { U 238 } & 0.060 & 0.022 & 0.053 & 0.002 & 1.130 & 0.41 & \text { A } \\ \text { U BO } & 0.131 & 0.033 & 0.107 & 0.003 & 1.220 & 0.31 & \text { A } \\ \text { U UG } & 4.150 & 0.420 & 4.310 & 0.100 & 0.960 & 0.10 & \text { A }\end{array}$

Matrix: So

1
1
1
1
1
1
1
1
1
1

$\begin{array}{lr}\text { AM241 } & 4.670 \\ \text { CS137 } & 407.000 \\ \text { K 40 } & 540.000 \\ \text { PU238 } & 45.600 \\ \text { PU239 } & 9.560 \\ \text { SR 90 } & 1040.000 \\ \text { U 234 } & 35.900 \\ \text { U 238 } & 35.300 \\ \text { U BO } & 72.900 \\ \text { U UG } & 1.420\end{array}$

$\begin{array}{rr}0.852 & 3.690 \\ 889.000 & 359.000 \\ 49.300 & 465.000 \\ 1.480 & 43.000 \\ 0.481 & 9.230 \\ 59.300 & 1340.000 \\ 2.040 & 34.200 \\ 2.040 & 35.900 \\ 2.900 & 71.700 \\ 0.142 & 2.900\end{array}$

0.454
10.000
30.000
2.440
0.346
113.000
3.520
3.630
4.150
0.290

1.270
1.130
1.160
1.060
1.040
0.780
1.050
0.980
1.020
0.490

0.28
2.48
0.13
0.07
0.07
0.08
0.12
0.12
0.07
0.07

Matrix: VE

$\begin{array}{rlr}1 & \text { AM241 } & 6.370 \\ 1 & \text { CM244 } & 4.190 \\ 1 & \text { CO 60 } & 67.400 \\ 1 & \text { CS137 } & 1120.000 \\ 1 & \text { K 40 } & 1330.000 \\ 1 & \text { PU239 } & 9.700 \\ 1 & \text { SR 90 } & 1310.000\end{array}$

$\begin{array}{rr}0.444 & 5.600 \\ 0.348 & 4.440 \\ 10.100 & 59.700 \\ 24.100 & 944.000 \\ 138.000 & 1030.000 \\ 0.556 & 9.820 \\ 74.100 & 1300.000\end{array}$

0.184
0.202
0.963
16.200
33.000
1.220
52.400

$$
\begin{aligned}
& 1.140 \\
& 0.940 \\
& 1.130 \\
& 1.190 \\
& 1.290 \\
& 0.990 \\
& 1.010
\end{aligned}
$$

$\begin{array}{ll}0.09 & A \\ 0.09 & A \\ 0.17 & A \\ 0.03 & A \\ 0.14 & \text { W } \\ 0.14 & A \\ 0.07 & A\end{array}$

Matrix: WA

$\begin{array}{llrrrrrrr}1 & \text { AM241 } & 0.844 & 0.064 & 0.766 & 0.013 & 1.100 & 0.09 & \text { A } \\ 1 & \text { CD 60 } & 33.700 & 0.496 & 32.800 & 0.580 & 1.030 & 0.02 & \text { A }\end{array}$

Units for matrices: $A \mid=B q / f i l t e r ~ S O=B q / k g ~ V E=B q / k g ~ W A=B q / L$. Values for elemental uranium are reported in $\mu g / f i l t e r, ~ g$, or $\mathrm{mL}$.

Evaluation: $A=A c c e p t a b l e, W=A c c e p t a b l e$ with Warning, $N=$ Not Acceptable $\mathrm{pCi}=\mathrm{Bq} \times 27$ 


\section{QAP44 Results by Laboratory}

Lab: CH California State Dept. Health Serv, Sanitation \& Radiation Laboratory

\begin{tabular}{|c|c|c|c|c|c|c|c|c|}
\hline $\begin{array}{l}\text { No. } \\
\text { Test }\end{array}$ & $\begin{array}{l}\text { Radio- } \\
\text { nuclide }\end{array}$ & $\begin{array}{l}\text { Reported } \\
\text { Value }\end{array}$ & $\begin{array}{l}\text { Reported } \\
\text { Error }\end{array}$ & $\begin{array}{c}\text { EML } \\
\text { Value }\end{array}$ & $\begin{array}{l}\text { EML } \\
\text { Error }\end{array}$ & $\frac{\text { Reported }}{\text { EML }}$ & $\begin{array}{l}\text { Ratio } \\
\text { Error }\end{array}$ & Evaluation \\
\hline
\end{tabular}

Matrix: WA

\begin{tabular}{|c|c|c|c|c|c|c|}
\hline CS137 & 42.100 & 0.556 & 38.300 & 0.881 & 1.100 & 0.03 \\
\hline GA 1 & 1650.000 & 126.000 & 1850.000 & 185.000 & 0.890 & 0.11 \\
\hline GB 2 & 534.000 & 72.200 & 744.000 & 74.000 & 0.720 & 0.12 \\
\hline H 3 & 227.000 & 2.610 & 251.000 & 11.400 & 0.900 & 0.04 \\
\hline MN 54 & 42.700 & 1.360 & 38.400 & 1.160 & 1.110 & 0.05 \\
\hline PU238 & 0.948 & 0.089 & 0.982 & 0.074 & 0.970 & 0.12 \\
\hline PU239 & 0.726 & 0.029 & 0.772 & 0.056 & 0.940 & 0.08 \\
\hline SR 90 & 1.510 & 0.385 & 1.450 & 0.034 & 1.040 & 0.27 \\
\hline U 234 & 0.356 & 0.019 & 0.274 & 0.019 & 1.300 & 0.11 \\
\hline U 238 & 0.331 & 0.031 & 0.275 & 0.024 & 1.200 & 0.16 \\
\hline $\mathrm{UBQ}$ & 0.687 & 0.036 & 0.559 & 0.047 & 1.230 & 0.12 \\
\hline U UG & 0.023 & 0.002 & 0.022 & 0.003 & 1.020 & 0.16 \\
\hline
\end{tabular}

Units for matrices: $A \mid=B q /$ filter $S O=B q \mid k g$ VE $=B q / k g ~ W A=B q \mid L$. Values for elemental uranium are reported in $\mu g / f i l t e r, g$, or $\mathrm{mL}$. 
QAP44 Results by Laboratory

Lab: CL Core Laboratories, Casper, WY

\begin{tabular}{lllllllll} 
No. & $\begin{array}{l}\text { Radio. } \\
\text { nuclide }\end{array}$ & $\begin{array}{l}\text { Reported } \\
\text { Value }\end{array}$ & $\begin{array}{c}\text { Reported } \\
\text { Error }\end{array}$ & $\begin{array}{c}\text { EML } \\
\text { Value }\end{array}$ & $\begin{array}{c}\text { EML } \\
\text { Error }\end{array}$ & $\begin{array}{c}\text { Reported } \\
\text { EML }\end{array}$ & $\begin{array}{c}\text { Ratio } \\
\text { Error }\end{array}$ & Evaluation \\
\hline
\end{tabular}

Matrix: Al

$\begin{array}{lrrrrrrr}\text { AM241 } & 0.220 & 0.040 & 0.189 & 0.007 & 1.160 & 0.22 & \text { A } \\ \text { CE144 } & 20.000 & 1.750 & 33.300 & 3.300 & 0.600 & 0.08 & \text { N } \\ \text { C0 57 } & 6.500 & 0.230 & 8.900 & 0.900 & 0.730 & 0.08 & \text { A } \\ \text { C0 60 } & 22.900 & 0.740 & 29.500 & 2.900 & 0.780 & 0.08 & \text { W } \\ \text { CS134 } & 13.100 & 0.450 & 14.700 & 1.460 & 0.890 & 0.09 & \text { A } \\ \text { CS137 } & 5.840 & 0.370 & 6.640 & 0.700 & 0.880 & 0.11 & \text { A } \\ \text { MN 54 } & 2.890 & 0.400 & 3.440 & 0.380 & 0.840 & 0.15 & \text { W } \\ \text { PU238 } & 0.100 & 0.060 & 0.096 & 0.002 & 1.040 & 0.62 & \text { A } \\ \text { PU239 } & 0.140 & 0.040 & 0.093 & 0.003 & 1.510 & 0.43 & \text { W } \\ \text { RU106 } & 11.400 & 2.080 & 11.600 & 1.440 & 0.980 & 0.22 & \text { A } \\ \text { SB125 } & 9.050 & 0.770 & 9.780 & 1.030 & 0.930 & 0.13 & \text { A } \\ \text { SR 90 } & 0.970 & 0.160 & 1.060 & 0.037 & 0.920 & 0.15 & \text { A } \\ \text { U 234 } & 0.070 & 0.030 & 0.052 & 0.002 & 1.350 & 0.58 & \text { A } \\ \text { U 238 } & 0.070 & 0.030 & 0.053 & 0.002 & 1.310 & 0.56 & \text { A } \\ \text { U BQ } & 0.120 & 0.020 & 0.107 & 0.003 & 1.120 & 0.19 & \text { A }\end{array}$

Matrix: So

$\begin{array}{llrr}1 & \text { AM241 } & 5.640 & 3.700 \\ 1 & \text { CS137 } & 355.000 & 14.800 \\ 1 & \text { K 40 } & 419.000 & 75.5 \\ 1 & \text { PU238 } & 38.100 & 6.300 \\ 1 & \text { PU239 } & 12.300 & 2.740 \\ 1 & \text { SR 90 } & 467.000 & 11.100 \\ 1 & \text { U 234 } & 40.200 & 5.2 \\ 1 & \text { U 238 } & 38.700 & 4.7 \\ 1 & \text { U BO } & 79.400 & 8.000\end{array}$

\subsection{0}

14.800

5.500

6.300

2.740

1.100

4.700
3.690
359.000
465.000
43.000
9.230
1340.000
34.200
35.900
71.700

$\begin{array}{rr}0.454 & 1.530 \\ 10.000 & 0.990 \\ 30.000 & 0.900 \\ 2.440 & 0.890 \\ 0.346 & 1.330 \\ 113.000 & 0.350 \\ 3.520 & 1.170 \\ 3.630 & 1.080 \\ 4.150 & 1.110\end{array}$

1.02

0.05

0.17

0.16

0.30

0.03

0.19

0.17

0.13

$\mathbf{W}$
$\mathbf{A}$
$\mathbf{A}$
$\mathbf{A}$
$\mathbf{W}$
$\mathbf{N}$
$\mathbf{W}$
$\mathbf{A}$
$\mathbf{W}$

Matrix: VE

$\begin{array}{rlrrrrrrr}1 & \text { AM241 } & 6.270 & 1.500 & 5.600 & 0.184 & 1.120 & 0.27 & \text { A } \\ 1 & \text { CM244 } & 3.880 & 1.000 & 4.440 & 0.202 & 0.870 & 0.23 & \text { A } \\ 1 & \text { CO 60 } & 53.800 & 5.900 & 59.700 & 0.963 & 0.900 & 0.10 & \text { A } \\ 1 & \text { CS137 } & 949.000 & 39.200 & 944.000 & 16.200 & 1.010 & 0.05 & \text { A } \\ 1 & \text { K 40 } & 974.000 & 118.000 & 1030.000 & 33.000 & 0.950 & 0.12 & \text { A } \\ 1 & \text { PU239 } & 11.200 & 1.300 & 9.820 & 1.220 & 1.140 & 0.19 & \text { A } \\ 1 & \text { SR 90 } & 437.000 & 11.100 & 1300.000 & 52.400 & 0.340 & 0.02 & \text { N }\end{array}$

Matrix: WA

\begin{tabular}{llrrrrrrr}
1 & AM241 & 0.830 & 0.150 & 0.766 & 0.013 & 1.080 & 0.20 & A \\
1 & CO 60 & 33.600 & 1.730 & 32.800 & 0.580 & 1.020 & 0.06 & A \\
1 & CS137 & 44.000 & 2.020 & 38.300 & 0.881 & 1.150 & 0.06 & A \\
1 & $H 3$ & 201.000 & 11.500 & 251.000 & 11.400 & 0.800 & 0.06 & W \\
1 & MN 54 & 40.900 & 1.800 & 38.400 & 1.160 & 1.070 & 0.06 & A \\
1 & PU238 & 0.910 & 0.220 & 0.982 & 0.074 & 0.930 & 0.24 & A \\
\hline
\end{tabular}

Units for matrices: $\mathrm{Al}=\mathrm{Bq} /$ filter $\mathrm{SO}=\mathrm{Bq} / \mathrm{kg}$ VE=Bq/kg $W \mathrm{~A}=\mathrm{Bq} / \mathrm{L}$. Values for elemental uranium are reported in $\mu \mathrm{g} /$ filter, $\mathrm{g}$, or $\mathrm{mL}$.

Evaluation: $A=A c c e p t a b l e, W=A c c e p t a b l e$ with Warning, $N=$ Not Acceptable

$\mathrm{pCi}=\mathrm{Bq} \times 27$ 


\section{QAP44 Results by Laboratory}

Lab: CL Core Laboratories, Casper, WY

\begin{tabular}{|c|c|c|c|c|c|c|c|c|}
\hline $\begin{array}{l}\text { No. } \\
\text { Test }\end{array}$ & $\begin{array}{l}\text { Radio- } \\
\text { nuclide }\end{array}$ & $\begin{array}{l}\text { Reported } \\
\text { Value }\end{array}$ & $\begin{array}{l}\text { Reported } \\
\text { Error }\end{array}$ & $\begin{array}{l}\text { EML } \\
\text { Value }\end{array}$ & $\begin{array}{l}\text { EML } \\
\text { Error }\end{array}$ & $\frac{\text { Reported }}{E M L}$ & $\begin{array}{l}\text { Ratio } \\
\text { Error }\end{array}$ & Evaluat \\
\hline Matrix: & WA & & & & & & & \\
\hline $\begin{array}{l}1 \\
1 \\
1 \\
1 \\
1\end{array}$ & $\begin{array}{l}\text { PU239 } \\
\text { SR 90 } \\
\text { U } 234 . \\
\text { U } 238 \\
\text { U BQ }\end{array}$ & $\begin{array}{l}0.880 \\
1.700 \\
0.370 \\
0.360 \\
0.730\end{array}$ & $\begin{array}{l}0.160 \\
0.470 \\
0.120 \\
0.110 \\
0.070\end{array}$ & $\begin{array}{l}0.772 \\
1.450 \\
0.274 \\
0.275 \\
0.559\end{array}$ & $\begin{array}{l}0.056 \\
0.034 \\
0.019 \\
0.024 \\
0.047\end{array}$ & $\begin{array}{l}1.140 \\
1.170 \\
1.350 \\
1.310 \\
1.310\end{array}$ & $\begin{array}{l}0.22 \\
0.33 \\
0.45 \\
0.42 \\
0.17\end{array}$ & $\begin{array}{l}A \\
A \\
W \\
W \\
W\end{array}$ \\
\hline
\end{tabular}

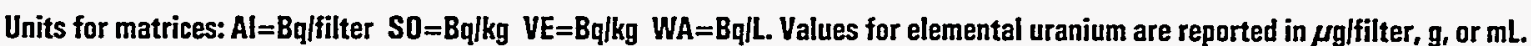

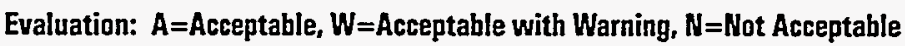
$\mathrm{pCi}=\mathrm{Bq} \times 27$ 
QAP44 Results by Laboratory

Lab: CP Controls for Environmental Pollution, Santa Fe

\begin{tabular}{llllllll} 
No. & $\begin{array}{l}\text { Radio- } \\
\text { nuclide }\end{array}$ & $\begin{array}{l}\text { Reported } \\
\text { Value }\end{array}$ & $\begin{array}{c}\text { Reported } \\
\text { Error }\end{array}$ & $\begin{array}{c}\text { EML } \\
\text { Value }\end{array}$ & $\begin{array}{c}\text { EML } \\
\text { Error }\end{array}$ & $\frac{\text { Reported }}{\text { EML }}$ & $\begin{array}{l}\text { Ratio } \\
\text { Error }\end{array}$ \\
\hline
\end{tabular}

Matrix: Al

$\begin{array}{llrlrllll}1 & \text { CE144 } & 30.600 & 4.300 & 33.300 & 3.300 & 0.920 & 0.16 & \text { A } \\ 1 & \text { CO 57 } & 8.770 & 1.190 & 8.900 & 0.900 & 0.990 & 0.17 & \text { A } \\ 1 & \text { CO 60 } & 29.400 & 3.000 & 29.500 & 2.900 & 1.000 & 0.14 & \text { A } \\ 1 & \text { CS134 } & 13.800 & 2.000 & 14.700 & 1.460 & 0.940 & 0.17 & \text { A } \\ 1 & \text { CS137 } & 7.100 & 0.830 & 6.640 & 0.700 & 1.070 & 0.17 & \text { A } \\ 1 & \text { GA 1 } & 1.810 & 0.030 & 1.620 & 0.150 & 1.120 & 0.11 & \text { A } \\ 1 & \text { GB 2 } & 1.170 & 0.010 & 1.770 & 0.150 & 0.660 & 0.06 & \text { N } \\ 1 & \text { MN 54 } & 3.710 & 0.840 & 3.440 & 0.380 & 1.080 & 0.27 & \text { A } \\ 1 & \text { RU106 } & 10.400 & 1.900 & 11.600 & 1.440 & 0.900 & 0.20 & \text { A } \\ 1 & \text { SB125 } & 10.900 & 2.000 & 9.780 & 1.030 & 1.120 & 0.24 & \text { A } \\ 1 & \text { SR 90 } & 0.755 & 0.057 & 1.060 & 0.037 & 0.710 & 0.06 & \text { W }\end{array}$

Matrix: So

$\begin{array}{llrrrrrrr}1 & \text { CS137 } & 388.000 & 42.000 & 359.000 & 10.000 & 1.080 & 0.12 & \text { A } \\ 1 & K K ~ 40 & 456.000 & 98.000 & 465.000 & 30.000 & 0.980 & 0.22 & \text { A } \\ 1 & \text { U BO } & 50.100 & 5.700 & 71.700 & 4.150 & 0.700 & 0.09 & \text { A }\end{array}$

Matrix: VE

$\begin{array}{rlrrrrrrr}1 & \text { CD 60 } & 67.900 & 8.100 & 59.700 & 0.963 & 1.140 & 0.14 & \text { A } \\ 1 & \text { CS137 } & 1120.000 & 111.000 & 944.000 & 16.200 & 1.190 & 0.12 & \text { A } \\ 1 & \text { K 40 } & 1440.000 & 227.000 & 1030.000 & 33.000 & 1.400 & 0.23 & \text { W } \\ 1 & \text { SR 90 } & 1040.000 & 13.000 & 1300.000 & 52.400 & 0.800 & 0.03 & \text { A }\end{array}$

Matrix: WA

$\begin{array}{llrrrrrrr}1 & \text { CD 60 } & 34.100 & 4.200 & 32.800 & 0.580 & 1.040 & 0.13 & \text { A } \\ 1 & \text { CS137 } & 39.500 & 4.800 & 38.300 & 0.881 & 1.030 & 0.13 & \text { A } \\ 1 & \text { GA 1 } & 1710.000 & 34.000 & 1850.000 & 185.000 & 0.920 & 0.09 & \text { A } \\ 1 & \text { GB 2 } & 843.000 & 16.000 & 744.000 & 74.000 & 1.130 & 0.12 & \text { A } \\ 1 & \text { MN 54 } & 41.300 & 15.500 & 38.400 & 1.160 & 1.080 & 0.41 & \text { A } \\ 1 & \text { SR 90 } & 0.815 & 0.211 & 1.450 & 0.034 & 0.560 & 0.15 & \text { N }\end{array}$

Units for matrices: $\mathrm{Al}=\mathrm{Bq} / \mathrm{filter} \mathrm{SO}=\mathrm{Bq} / \mathrm{kg} V \mathrm{VE}=\mathrm{Bq} / \mathrm{kg} W \mathrm{~W}=\mathrm{Bq} / \mathrm{L}$. Values for elemental uranium are reported in $\mu \mathrm{g} / \mathrm{filter}, \mathrm{g}$, or $\mathrm{mL}$.

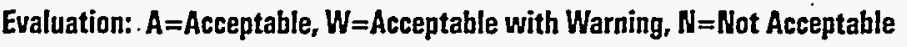
$\mathrm{pCi}=\mathrm{Bq} \times 27$ 


\section{OAP44 Results by Laboratory}

Lab: CS Rockwell International Corp., Canoga Park, CA

\begin{tabular}{lllllllll} 
No. & Radio- & $\begin{array}{l}\text { Reported } \\
\text { Talue }\end{array}$ & $\begin{array}{l}\text { Reported } \\
\text { Error }\end{array}$ & $\begin{array}{c}\text { EML } \\
\text { Value }\end{array}$ & $\begin{array}{c}\text { EML } \\
\text { Error }\end{array}$ & $\frac{\text { Reported }}{\text { EML }}$ & $\begin{array}{l}\text { Ratio } \\
\text { Error }\end{array}$ Evaluation \\
\hline
\end{tabular}

Matrix: Al

\begin{tabular}{|c|c|c|c|c|c|c|}
\hline AM241 & 0.180 & 0.010 & 0.189 & 0.007 & 0.950 & 0.06 \\
\hline CE144 & 16.000 & 0.690 & 33.300 & 3.300 & 0.480 & 0.05 \\
\hline CO 57 & 5.040 & 0.160 & 8.900 & 0.900 & 0.570 & 0.06 \\
\hline CO 60 & 20.900 & 0.640 & 29.500 & 2.900 & 0.710 & 0.07 \\
\hline CS134 & 8.960 & 0.190 & 14.700 & 1.460 & 0.610 & 0.06 \\
\hline CS137 & 4.470 & 0.190 & 6.640 & 0.700 & 0.670 & 0.08 \\
\hline MN 54 & 2.580 & 0.130 & 3.440 & 0.380 & 0.750 & 0.09 \\
\hline RU106 & 6.900 & 0.340 & 11.600 & 1.440 & 0.600 & 0.08 \\
\hline SB125 & 5.700 & 0.130 & 9.780 & 1.030 & 0.580 & 0.06 \\
\hline U 234 & 0.230 & 0.070 & 0.052 & 0.002 & 4.450 & 1.36 \\
\hline
\end{tabular}

Matrix: WA

$\begin{array}{llll}0.766 & 0.013 & 1.080 & 0.13\end{array}$

A

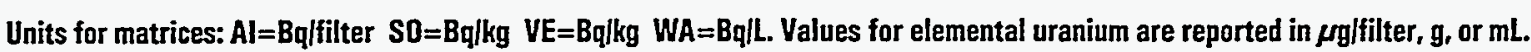




\section{QAP44 Results by Laboratory}

Lab: CW Carlshad Environmental Monitoring Research Center, NM

\begin{tabular}{llllllll} 
No. & $\begin{array}{l}\text { Radio- } \\
\text { nucst }\end{array}$ & $\begin{array}{l}\text { Reported } \\
\text { Value }\end{array}$ & $\begin{array}{c}\text { Reported } \\
\text { Error }\end{array}$ & $\begin{array}{c}\text { EML } \\
\text { Value }\end{array}$ & $\begin{array}{c}\text { EML } \\
\text { Error }\end{array}$ & $\begin{array}{c}\text { Reported } \\
\text { EML }\end{array}$ & $\begin{array}{l}\text { Ratio } \\
\text { Error }\end{array}$ \\
\hline
\end{tabular}

Matrix: Al

$\begin{array}{lllllllll}1 & \text { AM241 } & 0.196 & 0.007 & 0.189 & 0.007 & 1.040 & 0.05 & \text { A } \\ 1 & \text { PU238 } & 0.099 & 0.007 & 0.096 & 0.002 & 1.030 & 0.08 & \text { A } \\ 1 & \text { PU239 } & 0.097 & 0.010 & 0.093 & 0.003 & 1.050 & 0.11 & \text { A } \\ 1 & \text { U 234 } & 0.056 & 0.004 & 0.052 & 0.002 & 1.080 & 0.08 & \text { A } \\ 1 & \text { U 238 } & 0.056 & 0.004 & 0.053 & 0.002 & 1.050 & 0.08 & \text { A }\end{array}$

Matrix: So ,

$\begin{array}{llrlrllll}1 & \text { AM241 } & 3.520 & 0.170 & 3.690 & 0.454 & 0.950 & 0.13 & \text { A } \\ 1 & \text { PU238 } & 41.200 & 2.500 & 43.000 & 2.440 & 0.960 & 0.08 & \text { A } \\ 1 & \text { PU239 } & 8.840 & 0.620 & 9.230 & 0.346 & 0.960 & 0.08 & \text { A } \\ 1 & \text { U 234 } & 32.100 & 1.500 & 34.200 & 3.520 & 0.940 & 0.11 & \text { A } \\ 1 & \text { U 238 } & 33.300 & 1.500 & 35.900 & 3.630 & 0.930 & 0.10 & \text { A }\end{array}$

Matrix: VE

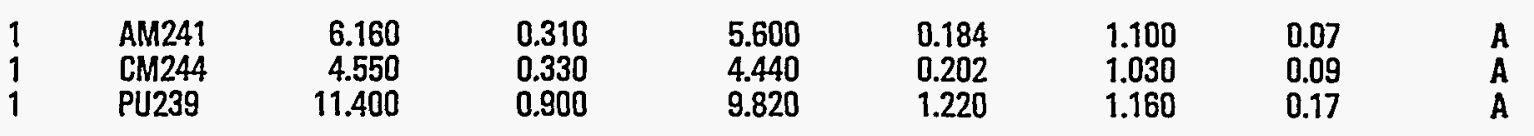

Matrix: WA

$\begin{array}{lllllllll}1 & \text { AM241 } & 0.827 & 0.037 & 0.766 & 0.013 & 1.080 & 0.05 & \text { A } \\ 1 & \text { PU238 } & 1.010 & 0.100 & 0.982 & 0.074 & 1.030 & 0.13 & \text { A } \\ 1 & \text { PU239 } & 0.812 & 0.060 & 0.772 & 0.056 & 1.050 & 0.11 & \text { A } \\ 1 & \text { U 234 } & 0.278 & 0.020 & 0.274 & 0.019 & 1.020 & 0.10 & \text { A } \\ 1 & \text { U 238 } & 0.278 & 0.020 & 0.275 & 0.024 & 1.010 & 0.12 & \text { A }\end{array}$

Units for matrices: $\mathrm{Al}=\mathrm{Bq} / \mathrm{filter} \mathrm{SO}=\mathrm{Bq} / \mathrm{kg}$ VE=Bqlkg WA=BqlL. Values for elemental uranium are reparted in $\mu \mathrm{g} / \mathrm{filter}, \mathrm{g}$, or $\mathrm{mL}$.

Evaluation: $A=A c c e p t a b l e, W=A c c e p t a b l e$ with Warning, $N=$ Not Acceptable

$\mathrm{pCi}=\mathrm{Bq} \times 27$ 


\section{QAP44 Results by Laboratory}

Lab: CZ ACZ Laboratories, Inc., Steamboat Springs, CO

\begin{tabular}{lllllllll} 
No. & Radio- & Reported & Reported & EML & EML & Reported & Ratio \\
Test & nuclide & Value & Error & Value & Error & EML & Error & Evaluation \\
\hline
\end{tabular}

Matrix: WA

$1 \quad U \cup G$

0.027

0.000

0.022

0.003

1.220

0.14

A

Units for matrices: $A l=B q /$ filter $S O=B q / k g V E=B q / k g W A=B q / L$. Values for elemental uranium are reported in $\mu g / f i l t e r, g$, or $\mathrm{mL}$. 
QAP44 Results by Laboratory

Lab: DC Datachem Laboratories, Salt Lake City

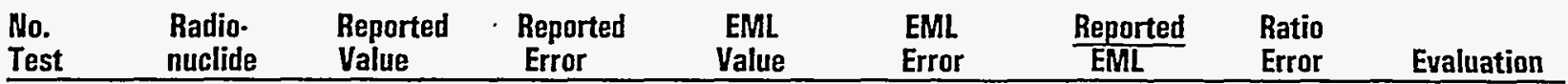

Matrix: Al

$\begin{array}{lrrrrrrr}\text { AM241 } & 0.220 & 0.051 & 0.189 & 0.007 & 1.160 & 0.27 & \text { A } \\ \text { CE144 } & 21.300 & 5.720 & 33.300 & 3.300 & 0.640 & 0.18 & \text { W } \\ \text { CO 57 } & 5.500 & 0.931 & 8.900 & 0.900 & 0.620 & 0.12 & \text { N } \\ \text { CO 60 } & 25.300 & 7.130 & 29.500 & 2.900 & 0.860 & 0.26 & \text { A } \\ \text { CS134 } & 16.000 & 1.910 & 14.700 & 1.460 & 1.090 & 0.17 & \text { W } \\ \text { CS137 } & 7.290 & 3.670 & 6.640 & 0.700 & 1.100 & 0.56 & \text { A } \\ \text { GA 1 } & 1.900 & 0.019 & 1.620 & 0.150 & 1.170 & 0.11 & \text { A } \\ \text { GB 2 } & 2.000 & 0.018 & 1.770 & 0.150 & 1.130 & 0.10 & \text { A } \\ \text { MN 54 } & 3.470 & 0.966 & 3.440 & 0.380 & 1.010 & 0.30 & \text { A } \\ \text { PU238 } & 0.087 & 0.030 & 0.096 & 0.002 & 0.910 & 0.31 & \text { A } \\ \text { PU239 } & 0.091 & 0.030 & 0.093 & 0.003 & 0.980 & 0.33 & \text { A } \\ \text { RU106 } & 13.700 & 4.810 & 11.600 & 1.440 & 1.180 & 0.44 & \text { W } \\ \text { SB125 } & 10.900 & 1.340 & 9.780 & 1.030 & 1.120 & 0.18 & \text { A } \\ \text { SR 90 } & 0.984 & 0.201 & 1.060 & 0.037 & 0.930 & 0.19 & \text { A } \\ \text { U 234 } & 0.074 & 0.033 & 0.052 & 0.002 & 1.440 & 0.64 & \text { W } \\ \text { U 238 } & 0.087 & 0.028 & 0.053 & 0.002 & 1.630 & 0.54 & \text { W } \\ \text { U UG } & 7.000 & 0.700 & 4.310 & 0.100 & 1.620 & 0.17 & \text { W }\end{array}$

Matrix: So

$\begin{array}{llrrrrrrr}1 & \text { AM241 } & 5.590 & 0.180 & 3.690 & 0.454 & 1.520 & 0.19 & \text { W } \\ 2 & & 5.590 & 0.180 & 3.690 & 0.454 & 1.520 & 0.19 & \text { W } \\ 3 & & 5.590 & 0.180 & 3.690 & 0.454 & 1.520 & 0.19 & \text { W } \\ 1 & \text { CS137 } & 399.000 & 96.300 & 359.000 & 10.000 & 1.110 & 0.27 & A \\ 2 & & 399.000 & 96.300 & 359.000 & 10.000 & 1.110 & 0.27 & A \\ 1 & \text { K 40 } & 418.000 & 101.000 & 465.000 & 30.000 & 0.900 & 0.23 & A \\ 2 & & 418.000 & 101.000 & 465.000 & 30.000 & 0.900 & 0.23 & A \\ 1 & \text { PU238 } & 44.300 & 1.800 & 43.000 & 2.440 & 1.030 & 0.07 & A \\ 2 & & 44.300 & 1.800 & 43.000 & 2.440 & 1.030 & 0.07 & A \\ 1 & \text { PU239 } & 9.970 & 0.400 & 9.230 & 0.346 & 1.080 & 0.06 & A \\ 2 & & 9.970 & 0.400 & 9.230 & 0.346 & 1.080 & 0.06 & A \\ 1 & \text { SR 90 } & 1190.000 & 144.000 & 1340.000 & 113.000 & 0.890 & 0.13 & A \\ 2 & & 1190.000 & 144.000 & 1340.000 & 113.000 & 0.890 & 0.13 & A \\ 1 & \text { U 234 } & 36.900 & 3.300 & 34.200 & 3.520 & 1.080 & 0.15 & \text { W } \\ 2 & & 36.900 & 3.300 & 34.200 & 3.520 & 1.080 & 0.15 & \text { W } \\ 1 & \text { U 238 } & 35.300 & 1.400 & 35.900 & 3.630 & 0.980 & 0.11 & A \\ 2 & & 35.300 & 1.400 & 35.900 & 3.630 & 0.980 & 0.11 & A \\ 1 & \text { U UG } & 2.400 & 0.240 & 2.900 & 0.290 & 0.830 & 0.12 & A \\ 2 & & 2.400 & 0.240 & 2.900 & 0.290 & 0.830 & 0.12 & A\end{array}$

Matrix: VE

$\begin{array}{rlrrrrrrr}1 & \text { AM241 } & 6.360 & 0.870 & 5.600 & 0.184 & 1.140 & 0.16 & \text { A } \\ 1 & \text { CM244 } & 4.770 & 1.700 & 4.440 & 0.202 & 1.070 & 0.39 & \text { A } \\ 1 & \text { CD 60 } & 54.500 & 10.400 & 59.700 & 0.963 & 0.910 & 0.18 & \text { A } \\ 1 & \text { CS137 } & 1100.000 & 315.000 & 944.000 & 16.200 & 1.170 & 0.33 & \text { A }\end{array}$

Units for matrices: $A \mathrm{I}=\mathrm{Bq} / \mathrm{filter} \mathrm{SO}=\mathrm{Bq} / \mathrm{kg} V E=B q / \mathrm{kg} W A=B q / \mathrm{L}$. Values for elemental uranium are reported in $\mu \mathrm{g} / \mathrm{filter}, \mathrm{g}$, or $\mathrm{mL}$.

Evaluation: $A=A c c e p t a b l e, W=A c c e p t a b l e$ with Warning, $N=$ Not Acceptable

$\mathrm{pCi}=\mathrm{Bq} \times 27$ 


\section{QAP44 Results by Laboratory}

Lab: DC Datachem Laboratories, Salt Lake City

\begin{tabular}{lllllllll} 
No. & $\begin{array}{l}\text { Radio- } \\
\text { nuclide }\end{array}$ & $\begin{array}{l}\text { Reported } \\
\text { Value }\end{array}$ & $\begin{array}{l}\text { Reported } \\
\text { Error }\end{array}$ & $\begin{array}{c}\text { EML } \\
\text { Value }\end{array}$ & $\begin{array}{c}\text { EML } \\
\text { Error }\end{array}$ & $\begin{array}{c}\text { Reported } \\
\text { EML }\end{array}$ & $\begin{array}{l}\text { Ratio } \\
\text { Error }\end{array}$ & Evaluation \\
\hline
\end{tabular}

Matrix: VE

$\begin{array}{llrrrrrrr}1 & \text { K 40 } & 976.000 & 300.000 & 1030.000 & 33.000 & 0.950 & 0.29 & \text { A } \\ 1 & \text { PU239 } & 9.370 & 0.870 & 9.820 & 1.220 & 0.950 & 0.15 & \text { A } \\ 1 & \text { SR 90 } & 826.000 & 149.000 & 1300.000 & 52.400 & 0.640 & 0.12 & \text { W }\end{array}$

Matrix: WA

$\begin{array}{llrrrrrrr}1 & \text { AM241 } & 0.884 & 0.066 & 0.766 & 0.013 & 1.150 & 0.09 & \text { A } \\ 1 & \text { CO 60 } & 35.100 & 5.260 & 32.800 & 0.580 & 1.070 & 0.16 & \text { A } \\ 1 & \text { CS137 } & 47.800 & 11.300 & 38.300 & 0.881 & 1.250 & 0.30 & \text { W } \\ 1 & \text { GA 1 } & 1750.000 & 15.000 & 1850.000 & 185.000 & 0.950 & 0.09 & A \\ 1 & \text { GB 2 } & 790.000 & 8.400 & 744.000 & 74.000 & 1.060 & 0.11 & A \\ 1 & \text { H 3 } & 244.000 & 27.300 & 251.000 & 11.400 & 0.970 & 0.12 & A \\ 1 & \text { MN 54 } & 46.800 & 10.300 & 38.400 & 1.160 & 1.220 & 0.27 & \text { N } \\ 1 & \text { PU238 } & 0.897 & 0.020 & 0.982 & 0.074 & 0.910 & 0.07 & A \\ 2 & & 0.897 & 0.020 & 0.982 & 0.074 & 0.910 & 0.07 & A \\ 1 & \text { PU239 } & 0.694 & 0.046 & 0.772 & .0 .056 & 0.900 & 0.09 & A \\ 2 & & 0.694 & 0.046 & 0.772 & 0.056 & 0.900 & 0.09 & A \\ 1 & \text { SR 90 } & 1.470 & 0.372 & 1.450 & 0.034 & 1.010 & 0.26 & A \\ 2 & & 1.470 & 0.372 & 1.450 & 0.034 & 1.010 & 0.26 & A \\ 1 & \text { U 234 } & 0.927 & 0.057 & 0.274 & 0.019 & 3.380 & 0.31 & \text { N } \\ 1 & \text { U 238 } & 0.327 & 0.036 & 0.275 & 0.024 & 1.190 & 0.17 & \text { W } \\ 1 & \text { U UG } & 0.023 & 0.002 & 0.022 & 0.003 & 1.040 & 0.16 & A\end{array}$

Units for matrices: $\mathrm{Al}=\mathrm{Bq} / \mathrm{ffilter} \mathrm{SO}=\mathrm{Bq} / \mathrm{kg}$ VE=Bq/kg WA=BqlL . Values for elemental uranium are reported in $\mu \mathrm{g} / \mathrm{filter}, \mathrm{g}$, or $\mathrm{mL}$. 
QAP44 Results by Laboratory

Lab: EG LITCO/INEL, Scoville

\begin{tabular}{llllccccc}
$\begin{array}{l}\text { No. } \\
\text { Test }\end{array}$ & $\begin{array}{l}\text { Radio- } \\
\text { nuclide }\end{array}$ & $\begin{array}{l}\text { Reported } \\
\text { Value }\end{array}$ & $\begin{array}{c}\text { Reported } \\
\text { Error }\end{array}$ & $\begin{array}{c}\text { EML } \\
\text { Value }\end{array}$ & $\begin{array}{c}\text { EML } \\
\text { Error }\end{array}$ & $\begin{array}{c}\text { Reported } \\
\text { EML }\end{array}$ & $\begin{array}{c}\text { Ratio } \\
\text { Error }\end{array}$ Evaluation \\
\hline
\end{tabular}

Matrix: Al

$\begin{array}{llrlrllll}1 & \text { CE144 } & 23.400 & 0.800 & 33.300 & 3.300 & 0.700 & 0.07 & \text { A } \\ 1 & \text { CO 57 } & 7.100 & 0.200 & 8.900 & 0.900 & 0.800 & 0.08 & \mathbf{A} \\ 1 & \text { CO 60 } & 26.700 & 0.040 & 29.500 & 2.900 & 0.910 & 0.09 & \mathbf{A} \\ 1 & \text { CS134 } & 13.800 & 0.300 & 14.700 & 1.460 & 0.940 & 0.10 & \mathbf{A} \\ 1 & \text { CS137 } & 5.800 & 0.200 & 6.640 & 0.700 & 0.870 & 0.10 & \mathbf{A} \\ 1 & \text { GA 1 } & 2.190 & 0.150 & 1.620 & 0.150 & 1.350 & 0.16 & \mathbf{W} \\ 1 & \text { GB 2 } & 2.190 & 0.140 & 1.770 & 0.150 & 1.240 & 0.13 & \mathbf{A} \\ 1 & \text { MN 54 } & 3.100 & 0.200 & 3.440 & 0.380 & 0.900 & 0.12 & \mathbf{A} \\ 1 & \text { RU106 } & 10.900 & 1.300 & 11.600 & 1.440 & 0.940 & 0.16 & \mathbf{A} \\ 1 & \text { SB125 } & 9.200 & 0.300 & 9.780 & 1.030 & 0.940 & 0.10 & \mathbf{A}\end{array}$

Matrix: So

$\begin{array}{ll}1 & \text { AM241 } \\ 1 & \text { CS } 137 \\ 1 & K 40 \\ 1 & \text { PU238 } \\ 1 & \text { PU239 } \\ 1 & \text { SR } 90 \\ 1 & U 234 \\ 1 & \text { U } 238\end{array}$

$\begin{array}{rrr}4.000 & 0.600 & 3.690 \\ 406.000 & 14.000 & 359.000 \\ 461.000 & 85.000 & 465.000 \\ 40.500 & 3.300 & 43.000 \\ 10.500 & 1.100 & 9.230 \\ 9960.000 & 30.000 & 1340.000 \\ 14.800 & 1.800 & 34.200 \\ 14.900 & 1.800 & 35.900\end{array}$

$\begin{array}{rr}0.454 & 1.080 \\ 10.000 & 1.130 \\ 30.000 & 0.990 \\ 2.440 & 0.940 \\ 0.346 & 1.140 \\ 113.000 & 7.390 \\ 3.520 & 0.430 \\ 3.630 & 0.420\end{array}$

0.21
0.05
0.19
0.09
0.13
0.62
0.07
0.07

A
$\mathbf{A}$
$\mathbf{A}$
$\mathbf{A}$
$\mathbf{A}$
$\mathbf{N}$
$\mathbf{W}$
$\mathbf{W}$

Matrix: VE

$\begin{array}{rlrrr}1 & \text { CD 60 } & 59.000 & 5.000 & \\ 1 & \text { CS137 } & 1030.000 & 30.000 & 9 \\ 1 & \text { K 40 } & 1030.000 & 130.000 & 1030 \\ 1 & \text { SR 90 } & 1250.000 & 30.000 & 130\end{array}$

59.700
944.000
1030.000
1300.000

$\begin{array}{rr}0.963 & 0.990 \\ 16.200 & 1.090 \\ 33.000 & 1.000 \\ 52.400 & 0.960\end{array}$

0.09
0.04
0.13
0.05

A
$\mathbf{A}$
$\mathbf{A}$
$\mathbf{A}$

Matrix: WA

$\begin{array}{llrr}1 & \text { AM241 } & 0.640 & 0.040 \\ 1 & \text { CO 60 } & 34.000 & 2.000 \\ 1 & \text { CS137 } & 43.000 & 2.000 \\ 1 & \text { FE 55 } & 62.000 & 17.000 \\ 1 & H 3 & 180.000 & 30.000 \\ 1 & \text { MN } 54 & 43.000 & 2.000 \\ 1 & \text { PU238 } & 0.830 & 0.060 \\ 1 & \text { PU239 } & 0.630 & 0.050 \\ 1 & \text { SR 90 } & 1.230 & 0.060 \\ 1 & \text { U 234 } & 0.250 & 0.030 \\ 1 & \text { U 238 } & 0.240 & 0.030\end{array}$

$\begin{array}{rrrr}0.040 & 0.766 & 0.013 & 0.840 \\ 2.000 & 32.800 & 0.580 & 1.040 \\ 2.000 & 38.300 & 0.881 & 1.120 \\ 17.000 & 83.000 & 3.440 & 0.750 \\ 30.000 & 251.000 & 11.400 & 0.720 \\ 2.000 & 38.400 & 1.160 & 1.120 \\ 0.060 & 0.982 & 0.074 & 0.850 \\ 0.050 & 0.772 & 0.056 & 0.820 \\ 0.060 & 1.450 & 0.034 & 0.850 \\ 0.030 & 0.274 & 0.019 & 0.910 \\ 0.030 & 0.275 & 0.024 & 0.870\end{array}$

0.05
0.06
0.06
0.21
0.12
0.06
0.09
0.09
0.05
0.13
0.13

$A$
$A$
$A$
$W$
$W$
$\mathbf{A}$
$\mathbf{W}$
$\mathbf{A}$
$\mathbf{W}$
$\mathbf{A}$
$\mathbf{W}$

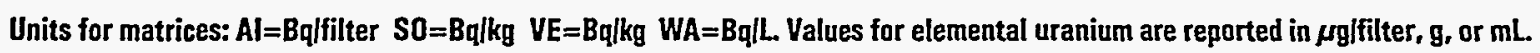




\section{QAP44 Results by Laboratory}

Lab: EI Eichrom Industries, Inc., Argonne

\begin{tabular}{llllllll} 
No. & $\begin{array}{l}\text { Radio- } \\
\text { nuclide }\end{array}$ & $\begin{array}{l}\text { Reported } \\
\text { Value }\end{array}$ & $\begin{array}{l}\text { Reported } \\
\text { Error }\end{array}$ & $\begin{array}{c}\text { EML } \\
\text { Value }\end{array}$ & $\begin{array}{c}\text { EML } \\
\text { Error }\end{array}$ & $\frac{\text { Reported }}{\text { EML }}$ & $\begin{array}{c}\text { Ratio } \\
\text { Error }\end{array}$ \\
\hline
\end{tabular}

Matrix: AI

$\begin{array}{lllllllll}1 & \text { AM241 } & 0.180 & 0.012 & 0.189 & 0.007 & 0.950 & 0.07 & \text { A } \\ 1 & \text { PU238 } & 0.110 & 0.008 & 0.096 & 0.002 & 1.140 & 0.09 & \text { A } \\ 1 & \text { PU239 } & 0.100 & 0.008 & 0.093 & 0.003 & 1.080 & 0.09 & \text { A } \\ 1 & \text { SR 90 } & 1.410 & 0.110 & 1.060 & 0.037 & 1.330 & 0.11 & \text { A } \\ 1 & \text { U 234 } & 0.050 & 0.005 & 0.052 & 0.002 & 0.970 & 0.10 & \text { A } \\ 1 & \text { U 238 } & 0.053 & 0.005 & 0.053 & 0.002 & 0.990 & 0.10 & \text { A }\end{array}$

Matrix: So

$\begin{array}{rrrrrrrrr}1 & \text { SR 90 } & 1580.000 & 216.000 & 1340.000 & 113.000 & 1.180 & 0.19 & \\ 1 & \text { U 234 } & 44.200 & 3.300 & 34.200 & 3.520 & 1.290 & 0.16 & \text { A } \\ 1 & \text { U 238 } & 44.200 & 3.500 & 35.900 & 3.630 & 1.230 & 0.16 & \text { W } \\ 2 & & 44.200 & 3.500 & 35.900 & 3.630 & 1.230 & 0.16 & \text { W }\end{array}$

Matrix: VE

$\begin{array}{rrrrrrrrr}1 & \text { AM241 } & 6.100 & 0.360 & 5.600 & 0.184 & 1.090 & 0.07 & \text { A } \\ 1 & \text { CM244 } & 5.170 & 0.320 & 4.440 & 0.202 & 1.160 & 0.09 & \text { A } \\ 1 & \text { PU239 } & 9.240 & 0.840 & 9.820 & 1.220 & 0.940 & 0.15 & \text { A } \\ 1 & \text { SR 90 } & 2060.000 & 193.000 & 1300.000 & 52.400 & 1.590 & 0.16 & \mathbb{N}\end{array}$

Matrix: WA

$\begin{array}{rlrrrrrrr}1 & \text { AM241 } & 0.630 & 0.035 & 0.766 & 0.013 & 0.820 & 0.05 & \text { W } \\ 1 & \text { H } 3 & 174.000 & 4.000 & 251.000 & 11.400 & 0.690 & 0.04 & \text { W } \\ 1 & \text { PU238 } & 0.880 & 0.053 & 0.982 & 0.074 & 0.900 & 0.09 & \text { W } \\ 1 & \text { PU239 } & 0.620 & 0.039 & 0.772 & 0.056 & 0.800 & 0.08 & \text { A } \\ 1 & \text { SR 90 } & 2.150 & 0.190 & 1.450 & 0.034 & 1.480 & 0.14 & W \\ 1 & \text { U 234 } & 0.280 & 0.016 & 0.274 & 0.019 & 1.020 & 0.09 & \text { A } \\ 1 & \text { U 238 } & 0.220 & 0.016 & 0.275 & 0.024 & 0.800 & 0.09 & W\end{array}$

Units for matrices: $\mathrm{Al}=\mathrm{Bq} /$ filter $\mathrm{SO}=\mathrm{Bq} / \mathrm{kg}$ VE=Bq/kg $W A=B q / L$. Values for elemental uranium are reported in $\mu \mathrm{g} / \mathrm{filter}, \mathrm{g}$, or $\mathrm{mL}$.

Evaluation: $A=$ Acceptable, $W=$ Acceptable with Warning, $N=$ Not Acceptable

$\mathrm{pCi}=\mathrm{Bq} \times 27$ 
QAP44 Results by Laboratory

Lab: EL Energy Laboratories, Inc., Casper, WY

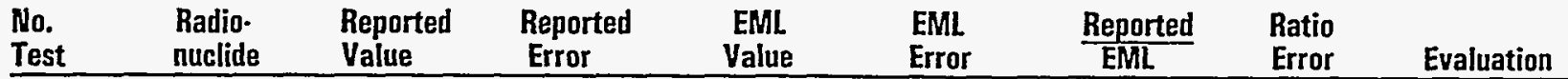

Matrix: Al

$\begin{array}{rlrlrllll}1 & \text { AM241 } & 0.245 & 0.020 & 0.189 & 0.007 & 1.300 & 0.12 & \text { W } \\ 1 & \text { CE144 } & 21.600 & 1.700 & 33.300 & 3.300 & 0.650 & 0.08 & \text { W } \\ 1 & \text { CO 57 } & 6.800 & 0.600 & 8.900 & 0.900 & 0.760 & 0.10 & \text { A } \\ 1 & \text { CO 60 } & 27.400 & 0.800 & 29.500 & 2.900 & 0.930 & 0.10 & \text { A } \\ 1 & \text { CS134 } & 12.800 & 0.500 & 14.700 & 1.460 & 0.870 & 0.09 & A \\ 1 & \text { CS137 } & 6.900 & 0.600 & 6.640 & 0.700 & 1.040 & 0.14 & A \\ 1 & \text { MN 54 } & 4.360 & 0.300 & 3.440 & 0.380 & 1.270 & 0.17 & \text { W } \\ 1 & \text { RU106 } & 14.100 & 1.200 & 11.600 & 1.440 & 1.220 & 0.18 & \text { W } \\ 1 & \text { SB125 } & 9.300 & 0.300 & 9.780 & 1.030 & 0.950 & 0.11 & A \\ 1 & \text { SR 90 } & 0.985 & 0.050 & 1.060 & 0.037 & 0.930 & 0.06 & A \\ 1 & \text { U 234 } & 0.070 & 0.010 & 0.052 & 0.002 & 1.350 & 0.20 & A \\ 1 & \text { U 238 } & 0.080 & 0.010 & 0.053 & 0.002 & 1.500 & 0.20 & \text { W } \\ 1 & \text { U BO } & 0.148 & 0.050 & 0.107 & 0.003 & 1.380 & 0.47 & A\end{array}$

Matrix: So

\begin{tabular}{|c|c|c|c|c|c|c|}
\hline $\begin{array}{l}\text { AM241 } \\
\text { CS137 } \\
\text { SR } 90 \\
\text { U } 234 \\
\text { U } 238 \\
\text { U BO }\end{array}$ & $\begin{array}{r}4.330 \\
378.000 \\
595.000 \\
40.000 \\
41.000 \\
80.400\end{array}$ & $\begin{array}{r}0.300 \\
20.000 \\
45.000 \\
4.000 \\
4.000 \\
5.500\end{array}$ & $\begin{array}{r}3.690 \\
359.000 \\
1340.000 \\
34.200 \\
35.900 \\
71.700\end{array}$ & $\begin{array}{r}0.454 \\
10.000 \\
113.000 \\
3.520 \\
3.630 \\
4.150\end{array}$ & $\begin{array}{l}1.170 \\
1.050 \\
0.440 \\
1.170 \\
1.140 \\
1.120\end{array}$ & $\begin{array}{l}0.17 \\
0.06 \\
0.05 \\
0.17 \\
0.16 \\
0.10\end{array}$ \\
\hline
\end{tabular}

Matrix: VE

$\begin{array}{llrrrrrrr}1 & \text { AM241 } & 7.150 & 0.700 & 5.600 & 0.184 & 1.280 & 0.13 & \text { A } \\ 1 & \text { CO 60 } & 27.600 & 3.000 & 59.700 & 0.963 & 0.460 & 0.05 & \mathbf{N} \\ 1 & \text { K 40 } & 955.000 & 59.000 & 1030.000 & 33.000 & 0.930 & 0.06 & \text { A } \\ 1 & \text { SR 90 } & 873.000 & 67.000 & 1300.000 & 52.400 & 0.670 & 0.06 & \text { W }\end{array}$

Matrix: WA

$\begin{array}{llrrrrrrr}1 & \text { AM241 } & 1.800 & 0.300 & 0.766 & 0.013 & 2.350 & 0.39 & \text { N } \\ 1 & \text { CO 60 } & 37.700 & 2.500 & 32.800 & 0.580 & 1.150 & 0.08 & \text { W } \\ 1 & \text { CS137 } & 43.800 & 2.800 & 38.300 & 0.881 & 1.140 & 0.08 & \text { A } \\ 1 & H 3 & 330.000 & 25.000 & 251.000 & 11.400 & 1.320 & 0.12 & \text { W } \\ 1 & \text { MN54 } & 38.200 & 3.100 & 38.400 & 1.160 & 1.000 & 0.09 & \text { A } \\ 1 & \text { SR 90 } & 1.550 & 0.200 & 1.450 & 0.034 & 1.070 & 0.14 & \text { A } \\ 1 & \text { U 234 } & 0.320 & 0.030 & 0.274 & 0.019 & 1.170 & 0.14 & \text { A } \\ 1 & \text { U 238 } & 0.330 & 0.030 & .0 .275 & 0.024 & 1.200 & 0.15 & \text { W } \\ 1 & \text { U BD } & 0.689 & 0.020 & 0.559 & 0.047 & 1.230 & 0.11 & \text { W }\end{array}$




\section{QAP44 Results by Laboratory}

Lab: EP US EPA, Las Vegas

\begin{tabular}{lllllllll} 
No. & $\begin{array}{l}\text { Radio- } \\
\text { nuclide }\end{array}$ & $\begin{array}{l}\text { Reported } \\
\text { Value }\end{array}$ & $\begin{array}{c}\text { Reported } \\
\text { Error }\end{array}$ & $\begin{array}{c}\text { EML } \\
\text { Value }\end{array}$ & $\begin{array}{c}\text { EML } \\
\text { Error }\end{array}$ & $\frac{\text { Reported }}{\text { EML }}$ & $\begin{array}{l}\text { Ratio } \\
\text { Error }\end{array}$ & Evaluation \\
\hline
\end{tabular}

Matrix: Al

$\begin{array}{llrlrllll}1 & \text { CE144 } & 26.900 & 0.472 & 33.300 & 3.300 & 0.810 & 0.08 & \text { A } \\ 1 & \text { CD 57 } & 7.710 & 0.609 & 8.900 & 0.900 & 0.870 & 0.11 & \text { A } \\ 1 & \text { CD 60 } & 29.800 & 1.100 & 29.500 & 2.900 & 1.010 & 0.11 & \text { A } \\ 1 & \text { CS134 } & 14.400 & 1.170 & 14.700 & 1.460 & 0.980 & 0.13 & \text { A } \\ 1 & \text { CS137 } & 6.190 & 0.515 & 6.640 & 0.700 & 0.930 & 0.13 & \text { A } \\ 1 & \text { MN 54 } & 3.240 & 0.437 & 3.440 & 0.380 & 0.940 & 0.16 & \text { A } \\ 1 & \text { PU238 } & 0.093 & 0.008 & 0.096 & 0.002 & 0.970 & 0.09 & \text { A } \\ 1 & \text { PU239 } & 0.099 & 0.008 & 0.093 & 0.003 & 1.060 & 0.09 & \text { A } \\ 1 & \text { RU106 } & 11.300 & 2.860 & 11.600 & 1.440 & 0.970 & 0.28 & \text { A } \\ 1 & \text { SB125 } & 9.350 & 1.330 & 9.780 & 1.030 & 0.960 & 0.17 & \text { A }\end{array}$

Matrix: So

$\begin{array}{rlrrrrrrr}1 & \text { PU238 } & 42.200 & 3.660 & 43.000 & 2.440 & 0.980 & 0.10 & \text { A } \\ 1 & \text { PU239 } & 8.990 & 0.946 & 9.230 & 0.346 & 0.970 & 0.11 & \text { A } \\ 1 & \text { SR 90 } & 1.220 & 0.023 & 1340.000 & 113.000 & 0.000 & 0.00 & \text { N }\end{array}$

Matrix: VE

$\begin{array}{llllrrrrr}1 & \text { PU239 } & 8.750 & 1.000 & 9.820 & 1.220 & 0.890 & 0.15 & \text { A } \\ 1 & \text { SR } 90 & 1.150 & 0.025 & 1300.000 & 52.400 & 0.000 & 0.00 & \text { N }\end{array}$

Matrix: WA

$\begin{array}{llrrrrrrr}1 & \text { CO 60 } & 36.100 & 3.280 & 32.800 & 0.580 & 1.100 & 0.10 & \text { A } \\ 1 & \text { CS137 } & 46.100 & 4.470 & 38.300 & 0.881 & 1.200 & 0.12 & \text { W } \\ 1 & \text { H } 3 & 222.000 & 7.990 & 251.000 & 11.400 & 0.880 & 0.05 & \text { A } \\ 1 & \text { MN 54 } & 45.400 & 5.040 & 38.400 & 1.160 & 1.180 & 0.14 & \text { W } \\ 1 & \text { PU238 } & 0.990 & 0.087 & 0.982 & 0.074 & 1.010 & 0.12 & \text { A } \\ 1 & \text { PU239 } & 0.778 & 0.074 & 0.772 & 0.056 & 1.010 & 0.12 & A \\ 1 & \text { SR 90 } & 1.290 & 0.343 & 1.450 & 0.034 & 0.890 & 0.24 & \text { A } \\ 1 & \text { U 234 } & 0.329 & 0.040 & 0.274 & 0.019 & 1.200 & 0.17 & \text { W } \\ 1 & \text { U 238 } & 0.313 & 0.039 & 0.275 & 0.024 & 1.140 & 0.17 & \text { A }\end{array}$

Units for matrices: $\mathrm{Al}=\mathrm{Bq} /$ filter $\mathrm{SO}=\mathrm{Bq} / \mathrm{kg} \quad \mathrm{VE}=\mathrm{Bq}|\mathrm{kg} W \mathrm{~W}=\mathrm{Bq}| \mathrm{L}$. Values for elemental uranium are reported in $\mu \mathrm{g} / \mathrm{filter}, \mathrm{g}$, or $\mathrm{mL}$.

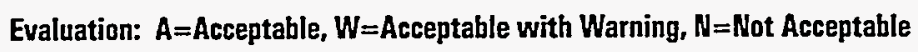
$\mathrm{pCi}=\mathrm{Bq} \times 27$ 


\section{QAP44 Results by Laboratory}

Lab: ES Environmental Sci. \& Engr., Inc., Gainesville, FL

\begin{tabular}{|c|c|c|c|c|c|c|c|c|}
\hline No. & $\begin{array}{l}\text { Radio- } \\
\text { nuclide }\end{array}$ & $\begin{array}{l}\text { Reported } \\
\text { Value }\end{array}$ & $\begin{array}{l}\text { Reported } \\
\text { Error }\end{array}$ & $\begin{array}{c}\text { EML } \\
\text { Value }\end{array}$ & $\begin{array}{l}\text { EML } \\
\text { Error }\end{array}$ & $\frac{\text { Reported }}{\text { EML }}$ & $\begin{array}{l}\text { Ratio } \\
\text { Error }\end{array}$ & Evaluation \\
\hline
\end{tabular}

Matrix: Al

$\begin{array}{lrrrrrrr}\text { AM241 } & 0.159 & 0.032 & 0.189 & 0.007 & 0.840 & 0.17 & . \\ \text { CE144 } & 20.600 & 2.970 & 33.300 & 3.300 & 0.620 & 0.11 & \text { A } \\ \text { C0 57 } & 6.800 & 0.830 & 8.900 & 0.900 & 0.760 & 0.12 & \text { A } \\ \text { C0 60 } & 25.300 & 2.880 & 29.500 & 2.900 & 0.860 & 0.13 & \text { A } \\ \text { CS134 } & 12.500 & 1.440 & 14.700 & 1.460 & 0.850 & 0.13 & \text { A } \\ \text { CS137 } & 5.140 & 0.760 & 6.640 & 0.700 & 0.770 & 0.14 & \text { W } \\ \text { GA 1 } & 1.660 & 0.290 & .1 .620 & 0.150 & 1.030 & 0.20 & \text { A } \\ \text { GB 2 } & 1.260 & 0.220 & 1.770 & 0.150 & 0.710 & 0.14 & \text { N } \\ \text { MN 54 } & 2.450 & 0.560 & 3.440 & 0.380 & 0.710 & 0.18 & \text { N } \\ \text { PU238 } & 0.098 & 0.020 & 0.096 & 0.002 & 1.020 & 0.21 & \text { A } \\ \text { PU239 } & 0.106 & 0.022 & 0.093 & 0.003 & 1.140 & 0.24 & \text { A } \\ \text { RU106 } & 11.000 & 3.630 & 11.600 & 1.440 & 0.950 & 0.33 & \text { A } \\ \text { SB125 } & 8.520 & 1.280 & 9.780 & 1.030 & 0.870 & 0.16 & \text { A } \\ \text { SR 90 } & 1.010 & 0.200 & 1.060 & 0.037 & 0.950 & 0.19 & \text { A } \\ \text { U 234 } & 0.061 & 0.014 & 0.052 & 0.002 . & 1.180 & 0.27 & \text { A } \\ \text { U 238 } & 0.062 & 0.014 & 0.053 & 0.002 & 1.160 & 0.27 & \text { A } \\ \text { U BQ } & 0.126 & 0.029 & 0.107 & 0.003 & 1.180 & 0.27 & \text { A } \\ \text { U UG } & 4.990 & 0.490 & 4.310 & 0.100 & 1.160 & 0.12 & \text { A }\end{array}$

Matrix: So

$\begin{array}{ll}1 & \text { A } 21 \\ 1 & \text { CS13 } \\ 1 & K \\ 1 & \text { PU23 } \\ 2 & \\ 1 & \text { PU23 } \\ 2 & \\ 1 & \text { SR } \\ 1 & U 234 \\ 1 & \text { U } \\ 1 & \text { U B } \\ 1 & \text { U } \\ 2 & \end{array}$

$\begin{array}{lr}\text { AM241 } & 3.310 \\ \text { CS137 } & 433.000 \\ \text { K 40 } & 516.000 \\ \text { PU238 } & 44.100 \\ & 44.100 \\ \text { PU239 } & 11.000 \\ & 9.320 \\ \text { SR 90 } & 1010.000 \\ \text { U 234 } & 31.300 \\ \text { U 238 } & 31.700 \\ \text { U BO } & 64.500 \\ \text { U UG } & 3.040 \\ & 2550.000\end{array}$

0.600

51.000

102.000

7.940

7.840

2.210

1.780

181.000

5.590

5.650

11.600

0.000

256.000

$$
\begin{array}{r}
3.690 \\
359.000 \\
465.000 \\
43.000 \\
43.000 \\
9.230 \\
9.230 \\
1340.000 \\
34.200 \\
35.900 \\
71.700 \\
2.900 \\
2.900
\end{array}
$$

$$
\begin{array}{r}
0.454 \\
10.000 \\
30.000 \\
2.440 \\
2.440 \\
0.346 \\
0.346 \\
113.000 \\
3.520 \\
3.630 \\
4.150 \\
0.290 \\
0.290
\end{array}
$$

\subsection{0 \\ 1.210 \\ 1.110 \\ 1.030 \\ 1.030 \\ 1.190 \\ 1.010 \\ 0.750 \\ 0.920 \\ 0.880 \\ 0.900 \\ 1.050}

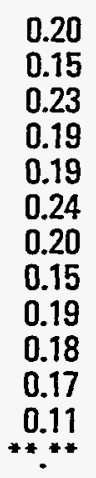

$A$
$A$
$A$
$A$
$A$
$A$
$A$
$W$
$A$
$A$
$A$
$A$
N

Matrix: VE

$\begin{array}{llr}1 & \text { AM241 } & 6.390 \\ 1 & \text { CM244 } & 6.250 \\ 1 & \text { CO 60 } & 68.200 \\ 1 & \text { CS137 } & 1260.000 \\ 1 & \text { K 40 } & 1240.000 \\ 1 & \text { PU239 } & 8.790 \\ 1 & \text { SR 90 } & 1170.000\end{array}$

1.430
1.140
12.400
145.000
220.000
1.310
164.000

5.600
4.440
59.700
944.000
1030.000
9.820
1300.000

0.184
0.202
0.963
16.200
33.000
1.220
52.400

1.140
1.410
1.140
1.340
1.200
0.900
0.900

0.26
0.27
0.21
0.16
0.22
0.17
0.13

$A$
$A$
$A$
$W$
$A$
$A$
$A$

Units for matrices: $\mathrm{Al}=\mathrm{Bq} / \mathrm{filter} \mathrm{SO}=\mathrm{Bq} / \mathrm{kg} \mathrm{VE}=\mathrm{Bq} / \mathrm{kg} W \mathrm{~W}=\mathrm{Bq} / \mathrm{L}$. Values for elemental uranium are reported in $\mu \mathrm{g} / \mathrm{filter}, \mathrm{g}$, or $\mathrm{mL}$. Evaluation: $A=A c c e p t a b l e, W=A c c e p t a b l e$ with Warning, $N=$ Not Acceptable $\mathrm{pCi}=\mathrm{Bq} \times 27$ 


\section{QAP44 Results by Laboratory}

Lab: ES Environmental Sci. \& Engr., Inc., Gainesville, FL

\begin{tabular}{|c|c|c|c|c|c|c|c|c|}
\hline $\begin{array}{l}\text { No. } \\
\text { Test }\end{array}$ & $\begin{array}{l}\text { Radio- } \\
\text { nuclide }\end{array}$ & $\begin{array}{l}\text { Reported } \\
\text { Value }\end{array}$ & $\begin{array}{l}\text { Reported } \\
\text { Error }\end{array}$ & $\begin{array}{l}\text { EML. } \\
\text { Value }\end{array}$ & $\begin{array}{l}\text { EML } \\
\text { Error }\end{array}$ & $\frac{\text { Reported }}{\text { EML }}$ & $\begin{array}{l}\text { Ratio } \\
\text { Error }\end{array}$ & Evaluation \\
\hline
\end{tabular}

Matrix: WA

\begin{tabular}{|c|c|c|c|c|c|c|c|}
\hline 1 & AM241 & 0.760 & 0.115 & 0.766 & 0.013 & 0.990 & 0.15 \\
\hline 2 & & 0.840 & 0.139 & 0.766 & 0.013 & 1.100 & 0.18 \\
\hline 1 & CO 60 & 36.000 & 4.790 & 32.800 & 0.580 & 1.100 & 0.15 \\
\hline 1 & CS137 & 47.600 & 6.470 & 38.300 & 0.881 & 1.240 & 0.17 \\
\hline 1 & H 3 & 204.000 & 36.600 & 251.000 & 11.400 & 0.810 & 0.15 \\
\hline 1 & MN 54 & 44.500 & 6.340 & 38.400 & 1.160 & 1.160 & 0.17 \\
\hline 1 & PU238 & 0.953 & 0.141 & 0.982 & 0.074 & 0.970 & 0.16 \\
\hline 2 & & 0.883 & 0.131 & 0.982 & 0.074 & 0.900 & 0.15 \\
\hline 1 & PU239 & 0.844 & 0.125 & 0.772 & 0.056 & 1.090 & 0.18 \\
\hline 2 & & 0.740 & 0.110 & 0.772 & 0.056 & 0.960 & 0.16 \\
\hline$\overline{1}$ & SR 90 & 1.760 & 0.300 & 1.450 & 0.034 & 1.210 & 0.21 \\
\hline 1 & U 234 & 0.286 & 0.047 & 0.274 & 0.019 & 1.040 & 0.19 \\
\hline 1 & U 238 & 0.290 & 0.047 & 0.275 & 0.024 & 1.050 & 0.20 \\
\hline 1 & $\mathrm{UBO}$ & 0.588 & 0.099 & 0.559 & 0.047 & 1.050 & 0.20 \\
\hline 1 & U UG & 0.023 & 0.000 & 0.022 & 0.003 & 1.050 & 0.12 \\
\hline 2 & & 23.200 & 2.350 & 0.022 & 0.003 & & ***** \\
\hline
\end{tabular}

Units for matrices: $A \mathrm{I}=\mathrm{Bq} / \mathrm{filter} \mathrm{SO}=\mathrm{Bq} / \mathrm{kg}$ VE=Bq/kg $W A=B q / \mathrm{L}$. Values for elemental uranium are reported in $\mu \mathrm{g} / \mathrm{filter}, \mathrm{g}$, or $\mathrm{mL}$.

Evaluation: $A=A c c e p t a b l e, W=A c c e p t a b l e$ with Warning, $N=$ Not Acceptable

$p C i=B q \times 27$ 


\section{OAP44 Results by Laboratory}

Lab: FG FGL Environmental, Santa Paula, CA

\begin{tabular}{llllllll} 
No. & Radio- & Reported & Reported & EML & EML & Reported & Ratio \\
Test & nuclide & Value & Error & Value & Error & $\frac{\text { EML }}{\text { Error }}$ & Evaluation \\
\hline
\end{tabular}

Matrix: Al

$\begin{array}{llrlrllll}1 & \text { AM241 } & 0.170 & 0.040 & 0.189 & 0.007 & 0.900 & 0.21 & \text { A } \\ 1 & \text { CE144 } & 33.900 & 3.300 & 33.300 & 3.300 & 1.020 & 0.14 & \text { A } \\ 1 & \text { CO 57 } & 9.370 & 1.060 & 8.900 & 0.900 & 1.050 & 0.16 & \text { A } \\ 1 & \text { CO 60 } & 32.200 & 8.090 & 29.500 & 2.900 & 1.090 & 0.29 & \text { A } \\ 1 & \text { CS134 } & 14.700 & 2.740 & 14.700 & 1.460 & 1.000 & 0.21 & \text { A } \\ 1 & \text { CS137 } & 7.420 & 1.410 & 6.640 & 0.700 & 1.120 & 0.24 & \text { A } \\ 1 & \text { GA 1 } & 2.010 & 0.130 & 1.620 & 0.150 & 1.240 & 0.14 & \text { A } \\ 1 & \text { GB 2 } & 1.480 & 0.130 & 1.770 & 0.150 & 0.840 & 0.10 & \text { W } \\ 1 & \text { MN 54 } & 3.210 & 0.790 & 3.440 & 0.380 & 0.930 & 0.25 & \text { A } \\ 1 & \text { RU106 } & 12.800 & 2.560 & 11.600 & 1.440 & 1.100 & 0.26 & \text { A } \\ 1 & \text { SB125 } & 11.600 & 2.290 & 9.780 & 1.030 & 1.190 & 0.27 & \text { W }\end{array}$

Matrix: So

$\begin{array}{rlrrrrrrr}1 & \text { AM241 } & 2.500 & 0.320 & 3.690 & 0.454 & 0.680 & 0.12 & \text { W } \\ 1 & \text { CS137 } & 370.000 & 6.600 & 359.000 & 10.000 & 1.030 & 0.03 & \text { A } \\ 1 & \text { K 40 } & 647.000 & 9.700 & 465.000 & 30.000 & 1.390 & 0.09 & W \\ 1 & \text { U BQ } & 42.500 & 5.200 & 71.700 & 4.150 & 0.590 & 0.08 & \text { A }\end{array}$

Matrix: WA

$\begin{array}{llrrrrrrr}1 & \text { AM241 } & 0.765 & 0.010 & 0.766 & 0.013 & 1.000 & 0.02 & \text { A } \\ 1 & \text { CO 60 } & 34.100 & 2.960 & 32.800 & 0.580 & 1.040 & 0.09 & \text { A } \\ 1 & \text { CS137 } & 38.700 & 2.640 & 38.300 & 0.881 & 1.010 & 0.07 & \text { A } \\ 1 & \text { GA 1 } & 1790.000 & 40.000 & 1850.000 & 185.000 & 0.970 & 0.10 & \text { A } \\ 1 & \text { GB 2 } & 437.000 & 32.000 & 744.000 & 74.000 & 0.590 & 0.07 & \text { N } \\ 1 & \text { H 3 } & 284.000 & 15.000 & 251.000 & 11.400 & 1.130 & 0.08 & \text { A } \\ 1 & \text { MN 54 } & 47.000 & 3.520 & 38.400 & 1.160 & 1.220 & 0.10 & \text { N } \\ 1 & \text { PU238 } & 0.925 & 0.010 & 0.982 & 0.074 & 0.940 & 0.07 & \text { A } \\ 1 & \text { PU239 } & 0.763 & 0.010 & 0.772 & 0.056 & 0.990 & 0.07 & \text { A } \\ 1 & \text { SR 90 } & 1.680 & 0.180 & 1.450 & 0.034 & 1.160 & 0.13 & \text { A } \\ 1 & \text { U BQ } & 0.720 & 0.010 & 0.559 & 0.047 & 1.290 & 0.11 & \text { W }\end{array}$




\section{QAP44 Results by Laboratory}

Lab: FL Florida Dept of Health \& Rehab. Serv., Orlando

\begin{tabular}{llllllll} 
No. & $\begin{array}{l}\text { Radio- } \\
\text { nuclide }\end{array}$ & $\begin{array}{l}\text { Reported } \\
\text { Value }\end{array}$ & $\begin{array}{c}\text { Reported } \\
\text { Error }\end{array}$ & $\begin{array}{c}\text { EML } \\
\text { Value }\end{array}$ & $\begin{array}{c}\text { EML } \\
\text { Error }\end{array}$ & $\begin{array}{c}\text { Reported } \\
\text { EML }\end{array}$ & $\begin{array}{l}\text { Ratio } \\
\text { Error }\end{array}$ \\
\hline
\end{tabular}

Matrix: Al

$\begin{array}{llrlrllll}1 & \text { AM241 } & 0.234 & 0.054 & 0.189 & 0.007 & 1.240 & 0.29 & \text { A } \\ 1 & \text { CE144 } & 26.700 & 1.100 & 33.300 & 3.300 & 0.800 & 0.09 & \text { A } \\ 1 & \text { CO 57 } & 7.810 & 0.020 & 8.900 & 0.900 & 0.880 & 0.09 & \text { A } \\ 1 & \text { CO 60 } & 28.800 & 0.120 & 29.500 & 2.900 & 0.980 & 0.10 & \text { A } \\ 1 & \text { CS134 } & 14.100 & 0.150 & 14.700 & 1.460 & 0.960 & 0.10 & \text { A } \\ 1 & \text { CS137 } & 6.720 & 0.110 & 6.640 & 0.700 & 1.010 & 0.11 & \text { A } \\ 1 & \text { GA 1 } & 1.500 & 0.070 & 1.620 & 0.150 & 0.930 & 0.10 & \text { A } \\ 1 & \text { GB 2 } & 2.160 & 0.070 & 1.770 & 0.150 & 1.220 & 0.11 & \text { A } \\ 1 & \text { MN 54 } & 3.610 & 0.250 & 3.440 & 0.380 & 1.050 & 0.14 & \text { A } \\ 1 & \text { PU238 } & 0.092 & 0.003 & 0.096 & 0.002 & 0.960 & 0.04 & \text { A } \\ 1 & \text { PU239 } & 0.091 & 0.003 & 0.093 & 0.003 & 0.980 & 0.04 & \text { A } \\ 1 & \text { RU106 } & 12.000 & 0.060 & 11.600 & 1.440 & 1.030 & 0.13 & \text { A } \\ 1 & \text { SB125 } & 10.600 & 0.100 & 9.780 & 1.030 & 1.080 & 0.12 & \text { A }\end{array}$

Matrix: So

$\begin{array}{llrlrrrrr}1 & \text { AM241 } & 2.800 & 0.500 & 3.690 & 0.454 & 0.760 & 0.17 & \text { A } \\ 1 & \text { CS137 } & 379.000 & 1.000 & 359.000 & 10.000 & 1.060 & 0.03 & \text { A } \\ 1 & \text { K 40 } & 479.000 & 2.000 & 465.000 & 30.000 & 1.030 & 0.07 & \text { A } \\ 1 & \text { PU238 } & 42.000 & 0.910 & 43.000 & 2.440 & 0.980 & 0.06 & \text { A } \\ 1 & \text { PU239 } & 9.070 & 0.360 & 9.230 & 0.346 & 0.980 & 0.05 & \text { A } \\ 1 & \text { U 238 } & 30.300 & 3.400 & 35.900 & 3.630 & 0.840 & 0.13 & \text { A }\end{array}$

Matrix: VE

$\begin{array}{llrrrrrrr}1 & \text { AM241 } & 6.300 & 1.300 & 5.600 & 0.184 & 1.130 & 0.24 & \text { A } \\ 1 & \text { CO 60 } & 65.000 & 1.000 & 59.700 & 0.963 & 1.090 & 0.02 & \text { A } \\ 1 & \text { CS137 } & 1160.000 & 8.000 & 944.000 & 16.200 & 1.230 & 0.02 & \text { A } \\ 1 & \text { K 40 } & 1200.000 & 15.000 & 1030.000 & 33.000 & 1.170 & 0.04 & \text { A }\end{array}$

Matrix: WA

\begin{tabular}{|c|c|c|c|c|c|c|}
\hline $\begin{array}{l}\text { AM241 } \\
\text { CO 60 } \\
\text { CS137 } \\
\text { GA } 1 \\
\text { GB } 2 \\
\text { H } 3 \\
\text { MN } 54 \\
\text { PU238 } \\
\text { PU239 }\end{array}$ & $\begin{array}{r}0.910 \\
32.100 \\
42.400 \\
2330.000 \\
915.000 \\
185.000 \\
42.100 \\
0.750 \\
0.592\end{array}$ & $\begin{array}{r}0.130 \\
0.600 \\
1.000 \\
29.000 \\
13.000 \\
2.710 \\
0.600 \\
0.017 \\
0.014\end{array}$ & $\begin{array}{r}0.766 \\
32.800 \\
38.300 \\
1850.000 \\
744.000 \\
251.000 \\
38.400 \\
0.982 \\
0.772\end{array}$ & $\begin{array}{r}0.013 \\
0.580 \\
0.881 \\
185.000 \\
74.000 \\
11.400 \\
1.160 \\
0.074 \\
0.056\end{array}$ & $\begin{array}{l}1.190 \\
0.980 \\
1.110 \\
1.260 \\
1.230 \\
0.740 \\
1.100 \\
0.760 \\
0.770\end{array}$ & $\begin{array}{l}0.17 \\
0.03 \\
0.04 \\
0.13 \\
0.12 \\
0.04 \\
0.04 \\
0.06 \\
0.06\end{array}$ \\
\hline
\end{tabular}

Units for matrices: Al=Bq/filter $\mathrm{SO}=\mathrm{Bq} / \mathrm{kg} \quad \mathrm{VE}=\mathrm{Bq} / \mathrm{kg} W \mathrm{~W}=\mathrm{Bq} / \mathrm{L}$. Values for elemental uranium are reported in $\mu \mathrm{g} / \mathrm{filter}, \mathrm{g}$, or $\mathrm{mL}$.

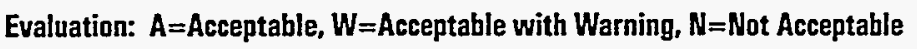
$\mathrm{pCi}=\mathrm{Bq} \times 27$ 


\section{QAP44 Results by Laboratory}

Lab: FM Florida Mobile Emergency Radiological Laboratory, Orlando

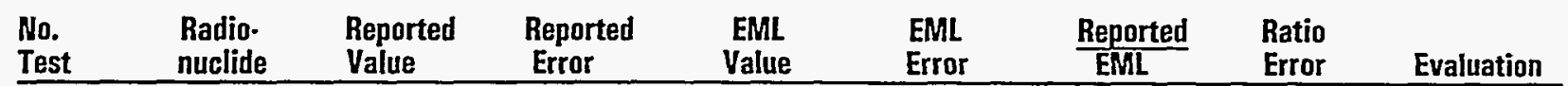

Matrix: Al

$\begin{array}{llrlrllll}1 & \text { AM241 } & 0.210 & 0.040 & 0.189 & 0.007 & 1.110 & 0.22 & \text { A } \\ 1 & \text { CE144 } & 25.100 & 0.560 & 33.300 & 3.300 & 0.750 & 0.08 & \text { A } \\ 1 & \text { CO 57 } & 7.390 & 0.130 & 8.900 & 0.900 & 0.830 & 0.09 & \text { A } \\ 1 & \text { CO 60 } & 27.300 & 0.230 & 29.500 & 2.900 & 0.930 & 0.09 & \text { A } \\ 1 & \text { CS134 } & 13.700 & 0.230 & 14.700 & 1.460 & 0.930 & 0.09 & \text { A } \\ 1 & \text { CS137 } & 6.300 & 0.140 & 6.640 & 0.700 & 0.950 & 0.10 & \text { A } \\ 1 & \text { MN 54 } & 3.340 & 0.120 & 3.440 & 0.380 & 0.970 & 0.11 & \text { A } \\ 1 & \text { RU106 } & 11.000 & 0.330 & 11.600 & 1.440 & 0.950 & 0.12 & \text { A } \\ 1 & \text { SB125 } & 9.480 & 0.200 & 9.780 & 1.030 & 0.970 & 0.10 & \text { A }\end{array}$

Matrix: WA

$\begin{array}{lllllllll}1 & \text { AM241 } & 1.140 & 0.200 & 0.766 & 0.013 & 1.490 & 0.26 & \text { W } \\ 1 & \text { CD 60 } & 32.100 & 0.510 & 32.800 & 0.580 & 0.980 & 0.02 & \text { A } \\ 1 & \text { CS137 } & 41.800 & 0.690 & 38.300 & 0.881 & 1.090 & 0.03 & \text { A } \\ 1 & \text { MN 54 } & 41.800 & 0.770 & 38.400 & 1.160 & 1.090 & 0.04 & \text { A }\end{array}$

Units for matrices: $\mathrm{Al}=\mathrm{Bq} / \mathrm{filter} \mathrm{SO}=\mathrm{Bq} / \mathrm{kg} \mathrm{VE}=\mathrm{Bq} / \mathrm{kg} W \mathrm{~W}=\mathrm{Bq} / \mathrm{L}$. Values for elemental uranium are reported in $\mu \mathrm{g} / \mathrm{filter}, \mathrm{g}$, or $\mathrm{mL}$. Evaluation: $A=$ Acceptable, $W=$ Acceptable with Warning, $N=$ Not Acceptable $\mathrm{pCi} \doteq \mathrm{Bq} \times 27$ 
QAP44 Results by Laboratory

Lab: FN Fermi Lab, Batavia, IL

\begin{tabular}{lllllllll} 
No. & Radio & $\begin{array}{l}\text { Reported } \\
\text { Value }\end{array}$ & $\begin{array}{l}\text { Reported } \\
\text { Error }\end{array}$ & $\begin{array}{c}\text { EML } \\
\text { Value }\end{array}$ & $\begin{array}{l}\text { EML } \\
\text { Error }\end{array}$ & $\frac{\text { Reported }}{\text { EML }}$ & $\begin{array}{l}\text { Ratio } \\
\text { Error }\end{array}$ \\
\hline
\end{tabular}

Matrix: Al

$\begin{array}{llrrrrrrr}1 & \text { CE144 } & 41.500 & 9.800 & 33.300 & 3.300 & 1.250 & 0.32 & \text { W } \\ 1 & \text { CO 57 } & 7.000 & 0.750 & 8.900 & 0.900 & 0.790 & 0.12 & \text { A } \\ 1 & \text { CO 60 } & 27.500 & 1.600 & 29.500 & 2.900 & 0.930 & 0.11 & \text { A } \\ 1 & \text { CS134 } & 14.400 & 0.700 & 14.700 & 1.460 & 0.980 & 0.11 & \text { A } \\ 1 & \text { CS137 } & 5.870 & 0.480 & 6.640 & 0.700 & 0.880 & 0.12 & \text { A } \\ 1 & \text { MN 54 } & 3.070 & 0.250 & 3.440 & 0.380 & 0.890 & 0.12 & \text { A } \\ 1 & \text { SB125 } & 9.390 & 0.500 & 9.780 & 1.030 & 0.960 & 0.11 & \text { A }\end{array}$

Matrix: So

$\begin{array}{ll}1 & \text { CS137 } \\ 1 & \text { K } 40 \\ 1 & \text { U } 238 \\ & \\ \text { atrix: } & \text { VE }\end{array}$

$\begin{array}{lrrrrrrr}\text { CS137 } & 363.000 & 36.000 & 359.000 & 10.000 & 1.010 & 0.10 & \text { A } \\ \text { K 40 } & 474.000 & 48.000 & 465.000 & 30.000 & 1.020 & 0.12 & \text { A } \\ \text { U } 238 & 29.300 & 1.800 & 35.900 & 3.630 & 0.820 & 0.10 & \text { A }\end{array}$

\begin{tabular}{|c|c|c|c|c|c|c|}
\hline $\begin{array}{l}\text { CO 60 } \\
\text { CS137 } \\
\text { K } 40\end{array}$ & $\begin{array}{r}56.800 \\
962.000 \\
982.000\end{array}$ & $\begin{array}{r}4.100 \\
96.000 \\
100.000\end{array}$ & $\begin{array}{r}59.700 \\
944.000 \\
1030.000\end{array}$ & $\begin{array}{r}0.963 \\
16.200 \\
33.000\end{array}$ & $\begin{array}{l}0.950 \\
1.020 \\
0.950\end{array}$ & $\begin{array}{l}0.07 \\
0.10 \\
0.10\end{array}$ \\
\hline
\end{tabular}

Matrix: WA

$\begin{array}{llrrrrrrr}1 & \text { CO } 60 & 32.200 & 2.300 & 32.800 & 0.580 & 0.980 & 0.07 & \text { A } \\ 1 & \text { CS137 } & 39.300 & 3.900 & 38.300 & 0.881 & 1.030 & 0.11 & \text { A } \\ 1 & \text { H } 3 & 228.000 & 9.000 & 251.000 & 11.400 & 0.910 & 0.05 & \text { A } \\ 1 & \text { MN 54 } & 41.100 & 4.100 & 38.400 & 1.160 & 1.070 & 0.11 & \text { A }\end{array}$

Units for matrices: $\mathrm{Al}=\mathrm{Bq} /$ filter $\mathrm{SO}=\mathrm{Bq} / \mathrm{kg}$ VE=Bq/kg $W A=B q / \mathrm{L}$. Values for elemental uranium are reported in $\mu \mathrm{g} / \mathrm{filter}, \mathrm{g}$, or $\mathrm{mL}$.

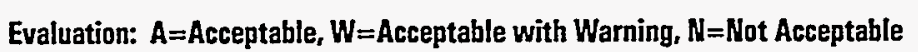

$\mathrm{pCi}=\mathrm{Bq} \times 27$ 


\section{QAP44 Results by Laboratory}

Lab: FS Florida State University, Tallahassee

\begin{tabular}{|c|c|c|c|c|c|c|c|c|}
\hline $\begin{array}{l}\text { No. } \\
\text { Test }\end{array}$ & $\begin{array}{l}\text { Radio. } \\
\text { nuclide }\end{array}$ & $\begin{array}{l}\text { Reported } \\
\text { Value }\end{array}$ & $\begin{array}{l}\text { Reported } \\
\text { Error }\end{array}$ & $\begin{array}{l}\text { EML } \\
\text { Value }\end{array}$ & $\begin{array}{l}\text { EML } \\
\text { Error }\end{array}$ & $\frac{\text { Reported }}{\text { EML }}$ & $\begin{array}{l}\text { Ratio } \\
\text { Error }\end{array}$ & iation \\
\hline
\end{tabular}

Matrix: So

$\begin{array}{llrrrrrrr}1 & \text { AM241 } & 3.770 & 0.260 & 3.690 & 0.454 & 1.020 & 0.14 & \text { A } \\ 1 & \text { CS137 } & 370.000 & 3.000 & 359.000 & 10.000 & 1.030 & 0.03 & \text { A } \\ 1 & \text { K 40 } & 478.000 & 18.000 & 465.000 & 30.000 & 1.030 & 0.08 & \text { A } \\ 1 & \text { PU238 } & 40.700 & 1.400 & 43.000 & 2.440 & 0.950 & 0.06 & \text { A } \\ 1 & \text { PU239 } & 8.640 & 0.530 & 9.230 & 0.346 & 0.940 & 0.07 & \text { A } \\ 1 & \text { U 234 } & 38.800 & 1.930 & 34.200 & 3.520 & 1.140 & 0.13 & \mathbf{W} \\ 1 & \text { U 238 } & 39.300 & 1.340 & 35.900 & 3.630 & 1.100 & 0.12 & \mathbf{W}\end{array}$

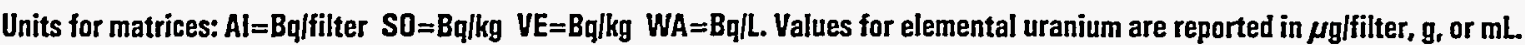




\section{QAP44 Results by Laboratory}

Lab: GA Lockheed Martin, Pikton, $\mathrm{OH}$

\begin{tabular}{|c|c|c|c|}
\hline $\begin{array}{l}\text { Wo. } \\
\text { Test }\end{array}$ & $\begin{array}{l}\text { Radio- } \\
\text { nuclide }\end{array}$ & $\begin{array}{l}\text { Reported } \\
\text { Value }\end{array}$ & $\begin{array}{l}\text { Reported } \\
\text { Error }\end{array}$ \\
\hline
\end{tabular}

Matrix: Al

$\begin{array}{llrlrllll}1 & \text { AM241 } & 0.210 & 0.000 & 0.189 & 0.007 & 1.110 & 0.04 & \text { A } \\ 1 & \text { CE144 } & 21.300 & 0.000 & 33.300 & 3.300 & 0.640 & 0.06 & \text { W } \\ 1 & \text { CO 57 } & 6.170 & 0.000 & 8.900 & 0.900 & 0.690 & 0.07 & \text { W } \\ 1 & \text { C } 60 & 24.500 & 0.000 & 29.500 & 2.900 & 0.830 & 0.08 & A \\ 1 & \text { CS134 } & 11.900 & 0.000 & 14.700 & 1.460 & 0.810 & 0.08 & \text { A } \\ 1 & \text { CS137 } & 5.150 & 0.000 & 6.640 & 0.700 & 0.780 & 0.08 & \text { W } \\ 1 & \text { MN 54 } & 2.940 & 0.000 & 3.440 & 0.380 & 0.860 & 0.09 & A \\ 1 & \text { PU238 } & 0.100 & 0.000 & 0.096 & 0.002 & 1.040 & 0.02 & A \\ 1 & \text { PU239 } & 0.100 & 0.000 & 0.093 & 0.003 & 1.080 & 0.03 & A \\ 1 & \text { RU106 } & 9.380 & 0.000 & 11.600 & 1.440 & 0.810 & 0.10 & A \\ 1 & \text { SB125 } & 7.250 & 0.000 & 9.780 & 1.030 & 0.740 & 0.08 & A \\ 1 & \text { SR 90 } & 0.930 & 0.078 & 1.060 & 0.037 & 0.880 & 0.08 & A \\ 1 & \text { U 234 } & 0.059 & 0.006 & 0.052 & 0.002 & 1.140 & 0.12 & A \\ 1 & \text { U 238 } & 0.054 & 0.007 & 0.053 & 0.002 & 1.010 & 0.14 & A \\ 1 & \text { U UG } & 4.310 & 0.000 & 4.310 & 0.100 & 1.000 & 0.02 & A\end{array}$

Matrix: So

$\begin{array}{llrlrrrrr}1 & \text { AM241 } & 3.300 & 0.000 & 3.690 & 0.454 & 0.890 & 0.11 & \text { A } \\ 1 & \text { CS137 } & 380.000 & 0.000 & 359.000 & 10.000 & 1.060 & 0.03 & \text { A } \\ 1 & \text { K 40 } & 486.000 & 0.000 & 465.000 & 30.000 & 1.040 & 0.07 & A \\ 1 & \text { PU238 } & 44.000 & 0.000 & 43.000 & 2.440 & 1.020 & 0.06 & A \\ 1 & \text { PU239 } & 9.800 & 0.000 & 9.230 & 0.346 & 1.060 & 0.04 & A \\ 1 & \text { U 234 } & 37.000 & 2.000 & 34.200 & 3.520 & 1.080 & 0.13 & \text { W } \\ 1 & \text { U 238 } & 37.000 & 1.100 & 35.900 & 3.630 & 1.030 & 0.11 & A \\ 1 & \text { U UG } & 3.200 & 0.000 & 2.900 & 0.290 & 1.100 & 0.11 & A\end{array}$

Matrix: VE

$\begin{array}{rlrrrrrrr}1 & \text { AM241 } & 6.000 & 0.000 & 5.600 & 0.184 & 1.070 & 0.04 & \text { A } \\ 1 & \text { CM244 } & 3.700 & .0 .000 & 4.440 & 0.202 & 0.830 & 0.04 & W \\ 1 & \text { CO 60 } & 66.000 & 0.000 & 59.700 & 0.963 & 1.110 & 0.02 & \text { A } \\ 1 & \text { CS137 } & 1140.000 & 0.000 & 944.000 & 16.200 & 1.210 & 0.02 & \text { A } \\ 1 & \text { K 40 } & 1200.000 & 0.000 & 1030.000 & 33.000 & 1.170 & 0.04 & \text { A } \\ 1 & \text { PU239 } & 10.900 & 0.000 & 9.820 & 1.220 & 1.110 & 0.14 & \text { A }\end{array}$

Matrix: WA

$\begin{array}{llrlrllll}1 & \text { AM241 } & 0.800 & 0.000 & 0.766 & 0.013 & 1.040 & 0.02 & \text { A } \\ 1 & \text { CO 60 } & 36.300 & 0.000 & 32.800 & 0.580 & 1.110 & 0.02 & A \\ 1 & \text { CS137 } & 41.700 & 0.000 & 38.300 & 0.881 & 1.090 & 0.03 & A \\ 1 & \text { MN 54 } & 43.400 & 0.000 & 38.400 & 1.160 & 1.130 & 0.03 & A \\ 1 & \text { PU238 } & 1.120 & 0.000 & 0.982 & 0.074 & 1.140 & 0.09 & A \\ 1 & \text { PU239 } & 0.890 & 0.000 & 0.772 & 0.056 & 1.150 & 0.08 & A \\ 1 & \text { SR 90 } & 1.640 & 0.310 & 1.450 & 0.034 & 1.130 & 0.22 & A \\ 1 & \text { U 234 } & 0.280 & 0.014 & 0.274 & 0.019 & 1.020 & 0.09 & A\end{array}$

Units for matrices: $\mathrm{Al}=\mathrm{Bq} / \mathrm{filter} \mathrm{SO}=\mathrm{Bq} / \mathrm{kg} \mathrm{VE}=\mathrm{Bq} / \mathrm{kg} W \mathrm{~W}=\mathrm{Bq} / \mathrm{L}$. Values for elemental uranium are reported in $\mu \mathrm{g} / \mathrm{filter}, \mathrm{g}$, or $\mathrm{mL}$.

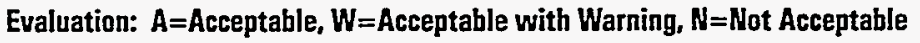
$\mathrm{pCi}=\mathrm{Bq} \times 27$ 


\section{QAP44 Results by Laboratory}

Lab: GA Lockheed Martin, Pikton, $\mathrm{OH}$

\begin{tabular}{lllllllll} 
No. & Radio- & $\begin{array}{l}\text { Reported } \\
\text { Test }\end{array}$ & nuclide & Reported & EML & EML & Reported & Ratio \\
Error & Value & Error & EML & Error & Evaluation \\
\hline
\end{tabular}

Matrix: WA

$\begin{array}{lllllllll}1 & \text { U } 238 & 0.260 & 0.024 & 0.275 & 0.024 & 0.950 & 0.12 & \text { A } \\ 1 & \text { U UG } & 0.021 & 0.000 & 0.022 & 0.003 & 0.950 & 0.11 & \text { A }\end{array}$

Units for matrices: $A=B q \mid$ filter $S O=B q / \mathrm{kg} V E=B q / \mathrm{kg} W A=B q / L$. Values for elemental uranium are reported in $\mu \mathrm{g} / \mathrm{filter}, \mathrm{g}$, or $\mathrm{mL}$. $\mathrm{pCi}=\mathrm{Bq} \times 27$ 


\section{OAP44 Results by Laboratory}

Lab: GE Environmental Physics, Inc., Charleston, SC

\begin{tabular}{|c|c|c|c|c|c|c|c|c|}
\hline $\begin{array}{l}\text { No. } \\
\text { Test }\end{array}$ & $\begin{array}{l}\text { Radio- } \\
\text { nuclide }\end{array}$ & $\begin{array}{l}\text { Reported } \\
\text { Value }\end{array}$ & $\begin{array}{l}\text { Reporte } \\
\text { Error }\end{array}$ & $\begin{array}{l}\text { EML } \\
\text { Valut }\end{array}$ & $\begin{array}{l}\text { EMI } \\
\text { Erro }\end{array}$ & $\frac{\text { Report }}{\text { EML }}$ & $\begin{array}{l}\text { Rat } \\
\text { Err }\end{array}$ & Evaluation \\
\hline
\end{tabular}

Matrix: Al

\begin{tabular}{|c|c|c|c|c|c|c|}
\hline $\begin{array}{l}\text { AM241 } \\
\text { CE144 } \\
\text { CO 57 } \\
\text { CO 60 } \\
\text { CS134 } \\
\text { CS137 } \\
\text { GA 1 } \\
\text { GB 2 } \\
\text { MN 54 } \\
\text { PU238 } \\
\text { PU239 } \\
\text { RU106 } \\
\text { SB125 } \\
\text { SR 90 } \\
\text { U 234 } \\
\text { U 238 } \\
\text { U UG }\end{array}$ & $\begin{array}{r}0.206 \\
23.700 \\
7.110 \\
26.300 \\
13.600 \\
5.900 \\
1.750 \\
1.640 \\
3.420 \\
0.088 \\
0.093 \\
9.890 \\
9.030 \\
0.788 \\
0.067 \\
0.055 \\
4.360\end{array}$ & $\begin{array}{l}0.030 \\
2.270 \\
0.460 \\
0.980 \\
0.600 \\
0.556 \\
0.026 \\
0.022 \\
0.570 \\
0.014 \\
0.014 \\
4.260 \\
1.420 \\
0.039 \\
0.011 \\
0.010 \\
0.060\end{array}$ & $\begin{array}{r}0.189 \\
33.300 \\
8.900 \\
29.500 \\
14.700 \\
6.640 \\
1.620 \\
1.770 \\
3.440 \\
0.096 \\
0.093 \\
11.600 \\
9.780 \\
1.060 \\
0.052 \\
0.053\end{array}$ & $\begin{array}{l}0.007 \\
3.300 \\
0.900 \\
2.900 \\
1.460 \\
0.700 \\
0.150 \\
0.150 \\
0.380 \\
0.002 \\
0.003 \\
1.440 \\
1.030 \\
0.037 \\
0.002 \\
0.002\end{array}$ & $\begin{array}{l}1.090 \\
0.710 \\
0.800 \\
0.890 \\
0.930 \\
0.890 \\
1.080 \\
0.930 \\
0.990 \\
0.920 \\
1.000 \\
0.850 \\
0.920 \\
0.740 \\
1.300 \\
1.030 \\
1010\end{array}$ & $\begin{array}{l}0.16 \\
0.10 \\
0.10 \\
0.09 \\
0.10 \\
0.13 \\
0.10 \\
0.08 \\
0.20 \\
0.15 \\
0.15 \\
0.38 \\
0.18 \\
0.04 \\
0.22 \\
0.19 \\
0.19\end{array}$ \\
\hline
\end{tabular}

Matrix: SO

$\begin{array}{llrr}1 & \text { AM241 } & 4.370 & 0.850 \\ 1 & \text { CS137 } & 374.000 & 3.700 \\ 1 & \text { K 40 } & 540.000 & 22.200 \\ 1 & \text { PU238 } & 50.300 & 0.890 \\ 1 & \text { PU239 } & 11.200 & 0.670 \\ 1 & \text { SR 90 } & 977.000 & 25.900 \\ 1 & \text { U 234 } & 35.200 & 4.000 \\ 1 & \text { U 238 } & 33.200 & 3.200 \\ 1 & \text { U UG } & 2.650 & 0.040\end{array}$

$\begin{array}{rrr}3.690 & 0.454 & 1.180 \\ 359.000 & 10.000 & 1.040 \\ 465.000 & 30.000 & 1.160 \\ 43.000 & 2.440 & 1.170 \\ 9.230 & 0.346 & 1.210 \\ 1340.000 & 113.000 & 0.730 \\ 34.200 & 3.520 & 1.030 \\ 35.900 & 3.630 & 0.930 \\ 2.900 & 0.290 & 0.910\end{array}$

0.27
0.03
0.09
0.07
0.09
0.06
0.16
0.13
0.09

A
A
A
A
A
$\mathbf{W}$
A
A
A

Matrix: VE

$\begin{array}{llrr}1 & \text { AM241 } & 6.440 & 0.770 \\ 1 & \text { CM244 } & 5.000 & 0.680 \\ 1 & \text { CD 60 } & 63.600 & 8.140 \\ 1 & \text { CS } 137 & 1050.000 & 114.000 \\ 1 & \text { K 40 } & 1210.000 & 155.000 \\ 1 & \text { PU239 } & 8.030 & 0.780 \\ 1 & \text { SR 90 } & 977.000 & 11.500\end{array}$

5.600
4.440
59.700
944.000
1030.000
9.820
1300.000

0.184
0.202
0.963
16.200
33.000
1.220
52.400

1.150

1.130

0.14

0.16

0.14

0.12

0.16

1.110

1.170

0.820

0.13

0.750

0.03

A
A
A
A
A
A
A

Matrix: WA

\begin{tabular}{llrrrrrrr}
1 & AM241 & 1.130 & 0.138 & 0.766 & 0.013 & 1.480 & 0.18 & W \\
1 & CO 60 & 34.900 & 2.780 & 32.800 & 0.580 & 1.060 & 0.09 & A \\
1 & CS137 & 46.400 & 3.920 & 38.300 & 0.881 & 1.210 & 0.11 & W \\
1 & FE 55 & 112.000 & 21.900 & 83.000 & 3.440 & 1.350 & 0.27 & W \\
\hline
\end{tabular}

Units for matrices: $\mathrm{Al}=\mathrm{Bq} / \mathrm{filter} \mathrm{SO}=\mathrm{Bq} / \mathrm{kg}$ VE=Bq/kg $W A=B q / \mathrm{L}$. Values for elemental uranium are reported in $\mu \mathrm{g} / \mathrm{filter}, \mathrm{g}$, or $\mathrm{mL}$.

Evaluation: $A=A c c e p t a b l e, W=A c c e p t a b l e$ with Warning, $N=$ Not Acceptable

$p C i=B q \times 27$ 


\section{QAP44 Results by Laboratory}

Lab: GE Environmental Physics, Inc., Charleston, SC

\begin{tabular}{llllllll} 
No. & $\begin{array}{llll}\text { Radio- } \\
\text { nuclide }\end{array}$ & $\begin{array}{l}\text { Reported } \\
\text { Value }\end{array}$ & $\begin{array}{l}\text { Reported } \\
\text { Error }\end{array}$ & $\begin{array}{c}\text { EML } \\
\text { Value }\end{array}$ & $\begin{array}{l}\text { EML } \\
\text { Error }\end{array}$ & $\frac{\text { Reported }}{\text { EML }}$ & $\begin{array}{l}\text { Ratio } \\
\text { Error }\end{array}$ \\
\hline
\end{tabular}

Matrix: WA

$\begin{array}{llrrrrrrr}1 & \text { GA 1 } & 1700.000 & 27.500 & 1850.000 & 185.000 & 0.920 & 0.09 & A \\ 1 & \text { GB 2 } & 733.000 & 15.200 & 744.000 & 74.000 & 0.990 & 0.10 & \text { A } \\ 1 & \text { H 3 } & 197.000 & 7.770 & 251.000 & 11.400 & 0.790 & 0.05 & W \\ 1 & \text { MN 54 } & 46.300 & 3.440 & 38.400 & 1.160 & 1.210 & 0.10 & W \\ 1 & \text { PU238 } & 0.890 & 0.090 & 0.982 & 0.074 & 0.910 & 0.11 & A \\ 1 & \text { PU239 } & 0.688 & 0.078 & 0.772 & 0.056 & 0.890 & 0.12 & \text { A } \\ 1 & \text { SR 90 } & 1.170 & 0.084 & 1.450 & 0.034 & 0.810 & 0.06 & W \\ 1 & \text { U 234 } & 0.335 & 0.058 & 0.274 & 0.019 & 1.220 & 0.23 & W \\ 1 & \text { U 238 } & 0.315 & 0.056 & 0.275 & 0.024 & 1.150 & 0.23 & W\end{array}$

Units for matrices: $\mathrm{Al}=\mathrm{Bq} / \mathrm{filter} \mathrm{SO}=\mathrm{Bq} / \mathrm{kg} \mathrm{VE}=\mathrm{Bq} / \mathrm{kg} W \mathrm{~W}=\mathrm{Bq} / \mathrm{l}$. Values for elemental uranium are reported in $\mu \mathrm{g} / \mathrm{filter}, \mathrm{g}$, or $\mathrm{mL}$. 


\section{QAP44 Results by Laboratory}

Lab: GS USGSINWOL, Arvada, CO

No.

Test

Radio- Reported Reported

EML

EML

Error

Value

Error

Reported

Ratio

EML

Error

Evaluation

Matrix: WA

$1 \quad \cup \cup G$

0.017

0.001

0.022

0.003

0.770

0.10

W

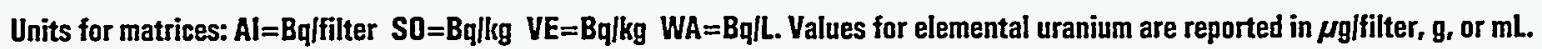




\section{QAP44 Results by Laboratory}

Lab: HA NUS Laboratory, Pittsburgh

No.

Radio-

- Reported

Reported.

EML

EML

Reported Ratio Error

Value

Error

EML

Error

Evaluation

Matrix: So

$\begin{array}{rlrrrrrrr}1 & \text { CS137 } & 407.000 & 40.000 & 359.000 & 10.000 & 1.130 & 0.12 & \text { A } \\ 1 & \text { K 40 } & 593.000 & 60.000 & 465.000 & 30.000 & 1.280 & 0.15 & \text { W } \\ 1 & \text { PU238 } & 36.600 & 4.000 & 43.000 & 2.440 & 0.850 & 0.11 & \text { A } \\ 1 & \text { PU239 } & 7.400 & 0.800 & 9.230 & 0.346 & 0.800 & 0.09 & \text { W } \\ 1 & \text { SR 90 } & 937.000 & 100.000 & 1340.000 & 113.000 & 0.700 & 0.10 & \text { W } \\ 1 & \text { U 234 } & 30.700 & 4.000 & 34.200 & 3.520 & 0.900 & 0.15 & \text { A } \\ 1 & \text { U 238 } & 31.800 & 4.000 & 35.900 & 3.630 & 0.890 & 0.14 & \text { A }\end{array}$

Matrix: WA

$\begin{array}{llr}1 & \text { AM241 } & 0.850 \\ 1 & \text { CD 60 } & 34.000 \\ 1 & \text { CS137 } & 40.700 \\ 1 & \text { FE 55 } & 81.400 \\ 1 & \text { GA 1 } & 2150.000 \\ 1 & \text { GB 2 } & 629.000 \\ 1 & \text { H 3 } & 226.000 \\ 1 & \text { MN 54 } & 44.400 \\ 1 & \text { PU238 } & 0.810 \\ 1 & \text { PU239 } & 0.730 \\ 1 & \text { SR 90 } & 1.240 \\ 1 & \text { U 234 } & 0.260 \\ 1 & \text { U 238 } & 0.300\end{array}$

$\begin{array}{rr}0.090 & 0.766 \\ 4.000 & 32.800 \\ 4.000 & 38.300 \\ 0.900 & 83.000 \\ 215.000 & 1850.000 \\ 63.000 & 744.000 \\ 30.000 & 251.000 \\ 5.000 & 38.400 \\ 0.090 & 0.982 \\ 0.080 & 0.772 \\ 0.200 & 1.450 \\ 0.030 & 0.274 \\ 0.030 & 0.275\end{array}$

0.013
0.580
0.881
3.440
185.000
74.000
11.400
1.160
0.074
0.056
0.034
0.019
0.024

1.110

1.040

1.060

0.980

1.160

0.850

0.900

1.160

0.830

0.950

0.860

0.950

1.090

0.12
0.12
0.11
0.04
0.16
0.12
0.13
0.14
0.11
0.12
0.14
0.13
0.15

$\mathbf{A}$
$\mathbf{A}$
$\mathbf{A}$
$\mathbf{A}$
$\mathbf{A}$
$\mathbf{W}$
$\mathbf{A}$
$\mathbf{W}$
$\mathbf{W}$
$\mathbf{A}$
$\mathbf{W}$
$\mathbf{A}$
$\mathbf{A}$ 


\section{QAP44 Results by Laboratory}

Lab: HC Lawrence Livermore Laboratory, California

No.

Test

Radionuclide

Reported

Value

Matrix: Al

$1 \quad G A$

$1 \quad \mathrm{~GB} 2$

1.820

1.710

0.200

0.200

Matrix: WA

$1 \quad G A$

$1 \quad G B 2$

1 H 3

1900.000

805.000

206.000

95.000

40.000

14.400
EML

Value

$$
1.620
$$

1.770

0.150

0.150

185.000

74.000

11.400

251.000
Reported EML

1.120

0.970

0.16

0.14

A

A

Ratio

Error Evaluation

1.030

1.080

0.820

0.12

0.12

0.07

A
A
W 


\section{QAP44 Results by Laboratory}

Lab: HL Heritage Laboratories, Inc., Romeoville, IL

\begin{tabular}{|c|c|c|c|c|c|c|c|c|}
\hline $\begin{array}{l}\text { No. } \\
\text { Test }\end{array}$ & $\begin{array}{l}\text { Radio- } \\
\text { nuclide }\end{array}$ & $\begin{array}{l}\text { Reported } \\
\text { Value }\end{array}$ & $\begin{array}{l}\text { Reported } \\
\text { Error }\end{array}$ & $\begin{array}{c}\text { EML } \\
\text { Value }\end{array}$ & $\begin{array}{l}\text { EML } \\
\text { Error }\end{array}$ & $\frac{\text { Reported }}{\text { EML }}$ & $\begin{array}{l}\text { Ratio } \\
\text { Error }\end{array}$ & Evaluation \\
\hline
\end{tabular}

Matrix: So

$\begin{array}{rlrrrrrrr}1 & \text { AM241 } & 5.500 & 1.900 & 3.690 & 0.454 & 1.490 & 0.55 & \text { A } \\ 1 & \text { CS137 } & 294.000 & 33.000 & 359.000 & 10.000 & 0.820 & 0.09 & W \\ 1 & \text { K 40 } & 600.000 & 149.000 & 465.000 & 30.000 & 1.290 & 0.33 & \text { W } \\ 1 & \text { SR 90 } & 1320.000 & 42.200 & 1340.000 & 113.000 & 0.990 & 0.09 & \text { A } \\ 1 & \text { U 234 } & 37.100 & 8.000 & 34.200 & 3.520 & 1.090 & 0.26 & W \\ 1 . & \text { U 238 } & 37.600 & 8.100 & 35.900 & 3.630 & 1.050 & 0.25 & A \\ 1 & \text { U BO } & 74.800 & 16.100 & 71.700 & 4.150 & 1.040 & 0.23 & A\end{array}$

Matrix: WA

$\begin{array}{llrlrrrrr}1 & \text { CO 60 } & \text { 35.900 } & 2.200 & 32.800 & 0.580 & 1.100 & 0.07 & \text { A } \\ 1 & \text { CS137 } & 45.300 & 4.300 & 38.300 & 0.881 & 1.180 & 0.12 & \text { W } \\ 1 & \text { H } 3 & 214.000 & 8.100 & 251.000 & 11.400 & 0.850 & 0.05 & \text { A } \\ 1 & \text { MN 54 } & 46.100 & 4.700 & 38.400 & 1.160 & 1.200 & 0.13 & \text { W } \\ 1 & \text { SR 90 } & 1.560 & 0.280 & 1.450 & 0.034 & 1.080 & 0.20 & \text { A } \\ 1 & \text { U 234 } & 0.260 & 0.110 & 0.274 & 0.019 & 0.950 & 0.41 & \text { A } \\ 1 & \text { U 238 } & 0.290 & 0.120 & 0.275 & 0.024 & 1.050 & 0.45 & \text { A } \\ 1 & \text { U BQ } & 0.540 & 0.220 & 0.559 & 0.047 & 0.970 & 0.40 & \text { A }\end{array}$




\section{QAP44 Results by Laboratory}

Lab: ID DPRA - IRD/CNEN, Rio de Janeiro, Brazil

\begin{tabular}{|c|c|c|c|c|c|c|c|c|}
\hline Test & $\begin{array}{l}\text { Radio- } \\
\text { nuclide }\end{array}$ & $\begin{array}{l}\text { Reported } \\
\text { Value }\end{array}$ & $\begin{array}{l}\text { Reported } \\
\text { Error }\end{array}$ & $\begin{array}{l}\text { EML } \\
\text { Value }\end{array}$ & $\begin{array}{l}\text { EML } \\
\text { Error }\end{array}$ & $\frac{\text { Reparted }}{\text { EML }}$ & $\begin{array}{l}\text { Ratio } \\
\text { Error }\end{array}$ & Fvaluation \\
\hline
\end{tabular}

Matrix: Al

$\begin{array}{lrrrrrrr}\text { CE144 } & 33.700 & 2.100 & 33.300 & 3.300 & 1.010 & 0.12 & \text { A } \\ \text { CO 57 } & 9.270 & 0.480 & 8.900 & 0.900 & 1.040 & 0.12 & \text { A } \\ \text { CO 60 } & 28.300 & 1.400 & 29.500 & 2.900 & 0.960 & 0.11 & \text { A } \\ \text { CS134 } & 15.900 & 0.800 & 14.700 & 1.460 & 1.080 & 0.12 & \text { A } \\ \text { CS137 } & 7.400 & 0.380 & 6.640 & 0.700 & 1.110 & 0.13 & \text { A } \\ \text { MN 54 } & 3.630 & 0.220 & 3.440 & 0.380 & 1.050 & 0.13 & \\ \text { RU106 } & 12.400 & 1.600 & 11.600 & 1.440 & 1.070 & 0.19 & \text { A } \\ \text { SB125 } & 11.000 & 0.600 & 9.780 & 1.030 & 1.130 & 0.13 & \text { W } \\ \text { SR 90 } & 2.330 & 0.130 & 1.060 & 0.037 & 2.200 & 0.14 & \text { W } \\ \text { U BD } & 0.115 & 0.006 & 0.107 & 0.003 & 1.080 & 0.06 & \text { A }\end{array}$

Matrix: So

$\begin{array}{llrr}1 & \text { AM241 } & 4.330 & 0.620 \\ 1 & \text { CS137 } & 363.000 & 19.000 \\ 1 & \text { K 40 } & 411.000 & 23.000 \\ 1 & \text { PU238 } & 41.700 & 3.700 \\ 1 & \text { PU239 } & 9.070 & 0.700 \\ 1 & \text { SR 90 } & 1050.000 & 124.000 \\ 1 & \text { U BO } & 69.100 & 4.600\end{array}$

$\begin{array}{rrr}3.690 & 0.454 & 1.170 \\ 359.000 & 10.000 & 1.010 \\ 465.000 & 30.000 & 0.880 \\ 43.000 & 2.440 & 0.970 \\ 9.230 & 0.346 & 0.980 \\ 1340.000 & 113.000 & 0.780 \\ 71.700 & 4.150 & 0.960\end{array}$

0.22
0.06
0.08
0.10
0.08
0.11
0.09

A
A
A
A
A
A
A

Matrix: VE

$\begin{array}{llr}1 & \text { AM241 } & 7.000 \\ 1 & \text { CD 60 } & 52.700 \\ 1 & \text { CS137 } & 1050.000 \\ 1 & \text { K 40 } & 997.000 \\ 1 & \text { PU239 } & 7.590 \\ 1 & \text { SR } 90 & 1180.000\end{array}$

1.060
2.800
53.000
60.000
1.030
90.000

5.600
59.700
944.000
1030.000
9.820
1300.000

0.184
0.963
16.200
33.000
1.220
52.400

1.250

0.880

0.19

0.05

1.110

0.970

0.770

0.06

0.07

0.14

0.910

0.08

Matrix: WA

$\begin{array}{llrrrrrrr}1 & \text { CO 60 } & 27.400 & 2.300 & 32.800 & 0.580 & 0.840 & 0.07 & \text { N } \\ 1 & \text { CS137 } & 37.500 & 2.300 & 38.300 & 0.881 & 0.980 & 0.06 & \text { A } \\ 1 & \text { H 3 } & 216.000 & 13.000 & 251.000 & 11.400 & 0.860 & 0.06 & \text { A } \\ 1 & \text { MN54 } & 37.800 & 2.200 & 38.400 & 1.160 & 0.980 & 0.06 & \text { A } \\ 1 & \text { PU238 } & 0.870 & 0.130 & 0.982 & 0.074 & 0.890 & 0.15 & \text { W } \\ 1 & \text { PU239 } & 0.700 & 0.050 & 0.772 & 0.056 & 0.910 & 0.09 & \text { A } \\ 1 & \text { SR 90 } & 1.710 & 0.150 & 1.450 & 0.034 & 1.180 & 0.11 & \text { A } \\ 1 & \text { U BO } & 0.727 & 0.040 & 0.559 & 0.047 & 1.300 & 0.13 & \text { W }\end{array}$

Units for matrices: $A l=B q / f i l t e r ~ S O=B q / k g \quad V E=B q / k g ~ W A=B q / L$. Values for elemental uranium are reported in $\mu g / f i l t e r, g$, or $\mathrm{mL}$. 
QAP44 Results by Laboratory

Lab: IE IEA, Inc., Morrisville, NC

\begin{tabular}{|c|c|c|c|c|c|c|c|c|}
\hline $\begin{array}{l}\text { No. } \\
\text { Test }\end{array}$ & $\begin{array}{l}\text { Radio. } \\
\text { nuclide }\end{array}$ & $\begin{array}{l}\text { Reported } \\
\text { Value }\end{array}$ & $\begin{array}{l}\text { Reported } \\
\text { Error }\end{array}$ & $\begin{array}{c}\text { EML } \\
\text { Value }\end{array}$ & $\begin{array}{l}\text { EML } \\
\text { Error }\end{array}$ & $\frac{\text { Reported }}{\text { EML }}$ & $\begin{array}{l}\text { Ratio } \\
\text { Error }\end{array}$ & Evaluation \\
\hline
\end{tabular}

Matrix: Al

$\begin{array}{lrrrrrrr}\text { AM241 } & 0.178 & 0.025 & 0.189 & 0.007 & 0.940 & 0.14 & \text { A } \\ \text { CE144 } & 22.000 & .1 .210 & 33.300 & 3.300 & 0.660 & 0.07 & \text { W } \\ \text { CO 57 } & 6.410 & 1.060 & 8.900 & 0.900 & 0.720 & 0.14 & \text { A } \\ \text { CD 60 } & 24.700 & 1.110 & 29.500 & 2.900 & 0.840 & 0.09 & \text { A } \\ \text { CS134 } & 12.300 & 0.180 & 14.700 & 1.460 & 0.840 & 0.08 & \text { A } \\ \text { CS137 } & 5.380 & 0.320 & 6.640 & 0.700 & 0.810 & 0.10 & \text { W } \\ \text { MN 54 } & 2.750 & 0.340 & 3.440 & 0.380 & 0.800 & 0.13 & \text { W } \\ \text { PU238 } & 0.094 & 0.015 & 0.096 & 0.002 & 0.980 & 0.16 & \text { A } \\ \text { PU239 } & 0.087 & 0.006 & 0.093 & 0.003 & 0.940 & 0.07 & \text { A } \\ \text { RU106 } & 8.000 & 0.800 & 11.600 & 1.440 & 0.690 & 0.11 & \text { A } \\ \text { SB125 } & 8.450 & 0.900 & 9.780 & 1.030 & 0.860 & 0.13 & \text { A } \\ \text { SR 90 } & 0.993 & 0.055 & 1.060 & 0.037 & 0.940 & 0.06 & \text { A } \\ \text { U 234 } & 0.056 & 0.012 & 0.052 & 0.002 & 1.080 & 0.24 & \text { A } \\ \text { U 238 } & 0.054 & 0.012 & 0.053 & 0.002 & 1.010 & 0.22 & \text { A } \\ \text { U UG } & 4.400 & 0.937 & 4.310 & 0.100 & 1.020 & 0.22 & \text { A }\end{array}$

Matrix: So

$\begin{array}{llr}1 & \text { AM241 } & 2.860 \\ 1 & \text { CS137 } & 388.000 \\ 1 & \text { K 40 } & 467.000 \\ 1 & \text { PU238 } & 40.000 \\ 1 & \text { PU239 } & 8.820 \\ 1 & \text { SR 90 } & 1140.000 \\ 1 & \text { U 234 } & 35.200 \\ 1 & \text { U 238 } & 34.800 \\ 1 & \text { U UG } & 2.820\end{array}$

0.199
18.700
46.400
0.620
0.419
248.000
2.240
2.570
0.210

3.690
359.000
465.000
43.000
9.230
1340.000
34.200
35.900
2.900

0.454
10.000
30.000
2.440
0.346
113.000
3.520
3.630
0.290

0.780
1.080
1.000
0.930
0.960
0.850
1.030
0.970
0.970

0.11

0.06

0.12

0.05

0.06

0.20

0.13

0.12

0.12

$\mathbf{A}$
$\mathbf{W}$
$\mathbf{A}$
$\mathbf{A}$
$\mathbf{A}$
$\mathbf{W}$
$\mathbf{W}$
$\mathbf{A}$
$\mathbf{A}$
$\mathbf{A}$
$\mathbf{A}$
$\mathbf{A}$
$\mathbf{A}$
$\mathbf{A}$
$\mathbf{A}$

Matrix: VE

$\begin{array}{llr}1 & \text { AM241 } & 5.670 \\ 1 & \text { CM244 } & 4.300 \\ 1 & \text { CO 60 } & 60.700 \\ 1 & \text { CS137 } & 1060.000 \\ 1 & \text { K 40 } & 1130.000 \\ 1 & \text { PU239 } & 9.400 \\ 1 & \text { SR 90 } & 1220.000\end{array}$

0.530
0.240
6.180
74.900
208.000
0.570
18.500

$\begin{array}{rr}5.600 & 0.184 \\ 4.440 & 0.202 \\ 59.700 & 0.963 \\ 944.000 & 16.200 \\ 1030.000 & 33.000 \\ 9.820 & 1.220 \\ 1300.000 & 52.400\end{array}$

$$
\begin{aligned}
& 1.010 \\
& 0.970 \\
& 1.020 \\
& 1.120 \\
& 1.100 \\
& 0.960 \\
& 0.940
\end{aligned}
$$

\subsection{0 \\ 0.07 \\ 0.11 \\ 0.08 \\ 0.21 \\ 0.13 \\ 0.04}

$\mathbf{A}$
$\mathbf{A}$
$\mathbf{A}$
$\mathbf{A}$
$\mathbf{A}$
$\mathbf{A}$
$\mathbf{A}$
$\mathbf{A}$
$\mathbf{A}$

Matrix: WA

$\begin{array}{llrrrrrrr}1 & \text { AM241 } & 0.750 & 0.113 & 0.766 & 0.013 & 0.980 & 0.15 & \text { A } \\ 1 & \text { CO 60 } & 34.000 & 1.450 & 32.800 & 0.580 & 1.040 & 0.05 & \text { A } \\ 1 & \text { CS137 } & 42.700 & 0.883 & 38.300 & 0.881 & 1.120 & 0.03 & \text { A } \\ 1 & \text { GA 1 } & 1970.000 & 65.300 & 1850.000 & 185.000 & 1.070 & 0.11 & \text { A } \\ 1 & \text { GB 2 } & 541.000 & 64.800 & 744.000 & 74.000 & 0.730 & 0.11 & \text { W } \\ 1 & \text { H 3 } & 208.000 & 55.200 & 251.000 & 11.400 & 0.830 & 0.22 & \text { W }\end{array}$

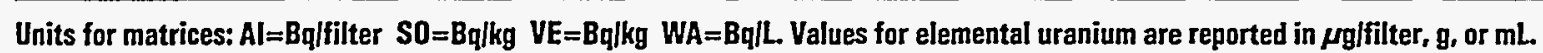


QAP44 Results by Laboratory

Lab: IE IEA, Inc., Morrisville, NC

\begin{tabular}{|c|c|c|c|c|c|c|c|c|}
\hline $\begin{array}{l}\text { No. } \\
\text { Test }\end{array}$ & $\begin{array}{l}\text { Radio: } \\
\text { nuclide }\end{array}$ & $\begin{array}{l}\text { Reported } \\
\text { Value }\end{array}$ & $\begin{array}{l}\text { Reported } \\
\text { Error }\end{array}$ & $\begin{array}{l}\text { EML } \\
\text { Value }\end{array}$ & $\begin{array}{l}\text { EML } \\
\text { Error }\end{array}$ & $\frac{\text { Reported }}{\text { EML }}$ & $\begin{array}{l}\text { Ratio } \\
\text { Error }\end{array}$ & Evaluation \\
\hline
\end{tabular}

Matrix: WA

$\begin{array}{lrrrrrrr}\text { MN 54 } & 43.300 & 2.820 & 38.400 & 1.160 & 1.130 & 0.08 & \text { A } \\ \text { PU238 } & 0.975 & 0.110 & 0.982 & 0.074 & 0.990 & 0.13 & \text { A } \\ \text { PU239 } & 0.730 & 0.022 & 0.772 & 0.056 & 0.950 & 0.07 & \text { A } \\ \text { SR 90 } & 1.330 & 0.060 & 1.450 & 0.034 & 0.920 & 0.05 & \text { A } \\ \text { U 234 } & 0.292 & 0.031 & 0.274 & 0.019 & 1.070 & 0.14 & \text { A } \\ \text { U 238 } & 0.277 & 0.014 & 0.275 & 0.024 & 1.010 & 0.10 & \text { A } \\ \text { U UG } & 22.500 & 1.160 & 0.022 & 0.003 & * * * * & * * * & \text { N }\end{array}$

Units for matrices: $\mathrm{Al}=\mathrm{Bq} / \mathrm{filter} \mathrm{SO}=\mathrm{Bq} / \mathrm{kg} \mathrm{VE}=\mathrm{Bq} / \mathrm{kg} \mathrm{WA}=\mathrm{Bq} / \mathrm{L}$. Values for elemental uranium are reported in $\mu \mathrm{g} / \mathrm{filter}, \mathrm{g}$, or $\mathrm{mL}$. 


\section{QAP44 Results by Laboratory}

Lab: IL ISU Environmental Monitoring Program, Pocatello, ID

\begin{tabular}{lllllllll} 
No. & Radio- & Reported & Reported & EML & EML & Reported & Ratio \\
Test & nuclide & Value & Error & Value & Error & EML & Error & Evaluation \\
\hline
\end{tabular}

Matrix: Al

$\begin{array}{lllllllll}1 & \text { GA } 1 & 2.520 & 0.200 & 1.620 & 0.150 & 1.560 & 0.19 & W \\ 1 & \text { GB } 2 & 1.620 & 0.160 & 1.770 & 0.150 & 0.920 & 0.12 & A\end{array}$

Matrix: $\quad$ SO

$\begin{array}{llrrrrrrr}1 & \text { CS137 } & 94.300 & 1.920 & 359.000 & 10.000 & 0.260 & 0.01 & N \\ 1 & K 40 & 133.000 & 4.450 & 465.000 & 30.000 & 0.290 & 0.02 & N\end{array}$

Matrix: VE

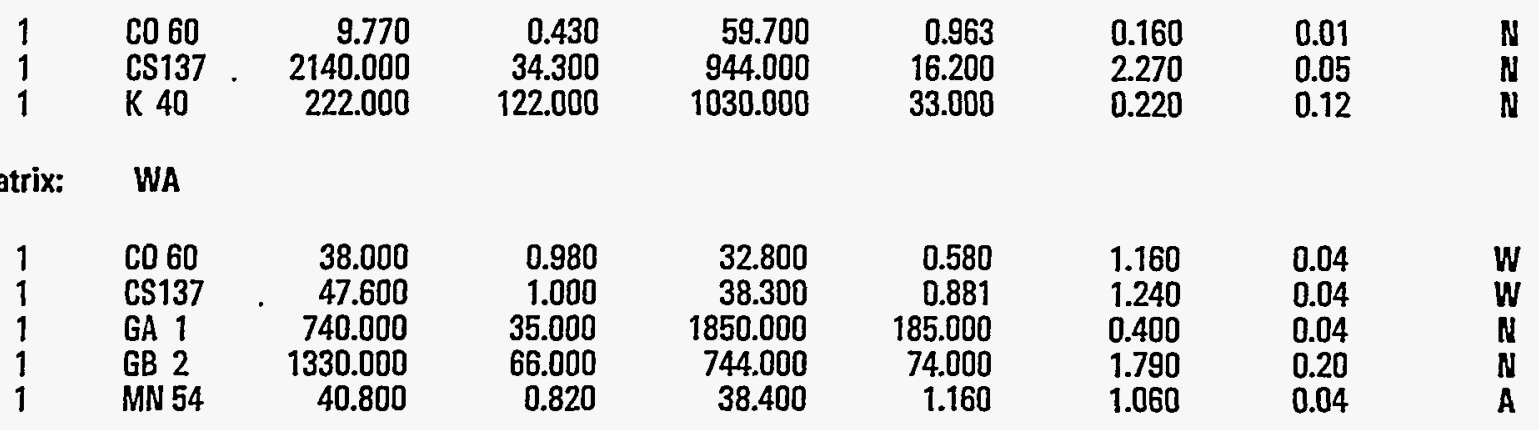

Units for matrices: $A I=B q / f i l t e r ~ S O=B q / k g ~ V E=B q / k g ~ W A=B q / L$. Values for elemental uranium are reported in $\mu g / f i l t e r, g$, or $\mathrm{mL}$. 
QAP44 Results by Laboratory

Lab: IN WINCO, Idaho Falls

\begin{tabular}{lllllllll} 
No. & $\begin{array}{l}\text { Radio- } \\
\text { nuclide }\end{array}$ & $\begin{array}{l}\text { Reported } \\
\text { Value }\end{array}$ & $\begin{array}{l}\text { Reported } \\
\text { Error }\end{array}$ & $\begin{array}{l}\text { EML } \\
\text { Value }\end{array}$ & $\begin{array}{l}\text { EML } \\
\text { Error }\end{array}$ & $\frac{\text { Reported }}{\text { EML }}$ & $\begin{array}{l}\text { Ratio } \\
\text { Error }\end{array}$ & Evaluation \\
\hline
\end{tabular}

Matrix: Al

$\begin{array}{llrlrllll}1 & \text { CE144 } & 21.500 & 0.630 & 33.300 & 3.300 & 0.650 & 0.07 & \text { W } \\ 1 & \text { CD 57 } & 6.360 & 0.160 & 8.900 & 0.900 & 0.720 & 0.07 & \text { A } \\ 1 & \text { CD 60 } & 23.700 & 0.220 & 29.500 & 2.900 & 0.800 & 0.08 & \text { W } \\ 1 & \text { CS134 } & 12.700 & 0.180 & 14.700 & 1.460 & 0.860 & 0.09 & \text { A } \\ 1 & \text { CS137 } & 4.950 & 0.250 & 6.640 & 0.700 & 0.750 & 0.09 & \text { W } \\ 1 & \text { MN 54 } & 2.580 & 0.010 & 3.440 & 0.380 & 0.750 & 0.08 & \mathbf{N} \\ 1 & \text { RU106 } & 10.300 & 1.300 & 11.600 & 1.440 & 0.890 & 0.16 & \text { A } \\ 1 & \text { SB125 } & 8.330 & 0.460 & 9.780 & 1.030 & 0.850 & 0.10 & \text { A }\end{array}$

Matrix: So

$\begin{array}{llrrrrrrr}1 & \text { AM241 } & 2.400 & 0.290 & 3.690 & 0.454 & 0.650 & 0.11 & \text { W } \\ 1 & \text { CS137 } & 376.000 & 4.970 & 359.000 & 10.000 & 1.050 & 0.03 & \text { A } \\ 1 & \text { K 40 } & 478.000 & 43.000 & 465.000 & 30.000 & 1.030 & 0.11 & \text { A } \\ 1 & \text { PU238 } & 44.000 & 5.000 & 43.000 & 2.440 & 1.020 & 0.13 & \text { A } \\ 1 & \text { PU239 } & 9.440 & 1.070 & 9.230 & 0.346 & 1.020 & 0.12 & \text { A } \\ 1 & \text { SR 90 } & 1010.000 & 50.500 & 1340.000 & 113.000 & 0.750 & 0.07 & \text { W }\end{array}$

Matrix: VE

$\begin{array}{rlrrrrrrr}1 & \text { CO 60 } & 66.000 & 2.800 & 59.700 & 0.963 & 1.110 & 0.05 & \text { A } \\ 1 & \text { CS137 } & 1080.000 & 27.100 & 944.000 & 16.200 & 1.140 & 0.03 & \text { A } \\ 1 & \text { K } 40 & 1250.000 & 60.800 & 1030.000 & 33.000 & 1.210 & 0.07 & \text { W }\end{array}$

Matrix: WA

$\begin{array}{llrlrllll}1 & \text { AM241 } & 0.800 & 0.090 & 0.766 & 0.013 & 1.040 & 0.12 & \text { A } \\ 1 & \text { CD 60 } & 35.600 & 0.500 & 32.800 & 0.580 & 1.090 & 0.02 & \text { A } \\ 1 & \text { CS137 } & 42.800 & 1.200 & 38.300 & 0.881 & 1.120 & 0.04 & \text { A } \\ 1 & \text { MN 54 } & 43.700 & 0.800 & 38.400 & 1.160 & 1.140 & 0.04 & \text { W } \\ 1 & \text { PU238 } & 0.960 & 0.110 & 0.982 & 0.074 & 0.980 & 0.13 & \text { A } \\ 1 & \text { PU239 } & 0.780 & 0.090 & 0.772 & 0.056 & 1.010 & 0.14 & A \\ 1 & \text { SR 90 } & 2.000 & 0.200 & 1.450 & 0.034 & 1.380 & 0.14 & \text { W }\end{array}$

Units for matrices: $A|=B q|$ filter $S O=B q / k g$ VE=Bq $/ \mathrm{kg} W A=B q \mid L$. Values for elemental uranium are reported in $\mu g / f i l t e r, g$, or $\mathrm{mL}$. 


\section{QAP44 Results by Laboratory}

Lab: IR Idaho National Engineering Laboratory

\begin{tabular}{|c|c|c|c|c|c|c|c|c|}
\hline $\begin{array}{l}\text { No. } \\
\text { Test }\end{array}$ & $\begin{array}{l}\text { Radio: } \\
\text { nuclide }\end{array}$ & $\begin{array}{l}\text { Reported } \\
\text { Value }\end{array}$ & $\begin{array}{l}\text { Reported } \\
\text { Error }\end{array}$ & $\begin{array}{c}\text { EML } \\
\text { Value }\end{array}$ & $\begin{array}{l}\text { EML } \\
\text { Error }\end{array}$ & $\frac{\text { Reported }}{\text { EML }}$ & $\begin{array}{l}\text { Ratio } \\
\text { Error }\end{array}$ & Evaluation \\
\hline
\end{tabular}

Matrix: Al

1 UUG

4.270

0.470

4.310

0.100

0.990

0.11

A

Matrix: WA

$1 \quad$ UUG

0.035

0.001

0.022

0.003

1.580

0.19

N

Units for matrices: $A \mathrm{~A}=\mathrm{Bq} /$ filter $\mathrm{SO}=\mathrm{Bq} / \mathrm{kg}$ VE=Bq/kg $W A=B q / L$. Values for elemental uranium are reported in $\mu \mathrm{g} /$ filter, $\mathrm{g}$, or $\mathrm{mL}$. 
QAP44 Results by Laboratory

Lab: IS Quanterra-St. Louis

\begin{tabular}{lllllllll} 
No. & $\begin{array}{l}\text { Radio- } \\
\text { nuclide }\end{array}$ & $\begin{array}{l}\text { Reported } \\
\text { Value }\end{array}$ & $\begin{array}{c}\text { Reported } \\
\text { Error }\end{array}$ & $\begin{array}{c}\text { EML } \\
\text { Value }\end{array}$ & $\begin{array}{c}\text { EML } \\
\text { Error }\end{array}$ & $\frac{\text { Reported }}{\text { EML }}$ & $\begin{array}{l}\text { Ratio } \\
\text { Error }\end{array}$ & Evaluation \\
\hline
\end{tabular}

Matrix: Al

$\begin{array}{llrlrllll}1 & \text { AM241 } & 0.156 & 0.029 & 0.189 & 0.007 & 0.830 & 0.16 & \text { A } \\ 1 & \text { CE144 } & 22.600 & 3.700 & 33.300 & 3.300 & 0.680 & 0.13 & \text { W } \\ 1 & \text { CO 57 } & 6.350 & 0.530 & 8.900 & 0.900 & 0.710 & 0.09 & \text { W } \\ 1 & \text { CO 60 } & 2.500 & 0.120 & 29.500 & 2.900 & 0.090 & 0.01 & \text { N } \\ 1 & \text { CS134 } & 12.300 & 0.800 & 14.700 & 1.460 & 0.840 & 0.10 & \text { A } \\ 1 & \text { CS137 } & 5.510 & 0.770 & 6.640 & 0.700 & 0.830 & 0.15 & \text { W } \\ 1 & \text { GA 1 } & 2.490 & 0.250 & 1.620 & 0.150 & 1.540 & 0.21 & \text { W } \\ 1 & \text { GB 2 } & 1.580 & 0.160 & 1.770 & 0.150 & 0.890 & 0.12 & \text { A } \\ 1 & \text { MN 54 } & 2.470 & 0.620 & 3.440 & 0.380 & 0.720 & 0.20 & \mathbf{N} \\ 1 & \text { PU238 } & 0.102 & 0.029 & 0.096 & 0.002 & 1.060 & 0.30 & \text { A } \\ 1 & \text { PU239 } & 0.108 & 0.030 & 0.093 & 0.003 & 1.170 & 0.33 & \mathbf{W} \\ 1 & \text { RU106 } & 14.700 & 5.100 & 11.600 & 1.440 & 1.270 & 0.47 & \mathbf{W} \\ 1 & \text { SB125 } & 8.640 & 1.130 & 9.780 & 1.030 & 0.880 & 0.15 & \mathbf{A} \\ 1 & \text { SR 90 } & 1.850 & 0.320 & 1.060 & 0.037 & 1.750 & 0.31 & \mathbf{W}\end{array}$

Matrix: So

$\begin{array}{llr}1 & \text { AM241 } & 2.860 \\ 1 & \text { CS137 } & 335.000 \\ 1 & \text { K } 40 & 450.000 \\ 1 & \text { PU238 } & 28.500 \\ 1 & \text { PU239 } & 7.670 \\ 1 & \text { SR 90 } & 660.000 \\ 1 & \text { U 234 } & 33.100 \\ 1 & \text { U 238 } & 38.700 \\ 1 & \text { U UG } & 3.140\end{array}$

0.890
43.000
54.000
5.000
1.580
66.000
13.100
14.800
1.200

$\begin{array}{rr}3.690 & 0.454 \\ 359.000 & 10.000 \\ 465.000 & 30.000 \\ 43.000 & 2.440 \\ 9.230 & 0.346 \\ 1340.000 & 113.000 \\ 34.200 & 3.520 \\ 35.900 & 3.630 \\ 2.900 & 0.290\end{array}$

0.780
0.930
0.970
0.660
0.830
0.490
0.970
1.080
1.080

0.26

0.12

0.13

0.12

0.17

0.06

0.40

0.43

0.43

$A$
$W$
$W$
$\mathbf{N}$
$\mathbf{A}$
$\mathbf{W}$
$\mathbf{W}$
$\mathbf{A}$
$\mathbf{N}$
$\mathbf{A}$
$\mathbf{W}$
$\mathbf{W}$
$\mathbf{A}$
$\mathbf{W}$

Matrix: VE

$\begin{array}{llr}1 & \text { AM241 } & 7.090 \\ 1 & \text { CO 60 } & 59.800 \\ 1 & \text { CS137 } & 1020.000 \\ 1 & \text { K 40 } & 1160.000 \\ 1 & \text { PU239 } & 9.540 \\ 1 & \text { SR 90 } & 1130.000\end{array}$

1.290
5.200
116.000
128.000
2.860
113.000

$\begin{array}{rr}5.600 & 0.184 \\ 59.700 & 0.963 \\ 944.000 & 16.200 \\ 1030.000 & 33.000 \\ 9.820 & 1.220 \\ 1300.000 & 52.400\end{array}$

1.270
1.000
1.080
1.130
0.970
0.870

0.23
0.09
0.12
0.13
0.32
0.09

A
A
A
A
A
A

Matrix: WA

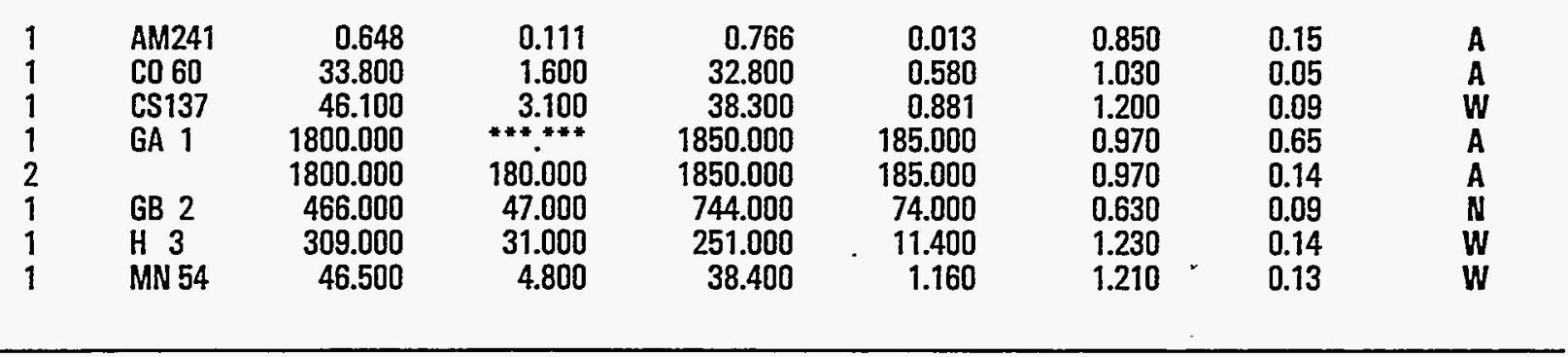

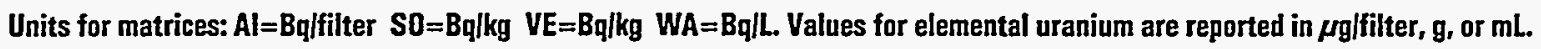

Evaluation: $A=A c c e p t a b l e, W=A c c e p t a b l e$ with Warning, $N=$ Not Acceptable

$\mathrm{pCi}=\mathrm{Bq} \times 27$ 
QAP44 Results by Laboratory

Lab: IS Quanterra-St. Louis

\begin{tabular}{lllllllll} 
No. & Radio- & Reported & Reported & & EML & EML & Reported & Ratio \\
Test & nuclide & Value & Error & Value & Error & EML & Error & Evaluation \\
\hline
\end{tabular}

Matrix: WA

$\begin{array}{lllllllll}1 & \text { PU238 } & 0.936 & 0.193 & 0.982 & 0.074 & 0.950 & 0.21 & \text { A } \\ 1 & \text { PU239 } & 0.822 . & 0.177 & 0.772 & 0.056 & 1.070 & 0.24 & \text { A } \\ 1 & \text { SR 90 } & 1.540 & 0.000 & 1.450 & 0.034 & 1.060 & 0.03 & \text { A } \\ 2 & & 1.540 & 0.520 & 1.450 & 0.034 & 1.060 & 0.36 & \text { A }\end{array}$

Units for matrices: $A l=B q / f i l t e r ~ S O=B q / k g$ VE=Bqlkg $W A=B q / L$. Values for elemental uranium are reported in $\mu g / f i l t e r, g$, or $\mathrm{mL}$.

$\mathrm{pCi}=\mathrm{Bq} \times 27$ 


\section{QAP44 Results by Laboratory}

Lab: IT Quanterra-Richland Laboratory

\begin{tabular}{lllllllll} 
No. & $\begin{array}{l}\text { Radio- } \\
\text { nuclide }\end{array}$ & $\begin{array}{l}\text { Reported } \\
\text { Value }\end{array}$ & $\begin{array}{l}\text { Reported } \\
\text { Error }\end{array}$ & $\begin{array}{c}\text { EML } \\
\text { Value }\end{array}$ & $\begin{array}{c}\text { EML } \\
\text { Error }\end{array}$ & $\begin{array}{c}\text { Reported } \\
\text { EML }\end{array}$ & $\begin{array}{l}\text { Ratio } \\
\text { Error }\end{array}$ & Evaluation \\
\hline
\end{tabular}

Matrix: Al

\begin{tabular}{|c|c|c|c|c|c|c|}
\hline $\begin{array}{l}\text { AM241 } \\
\text { CE144 } \\
\text { CO 57 } \\
\text { CO 60 } \\
\text { CS134 } \\
\text { CS137 } \\
\text { GA 1 } \\
\text { GB 2 } \\
\text { MN 54 } \\
\text { PU238 } \\
\text { PU239 } \\
\text { RU106 } \\
\text { SB125 } \\
\text { SR 90 } \\
\text { U UG }\end{array}$ & $\begin{array}{r}0.173 \\
20.000 \\
5.840 \\
22.900 \\
11.400 \\
5.050 \\
1.860 \\
2.990 \\
2.600 \\
0.091 \\
0.095 \\
9.650 \\
8.150 \\
1.060 \\
4.690\end{array}$ & $\begin{array}{l}0.006 \\
0.530 \\
0.340 \\
0.540 \\
0.380 \\
0.180 \\
0.030 \\
0.073 \\
0.230 \\
0.006 \\
0.006 \\
0.800 \\
0.250 \\
0.060 \\
0.640\end{array}$ & $\begin{array}{r}0.189 \\
33.300 \\
8.900 \\
29.500 \\
14.700 \\
6.640 \\
1.620 \\
1.770 \\
3.440 \\
0.096 \\
0.093 \\
11.600 \\
9.780 \\
1.060 \\
4.310\end{array}$ & $\begin{array}{l}0.007 \\
3.300 \\
0.900 \\
2.900 \\
1.460 \\
0.700 \\
0.150 \\
0.150 \\
0.380 \\
0.002 \\
0.003 \\
1.440 \\
1.030 \\
0.037 \\
0.100\end{array}$ & $\begin{array}{l}0.920 \\
0.600 \\
0.660 \\
0.780 \\
0.780 \\
0.760 \\
1.150 \\
1.690 \\
0.760 \\
0.950 \\
1.030 \\
0.830 \\
0.830 \\
1.000 \\
1.090\end{array}$ & $\begin{array}{l}0.04 \\
0.06 \\
0.08 \\
0.08 \\
0.08 \\
0.08 \\
0.11 \\
0.15 \\
0.11 \\
0.07 \\
0.07 \\
0.12 \\
0.09 \\
0.07 \\
0.15\end{array}$ \\
\hline
\end{tabular}

Matrix: So

$\begin{array}{llrrrrrrr}1 & \text { AM241 } & 3.430 & 0.830 & 3.690 & 0.454 & 0.930 & 0.25 & \text { A } \\ 1 & \text { CS137 } & 432.000 & 12.000 & 359.000 & 10.000 & 1.200 & 0.05 & \mathbf{A} \\ 1 & \text { K 40 } & 541.000 & 24.000 & 465.000 & 30.000 & 1.160 & 0.09 & \mathbf{A} \\ 1 & \text { PU238 } & 30.900 & 3.950 & 43.000 & 2.440 & 0.720 & 0.10 & \mathbf{A} \\ 1 & \text { PU239 } & 6.470 & 0.610 & 9.230 & 0.346 & 0.700 & 0.07 & \mathbf{W} \\ 1 & \text { SR 90 } & 1100.000 & 111.000 & 1340.000 & 113.000 & 0.820 & 0.11 & \mathbf{A} \\ 1 & \text { U UG } & 2.250 & 0.060 & 2.900 & 0.290 & 0.780 & 0.08 & \mathbf{A}\end{array}$

Matrix: VE

$\begin{array}{llrrrrrrr}1 & \text { AM241 } & 6.160 & 0.225 & 5.600 & 0.184 & 1.100 & 0.05 & \text { A } \\ 1 & \text { CM244 } & 4.490 & 0.036 & 4.440 & 0.202 & 1.010 & 0.05 & \mathbf{A} \\ 1 & \text { CO 60 } & 72.800 & 1.370 & 59.700 & 0.963 & 1.220 & 0.03 & \text { A } \\ 1 & \text { CS137 } & 1250.000 & 47.200 & 944.000 & 16.200 & 1.320 & 0.05 & \mathbf{W} \\ 1 & \text { K 40 } & 1360.000 & 24.000 & 1030.000 & 33.000 & 1.320 & 0.05 & \mathbf{W} \\ 1 & \text { PU239 } & 8.990 & 0.590 & 9.820 & 1.220 & 0.920 & 0.13 & \mathbf{A} \\ 1 & \text { SR 90 } & 1550.000 & 18.800 & 1300.000 & 52.400 & 1.190 & 0.05 & \mathbf{W}\end{array}$

Matrix: WA

$\begin{array}{lrrrrrrr}\text { AM241 } & 0.840 & 0.090 & 0.766 & 0.013 & 1.100 & 0.12 & \text { A } \\ \text { CO 60 } & 37.000 & 3.000 & 32.800 & 0.580 & 1.130 & 0.09 & \text { W } \\ \text { CS137 } & 46.200 & 4.100 & 38.300 & 0.881 & 1.210 & 0.11 & \text { W } \\ \text { GA 1 } & 1680.000 & 217.000 & 1850.000 & 185.000 & 0.910 & 0.15 & \text { A } \\ \text { GB 2 } & 886.000 & 109.000 & 744.000 & 74.000 & 1.190 & 0.19 & \text { A } \\ \text { H 3 } & 209.000 & 6.100 & 251.000 & 11.400 & 0.830 & 0.05 & \text { W } \\ \text { MN 54 } & 46.500 & 5.000 & 38.400 & 1.160 & 1.210 & 0.14 & \text { W } \\ \text { PU238 } & 1.000 & 0.020 & 0.982 & 0.074 & 1.020 & 0.08 & \text { A }\end{array}$

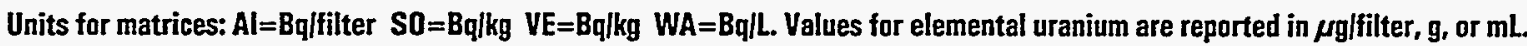

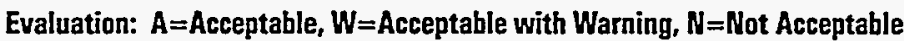

$\mathrm{pCi}=\mathrm{Bq} \times 27$ 


\section{QAP44 Results by Laboratory}

Lab: IT Quanterra- Richland Laboratory

\begin{tabular}{llllllll} 
No. & $\begin{array}{llll}\text { Radio- } \\
\text { Test }\end{array}$ & $\begin{array}{l}\text { Reported } \\
\text { nuclide }\end{array}$ & $\begin{array}{l}\text { Reported } \\
\text { Ealue }\end{array}$ & $\begin{array}{l}\text { EML } \\
\text { Eluror }\end{array}$ & $\begin{array}{l}\text { EML } \\
\text { Error }\end{array}$ & $\begin{array}{l}\text { Reported } \\
\text { EML }\end{array}$ & $\begin{array}{l}\text { Ratio } \\
\text { Error }\end{array}$ \\
\hline
\end{tabular}

Matrix: WA

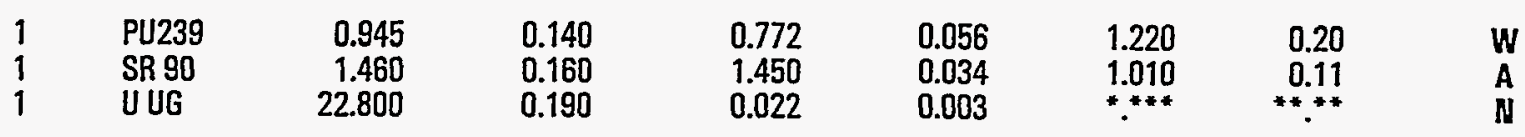

Units for matrices: $A I=B q / f i l t e r ~ S O=B q / k g$ VE=Bq/kg $W A=B q / L$. Values for elemental uranium are reported in $\mu g / f i l t e r, g$, or $\mathrm{mL}$. 


\section{QAP44 Results by Laboratory}

Lab: JP Japan Chemical Analysis Center

\begin{tabular}{|c|c|c|c|c|c|c|c|c|}
\hline $\begin{array}{l}\text { No. } \\
\text { Test }\end{array}$ & $\begin{array}{l}\text { Radio- } \\
\text { nuclide }\end{array}$ & $\begin{array}{l}\text { Reported } \\
\text { Value }\end{array}$ & $\begin{array}{l}\text { Reported } \\
\text { Error }\end{array}$ & $\begin{array}{c}\text { EML } \\
\text { Value }\end{array}$ & $\begin{array}{l}\text { EM } \\
\text { Errc }\end{array}$ & $\frac{\text { Report }}{\text { EML }}$ & $\begin{array}{l}\text { Rati } \\
\text { Erro }\end{array}$ & Evaluation \\
\hline
\end{tabular}

Matrix: Al

$\begin{array}{llrlrllll}1 & \text { AM241 } & 0.180 & 0.005 & 0.189 & 0.007 & 0.950 & 0.04 & \text { A } \\ 1 & \text { CE144 } & 23.000 & 0.200 & 33.300 & 3.300 & 0.690 & 0.07 & \text { A } \\ 1 & \text { CO 57 } & 7.100 & 0.040 & 8.900 & 0.900 & 0.800 & 0.08 & \text { A } \\ 1 & \text { CO 60 } & 24.000 & 0.200 & 29.500 & 2.900 & 0.810 & 0.08 & \text { W } \\ 1 & \text { CS134 } & 13.000 & 0.100 & 14.700 & 1.460 & 0.880 & 0.09 & \text { A } \\ 1 & \text { CS137 } & 5.700 & 0.070 & 6.640 & 0.700 & 0.860 & 0.09 & \text { A } \\ 1 & \text { GA 1 } & 2.100 & 0.020 & 1.620 & 0.150 & 1.300 & 0.12 & \text { A } \\ 1 & \text { MN 54 } & 3.100 & 0.110 & 3.440 & 0.380 & 0.900 & 0.11 & A \\ 1 & \text { PU238 } & 0.088 & 0.004 & 0.096 & 0.002 & 0.920 & 0.04 & A \\ 1 & \text { PU239 } & 0.091 & 0.004 & 0.093 & 0.003 & 0.980 & 0.05 & \text { A } \\ 1 & \text { RU106 } & 10.000 & 0.400 & 11.600 & 1.440 & 0.860 & 0.11 & \text { A } \\ 1 & \text { SB125 } & 9.200 & 0.150 & 9.780 & 1.030 & 0.940 & 0.10 & \text { A } \\ 1 & \text { SR 90 } & 0.670 & 0.029 & 1.060 & 0.037 & 0.630 & 0.04 & \text { W } \\ 1 & \text { U 234 } & 0.051 & 0.003 & 0.052 & 0.002 & 0.990 & 0.06 & A \\ 1 & \text { U 238 } & 0.053 & 0.003 & 0.053 & 0.002 & 0.990 & 0.07 & \mathbf{A}\end{array}$

Matrix: So

$\begin{array}{llrrrrrrr}1 & \text { AM241 } & 3.700 & 0.230 & 3.690 & 0.454 & 1.000 & 0.14 & \text { A } \\ 1 & \text { CS137 } & 390.000 & 3.000 & 359.000 & 10.000 & 1.090 & 0.03 & \text { A } \\ 1 & \text { K 40 } & 480.000 & 12.000 & 465.000 & 30.000 & 1.030 & 0.07 & \text { A } \\ 1 & \text { PU238 } & 45.000 & 1.500 & 43.000 & 2.440 & 1.050 & 0.07 & \text { A } \\ 1 & \text { PU239 } & 9.300 & 0.340 & 9.230 & 0.346 & 1.010 & 0.05 & \text { A } \\ 1 & \text { SR 90 } & 1200.000 & 10.000 & 1340.000 & 113.000 & 0.900 & 0.08 & \text { A } \\ 1 & \text { U 234 } & 33.000 & 1.400 & 34.200 & 3.520 & 0.970 & 0.11 & \text { A } \\ 1 & \text { U 238 } & 33.000 & 1.400 & 35.900 & 3.630 & 0.920 & 0.10 & \text { A }\end{array}$

Matrix: VE

$\begin{array}{llrrrrrrr}1 & \text { AM241 } & 6.400 & 0.150 & 5.600 & 0.184 & 1.140 & 0.05 & \text { A } \\ 1 & \text { CM244 } & 5.000 & 0.120 & 4.440 & 0.202 & 1.130 & 0.06 & \text { A } \\ 1 & \text { CO 60 } & 62.000 & 1.400 & 59.700 & 0.963 & 1.040 & 0.03 & \text { A } \\ 1 & \text { CS137 } & 1100.000 & 6.000 & 944.000 & 16.200 & 1.170 & 0.02 & \text { A } \\ 1 & \text { K 40 } & 1100.000 & 30.000 & 1030.000 & 33.000 & 1.070 & 0.04 & \text { A } \\ 1 & \text { PU239 } & 9.600 & 0.530 & 9.820 & 1.220 & 0.980 & 0.13 & \text { A } \\ 1 & \text { SR 90 } & 1400.000 & 10.000 & 1300.000 & 52.400 & 1.080 & 0.04 & \text { A }\end{array}$

Matrix: WA

$\begin{array}{llrrrrrrr}1 & \text { AM241 } & 0.840 & 0.035 & 0.766 & 0.013 & 1.100 & 0.05 & \text { A } \\ 1 & \text { CO 60 } & 33.000 & 0.800 & 32.800 & 0.580 & 1.010 & 0.03 & \text { A } \\ 1 & \text { CS137 } & 43.000 & 1.200 & 38.300 & 0.881 & 1.120 & 0.04 & \text { A } \\ 1 & \text { GA 1 } & 1500.000 & 10.000 & 1850.000 & 185.000 & 0.810 & 0.08 & \text { A } \\ 1 & \text { GB 2 } & 850.000 & 20.000 & 744.000 & 74.000 & 1.140 & 0.12 & \text { A } \\ 1 & \text { H 3 } & 220.000 & 1.000 & 251.000 & 11.400 & 0.880 & 0.04 & \text { A } \\ 1 & \text { MN 54 } & 42.000 & 1.600 & 38.400 & 1.160 & 1.090 & 0.05 & \text { A }\end{array}$

Units for matrices: $A \mathrm{~A}=\mathrm{Bq} /$ filter $\mathrm{SO}=\mathrm{Bq} \mid \mathrm{kg}$ VE=Bq/kg WA=Bq/L. Values for elemental uranium are reported in $\mu \mathrm{g} /$ filter, $\mathrm{g}$, or $\mathrm{mL}$.

Evaluation: $A=A c c e p t a h l e, W=A c c e p t a b l e$ with Warning, $N=$ Not Acceptable $\mathrm{pCi}=\mathrm{Bq} \times 27$ 


\section{QAP44 Results by Laboratory}

Lab: JP Japan Chemical Analysis Center

\begin{tabular}{|c|c|c|c|c|c|c|c|c|}
\hline $\begin{array}{l}\text { No. } \\
\text { Test }\end{array}$ & $\begin{array}{l}\text { Radio- } \\
\text { nuclide }\end{array}$ & $\begin{array}{l}\text { Reported } \\
\text { Value }\end{array}$ & $\begin{array}{l}\text { Reported } \\
\text { Error }\end{array}$ & $\begin{array}{c}\text { EML } \\
\text { Value }\end{array}$ & $\begin{array}{l}\text { EML } \\
\text { Error }\end{array}$ & $\frac{\text { Reported }}{\text { EML }}$ & $\begin{array}{l}\text { Ratio } \\
\text { Error }\end{array}$ & Evaluation \\
\hline
\end{tabular}

Matrix: WA

$\begin{array}{lllllllll}1 & \text { PU238 } & 1.100 & 0.060 & 0.982 & 0.074 & 1.120 & 0.10 & \text { A } \\ 1 & \text { PU239 } & 0.840 & 0.045 & 0.772 & 0.056 & 1.090 & 0.10 & \text { A } \\ 1 & \text { SR 90 } & 1.600 & 0.140 & 1.450 & 0.034 & 1.100 & 0.10 & \text { A } \\ 1 & \text { U 234 } & 0.310 & 0.015 & 0.274 & 0.019 & 1.130 & 0.10 & \text { A } \\ 1 & \text { U 238 } & 0.310 & 0.015 & 0.275 & 0.024 & 1.130 & 0.11 & \text { A }\end{array}$

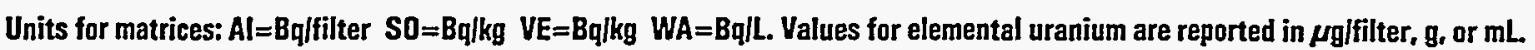

$\mathrm{pCi}=\mathrm{Bq} \times 27$ 


\section{QAP44 Results by Laboratory}

Lab: KA Knolls Atomic Power Lab, Schenectady

No.
Test

Radio- Reported Reported EML nuclide Value

Error

Value

EML
Error

Reporte EML

Ratio Error Evaluation

Matrix: AI

$1 \quad G A$

GA 1

2.420

0.090

1.620

1.770

0.150

1.490

0.150

1.110

0.15

0.10

W

Matrix: $\quad$ so

$\begin{array}{ll}1 & \text { CS137 } \\ 1 & \text { K 40 } \\ 1 & \text { PU238 } \\ 1 & \text { PU239 } \\ 1 & \text { SR 90 }\end{array}$

434.000

20.000

498.000

45.000

359.000

47.000

5.000

465.000

10.100

0.500

43.000

9.230

1450.000

147.000

1340.000

$\begin{array}{rr}10.000 & 1.210 \\ 30.000 & 1.070 \\ 2.440 & 1.090 \\ 0.346 & 1.090 \\ 113.000 & 1.080\end{array}$

0.07

0.12

0.13

0.07

0.14

Matrix: WA

$\begin{array}{llr}1 & \text { CO 60 } & 33.000 \\ 1 & \text { CS137 } & 42.000 \\ 1 & \text { FE 55 } & 108.000 \\ 1 & \text { GA 1 } & 2040.000 \\ 1 & \text { GB 2 } & 912.000 \\ 1 & \text { H 3 } & 239.000 \\ 1 & \text { MN 54 } & 41.000 \\ 1 & \text { PU238 } & 1.010 \\ 1 & \text { PU239 } & 0.753 \\ 1 & \text { SR 90 } & 1.460 \\ 1 & \text { U UG } & 0.023\end{array}$

1.000
2.000
6.000
167.000
74.000
13.000
2.000
0.010
0.025
0.240
0.001

32.800
38.300
83.000
1850.000
744.000
251.000
38.400
0.982
0.772
1.450
0.022

0.580
0.881
3.440
185.000
74.000
11.400
1.160
0.074
0.056
0.034
0.003

1.010

1.100

1.300

1.100

1.230

0.950

1.070

1.030

0.980

1.010

1.020

$\begin{array}{ll}0.04 & \text { A } \\ 0.06 & \text { A } \\ 0.09 & \text { W } \\ 0.14 & \text { A } \\ 0.16 & \text { A } \\ 0.07 & \text { A } \\ 0.06 & \text { A } \\ 0.08 & \text { A } \\ 0.08 & \text { A } \\ 0.17 & \text { A } \\ 0.12 & \text { A }\end{array}$




\section{QAP44 Results by Laboratory}

Lab: LA Los Alamos National Laboratory, NM

\begin{tabular}{llllllll}
$\begin{array}{l}\text { No. } \\
\text { Test }\end{array}$ & $\begin{array}{l}\text { Radio- } \\
\text { nuclide }\end{array}$ & $\begin{array}{l}\text { Reported } \\
\text { Value }\end{array}$ & $\begin{array}{l}\text { Reported } \\
\text { Error }\end{array}$ & $\begin{array}{c}\text { EML } \\
\text { Value }\end{array}$ & $\begin{array}{c}\text { EML } \\
\text { Error }\end{array}$ & $\begin{array}{l}\text { Reported } \\
\text { EML }\end{array}$ & $\begin{array}{l}\text { Ratio } \\
\text { Error }\end{array}$ \\
\hline
\end{tabular}

Matrix: Al

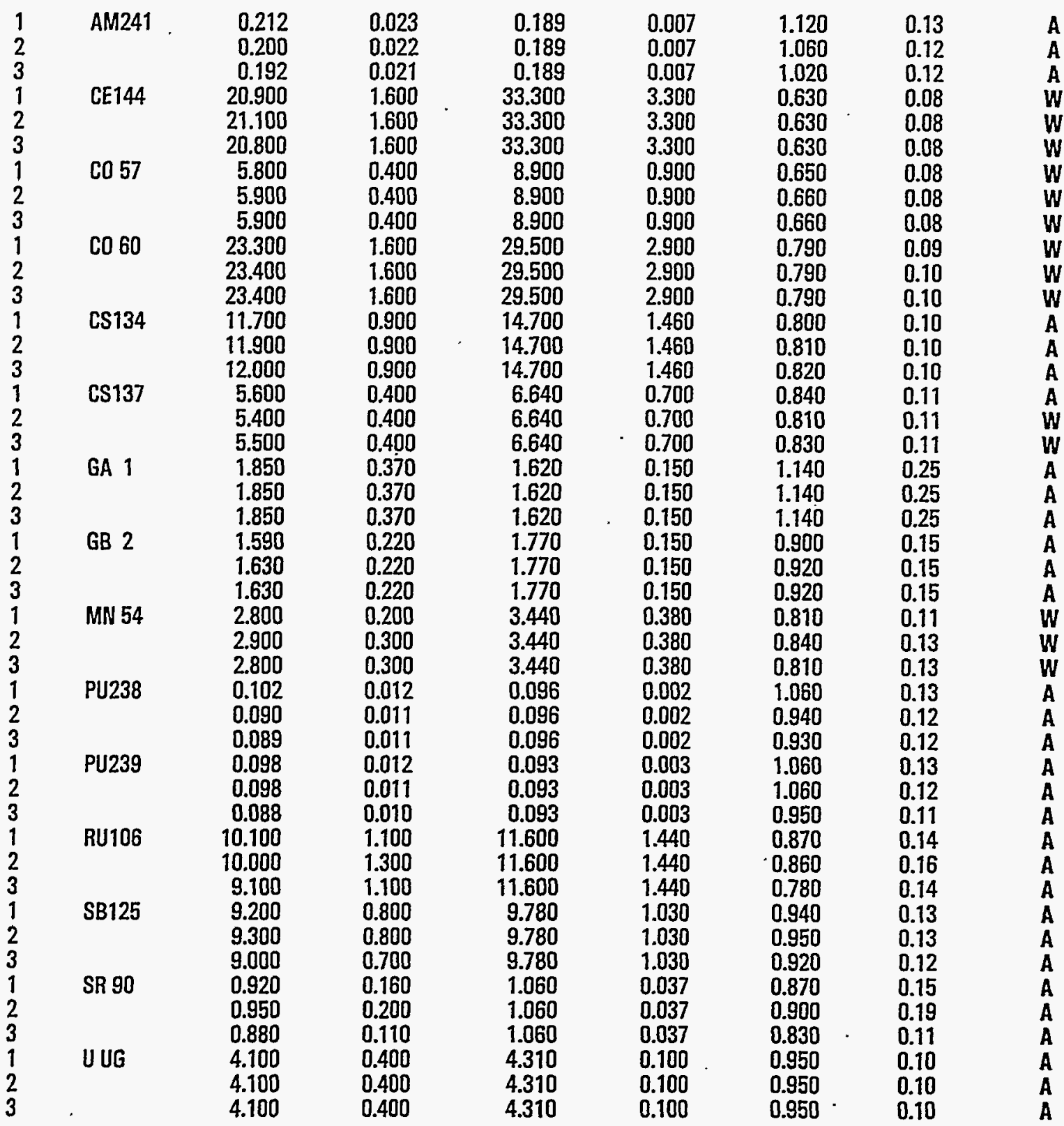

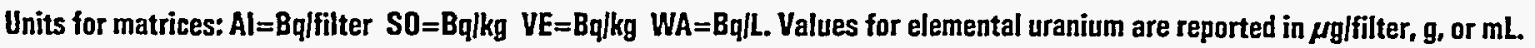

Evaluation: $A=$ Acceptable, $W=A c c e p t a b l e$ with Warning, $N=$ Not Acceptable

$\mathrm{pCi}=\mathrm{Bq} \times 27$ 


\section{QAP44 Results by Laboratory}

Lab: LA Los Alamos National Laboratory, NM

\begin{tabular}{lllllllll} 
No. & $\begin{array}{l}\text { Radio- } \\
\text { nuclide }\end{array}$ & $\begin{array}{l}\text { Reported } \\
\text { Value }\end{array}$ & $\begin{array}{c}\text { Reported } \\
\text { Error }\end{array}$ & $\begin{array}{c}\text { EML } \\
\text { Value }\end{array}$ & $\begin{array}{c}\text { EML } \\
\text { Error }\end{array}$ & $\frac{\text { Reported }}{\text { EML }}$ & $\begin{array}{l}\text { Ratio } \\
\text { Error }\end{array}$ & Evaluation \\
\hline
\end{tabular}

Matrix: $\quad$ So

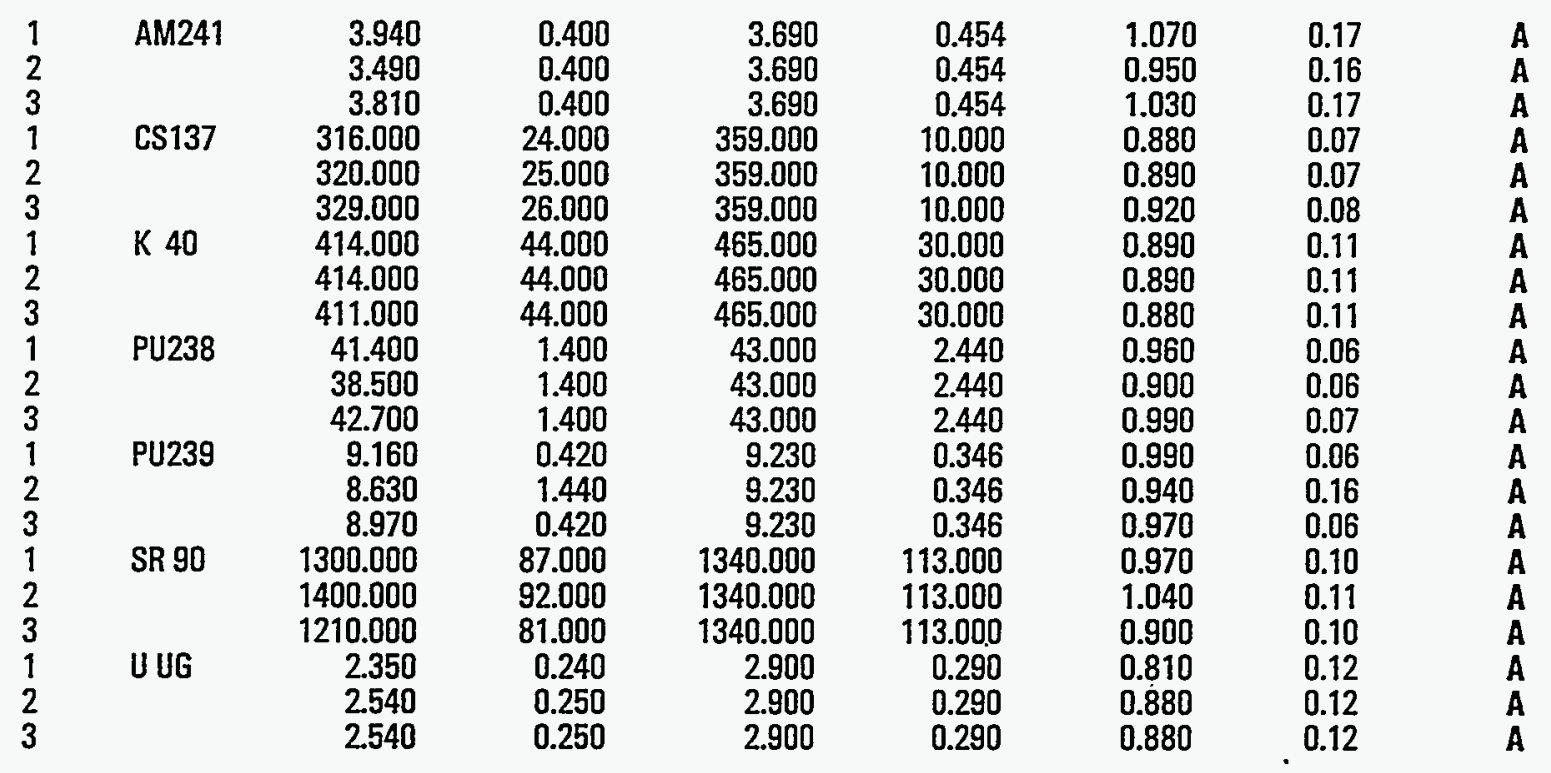

Matrix: VE

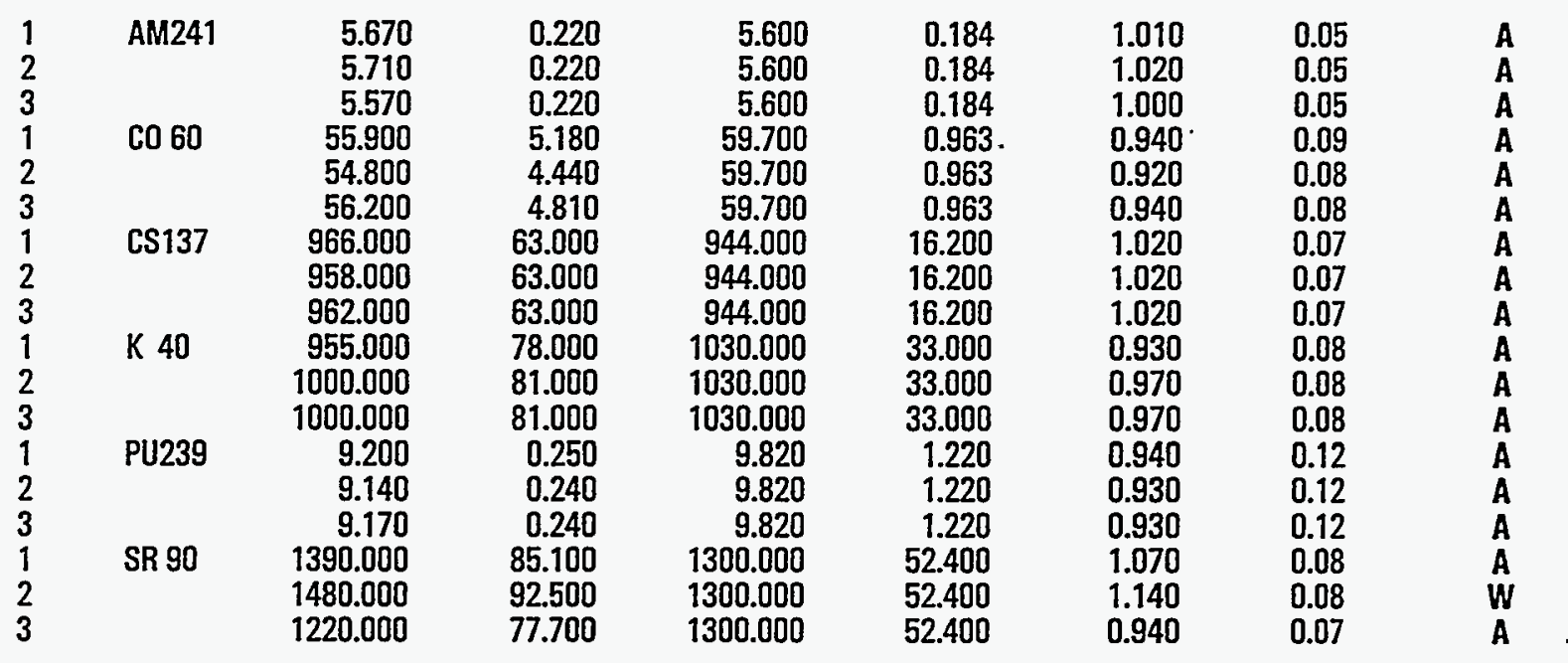

Matrix: WA

$1 \quad$ AM241

0.810

0.180

0.766

0.013

1.060

0.24

A

Units for matrices: $A|=B q|$ filter $S O=B q / k g ~ V E=B q / k g ~ W A=B q \mid L$. Values for elemental uranium are reported in $\mu g / f i l t e r, g$, or ml.

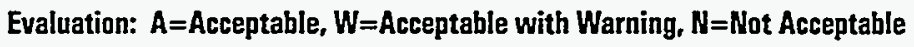
$p C i=B q \times 27$ 


\section{OAP44 Results by Laboratory}

Lab: LA Los Alamos National Laboratory, NM

\begin{tabular}{llllllll} 
No. & $\begin{array}{l}\text { Radio- } \\
\text { nuclide }\end{array}$ & $\begin{array}{l}\text { Reported } \\
\text { Value }\end{array}$ & $\begin{array}{l}\text { Reported } \\
\text { Error }\end{array}$ & $\begin{array}{c}\text { EML } \\
\text { Value }\end{array}$ & $\begin{array}{c}\text { EML } \\
\text { Error }\end{array}$ & $\frac{\text { Reported }}{\text { EML }}$ & $\begin{array}{l}\text { Ratio } \\
\text { Error }\end{array}$ \\
\hline
\end{tabular}

Matrix: WA .

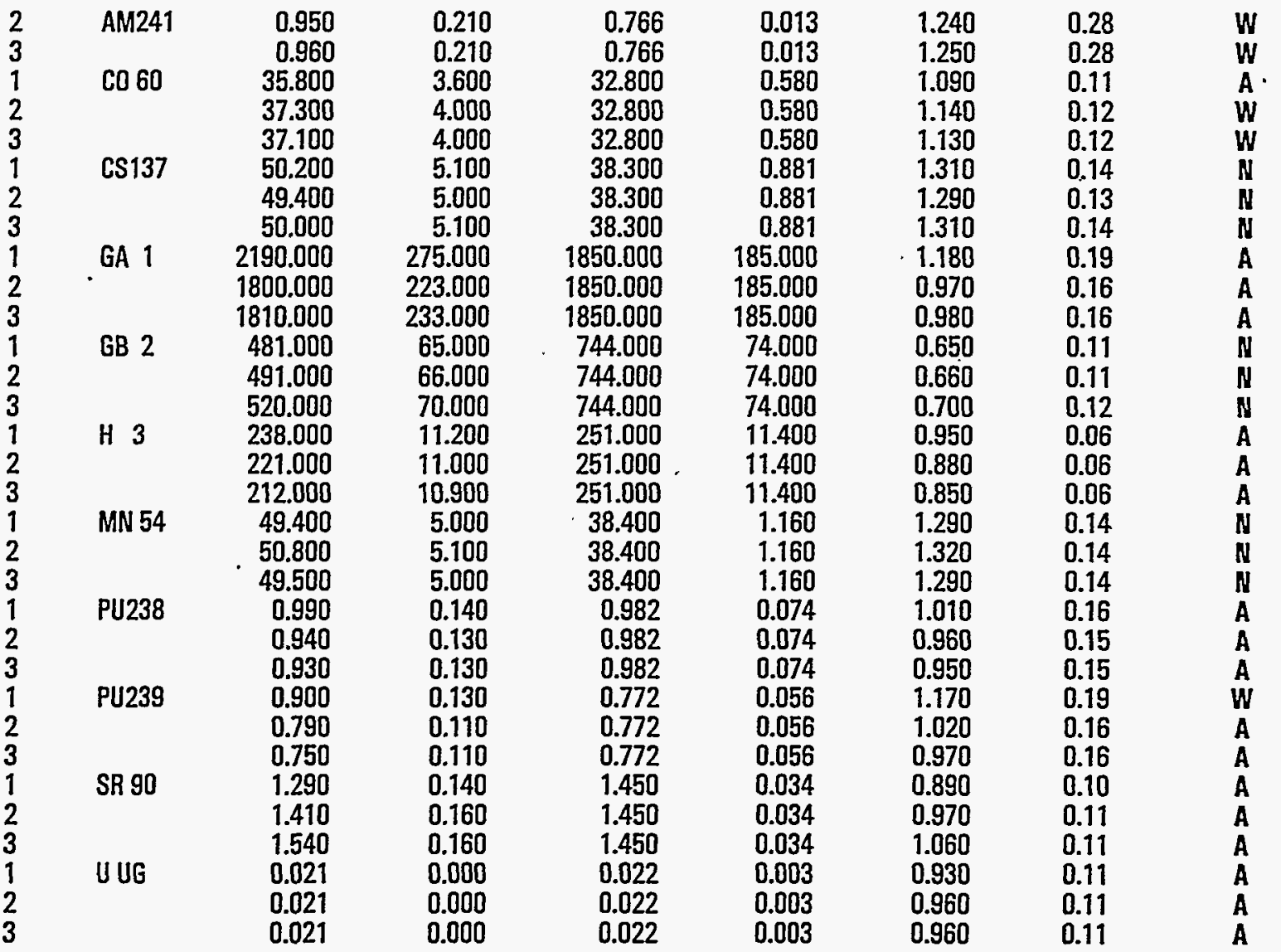




\section{QAP44 Results by Laboratory}

Lab: LB Lawrence Berkeley Lab UCB

Value Error Value Error EML Error Evaluation

Matrix: Al

$\begin{array}{lrrrrrrr}\text { AM241 } & 0.159 & 0.012 & 0.189 & 0.007 & 0.840 & 0.07 & \text { A } \\ \text { CE144 } & 28.500 & 2.310 & 33.300 & 3.300 & 0.860 & 0.11 & \text { A } \\ \text { CO 57 } & 6.630 & 0.186 & 8.900 & 0.900 & 0.750 & 0.08 & \text { A } \\ \text { CO 60 } & 25.700 & 6.160 & 29.500 & 2.900 & 0.870 & 0.23 & \text { A } \\ \text { CS134 } & 12.900 & 2.480 & 14.700 & 1.460 & 0.880 & 0.19 & \text { A } \\ \text { CS137 } & 5.280 & 0.940 & 6.640 & 0.700 & 0.800 & 0.17 & \text { W } \\ \text { GA 1 } & 1.590 & 0.160 & 1.620 & 0.150 & 0.980 & 0.13 & \text { A } \\ \text { GB 2 } & 1.480 & 0.150 & 1.770 & 0.150 & 0.840 & 0.11 & \text { W } \\ \text { MN 54 } & 2.840 & 0.550 & 3.440 & 0.380 & 0.830 & 0.18 & \text { W } \\ \text { SB125 } & 8.230 & 0.913 & 9.780 & 1.030 & 0.840 & 0.13 & \text { A }\end{array}$

Matrix: So

$\begin{array}{llr}1 & \text { AM241 } & 1.940 \\ 1 & \text { CS137 } & 332.00 \\ 1 & \text { K } 40 & 496.00\end{array}$

$\begin{array}{rr}0.868 & 3.690 \\ 13.700 & 359.000\end{array}$

0.454

10.000

0.530

33.400

465.000

30.000

1.070

0.24

0.05

0.10

W
A
A

Matrix: VE

$\begin{array}{rlr}1 & \text { AM241 } & 4.72 \\ 1 & \text { CO 60 } & 57.400 \\ 1 & \text { CS137 } & 80.90 \\ 1 & \text { K } 40 & 1120.00\end{array}$

1.880

5.600
59.700
944.000
1030.000

0.184

0.963

36.300

74.000

16.200

33.000

0.960

0.090

1.090

0.34

0.06

0.04

0.08

Matrix: WA

$\begin{array}{llr}1 & \text { AM241 } & 0.552 \\ 1 & \text { CO 60 } & 36.300 \\ 1 & \text { CS137 } & 46.200 \\ 1 & \text { MN 54 } & 47.500\end{array}$

0.552

0.215

23.100

0.766

32.800

0.013

0.580

0.720

38.300

0.881

1.160

1.110

1.210

25.200

38.400

1.240

0.28
0.71
0.58
0.66

$\mathbf{W}$
$\mathbf{A}$
$\mathbf{W}$
$\mathbf{N}$

Units for matrices: $A I=B q / f i l t e r ~ S O=B q / k g ~ V E=B q / k g ~ W A=B q / L$. Values for elemental uranium are reported in $\mu g / f i l f e r, g$, or $\mathrm{mL}$. Evaluation: $A=A c c e p t a b l e, W=A c c e p t a b l e$ with Warning, $N=$ Not Acceptable $\mathrm{pCi}=\mathrm{Bq} \times 27$ 
QAP44 Results by Laboratory

Lab: LH Lockheed Analytical Laboratory, Las Vegas

\begin{tabular}{|c|c|c|c|c|c|c|c|}
\hline $\begin{array}{l}\text { No. } \\
\text { Test }\end{array}$ & $\begin{array}{l}\text { Radio- } \\
\text { nuclide }\end{array}$ & $\begin{array}{l}\text { Reported } \\
\text { Value }\end{array}$ & $\begin{array}{l}\text { Reported } \\
\text { Error }\end{array}$ & $\begin{array}{c}\text { EML } \\
\text { Value }\end{array}$ & $\begin{array}{l}\text { EML } \\
\text { Error }\end{array}$ & $\frac{\text { Reported }}{\text { EML }}$ & $\begin{array}{l}\text { Ratio } \\
\text { Error }\end{array}$ \\
\hline
\end{tabular}

Matrix: Al

$\begin{array}{lrrrrrrr}\text { AM241 } & 0.140 & 0.020 & 0.189 & 0.007 & 0.740 & 0.11 & \text { W } \\ \text { CE144 } & 20.000 & 1.900 & 33.300 & 3.300 & 0.600 & 0.08 & \text { N } \\ \text { CD 57 } & 5.900 & 0.600 & 8.900 & 0.900 & 0.660 & 0.10 & \text { W } \\ \text { CD 60 } & 21.800 & 1.600 & 29.500 & 2.900 & 0.740 & 0.09 & \text { W } \\ \text { CS134 } & 10.700 & 0.800 & 14.700 & 1.460 & 0.730 & 0.09 & \text { W } \\ \text { CS137 } & 5.200 & 0.500 & 6.640 & 0.700 & 0.780 & 0.11 & \text { W } \\ \text { GA 1 } & 1.990 & 0.130 & 1.620 & 0.150 & 1.230 & 0.14 & \text { A } \\ \text { GB 2 } & 1.740 & 0.100 & 1.770 & 0.150 & 0.980 & 0.10 & \text { A } \\ \text { MN 54 } & 2.700 & 0.300 & 3.440 & 0.380 & 0.790 & 0.12 & \text { W } \\ \text { PU238 } & 0.100 & 0.020 & 0.096 & 0.002 & 1.040 & 0.21 & \text { A } \\ \text { PU239 } & 0.080 & 0.020 & 0.093 & 0.003 & 0.860 & 0.22 & \text { A } \\ \text { RU106 } & 8.100 & 1.200 & 11.600 & 1.440 & 0.700 & 0.14 & \text { A } \\ \text { SB125 } & 7.700 & 0.500 & 9.780 & 1.030 & 0.790 & 0.10 & \text { A } \\ \text { SR 90 } & 0.920 & 0.110 & 1.060 & 0.037 & 0.870 & 0.11 & \text { A } \\ \text { U 234 } & 0.095 & 0.025 & 0.052 & 0.002 & 1.840 & 0.49 & \text { W } \\ \text { U 238 } & 0.086 & 0.023 & 0.053 & 0.002 & 1.610 & 0.44 & \text { W }\end{array}$

Matrix: So

$\begin{array}{llrrrrrrr}1 & \text { AM241 } & 3.400 & 0.500 & 3.690 & 0.454 & 0.920 & 0.18 & \text { A } \\ 1 & \text { CS137 } & 419.000 & 60.000 & 359.000 & 10.000 & 1.170 & 0.17 & \text { A } \\ 1 & \text { K 40 } & 497.000 & 82.000 & 465.000 & 30.000 & 1.070 & 0.19 & \text { A } \\ 1 & \text { PU238 } & 42.900 & 3.300 & 43.000 & 2.440 & 1.000 & 0.10 & \text { A } \\ 1 & \text { PU239 } & 9.670 & 0.940 & 9.230 & 0.346 & 1.050 & 0.11 & \text { A } \\ 1 & \text { SR 90 } & 1140.000 & 86.000 & 1340.000 & 113.000 & 0.850 & 0.10 & \text { A } \\ 1 & \text { U 234 } & 33.800 & 5.400 & 34.200 & 3.520 & 0.990 & 0.19 & \text { A } \\ 1 & \text { U 238 } & 32.000 & 5.300 & 35.900 & 3.630 & 0.890 & 0.17 & \text { A }\end{array}$

Matrix: VE

$\begin{array}{llrrrrrrr}1 & \text { AM241 } & 5.230 & 0.440 & 5.600 & 0.184 & 0.930 & 0.08 & \text { A } \\ 1 & \text { CM244 } & 3.610 & 0.560 & 4.440 & 0.202 & 0.810 & 0.13 & \text { W } \\ 1 & \text { CO 60 } & 61.700 & 7.300 & 59.700 & 0.963 & 1.030 & 0.12 & \text { A } \\ 1 & \text { CS137 } & 1150.000 & 163.000 & 944.000 & 16.200 & 1.220 & 0.17 & \text { A } \\ 1 & \text { K 40 } & 1180.000 & 184.000 & 1030.000 & .33 .000 & 1.150 & 0.18 & \text { A } \\ 1 & \text { PU239 } & 9.160 & 0.870 & 9.820 & 1.220 & 0.930 & 0.15 & \text { A } \\ 1 & \text { SR 90 } & 1220.000 & 97.000 & 1300.000 & 52.400 & 0.940 & 0.08 & \text { A }\end{array}$

Matrix: WA

\begin{tabular}{rlrrrrrrr}
1 & AM241 & 0.790 & 0.140 & 0.766 & 0.013 & 1.030 & 0.18 & A \\
1 & CO 60 & 31.600 & 3.500 & 32.800 & 0.580 & 0.960 & 0.11 & A \\
1 & CS137 & 41.800 & 6.400 & 38.300 & 0.881 & 1.090 & 0.17 & A \\
1 & FE 55 & 68.900 & 12.400 & 83.000 & 3.440 & 0.830 & 0.15 & A \\
1 & GA 1 & 1980.000 & 175.000 & 1850.000 & 185.000 & 1.070 & 0.14 & A \\
1 & GB 2 & 539.000 & 63.000 & 744.000 & 74.000 & 0.720 & 0.11 & N \\
\hline
\end{tabular}

Units for matrices: $A I=B q / f i l t e r ~ S O=B q / k g ~ V E=B q / k g ~ W A=B q \mid L$. Values for elemental uranium are reported in $\mu g / f i l t e r, g$, or $\mathrm{mL}$.

Evaluation: $A=A c c e p t a b l e, W=A c c e p t a b l e$ with Warning, $N=$ Not Acceptahle

$\mathrm{pCi}=\mathrm{Bq} \times 27$ 


\section{QAP44 Results by Laboratory}

Lab: LH Lockheed Analytical Laboratory, Las Vegas

\begin{tabular}{lllllllll} 
No. & Radio- & Reported & Reported & EML & EML & Reported & Ratio \\
Test & nuclide & Value & Error & Value & Error & EML & Error & Evaluation \\
\hline
\end{tabular}

Matrix: WA

$\begin{array}{llrrrrrrr}1 & \text { H 3 } & 214.000 & 17.000 & 251.000 & 11.400 & 0.850 & 0.08 & \\ 1 & \text { MN 54 } & 41.500 & 6.400 & 38.400 & 1.160 & 1.080 & \mathbf{0 . 1 7} & \text { A } \\ 1 & \text { PU238 } & 0.930 & 0.120 & 0.982 & 0.074 & 0.950 & 0.14 & \text { A } \\ 1 & \text { PU239 } & 0.800 & 0.150 & 0.772 & 0.056 & 1.040 & 0.21 & \text { A } \\ 1 & \text { SR 90 } & 1.420 & 0.360 & 1.450 & 0.034 & 0.980 & 0.25 & \text { A } \\ 1 & \text { U 234 } & 0.320 & 0.080 & 0.274 & 0.019 & 1.170 & 0.30 & \text { A } \\ 1 & \text { U 238 } & 0.290 & 0.070 & 0.275 & 0.024 & 1.050 & 0.27 & \text { A }\end{array}$

Units for matrices: $A \mathrm{l}=\mathrm{Bq} / \mathrm{filter} \mathrm{SO}=\mathrm{Bq} / \mathrm{kg} \mathrm{VE}=\mathrm{Bq} / \mathrm{kg} W A=\mathrm{Bq} / \mathrm{L}$. Values for elemental uranium are reported in $\mu \mathrm{g} / \mathrm{filter}, \mathrm{g}$, or $\mathrm{mL}$. 


\section{QAP44 Results by Laboratory}

Lab: LL Lawrence Livermore National Lab, CA

\begin{tabular}{|c|c|c|c|c|}
\hline $\begin{array}{l}\text { Radio- } \\
\text { nuclide }\end{array}$ & $\begin{array}{l}\text { Reported } \\
\text { Value }\end{array}$ & $\begin{array}{l}\text { Reported } \\
\text { Error }\end{array}$ & $\begin{array}{c}\text { EML } \\
\text { Value }\end{array}$ & $\begin{array}{l}\text { EM } \\
\text { Err }\end{array}$ \\
\hline
\end{tabular}

Matrix: Al

$\begin{array}{llrrrrrrr}1 & \text { CE144 } & 24.700 & 4.200 & 33.300 & 3.300 & 0.740 & 0.15 & \text { A } \\ 1 & \text { CO 57 } & 7.120 & 3.600 & 8.900 & 0.900 & 0.800 & 0.41 & \text { A } \\ 1 & \text { CO 60 } & 28.700 & 1.800 & 29.500 & 2.900 & 0.970 & 0.11 & \text { A } \\ 1 & \text { CS134 } & 15.800 & 1.800 & 14.700 & 1.460 & 1.080 & 0.16 & \text { A } \\ 1 & \text { CS137 } & 6.320 & 4.000 & 6.640 & 0.700 & 0.950 & 0.61 & \text { A } \\ 1 & \text { GA 1 } & 1.690 & 1.110 & 1.620 & 0.150 & 1.040 & 0.69 & \text { A } \\ 1 & \text { GB 2 } & 2.310 & 0.860 & 1.770 & 0.150 & 1.310 & 0.50 & \text { A } \\ 1 & \text { MN 54 } & 3.130 & 6.400 & 3.440 & 0.380 & 0.910 & 1.86 & \text { A } \\ 1 & \text { PU238 } & 0.098 & 0.081 & 0.096 & 0.002 & 1.020 & 0.84 & \text { A } \\ 1 & \text { PU239 } & 0.098 & 0.008 & 0.093 & 0.003 & 1.060 & 0.09 & \text { A } \\ 1 & \text { RU106 } & 14.700 & 12.400 & 11.600 & 1.440 & 1.270 & 1.08 & \text { W } \\ 1 & \text { SB125 } & 9.810 & 4.200 & 9.780 & 1.030 & 1.000 & 0.44 & \mathbf{A} \\ 1 & \text { U 234 } & 4.330 & 0.084 & 0.052 & 0.002 & \because * * & 2.92 & \mathbf{N} \\ 1 & \text { U 238 } & 4.290 & 0.084 & 0.053 & 0.002 & \because * * & 3.54 & \mathbf{N}\end{array}$

Matrix: So

$\begin{array}{llrrrrrrr}1 & \text { CS137 } & 400.000 & 1.800 & 359.000 & 10.000 & 1.110 & 0.03 & \text { A } \\ 1 & \text { K 40 } & 496.000 & 6.800 & 465.000 & 30.000 & 1.070 & 0.07 & \text { A } \\ 1 & \text { PU238 } & 44.700 & 3.210 & 43.000 & 2.440 & 1.040 & 0.10 & \text { A } \\ 1 & \text { PU239 } & 9.660 & 0.941 & 9.230 & 0.346 & 1.050 & 0.11 & \text { A }\end{array}$

Matrix: VE

$\begin{array}{llrrrrrrr}1 & \text { CO 60 } & 64.000 & 4.400 & 59.700 & 0.963 & 1.070 & 0.08 & \text { A } \\ 1 & \text { CS137 } & 1140.000 & 2.400 & 944.000 & 16.200 & 1.210 & 0.02 & \text { A } \\ 1 & \text { K 40 } & 1130.000 & 5.200 & 1030.000 & 33.000 & 1.100 & 0.04 & \text { A } \\ 1 & \text { PU239 } & 9.480 & 0.897 & 9.820 & 1.220 & 0.970 & 0.15 & \text { A }\end{array}$

Matrix: WA

$\begin{array}{llrrrrrrr}1 & \text { CO 60 } & 30.800 & 4.000 & 32.800 & 0.580 & 0.940 & 0.12 & \text { W } \\ 1 & \text { CS137 } & 40.200 & 3.870 & 38.300 & 0.881 & 1.050 & 0.10 & \text { A } \\ 1 & \text { GA 1 } & 2420.000 & 1.040 & 1850.000 & 185.000 & 1.310 & 0.13 & \text { W } \\ 1 & \text { GB 2 } & 1000.000 & 1.210 & 744.000 & 74.000 & 1.340 & 0.13 & \text { A } \\ 1 & \text { H 3 } & 217.000 & 1.600 & 251.000 & 11.400 & 0.870 & 0.04 & \text { A } \\ 1 & \text { MN 54 } & 4.200 & 3.800 & 38.400 & 1.160 & 0.110 & 0.10 & \text { N } \\ 1 & \text { PU238 } & 1.040 & 0.104 & 0.982 & 0.074 & 1.060 & 0.13 & \text { A } \\ 1 & \text { PU239 } & 0.840 & 0.090 & 0.772 & 0.056 & 1.090 & 0.14 & \text { A } \\ 1 & \text { U 234 } & 0.022 & 0.001 & 0.274 & 0.019 & 0.080 & 0.01 & \text { N } \\ 1 & \text { U 238 } & 0.022 & 0.001 & 0.275 & 0.024 & 0.080 & 0.01 & \text { N }\end{array}$

Units for matrices: $\mathrm{Al}=\mathrm{Bq} / \mathrm{filter} \mathrm{SO}=\mathrm{Bq} / \mathrm{kg} \mathrm{VE}=\mathrm{Bq} / \mathrm{kg} W \mathrm{~W}=\mathrm{Bq} / \mathrm{L}$. Values for elemental uranium are reported in $\mu \mathrm{g} / \mathrm{filter}, \mathrm{g}$, or $\mathrm{mL}$.

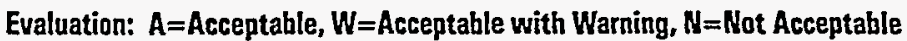
$\mathrm{pCi}=\mathrm{Bq} \times 27$ 


\section{QAP44 Results by Laboratory}

Lab: LM Los Alamos National Lab, Mercury, NV

\begin{tabular}{lllllllll} 
No. & Radio- & $\begin{array}{l}\text { Reported } \\
\text { Value }\end{array}$ & $\begin{array}{l}\text { Reported } \\
\text { Error }\end{array}$ & $\begin{array}{c}\text { EML } \\
\text { Value }\end{array}$ & $\begin{array}{l}\text { EML } \\
\text { Error }\end{array}$ & $\frac{\text { Reported }}{\text { EML }}$ & $\begin{array}{l}\text { Ratio } \\
\text { Error }\end{array}$ & Evaluation \\
\hline
\end{tabular}

Matrix: Al

$\begin{array}{llrlrllll}1 & \text { CE144 } & 46.600 & 1.600 & 33.300 & 3.300 & 1.400 & 0.15 & N \\ 1 & \text { CO 57 } & 11.400 & 0.300 & 8.900 & 0.900 & 1.280 & 0.13 & W \\ 1 & \text { CO 60 } & 40.900 & 0.900 & 29.500 & 2.900 & 1.390 & 0.14 & \text { N } \\ 1 & \text { CS134 } & 23.700 & 0.600 & 14.700 & 1.460 & 1.610 & 0.17 & \text { N } \\ 1 & \text { CS137 } & 9.100 & 0.500 & 6.640 & 0.700 & 1.370 & 0.16 & \mathbf{N} \\ 1 & \text { GA 1 } & 1.900 & 0.500 & 1.620 & 0.150 & 1.170 & 0.33 & \mathbf{A} \\ 1 & \text { GB 2 } & 2.000 & 0.500 & 1.770 & 0.150 & 1.130 & 0.30 & \mathbf{N} \\ 1 & \text { MN 54 } & 5.000 & 0.500 & 3.440 & 0.380 & 1.450 & 0.22 & \mathbf{N} \\ 1 & \text { RU106 } & 15.100 & 3.100 & 11.600 & 1.440 & 1.300 & 0.31 & W \\ 1 & \text { SB125 } & 13.700 & 1.200 & 9.780 & 1.030 & 1.400 & 0.19 & W\end{array}$

Matrix: SO

$\begin{array}{llrrrrrrr}1 & C S 137 & 850.000 & 13.000 & 359.000 & 10.000 & 2.370 & 0.08 & N \\ 1 & K 40 & 1030.000 & 65.000 & 465.000 & 30.000 & 2.210 & 0.20 & N\end{array}$

Matrix: WA

$\begin{array}{llrrrrrrr}1 & \text { CO 60 } & 45.200 & 2.600 & 32.800 & 0.580 & 1.380 & 0.08 & \text { N } \\ 1 & \text { CS137 } & 58.000 & 2.800 & 38.300 & 0.881 & 1.510 & 0.08 & \text { N } \\ 1 & \text { H } 3 & 244.000 & 24.000 & 251.000 & 11.400 & 0.970 & 0.11 & \text { A } \\ 1 & \text { MN 54 } & 58.500 & 3.000 & 38.400 & 1.160 & 1.520 & 0.09 & N\end{array}$

Units for matrices: $A \mathrm{I}=\mathrm{Bq} /$ filter $\mathrm{SO}=\mathrm{Bq} / \mathrm{kg} V E=\mathrm{Bq} / \mathrm{kg} W \mathrm{~W}=\mathrm{Bq} / \mathrm{l}$. Values for elemental uranium are reported in $\mu \mathrm{g} / \mathrm{filter}, \mathrm{g}$, or $\mathrm{mL}$. 


\section{OAP44 Results by Laboratory}

Lah: LV UNLV, Dept of Health Physics

\begin{tabular}{|c|c|c|c|c|c|c|c|c|}
\hline $\begin{array}{l}\text { No. } \\
\text { Test }\end{array}$ & $\begin{array}{l}\text { Radio- } \\
\text { nuclide }\end{array}$ & $\begin{array}{l}\text { Reported } \\
\text { Value }\end{array}$ & $\begin{array}{l}\text { Reported } \\
\text { Error }\end{array}$ & $\begin{array}{c}\text { EML } \\
\text { Value }\end{array}$ & $\begin{array}{l}\text { EML } \\
\text { Error }\end{array}$ & $\frac{\text { Reported }}{\text { EML }}$ & $\begin{array}{l}\text { Ratio } \\
\text { Error }\end{array}$ & Evaluation \\
\hline
\end{tabular}

Matrix: Al

$\begin{array}{lrrrrrrr}\text { AM241 } & 0.249 & 0.050 & 0.189 & 0.007 & 1.320 & 0.27 & \text { W } \\ \text { CE144 } & 23.500 & 0.700 & 33.300 & 3.300 & 0.710 & 0.07 & \text { A } \\ \text { CD 57 } & 6.600 & 0.100 & 8.900 & 0.900 & 0.740 & 0.08 & \text { A } \\ \text { CO 60 } & 25.100 & 0.200 & 29.500 & 2.900 & 0.850 & 0.08 & \text { A } \\ \text { CS134 } & 13.000 & 0.200 & 14.700 & 1.460 & 0.880 & 0.09 & \text { A } \\ \text { CS137 } & 5.630 & 0.070 & 6.640 & 0.700 & 0.850 & 0.09 & \text { A } \\ \text { MN 54 } & 2.760 & 0.100 & 3.440 & 0.380 & 0.800 & 0.09 & \text { W } \\ \text { RU106 } & 10.400 & 0.800 & 11.600 & 1.440 & 0.900 & 0.13 & \text { A } \\ \text { SB125 } & 7.650 & 0.220 & 9.780 & 1.030 & 0.780 & 0.09 & \text { A }\end{array}$

Matrix: So

$\begin{array}{rrr}1 & \text { AM241 } & 5.960 \\ 1 & \text { CS137 } & 370.000\end{array}$

$\begin{array}{rr}0.670 & 3.690 \\ 2.000 & 359.000\end{array}$

0.454

10.000

1.620

465.000

30.000

1.030

0.27

0.03

0.06

W
A

Matrix: VE

\begin{tabular}{|c|c|c|c|c|c|c|c|c|}
\hline 1 & $\begin{array}{l}\text { AM241 } \\
\text { CO 60 } \\
\text { CS137 } \\
\text { K } 40\end{array}$ & $\begin{array}{r}12.000 \\
70.200 \\
1240.000 \\
1160.000\end{array}$ & $\begin{array}{r}2.000 \\
2.400 \\
7.000 \\
20.000\end{array}$ & $\begin{array}{r}5.600 \\
59.700 \\
944.000 \\
1030.000\end{array}$ & $\begin{array}{r}0.184 \\
0.963 \\
16.200 \\
33.000\end{array}$ & $\begin{array}{l}2.140 \\
1.180 \\
1.310\end{array}$ & $\begin{array}{l}0.36 \\
0.04 \\
0.02\end{array}$ & $\begin{array}{l}W \\
\text { A } \\
W \\
\text { A }\end{array}$ \\
\hline
\end{tabular}

Matrix: WA

$\begin{array}{lllllllll}1 & \text { AM241 } & 0.946 & 0.530 & 0.766 & 0.013 & 1.240 & 0.69 & \text { W } \\ 1 & \text { CO 60 } & 32.300 & 0.700 & 32.800 & 0.580 & 0.990 & 0.03 & A \\ 1 & \text { CS137 } & 43.300 & 0.700 & 38.300 & 0.881 & 1.130 & 0.03 & A \\ 1 & \text { MN 54 } & 45.500 & 0.900 & 38.400 & 1.160 & 1.180 & 0.04 & \text { W }\end{array}$

Units for matrices: $A \mid=B q /$ filter $S O=B q / k g$ VE=Bq/kg $W A=B q / L$. Values for elemental uranium are reported in $\mu g / f i l t e r, g$, or $\mathrm{mL}$.

Evaluation: $A=A c c e p t a b l e, W=A c c e p t a b l e$ with Warning, $N=N$ ot Acceptable

$\mathrm{pCi}=\mathrm{Bq} \times 27$ 


\section{QAP44 Results by Laboratory}

Lab: IW Lawrence Livermore National Lab, CA

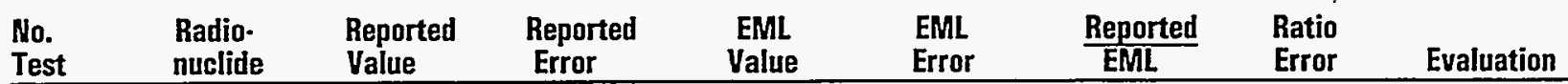

Matrix: So

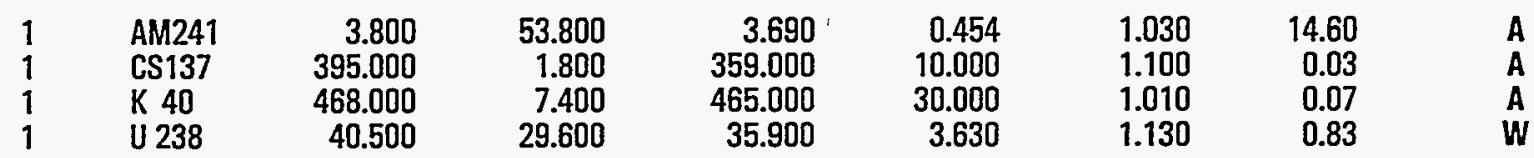

Matrix: VE

\begin{tabular}{|c|c|c|c|c|c|c|}
\hline $\begin{array}{l}\text { AM241 } \\
\text { CO 60 } \\
\text { CS137 } \\
\text { K } 40\end{array}$ & $\begin{array}{r}5.300 \\
59.600 \\
1050.000 \\
1060.000\end{array}$ & $\begin{array}{r}57.400 \\
5.600 \\
2.000 \\
4.400\end{array}$ & $\begin{array}{r}5.600 \\
59.700 \\
944.000 \\
1030.000\end{array}$ & $\begin{array}{r}0.184 \\
0.963 \\
16.200 \\
33.000\end{array}$ & $\begin{array}{l}0.950 \\
1.000 \\
1.110 \\
1.030\end{array}$ & $\begin{array}{r}10.30 \\
0.10 \\
0.02 \\
0.03\end{array}$ \\
\hline
\end{tabular}

Matrix: WA

$\begin{array}{llrlrrrrr}1 & \text { CO 60 } & 30.200 & 3.200 & 32.800 & 0.580 & 0.920 & 0.10 & \text { W } \\ 1 & \text { CS137 } & 40.800 & 3.200 & 38.300 & 0.881 & 1.070 & 0.09 & \text { A } \\ 1 & \text { GA 1 } & 1880.000 & 2.800 & 1850.000 & 185.000 & 1.020 & 0.10 & \text { A } \\ 1 & \text { GB 2 } & 784.000 & 2.000 & 744.000 & 74.000 & 1.050 & 0.11 & \text { A } \\ 1 & \text { H 3 } & 228.000 & 6.200 & 251.000 & 11.400 & .0 .910 & 0.05 & \text { A } \\ 1 & \text { MN 54 } & 40.200 & 3.400 & 38.400 & 1.160 & 1.050 & 0.09 & \text { A }\end{array}$

Units for matrices: $A l=B q / f i l t e r ~ S O=B q / k g$ VE=Bq/kg $W A=B q / L$. Values for elemental uranium are reported in $\mu \mathrm{gg} / \mathrm{filter}, \mathrm{g}, \mathrm{or} \mathrm{mL}$. 


\section{OAP44 Results by Laboratory}

Lab: MA ORNL Health Sciences Research Div.

\begin{tabular}{|c|c|c|c|c|c|c|c|c|}
\hline $\begin{array}{l}\text { No. } \\
\text { Test } \\
\end{array}$ & $\begin{array}{l}\text { Radio- } \\
\text { nuclide }\end{array}$ & $\begin{array}{l}\text { Reported } \\
\text { Value } \\
\end{array}$ & $\begin{array}{c}\text { Reported } \\
\text { Error }\end{array}$ & $\begin{array}{c}\text { EML } \\
\text { Value }\end{array}$ & $\begin{array}{l}\text { EML } \\
\text { Error }\end{array}$ & $\frac{\text { Reported }}{\text { EML }}$ & $\begin{array}{l}\text { Ratio } \\
\text { Error } \\
\end{array}$ & Evaluation \\
\hline Matrix: & SO & & & & & & & \\
\hline $\begin{array}{l}1 \\
1 \\
1\end{array}$ & $\begin{array}{l}\text { AM241 } \\
\text { CS137 } \\
\text { K } 40\end{array}$ & $\begin{array}{r}5.200 \\
444.000 \\
518.000\end{array}$ & $\begin{array}{r}2.200 \\
33.000 \\
59.000\end{array}$ & $\begin{array}{r}3.690 \\
359.000 \\
465.000\end{array}$ & $\begin{array}{r}0.454 \\
10.000 \\
30.000\end{array}$ & $\begin{array}{l}1.410 \\
1.240 \\
1.110\end{array}$ & $\begin{array}{l}0.62 \\
0.10 \\
0.15\end{array}$ & $\begin{array}{l}A \\
W \\
A\end{array}$ \\
\hline Matrix: & VE & & & & & & & \\
\hline $\begin{array}{l}1 \\
1 \\
1 \\
1\end{array}$ & $\begin{array}{l}\text { AM241 } \\
\text { CO } 60 \\
\text { CS137 } \\
\text { K } 40\end{array}$ & $\begin{array}{r}5.200 \\
67.000 \\
1290.000 \\
1220.000\end{array}$ & $\begin{array}{r}1.500 \\
7.400 \\
93.000 \\
150.000\end{array}$ & $\begin{array}{r}5.600 \\
59.700 \\
944.000 \\
1030.000\end{array}$ & $\begin{array}{r}0.184 \\
0.963 \\
16.200 \\
33.000\end{array}$ & $\begin{array}{l}0.930 \\
1.120 \\
1.370 \\
1.180\end{array}$ & $\begin{array}{l}0.27 \\
0.13 \\
0.10 \\
0.15\end{array}$ & $\begin{array}{l}A \\
A \\
W \\
A\end{array}$ \\
\hline
\end{tabular}


QAP44 Results by Laboratory

Lab: ME Radiation Control Program, Jamaica Plain, MA

\begin{tabular}{lllllllll} 
No. & $\begin{array}{l}\text { Radio- } \\
\text { nuclide }\end{array}$ & $\begin{array}{l}\text { Reported } \\
\text { Value }\end{array}$ & $\begin{array}{l}\text { Reported } \\
\text { Error }\end{array}$ & $\begin{array}{c}\text { EML } \\
\text { Value }\end{array}$ & $\begin{array}{l}\text { EML } \\
\text { Error }\end{array}$ & $\begin{array}{l}\text { Reported } \\
\text { EML }\end{array}$ & $\begin{array}{l}\text { Ratio } \\
\text { Error }\end{array}$ & Evaluation \\
\hline
\end{tabular}

Matrix: AI

$\begin{array}{llrlrllll}1 & \text { CE144 } & 29.400 & 0.246 & 33.300 & 3.300 & 0.880 & 0.09 & \text { A } \\ 1 & \text { CO 57 } & 6.700 & 0.035 & 8.900 & 0.900 & 0.750 & 0.08 & \text { A } \\ 1 & \text { CO 60 } & 28.500 & 0.154 & 29.500 & 2.900 & 0.970 & 0.10 & \text { A } \\ 1 & \text { CS134 } & 13.400 & 0.094 & 14.700 & 1.460 & 0.910 & 0.09 & \text { A } \\ 1 & \text { CS137 } & 5.980 & 0.062 & 6.640 & 0.700 & 0.900 & 0.10 & \text { A } \\ 1 & \text { GA 1 } & 3.500 & 0.070 & 1.620 & 0.150 & 2.160 & 0.21 & \text { N } \\ 1 & \text { GB 2 } & 2.490 & 0.046 & 1.770 & 0.150 & 1.410 & 0.12 & \text { A } \\ 1 & \text { MN 54 } & 3.380 & 0.064 & 3.440 & 0.380 & 0.980 & 0.11 & \text { A }\end{array}$

Matrix: So

$\begin{array}{lllllllll}1 & \text { CS137 } & 370.000 & 12.100 & 359.000 & 10.000 & 1.030 & 0.04 & A \\ 1 & K ~ 40 & 622.000 & 21.400 & 465.000 & 30.000 & 1.340 & 0.10 & W\end{array}$

Matrix: VE

$\begin{array}{llrrrrrrr}1 & C 060 & 64.300 & 1.660 & 59.700 & 0.963 & 1.080 & 0.03 & A \\ 1 & C S 137 & 1170.000 & 37.600 & 944.000 & 16.200 & 1.240 & 0.05 & A \\ 1 & K ~ 40 & 1390.000 & 47.400 & 1030.000 & 33.000 & 1.350 & 0.06 & W\end{array}$

Matrix: WA

$\begin{array}{lllllllll}1 & \text { C } 60 & 34.700 & 0.912 & 32.800 & 0.580 & 1.060 & 0.03 & A \\ 1 & \text { CS137 } & 44.500 & 1.750 & 38.300 & 0.881 & 1.160 & 0.05 & \text { W } \\ 1 & \text { MN 54 } & 46.100 & 1.560 & 38.400 & 1.160 & 1.200 & 0.05 & \mathbf{W}\end{array}$

Units for matrices: $\mathrm{Al}=\mathrm{Bq} / \mathrm{filter} \mathrm{SO}=\mathrm{B} q \mathrm{~kg} \quad \mathrm{VE}=\mathrm{Bq} / \mathrm{kg} \quad W \mathrm{~A}=\mathrm{Bq} / \mathrm{l}$. Values for elemental uranium are reported in $\mu \mathrm{g} /$ filter, $\mathrm{g}$, or $\mathrm{mL}$.

$\mathrm{pCi}=\mathrm{Bq} \times 27$ 


\section{QAP44 Results by Laboratory}

Lab: MI Massachusetts Institute of Technology

\begin{tabular}{llllllll} 
No. & $\begin{array}{l}\text { Radio. } \\
\text { nuclide }\end{array}$ & $\begin{array}{l}\text { Reported } \\
\text { Value }\end{array}$ & $\begin{array}{c}\text { Reported } \\
\text { Error }\end{array}$ & $\begin{array}{c}\text { EML } \\
\text { Value }\end{array}$ & $\begin{array}{l}\text { EML } \\
\text { Error }\end{array}$ & $\frac{\text { Reported }}{\text { EML }}$ & $\begin{array}{l}\text { Ratio } \\
\text { Error }\end{array}$ \\
\hline
\end{tabular}

Matrix: Al

$\begin{array}{rrrrrrrrr}1 & \text { CO 57 } & 5.820 & 0.181 & 8.900 & 0.900 & 0.650 & 0.07 & \text { W } \\ 1 & \text { CO 60 } & 26.800 & 0.865 & 29.500 & 2.900 & 0.910 & 0.09 & \text { A } \\ 1 & \text { CS134 } & 11.100 & 0.251 & 14.700 & 1.460 & 0.760 & 0.08 & \text { W } \\ 1 & \text { CS137 } & 5.210 & 0.249 & 6.640 & 0.700 & 0.790 & 0.09 & \text { W } \\ 1 & \text { MN 54 } & 2.980 & 0.145 & 3.440 & 0.380 & 0.870 & 0.11 & \text { A }\end{array}$

Matrix: WA

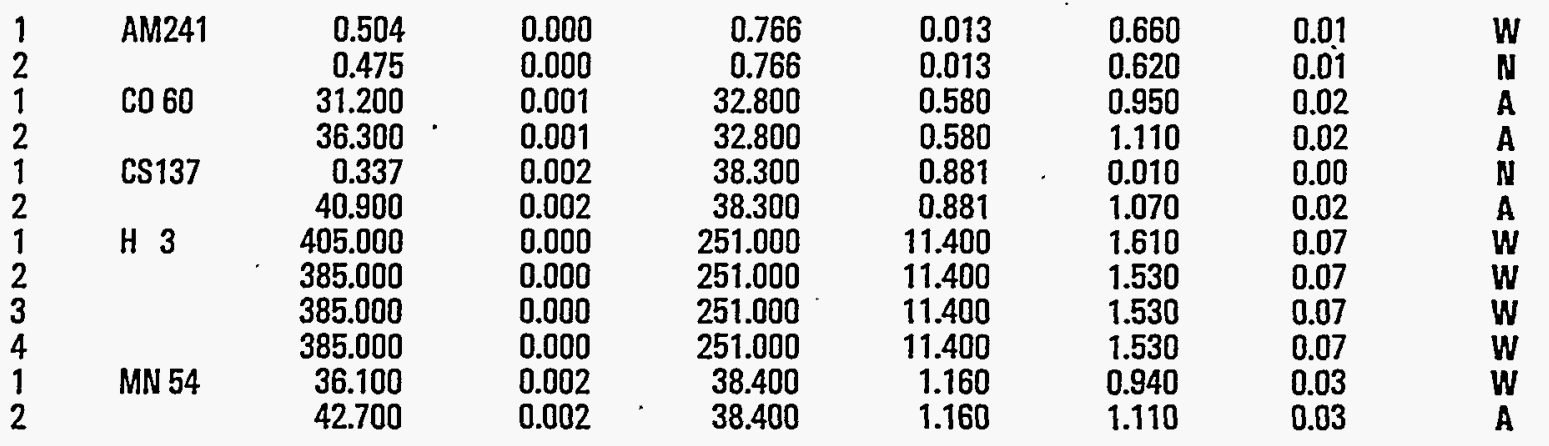

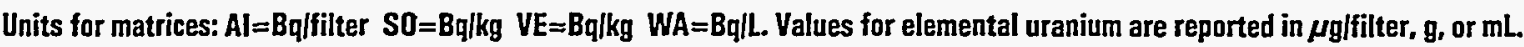




\section{QAP44 Results by Laboratory}

Lab: ML EG\&G Mound Applied Technologies, Miamisburg, $\mathrm{OH}$

\begin{tabular}{|c|c|c|c|c|c|}
\hline $\begin{array}{l}\text { No. } \\
\text { Test }\end{array}$ & $\begin{array}{l}\text { Radio- } \\
\text { nuclide }\end{array}$ & $\begin{array}{l}\text { Reported } \\
\text { Value }\end{array}$ & $\begin{array}{l}\text { Reported } \\
\text { Error }\end{array}$ & $\begin{array}{l}\text { EML } \\
\text { Value }\end{array}$ & $\begin{array}{l}\text { EML } \\
\text { Error }\end{array}$ \\
\hline
\end{tabular}

Matrix: Al

\begin{tabular}{|c|c|c|c|c|c|c|}
\hline CE144 & 27.600 & 0.800 & 33.300 & 3.300 & 0.830 & 0.09 \\
\hline CO 57 & 6.700 & 0.200 & 8.900 & 0.900 & 0.750 & 0.08 \\
\hline CO 60 & 25.800 & 0.400 & 29.500 & 2.900 & 0.880 & 0.09 \\
\hline CS134 & 13.900 & 0.400 & 14.700 & 1.460 & 0.950 & 0.10 \\
\hline CS137 & 5.600 & 0.200 & 6.640 & 0.700 & 0.840 & 0.09 \\
\hline MN 54 & 2.800 & 0.200 & 3.440 & 0.380 & 0.810 & 0.11 \\
\hline PU238 & 0.091 & 0.005 & 0.096 & 0.002 & 0.950 & 0.06 \\
\hline PU239 & 0.100 & 0.005 & 0.093 & 0.003 & 1.080 & 0.06 \\
\hline RU106 & 11.400 & 1.000 & 11.600 & 1.440 & 0.980 & 0.15 \\
\hline SB125 & 9.300 & 0.400 & 9.780 & 1.030 & 0.950 & 0.11 \\
\hline U 234 & 0.058 & 0.004 & 0.052 & 0.002 & 1.120 & 0.08 \\
\hline U 238 & 0.056 & 0.004 & 0.053 & 0.002 & 1.050 & 0.09 \\
\hline
\end{tabular}

Matrix: SO

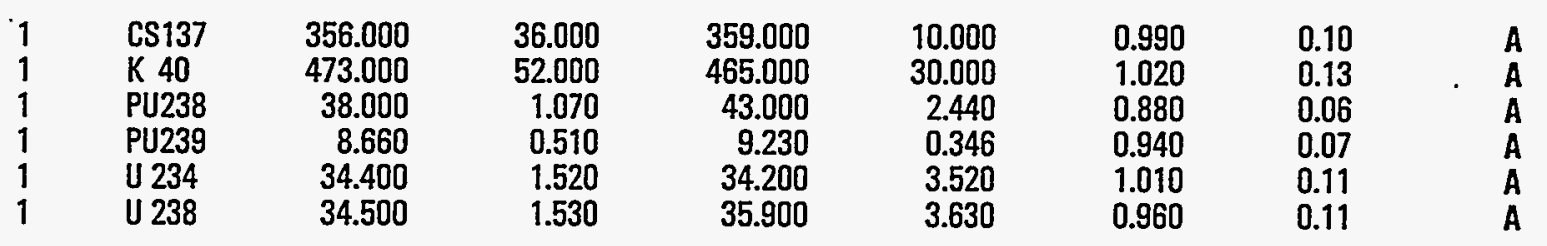

Matrix: VE

$\begin{array}{rlrrrrrrr}1 & \text { CO 60 } & 61.500 & 12.800 & 59.700 & 0.963 & 1.030 & \mathbf{0 . 2 2} & \\ 1 & \text { CS137 } & 1020.000 & 20.000 & 944.000 & 16.200 & 1.080 & 0.03 & \text { A } \\ 1 & \text { K 40 } & 1190.000 & 244.000 & 1030.000 & 33.000 & 1.160 & 0.24 & \text { A } \\ 1 & \text { PU239 } & 8.240 & 0.350 & 9.820 & 1.220 & 0.840 & \mathbf{0 . 1 1} & \mathbf{A}\end{array}$

Matrix: WA

$\begin{array}{llrrrrrrr}1 & \text { CO 60 } & 32.200 & 6.600 & 32.800 & 0.580 & 0.980 & 0.20 & \text { A } \\ 1 & \text { CS137 } & 40.000 & 8.200 & 38.300 & 0.881 & 1.040 & 0.22 & \text { A } \\ 1 & \text { H 3 } & 197.000 & 20.700 & 251.000 & 11.400 & 0.790 & 0.09 & \text { W } \\ 1 & \text { MN 54 } & 41.100 & 8.400 & 38.400 & 1.160 & 1.070 & 0.22 & \text { A } \\ 1 & \text { PU238 } & 0.980 & 0.040 & 0.982 & 0.074 & 1.000 & 0.09 & \text { A } \\ 1 & \text { PU239 } & 0.790 & 0.040 & 0.772 & 0.056 & 1.020 & 0.09 & \text { A } \\ 1 & \text { U 234 } & 0.300 & 0.020 & 0.274 & 0.019 & 1.100 & 0.11 & \text { A } \\ 1 & \text { U 238 } & 0.298 & 0.005 & 0.275 & 0.024 & 1.080 & 0.10 & \text { A }\end{array}$

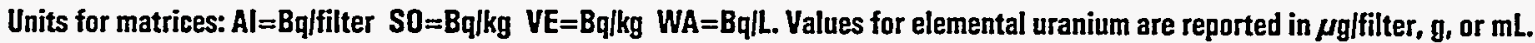

Evaluation: $A=A c c e p t a b l e, W=A c c e p t a b l e$ with Warning, $N=$ Not Acceptable $\mathrm{pCi}=\mathrm{Bq} \times 27$ 
QAP44 Results by Laboratory

Lab: MS Manufacturing Sciences Corporation, Oak Ridge

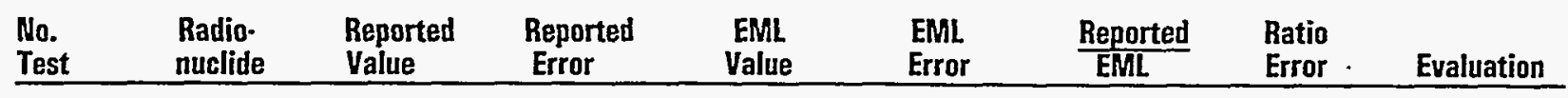

Matrix: Al

$\begin{array}{rrrrrrrrr}1 & \text { CE144 } & 33.300 & 3.300 & 33.300 & 3.300 & 1.000 & 0.14 & \text { A } \\ 1 & \text { CO 57 } & 9.900 & 0.990 & 8.900 & 0.900 & 1.110 & 0.16 & \text { W } \\ 1 & \text { CO 60 } & 30.400 & 3.000 & 29.500 & 2.900 & 1.030 & 0.14 & \text { A } \\ 1 & \text { CS134 } & 15.800 & 1.600 & 14.700 & 1.460 & 1.080 & 0.15 & \text { A } \\ 1 & \text { CS137 } & 6.700 & 0.670 & 6.640 & 0.700 & 1.010 & 0.15 & \text { A } \\ 1 & \text { MN 54 } & 3.400 & 0.340 & 3.440 & 0.380 & 0.990 & 0.15 & \text { A } \\ 1 & \text { SB125 } & 10.000 & 1.000 & 9.780 & 1.030 & 1.020 & 0.15 & \text { A }\end{array}$

Matrix: So

$\begin{array}{lllllllll}1 & \text { CS137 } & 362.000 & 36.000 & 359.000 & 10.000 & 1.010 & 0.10 & \text { A } \\ 1 & K 40 & 458.000 & 46.000 & 465.000 & 30.000 & 0.990 & 0.12 & \text { A }\end{array}$

Matrix: WA

$\begin{array}{lllllllll}1 & \text { AM241 } & 0.980 & 0.100 & 0.766 & 0.013 & 1.280 & 0.13 & \text { W } \\ 1 & \text { CD 60 } & 34.000 & 3.400 & 32.800 & 0.580 & 1.040 & 0.11 & \text { A } \\ 1 & \text { CS137 } & 40.900 & 4.100 & 38.300 & 0.881 & 1.070 & 0.11 & \text { A } \\ 1 & \text { MN 54 } & 42.500 & 4.300 & .38 .400 & 1.160 & 1.110 & 0.12 & \text { A }\end{array}$

Units for matrices: $A|=B q| f i l t e r ~ S O=B q / k g ~ V E=B q / k g ~ W A=B q l L$. Values for elemental uranium are reported in $\mu \mathrm{g} / f i l t e r, g$, or $\mathrm{mL}$.

Evaluation: $A=A c c e p t a b l e, W=A c c e p t a b l e$ with Warning, $N=N o t$ Acceptable

$\mathrm{pCi}=\mathrm{Bq} \times 27$ 


\section{QAP44 Results by Laboratory}

Lab: NA US EPA NAREL, Montgomery, AL

\begin{tabular}{lllllllll}
$\begin{array}{l}\text { No. } \\
\text { Test }\end{array}$ & $\begin{array}{l}\text { Radio- } \\
\text { nuclide }\end{array}$ & $\begin{array}{l}\text { Reported } \\
\text { Value }\end{array}$ & $\begin{array}{l}\text { Reported } \\
\text { Error }\end{array}$ & $\begin{array}{c}\text { EML } \\
\text { Value }\end{array}$ & $\begin{array}{l}\text { EML } \\
\text { Error }\end{array}$ & $\begin{array}{c}\text { Reported } \\
\text { EML }\end{array}$ & $\begin{array}{l}\text { Ratio } \\
\text { Error }\end{array}$ & Evaluation \\
\hline
\end{tabular}

Matrix: Al

$\begin{array}{llrlrllll}1 & \text { AM241 } & 0.182 & 0.015 & 0.189 & 0.007 & 0.960 & 0.09 & \text { A } \\ 1 & \text { CE144 } & 27.300 & 0.290 & 33.300 & 3.300 & 0.820 & 0.08 & \text { A } \\ 1 & \text { CO 57 } & 7.930 & 0.050 & 8.900 & 0.900 & 0.890 & 0.09 & \text { A } \\ 1 & \text { CO 60 } & 27.600 & 0.130 & 29.500 & 2.900 & 0.940 & 0.09 & \text { A } \\ 1 & \text { CS134 } & 13.800 & 0.080 & 14.700 & 1.460 & 0.940 & 0.09 & \text { A } \\ 1 & \text { CS137 } & 6.630 & 0.090 & 6.640 & 0.700 & 1.000 & 0.11 & \text { A } \\ 1 & \text { MN 54 } & 3.400 & 0.080 & 3.440 & 0.380 & 0.990 & 0.11 & \text { A } \\ 1 & \text { PU238 } & 0.103 & 0.012 & 0.096 & 0.002 & 1.070 & 0.13 & \text { A } \\ 1 & \text { PU239 } & 0.098 & 0.011 & 0.093 & 0.003 & 1.060 & 0.12 & \text { A } \\ 1 & \text { RU106 } & 10.300 & 0.560 & 11.600 & 1.440 & 0.890 & 0.12 & \text { A } \\ 1 & \text { SB125 } & 10.500 & 0.150 & 9.780 & 1.030 & 1.070 & 0.11 & \text { A } \\ 1 & \text { SR 90 } & 1.030 & 0.180 & 1.060 & 0.037 & 0.970 & 0.17 & \text { A }\end{array}$

Matrix: So

$\begin{array}{llrrrrrrr}1 & \text { CS137 } & 382.000 & 1.940 & 359.000 & 10.000 & 1.060 & 0.03 & \text { A } \\ 1 & K ~ 40 & 480.000 & 9.460 & 465.000 & 30.000 & 1.030 & 0.07 & \text { A } \\ 1 & \text { PU238 } & 40.000 & 3.260 & 43.000 & 2.440 & 0.930 & .0 .09 & \text { A } \\ 1 & \text { PU239 } & 9.640 & 1.450 & 9.230 & 0.346 & 1.040 & 0.16 & \text { A } \\ 1 & \text { SR 90 } & 1450.000 & 78.000 & 1340.000 & 113.000 & 1.080 & 0.11 & \text { A } \\ 1 & \text { U 234 } & 35.900 & 2.830 & 34.200 & 3.520 & 1.050 & 0.14 & \text { A } \\ 1 & \text { U 238 } & 35.400 & 2.810 & 35.900 & 3.630 & 0.990 & 0.13 & \text { A }\end{array}$

Matrix: VE

$\begin{array}{llrrrrrrr}1 & \text { AM241 } & 5.810 & 0.570 & 5.600 & 0.184 & 1.040 & 0.11 & \text { A } \\ 2 & & 5.810 & 0.570 & 5.600 & 0.184 & 1.040 & 0.11 & \text { A } \\ 1 & \text { CO 60 } & 63.000 & 1.100 & 59.700 & 0.963 & 1.050 & 0.03 & \text { A } \\ 1 & \text { CS137 } & 1130.000 & 4.500 & 944.000 & 16.200 & 1.200 & 0.02 & \text { A } \\ 1 & \text { K 40 } & 1180.000 & 20.000 & 1030.000 & 33.000 & 1.150 & 0.04 & \text { A } \\ 1 & \text { PU239 } & 8.410 & 0.610 & 9.820 & 1.220 & 0.860 & 0.12 & \text { A } \\ 1 & \text { SR 90 } & 1230.000 & 27.000 & 1300.000 & 52.400 & 0.950 & 0.04 & \text { A }\end{array}$

Matrix: WA

$\begin{array}{llrrrrrl}1 & \text { AM241 } & 0.732 & 0.045 & 0.766 & 0.013 & 0.960 & 0.06 \\ 1 & \text { CO 60 } & 32.900 & 0.410 & 32.800 & 0.580 & 1.000 & 0.02 \\ 1 & \text { CS137 } & 41.000 & 0.560 & 38.300 & 0.881 & 1.070 & 0.03 \\ 1 & H 3 & 199.000 & 4.200 & 251.000 & 11.400 & 0.790 & 0.04 \\ 1 & \text { MN 54 } & 41.300 & 0.580 & 38.400 & 1.160 & 1.080 & 0.04 \\ 1 & \text { SR 90 } & 1.550 & 0.260 & 1.450 & 0.034 & 1.070 & 0.18 \\ 1 & \text { U 234 } & 0.416 & 0.037 & 0.274 & 0.019 & 1.520 & 0.17 \\ 1 & \text { U 238 } & 0.355 & 0.033 & 0.275 & 0.024 & 1.290 & 0.17\end{array}$

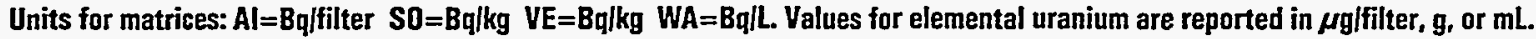

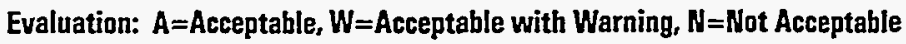
$\mathrm{pCi}=\mathrm{Bq} \times 27$ 


\section{QAP44 Results by Laboratory}

Lab: NC Nuclear Services North Carolina State University, Raleigh

\begin{tabular}{llllllll} 
No. & $\begin{array}{l}\text { Radio- } \\
\text { nuclide }\end{array}$ & $\begin{array}{l}\text { Reported } \\
\text { Value }\end{array}$ & $\begin{array}{l}\text { Reported } \\
\text { Error }\end{array}$ & $\begin{array}{c}\text { EML } \\
\text { Value }\end{array}$ & $\begin{array}{c}\text { EML } \\
\text { Error }\end{array}$ & $\frac{\text { Reported }}{\text { EML }}$ & $\begin{array}{l}\text { Ratio } \\
\text { Error }\end{array}$ \\
\hline
\end{tabular}

Matrix: Al

$\begin{array}{llrlrllll}1 & \text { CE144 } & 30.000 & 1.480 & 33.300 & 3.300 & 0.900 & 0.10 & \text { A } \\ 1 & \text { CO 57 } & 9.090 & 0.178 & 8.900 & 0.900 & 1.020 & 0.11 & \text { A } \\ 1 & \text { CO 60 } & 28.700 & 0.470 & 29.500 & 2.900 & 0.970 & 0.10 & \text { A } \\ 1 & \text { CS134 } & 14.100 & 0.389 & 14.700 & 1.460 & 0.960 & 0.10 & \text { A } \\ 1 & \text { CS137 } & 6.650 & 0.251 & 6.640 & 0.700 & 1.000 & 0.11 & \text { A } \\ 1 & \text { MN 54 } & 3.700 & 0.245 & 3.440 & 0.380 & 1.080 & 0.14 & \text { A } \\ 1 & \text { SB125 } & 10.000 & 0.512 & 9.780 & 1.030 & 1.020 & 0.12 & \text { A }\end{array}$

Matrix: $\quad$ So

$\begin{array}{lllllllll}1 & \operatorname{CS} 137 & 366.000 & 2.230 & 359.000 & 10.000 & 1.020 & 0.03 & \text { A }\end{array}$

Matrix: VE

\begin{tabular}{|c|c|c|c|c|c|c|c|}
\hline $\begin{array}{l}1 \\
1\end{array}$ & $\begin{array}{l}\text { CO } 60 \\
\text { CS137 }\end{array}$ & $\begin{array}{r}58.300 \\
1060.000\end{array}$ & $\begin{array}{l}1.870 \\
4.960\end{array}$ & $\begin{array}{r}59.700 \\
944.000\end{array}$ & $\begin{array}{r}0.963 \\
16.200\end{array}$ & $\begin{array}{l}0.980 \\
1.120\end{array}$ & $\begin{array}{l}0.04 \\
0.02\end{array}$ \\
\hline
\end{tabular}

Matrix: WA

$\begin{array}{lllllllll}1 & \text { C } 60 & 33.700 & 1.300 & 32.800 & 0.580 & 1.030 & 0.04 & \text { A } \\ 1 & \text { CS137 } & 39.100 & 1.020 & 38.300 & 0.881 & 1.020 & 0.04 & \text { A } \\ 1 & \text { MN } 54 & 40.600 & 1.350 & 38.400 & 1.160 & 1.060 & 0.05 & \text { A }\end{array}$

Units for matrices: $A \mathrm{l}=\mathrm{Bq} /$ filter $\mathrm{SO}=\mathrm{Bq} / \mathrm{kg} V E=B q / \mathrm{kg} W A=B q / \mathrm{L}$. Values for elemental uranium are reported in $\mu \mathrm{g} / \mathrm{filter}, \mathrm{g}$, or $\mathrm{mL}$.

Evaluation: $A=$ Acceptable, $W=A c c e p t a b l e$ with Warning, $N=$ Not Acceptable

$\mathrm{pCi}=\mathrm{Bq} \times 27$ 


\section{OAP44 Results by Laboratory}

Lab: NL FERMCO, Cincinnati, OH

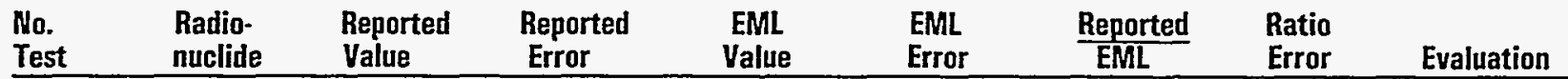

Matrix: Al

$\begin{array}{llrlrllll}1 & \text { CE144 } & 30.900 & 2.000 & 33.300 & 3.300 & 0.930 & 0.11 & \text { A } \\ 1 & \text { CO 57 } & 8.640 & 0.680 & 8.900 & 0.900 & 0.970 & 0.12 & \text { A } \\ 1 & \text { CO 60 } & 28.400 & 1.100 & 29.500 & 2.900 & 0.960 & 0.10 & \text { A } \\ 1 & \text { CS134 } & 13.400 & 0.600 & 14.700 & 1.460 & 0.910 & 0.10 & \text { A } \\ 1 & \text { CS137 } & 6.430 & 0.390 & 6.640 & 0.700 & 0.970 & 0.12 & \text { A } \\ 1 & \text { MN 54 } & 3.580 & 0.240 & 3.440 & 0.380 & 1.040 & 0.13 & \text { A } \\ 1 & \text { PU238 } & 0.084 & 0.020 & 0.096 & 0.002 & 0.870 & 0.21 & A \\ 1 & \text { PU239 } & 0.084 & 0.020 & 0.093 & 0.003 & 0.910 & 0.22 & A \\ 1 & \text { RU106 } & 10.600 & 1.100 & 11.600 & 1.440 & 0.910 & 0.15 & \text { A } \\ 1 & \text { SB125 } & 10.600 & 0.900 & 9.780 & 1.030 & 1.080 & 0.15 & \text { A } \\ 1 & \text { U 234 } & 0.053 & 0.010 & 0.052 & 0.002 & 1.030 & 0.20 & \text { A } \\ 1 & \text { U 238 } & 0.046 & 0.012 & 0.053 & 0.002 & 0.860 & 0.23 & \text { A } \\ 1 & \text { U UG } & 4.300 & 0.500 & 4.310 & 0.100 & 1.000 & 0.12 & \text { A }\end{array}$

Matrix: So

$\begin{array}{llrrrrrrr}1 & \text { CS137 } & 399.000 & 8.000 & 359.000 & 10.000 & 1.110 & 0.04 & \text { A } \\ 1 & \text { K 40 } & 584.000 & 80.000 & 465.000 & 30.000 & 1.260 & 0.19 & \text { W } \\ 1 & \text { PU238 } & 43.600 & 10.000 & 43.000 & 2.440 & 1.010 & 0.24 & \text { A } \\ 1 & \text { PU239 } & 9.350 & 2.100 & 9.230 & 0.346 & 1.010 & 0.23 & \text { A } \\ 1 & \text { U 234 } & 34.400 & 5.600 & 34.200 & 3.520 & 1.010 & 0.19 & \text { A } \\ 2 & \text { U 238 } & 35.500 & 5.800 & 35.900 & 3.630 & 0.990 & 0.19 & \text { A }\end{array}$

Matrix: WA

$\begin{array}{llrlrllll}1 & \text { CO 60 } & 34.600 & 0.900 & 32.800 & 0.580 & 1.050 & 0.03 & \text { A } \\ 1 & \text { CS137 } & 42.900 & 0.800 & 38.300 & 0.881 & 1.120 & 0.03 & \text { A } \\ 1 & \text { MN 54 } & 46.200 & 0.200 & 38.400 & 1.160 & 1.200 & 0.04 & \text { W } \\ 1 & \text { PU238 } & 0.864 & 0.210 & 0.982 & 0.074 & 0.880 & 0.22 & \text { W } \\ 1 & \text { PU239 } & 0.726 & 0.170 & 0.772 & 0.056 & 0.940 & 0.23 & \text { A } \\ 1 & \text { U 234 } & 0.280 & 0.051 & 0.274 & 0.019 & 1.020 & 0.20 & \text { A } \\ 1 & \text { U 238 } & 0.291 & 0.051 & 0.275 & 0.024 & 1.060 & 0.21 & \text { A } \\ 1 & \text { U UG } & 0.023 & 0.003 & 0.022 & 0.003 & 1.040 & 0.18 & \text { A }\end{array}$

Units for matrices: $\mathrm{Al}=\mathrm{Bq} / \mathrm{filter} \mathrm{SO}=\mathrm{Bq} / \mathrm{kg} \mathrm{VE}=\mathrm{Bq} / \mathrm{kg} \mathrm{WA}=\mathrm{Bq} / \mathrm{L}$. Values for elemental uranium are reported in $\mu \mathrm{rg} / \mathrm{filter}, \mathrm{g}$, or $\mathrm{mL}$.

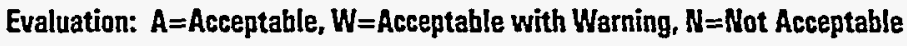
$p \mathrm{Ci}=\mathrm{Bq} \times 27$ 
QAP44 Results by Laboratory

Lab: NM Environmental Evaluation Group, Carlsbad, NM

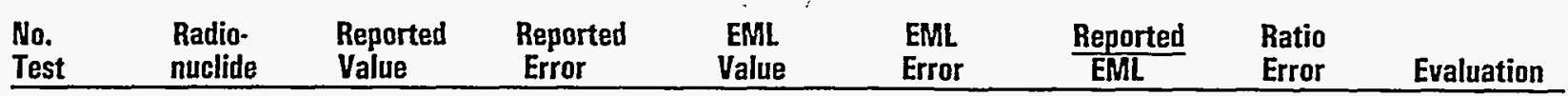

Matrix: Al

$\begin{array}{llrlrllll}1 & \text { AM241 } & 0.186 & 0.012 & 0.189 & 0.007 & 0.980 & 0.07 & \text { A } \\ 1 & \text { CD 60 } & 20.400 & 0.600 & 29.500 & 2.900 & 0.690 & 0.07 & \text { N } \\ 1 & \text { CS137 } & 4.500 & 0.260 & 6.640 & 0.700 & 0.680 & 0.08 & \text { N. } \\ 1 & \text { GA 1 } & 1.890 & 0.060 & 1.620 & 0.150 & 1.170 & 0.11 & \text { A } \\ 1 & \text { GB 2 } & 1.870 & 0.090 & 1.770 & 0.150 & 1.060 & 0.10 & \text { A } \\ 1 & \text { PU238 } & 0.108 & 0.007 & 0.096 & 0.002 & 1.120 & 0.08 & \text { A } \\ 1 & \text { PU239 } & 0.102 & 0.007 & 0.093 & 0.003 & 1.100 & 0.08 & \text { A } \\ 1 & \text { U 234 } & 0.055 & 0.004 & 0.052 & 0.002 & 1.060 & 0.08 & \text { A } \\ 1 & \text { U 238 } & 0.060 & 0.005 & 0.053 & 0.002 & 1.130 & 0.10 & \text { A }\end{array}$

Matrix: So

$\begin{array}{llrrrr}1 & \text { AM241 } & 4.900 & 0.800 & 3.690 & 0.454 \\ 1 & \text { PU238 } & 43.000 & 2.000 & 43.000 & 2.44 \\ 1 & \text { PU239 } & 22.000 & 1.000 & 9.230 & 0.34 \\ 1 & \text { U 234 } & 36.000 & 1.000 & 34.200 & 3.52 \\ 1 & \text { U 238 } & 36.000 & 1.000 & 35.900 & 3.63\end{array}$

Matrix: WA

$\begin{array}{llrr}1 & \text { AM241 } & 0.735 & 0.040 \\ 1 & \text { CO 60 } & 34.500 & 0.300 \\ 1 & \text { CS137 } & 46.900 & 0.500 \\ 1 & \text { GA 1 } & 1740.000 & 95.000 \\ 1 & \text { GB 2 } & 872.000 & 43.000 \\ 1 & \text { PU238 } & 0.940 & 0.038 \\ 1 & \text { PU239 } & 0.711 & 0.031 \\ 1 & \text { U 234 } & 0.274 & 0.017 \\ 1 & \text { U 238 } & 0.294 & 0.018\end{array}$

0.040

0.300

95.000

43.000

0.038

0.031

0.018

0.766
32.800
38.300
1850.000
744.000
0.982
0.772
0.274
0.275

0.960
1.050
1.230
0.940
1.170
0.960
0.920
1.000
1.070

1.330

1.000

2.380

1.050

1.000

0.27

0.07

0.14

0.11

0.11

$$
\begin{aligned}
& \text { A } \\
& \text { A } \\
& \text { N } \\
& \text { A } \\
& \text { A }
\end{aligned}
$$

0.05
0.02
0.03
0.11
0.13
0.08
0.08
0.09
0.12

$A$
$A$
$W$
$A$
$A$
$A$
$A$
$A$
$A$ 


\section{QAP44 Results by Laboratory}

Lab: NR Naval Reactors Facility Chemistry, Scoville, ID

No.

Test

Radionuclide

Reported

Value

Matrix: So

$1 \quad \operatorname{cs} 137$

$1 \quad K 40$

312.000

407.000

62.000

81.000

359.000

465.000

10.000

30.000

Matrix: VE

$\begin{array}{rrr}1 & C 060 & 47.700 \\ 1 & C S 137 & 799.000\end{array}$

$\begin{array}{ll}\text { CS137 } & 799.000 \\ \mathrm{~K} 40 & 918.000\end{array}$
Reported

$\begin{array}{rr}9.500 & 59.700 \\ 160.000 & 944.000 \\ 184.000 & 1030.000\end{array}$

184.000

0.963
16.200
33.000

33.000
EML

Error

Reported

EML

Ratio

Error

Evaluation

0.850

0.870

0.880

0.17

0.18

W

A

$K 40.018 .000 .000$

0.890

0.16
0.17
0.18

w

W

A

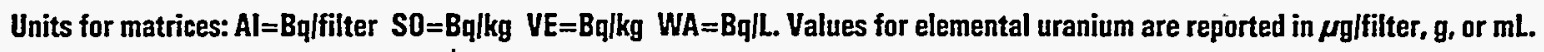




\section{QAP44 Results by Laboratory}

Lab: OB OBG Laboratories, East Syracuse, NY

\begin{tabular}{lllllllll} 
No. & Radio & Reported & Reported & EML & EML & Reported & Ratio \\
Test & nuclide & Value & Error & Value & Error & EML & Error & Evaluation \\
\hline
\end{tabular}

Matrix: Al

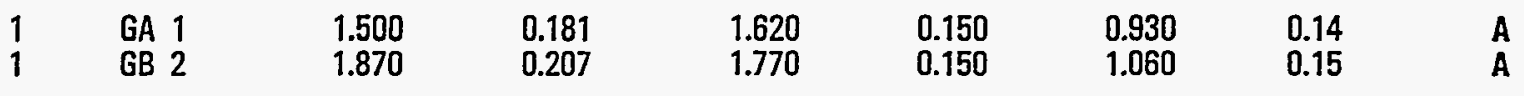

Matrix:' So

$1 \quad 0234$

$1 \quad$ U 238

14.100

14.100

0.000

0.000

34.200

35.900

3.520

3.630

Matrix: WA

$1 \quad$ GA $1 \quad 2140.000$

1 GB $2 \quad 561.000$

81.600

37.600

1850.000

744.000

185.000

74.000

1.160

0.750

0.410

0.390

0.04

0.04

W

A

A


QAP44 Results by Laboratory

Lab: OD ORNL, Radiobioassay Lab

\begin{tabular}{llllllll} 
No. & $\begin{array}{l}\text { Radio- } \\
\text { Test }\end{array}$ & $\begin{array}{l}\text { Reported } \\
\text { nalide }\end{array}$ & $\begin{array}{l}\text { Reported } \\
\text { Error }\end{array}$ & $\begin{array}{l}\text { EML } \\
\text { Value }\end{array}$ & $\begin{array}{l}\text { EML } \\
\text { Error }\end{array}$ & $\frac{\text { Reported }}{\text { EML }}$ & $\begin{array}{l}\text { Ratio } \\
\text { Error }\end{array}$ \\
\hline
\end{tabular}

Matrix: Al

$\begin{array}{lrrrrrrr}\text { AM241 } & 0.353 & 0.057 & 0.189 & 0.007 & 1.870 & 0.31 & \text { W } \\ \text { CE144 } & 25.100 & 0.720 & 33.300 & 3.300 & 0.750 & 0.08 & \text { A } \\ \text { CO 57 } & 7.490 & 0.181 & 8.900 & 0.900 & 0.840 & 0.09 & \text { A } \\ \text { CO 60 } & 27.100 & 0.290 & 29.500 & 2.900 & 0.920 & 0.09 & \text { A } \\ \text { CS134 } & 14.000 & 0.422 & 14.700 & 1.460 & 0.950 & 0.10 & . A \\ \text { CS137 } & 6.410 & 0.379 & 6.640 & 0.700 & 0.970 & 0.12 & \text { A } \\ \text { GA 1 } & 1.510 & 0.050 & 1.620 & 0.150 & 0.930 & 0.09 & \text { A } \\ \text { GB 2 } & 2.180 & 0.060 & 1.770 & 0.150 & 1.230 & 0.11 & \text { A } \\ \text { MN 54 } & 3.160 & 0.214 & 3.440 & 0.380 & 0.920 & 0.12 & \text { A } \\ \text { RU106 } & 11.400 & 0.635 & 11.600 & 1.440 & 0.980 & 0.13 & \text { A } \\ \text { SB125 } & 9.560 & 0.377 & 9.780 & 1.030 & 0.980 & 0.11 & \text { A }\end{array}$

Matrix: WA

$\begin{array}{llrrrrrrr}1 & \text { AM241 } & 0.679 & 0.072 & 0.766 & 0.013 & 0.890 & 0.10 & \text { A } \\ 1 & \text { H 3 } & 207.000 & 15.700 & 251.000 & 11.400 & 0.830 & 0.07 & \text { W } \\ 1 & \text { PU238 } & 0.881 & 0.091 & 0.982 & 0.074 & 0.900 & 0.11 & \text { W } \\ 1 & \text { PU239 } & 0.725 & 0.075 & 0.772 & 0.056 & 0.940 & 0.12 & A \\ 1 & \text { U 234 } & 0.280 & 0.024 & 0.274 & 0.019 & 1.020 & 0.11 & A \\ 1 & \text { U 238 } & 0.278 & 0.024 & 0.275 & 0.024 & 1.010 & 0.13 & A\end{array}$

Units for matrices: $\mathrm{Al}=\mathrm{Bq} /$ filter $\mathrm{SO}=\mathrm{Bq} / \mathrm{kg} \mathrm{VE}=\mathrm{Bq} / \mathrm{kg} \mathrm{WA}=\mathrm{Bq} / \mathrm{L}$. Values for elemental uranium are reported in $\mu \mathrm{g} / \mathrm{filter}, \mathrm{g}$, or $\mathrm{mL}$.

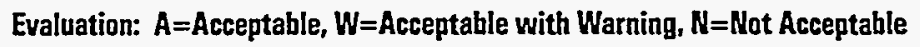
$\mathrm{pCi}=\mathrm{Bq} \times 27$ 


\section{QAP44 Results by Laboratory}

Lab: OL ORNL Environmental Sciences Div.

\begin{tabular}{|c|c|c|c|c|c|c|c|c|}
\hline $\begin{array}{l}\text { No. } \\
\text { Test } \\
\end{array}$ & $\begin{array}{l}\text { Radio- } \\
\text { nuclide }\end{array}$ & $\begin{array}{l}\text { Reported } \\
\text { Value }\end{array}$ & $\begin{array}{l}\text { Reported } \\
\text { Error }\end{array}$ & $\begin{array}{c}\text { EML } \\
\text { Value }\end{array}$ & $\begin{array}{l}\text { EML } \\
\text { Error }\end{array}$ & $\frac{\text { Reported }}{\text { EML }}$ & $\begin{array}{l}\text { Ratio } \\
\text { Error }\end{array}$ & Evaluat \\
\hline Matrix: & Al & & & & & & & \\
\hline $\begin{array}{l}1 \\
1 \\
1 \\
1 \\
1 \\
1 \\
1 \\
1\end{array}$ & $\begin{array}{l}\text { CE144 } \\
\text { CO } 57 \\
\text { CO 60 } \\
\text { CS134 } \\
\text { CS137 } \\
\text { MN 54 } \\
\text { RU106 } \\
\text { SB125 }\end{array}$ & $\begin{array}{r}23.400 \\
6.890 \\
25.600 \\
11.200 \\
6.060 \\
3.140 \\
9.000 \\
8.600\end{array}$ & $\begin{array}{l}1.720 \\
0.190 \\
0.400 \\
0.960 \\
0.190 \\
0.100 \\
0.930 \\
0.540\end{array}$ & $\begin{array}{r}33.300 \\
8.900 \\
29.500 \\
14.700 \\
6.640 \\
3.440 \\
11.600 \\
9.780\end{array}$ & $\begin{array}{l}3.300 \\
0.900 \\
2.900 \\
1.460 \\
0.700 \\
0.380 \\
1.440 \\
1.030\end{array}$ & $\begin{array}{l}0.700 \\
0.770 \\
0.870 \\
0.760 \\
0.910 \\
0.910 \\
0.780 \\
0.880\end{array}$ & $\begin{array}{l}0.09 \\
0.08 \\
0.09 \\
0.10 \\
0.10 \\
0.11 \\
0.13 \\
0.11\end{array}$ & $\begin{array}{c}\mathbf{A} \\
\mathbf{A} \\
\mathbf{A} \\
\mathbf{W} \\
\mathbf{A}\end{array}$ \\
\hline
\end{tabular}

Matrix: So

$\begin{array}{lllllllll}1 & \text { CS137 } & 391.000 & 11.300 & 359.000 & 10.000 & 1.090 & 0.04 & \text { A } \\ 1 & K 40 & 524.000 & 36.500 & 465.000 & 30.000 & 1.130 & 0.11 & \text { A }\end{array}$

Matrix: VE

$\begin{array}{llrrrrrrr}1 & \text { CO 60 } & 64.100 & 2.620 & 59.700 & 0.963 & 1.070 & 0.05 & \text { A } \\ 1 & \text { CS137 } & 1110.000 & 33.000 & 944.000 & 16.200 & 1.180 & 0.04 & \text { A } \\ 1 & \text { K 40 } & 1200.000 & 75.400 & 1030.000 & 33.000 & 1.170 & 0.08 & \text { A }\end{array}$

Matrix: WA

$\begin{array}{lllllllll}1 & \text { CO 60 } & 32.800 & 1.160 & 32.800 & 0.580 & 1.000 & 0.04 & \text { A } \\ 1 & \text { CS137 } & 42.000 & 1.370 & 38.300 & 0.881 & 1.100 & 0.04 & \text { A } \\ 1 & \text { MN 54 } & 42.700 & 1.250 & 38.400 & 1.160 & 1.110 & 0.05 & \text { A }\end{array}$

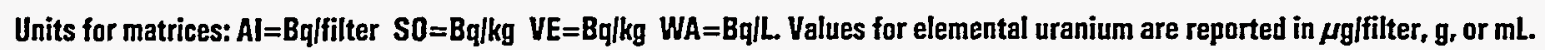

Evaluation: $A=$ Acceptable, $W=$ Acceptable with Warning, $N=$ Not Acceptable

$p C i=B q \times 27$ 
OAP44 Results by Laboratory

Lab: OR Oak Ridge National Lab

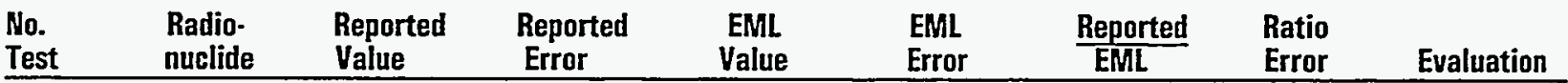

Matrix: Al

$\begin{array}{llrlrllll}1 & \text { AM241 } & 0.190 & 0.020 & 0.189 & 0.007 & 1.010 & 0.11 & \text { A } \\ 1 & \text { CE144 } & 27.000 & 1.000 & 33.300 & 3.300 & 0.810 & 0.09 & \text { A } \\ 1 & \text { CO 57 } & 7.800 & 0.200 & 8.900 & 0.900 & 0.880 & 0.09 & \text { A } \\ 1 & \text { CO 60 } & 29.000 & 1.000 & 29.500 & 2.900 & 0.980 & 0.10 & \text { A } \\ 1 & \text { CS134 } & 13.000 & 1.000 & 14.700 & 1.460 & 0.880 & 0.11 & \text { A } \\ 1 & \text { CS137 } & 6.700 & 0.400 & 6.640 & 0.700 & 1.010 & 0.12 & \text { A } \\ 1 & \text { MN 54 } & 3.400 & 0.400 & 3.440 & 0.380 & 0.990 & 0.16 & \text { A } \\ 1 & \text { PU238 } & 0.093 & 0.010 & 0.096 & 0.002 & 0.970 & 0.11 & \text { A } \\ 1 & \text { PU239 } & 0.094 & 0.010 & 0.093 & 0.003 & 1.010 & 0.11 & \text { A } \\ 1 & \text { RU106 } & 11.000 & 3.000 & 11.600 & 1.440 & 0.950 & 0.28 & \text { A } \\ 1 & \text { SB125 } & 10.000 & 1.000 & 9.780 & 1.030 & 1.020 & 0.15 & \text { A } \\ 1 & \text { SR 90 } & 0.830 & 0.080 & 1.060 & 0.037 & 0.780 & 0.08 & \text { W } \\ 1 & \text { U BO } & 0.133 & 0.020 & 0.107 & 0.003 & 1.240 & \mathbf{0 . 1 9} & \text { A }\end{array}$

Matrix: SO

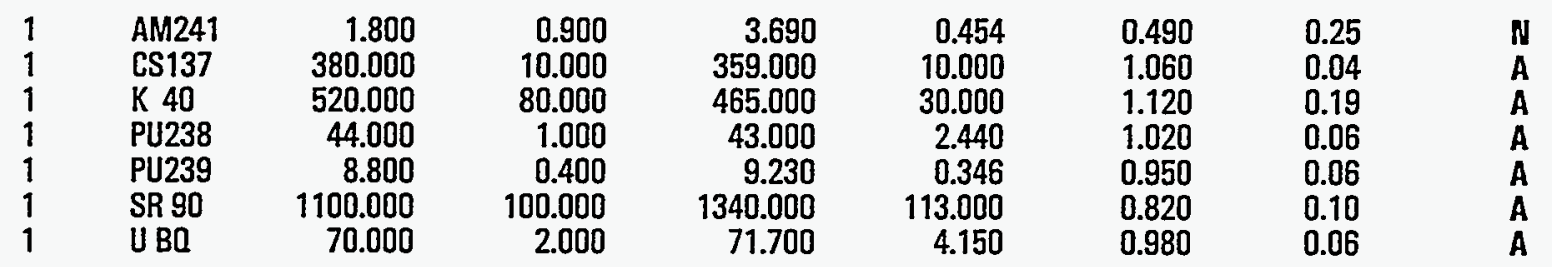

Matrix: VE

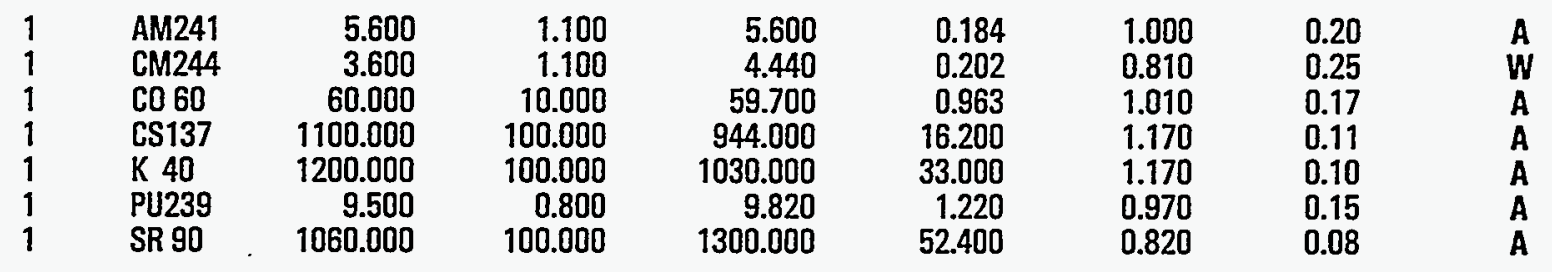

Matrix: WA

$\begin{array}{rlrr}1 & \text { AM2241 } & 0.780 & 0.080 \\ 1 & \text { CO 60 } & 32.000 & 1.000 \\ 1 & \text { CS137 } & 40.000 & 1.000 \\ 1 & \text { H } 3 & 220.000 & 30.000 \\ 1 & \text { MN } 54 & 41.000 & 1.000 \\ 1 & \text { PU238 } & 0.970 & 0.050 \\ 1 & \text { PU239 } & 0.730 & 0.050 \\ 1 & \text { SR 90 } & 1.370 & 0.300 \\ 1 & \text { U BO } & 0.690 & 0.080\end{array}$

$\begin{array}{rrr}0.766 & 0.013 & 1.020 \\ 32.800 & 0.580 & 0.980 \\ 38.300 & 0.881 & 1.040 \\ 251.000 & 11.400 & 0.880 \\ 38.400 & 1.160 & 1.070 \\ 0.982 & 0.074 & 0.990 \\ 0.772 & 0.056 & 0.950 \\ 1.450 & 0.034 & 0.950 \\ 0.559 & 0.047 & 1.230\end{array}$

0.11
0.04
0.04
0.13
0.04
0.09
0.09
0.21
0.18

$A$
$A$
$A$
$A$
$A$
$A$
$A$
$A$
$W$

Units for matrices: $A l=B q / f i l t e r ~ S B=B q / k g V E=B q / k g ~ W A=B q / L$. Values for elemental uranium are reported in $\mu \mathrm{g} / \mathrm{filter}, \mathrm{g}$, or $\mathrm{mL}$.

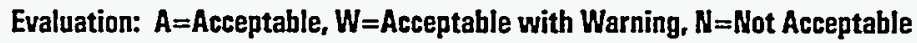
$\mathrm{pCi}=\mathrm{Bq} \times 27$ 


\section{QAP44 Results by Laboratory}

Lab: OS Oregon Health Division Radiation Controls Section, Portland

\begin{tabular}{lllllllll} 
No. & Radio. & Reported & Reported & EML & EML & Reported & Ratio \\
Test & nuclide & Value & Error & Value & Error & $\frac{\text { EML }}{\text { Error }}$ & Evaluation \\
\hline
\end{tabular}

Matrix: So

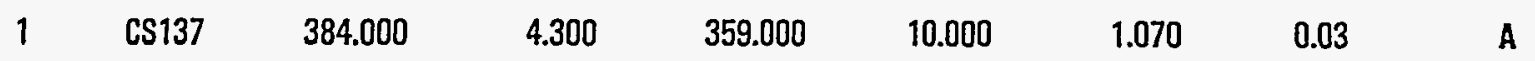

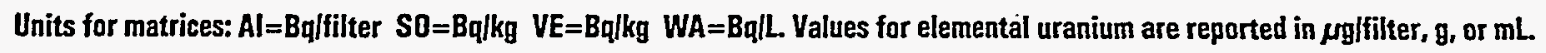
Evaluation: $A=A c c e p t a b l e, W=A c c e p t a b l e$ with Warning, $N=$ Not Acceptable $p C i=B q \times 27$ 


\section{QAP44 Results by Laboratory}

Lab: OT ORNL Radioactive Material Analysis Lab

\begin{tabular}{lllllllll}
$\begin{array}{l}\text { No. } \\
\text { Test }\end{array}$ & $\begin{array}{l}\text { Radio- } \\
\text { nuclide }\end{array}$ & $\begin{array}{l}\text { Reported } \\
\text { Value }\end{array}$ & $\begin{array}{c}\text { Reported } \\
\text { Error }\end{array}$ & $\begin{array}{c}\text { EML } \\
\text { Value }\end{array}$ & $\begin{array}{c}\text { EML } \\
\text { Error }\end{array}$ & $\frac{\text { Reported }}{\text { EML }}$ & $\begin{array}{l}\text { Ratio } \\
\text { Error }\end{array}$ Evaluation \\
\hline
\end{tabular}

Matrix: Al

$\begin{array}{llrl}1 & \text { AM241 } & 0.280 & 0.080 \\ 1 & \text { CE144 } & 27.000 & 1.000 \\ 1 & \text { CD 57 } & 7.200 & 0.100 \\ 1 & \text { CO 60 } & 26.000 & 1.000 \\ 1 & \text { CS134 } & 13.000 & 1.000 \\ 1 & \text { CS137 } & 6.200 & 0.200 \\ 1 & \text { GA 1 } & 0.630 & 0.080 \\ 1 & \text { GB 2 } & 0.970 & 0.140 \\ 1 & \text { MN 54 } & 3.100 & 0.200 \\ 1 & \text { PU238 } & 0.070 & 0.020 \\ 1 & \text { PU239 } & 0.094 & 0.028 \\ 1 & \text { RU106 } & 9.800 & 1.100 \\ 1 & \text { SB125 } & 9.200 & . \\ 1 & \text { SR 90 } & 0.380 & 0.190 \\ 1 & \text { U BO } & 0.680 & 0.070\end{array}$

$\begin{array}{lrll}0.080 & 0.189 & 0.007 & 1.480 \\ 1.000 & 33.300 & 3.300 & 0.810 \\ 0.100 & 8.900 & 0.900 & 0.810 \\ 1.000 & 29.500 & 2.900 & 0.880 \\ 1.000 & 14.700 & 1.460 & 0.880 \\ 0.200 & 6.640 & 0.700 & 0.930 \\ 0.080 & 1.620 & 0.150 & 0.390 \\ 0.140 & 1.770 & 0.150 & 0.550 \\ 0.200 & 3.440 & 0.380 & 0.900 \\ 0.026 & 0.096 & 0.002 & 0.730 \\ 0.028 & 0.093 & 0.003 & 1.010 \\ 1.100 & 11.600 & 1.440 & 0.850 \\ 0.400 & 9.780 & 1.030 & 0.940 \\ 0.190 & 1.060 & 0.037 & 0.360 \\ 0.070 & 0.107 & 0.003 & 6.360\end{array}$

0.43
0.09
0.08
0.09
0.11
0.10
0.06
0.09
0.12
0.27
0.30
0.14
0.11
0.18
0.67

W
A
$A$
$A$
$A$
$A$
$A$
$N$
$N$
A
$W$
$A$
$A$
$A$
N
N

Matrix: So

1
1
1
1
1

$\begin{array}{lr}\text { CS137 } & 380.000 \\ \text { K 40 } & 480.000 \\ \text { PU238 } & 50.000 \\ \text { PU239 } & 13.000 \\ \text { SR 90 } & 1200.000\end{array}$

10.000
60.000
6.000
3.000
100.000

$\begin{array}{rr}359.000 & 10.000 \\ 465.000 & 30.000 \\ 43.000 & 2.440 \\ 9.230 & 0.346 \\ 1340.000 & 113.000\end{array}$

1.060
1.030
1.160
1.410
0.900

0.04

0.15

0.15

0.33

0.11

$\mathbf{A}$
$\mathbf{A}$
$\mathbf{A}$
$\mathbf{W}$
$\mathbf{A}$

Matrix: VE

$\begin{array}{llrrrrrrr}1 & \text { CO 60 } & 60.000 & 8.000 & 59.700 & 0.963 & 1.010 & 0.14 & \text { A } \\ 1 & \text { CS137 } & 1000.000 & 100.000 & 944.000 & 16.200 & 1.060 & 0.11 & \text { A } \\ 1 & \text { K 40 } & 1100.000 & 100.000 & 1030.000 & 33.000 & 1.070 & 0.10 & \text { A } \\ 1 & \text { PU239 } & 20.000 & 6.000 & 9.820 & 1.220 & 2.040 & 0.66 & \text { N } \\ 1 & \text { SR 90 } & 1400.000 & 100.000 & 1300.000 & 52.400 & 1.080 & 0.09 & \text { A }\end{array}$

Matrix: WA

$\begin{array}{llrr}1 & \text { AM241 } & 1.100 & 0.300 \\ 1 & \text { CO 60 } & 34.000 & 1.000 \\ 1 & \text { CS137 } & 43.000 & 1.000 \\ 1 & \text { GA 1 } & 1900.000 & 100.000 \\ 1 & \text { GB 2 } & 910.000 & 60.000 \\ 1 & \text { H 3 } & 370.000 & 30.000 \\ 1 & \text { MN 54 } & 44.000 & 1.000 \\ 1 & \text { PU238 } & 1.000 & 0.100 \\ 1 & \text { PU239 } & 0.740 & 0.110 \\ 1 & \text { SR 90 } & 2.400 & 0.600 \\ 1 & \text { U BQ } & 0.400 & 0.160\end{array}$

$\begin{array}{rr}0.766 & 0.013 \\ 32.800 & 0.580 \\ 38.300 & 0.881 \\ 1850.000 & 185.000 \\ 744.000 & 74.000 \\ 251.000 & 11.400 \\ 38.400 & 1.160 \\ 0.982 & 0.074 \\ 0.772 & 0.056 \\ 1.450 & 0.034 \\ 0.559 & 0.047\end{array}$

1.440
1.040
1.120
1.030
1.220
1.470
1.150
1.020
0.960
1.660
0.720

0.39

0.04

0.04

0.12

0.15

0.14

0.04

0.13

0.16

0.42

0.29

A
A
$\mathbf{A}$
$\mathbf{N}$
$\mathbf{A}$

Units for matrices: $A \mid=B q / f i l t e r ~ S O=B q / k g ~ V E=B q / k g ~ W A=B q / L$. Values for elemental uranium are reported in $\mu g / f i l t e r, g$, or $\mathrm{mL}$.

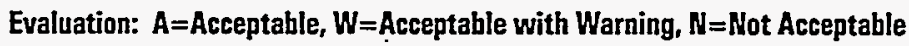
$\mathrm{pCi}=\mathrm{Bq} \times 27$ 


\section{OAP44 Results by Laboratory}

Lab: PA Mason \& Hanger-Silas Mason Co., Inc., Battelle Pantex, Amarillo, TX

\begin{tabular}{lllcllll} 
No. & Radio & Reported & Reported & EML & EML & Reported & Ratio \\
Test & nuclide & Value & Error & Value & Error & EML & Error \\
\hline
\end{tabular}

Matrix: Al

$\begin{array}{lllllllll}1 & \text { GA 1 } & 1.490 & 0.040 & 1.620 & 0.150 & 0.920 & 0.09 & \text { A } \\ 1 & \text { GB 2 } & 1.760 & 0.030 & 1.770 & 0.150 & 0.990 & 0.09 & \text { A } \\ 1 & \text { PU239 } & 0.100 & 0.013 & 0.093 & 0.003 & 1.080 & 0.14 & \text { A } \\ 1 & \text { U 234 } & 0.027 & 0.067 & 0.052 & 0.002 & 0.520 & 1.30 & \mathbf{N} \\ 1 & \text { U 238 } & 0.023 & 0.067 & 0.053 & 0.002 & 0.440 & 1.26 & \mathbf{N}\end{array}$

Matrix: So

$\begin{array}{lllllllll}1 & \text { PU239 } & 10.700 & 1.480 & 9.230 & 0.346 & 1.160 & 0.17 & \text { A } \\ 1 & \text { U } 234 & 29.300 & 2.960 & 34.200 & 3.520 & 0.860 & 0.12 & \text { A } \\ 1 & \text { U 238 } & 27.400 & 2.590 & 35.900 & 3.630 & 0.760 & 0.11 & \text { A }\end{array}$

Matrix: VE

$1 \quad$ PU239

7.410

3.700

9.820

1.220

0.760

0.39

w

Matrix: WA

$\begin{array}{llrrrrrrr}1 & \text { GA 1 } & 1930.000 & 211.000 & 1850.000 & 185.000 & 1.040 & 0.16 & \text { A } \\ 1 & \text { GB 2 } & 518.000 & 66.700 & 744.000 & 74.000 & 0.700 & 0.11 & \text { N } \\ 1 & \text { H 3 } & 222.000 & 7.040 & 251.000 & 11.400 & 0.880 & 0.05 & \text { A } \\ 1 & \text { PU239 } & 0.815 & 0.119 & 0.772 & 0.056 & 1.060 & 0.17 & \text { A } \\ 1 & \text { U 234 } & 0.310 & 0.100 & 0.274 & 0.019 & 1.130 & 0.37 & \text { A } \\ 1 & \text { U 238 } & 0.300 & 0.096 & 0.275 & 0.024 & 1.090 & 0.36 & \text { A }\end{array}$

Units for matrices: $A \mathrm{~A}=\mathrm{Bq} / \mathrm{filter} \mathrm{SB}=\mathrm{Bq} / \mathrm{kg} \mathrm{VE}=\mathrm{Bq} / \mathrm{kg} W \mathrm{~W}=\mathrm{Bq} / \mathrm{L}$. Values for elemental uranium are reported in $\mu \mathrm{g} / \mathrm{filter}, \mathrm{g}$, or $\mathrm{mL}$. 


\section{QAP44 Results by Laboratory}

Lab: PB Mason \& Hanger-Silas Mason Co., Inc., Battelle Pantex, Amarillo, TX

\begin{tabular}{|c|c|c|c|c|c|c|c|c|}
\hline $\begin{array}{l}\text { No. } \\
\text { Test }\end{array}$ & $\begin{array}{l}\text { Radio- } \\
\text { nuclide }\end{array}$ & $\begin{array}{l}\text { Reported } \\
\text { Value }\end{array}$ & $\begin{array}{l}\text { Reported } \\
\text { Error }\end{array}$ & $\begin{array}{c}\text { EML } \\
\text { Value }\end{array}$ & $\begin{array}{l}\text { EML } \\
\text { Error }\end{array}$ & $\frac{\text { Reported }}{\text { EML }}$ & $\begin{array}{l}\text { Ratio } \\
\text { Error }\end{array}$ & Evaluation \\
\hline
\end{tabular}

Matrix: So

$\begin{array}{lllllllll}1 & \text { PU239 } & 8.890 & 1.480 & 9.230 & 0.346 & 0.960 & 0.16 & \text { A } \\ 1 & \text { U } 234 & 34.400 & 2.590 & 34.200 & 3.520 & 1.010 & 0.13 & \text { A } \\ 1 & \text { U } 238 & 34.800 & 2.590 & 35.900 & 3.630 & 0.970 & 0.12 & \text { A }\end{array}$

Matrix: VE

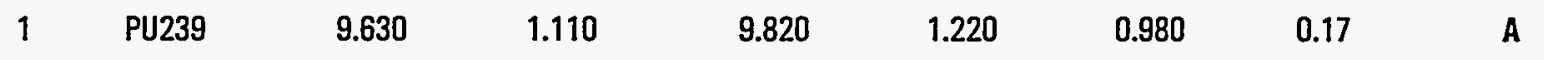

Matrix: WA

$\begin{array}{llrrrrrrr}2 & \text { GA 1 } & 2000.000 & 64.200 & 1850.000 & 185.000 & 1.080 & 0.11 & \text { A } \\ 2 & \text { GB 2 } & 506.000 & 26.900 & 744.000 & 74.000 & 0.680 & 0.08 & \text { N } \\ 1 & \text { H 3 } & 222.000 & 11.700 & 251.000 & 11.400 & 0.880 & 0.06 & \text { A } \\ 1 & \text { PU239 } & 0.731 & 0.082 & 0.772 & 0.056 & 0.950 & 0.13 & \text { A } \\ 1 & \text { U 234 } & 0.281 & 0.039 & 0.274 & 0.019 & 1.030 & 0.16 & \text { A } \\ 1 & \text { U 238 } & 0.263 & 0.037 & 0.275 & 0.024 & 0.960 & 0.16 & \text { A }\end{array}$

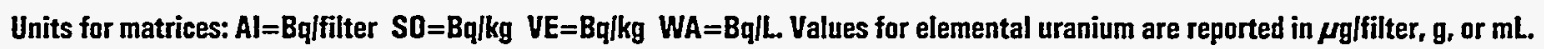

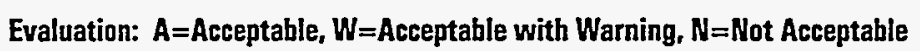

$\mathrm{pCi}=\mathrm{Bq} \times 27$ 
OAP44 Results by Laboratory

Lab: PI Lockheed Martin Specialty Components, Largo, FL

\begin{tabular}{|c|c|c|c|c|c|c|c|c|}
\hline $\begin{array}{l}\text { No. } \\
\text { Test }\end{array}$ & $\begin{array}{l}\text { Radio- } \\
\text { nuclide }\end{array}$ & $\begin{array}{l}\text { Reported } \\
\text { Value }\end{array}$ & $\begin{array}{l}\text { Reported } \\
\text { Error }\end{array}$ & $\begin{array}{c}\text { EML } \\
\text { Value }\end{array}$ & $\begin{array}{l}\text { EML } \\
\text { Error }\end{array}$ & $\frac{\text { Reported }}{\text { EML }}$ & $\begin{array}{l}\text { Ratio } \\
\text { Error }\end{array}$ & ation \\
\hline
\end{tabular}

Matrix: Al

$\begin{array}{lllllllll}1 & \text { PU238 } & 0.096 & 0.008 & 0.096 & 0.002 & 1.000 & 0.09 & A \\ 1 & \text { PU239 } & 0.100 & 0.008 & 0.093 & 0.003 & 1.080 & 0.09 & A\end{array}$

Matrix: $\quad$ So

$\begin{array}{llllrrrrr}1 & \text { PU238 } & 47.000 & 4.500 & 43.000 & 2.440 & 1.090 & 0.12 & \text { A } \\ 1 & \text { PU239 } & 10.000 & 1.300 & 9.230 & 0.346 & 1.080 & 0.15 & \text { A }\end{array}$

Matrix: WA

$\begin{array}{llrrrrrrr}1 & \text { H 3 } 3 & 214.000 & 11.000 & 251.000 & 11.400 & 0.850 & 0.06 & \text { A } \\ 1 & \text { PU238 } & 0.950 & 0.070 & 0.982 & 0.074 & 0.970 & 0.10 & \text { A } \\ 1 & \text { PU239 } & 0.740 & 0.060 & 0.772 & 0.056 & 0.960 & 0.10 & \text { A. } \\ 1 & \text { U 234 } & 0.290 & 0.030 & 0.274 & 0.019 & 1.060 & 0.13 & \text { A } \\ 1 & \text { U 238 } & 0.310 & 0.030 & 0.275 & 0.024 & 1.130 & 0.15 & \text { A }\end{array}$

Units for matrices: $A l=B q \mid$ filter $S O=B q / \mathrm{kg}$ VE $=B q / \mathrm{kg} W A=B q / L$. Values for elemental uranium are reported in $\mu \mathrm{g} /$ filter, $\mathrm{g}$, or $\mathrm{mL}$.

Evaluation: $A=A c c e p t a b l e, W=A c c e p t a b l e$ with Warning, $N=$ Not Acceptable $\mathrm{pCi}=\mathrm{Bq} \times 27$ 


\section{QAP44 Results by Laboratory}

Lab: RA V. G. Khlopin Radium Institute, St. Petersburg, Russia

No.

Radio-

Reported Reported

EML

EML

Reported

Ratio

Test nuclide Value

Error

Value

Error EML

Error

Evaluation

Matrix: Al

$\begin{array}{llr}1 & \text { CE144 } & 31.30 \\ 1 & \text { CO 57 } & 9.250 \\ 1 & \text { CO 60 } & 22.900 \\ 1 & \text { CS134 } & 14.200 \\ 1 & \text { CS137 } & 6.210 \\ 1 & \text { MN 54 } & 2.900 \\ 1 & \text { PU238 } & 0.100 \\ 1 & \text { PU239 } & 0.100 \\ 1 & \text { RU106 } & 12.300 \\ 1 & \text { SB125 } & 11.000 \\ 1 & \text { SR 90 } & 0.300\end{array}$

1.900
0.390
0.800
0.600
0.320
0.140
0.010
0.010
1.400
0.400
0.100

33.300

8.900

29.500

14.700

6.640

3.440

0.096

0.093

11.600

9.780

1.060

Matrix: SO

1
1
1
2
1
2
1
2
1

$\begin{array}{lr}\text { CS137 } & 368.000 \\ \text { K 40 } & 550.000 \\ \text { PU238 } & 47.000 \\ & 45.000 \\ \text { PU239 } & 9.000 \\ & 9.000 \\ \text { SR 90 } & 700.000 \\ & 650.000 \\ \text { U UG } & 2.500\end{array}$

368.000

(50.000

(57.000

9.000

9.000

2.500

11.000
50.000
2.000
2.000
1.000
1.000
100.000
100.000
0.220

359.000
465.000
43.000
43.000
9.230
9.230
1340.000
1340.000

1340.000
2.900

3.300
0.900
2.900
1.460
0.700
0.380
0.002
0.003
1.440
1.030
0.037

0.940

1.040

0.780

0.970

0.940

0.840

1.040

1.080

1.060

1.130

0.280

10.000
30.000
2.440
2.440
0.346
0.346
113.000
113.000
0.290

1.030

1.180

1.090

1.050

0.980

0.980

0.520

0.490

0.860

2.200
50.000
70.000
1.000
1.000
250.000
250.000

59.700
944.000
1030.000
9.820
9.820
1300.000
1300.000

0.963
16.200
33.000
1.220
1.220
52.400
52.400

0.930
1.060
1.020
1.120
1.020
1.310
1.460

0.04

0.06

0.08

0.17

0.16

0.20

1900.000

$\begin{array}{lr}\text { CO 60 } & 55.400 \\ \text { CS137 } & 1000.000 \\ \text { K 40 } & 1050.000 \\ \text { PU239 } & 11.000 \\ & 10.000 \\ \text { SR 90 } & 1700.000 \\ & 1900.000\end{array}$

250.000

0.20

Matrix: VE

$\begin{array}{ll}0.11 & \text { A } \\ 0.11 & \text { A } \\ 0.08 & \text { W } \\ 0.10 & A \\ 0.11 & A \\ 0.10 & \text { W } \\ 0.11 & A \\ 0.11 & A \\ 0.18 & A \\ 0.13 & \text { W } \\ 0.09 & \text { N }\end{array}$

$A$
$A$
$W$
$A$
$A$
$W$
$A$
$A$
$A$
$W$
$W$ 


\section{QAP44 Results by Laboratory}

Lab: RE Bechtel Nevada, Mercury, NV

\begin{tabular}{|c|c|c|c|c|c|c|c|}
\hline $\begin{array}{l}\text { Wo. } \\
\text { Test }\end{array}$ & $\begin{array}{l}\text { Radio. } \\
\text { nuclide }\end{array}$ & $\begin{array}{l}\text { Reported } \\
\text { Value }\end{array}$ & $\begin{array}{l}\text { Reported } \\
\text { Error }\end{array}$ & $\begin{array}{c}\text { EML } \\
\text { Value }\end{array}$ & $\begin{array}{l}\text { EML } \\
\text { Error }\end{array}$ & $\frac{\text { Reported }}{\text { EML }}$ & $\begin{array}{l}\text { Ratio } \\
\text { Error }\end{array}$ \\
\hline
\end{tabular}

Matrix: Al

\begin{tabular}{|c|c|c|c|c|c|c|}
\hline $\begin{array}{l}\text { AM241 } \\
\text { CE144 } \\
\text { CO } 57 \\
\text { CO } 60 \\
\text { CS134 } \\
\text { CS137 } \\
\text { GA } 1 \\
\text { GB } 2 \\
\text { MN 54 } \\
\text { PU238 } \\
\text { PU239 } \\
\text { SB125 } \\
\text { SR 90 } \\
\text { U 234 } \\
\text { U 238 } \\
\text { U BO }\end{array}$ & $\begin{array}{r}0.139 \\
24.600 \\
6.700 \\
24.900 \\
11.600 \\
5.470 \\
2.640 \\
5.350 \\
2.920 \\
0.074 \\
0.081 \\
7.860 \\
0.947 \\
0.051 \\
0.057 \\
0.113\end{array}$ & $\begin{array}{l}0.011 \\
3.200 \\
0.740 \\
2.370 \\
1.160 \\
0.710 \\
0.550 \\
0.840 \\
0.470 \\
0.008 \\
0.008 \\
1.340 \\
0.047 \\
0.006 \\
0.006 \\
0.006\end{array}$ & $\begin{array}{r}0.189 \\
33.300 \\
8.900 \\
29.500 \\
14.700 \\
6.640 \\
1.620 \\
1.770 \\
3.440 \\
0.096 \\
0.093 \\
9.780 \\
1.060 \\
0.052 \\
0.053 \\
0.107\end{array}$ & $\begin{array}{l}0.007 \\
3.300 \\
0.900 \\
2.900 \\
1.460 \\
0.700 \\
0.150 \\
0.150 \\
0.380 \\
0.002 \\
0.003 \\
1.030 \\
0.037 \\
0.002 \\
0.002 \\
0.003\end{array}$ & $\begin{array}{l}0.740 \\
0.740 \\
0.750 \\
0.840 \\
0.790 \\
0.820 \\
1.630 \\
3.020 \\
0.850 \\
0.770 \\
0.870 \\
0.800 \\
0.890 \\
0.990 \\
1.060 \\
1.060\end{array}$ & $\begin{array}{l}0.06 \\
0.12 \\
0.11 \\
0.12 \\
0.11 \\
0.14 \\
0.37 \\
0.54 \\
0.17 \\
0.08 \\
0.09 \\
0.16 \\
0.05 \\
0.12 \\
0.13 \\
0.06\end{array}$ \\
\hline
\end{tabular}

Matrix: SO

$\begin{array}{llrrrrrrr}1 & \text { CS137 } & 309.000 & 26.600 & 359.000 & 10.000 & 0.860 & 0.08 & \text { W } \\ 1 & \text { K 40 } & 392.000 & 50.900 & 465.000 & 30.000 & 0.840 & 0.12 & \text { A } \\ 1 & \text { PU238 } & 34.800 & 4.100 & 43.000 & 2.440 & 0.810 & 0.11 & \text { A } \\ 1 & \text { PU239 } & 8.470 & 1.140 & 9.230 & 0.346 & 0.920 & 0.13 & \text { A } \\ 1 & \text { SR 90 } & 1130.000 & 210.000 & 1340.000 & 113.000 & 0.840 & 0.17 & \text { A } \\ 1 & \text { U 234 } & 30.900 & 2.900 & 34.200 & 3.520 & 0.900 & 0.13 & \text { A } \\ 1 & \text { U 238 } & 33.100 & 3.100 & 35.900 & 3.630 & 0.920 & 0.13 & \text { A }\end{array}$

Matrix: VE

$\begin{array}{rlrrr}1 & \text { AM241 } & 7.150 & 0.650 & 5.600 \\ 1 & \text { CO 60 } & 62.200 & 9.330 & 59.700 \\ 1 & \text { CS137 } & 1080.000 & 90.000 & 944.000 \\ 1 & \text { K 40 } & 1120.000 & 146.000 & 1030.000 \\ 1 & \text { PU239 } & 8.380 & 0.730 & 9.820 \\ 1 & \text { SR 90 } & 1240.000 & 62.000 & 1300.000\end{array}$

Matrix: WA

$\begin{array}{llrrrrrrr}1 & \text { AM241 } & 0.750 & 0.077 & 0.766 & 0.013 & 0.980 & 0.10 & \text { A } \\ 1 & \text { CD 60 } & 33.200 & 3.980 & 32.800 & 0.580 & 1.010 & 0.12 & \text { A } \\ 1 & \text { CS137 } & 41.800 & 5.010 & 38.300 & 0.881 & 1.090 & 0.13 & \text { A } \\ 1 & \text { GA 1 } & 1950.000 & 98.000 & 1850.000 & 185.000 & 1.050 & 0.12 & \text { A } \\ 1 & \text { GB 2 } & 773.000 & 39.000 & 744.000 & 74.000 & 1.040 & 0.12 & \text { A } \\ 1 & \text { H 3 } & 208.000 & 20.000 & 251.000 & 11.400 & 0.830 & 0.09 & \text { W } \\ 1 & \text { MN 54 } & 41.300 & 4.540 & 38.400 & 1.160 & 1.080 & 0.12 & \text { A } \\ 1 & \text { PU238 } & 0.860 & 0.090 & 0.982 & 0.074 & 0.880 & 0.11 & \text { W } \\ \end{array}$

Units for matrices: $\mathrm{Al}=\mathrm{Bq}$ qfilter $\mathrm{SO}=\mathrm{Bq} / \mathrm{kg} V E=\mathrm{Bq} / \mathrm{kg} W \mathrm{~W}=\mathrm{Bq} / \mathrm{L}$. Values for elemental uranium are reported in $\mu \mathrm{g} / \mathrm{filt}$ er, $\mathrm{g}$, or $\mathrm{mL}$.

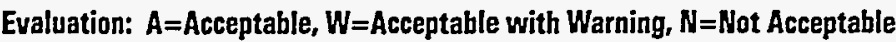

$\mathrm{pCi}=\mathrm{Bq} \times 27$ 


\section{QAP44 Results by Laboratory}

Lab: RE Bechtel Nevada, Mercury, NV

\begin{tabular}{lllllllll} 
No. & Radio- & Reported & Reported & EML & EML & Reported & Ratio \\
Test & nuclide & Value & Error & Value & Error & EML & Error & Evaluation \\
\hline
\end{tabular}

Matrix: WA

$\begin{array}{lllllllll}1 & \text { PU239 } & 0.696 & 0.075 & 0.772 & 0.056 & 0.900 & 0.12 & \text { A } \\ 1 & \text { SR 90 } & 1.470 & 0.150 & 1.450 & 0.034 & 1.010 & 0.11 & \text { A } \\ 1 & \text { U 234 } & 0.277 & 0.035 & 0.274 & 0.019 & 1.010 & 0.15 & \text { A } \\ 1 & \text { U 238 } & 0.290 & 0.036 & 0.275 & 0.024 & 1.050 & 0.16 & \text { A } \\ 1 & \text { U BQ } & 0.510 & 0.026 & 0.559 & 0.047 & 0.910 & 0.09 & \text { A }\end{array}$

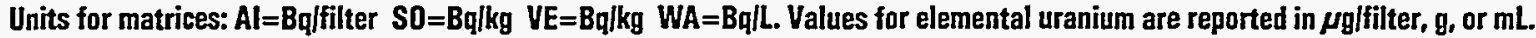




\section{QAP44 Results by Laboratory}

Lab: RF EG\&G Rocky Flats Plant, Golden

\begin{tabular}{|c|c|c|c|c|c|c|c|c|}
\hline $\begin{array}{l}\text { No. } \\
\text { Test }\end{array}$ & $\begin{array}{l}\text { Radio- } \\
\text { nuclide }\end{array}$ & $\begin{array}{l}\text { Reported } \\
\text { Value }\end{array}$ & $\begin{array}{l}\text { Reported } \\
\text { Error }\end{array}$ & $\begin{array}{c}\text { EML } \\
\text { Value }\end{array}$ & $\begin{array}{l}\text { EML } \\
\text { Error }\end{array}$ & $\frac{\text { Reported }}{\text { EML }}$ & $\begin{array}{l}\text { Ratio } \\
\text { Error }\end{array}$ & Evaluation \\
\hline
\end{tabular}

Matrix: Al

$\begin{array}{lllllllll}1 & \text { GA } 1 & 0.926 & 0.140 & 1.620 & 0.150 & 0.570 & 0.10 & N \\ 1 & \text { GB } 2 & 1.180 & 0.210 & 1.770 & 0.150 & 0.670 & 0.13 & \text { N }\end{array}$

Matrix: So

$\begin{array}{llrlrllll}1 & \text { AM2241 } & 4.240 & 0.460 & 3.690 & 0.454 & 1.150 & 0.19 & \text { A } \\ 1 & \text { PU239 } & 9.830 & 0.970 & 9.230 & 0.346 & 1.070 & 0.11 & \text { A } \\ 1 & \text { U 2234 } & 37.800 & 3.100 & 34.200 & 3.520 & 1.110 & 0.15 & \text { W } \\ 1 & \text { U 238 } & 37.400 & 3.100 & 35.900 & 3.630 & 1.040 & 0.14 & \text { A }\end{array}$

Matrix: VE

- 1 PU239

9.130

0.690

9.820

1.220

0.930

0.14

A

Matrix: WA

$\begin{array}{llr}1 & \text { AM241 } & 0.784 \\ 1 & \text { CO } 60 & 33.900 \\ 1 & \text { CS137 } & 42.200 \\ 1 & H 3 & 240.000 \\ 1 & \text { MN } 54 & 42.700 \\ 1 & \text { PU239 } & 0.763 \\ 1 & \text { U } 234 & 0.331 \\ 1 & \text { U 238 } & 0.316\end{array}$

0.042
1.100
1.600
5.300
1.500
0.043
0.023
0.023

$\begin{array}{rr}0.766 & 0.013 \\ 32.800 & 0.580 \\ 38.300 & 0.881 \\ 251.000 & 11.400 \\ 38.400 & 1.160 \\ 0.772 & 0.056 \\ 0.274 & . \\ 0.275 & 0.019 \\ 0.024\end{array}$

1.020
1.030
1.100
0.960
1.110
0.990
1.210
1.150

0.06
0.04
0.05
0.05
0.05
0.09
0.12
0.13

$A$
$A$
$A$
$A$
$A$
$A$
$W$
$W$

Units for matrices: $A I=B q / f i l t e r ~ S O=B q / k g ~ V E=B q / k g ~ W A=B q / L$. Values for elemental uranium are reported in $\mu g / f i l t e r, g$, or $\mathrm{mL}$. 


\section{QAP44 Results by Laboratory}

Lab: RG EG\&G Rocky Flats Plant, Golden

No.

Radio-

Reported- Reported

EML

EML

Reported Ratio

Test nuclide Value

Error

Value

Error

EML

Error

Evaluation

Matrix: So

$\begin{array}{llrlrllll}1 & \text { AM221 } & 3.890 & 0.420 & 3.690 & 0.454 & 1.050 & 0.17 & \text { A } \\ 1 & \text { PU238 } & 42.300 & 1.700 & 43.000 & 2.440 & 0.980 & 0.07 & \text { A } \\ 1 & \text { PU239 } & 8.340 & 0.570 & 9.230 & 0.346 & 0.900 & 0.07 & \text { A } \\ 1 & \text { U 234 } & 35.500 & 1.900 & 34.200 & 3.520 & 1.040 & 0.12 & \text { A } \\ 1 & \text { U 238 } & 37.000 & 2.000 & 35.900 & 3.630 & 1.030 & 0.12 & \text { A } \\ 1 & \text { U BO } & 73.900 & 4.200 & 71.700 & 4.150 & 1.030 & 0.08 & \text { A }\end{array}$

Matrix: WA

$\begin{array}{llr}1 & \text { AM241 } & 0.89 \\ 1 & \text { GA 1 } & 2110.00 \\ 1 & \text { GB 2 } & 590.00 \\ 1 & \text { H 3 } & 210.000 \\ 1 & \text { PU238 } & 0.94 \\ 1 & \text { PU239 } & 0.76 \\ 1 & \text { U 234 } & 0.328 \\ 1 & \text { U 238 } & 0.295 \\ 1 & \text { U BO } & 0.636\end{array}$

$\begin{array}{rrr}0.027 & 0.766 & 0.013 \\ 109.000 & 1850.000 & 185.000 \\ 29.500 & 744.000 & 74.000 \\ 5.500 & 251.000 & 11.400 \\ 0.027 & 0.982 & 0.074 \\ 0.023 & 0.772 & 0.056 \\ 0.018 & 0.274 & 0.019 \\ 0.017 & 0.275 & 0.024 \\ 0.038 & 0.559 & 0.047\end{array}$

1.160

1.140

0.790

0.840

0.960

0.990

1.200

1.070

1.140

0.04
0.13
0.09
0.04
0.08
0.08
0.11
0.11
0.12

$A$
$A$
$W$
$A$
$A$
$A$
$W$
$A$
$A$

Units for matrices: $A l=B q / f i l t e r ~ S D=B q / k g ~ V E=B q / k g ~ W A=B q / L$. Values for elemental uranium are reported in $\mu g / f i l t e r, g$, or $m L$. 
QAP44 Results by Laboratory

Lab: RI Westinghouse Hanford Co. Analytical Labs

\begin{tabular}{lllllllll} 
No. & $\begin{array}{l}\text { Radio- } \\
\text { Test }\end{array}$ & $\begin{array}{l}\text { Reported } \\
\text { nuclide }\end{array}$ & $\begin{array}{l}\text { Reported } \\
\text { Value }\end{array}$ & Error & $\begin{array}{c}\text { EML } \\
\text { Value }\end{array}$ & $\begin{array}{c}\text { EML } \\
\text { Error }\end{array}$ & $\begin{array}{l}\text { Reported } \\
\text { EML }\end{array}$ & $\begin{array}{l}\text { Ratio } \\
\text { Error }\end{array}$ Evaluation \\
\hline
\end{tabular}

Matrix: Al

$\begin{array}{llrlrllll}1 & \text { AM241 } & 0.175 & 0.016 & 0.189 & 0.007 & 0.930 & 0.09 & \text { A } \\ 1 & \text { CE144 } & 18.900 & 2.000 & 33.300 & 3.300 & 0.570 & 0.08 & \mathbf{N} \\ 1 & \text { CO 57 } & 5.020 & 0.340 & 8.900 & 0.900 & 0.560 & 0.07 & \mathbf{N} \\ 1 & \text { CO 60 } & 2.230 & 0.700 & 29.500 & 2.900 & 0.080 & 0.02 & \mathbf{N} \\ 1 & \text { CS134 } & 11.600 & 0.440 & 14.700 & 1.460 & 0.790 & 0.08 & \mathbf{W} \\ 1 & \text { CS137 } & 5.210 & 0.710 & 6.640 & 0.700 & 0.790 & 0.14 & \mathbf{W} \\ 1 & \text { MN 54 } & 2.220 & 0.560 & 3.440 & 0.380 & 0.650 & 0.18 & \mathbf{N} \\ 1 & \text { PU238 } & 0.086 & 0.008 & 0.096 & 0.002 & 0.900 & 0.09 & \mathbf{A} \\ 1 & \text { PU239 } & 0.077 & 0.007 & 0.093 & 0.003 & 0.830 & 0.08 & \mathbf{W} \\ 1 & \text { RU106 } & 8.780 & 3.900 & 11.600 & 1.440 & 0.760 & 0.35 & \mathbf{A} \\ 1 & \text { SB125 } & 8.270 & 1.100 & 9.780 & 1.030 & 0.850 & 0.14 & \mathbf{A} \\ 1 & \text { SR 90 } & 1.070 & 0.064 & 1.060 & 0.037 & 1.010 & 0.07 & \mathbf{A}\end{array}$

Matrix: So

$\begin{array}{lllrlllll}1 & C S 137 & 185.000 & 5.000 & 359.000 & 10.000 & 0.520 & 0.02 & N \\ 1 & K ~ 40 & 253.000 & 43.000 & 465.000 & 30.000 & 0.540 & 0.10 & N\end{array}$

Matrix: VE

$\begin{array}{rlrrrrrrr}1 & \text { AM241 } & 5.440 & 0.510 & 5.600 & 0.184 & 0.970 & 0.10 & \text { A } \\ 1 & \text { CM244 } & 4.590 & 0.440 & 4.440 & 0.202 & 1.030 & 0.11 & \text { A } \\ 1 & \text { CD 60 } & 30.200 & 2.600 & 59.700 & 0.963 & 0.510 & 0.04 & \text { N } \\ 1 & \text { CS137 } & 532.000 & 8.000 & 944.000 & 16.200 & 0.560 & 0.01 & \text { N } \\ 1 & \text { K 40 } & 517.000 & 66.000 & 1030.000 & 33.000 & 0.500 & 0.07 & \text { W } \\ 1 & \text { PU239 } & 8.700 & 0.670 & 9.820 & 1.220 & 0.890 & 0.13 & \text { A }\end{array}$

Matrix: WA

$\begin{array}{llrrrrrrr}1 & \text { AM241 } & 0.821 & 0.075 & 0.766 & 0.013 & 1.070 & 0.10 & \mathbf{A} \\ 1 & \text { CO 60 } & 3.360 & 0.160 & 32.800 & 0.580 & 0.100 & 0.01 & \mathbf{N} \\ 1 & \text { CS137 } & 42.300 & 2.200 & 38.300 & 0.881 & 1.100 & 0.06 & \mathbf{A} \\ 1 & H 3 & 304.000 & 18.000 & 251.000 & 11.400 & 1.210 & 0.09 & \mathbf{W} \\ 1 & \text { MN 54 } & 38.700 & 1.700 & 38.400 & 1.160 & 1.010 & 0.05 & \mathbf{A} \\ 1 & \text { PU238 } & 0.810 & 0.065 & 0.982 & 0.074 & 0.830 & 0.09 & \mathbf{W} \\ 1 & \text { PU239 } & 0.633 & 0.057 & 0.772 & 0.056 & 0.820 & 0.09 & \mathbf{A} \\ 1 & \text { SR 90 } & 1.740 & 0.370 & 1.450 & 0.034 & 1.200 & 0.26 & \mathbf{A} \\ 1 & \text { U UG } & 0.006 & 0.000 & 0.022 & 0.003 & 0.270 & 0.03 & \mathbf{N}\end{array}$




\section{OAP44 Results by Laboratory}

Lab: SA Sandia Labs Radioactive Sample Diag. Prog., NM

No.

Radio-

Reported Reported

EML

Error

Value

EML

Reported Ratio

Test nuclide Value

Error

EML

Error

Evaluation

Matrix: Al

$\begin{array}{llr}1 & \text { CE144 } & 37.900 \\ 1 & \text { CO 57 } & 11.000 \\ 1 & \text { CO 60 } & 33.900 \\ 1 & \text { CS134 } & 16.900 \\ 1 & \text { CS137 } & 8.400 \\ 1 & \text { MN 54 } & 4.300 \\ 1 & \text { SB125 } & 12.50\end{array}$

37.900

11.000

3.500

33.300

1.100

8.900

1.800

29.500

0.800

14.700

0.400

6.640

0.300 .

3.440

1.700

9.780

$\begin{array}{ll}3.300 & 1.140 \\ 0.900 & 1.240 \\ 2.900 & 1.150 \\ 1.460 & 1.150 \\ 0.700 & 1.270 \\ 0.380 & 1.250 \\ 1.030 & 1.280\end{array}$

0.15

0.18

0.13

0.13

0.15

0.16

0.22

Matrix: $\quad$ So

$1 \quad$ CS137

372.000

20.000

359.000

10.000

1.040

1.020

0.06

465.000

30.000

0.14

A

Matrix: WA

$\begin{array}{llr}1 & \text { CO 60 } & 32.000 \\ 1 & \text { CS137 } & 42.000 \\ 1 & \text { GA 1 } & 1650.000 \\ 1 & \text { GB 2 } & 1090.000 \\ 1 & \text { MN 54 } & 41.000\end{array}$

2.000
3.000
388.000
200.000
3.000

32.800

38.300

1850.000

744.000

38.400

0.580
0.881
185.000
74.000
1.160

0.980

1.100

0.890

1.470

1.070

0.06
0.08
0.23
0.31
0.08

$W$
$W$
$W$
$W$
$W$
$W$ 
QAP44 Results by Laboratory

Lab: SC S.Cubed Division Maxwell Labs, La Jolla, CA

\begin{tabular}{lllllllll} 
No. & $\begin{array}{l}\text { Radio- } \\
\text { nuclide }\end{array}$ & $\begin{array}{l}\text { Reported } \\
\text { Value }\end{array}$ & $\begin{array}{c}\text { Reported } \\
\text { Error }\end{array}$ & $\begin{array}{c}\text { EML } \\
\text { Value }\end{array}$ & $\begin{array}{c}\text { EML } \\
\text { Error }\end{array}$ & $\begin{array}{c}\text { Reported } \\
\text { EML }\end{array}$ & $\begin{array}{l}\text { Ratio } \\
\text { Error }\end{array}$ & Evaluation \\
\hline
\end{tabular}

Matrix: Al

$1 \quad G A$

GA

1.620

0.020

1.620

0.150

1.000

1.090

0.09

0.09

A

Matrix: SO

$\begin{array}{llrrrrrrr}1 & \text { CS137 } & 256.000 & 1.000 & 359.000 & 10.000 & 0.710 & 0.02 & \text { N } \\ 1 & \text { K 40 } & 387.000 & 4.000 & 465.000 & 30.000 & 0.830 & 0.05 & \text { W } \\ 1 & \text { PU238 } & 50.000 & 1.000 & 43.000 & 2.440 & 1.160 & 0.07 & \text { A } \\ 1 & \text { PU239 } & 8.150 & 0.500 & 9.230 & 0.346 & 0.880 & 0.06 & \text { A } \\ 1 & \text { U 234 } & 36.400 & 1.400 & 34.200 & 3.520 & 1.060 & 0.12 & \text { A } \\ 1 & \text { U 238 } & 37.300 & 1.400 & 35.900 & 3.630 & 1.040 & 0.11 & \text { A } \\ 1 & \text { U BD } & 73.700 & 2.800 & 71.700 & 4.150 & 1.030 & 0.07 & \text { A }\end{array}$

Matrix: WA

\begin{tabular}{|c|c|c|c|c|c|c|}
\hline $\begin{array}{l}\text { AM241 } \\
\text { CD 60 } \\
\text { CS137 } \\
\text { GA } 1 \\
\text { GB } 2 \\
H 3 \\
\text { MN 54 } \\
\text { PU238 } \\
\text { PU239 } \\
\text { SR 90 } \\
\text { U } 234 \\
\text { U } 238 \\
\text { U BO }\end{array}$ & $\begin{array}{r}0.600 \\
34.000 \\
40.000 \\
1880.000 \\
495.000 \\
227.000 \\
41.000 \\
1.140 \\
0.814 \\
2.570 \\
0.300 \\
0.300 \\
0.600\end{array}$ & $\begin{array}{r}0.050 \\
1.000 \\
1.000 \\
19.000 \\
10.000 \\
7.000 \\
1.000 \\
0.016 \\
0.013 \\
0.060 \\
0.020 \\
0.020 \\
0.050\end{array}$ & $\begin{array}{r}0.766 \\
32.800 \\
38.300 \\
1850.000 \\
744.000 \\
251.000 \\
38.400 \\
0.982 \\
0.772 \\
1.450 \\
0.274 \\
0.275 \\
0.559\end{array}$ & $\begin{array}{r}0.013 \\
0.580 \\
0.881 \\
185.000 \\
74.000 \\
11.400 \\
1.160 \\
0.074 \\
0.056 \\
0.034 \\
0.019 \\
0.024 \\
0.047\end{array}$ & $\begin{array}{l}0.780 \\
1.040 \\
1.040 \\
1.020 \\
0.670 \\
0.900 \\
1.070 \\
1.160 \\
1.050 \\
1.770 \\
1.100 \\
1.090 \\
1.070\end{array}$ & $\begin{array}{l}0.07 \\
0.04 \\
0.04 \\
0.10 \\
0.07 \\
0.05 \\
0.04 \\
0.09 \\
0.08 \\
0.06 \\
0.11 \\
0.12 \\
0.13\end{array}$ \\
\hline
\end{tabular}

Units for matrices: $A \mathrm{~A}=\mathrm{Bq} / \mathrm{filter} \mathrm{SO}=\mathrm{Bq} / \mathrm{kg}$ VE=Bq/kg $W \mathrm{~A}=\mathrm{Bq} / \mathrm{L}$. Values for elemental uranium are reported in $\mu \mathrm{g} / \mathrm{filter}, \mathrm{g}$, or $\mathrm{mL}$.

$\mathrm{pCi}=\mathrm{Bq} \times 27$ 


\section{QAP44 Results by Laboratory}

Lab: SE Shealy Environmental Services Inc., Cayce, SC

\begin{tabular}{lllllllll} 
No. & $\begin{array}{l}\text { Radio- } \\
\text { nuclide }\end{array}$ & $\begin{array}{l}\text { Reported } \\
\text { Value }\end{array}$ & $\begin{array}{l}\text { Reported } \\
\text { Error }\end{array}$ & $\begin{array}{c}\text { EML } \\
\text { Value }\end{array}$ & $\begin{array}{c}\text { EML } \\
\text { Error }\end{array}$ & $\frac{\text { Reported }}{\text { EML }}$ & $\begin{array}{l}\text { Ratio } \\
\text { Error }\end{array}$ & Evaluation \\
\hline
\end{tabular}

Matrix: Al

$\begin{array}{llrrrrrrr}1 & \text { AM241 } & 0.280 & 0.020 & 0.189 & 0.007 & 1.480 & 0.12 & \text { W } \\ 1 & \text { CE144 } & 32.700 & 2.770 & 33.300 & 3.300 & 0.980 & 0.13 & \text { A } \\ 1 & \text { CO 57 } & 8.470 & 0.160 & 8.900 & 0.900 & 0.950 & 0.10 & \text { A } \\ 1 & \text { CO 60 } & 29.200 & 1.640 & 29.500 & 2.900 & 0.990 & 0.11 & \text { A } \\ 1 & \text { CS134 } & 15.600 & 0.500 & 14.700 & 1.460 & 1.060 & 0.11 & \text { A } \\ 1 & \text { CS137 } & 6.750 & 0.680 & 6.640 & 0.700 & 1.020 & 0.15 & \text { A } \\ 1 & \text { MN 54 } & 3.450 & 0.170 & 3.440 & 0.380 & 1.000 & 0.12 & \text { A } \\ 1 & \text { RU106 } & 11.000 & 1.230 & 11.600 & 1.440 & 0.950 & 0.16 & \text { A } \\ 1 & \text { SB125 } & 10.100 & 0.420 & 9.780 & 1.030 & 1.030 & 0.12 & \text { A }\end{array}$

Matrix: So

$\begin{array}{llrrrr}1 & \text { AM241 } & 7.830 & 1.930 & 3.690 & 0.454 \\ 1 & \text { CS137 } & 423.000 & 63.600 & 359.000 & 10.000 \\ 1 & \text { K 40 } & 693.000 & 25.300 & 465.000 & 30.000 \\ 1 & \text { PU238 } & 56.000 & 2.590 & 43.000 & 2.440 \\ 1 & \text { PU239 } & 12.400 & 1.620 & 9.230 & 0.346 \\ 1 & \text { U UG } & 1.280 & 0.060 & 2.900 & 0.290\end{array}$

Matrix: VE

$\begin{array}{rlrrrrrrr}1 & \text { AM241 } & 6.280 & 0.340 & 5.600 & 0.184 & 1.120 & 0.07 & \text { A } \\ 1 & \text { CM244 } & 3.070 & 0.190 & 4.440 & 0.202 & 0.690 & 0.05 & \text { W } \\ 1 & \text { CO 60 } & 71.100 & 2.410 & 59.700 & 0.963 & 1.190 & 0.04 & \text { A } \\ 1 & \text { CS137 } & 1130.000 & 84.400 & 944.000 & 16.200 & 1.200 & 0.09 & \text { A } \\ 1 & \text { K 40 } & 1430.000 & 49.400 & 1030.000 & 33.000 & 1.390 & 0.07 & \text { W } \\ 1 & \text { PU238 } & 1.160 & 8.370 & 0.820 & 0.104 & 1.420 & 10.20 & \text { A } \\ 1 & \text { PU239 } & 8.370 & 1.160 & 9.820 & 1.220 & 0.850 & 0.16 & \text { A }\end{array}$

Matrix: WA

$\begin{array}{llrr}1 & \text { AM241 } & 0.830 & 0.040 \\ 1 & \text { CO 60 } & 34.200 & 1.120 \\ 1 & \text { CS137 } & 43.600 & 2.140 \\ 1 & \text { GA } 1 & 2120.000 & 101.000 \\ 1 & \text { GB 2 } & 881.000 & 40.900 \\ 1 & \text { H } 3 & 191.000 & 88.500 \\ 2 & & 191.000 & 5.960 \\ 1 & \text { PU238 } & 0.740 & 0.060 \\ 1 & \text { PU239 } & 0.480 & 0.040 \\ 1 & \text { U BO } & 0.260 & 0.010 \\ 1 & \text { U UG } & 0.000 & 0.000\end{array}$

$\begin{array}{rrrr}0.040 & 0.766 & 0.013 & 1.080 \\ 1.120 & 32.800 & 0.580 & 1.040 \\ 2.140 & 38.300 & 0.881 & 1.140 \\ 101.000 & 1850.000 & 185.000 & 1.150 \\ 40.900 & 744.000 & 74.000 & 1.180 \\ 88.500 & 251.000 & 11.400 & 0.760 \\ 5.960 & 251.000 & 11.400 & 0.760 \\ 0.060 & 0.982 & 0.074 & 0.750 \\ 0.040 & 0.772 & 0.056 & 0.620 \\ 0.010 & 0.559 & 0.047 & 0.470 \\ 0.000 & 0.022 & 0.003 & 0.000\end{array}$

$\begin{array}{ll}2.120 & 0.59 \\ 1.180 & 0.18 \\ 1.490 & 0.11 \\ 1.300 & 0.10 \\ 1.340 & 0.18 \\ 0.440 & 0.05\end{array}$

$\mathbf{W}$
$\mathbf{W}$
$\mathbf{W}$
$\mathbf{W}$
$\mathbf{W}$

$\mathbf{A}$
$\mathbf{W}$
$\mathbf{W}$
$\mathbf{W}$


QAP44 Results by Laboratory

Lab: SK Savannah River Plant

\begin{tabular}{llllllll} 
No. & Radio- & Reported & Reported & EML & EML & Reported & Ratio \\
Test & nuclide & Value & Error & Value & Error & EML & Error \\
\hline
\end{tabular}

Matrix: AI

\begin{tabular}{|c|c|c|c|c|c|c|}
\hline CE144 & 22.600 & 0.800 & 33.300 & 3.300 & 0.680 & 0.07 \\
\hline CO 57 & 6.230 & 0.090 & 8.900 & 0.900 & 0.700 & 0.07 \\
\hline CO 60 & 27.700 & 0.900 & 29.500 & 2.900 & 0.940 & 0.10 \\
\hline CS134 & 16.800 & 0.300 & 14.700 & 1.460 & 1.140 & 0.12 \\
\hline CS137 & 5.070 & 0.040 & 6.640 & 0.700 & 0.760 & 0.08 \\
\hline MN 54 & 2.410 & 0.040 & 3.440 & 0.380 & 0.700 & 0.08 \\
\hline RU106 & 12.600 & 0.400 & 11.600 & 1.440 & 1.090 & 0.14 \\
\hline SB125 & 8.100 & 0.300 & 9.780 & 1.030 & 0.830 & 0.09 \\
\hline
\end{tabular}

Matrix: So

$\begin{array}{lllllllll}1 & \text { CS137 } & 443.000 & 13.000 & 359.000 & 10.000 & 1.230 & 0.05 & \text { A } \\ 1 & K ~ 40 & 525.000 & 17.000 & 465.000 & 30.000 & 1.130 & 0.08 & \text { A }\end{array}$

Matrix: VE

$\begin{array}{rlrrrrrrr}1 & \text { CO 60 } & 66.500 & 5.100 & 59.700 & 0.963 & 1.110 & 0.09 & \text { A } \\ 1 & \text { CS137 } & 1140.000 & 47.000 & 944.000 & 16.200 & 1.210 & 0.05 & \text { A } \\ 1 & K ~ 40 & 1120.000 & 25.000 & 1030.000 & 33.000 & 1.090 & 0.04 & \text { A }\end{array}$

Matrix: WA

$\begin{array}{llrrrrrrr}1 & \text { CO 60 } & 34.300 & 0.700 & 32.800 & 0.580 & 1.050 & 0.03 & \text { A } \\ 1 & \text { CS137 } & 42.000 & 1.000 & 38.300 & 0.881 & 1.100 & 0.04 & \text { A } \\ 1 & H 3 & 223.000 & 3.000 & 251.000 & 11.400 & 0.890 & 0.04 & \text { A } \\ 1 & \text { MN } 54 & 41.300 & 1.400 & 38.400 & 1.160 & 1.080 & 0.05 & \text { A }\end{array}$

Units for matrices: $\mathrm{Al}=\mathrm{Bq} / \mathrm{filter} \mathrm{SO}=\mathrm{Bq} / \mathrm{kg} \mathrm{VE}=\mathrm{Bq} / \mathrm{kg} \mathrm{WA}=\mathrm{Bq} / \mathrm{L}$. Values for elemental uranium are reported in $\mu \mathrm{g} / f i l t e r, \mathrm{~g}$, or $\mathrm{mL}$. 


\section{QAP44 Results by Laboratory}

Lab: SN Sanford Cohen Associates, Inc., Montgomery, AL

\begin{tabular}{|c|c|c|c|c|c|c|c|c|}
\hline $\begin{array}{l}\text { No. } \\
\text { Test }\end{array}$ & $\begin{array}{l}\text { Radio- } \\
\text { nuclide } \\
\end{array}$ & $\begin{array}{l}\text { Reported } \\
\text { Value } \\
\end{array}$ & $\begin{array}{l}\text { Reported } \\
\text { Error }\end{array}$ & $\begin{array}{l}\text { EML } \\
\text { Value }\end{array}$ & $\begin{array}{l}\text { EML } \\
\text { Error }\end{array}$ & $\frac{\text { Reported }}{\text { EML }}$ & $\begin{array}{l}\text { Ratio } \\
\text { Error } \\
\end{array}$ & Evalu \\
\hline Matrix: & Al & & & & & & & \\
\hline $\begin{array}{l}1 \\
1 \\
1 \\
1\end{array}$ & $\begin{array}{l}\text { PU238 } \\
\text { PU239 } \\
\text { U } 234 \\
\text { U } 238\end{array}$ & $\begin{array}{l}0.079 \\
0.087 \\
0.071 \\
0.068\end{array}$ & $\begin{array}{l}0.009 \\
0.010 \\
0.010 \\
0.009\end{array}$ & $\begin{array}{l}0.096 \\
0.093 \\
0.052 \\
0.053\end{array}$ & $\begin{array}{l}0.002 \\
0.003 \\
0.002 \\
0.002\end{array}$ & $\begin{array}{l}0.820 \\
0.940 \\
1.370 \\
1.280\end{array}$ & $\begin{array}{l}0.10 \\
0.11 \\
0.20 \\
0.18\end{array}$ & $\begin{array}{l}\text { A } \\
\text { A } \\
\text { A }\end{array}$ \\
\hline
\end{tabular}

Matrix: So

$\begin{array}{llrrrrrrr}1 & \text { CS137 } & 399.000 & 40.000 & 359.000 & 10.000 & 1.110 & 0.12 & \text { A } \\ 1 & \text { K 40 } & 528.000 & 50.900 & 465.000 & 30.000 & 1.140 & 0.13 & \text { A } \\ 1 & \text { PU238 } & 41.100 & 8.160 & 43.000 & 2.440 & 0.960 & 0.20 & \text { A } \\ 1 & \text { PU239 } & 8.460 & 3.290 & 9.230 & 0.346 & 0.920 & 0.36 & \text { A } \\ 1 & \text { U 234 } & 57.400 & 10.400 & 34.200 & 3.520 & 1.680 & 0.35 & N \\ 1 & \text { U 238 } & 48.200 & 9.270 & 35.900 & 3.630 & 1.340 & 0.29 & \text { W }\end{array}$

Matrix: VE

$\begin{array}{rlr}1 & \text { CO 60 } & 68.500 \\ 1 & \text { CS137 } & 1110.000 \\ 1 & \text { K 40 } & 1260.000 \\ 1 & \text { PU239 } & 8.150\end{array}$

6.320

111.000

117.000

2.420

Matrix: WA

$\begin{array}{ll}1 & \text { CO 60 } \\ 1 & \text { CS137 } \\ 1 & \text { MN } 54 \\ 1 & \text { PU238 } \\ 1 & \text { PU239 } \\ 1 & \text { U 234 } \\ 1 & \text { U } 238\end{array}$

32.400
38.000
41.100
0.847
0.653
0.314
0.303

2.950
3.420
4.340
0.094
0.075
0.043
0.041

2.950

3.420

0.094

0.075

0.041
59.700
944.000
1030.000

9.820

$\begin{array}{rr}32.800 & 0.580 \\ 38.300 & 0.881 \\ 38.400 & 1.160 \\ 0.982 & 0.074 \\ 0.772 & 0.056 \\ 0.274 & 0.019 \\ 0.275 & 0.024\end{array}$

1.150

1.180

1.220

0.830

0.990
0.990
1.070
0.860
0.850
1.150
1.100
0.11

0.12

0.12

0.27

A

A 


\section{OAP44 Results by Laboratory}

Lab: SR Savannah River Plant

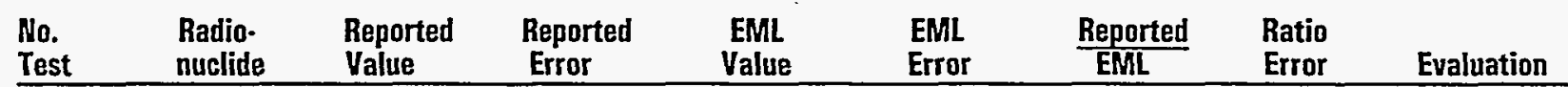

Matrix: Al

$\begin{array}{lrrrrrrr}\text { AM241 } & 0.158 & 0.022 & 0.189 & 0.007 & 0.840 & 0.12 & \text { A } \\ \text { CE144 } & 27.000 & 4.000 & 33.300 & 3.300 & 0.810 & 0.15 & \text { A } \\ \text { C0 57 } & 7.600 & 0.600 & 8.900 & 0.900 & 0.850 & 0.11 & \text { A } \\ \text { CO 60 } & 27.000 & 1.000 & 29.500 & 2.900 & 0.920 & 0.10 & \text { A } \\ \text { CS134 } & 13.400 & 0.400 & 14.700 & 1.460 & 0.910 & 0.09 & \text { A } \\ \text { CS137 } & 6.000 & 2.000 & 6.640 & 0.700 & 0.900 & 0.32 & \text { A } \\ \text { GA 1 } & 1.800 & 0.130 & 1.620 & 0.150 & 1.110 & 0.13 & \text { A } \\ \text { GB 2 } & 1.780 & 0.110 & 1.770 & 0.150 & 1.010 & 0.11 & \text { A } \\ \text { MN 54 } & 3.000 & 1.000 & 3.440 & 0.380 & 0.870 & 0.31 & \text { A } \\ \text { PU238 } & 0.085 & 0.014 & 0.096 & 0.002 & 0.880 & 0.15 & \text { A } \\ \text { PU239 } & 0.094 & 0.016 & 0.093 & 0.003 & 1.010 & 0.18 & \text { A } \\ \text { RU106 } & 16.000 & 11.000 & 11.600 & 1.440 & 1.380 & 0.96 & \text { W } \\ \text { SB125 } & 9.700 & 0.500 & 9.780 & 1.030 & 0.990 & 0.12 & \text { A } \\ \text { SR 90 } & 0.630 & 0.090 & 1.060 & 0.037 & 0.590 & 0.09 & \text { N } \\ \text { U 234 } & 0.045 & 0.009 & 0.052 & 0.002 & 0.870 & 0.18 & \text { W } \\ \text { U 238 } & 0.048 & 0.011 & 0.053 & 0.002 & 0.900 & 0.21 & \text { A }\end{array}$

Matrix: So

$\begin{array}{llrrrrrrr}1 & \text { CS137 } & 395.000 & 23.000 & 359.000 & 10.000 & 1.100 & 0.07 & \text { A } \\ 1 & K 40 & 502.000 & 60.000 & 465.000 & 30.000 & 1.080 & 0.15 & \text { A } \\ 1 & \text { PU238 } & 42.600 & 7.200 & 43.000 & 2.440 & 0.990 & 0.18 & \text { A } \\ 1 & \text { PU239 } & 9.290 & 1.930 & 9.230 & 0.346 & 1.010 & 0.21 & \text { A } \\ 1 & \text { SR 90 } & 695.000 & 12.000 & 1340.000 & 113.000 & 0.520 & 0.04 & \mathbf{N}\end{array}$

Matrix: VE

$\begin{array}{rlrrrrrrr}1 & \text { CO 60 } & 66.000 & 6.000 & 59.700 & 0.963 & 1.110 & 0.10 & \text { A } \\ 1 & \text { CS137 } & 85.000 & 44.000 & 944.000 & 16.200 & 0.090 & 0.05 & \text { N } \\ 1 & \text { K 40 } & 166.000 & 24.000 & 1030.000 & 33.000 & 0.160 & 0.02 & \text { N } \\ 1 & \text { PU239 } & 9.450 & 1.370 & 9.820 & 1.220 & 0.960 & 0.18 & \text { A } \\ 1 & \text { SR 90 } & 806.000 & 35.000 & 1300.000 & 52.400 & 0.620 & 0.04 & \mathbf{W}\end{array}$

Matrix: WA

\begin{tabular}{llrrrrrrr}
1 & AM241 & 0.832 & 0.116 & 0.766 & 0.013 & 1.090 & 0.15 & A \\
1 & CO 60 & 34.000 & 1.000 & 32.800 & 0.580 & 1.040 & 0.04 & A \\
1 & CS137 & 44.000 & 1.000 & 38.300 & 0.881 & 1.150 & 0.04 & A \\
1 & GA 1 & 1820.000 & 41.000 & 1850.000 & 185.000 & 0.980 & 0.10 & A \\
1 & GB 2 & 600.000 & 18.000 & 744.000 & 74.000 & 0.810 & 0.08 & W \\
1 & H 3 & 214.000 & 5.400 & 251.000 & 11.400 & 0.850 & 0.04 & A \\
1 & MN 54 & 44.000 & 1.000 & 38.400 & 1.160 & 1.150 & 0.04 & W \\
1 & PU238 & 1.040 & 0.140 & 0.982 & 0.074 & 1.060 & 0.16 & A \\
1 & PU239 & 0.810 & 0.120 & 0.772 & 0.056 & 1.050 & 0.17 & A \\
1 & SR 90 & 1.630 & 0.240 & 1.450 & 0.034 & 1.120 & 0.17 & A \\
1 & U 234 & 0.289 & 0.052 & 0.274 & 0.019 & 1.050 & 0.20 & A \\
\hline
\end{tabular}

Units for matrices: $A]=B q / f i l t e r ~ S O=B q / k g$ VE=Bq/kg $W A=B q / L$. Values for elemental uranium are reported in $\mu$ glfilter, $g$, or $\mathrm{mL}$.

Evaluation: $A=A c c e p t a b l e, W=A c c e p t a b l e$ with Warning, $N=$ Not Acceptable $p C i=B q \times 27$ 
OAP44 Results by Laboratory

Lab: SR Savannah River Plant

\begin{tabular}{lllllllll} 
No. & Radio- & $\begin{array}{l}\text { Reported } \\
\text { nest }\end{array}$ & $\begin{array}{l}\text { Reported } \\
\text { nuclide }\end{array}$ & Value & EML & EML & Reported & Ratio \\
Error & Value & Error & $\frac{\text { EML }}{\text { Evaluation }}$ & Error \\
\hline
\end{tabular}

Matrix: WA

0.285

0.051

0.275

0.024

1.040

0.21

A

Units for matrices: $A \mathrm{l}=\mathrm{Bq} / \mathrm{filter} \mathrm{SO}=\mathrm{Bq} / \mathrm{kg}$ VE=Bq/kg $W A=B q / L$. Values for elemental uranium are reported in $\mu \mathrm{g} / \mathrm{filter}, \mathrm{g}$, or $\mathrm{mL}$.

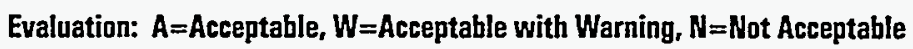
$\mathrm{pCi}=\mathrm{Bq} \times 27$ 


\section{QAP44 Results by Laboratory.}

Lab: SS Savannah River Tech Center

\begin{tabular}{lllllllll} 
No. & $\begin{array}{l}\text { Radio. } \\
\text { nuclide }\end{array}$ & $\begin{array}{l}\text { Reported } \\
\text { Value }\end{array}$ & $\begin{array}{l}\text { Reported } \\
\text { Error }\end{array}$ & $\begin{array}{l}\text { EML } \\
\text { Value }\end{array}$ & $\begin{array}{l}\text { EML } \\
\text { Error }\end{array}$ & $\frac{\text { Reported }}{\text { EML }}$ & $\begin{array}{l}\text { Ratio } \\
\text { Error }\end{array}$ & Evaluation \\
\hline
\end{tabular}

Matrix: Al

$\begin{array}{llrlrllll}1 & \text { CE144 } & 20.400 & 2.200 & 33.300 & 3.300 & 0.610 & 0.09 & \text { W } \\ 1 & \text { CO 57 } & 5.740 & 0.940 & 8.900 & 0.900 & 0.650 & 0.12 & \text { W } \\ 1 & \text { CO 60 } & 24.600 & 0.550 & 29.500 & 2.900 & 0.830 & 0.08 & \text { A } \\ 1 & \text { CS134 } & 13.200 & 0.500 & 14.700 & 1.460 & 0.900 & 0.10 & \text { A } \\ 1 & \text { CS137 } & 5.400 & 0.400 & 6.640 & 0.700 & 0.810 & 0.11 & \text { W } \\ 1 & \text { MN 54 } & 3.000 & 0.400 & 3.440 & 0.380 & 0.870 & 0.15 & \text { A } \\ 1 & \text { RU106 } & 9.500 & 3.400 & 11.600 & 1.440 & 0.820 & 0.31 & \text { A } \\ 1 & \text { SB125 } & 7.900 & 0.700 & 9.780 & 1.030 & 0.810 & \mathbf{0 . 1 1} & \mathbf{A}\end{array}$

Matrix: So

$\begin{array}{lllllllll}1 & \text { CS137 } & 445.000 & 23.000 & 359.000 & 10.000 & 1.240 & 0.07 & W \\ 1 & K ~ 40 & 554.000 & 46.000 & 465.000 & 30.000 & 1.190 & 0.13 & A\end{array}$

Matrix: VE

$\begin{array}{llrrrrrrr}1 & \text { C0 60 } & 64.900 & 3.800 & 59.700 & 0.963 & 1.090 & 0.07 & \text { A } \\ 1 & \text { CS137 } & 1100.000 & 48.000 & 944.000 & 16.200 & 1.170 & 0.05 & \text { A } \\ 1 & \text { K 40 } & 1150.000 & 99.000 & 1030.000 & 33.000 & 1.120 & 0.10 & \text { A } \\ & & & & & & . & & \\ \text { trix: } & \text { WA } & & & & & & & \\ & & & & & & & & \\ 1 & \text { CO 60 } & 33.600 & 1.900 & 32.800 & 0.580 & 1.020 & 0.06 & \text { A } \\ 1 & \text { CS137 } & 41.800 & 1.800 & 38.300 & 0.881 & 1.090 & 0.05 & \text { A } \\ 1 & \text { MN 54 } & 41.700 & 1.600 & 38.400 & 1.160 & 1.090 & 0.05 & \text { A }\end{array}$

Units for matrices: $A \mathrm{I}=\mathrm{Bq} / \mathrm{filter} \mathrm{SO}=\mathrm{Bq} / \mathrm{kg}$ VE=Bq/kg $W A=B q / \mathrm{L}$. Values for elemental uranium are reported in $\mu \mathrm{g} / f i l t e r, \mathrm{~g}$, or $\mathrm{mL}$.

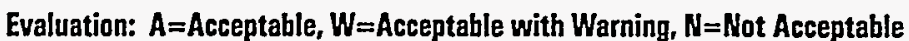

$\mathrm{pCi}=\mathrm{Bq} \times 27$ 


\section{QAP44 Results by Laboratory}

Lab: SV Savannah Lab \& Environmental Services, Inc., Tampa, FL

\begin{tabular}{|c|c|c|c|c|c|c|c|c|}
\hline $\begin{array}{l}\text { No. } \\
\text { Test }\end{array}$ & $\begin{array}{l}\text { Radio- } \\
\text { nuclide }\end{array}$ & $\begin{array}{l}\text { Reported } \\
\text { Value }\end{array}$ & $\begin{array}{l}\text { Reported } \\
\text { Error }\end{array}$ & $\begin{array}{c}\text { EML } \\
\text { Value }\end{array}$ & $\begin{array}{l}\text { EML } \\
\text { Error }\end{array}$ & $\frac{\text { Reported }}{\text { EML }}$ & $\begin{array}{l}\text { Ratio } \\
\text { Error }\end{array}$ & Evalua \\
\hline . & & & " & & & & & \\
\hline Matrix: & WA & & & & & & & \\
\hline $\begin{array}{l}1 \\
2 \\
1 \\
2 \\
1\end{array}$ & $\begin{array}{l}\text { GA } 1 \\
\text { GB } 2 \\
\text { H } 3\end{array}$ & $\begin{array}{r}2110.000 \\
1910.000 \\
874.000 \\
930.000 \\
267.000\end{array}$ & $\begin{array}{l}89.800 \\
87.900 \\
41.800 \\
43.100 \\
10.000\end{array}$ & $\begin{array}{r}1850.000 \\
1850.000 \\
744.000 \\
744.000 \\
251.000\end{array}$ & $\begin{array}{r}185.000 \\
185.000 \\
74.000 \\
74.000 \\
11.400\end{array}$ & $\begin{array}{l}1.140 \\
1.030 \\
1.170 \\
1.250 \\
1.060\end{array}$ & $\begin{array}{l}0.12 \\
0.11 \\
0.13 \\
0.14 \\
0.06\end{array}$ & $\begin{array}{l}A \\
A \\
A \\
A \\
A\end{array}$ \\
\hline
\end{tabular}

Units for matrices: $A l=B q / f i l t e r ~ S O=B q / k g ~ V E=B q / k g ~ W A=B q / L$. Values for elemental uranium are reported in $\mu g / f i l t e r, g$, or $\mathrm{mL}$. 


\section{OAP44 Results by Laboratory}

Lab: SW Southwest Research Institute, San Antonio, TX

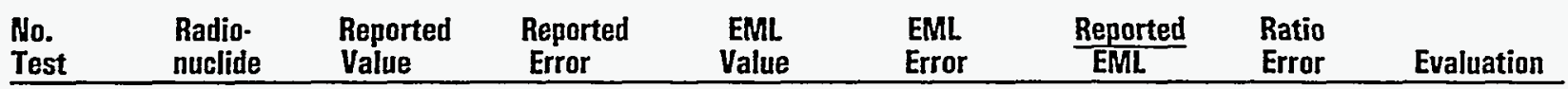

Matrix: Al

$\begin{array}{llrlrllll}1 & \text { AM241 } & 0.570 & 0.010 & 0.189 & 0.007 & 3.020 & 0.12 & \mathbf{N} \\ 1 & \text { CE144 } & 22.000 & 1.090 & 33.300 & 3.300 & 0.660 & 0.07 & \mathbf{W} \\ 1 & \text { C0 57 } & 6.510 & 0.220 & 8.900 & 0.900 & 0.730 & 0.08 & \mathbf{A} \\ 1 & \text { C0 60 } & 24.000 & 0.510 & 29.500 & 2.900 & 0.810 & 0.08 & \mathbf{W} \\ 1 & \text { CS134 } & 11.900 & 0.290 & 14.700 & 1.460 & 0.810 & 0.08 & \mathbf{A} \\ 1 & \text { CS137 } & 5.320 & 0.350 & 6.640 & 0.700 & 0.800 & 0.10 & \mathbf{W} \\ 1 & \text { MN 54 } & 2.540 & 0.350 & 3.440 & 0.380 & 0.740 & 0.13 & \mathbf{N} \\ 1 & \text { PU238 } & 0.090 & 0.010 & 0.096 & 0.002 & 0.940 & 0.11 & \mathbf{A} \\ 1 & \text { PU239 } & 0.070 & 0.010 & 0.093 & 0.003 & 0.760 & 0.11 & \mathbf{W} \\ 1 & \text { RU106 } & 10.400 & 2.570 & 11.600 & 1.440 & 0.900 & 0.25 & \mathbf{A} \\ 1 & \text { SB125 } & 8.510 & 0.560 & 9.780 & 1.030 & 0.870 & 0.11 & \mathbf{A} \\ 1 & \text { SR 90 } & 1.030 & 0.110 & 1.060 & 0.037 & 0.970 & 0.11 & \mathbf{A} \\ 1 & \text { UUG } & 5.450 & 0.960 & 4.310 & 0.100 & 1.270 & 0.23 & \mathbf{W}\end{array}$

Matrix: SO

$\begin{array}{llrrrrrrr}1 & \text { AM241 } & 5.700 & 1.850 & 3.690 & 0.454 & 1.550 & 0.54 & W \\ 1 & \text { CS137 } & 483.000 & 14.300 & 359.000 & 10.000 & 1.350 & 0.05 & W \\ 1 & \text { K 40 } & 848.000 & 121.000 & 465.000 & 30.000 & 1.820 & 0.29 & \text { N } \\ 1 & \text { U UG } & 2.300 & 0.290 & 2.900 & 0.290 & 0.790 & 0.13 & \text { A }\end{array}$

Matrix: VE

$\begin{array}{rlrrrrrrr}1 & \text { AM241 } & 10.300 & 3.340 & 5.600 & 0.184 & 1.840 & 0.60 & \text { W } \\ 1 & \text { CM244 } & 4.740 & 2.070 & 4.440 & 0.202 & 1.070 & 0.47 & \text { A } \\ 1 & \text { CO 60 } & 58.100 & 4.750 & 59.700 & 0.963 & 0.970 & 0.08 & \text { A } \\ 1 & \text { CS137 } & 868.000 & 14.700 & 944.000 & 16.200 & 0.920 & 0.02 & \text { A } \\ 1 & \text { K 40 } & 1030.000 & 129.000 & 1030.000 & 33.000 & 1.000 & 0.13 & \text { A } \\ 1 & \text { PU239 } & 16.900 & 1.840 & 9.820 & 1.220 & 1.720 & 0.28 & \text { W } \\ 1 & \text { SR 90 } & 1320.000 & 15.300 & 1300.000 & 52.400 & 1.020 & 0.04 & \text { A }\end{array}$

Matrix: WA

$\begin{array}{llrrrrrrr}1 & \text { AM241 } & 2.950 & 0.270 & 0.766 & 0.013 & 3.850 & 0.36 & \mathbf{N} \\ 1 & \text { CD 60 } & 31.800 & 0.570 & 32.800 & 0.580 & 0.970 & 0.02 & \mathbf{A} \\ 1 & \text { CS137 } & 39.500 & 0.750 & 38.300 & 0.881 & 1.030 & 0.03 & \text { A } \\ 1 & \text { H } 3 & 290.000 & 8.640 & 251.000 & 11.400 & 1.160 & 0.06 & \text { A } \\ 1 & \text { MN 54 } & 39.900 & 0.760 & 38.400 & 1.160 & 1.040 & 0.04 & \text { A } \\ 1 & \text { SR 90 } & 1.650 & 0.220 & 1.450 & 0.034 & 1.140 & 0.15 & \text { A } \\ 1 & \text { U UG } & 2.040 & 0.230 & 0.022 & 0.003 & \because * * & 14.90 & \mathbf{N}\end{array}$

Units for matrices: $A \mid=B q / f i l t e r ~ S O=B q / k g ~ V E=B q / k g ~ W A=B q / L$. Values for elemental uranium are reported in $\mu g / f i l t e r, g$, or $\mathrm{mL}$.

Evaluation: $A=A c c e p t a b l e, W=A c c e p t a b l e$ with Warning, $N=$ Not Acceptable

$\mathrm{pCi}=\mathrm{Bq} \times 27$ 


\section{QAP44 Results by Laboratory}

Lab: TE Teledyne Isotopes Midwest Lab, Northbrook, IL

\begin{tabular}{|c|c|c|c|c|c|c|c|c|}
\hline $\begin{array}{l}\text { No. } \\
\text { Test }\end{array}$ & $\begin{array}{l}\text { Radio- } \\
\text { nuclide }\end{array}$ & $\begin{array}{l}\text { Reported } \\
\text { Value }\end{array}$ & $\begin{array}{l}\text { Reported } \\
\text { Error }\end{array}$ & $\begin{array}{l}\text { EML } \\
\text { Value }\end{array}$ & $\begin{array}{l}\text { EML } \\
\text { Error }\end{array}$ & $\frac{\text { Reporte }}{\text { EML }}$ & $\begin{array}{l}\text { Rati } \\
\text { Erro }\end{array}$ & Evaluation \\
\hline
\end{tabular}

Matrix: Al

$\begin{array}{lrrrrrrr}\text { AM241 } & 0.270 & 0.040 & 0.189 & 0.007 & 1.430 & 0.22 & \text { W } \\ \text { CE144 } & 23.200 & 1.020 & 33.300 & 3.300 & 0.700 & 0.08 & \text { A } \\ \text { CO 57 } & 6.050 & 0.130 & 8.900 & 0.900 & 0.680 & 0.07 & \text { W } \\ \text { CO 60 } & 26.500 & 0.430 & 29.500 & 2.900 & 0.900 & 0.09 & \text { A } \\ \text { CS134 } & 12.900 & 0.280 & 14.700 & 1.460 & 0.880 & 0.09 & \text { A } \\ \text { CS137 } & 6.200 & 0.900 & 6.640 & 0.700 & 0.930 & 0.17 & \text { A } \\ \text { GA 1 } & 2.210 & 0.050 & 1.620 & 0.150 & 1.360 & 0.13 & \text { W } \\ \text { GB 2 } & 1.950 & 0.040 & 1.770 & 0.150 & 1.100 & 0.10 & \text { A } \\ \text { MN 54 } & 3.270 & 0.350 & 3.440 & 0.380 & 0.950 & 0.15 & \text { A } \\ \text { PU238 } & 0.080 & 0.020 & 0.096 & 0.002 & 0.830 & 0.21 & \text { A } \\ \text { PU239 } & 0.090 & 0.020 & 0.093 & 0.003 & 0.970 & 0.22 & \text { A } \\ \text { RU106 } & 10.200 & 1.850 & 11.600 & 1.440 & 0.880 & 0.19 & \text { A } \\ \text { SB125 } & 10.100 & 0.750 & 9.780 & 1.030 & 1.030 & 0.13 & \text { A } \\ \text { SR 90 } & 1.070 & 0.230 & 1.060 & 0.037 & 1.010 & 0.22 & \text { A } \\ \text { U BQ } & 0.118 & 0.020 & 0.107 & 0.003 & 1.100 & 0.19 & \text { A }\end{array}$

Matrix: So

$\begin{array}{llrr}1 & \text { AM241 } & 6.230 & 2.880 \\ 1 & \text { CS137 } & 405.000 & 4.150 \\ 1 & \text { K 40 } & 525.000 & 23.300 \\ 1 & \text { PU238 } & 42.300 & 1.590 \\ 1 & \text { PU239 } & 9.000 & 0.700 \\ 1 & \text { SR 90 } & 1200.000 & 32.300 \\ 1 & \text { U BO } & 68.200 & 2.400\end{array}$

$\begin{array}{rrr}3.690 & 0.454 & 1.690 \\ 359.000 & 10.000 & 1.130 \\ 465.000 & 30.000 & 1.130 \\ 43.000 & 2.440 & 0.980 \\ 9.230 & 0.346 & 0.980 \\ 1340.000 & 113.000 & 0.900 \\ 71.700 & 4.150 & 0.950\end{array}$

0.81
0.03
0.09
0.07
0.08
0.08
0.06

W
A
A
A
A
A
A

Matrix: VE

$\begin{array}{llrrrrrrr}1 & \text { AM241 } & 6.100 & 1.300 & 5.600 & 0.184 & 1.090 & 0.24 & \text { A } \\ 1 & \text { CM244 } & 6.000 & 1.200 & 4.440 & 0.202 & 1.350 & 0.28 & \text { A } \\ 1 & \text { CO 60 } & 65.600 & 4.000 & 59.700 & 0.963 & 1.100 & 0.07 & \text { A } \\ 1 & \text { CS137 } & 1100.000 & 12.600 & 944.000 & 16.200 & 1.170 & 0.02 & \text { A } \\ 1 & \text { K 40 } & 1190.000 & 61.600 & 1030.000 & 33.000 & 1.160 & 0.07 & \text { A } \\ 1 & \text { PU239 } & 9.200 & 1.300 & 9.820 & 1.220 & 0.940 & 0.18 & \text { A } \\ 1 & \text { SR 9D } & 1210.000 & 32.200 & 1300.000 & 52.400 & 0.930 & 0.05 & \text { A }\end{array}$

Matrix: WA

\begin{tabular}{llrrrrrrr}
1 & AM241 & 0.800 & 0.100 & 0.766 & 0.013 & 1.040 & 0.13 & A \\
1 & CO 60 & 33.600 & 1.000 & 32.800 & 0.580 & 1.020 & 0.04 & A \\
1 & CS137 & 42.800 & 1.300 & 38.300 & 0.881 & 1.120 & 0.04 & A \\
1 & FE 55 & 109.000 & 21.700 & 83.000 & 3.440 & 1.310 & 0.27 & W \\
1 & GA 1 & 2180.000 & 53.500 & 1850.000 & 185.000 & 1.180 & 0.12 & A \\
1 & GB 2 & 872.000 & 27.000 & 744.000 & 74.000 & 1.170 & 0.12 & W \\
1 & H 3 & 434.000 & 34.100 & 251.000 & 11.400 & 1.730 & 0.16 & A \\
1 & MN 54 & 41.900 & 1.400 & 38.400 & 1.160 & 1.090 & $\mathbf{0 . 0 5}$ & A \\
\hline
\end{tabular}

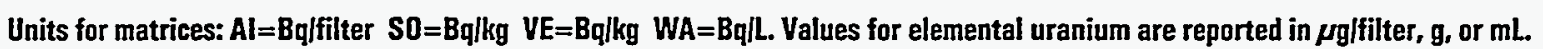

Evaluation: $A=A c c e p t a b l e, W=A c c e p t a b l e$ with Warning, $N=$ Not Acceptable $\mathrm{pCi}=\mathrm{Bq} \times 27$ 


\section{QAP44 Results by Laboratory}

Lab: TE Teledyne Isotopes Midwest Lab, Northbrook, IL

\begin{tabular}{|c|c|c|c|c|c|c|c|c|}
\hline $\begin{array}{l}\text { No. } \\
\text { Test }\end{array}$ & $\begin{array}{l}\text { Radio- } \\
\text { nuclide }\end{array}$ & $\begin{array}{l}\text { Reported } \\
\text { Value }\end{array}$ & $\begin{array}{c}\text { Reported } \\
\text { Error } \\
\end{array}$ & $\begin{array}{c}\text { EML } \\
\text { Value }\end{array}$ & $\begin{array}{l}\text { EML } \\
\text { Error }\end{array}$ & $\frac{\text { Reported }}{\text { EML }}$ & $\begin{array}{l}\text { Ratio } \\
\text { Error }\end{array}$ & Evaluat \\
\hline Matrix: & WA & . & & & & & & \\
\hline $\begin{array}{l}1 \\
1 \\
1 \\
1\end{array}$ & $\begin{array}{l}\text { PU238 } \\
\text { PU239 } \\
\text { SR 90 } \\
\text { U BQ }\end{array}$ & $\begin{array}{l}0.900 \\
0.700 \\
2.200 \\
0.600\end{array}$ & $\begin{array}{l}0.100 \\
0.100 \\
0.700 \\
0.100\end{array}$ & $\begin{array}{l}0.982 \\
0.772 \\
1.450 \\
0.559\end{array}$ & $\begin{array}{l}0.074 \\
0.056 \\
0.034 \\
0.047\end{array}$ & $\begin{array}{l}0.920 \\
0.910 \\
1.520 \\
1.070\end{array}$ & $\begin{array}{l}0.12 \\
0.15 \\
0.48 \\
0.20\end{array}$ & $\begin{array}{c}\mathbf{A} \\
\mathbf{A} \\
\mathbf{W} \\
\mathbf{A}\end{array}$ \\
\hline
\end{tabular}

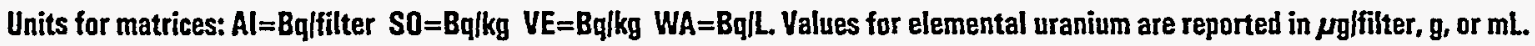




\section{QAP44 Results by Laboratory}

Lab: TI Teledyne Brown Engineering Environmental Services, Westwood, NJ

\begin{tabular}{|c|c|c|c|c|c|c|c|c|}
\hline $\begin{array}{l}\text { No. } \\
\text { Test }\end{array}$ & $\begin{array}{l}\text { Radio- } \\
\text { nuclide }\end{array}$ & $\begin{array}{l}\text { Reported } \\
\text { Value }\end{array}$ & $\begin{array}{l}\text { Reported } \\
\text { Error }\end{array}$ & $\begin{array}{c}\text { EML } \\
\text { Value }\end{array}$ & $\begin{array}{l}\text { EML } \\
\text { Error }\end{array}$ & $\frac{\text { Reportec }}{\text { EML }}$ & $\begin{array}{l}\text { Ratio } \\
\text { Error }\end{array}$ & Evaluation \\
\hline
\end{tabular}

Matrix: Al

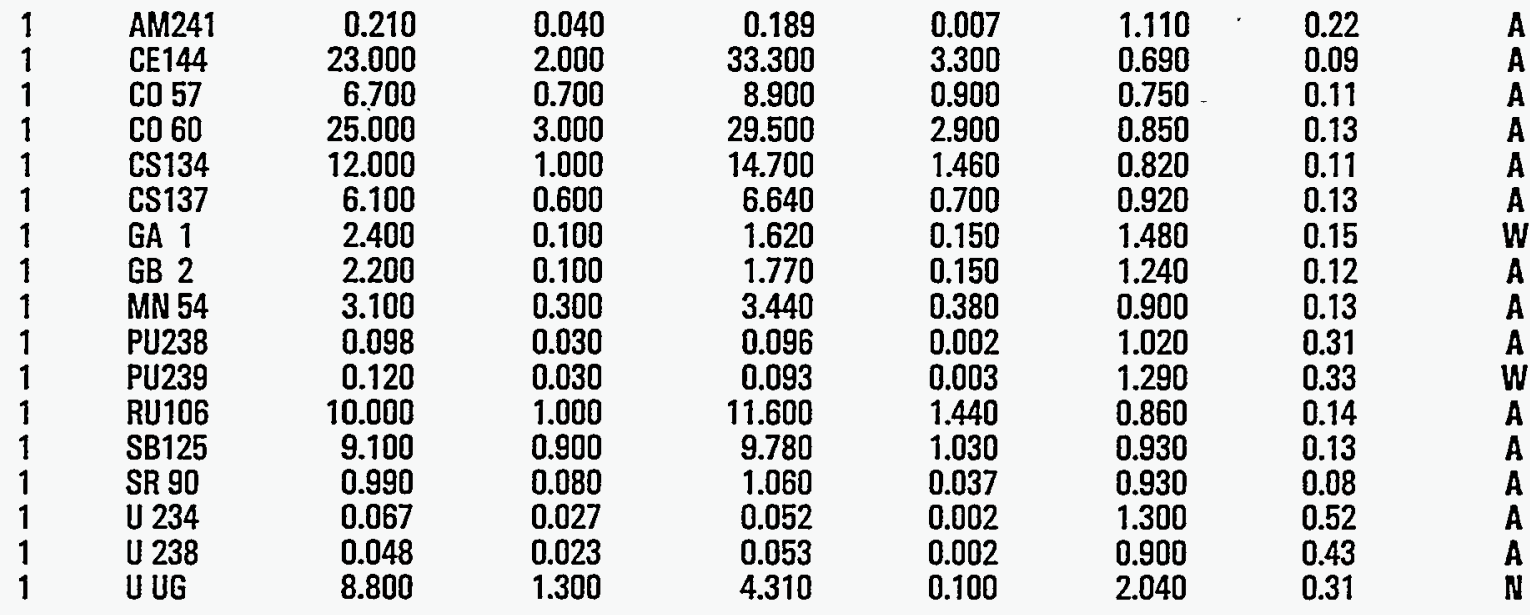

Matrix: So

$\begin{array}{llr}1 & \text { AM241 } & 5.000 \\ 1 & \text { CS137 } & 430.000 \\ 1 & \text { K 40 } & 540.000 \\ 1 & \text { PU238 } & 44.000 \\ 1 & \text { PU239 } & 11.000 \\ 1 & \text { SR } 90 & 1300.000 \\ 1 & \text { U } 234 & 28.000 \\ 1 & \text { U 238 } & 35.000 \\ 1 & \text { U UG } & 2.500\end{array}$

2.100
40.000
50.000
6.000
2.000
100.000
8.000
9.000
0.400

$\begin{array}{rr}3.690 & 0.454 \\ 359.000 & 10.000 \\ 465.000 & 30.000 \\ 43.000 & 2.440 \\ 9.230 & 0.346 \\ 1340.000 & 113.000 \\ 34.200 & 3.520 \\ 35.900 & 3.630 \\ 2.900 & 0.290\end{array}$

1.360
1.200
1.160
1.020
1.190
0.970
0.820
0.980
0.860

0.59

0.12

0.13

0.15

0.22

0.11

0.25

0.27

0.16

$A$
$A$
$A$
$A$
$A$
$A$
$W$
$A$
$A$
$A$
$W$
$A$
$A$
$A$
$A$
$A$
$N$

Matrix: VE

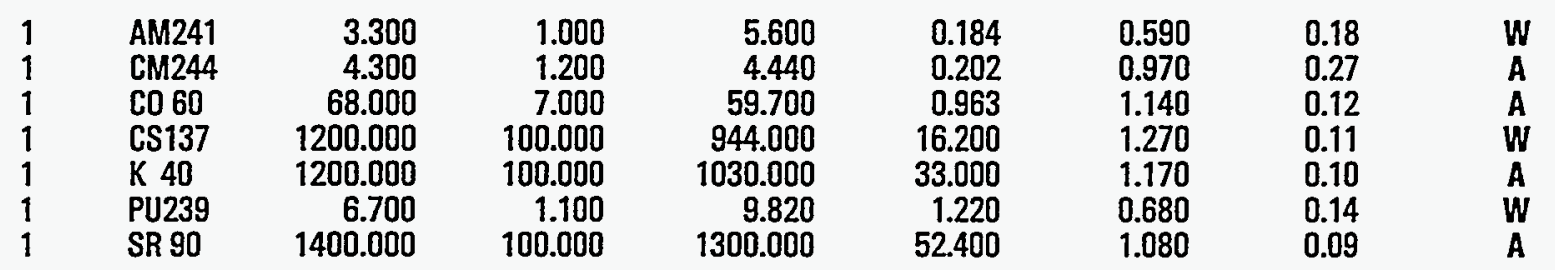

Matrix: WA

$\begin{array}{llrrrrrrr}1 & \text { AM241 } & 0.770 & 0.160 & 0.766 & 0.013 & 1.010 & 0.21 & \text { A } \\ 1 & \text { CO 60 } & 31.000 & 3.000 & 32.800 & 0.580 & 0.950 & 0.09 & A \\ 1 & \text { CS137 } & 42.000 & 4.000 & 38.300 & 0.881 & 1.100 & 0.11 & \text { A } \\ 1 & \text { FE 55 } & 110.000 & 20.000 & 83.000 & 3.440 & 1.330 & 0.25 & \text { W }\end{array}$

Units for matrices: $\mathrm{Al}=\mathrm{Bq} / \mathrm{filter} \mathbf{S O}=\mathrm{B} q / \mathrm{kg} \quad \mathrm{VE}=\mathrm{Bq} / \mathrm{kg} \quad \mathrm{WA}=\mathrm{Bq} / \mathrm{L}$. Values for elemental uranium are reported in $\mu \mathrm{g} / \mathrm{filter}, \mathrm{g}$, or $\mathrm{mL}$.

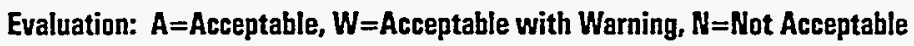
$\mathrm{pCi}=\mathrm{Bq} \times 27$ 


\section{QAP44 Results by Laboratory}

Lab: TI Teledyne Brown Engineering Environmental Services, Westwood, NJ

\begin{tabular}{|c|c|c|c|c|c|c|c|c|}
\hline $\begin{array}{l}\text { No. } \\
\text { Test }\end{array}$ & $\begin{array}{l}\text { Radio- } \\
\text { nuclide }\end{array}$ & $\begin{array}{l}\text { Reported } \\
\text { Value }\end{array}$ & $\begin{array}{l}\text { Reported } \\
\text { Error }\end{array}$ & $\begin{array}{c}\text { EML } \\
\text { Value }\end{array}$ & $\begin{array}{l}\text { EML } \\
\text { Error }\end{array}$ & $\frac{\text { Reported }}{\text { EML }}$ & $\begin{array}{l}\text { Ratio } \\
\text { Error }\end{array}$ & Evaluation \\
\hline
\end{tabular}

Matrix: WA

$\begin{array}{llrrrrrrr}1 & \text { GA 1 } & 1900.000 & 100.000 & 1850.000 & 185.000 & 1.030 & 0.12 & \text { A } \\ 1 & \text { GB 2 } & 530.000 & 50.000 & 744.000 & 74.000 & 0.710 & 0.10 & \text { N } \\ 1 & \text { H 3 } & 210.000 & 10.000 & 251.000 & 11.400 & 0.840 & 0.06 & \text { A } \\ 1 & \text { MN 54 } & 41.000 & 4.000 & 38.400 & 1.160 & 1.070 & 0.11 & \text { A } \\ 1 & \text { PU238 } & 0.970 & 0.170 & 0.982 & 0.074 & 0.990 & 0.19 & \text { A } \\ 1 & \text { PU239 } & 0.810 & 0.150 & 0.772 & 0.056 & 1.050 & 0.21 & \text { A } \\ 1 & \text { SR 90 } & 1.600 & 0.300 & 1.450 & 0.034 & 1.100 & 0.21 & \text { A } \\ 1 & \text { U 234 } & 0.520 & 0.280 & 0.274 & 0.019 & 1.900 & 1.03 & \text { N } \\ 1 & \text { U 238 } & 1.300 & 0.500 & 0.275 & 0.024 & 4.730 & 1.87 & \text { N } \\ 1 & \text { U UG } & 0.040 & 0.000 & 0.022 & 0.003 & 1.800 & 0.21 & \text { N }\end{array}$

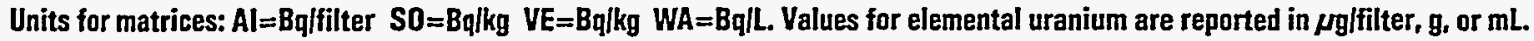

$\mathrm{pCi}=\mathrm{Bq} \times 27$ 


\section{QAP44 Results by Laboratory}

Lab: TM TMA/Eberline-Albuquerque Lab, NM

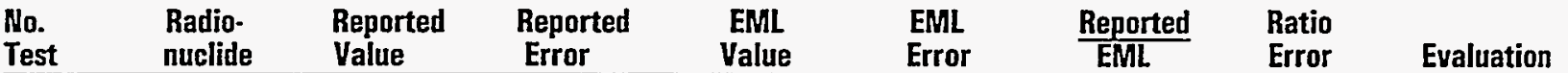

Matrix: Al

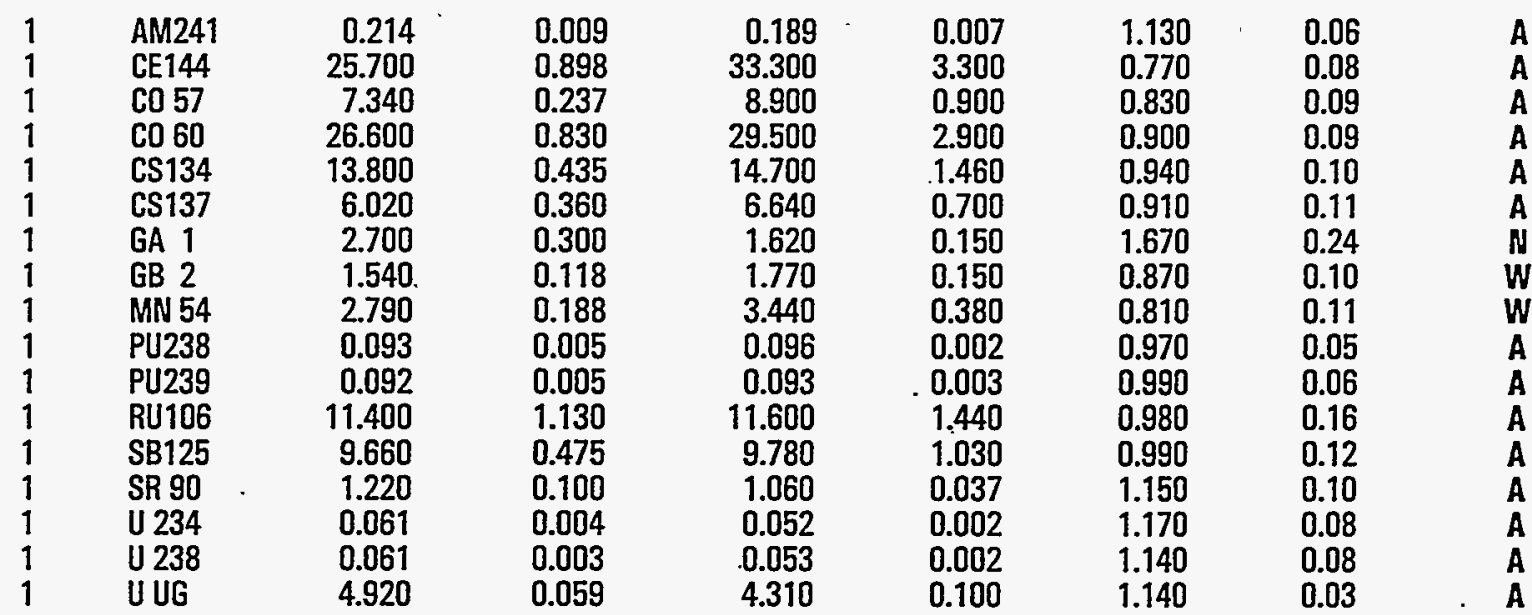

Matrix: So

$\begin{array}{llrr}1 & \text { AM241 } & 3.480 & 0.259 \\ 1 & \text { CS } 137 & 413.000 & 13.300 \\ 1 & \text { K } 40 & 439.000 & 47.000 \\ 1 & \text { PU238 } & 42.800 & 2.360 \\ 1 & \text { PU239 } & 8.530 & 0.888 \\ 1 & \text { SR } 90 & 887.000 & 136.000 \\ 1 & \text { U } 234 & 35.000 & 1.880 \\ 1 & \text { U 238 } & 35.100 & 1.880 \\ 1 & \text { U UG } & 2.620 & 0.142\end{array}$

$\begin{array}{rrr}3.690 & 0.454 & 0.940 \\ 359.000 & 10.000 & 1.150 \\ 465.000 & 30.000 & 0.940 \\ 43.000 & 2.440 & 1.000 \\ 9.230 & 0.346 & 0.920 \\ 1340.000 & 113.000 & 0.660 \\ 34.200 & 3.520 & 1.020 \\ 35.900 & 3.630 & 0.980 \\ 2.900 & 0.290 & 0.900\end{array}$

0.14

0.05

0.12

0.08

0.10

0.12

0.12

0.11

0.10

A
$A$
$A$
$A$
$A$
$A$
$A$
$N$
$W$
$W$
$A$
$A$
$A$
$A$
$A$
$A$
$A$

Matrix: VE

\begin{tabular}{|c|c|c|c|c|c|c|}
\hline $\begin{array}{l}\text { AM241 } \\
\text { CM244 } \\
\text { CD 60 } \\
\text { CS137 } \\
\text { K 40 } \\
\text { PU239 } \\
\text { SR 90 }\end{array}$ & $\begin{array}{r}6.700 \\
5.490 \\
66.500 \\
1190.000 \\
1180.000 \\
8.810 \\
1370.000\end{array}$ & $\begin{array}{r}0.506 \\
0.754 \\
6.060 \\
36.200 \\
77.300 \\
0.638 \\
167.000\end{array}$ & $\begin{array}{r}5.600 \\
4.440 \\
59.700 \\
944.000 \\
1030.000 \\
9.820 \\
1300.000\end{array}$ & $\begin{array}{r}0.184 \\
0.202 \\
0.963 \\
16.200 \\
33.000 \\
1.220 \\
52.400\end{array}$ & $\begin{array}{l}1.200 \\
1.240 \\
1.110 \\
1.260 \\
1.150 \\
0.900 \\
1.050\end{array}$ & $\begin{array}{l}0.10 \\
0.18 \\
0.10 \\
0.04 \\
0.08 \\
0.13 \\
0.14\end{array}$ \\
\hline
\end{tabular}

Matrix: WA

$\begin{array}{rlrrrrrrr}1 & \text { AM241 } & 0.855 & 0.420 & 0.766 & 0.013 & 1.120 & 0.55 & \text { A } \\ 1 & \text { CO 60 } & 34.300 & 1.250 & 32.800 & 0.580 & 1.050 & 0.04 & \text { A } \\ 1 & \text { CS137 } & 44.800 & 1.820 & 38.300 & 0.881 & 1.170 & 0.05 & \text { W } \\ 1 & \text { GA 1 } & 1950.000 & 218.000 & 1850.000 & 185.000 & 1.050 & 0.16 & \text { A }\end{array}$

Units for matrices: $\mathrm{Al}=\mathrm{Bq} / \mathrm{filter} \mathrm{SO}=\mathrm{Bq} / \mathrm{kg} \quad \mathrm{VE}=\mathrm{Bq} / \mathrm{kg} W A=B q / \mathrm{L}$. Yalues for elemental uranium are reported in $\mu \mathrm{g} / \mathrm{filter}, \mathrm{g}$, or $\mathrm{mL}$.

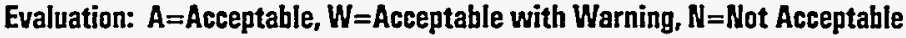
$\mathrm{pCi}=\mathrm{Bq} \times 27$ 
QAP44 Results by Laboratory

Lab: TM TMA/Eberline-Albuquerque Lab, NM

\begin{tabular}{llllclll} 
No. & Radio- & Reported & Reported & EML & EML & Reported & Ratio \\
nest & nuclide & Value & Error & Value & Error & EML & Error \\
\hline
\end{tabular}

Matrix: WA

$\begin{array}{llrrrrrrr}1 & \text { GB 2 } & 877.000 & 69.200 & 744.000 & 74.000 & 1.180 & 0.15 & \text { A } \\ 1 & \text { H } 3 & 206.000 & 10.900 & 251.000 & 11.400 & 0.820 & 0.06 & \text { W } \\ 1 & \text { MN 54 } & 44.200 & 1.490 & 38.400 & 1.160 & 1.150 & 0.05 & \text { W } \\ 1 & \text { PU238 } & 0.984 & 0.043 & 0.982 & 0.074 & 1.000 & 0.09 & \text { A } \\ 1 & \text { PU239 } & 0.761 & 0.035 & 0.772 & 0.056 & 0.990 & 0.08 & A \\ 1 & \text { SR 90 } & 1.450 & 0.142 & 1.450 & 0.034 & 1.000 & 0.10 & A \\ 1 & \text { U 234 } & 0.311 & 0.026 & 0.274 & 0.019 & 1.140 & 0.12 & A \\ 1 & \text { U 238 } & 0.259 & 0.024 & 0.275 & 0.024 & 0.940 & 0.12 & A \\ 1 & \text { U UG } & 0.025 & 0.001 & 0.022 & 0.003 & 1.130 & 0.14 & \text { A }\end{array}$

Units for matrices: $A \mid=B q / f i l t e r ~ S O=B q / k g$ VE=Bq/kg $W A=B q / L$. Values for elemental uranium are reported in $\mu g / f i l t e r, g$, or $\mathrm{mL}$. 


\section{QAP44 Results by Laboratory}

Lab: TN TMA/NORCAL, Richmond, CA

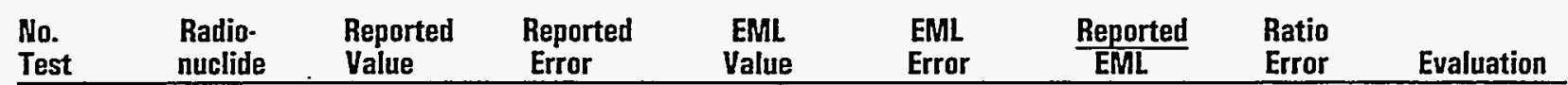

Matrix: Al

$\begin{array}{llrlrllll}1 & \text { AM241 } & 0.181 & 0.012 & 0.189 & 0.007 & 0.960 & 0.07 & \text { A } \\ 1 & \text { CE144 } & 22.900 & 1.070 & 33.300 & 3.300 & 0.690 & 0.08 & \text { A } \\ 1 & \text { CO 57 } & 6.800 & 0.286 & 8.900 & 0.900 & 0.760 & 0.08 & \text { A } \\ 1 & \text { CO 60 } & 25.400 & 0.864 & 29.500 & 2.900 & 0.860 & 0.09 & \text { A } \\ 1 & \text { CS134 } & 14.300 & 1.340 & 14.700 & 1.460 & 0.970 & 0.13 & \text { A } \\ 1 & \text { CS137 } & 5.530 & 0.498 & 6.640 & 0.700 & 0.830 & 0.12 & \text { W } \\ 1 & \text { GA 1 } & 2.010 & 0.033 & 1.620 & 0.150 & 1.240 & 0.12 & \text { A } \\ 1 & \text { GB 2 } & 1.920 & 0.032 & 1.770 & 0.150 & 1.090 & 0.09 & \text { A } \\ 1 & \text { MN 54 } & 3.020 & 0.520 & 3.440 & 0.380 & 0.880 & 0.18 & \text { A } \\ 1 & \text { PU238 } & 0.095 & 0.008 & 0.096 & 0.002 & 0.990 & 0.09 & \text { A } \\ 1 & \text { PU239 } & 0.089 & 0.008 & 0.093 & 0.003 & 0.960 & 0.09 & \text { A } \\ 1 & \text { RU106 } & 11.700 & 3.190 & 11.600 & 1.440 & 1.010 & 0.30 & \text { A } \\ 1 & \text { SB125 } & 8.230 & 1.150 & 9.780 & 1.030 & 0.840 & 0.15 & \text { A } \\ 1 & \text { SR 90 } & 1.060 & 0.061 & 1.060 & 0.037 & 1.000 & 0.07 & \text { A } \\ 1 & \text { U 234 } & 0.061 & 0.008 & 0.052 & 0.002 & 1.180 & 0.16 & \text { A } \\ 1 & \text { U 238 } & 0.054 & 0.008 & 0.053 & 0.002 & 1.020 & 0.16 & \text { A } \\ 1 & \text { U UG } & 4.560 & 1.190 & 4.310 & 0.100 & 1.060 & 0.28 & \text { A }\end{array}$

Matrix: So

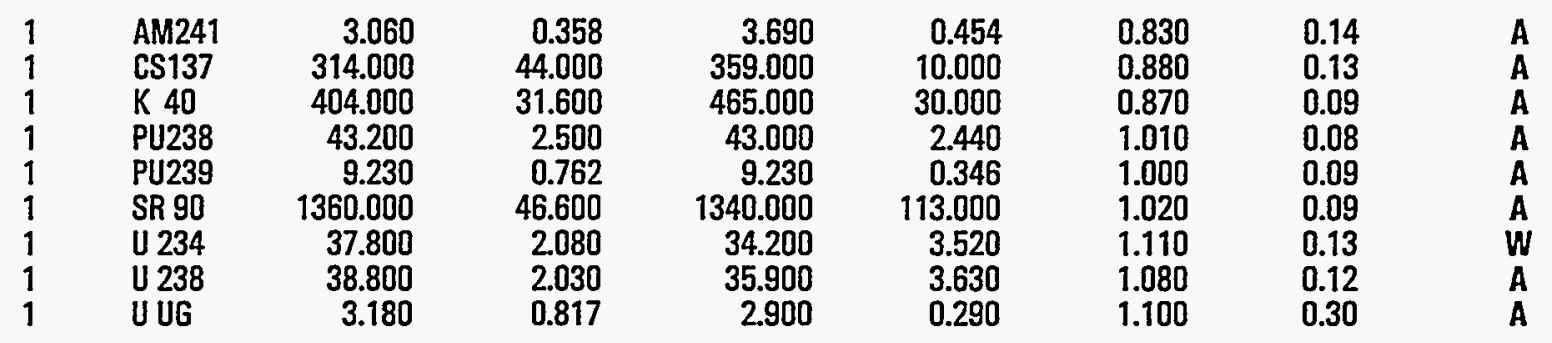

Matrix: VE

$\begin{array}{llrrrrrrr}1 & \text { AM241 } & 5.930 & 0.479 & 5.600 & 0.184 & 1.060 & 0.09 & \text { A } \\ 1 & \text { CM244 } & 4.900 & 0.582 & 4.440 & 0.202 & 1.100 & 0.14 & \text { A } \\ 1 & \text { CD 60 } & 48.700 & 3.990 & 59.700 & 0.963 & 0.820 & 0.07 & \text { A } \\ 1 & \text { CS137 } & 847.000 & 10.200 & 944.000 & 16.200 & 0.900 & 0.02 & \text { W } \\ 1 & \text { K 40 } & 884.000 & 44.200 & 1030.000 & 33.000 & 0.860 & 0.05 & \text { A } \\ 1 & \text { PU239 } & 9.580 & 0.700 & 9.820 & 1.220 & 0.980 & 0.14 & \text { A } \\ 1 & \text { SR 9D } & 1430.000 & 47.400 & 1300.000 & 52.400 & 1.100 & 0.06 & \text { A }\end{array}$

Matrix: WA

$\begin{array}{lllllllll}1 & \text { AM241 } & 0.731 & 0.037 & 0.766 & 0.013 & 0.950 & 0.05 & \text { A } \\ 1 & \text { CO 60 } & 35.600 & 1.990 & 32.800 & 0.580 & 1.090 & 0.06 & \text { A } \\ 1 & \text { CS137 } & 44.300 & 2.040 & 38.300 & 0.881 & 1.160 & 0.06 & \\ 1 & \text { FE 55 } & 94.700 & 3.440 & 83.000 & 3.440 & 1.140 & 0.06 & \text { A }\end{array}$

Units for matrices: $\mathrm{Al}=\mathrm{Bq} \mid \mathrm{filter} \mathrm{SB}=\mathrm{Bq} / \mathrm{kg} \quad \mathrm{VE}=\mathrm{Bq} / \mathrm{kg} \quad \mathrm{WA}=\mathrm{Bq} / \mathrm{L}$. Values for elemental uranium are reported in $\mu \mathrm{g} / \mathrm{filter}$, $\mathrm{g}$, or $\mathrm{mL}$.

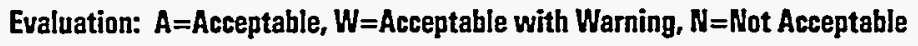
$\mathrm{pCi}=\mathrm{Bq} \times 27$ 


\section{QAP44 Results by Laboratory}

Lab: TN TMA/NORCAL, Richmond, CA

\begin{tabular}{llllllll} 
No. & Radio- & Reported & Reported & EML & EML & Reported & Ratio \\
Test & nuclide & Value & Error & Value & Error & EML & Error \\
\hline
\end{tabular}

Matrix: WA

$\begin{array}{llrrrrrrr}1 & \text { GA 1 } & 2020.000 & 40.400 & 1850.000 & 185.000 & 1.090 & 0.11 & \text { A } \\ 1 & \text { GB 2 } & 590.000 & 15.200 & 744.000 & 74.000 & 0.790 & 0.08 & \mathbf{W} \\ 2 & & 590.000 & 15.200 & 744.000 & 74.000 & 0.790 & 0.08 & \mathbf{W} \\ 1 & \text { H 3 } & 221.000 & 17.000 & 251.000 & 11.400 & 0.880 & 0.08 & \mathbf{A} \\ 1 & \text { MN 54 } & 45.100 & 2.160 & 38.400 & 1.160 & 1.170 & 0.07 & \mathbf{W} \\ 1 & \text { PU238 } & 0.942 & 0.045 & 0.982 & 0.074 & 0.960 & 0.09 & \mathbf{A} \\ 1 & \text { PU239 } & 0.770 & 0.038 & 0.772 & 0.056 & 1.000 & 0.09 & \mathbf{A} \\ 1 & \text { SR 90 } & 1.620 & 0.093 & 1.450 & 0.034 & 1.120 & 0.07 & \mathbf{A} \\ 1 & \text { U 234 } & 0.289 & 0.022 & 0.274 & 0.019 & 1.050 & 0.11 & \text { A } \\ 1 & \text { U 238 } & 0.299 & 0.022 & 0.275 & 0.024 & 1.090 & 0.13 & \text { A } \\ 1 & \text { U UG } & 0.013 & 0.004 & 0.022 & 0.003 & 0.570 & 0.18 & \mathbf{N}\end{array}$

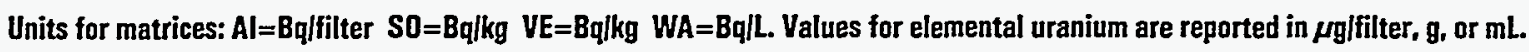




\section{QAP44 Results by Laboratory}

Lab: TO TMA/Eberline Oak Ridge Laboratory

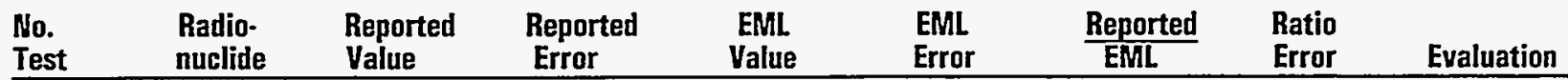

Matrix: Al

$\begin{array}{llrrrrrrr}1 & \text { AM241 } & 0.215 & 0.126 & 0.189 & 0.007 & 1.140 & 0.67 & \text { A } \\ 1 & \text { CE144 } & 29.600 & 2.530 & 33.300 & 3.300 & 0.890 & 0.12 & \text { A } \\ 1 & \text { CO 57 } & 7.990 & 0.461 & 8.900 & 0.900 & 0.900 & 0.11 & \text { A } \\ 1 & \text { CO 60 } & 27.800 & 1.270 & 29.500 & 2.900 & 0.940 & 0.10 & \text { A } \\ 1 & \text { CS134 } & 15.700 & 0.448 & 14.700 & 1.460 & 1.070 & 0.11 & \text { A } \\ 1 & \text { CS137 } & 6.030 & 0.490 & 6.640 & 0.700 & 0.910 & 0.12 & \text { A } \\ 1 & \text { GA 1 } & 2.090 & 0.060 & 1.620 & 0.150 & 1.290 & 0.13 & \text { A } \\ 1 & \text { GB 2 } & 1.560 & 0.050 & 1.770 & 0.150 & 0.880 & 0.08 & \text { W } \\ 1 & \text { MN 54 } & 3.180 & 0.593 & 3.440 & 0.380 & 0.920 & 0.20 & \text { A } \\ 1 & \text { PU238 } & 0.104 & 0.036 & 0.096 & 0.002 & 1.080 & 0.38 & \text { A } \\ 1 & \text { PU239 } & 0.110 & 0.037 & 0.093 & 0.003 & 1.190 & 0.40 & \text { W } \\ 1 & \text { RU106 } & 11.400 & 3.570 & 11.600 & 1.440 & 0.980 & 0.33 & \text { A } \\ 1 & \text { SB125 } & 9.650 & 1.010 & 9.780 & 1.030 & 0.990 & 0.15 & \text { A } \\ 1 & \text { SR 90 } & 1.080 & 0.081 & 1.060 & 0.037 & 1.020 & 0.08 & \text { A } \\ 1 & \text { U 234 } & 0.049 & 0.003 & 0.052 & 0.002 & 0.950 & 0.06 & \text { A } \\ 1 & \text { U 238 } & 0.048 & 0.005 & 0.053 & 0.002 & 0.910 & 0.10 & \text { A } \\ 1 & \text { U UG } & 3.980 & 0.251 & 4.310 & 0.100 & 0.920 & 0.06 & \text { A }\end{array}$

Matrix: So

$\begin{array}{llrrrrrrr}1 & \text { AM241 } & 3.230 & 2.210 & 3.690 & 0.454 & 0.880 & 0.61 & \text { A } \\ 1 & \text { CS137 } & 405.000 & 7.590 & 359.000 & 10.000 & 1.130 & 0.04 & \text { A } \\ 1 & \text { K 40 } & 411.000 & 32.900 & 465.000 & 30.000 & 0.880 & 0.09 & \text { A } \\ 1 & \text { PU238 } & 43.800 & 13.700 & 43.000 & 2.440 & 1.020 & 0.32 & \text { A } \\ 1 & \text { PU239 } & 9.220 & 3.590 & 9.230 & 0.346 & 1.000 & 0.39 & \text { A } \\ 1 & \text { SR 90 } & 983.000 & 41.700 & 1340.000 & 113.000 & 0.730 & 0.07 & W \\ 1 & \text { U 234 } & 38.700 & 0.907 & 34.200 & 3.520 & 1.130 & 0.12 & \text { W } \\ 1 & \text { U 238 } & 38.300 & 0.889 & 35.900 & 3.630 & 1.070 & 0.11 & \text { A } \\ 1 & \text { U UG } & 3.160 & 0.073 & 2.900 & 0.290 & 1.090 & 0.11 & \text { A }\end{array}$

Matrix: VE

\begin{tabular}{|c|c|c|c|c|c|c|}
\hline $\begin{array}{l}\text { AM241 } \\
\text { CM244 } \\
\text { CO 60 } \\
\text { CS137 } \\
\text { K 40 } \\
\text { PU239 } \\
\text { SR 90 }\end{array}$ & $\begin{array}{r}5.970 \\
2.250 \\
66.600 \\
1090.000 \\
1050.000 \\
9.830 \\
1370.000\end{array}$ & $\begin{array}{r}0.288 \\
0.222 \\
3.900 \\
16.100 \\
59.700 \\
3.480 \\
45.900\end{array}$ & $\begin{array}{r}5.600 \\
4.440 \\
59.700 \\
944.000 \\
1030.000 \\
9.820 \\
1300.000\end{array}$ & $\begin{array}{r}0.184 \\
0.202 \\
0.963 \\
16.200 \\
33.000 \\
1.220 \\
52.400\end{array}$ & $\begin{array}{l}1.070 \\
0.510 \\
1.120 \\
1.160 \\
1.020 \\
1.000 \\
1.050\end{array}$ & $\begin{array}{l}0.06 \\
0.06 \\
0.07 \\
0.03 \\
0.07 \\
0.38 \\
0.06\end{array}$ \\
\hline
\end{tabular}

Matrix: WA

$\begin{array}{rlrrrrrrr}1 & \text { AM241 } & 1.710 & 0.343 & 0.766 & 0.013 & 2.230 & 0.45 & \text { N } \\ 1 & \text { CO 60 } & 30.700 & 0.693 & 32.800 & 0.580 & 0.940 & 0.03 & \text { W } \\ 1 & \text { CS137 } & 39.500 & 0.953 & 38.300 & 0.881 & 1.030 & 0.03 & \text { A } \\ 1 & \text { GA 1 } & 1870.000 & 45.100 & 1850.000 & 185.000 & 1.010 & 0.10 & \text { A }\end{array}$

Units for matrices: $\mathrm{Al}=\mathrm{Bq} /$ filter $\mathrm{SO}=\mathrm{Bq} / \mathrm{kg}$ VE=Bq/kg WA=BqlL. Values for elemental uranium are reported in $\mu \mathrm{g} /$ filter, $\mathrm{g}$, or $\mathrm{mL}$.

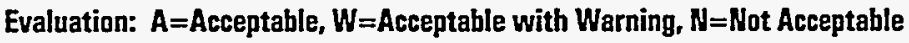
$\mathrm{pCi}=\mathrm{Bq} \times 27$ 
OAP44 Results by Laboratory

Lab: TO TMA/Eberline Oak Ridge Laboratory

\begin{tabular}{|c|c|c|c|c|c|c|c|c|}
\hline $\begin{array}{l}\text { Mo. } \\
\text { Test }\end{array}$ & $\begin{array}{l}\text { Radio- } \\
\text { nuclide }\end{array}$ & $\begin{array}{l}\text { Reported } \\
\text { Value }\end{array}$ & $\begin{array}{c}\text { Reported } \\
\text { Error }\end{array}$ & $\begin{array}{c}\text { EML } \\
\text { Value }\end{array}$ & $\begin{array}{l}\text { EML } \\
\text { Error }\end{array}$ & $\frac{\text { Reported }}{E M L}$ & $\begin{array}{l}\text { Ratio } \\
\text { Error }\end{array}$ & Evaluat \\
\hline Matrix: & WA & & & & & & & \\
\hline $\begin{array}{l}1 \\
1 \\
1 \\
1 \\
1 \\
1 \\
1 \\
1 \\
1\end{array}$ & $\begin{array}{l}\text { GB } 2 \\
H 3 \\
\text { MN } 54 \\
\text { PU238 } \\
\text { PU239 } \\
\text { SR 90 } \\
\text { U } 234 \\
\text { U 238 } \\
\text { U UG }\end{array}$ & $\begin{array}{r}903.000 \\
219.000 \\
40.100 \\
1.010 \\
0.746 \\
1.450 \\
0.257 \\
0.262 \\
0.021\end{array}$ & $\begin{array}{r}25.200 \\
42.200 \\
1.070 \\
0.332 \\
0.255 \\
0.247 \\
0.024 \\
0.024 \\
0.001\end{array}$ & $\begin{array}{r}744.000 \\
251.000 \\
38.400 \\
0.982 \\
0.772 \\
1.450 \\
0.274 \\
0.275 \\
0.022\end{array}$ & $\begin{array}{r}74.000 \\
11.400 \\
1.160 \\
0.074 \\
0.056 \\
0.034 \\
0.019 \\
0.024 \\
0.003\end{array}$ & $\begin{array}{l}1.210 \\
0.870 \\
1.040 \\
1.030 \\
0.970 \\
1.000 \\
0.940 \\
0.950 \\
0.950\end{array}$ & $\begin{array}{l}0.13 \\
0.17 \\
0.04 \\
0.35 \\
0.34 \\
0.17 \\
0.11 \\
0.12 \\
0.12\end{array}$ & $\begin{array}{l}\mathbf{A} \\
\mathbf{A} \\
\mathbf{A} \\
\mathbf{A} \\
\mathbf{A} \\
\mathbf{A} \\
\mathbf{A} \\
\mathbf{A} \\
\mathbf{A}\end{array}$ \\
\hline
\end{tabular}

Units for matrices: $A \mid=B q / f i l t e r ~ S O=B q / k g ~ V E=B q / k g ~ W A=B q / L$. Values for elemental uranium are reported in $\mu g / f i l t e r, g$, or $\mathrm{mL}$. 


\section{OAP44 Results by Laboratory}

Lab: TW Taiwan Radiation Monitoring Center

\begin{tabular}{lllllllll} 
No. & $\begin{array}{l}\text { Radio- } \\
\text { Test }\end{array}$ & $\begin{array}{l}\text { Reported } \\
\text { nuclide }\end{array}$ & $\begin{array}{c}\text { Reported } \\
\text { Value }\end{array}$ & $\begin{array}{c}\text { EML } \\
\text { Ealue }\end{array}$ & $\begin{array}{l}\text { EML. } \\
\text { Error }\end{array}$ & $\frac{\text { Reported }}{\text { EML }}$ & $\begin{array}{l}\text { Ratio } \\
\text { Error }\end{array}$ & Evaluation \\
\hline
\end{tabular}

Matrix: Al

$\begin{array}{llrlrllll}1 & \text { AM241 } & 0.175 & 0.009 & 0.189 & 0.007 & 0.930 & 0.06 & \text { A } \\ 1 & \text { CE144 } & 24.000 & 0.700 & 33.300 & 3.300 & 0.720 & 0.07 & \text { A } \\ 1 & \text { CO 57 } & 7.400 & 0.100 & 8.900 & 0.900 & 0.830 & 0.08 & \text { A } \\ 1 & \text { CO 60 } & 27.000 & 0.500 & 29.500 & 2.900 & 0.920 & 0.09 & \text { A } \\ 1 & \text { CS134 } & 12.000 & 0.100 & 14.700 & 1.460 & 0.820 & 0.08 & \text { A } \\ 1 & \text { CS137 } & 6.200 & 0.200 & 6.640 & 0.700 & 0.930 & 0.10 & \text { A } \\ 1 & \text { GA 1 } & 2.230 & 0.060 & 1.620 & 0.150 & 1.380 & 0.13 & \mathbf{W} \\ 1 & \text { GB 2 } & 1.790 & 0.040 & 1.770 & 0.150 & 1.010 & 0.09 & \mathbf{A} \\ 1 & \text { MN 54 } & 3.150 & 0.090 & 3.440 & 0.380 & 0.920 & 0.10 & \mathbf{A} \\ 1 & \text { PU238 } & 0.095 & 0.008 & 0.096 & 0.002 & 0.990 & 0.09 & \mathbf{A} \\ 1 & \text { PU239 } & 0.086 & 0.008 & 0.093 & 0.003 & 0.930 & 0.09 & \mathbf{A} \\ 1 & \text { RU106 } & 10.000 & 0.400 & 11.600 & 1.440 & 0.860 & 0.11 & \mathbf{A} \\ 1 & \text { SB125 } & 9.300 & 0.200 & 9.780 & 1.030 & 0.950 & 0.10 & \mathbf{A} \\ 1 & \text { SR 90 } & 0.900 & 0.040 & 1.060 & 0.037 & 0.850 & 0.05 & \mathbf{A} \\ 1 & \text { U BQ } & 0.430 & 0.020 & 0.107 & 0.003 & 4.020 & 0.21 & \mathbf{N} \\ 1 & \text { U UG } & 17.400 & 0.900 & 4.310 & 0.100 & 4.040 & 0.23 & \mathbf{N}\end{array}$

Matrix: So

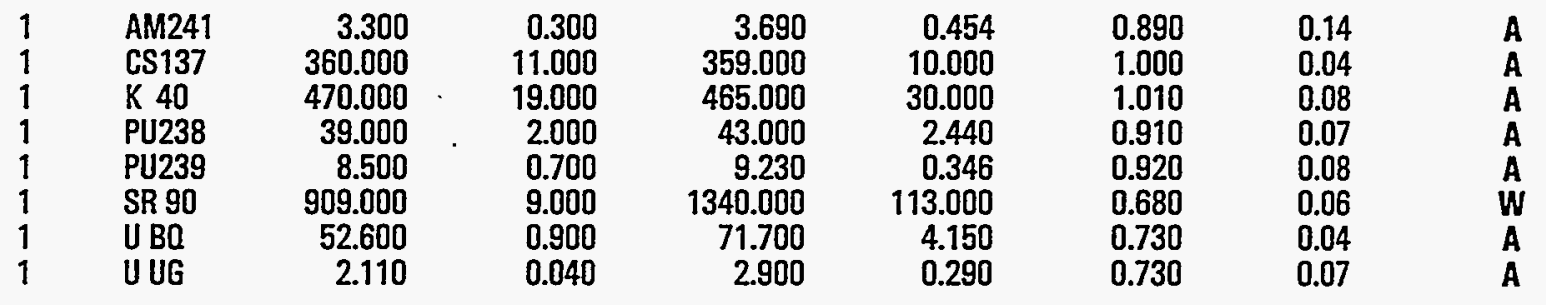

Matrix: VE

\begin{tabular}{|c|c|c|c|c|c|c|c|}
\hline 1 & AM241 & 5.800 & 0.200 & 5.600 & 0.184 & 1.040 & 0.05 \\
\hline 1 & CM244 & 4.000 & 0.100 & 4.440 & 0.202 & 0.900 & 0.05 \\
\hline 1 & CO 60 & 62.000 & 1.000 & 59.700 & 0.963 & 1.040 & 0.02 \\
\hline & CS137 & 1010.000 & 30.000 & 944.000 & 16.200 & 1.070 & 0.04 \\
\hline & K 40 & 1070.000 & 43.000 & 1030.000 & 33.000 & 1.040 & 0.05 \\
\hline & PU239 & 8.400 & . 0.400 & 9.820 & 1.220 & 0.860 & 0.11 \\
\hline & SR 90 & 930.000 & 10.000 & 1300.000 & 52.400 & 0.720 & 0.03 \\
\hline
\end{tabular}

Matrix: WA

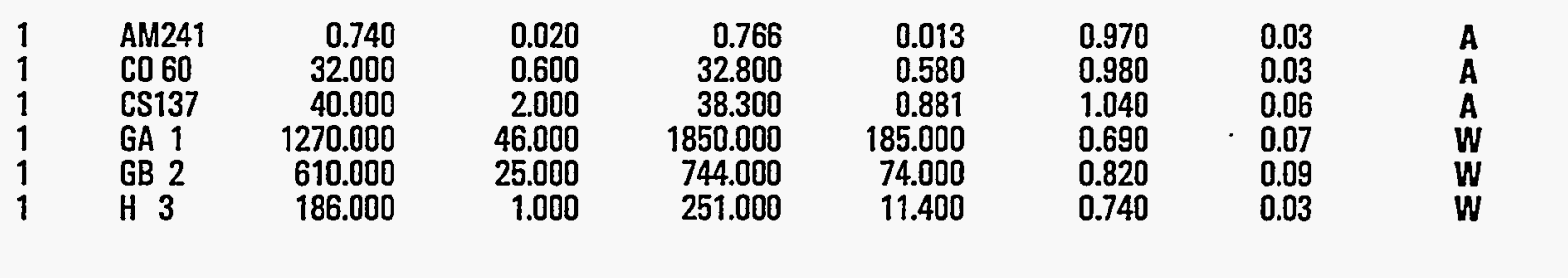

Units for matrices: $\mathrm{Al}=\mathrm{Bq} / \mathrm{filter} \mathrm{SO}=\mathrm{Bq} / \mathrm{kg} \mathrm{VE}=\mathrm{Bq} / \mathrm{kg} \mathrm{WA}=\mathrm{Bq} / \mathrm{L}$. Values for elemental uranium are reported in $\mu \mathrm{g} / \mathrm{filter}, \mathrm{g}$, or $\mathrm{mL}$.

Evaluation: $A=A c c e p t a b l e, W=A c c e p t a b l e$ with Warning, $N=$ Not Acceptable

$\mathrm{pCi}=\mathrm{Bq} \times 27$ 


\section{QAP44 Results by Laboratory}

Lab: TW Taiwan Radiation Monitoring Center

\begin{tabular}{lllllllll} 
No. & Radio- & $\begin{array}{l}\text { Reported } \\
\text { Value }\end{array}$ & $\begin{array}{l}\text { Reported } \\
\text { Error }\end{array}$ & $\begin{array}{c}\text { EML } \\
\text { Value }\end{array}$ & $\begin{array}{l}\text { EML } \\
\text { Error }\end{array}$ & $\frac{\text { Reported }}{\text { EML }}$ & $\begin{array}{l}\text { Ratio } \\
\text { Error }\end{array}$ Evaluation \\
\hline
\end{tabular}

Matrix: WA

$\begin{array}{llrlrllll}1 & \text { MN 54 } & 40.000 & 0.800 & 38.400 & 1.160 & 1.040 & 0.04 & \text { A } \\ 1 & \text { PU238 } & 0.930 & 0.070 & 0.982 & 0.074 & 0.950 & \mathbf{0 . 1 0} & \mathbf{A} \\ 1 & \text { PU239 } & 0.580 & 0.060 & 0.772 & 0.056 & 0.750 & 0.09 & \text { W } \\ 1 & \text { SR 90 } & 1.300 & 0.100 & 1.450 & 0.034 & 0.900 & 0.07 & \text { A } \\ 1 & \text { U BQ } & 1.690 & 0.080 & 0.559 & 0.047 & 3.020 & 0.29 & \mathbf{N} \\ 1 & \text { U UG } & 0.068 & 0.003 & 0.022 & 0.003 & 3.060 & 0.38 & \mathbf{N}\end{array}$

Units for matrices: $A|=B q / f i l t e r ~ S O=B q / k g ~ V E=B q| k g ~ W A=B q \mid L$. Values for elemental uranium are reported in $\mu g / f i l t e r, g$, or $\mathrm{mL}$. 


\section{QAP44 Results by Laboratory}

Lab: TX Texas Dept. of Health/Laboratories, Austin

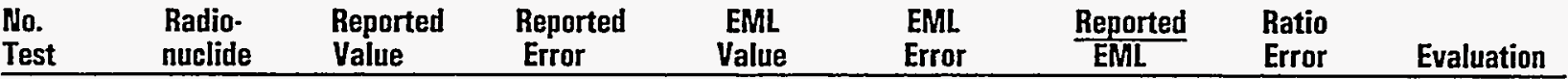

Matrix: Al

$\begin{array}{llrlrllll}1 & \text { AM241 } & 0.194 & 0.014 & 0.189 & 0.007 & 1.030 & 0.08 & A \\ 1 & \text { CE144 } & 22.900 & 0.200 & 33.300 & 3.300 & 0.690 & 0.07 & \text { A } \\ 1 & \text { CD 57 } & 6.120 & 0.040 & 8.900 & 0.900 & 0.690 & 0.07 & \text { W } \\ 1 & \text { CD 60 } & 24.000 & 0.200 & 29.500 & 2.900 & 0.810 & 0.08 & \text { W } \\ 1 & \text { CS134 } & 11.800 & 0.200 & 14.700 & 1.460 & 0.800 & 0.08 & A \\ 1 & \text { CS137 } & 5.350 & 0.080 & 6.640 & 0.700 & 0.810 & 0.09 & \text { W } \\ 1 & \text { MN 54 } & 3.170 & 0.110 & 3.440 & 0.380 & 0.920 & 0.11 & A \\ 1 & \text { PU238 } & 0.103 & 0.007 & 0.096 & 0.002 & 1.070 & 0.08 & A \\ 1 & \text { PU239 } & 0.105 & 0.007 & 0.093 & 0.003 & 1.130 & 0.08 & A \\ 1 & \text { RU106 } & 8.190 & 0.490 & 11.600 & 1.440 & 0.710 & 0.10 & A \\ 1 & \text { SB125 } & 7.460 & 0.190 & 9.780 & 1.030 & 0.760 & 0.08 & A \\ 1 & \text { U 234 } & 0.072 & 0.006 & 0.052 & 0.002 & 1.390 & 0.12 & \text { W } \\ 1 & \text { U 238 } & 0.068 & 0.004 & 0.053 & 0.002 & 1.280 & 0.09 & A\end{array}$

Matrix: So

$\begin{array}{llrr}1 & \text { AM241 } & 5.920 & 0.930 \\ 1 & \text { CS137 } & 393.000 & 4.000 \\ 1 & \text { K 40 } & 505.000 & 11.000 \\ 1 & \text { PU238 } & 42.900 & 2.600 \\ 1 & \text { PU239 } & 10.300 & 0.900 \\ 1 & \text { SR 90 } & 1340.000 & 52.000 \\ 1 & \text { U 234 } & 40.700 & 3.000 \\ 1 & \text { U 238 } & 41.600 & 3.000\end{array}$

$0.930 \quad 3.690$

4.000

11.000

2.600

0.900

2.000

3.000

$$
\begin{array}{r}
3.690 \\
359.000 \\
465.000 \\
43.000 \\
9.230 \\
1340.000 \\
34.200 \\
35.900
\end{array}
$$

0.454
10.000
30.000
2.440
0.346
113.000
3.520
3.630

1.600

1.100

1.090

1.000

1.120

1.000

1.190

1.160
0.32

0.03

0.07

0.08

0.11

0.09

0.15

0.14
$A$
$A$
$W$
$W$
$A$
$W$
$A$
$A$
$A$
$A$
$A$
$W$
$A$

Matrix: VE

$\begin{array}{llrrrrrrr}1 & \text { AM241 } & 6.660 & 0.370 & 5.600 & 0.184 & 1.190 & 0.08 & \text { A } \\ 1 & \text { CO 60 } & 73.300 & 2.200 & 59.700 & 0.963 & 1.230 & 0.04 & \text { A } \\ 1 & \text { CS137 } & 1260.000 & 8.000 & 944.000 & 16.200 & 1.340 & 0.02 & \text { W } \\ 1 & \text { K 40 } & 1350.000 & 26.000 & 1030.000 & 33.000 & 1.310 & 0.05 & \text { W } \\ 1 & \text { PU239 } & 10.300 & 0.600 & 9.820 & 1.220 & 1.050 & 0.14 & \text { A } \\ 1 & \text { SR 90 } & 1310.000 & 60.000 & 1300.000 & 52.400 & 1.010 & 0.06 & \text { A }\end{array}$

Matrix: WA

\begin{tabular}{llrrrrrrr}
1 & AM241 & 1.000 & 0.093 & 0.766 & 0.013 & 1.310 & 0.12 & $\mathbf{W}$ \\
1 & CO 60 & 37.600 & 0.700 & 32.800 & 0.580 & 1.150 & 0.03 & $\mathbf{W}$ \\
1 & CS137 & 47.400 & 0.700 & 38.300 & 0.881 & 1.240 & 0.03 & $\mathbf{W}$ \\
1 & H 3 & 209.000 & 11.000 & 251.000 & 11.400 & 0.830 & 0.06 & $\mathbf{W}$ \\
1 & MN 54 & 48.300 & 0.800 & 38.400 & 1.160 & 1.260 & 0.04 & $\mathbf{N}$ \\
1 & PU238 & 0.970 & 0.071 & 0.982 & 0.074 & 0.990 & 0.10 & $\mathbf{A}$ \\
1 & PU239 & 0.840 & 0.069 & 0.772 & 0.056 & 1.090 & 0.12 & $\mathbf{A}$ \\
1 & SR 90 & 1.920 & 0.620 & 1.450 & 0.034 & 1.320 & 0.43 & $\mathbf{W}$ \\
1 & U 234 & 0.440 & 0.026 & 0.274 & 0.019 & 1.610 & 0.15 & $\mathbf{N}$ \\
1 & U 238 & 0.440 & 0.026 & 0.275 & 0.024 & 1.600 & 0.17 & $\mathbf{N}$ \\
& & & & & & & & \\
\hline
\end{tabular}

Units for matrices: $\mathrm{Al}=\mathrm{Bq} \mid \mathrm{filter} \mathrm{SO}=\mathrm{Bq} / \mathrm{kg}$ VE=Bq/kg WA=Bq/L . Values for elemental uranium are reported in $\mu \mathrm{g} \mid$ filter, $\mathrm{g}$, or $\mathrm{mL}$.

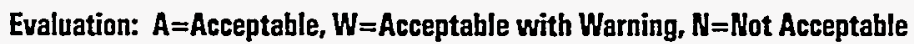

$\mathrm{pCi}=\mathrm{Bq} \times 27$ 
QAP44 Results by Laboratory

Lab: TY Scientific Production Association, Russia

\begin{tabular}{llllllll} 
No. & $\begin{array}{l}\text { Radio- } \\
\text { nuclide }\end{array}$ & $\begin{array}{l}\text { Reported } \\
\text { Value }\end{array}$ & $\begin{array}{l}\text { Reported } \\
\text { Error }\end{array}$ & $\begin{array}{c}\text { EML } \\
\text { Value }\end{array}$ & $\begin{array}{c}\text { EML } \\
\text { Error }\end{array}$ & $\frac{\text { Reported }}{\text { EML }}$ & $\begin{array}{l}\text { Ratio } \\
\text { Error }\end{array}$ \\
\hline
\end{tabular}

Matrix: Al

$\begin{array}{llrlrllll}1 & \text { CE144 } & 19.200 & 0.800 & 33.300 & 3.300 & 0.580 & 0.06 & \mathbf{N} \\ 1 & \text { CO 57 } & 4.300 & 0.400 & 8.900 & 0.900 & 0.480 & 0.07 & \mathbf{N} \\ 1 & \text { CO 60 } & 18.500 & 0.600 & 29.500 & 2.900 & 0.630 & 0.06 & \mathbf{N} \\ 1 & \text { CS134 } & 10.200 & 0.300 & 14.700 & 1.460 & 0.690 & 0.07 & \mathbf{N} \\ 1 & \text { CS137 } & 5.300 & 0.300 & 6.640 & 0.700 & 0.800 & 0.10 & W \\ 1 & \text { MN 54 } & 2.700 & 0.200 & 3.440 & 0.380 & 0.790 & 0.10 & \mathbf{W} \\ 1 & \text { RU106 } & 8.600 & 0.700 & 11.600 & 1.440 & 0.740 & 0.11 & \mathbf{A} \\ 1 & \text { SB125 } & 8.200 & 0.500 & 9.780 & 1.030 & 0.840 & 0.10 & \mathbf{A}\end{array}$

Matrix: So

$\begin{array}{llrrrrrrr}1 & \text { CS137 } & 359.000 & 4.000 & 359.000 & 10.000 & 1.000 & 0.03 & \\ 1 & K ~ 40 & 478.000 & 15.000 & 465.000 & 30.000 & 1.030 & 0.07 & A \\ 1 & U 238 & 106.000 & 20.000 & 35.900 & 3.630 & 2.950 & 0.63 & \text { N }\end{array}$

Matrix: VE

$\begin{array}{rlrrrrrrr}1 & \text { CO 60 } & 56.000 & 3.000 & 59.700 & 0.963 & 0.940 & 0.05 & \text { A } \\ 1 & \text { CS137 } & 978.000 & 10.000 & 944.000 & 16.200 & 1.040 & 0.02 & \text { A } \\ 1 & \text { K 40 } & 1120.000 & 50.000 & 1030.000 & 33.000 & 1.090 & 0.06 & \text { A } \\ 1 & \text { PU239 } & 8.700 & 1.400 & 9.820 & 1.220 & 0.890 & 0.18 & \text { A } \\ 1 & \text { SR 90 } & 1500.000 & 320.000 & 1300.000 & 52.400 & 1.150 & 0.25 & \end{array}$

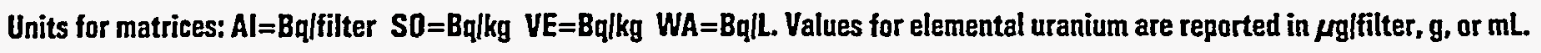
$p C i=B q \times 27$ 
QAP44 Results by Laboratory

Lab: UC Lockheed Martin, Paducah, KY

\begin{tabular}{|c|c|c|c|c|c|c|c|c|}
\hline $\begin{array}{l}\text { No. } \\
\text { Test }\end{array}$ & $\begin{array}{l}\text { Radio- } \\
\text { nuclide }\end{array}$ & $\begin{array}{l}\text { Reported } \\
\text { Value }\end{array}$ & $\begin{array}{l}\text { Reported } \\
\text { Error }\end{array}$ & $\begin{array}{c}\text { EML } \\
\text { Value }\end{array}$ & $\begin{array}{l}\text { EML } \\
\text { Error }\end{array}$ & $\frac{\text { Reported }}{\text { EML }}$ & $\begin{array}{l}\text { Ratio } \\
\text { Error }\end{array}$ & Evaluation \\
\hline
\end{tabular}

Matrix: Al

$\begin{array}{llrlrllll}1 & \text { CO 60 } & 25.100 & 0.130 & 29.500 & 2.900 & 0.850 & 0.08 & A \\ 1 & \text { CS137 } & 6.960 & 0.310 & 6.640 & 0.700 & 1.050 & 0.12 & A \\ 1 & \text { GA } 1 & 1.690 & 0.090 & 1.620 & 0.150 & 1.040 & 0.11 & A \\ 1 & \text { GB 2 } & 1.640 & 0.060 & 1.770 & 0.150 & 0.930 & 0.09 & A\end{array}$

Matrix: So

$\begin{array}{lllllllll}1 & \text { CS137 } & 404.000 & 17.900 & 359.000 & 10.000 & 1.130 & 0.06 & \text { A } \\ 1 & \text { K } 40 & 552.000 & 24.900 & 465.000 & 30.000 & 1.190 & 0.09 & \text { A }\end{array}$

Matrix: VE

$\begin{array}{llrrrrrrr}1 & \text { CO 60 } & 66.200 & 2.370 & 59.700 & 0.963 & 1.110 & 0.04 & \text { A } \\ 1 & \text { CS137 } & 1160.000 & 77.200 & 944.000 & 16.200 & 1.230 & 0.08 & \text { A } \\ 1 & \text { K 40 } & 1210.000 & 60.200 & 1030.000 & 33.000 & 1.170 & 0.07 & \text { A }\end{array}$

Matrix: WA

$\begin{array}{llrrrrrrr}1 & \text { CO 60 } & 35.100 & 0.700 & 32.800 & 0.580 & 1.070 & 0.03 & \text { A } \\ 1 & \text { CS137 } & 41.500 & 1.690 & 38.300 & 0.881 & 1.080 & 0.05 & \text { A } \\ 1 & \text { GA 1 } & 1970.000 & 92.000 & 1850.000 & 185.000 & 1.070 & 0.12 & \text { A } \\ 1 & \text { GB 2 } & 634.000 & 28.000 & 744.000 & 74.000 & 0.850 & 0.09 & \text { W } \\ 1 & \text { PU239 } & 0.780 & 0.110 & 0.772 & 0.056 & 1.010 & 0.16 & \text { A } \\ 1 & \text { U UG } & 0.024 & 0.002 & 0.022 & 0.003 & 1.080 & 0.17 & \text { A }\end{array}$

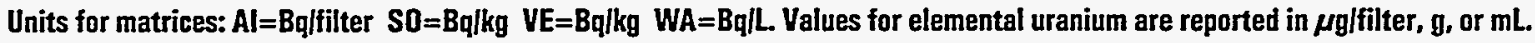

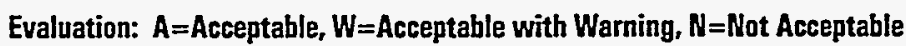

$p C i=B q \times 27$ 


\section{QAP44 Results by Laboratory}

Lab: UK Lockheed Martin Energy Systems, Oak Ridge

\begin{tabular}{lllllllll} 
No. & $\begin{array}{l}\text { Radio- } \\
\text { nuclide }\end{array}$ & $\begin{array}{l}\text { Reported } \\
\text { Value }\end{array}$ & $\begin{array}{c}\text { Reported } \\
\text { Error }\end{array}$ & $\begin{array}{c}\text { EML } \\
\text { Value }\end{array}$ & $\begin{array}{c}\text { EML } \\
\text { Error }\end{array}$ & $\frac{\text { Reported }}{\text { EML }}$ & $\begin{array}{l}\text { Ratio } \\
\text { Error }\end{array}$ & Evaluation \\
\hline
\end{tabular}

Matrix: Al

$\begin{array}{llrlrllll}1 & \text { AM241 } & 0.208 & 0.030 & 0.189 & 0.007 & 1.100 & 0.16 & \text { A } \\ 1 & \text { CE144 } & 21.800 & 0.720 & 33.300 & 3.300 & 0.660 & 0.07 & \text { W } \\ 1 & \text { CO 57 } & 6.380 & 0.130 & 8.900 & 0.900 & 0.720 & 0.07 & \text { A } \\ 1 & \text { CO 60 } & 24.900 & 0.370 & 29.500 & 2.900 & 0.840 & 0.08 & \text { A } \\ 1 & \text { CS134 } & 12.500 & 0.220 & 14.700 & 1.460 & 0.850 & 0.09 & \text { A } \\ 1 & \text { CS137 } & 5.190 & 0.260 & 6.640 & 0.700 & 0.780 & 0.09 & \text { W } \\ 1 & \text { MN 54 } & 2.770 & 0.270 & 3.440 & 0.380 & 0.810 & 0.12 & \text { W } \\ 1 & \text { PU238 } & 0.103 & 0.032 & 0.096 & 0.002 & 1.070 & 0.33 & \text { A } \\ 1 & \text { PU239 } & 0.111 & 0.033 & 0.093 & 0.003 & 1.200 & 0.36 & \text { W } \\ 1 & \text { U BO } & 0.184 & 0.039 & 0.107 & 0.003 & 1.720 & 0.37 & \text { W }\end{array}$

Matrix: So

$\begin{array}{llrrrrrrr}1 & \text { AM241 } & 10.100 & 0.810 & 3.690 & 0.454 & 2.740 & 0.40 & \text { N } \\ 1 & \text { CS137 } & 375.000 & 2.600 & 359.000 & 10.000 & .1 .040 & 0.03 & \text { A } \\ 1 & \text { K 40 } & 506.000 & 15.000 & 465.000 & 30.000 & 1.090 & 0.08 & \text { A } \\ 1 & \text { PU238 } & 36.900 & 2.200 & 43.000 & 2.440 & 0.860 & 0.07 & \text { A } \\ 1 & \text { PU239 } & 7.110 & 0.980 & 9.230 & 0.346 & 0.770 & 0.11 & \text { W } \\ 1 & \text { U BQ } & 48.000 & 4.100 & 71.700 & 4.150 & 0.670 & 0.07 & \text { A }\end{array}$

Matrix: WA

$\begin{array}{llrlrllll}1 & \text { AM241 } & 0.835 & 0.091 & 0.766 & 0.013 & 1.090 & 0.12 & \text { A } \\ 1 & \text { CO 60 } & 36.100 & 0.760 & 32.800 & 0.580 & 1.100 & 0.03 & \text { A } \\ 1 & \text { CS137 } & 43.200 & 0.990 & 38.300 & 0.881 & 1.130 & 0.04 & \text { A } \\ 1 & \text { MN 54 } & 48.100 & 1.100 & 38.400 & 1.160 & 1.250 & 0.05 & \text { N } \\ 1 & \text { PU238 } & 0.921 & 0.100 & 0.982 & 0.074 & 0.940 & 0.12 & \text { A } \\ 1 & \text { PU239 } & 0.762 & 0.092 & 0.772 & 0.056 & 0.990 & 0.14 & \text { A } \\ 1 & \text { U BO } & 0.595 & 0.100 & 0.559 & 0.047 & 1.060 & 0.20 & \text { A }\end{array}$

Units for matrices: $\mathrm{Al}=\mathrm{Bq} / \mathrm{filter} \mathrm{SO}=\mathrm{Bq} / \mathrm{kg}$ VE=Bq/kg $W A=B \mathrm{q} / \mathrm{L}$. Values for elemental uranium are reported in $\mu g / f i l t e r, g$, or $\mathrm{mL}$. 


\section{QAP44 Results by Laboratory}

Lab: UP Lockheed Martin Energy Systems, Y.12 Plant, Oak Ridge

\begin{tabular}{|c|c|c|c|c|c|c|c|c|}
\hline $\begin{array}{l}\text { No. } \\
\text { Test }\end{array}$ & $\begin{array}{l}\text { Radio- } \\
\text { nuclide }\end{array}$ & $\begin{array}{l}\text { Reported } \\
\text { Value }\end{array}$ & $\begin{array}{l}\text { Reported } \\
\text { Error }\end{array}$ & $\begin{array}{l}\text { EML } \\
\text { Value }\end{array}$ & $\begin{array}{l}\text { EML } \\
\text { Error }\end{array}$ & $\frac{\text { Reported }}{\text { EML }}$ & $\begin{array}{l}\text { Ratio } \\
\text { Error }\end{array}$ & Evaluation \\
\hline
\end{tabular}

Matrix: Al

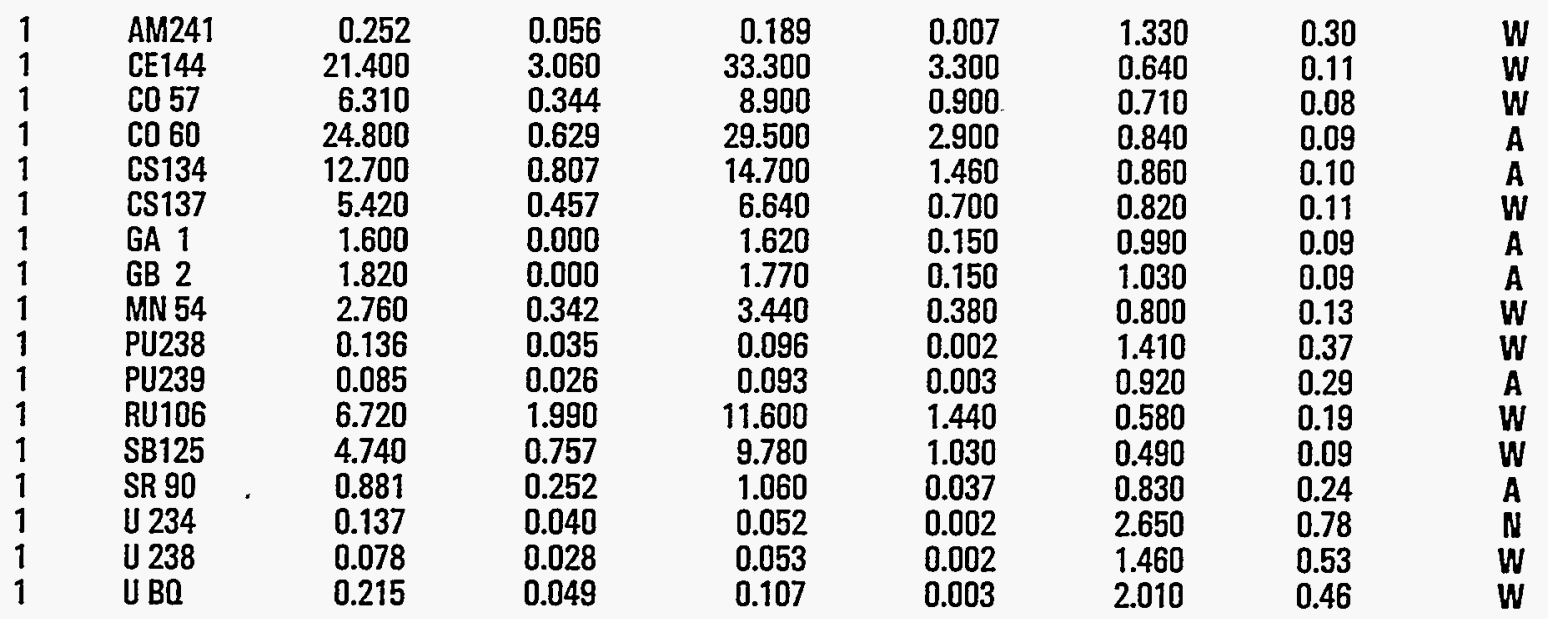

Matrix: So

$\begin{array}{llrrrrrrr}1 & \text { CS137 } & 325.000 & 34.900 & 359.000 & 10.000 & 0.910 & 0.10 & \text { A } \\ 1 & \text { PU238 } & 41.400 & 6.020 & 43.000 & 2.440 & 0.960 & 0.15 & \text { A } \\ 1 & \text { PU239 } & 8.450 & 1.590 & 9.230 & 0.346 & 0.920 & 0.18 & \text { A } \\ 1 & \text { SR 90 } & 1100.000 & 149.000 & 1340.000 & 113.000 & 0.820 & 0.13 & \text { A } \\ 1 & \text { U 234 } & 36.800 & 6.160 & 34.200 & 3.520 & 1.080 & 0.21 & W \\ 1 & \text { U 238 } & 37.100 & 6.220 & 35.900 & 3.630 & 1.030 & 0.20 & \text { A } \\ 1 & \text { U UG } & 2.710 & 0.000 & 2.900 & 0.290 & 0.930 & 0.09 & \text { A }\end{array}$

Matrix: WA

$\begin{array}{llrrrrrrr}1 & \text { AM241 } & 0.908 & 0.178 & 0.766 & 0.013 & 1.180 & 0.23 & \text { A } \\ 1 & \text { CO 60 } & 33.600 & 1.180 & 32.800 & 0.580 & 1.020 & 0.04 & \text { A } \\ 1 & \text { CS137 } & 43.600 & 3.020 & 38.300 & 0.881 & 1.140 & 0.08 & \text { A } \\ 1 & \text { GA 1 } & 1490.000 & 108.000 & 1850.000 & 185.000 & 0.810 & 0.10 & \text { A } \\ 1 & \text { GB 2 } & 700.000 & 67.200 & 744.000 & 74.000 & 0.940 & 0.13 & \text { A } \\ 1 & \text { H 3 } & 227.000 & 15.400 & 251.000 & 11.400 & 0.900 & 0.07 & \text { A } \\ 1 & \text { MN 54 } & 43.300 & 3.290 & 38.400 & 1.160 & 1.130 & 0.09 & \text { A } \\ 1 & \text { PU238 } & 0.992 & 0.205 & 0.982 & 0.074 & 1.010 & 0.22 & \text { A } \\ 1 & \text { PU239 } & 0.767 & 0.166 & 0.772 & 0.056 & 0.990 & 0.23 & \text { A } \\ 1 & \text { SR 90 } & 1.190 & 0.670 & 1.450 & 0.034 & 0.820 & 0.46 & \text { W } \\ 1 & \text { U 234 } & 0.349 & 0.113 & 0.274 & 0.019 & 1.270 & 0.42 & \text { W } \\ 1 & \text { U 238 } & 0.303 & 0.157 & 0.275 & 0.024 & 1.100 & 0.58 & \text { A } \\ 1 & \text { U UG } & 0.024 & 0.000 & 0.022 & 0.003 & 1.080 & 0.13 & \text { A }\end{array}$

Units for matrices: $\mathrm{Al}=\mathrm{Bq} / \mathrm{filter} \mathrm{SO}=\mathrm{Bq} / \mathrm{kg}$ VE $=\mathrm{Bq} / \mathrm{kg} W \mathrm{WA}=\mathrm{Bq} / \mathrm{L}$. Values for elemental uranium are reported in $\mu \mathrm{g} /$ filter, $\mathrm{g}$, or $\mathrm{mL}$. 
QAP44 Results by Laboratory

Lab: UY Lockheed Martin Energy Systems, Y-12 Plant, Oak Ridge

\begin{tabular}{|c|c|c|c|c|c|c|c|c|}
\hline $\begin{array}{l}\text { No. } \\
\text { Test }\end{array}$ & $\begin{array}{l}\text { Radio. } \\
\text { nuclide }\end{array}$ & $\begin{array}{l}\text { Reported } \\
\text { Value }\end{array}$ & $\begin{array}{l}\text { Reported } \\
\text { Error }\end{array}$ & $\begin{array}{l}\text { EML } \\
\text { Value }\end{array}$ & $\begin{array}{l}\text { EML } \\
\text { Error }\end{array}$ & $\frac{\text { Reported }}{\text { EML }}$ & $\begin{array}{l}\text { Ratio } \\
\text { Error }\end{array}$ & Evaluation \\
\hline
\end{tabular}

Matrix: Al

$\begin{array}{llrlrllll}1 & \text { AM241 } & 0.270 & 0.060 & 0.189 & 0.007 & 1.430 & 0.32 & \text { W } \\ 1 & \text { CE144. } & 25.300 & 0.950 & 33.300 & 3.300 & 0.760 & 0.08 & \text { A } \\ 1 & \text { CO 57 } & 7.200 & 0.200 & 8.900 & 0.900 & 0.810 & 0.08 & \text { A } \\ 1 & \text { CO 60 } & 27.000 & 1.700 & 29.500 & 2.900 & 0.920 & 0.11 & \text { A } \\ 1 & \text { CS134 } & 14.300 & 0.510 & 14.700 & 1.460 & 0.970 & 0.10 & \text { A } \\ 1 & \text { CS137 } & 5.800 & 0.410 & 6.640 & 0.700 & 0.870 & 0.11 & \text { A } \\ 1 & \text { GA 1 } & 1.950 & 0.000 & 1.620 & 0.150 & 1.200 & 0.11 & \text { A } \\ 1 & \text { GB 2 } & 2.150 & 0.000 & 1.770 & 0.150 & 1.220 & 0.10 & \text { A } \\ 1 & \text { MN 54 } & 3.200 & 0.320 & 3.440 & 0.380 & 0.930 & 0.14 & \text { A } \\ 1 & \text { PU238 } & 0.100 & 0.024 & 0.096 & 0.002 & 1.040 & 0.25 & \text { A } \\ 1 & \text { PU239 } & 0.094 & 0.023 & 0.093 & 0.003 & 1.010 & 0.25 & \text { A } \\ 1 & \text { RU106 } & 11.300 & 1.900 & 11.600 & 1.440 & 0.970 & 0.20 & \text { A } \\ 1 & \text { SB125 } & 9.530 & 0.560 & 9.780 & 1.030 & 0.970 & 0.12 & \text { A } \\ 1 & \text { SR 90 } & 1.120 & 0.200 & 1.060 & 0.037 & 1.060 & 0.19 & \text { A } \\ 1 & \text { U 234 } & 0.095 & 0.030 & 0.052 & 0.002 & 1.840 & 0.58 & \text { W } \\ 1 & \text { U 238 } & 0.076 & 0.027 & 0.053 & 0.002 & 1.430 & 0.51 & \text { W }\end{array}$

Matrix: So

$\begin{array}{llrrrrrrr}1 & \text { CS137 } & 377.000 & 40.000 & 359.000 & 10.000 & 1.050 & 0.12 & \text { A } \\ 1 & \text { K 40 } & 544.000 & 136.000 & 465.000 & 30.000 & 1.170 & 0.30 & A \\ 1 & \text { PU238 } & 49.600 & 10.000 & 43.000 & 2.440 & 1.150 & 0.24 & \text { A } \\ 1 & \text { PU239 } & 11.000 & 3.500 & 9.230 & 0.346 & 1.190 & 0.38 & A \\ 1 & \text { SR 90 } & 1140.000 & 300.000 & 1340.000 & 113.000 & 0.850 & 0.24 & \text { A } \\ 1 & \text { U 234 } & 37.000 & 9.000 & 34.200 & 3.520 & 1.080 & 0.29 & \text { W } \\ 1 & \text { U 238 } & 36.000 & 9.100 & 35.900 & 3.630 & 1.000 & 0.27 & A \\ 1 & \text { U UG } & 2.810 & 0.000 & 2.900 & 0.290 & 0.970 & 0.10 & A\end{array}$

Matrix: WA

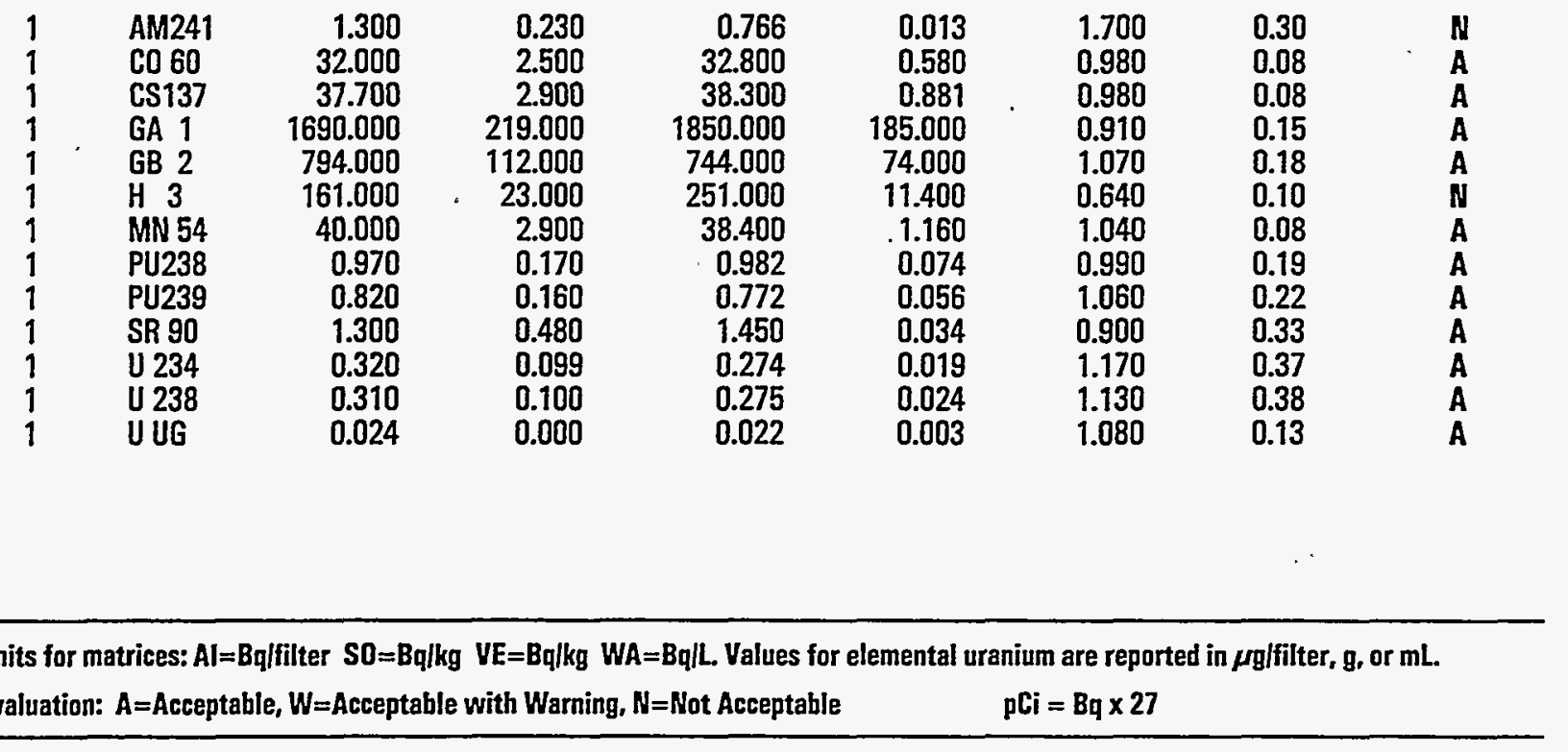




\section{QAP44 Results by Laboratory}

Lab: WA Environmental Radiation Lab, Off. of Public Health Labs. Seattle

\begin{tabular}{lllllllll} 
No. & Radio- & Reported & Reported & EML & EML & Reported & Ratio \\
nuclide & Value & Error & Value & Error & EML & Error & Evaluation \\
\hline
\end{tabular}

Matrix: Al

\begin{tabular}{|c|c|c|c|c|c|c|}
\hline AM241 & 0.162 & 0.016 & 0.189 & 0.007 & 0.860 & 0.09 \\
\hline CE144 & 25.500 & 2.400 . & 33.300 & 3.300 & 0.770 & 0.11 \\
\hline CO 57 & 7.300 & 0.370 & 8.900 & 0.900 & 0.820 & 0.09 \\
\hline CO 60 & 27.400 & 0.700 & 29.500 & 2.900 & 0.930 & 0.09 \\
\hline CS134 & 11.600 & 0.700 & 14.700 & 1.460 & 0.790 & 0.09 \\
\hline CS137 & 6.200 & 1.000 & 6.640 & 0.700 & 0.930 & 0.18 \\
\hline GA 1 & 2.200 & 0.080 & 1.620 & 0.150 & 1.360 & 0.14 \\
\hline GB 2 & 2.170 & 0.060 & 1.770 & 0.150 & 1.230 & 0.11 \\
\hline MN 54 & 3.420 & 0.260 & 3.440 & 0.380 & 0.990 & 0.13 \\
\hline PU238 & 0.095 & 0.023 & 0.096 & 0.002 & 0.990 & 0.24 \\
\hline PU239 & 0.084 & 0.019 & 0.093 & 0.003 & 0.910 & 0.21 \\
\hline RU106 & 9.300 & 1.600 & 11.600 & 1.440 & 0.800 & 0.17 \\
\hline SB125 & 10.100 & 1.000 & 9.780 & 1.030 & 1.030 & 0.15 \\
\hline SR 90 & 1.180 & 0.150 & 1.060 & 0.037 & 1.110 & 0.15 \\
\hline U 234 & 0.043 & 0.010 & 0.052 & 0.002 & 0.830 & 0.20 \\
\hline U 238 & 0.052 & 0.010 & 0.053 & 0.002 & 0.980 & 0.19 \\
\hline U BQ & 0.106 & 0.015 & 0.107 & 0.003 & 0.990 & 0.14 \\
\hline
\end{tabular}

Matrix: So

$\begin{array}{llrrrrrrr}1 & \text { AM241 } & 2.850 & 0.320 & 3.690 & 0.454 & 0.770 & 0.13 & \text { A } \\ 1 & \text { CS137 } & 401.000 & 31.000 & 359.000 & 10.000 & 1.120 & 0.09 & \text { A } \\ 1 & \text { K 40 } & 549.000 & 31.000 & 465.000 & 30.000 & 1.180 & 0.10 & \text { A } \\ 1 & \text { PU238 } & 43.300 & 1.200 & 43.000 & 2.440 & 1.010 & 0.06 & \text { A } \\ 1 & \text { PU239 } & 8.990 & 0.600 & 9.230 & 0.346 & 0.970 & 0.07 & \text { A } \\ 1 & \text { SR 90 } & 1540.000 & 20.000 & 1340.000 & 113.000 & 1.150 & 0.10 & \text { A } \\ 1 & \text { U 234 } & 45.500 & 4.100 & 34.200 & 3.520 & 1.330 & 0.18 & \text { N } \\ 1 & \text { U 238 } & 45.900 & 4.000 & 35.900 & 3.630 & 1.280 & 0.17 & \text { W } \\ 1 & \text { U BO } & 93.200 & 5.800 & 71.700 & 4.150 & 1.300 & 0.11 & \text { W }\end{array}$

Matrix: VE

$\begin{array}{llrrrrrrr}1 & \text { AM241 } & 5.620 & 0.630 & 5.600 & 0.184 & 1.000 & 0.12 & \text { A } \\ 1 & \text { CO 60 } & 59.900 & 3.000 & 59.700 & 0.963 & 1.000 & 0.05 & \text { A } \\ 1 & \text { CS137 } & 99.200 & 7.400 & 944.000 & 16.200 & 0.110 & 0.01 & \text { N } \\ 1 & \text { K 40 } & 1090.000 & 60.000 & 1030.000 & 33.000 & 1.060 & 0.07 & \text { A } \\ 1 & \text { PU239 } & 9.950 & 0.520 & 9.820 & 1.220 & 1.010 & 0.14 & \text { A } \\ 1 & \text { SR 90 } & 1470.000 & 26.000 & 1300.000 & 52.400 & 1.130 & 0.05 & W\end{array}$

Matrix: WA

$\begin{array}{llrrrrrrr}1 & \text { AM241 } & 0.730 & 0.110 & 0.766 & 0.013 & 0.950 & 0.14 & \text { A } \\ 1 & \text { CD 60 } & 33.800 & 1.400 & 32.800 & 0.580 & 1.030 & 0.05 & \text { A } \\ 1 & \text { CS137 } & 43.100 & 3.700 & 38.300 & 0.881 & 1.130 & 0.10 & \text { A } \\ 1 & \text { GA 1 } & 111.000 & 4.000 & 1850.000 & 185.000 & 0.060 & 0.01 & \mathbf{N} \\ 1 & \text { GB 2 } & 44.900 & 2.000 & 744.000 & 74.000 & 0.060 & 0.01 & \mathbf{N}\end{array}$

Units for matrices: $A \mathrm{l}=\mathrm{Bq} / \mathrm{filter} \mathrm{SO}=\mathrm{Bq} / \mathrm{kg}$ VE=Bq/kg $\mathrm{WA}=\mathrm{Bq} / \mathrm{L}$. Values for elemental uranium are reported in $\mu \mathrm{g} / \mathrm{filter}, \mathrm{g}$, or $\mathrm{mL}$.

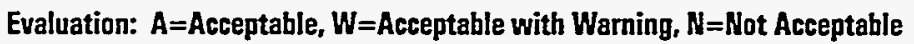

$\mathrm{pCi}=\mathrm{Bq} \times 27$ 
QAP44 Results by Lahoratory

Lab: WA Environmental Radiation Lab, Off. of Public Health Labs. Seattle

\begin{tabular}{|c|c|c|c|c|c|c|c|c|}
\hline $\begin{array}{l}\text { No. } \\
\text { Test } \\
\end{array}$ & $\begin{array}{l}\text { Radio- } \\
\text { nuclide }\end{array}$ & $\begin{array}{l}\text { Reported } \\
\text { Value } \\
\end{array}$ & $\begin{array}{l}\text { Reported } \\
\text { Error }\end{array}$ & $\begin{array}{c}\text { EML } \\
\text { Value }\end{array}$ & $\begin{array}{l}\text { EML } \\
\text { Error } \\
\end{array}$ & $\frac{\text { Reported }}{\text { EML }}$ & $\begin{array}{l}\text { Ratio } \\
\text { Error } \\
\end{array}$ & Evaluatio \\
\hline Matrix: & WA & & & & & & & \\
\hline $\begin{array}{l}1 \\
1 \\
1 \\
1 \\
1 \\
1 \\
1 \\
1\end{array}$ & $\begin{array}{l}H \quad 3 \\
\text { MN 54 } \\
\text { PU238 } \\
\text { PU239 } \\
\text { SR 90 } \\
\text { U } 234 \\
\text { U } 238 \\
\text { U BQ }\end{array}$ & $\begin{array}{r}214.000 \\
42.700 \\
0.870 \\
0.680 \\
1.630 \\
0.290 \\
0.300 \\
0.640\end{array}$ & $\begin{array}{r}25.000 \\
3.700 \\
0.120 \\
0.100 \\
0.240 \\
0.040 \\
0.050 \\
0.070\end{array}$ & $\begin{array}{r}251.000 \\
38.400 \\
0.982 \\
0.772 \\
1.450 \\
0.274 \\
0.275 \\
0.559\end{array}$ & $\begin{array}{r}11.400 \\
1.160 \\
0.074 \\
0.056 \\
0.034 \\
0.019 \\
0.024 \\
0.047\end{array}$ & $\begin{array}{l}0.850 \\
1.110 \\
0.890 \\
0.880 \\
1.120 \\
1.060 \\
1.090 \\
1.150\end{array}$ & $\begin{array}{l}0.11 \\
0.10 \\
0.14 \\
0.14 \\
0.17 \\
0.16 \\
0.21 \\
0.16\end{array}$ & $\begin{array}{l}A \\
A \\
W \\
A \\
A \\
A \\
A \\
A\end{array}$ \\
\hline
\end{tabular}

Units for matrices: $A \mathrm{~A}=\mathrm{Bq} / \mathrm{filter} \mathrm{SO}=\mathrm{Bq} / \mathrm{kg}$ VE=Bq/kg $W A=B q / \mathrm{L}$. Values for elemental uranium are reported in $\mu \mathrm{g} / \mathrm{filter}, \mathrm{g}$, or $\mathrm{mL}$. 


\section{QAP44 Results by Laboratory}

Lab: WC Westinghouse Hanford Co.

No. Radio- Reported Reported EML EML Reported Ratio

Test nuclide Value Error Value Error EML

Matrix: Al

$\begin{array}{llrlrllll}1 & \text { AM241 } & 0.226 & 0.049 & 0.189 & 0.007 & 1.200 & 0.26 & \text { A } \\ 1 & \text { CE144 } & 19.900 & 2.560 & 33.300 & 3.300 & 0.600 & 0.10 & \text { N } \\ 1 & \text { CO 57 } & 5.820 & 0.588 & 8.900 & 0.900 & 0.650 & 0.09 & \text { W } \\ 1 & \text { CO 60 } & 23.400 & 1.820 & 29.500 & 2.900 & 0.790 & 0.10 & \text { W } \\ 1 & \text { CS134 } & 11.200 & 0.825 & 14.700 & 1.460 & 0.760 & 0.09 & W \\ 1 & \text { CS137 } & 5.190 & 0.741 & 6.640 & 0.700 & 0.780 & 0.14 & \text { W } \\ 1 & \text { GA 1 } & 1.530 & 0.031 & 1.620 & 0.150 & 0.940 & 0.09 & A \\ 1 & \text { GB 2 } & 1.880 & 0.038 & 1.770 & 0.150 & 1.060 & 0.09 & A \\ 1 & \text { MN 54 } & 2.790 & 0.459 & 3.440 & 0.380 & .0 .810 & 0.16 & W \\ 1 & \text { PU238 } & 0.094 & 0.022 & 0.096 & 0.002 & 0.980 & 0.23 & A \\ 1 & \text { PU239 } & 0.097 & 0.023 & 0.093 & 0.003 & 1.050 & 0.25 & A \\ 1 & \text { RU106 } & 9.820 & 2.150 & 11.600 & 1.440 & 0.850 & 0.21 & A \\ 1 & \text { SB125 } & 8.260 & 0.687 & 9.780 & 1.030 & 0.850 & 0.11 & A \\ 1 & \text { SR 90 } & 0.768 & 0.174 & 1.060 & 0.037 & 0.730 & 0.17 & \text { W } \\ 1 & \text { U 234 } & 0.064 & 0.020 & 0.052 & 0.002 & 1.250 & 0.38 & A \\ 1 & \text { U 238 } & 0.057 & 0.018 & 0.053 & 0.002 & 1.080 & 0.34 & A\end{array}$

Matrix: So

$\begin{array}{lllllllll}1 & \text { CS137 } & 407.000 & 44.200 & 359.000 & 10.000 & 1.130 & 0.13 & \text { A } \\ 1 & \text { K 40 } & 518.000 & 73.600 & 465.000 & 30.000 & 1.110 & 0.17 & \text { A }\end{array}$

Matrix: VE

$\begin{array}{llrrrrrrr}1 & \text { CO } 60 & 65.300 & 6.190 & 59.700 & 0.963 & 1.090 & 0.11 & \text { A } \\ 1 & \text { CS137 } & 1140.000 & 124.000 & 944.000 & 16.200 & 1.210 & 0.13 & \text { A } \\ 1 & \text { K 40 } & 1230.000 & 173.000 & 1030.000 & 33.000 & 1.190 & 0.17 & \text { A }\end{array}$

Matrix: WA

$\begin{array}{llrrrrrrr}1 & \text { AM241 } & 0.952 & 0.185 & 0.766 & 0.013 & 1.240 & 0.24 & \mathbf{W} \\ 1 & \text { CO 60 } & 34.300 & 2.660 & 32.800 & 0.580 & 1.050 & 0.08 & \text { A } \\ 1 & \text { CS137 } & 44.900 & 6.140 & 38.300 & 0.881 & 1.170 & 0.16 & \mathbf{W} \\ 1 & \text { GA 1 } & 1400.000 & 144.000 & 1850.000 & 185.000 & 0.760 & 0.11 & \mathbf{W} \\ 1 & \text { GB 2 } & 655.000 & 67.500 & 744.000 & 74.000 & 0.880 & 0.13 & \mathbf{W} \\ 1 & \text { H 3 } & 186.000 & 20.500 & 251.000 & 11.400 & 0.740 & 0.09 & \mathbf{W} \\ 1 & \text { MN 54 } & 44.900 & 6.470 & 38.400 & 1.160 & 1.170 & 0.17 & \mathbf{W} \\ 1 & \text { PU238 } & 0.951 & 0.163 & 0.982 & 0.074 & 0.970 & 0.18 & \mathbf{A} \\ 1 & \text { PU239 } & 0.782 & 0.137 & 0.772 & 0.056 & 1.010 & 0.19 & \mathbf{A} \\ 1 & \text { SR 90 } & 1.010 & 0.250 & 1.450 & 0.034 & 0.700 & 0.17 & \mathbf{W} \\ 1 & \text { U 234 } & 0.350 & 0.081 & 0.274 & 0.019 & 1.280 & 0.31 & \mathbf{W} \\ 1 & \text { U 238 } & 0.300 & 0.072 & 0.275 & 0.024 & 1.090 & 0.28 & \mathbf{A}\end{array}$

Units for matrices: $A I=B q / f i l t e r ~ S O=B q / k g$ VE=Bqlkg WA=BqlL. Values for elemental uranium are reported in $\mu g \mid f i l t e r, g$, or $\mathrm{mL}$.

Evaluation: $A=A c c e p t a b l e, W=A c c e p t a b l e$ with Warning, $N=$ Not Acceptable

$\mathrm{pCi}=\mathrm{Bq} \times 27$ 


\section{QAP44 Results by Laboratory}

Lab: WE Westinghouse Electric Corp., Madison, PA

\begin{tabular}{llllllll} 
No. & $\begin{array}{l}\text { Radio: } \\
\text { nuclide }\end{array}$ & $\begin{array}{l}\text { Reported } \\
\text { Value }\end{array}$ & $\begin{array}{l}\text { Reported } \\
\text { Error }\end{array}$ & $\begin{array}{c}\text { EML } \\
\text { Value }\end{array}$ & $\begin{array}{l}\text { EML } \\
\text { Error }\end{array}$ & $\frac{\text { Reported }}{\text { EML }}$ & $\begin{array}{l}\text { Ratio } \\
\text { Error }\end{array}$ \\
\hline
\end{tabular}

Matrix: Al

$\begin{array}{llrlrllll}1 & \text { CE144 } & 15.400 & 1.830 & 33.300 & 3.300 & 0.460 & 0.07 & \mathbf{N} \\ 1 & \text { CO 57 } & 4.440 & 0.296 & 8.900 & 0.900 & 0.500 & 0.06 & \mathbf{N} \\ 1 & \text { CO 60 } & 47.100 & 1.570 & 29.500 & 2.900 & 1.600 & 0.17 & \mathbf{N} \\ 1 & \text { CS134 } & 11.000 & 0.979 & 14.700 & 1.460 & 0.750 & 0.10 & \mathbf{W} \\ 1 & \text { CS137 } & 4.510 & 0.663 & 6.640 & 0.700 & 0.680 & 0.12 & \mathbf{N} \\ 1 & \text { MN 54 } & 4.250 & 0.799 & 3.440 & 0.380 & 1.240 & 0.27 & \mathbf{W} \\ 1 & \text { PU238 } & 0.065 & 0.011 & 0.096 & 0.002 & 0.680 & 0.11 & \mathbf{W} \\ 1 & \text { PU239 } & 0.057 & 0.010 & 0.093 & 0.003 & 0.610 & 0.11 & \mathbf{N} \\ 1 & \text { RU106 } & 12.500 & 5.970 & 11.600 & -1.440 & 1.080 & 0.53 & \mathbf{A} \\ 1 & \text { SB125 } & 7.050 & 1.310 & 9.780 & 1.030 & 0.720 & 0.15 & \mathbf{A} \\ 1 & \text { SR 90 } & 0.002 & 0.001 & 1.060 & 0.037 & 0.000 & 0.00 & \mathbf{N} \\ 1 & \text { U 234 } & 0.047 & 0.010 & 0.052 & 0.002 & 0.910 & 0.19 & \mathbf{A} \\ 1 & \text { U 238 } & 0.047 & 0.009 & 0.053 & 0.002 & 0.880 & 0.17 & \mathbf{N}\end{array}$

Matrix: So

\begin{tabular}{|c|c|c|c|c|c|c|}
\hline $\begin{array}{l}\text { CS137 } \\
\text { K 40 } \\
\text { PU238 } \\
\text { PU239 } \\
\text { SR } 90 \\
\text { U } 234 \\
\text { U } 238\end{array}$ & $\begin{array}{r}372.000 \\
431.000 \\
17.200 \\
3.460 \\
2.640 \\
28.200 \\
28.800\end{array}$ & $\begin{array}{r}9.500 \\
59.500 \\
0.926 \\
0.421 \\
0.139 \\
1.250 \\
1.260\end{array}$ & $\begin{array}{r}359.000 \\
465.000 \\
43.000 \\
9.230 \\
1340.000 \\
34.200 \\
35.900\end{array}$ & $\begin{array}{r}10.000 \\
30.000 \\
2.440 \\
0.346 \\
113.000 \\
3.520 \\
3.630\end{array}$ & $\begin{array}{l}1.040 \\
0.930 \\
0.400 \\
0.380 \\
0.000 \\
0.830 \\
0.800\end{array}$ & $\begin{array}{l}0.04 \\
0.14 \\
0.03 \\
0.05 \\
0.00 \\
0.09 \\
0.09\end{array}$ \\
\hline
\end{tabular}

Matrix: VE

$\begin{array}{rlrrrrrrr}1 & \text { CO 60 } & 61.300 & 8.320 & 59.700 & 0.963 & 1.030 & 0.14 & \text { A } \\ 1 & \text { CS137 } & 1040.000 & 15.100 & 944.000 & 16.200 & 1.100 & 0.02 & \text { A } \\ 1 & \text { K 40 } & 1000.000 & 89.900 & 1030.000 & 33.000 & 0.970 & 0.09 & \text { A } \\ 1 & \text { PU239 } & 0.529 & 0.262 & 9.820 & 1.220 & 0.050 & 0.03 & \text { N } \\ 1 & \text { SR 90 } & 2.810 & 0.198 & 1300.000 & 52.400 & 0.000 & 0.00 & \text { N }\end{array}$

Matrix: WA

$\begin{array}{llr}1 & \text { CO 60 } & 30.900 \\ 1 & \text { CS137 } & 39.000 \\ 1 & \text { MN 54 } & 39.700 \\ 1 & \text { PU238 } & 0.842 \\ 1 & \text { PU239 } & 0.526 \\ 1 & \text { SR } 90 & 0.004 \\ 1 & \text { U 234 } & 0.278 \\ 1 & \text { U 238 } & 0.316\end{array}$

$\begin{array}{lr}1.170 & 32.800 \\ 1.080 & 38.300 \\ 1.190 & 38.400 \\ 0.079 & 0.982 \\ 0.063 & 0.772 \\ 0.001 & 1.450 \\ 0.057 & 0.274 \\ 0.059 & 0.275\end{array}$

$\begin{array}{ll}0.580 & 0.940 \\ 0.881 & 1.020 \\ 1.160 & 1.030 \\ 0.074 & 0.860 \\ 0.056 & 0.680 \\ 0.034 & 0.000 \\ 0.019 & 1.020 \\ 0.024 & 1.150\end{array}$

0.04
0.04
0.04
0.10
0.10
0.00
0.22
0.24

$\mathbf{W}$
$\mathbf{A}$
$\mathbf{A}$
$\mathbf{W}$
$\mathbf{W}$
$\mathbf{W}$
$\mathbf{A}$
$\mathbf{W}$

Units for matrices: $A \mathrm{I}=\mathrm{Bq} / \mathrm{filter} \mathrm{SO}=\mathrm{Bq} / \mathrm{kg} \mathrm{VE}=\mathrm{Bq} / \mathrm{kg} W \mathrm{~W}=\mathrm{Bq} / \mathrm{L}$. Values for elemental uranium are reported in $\mu \mathrm{g} / \mathrm{filter}, \mathrm{g}$, or $\mathrm{mL}$. Evaluation: $A=A c c e p t a b l e, W=A c c e p t a b l e$ with Warning, $N=$ Not Acceptable $\mathrm{pCi}=\mathrm{Bq} \times 27$ 


\section{QAP44 Results by Laboratory}

Lab: WI WIPP Site, Westinghouse Electric Corp.

\begin{tabular}{lllllllll} 
No. & Radio- & Reported & Reported & EML & EML & Reported & Ratio \\
Test & nuclide & Value & Error & Value & Error & EML & Error & Evaluation \\
\hline
\end{tabular}

Matrix: Al

$\begin{array}{llrl}1 & \text { CE144 } & 29.000 & 3.530 \\ 1 & \text { CO 57 } & 8.630 & 0.750 \\ 1 & \text { CO 60 } & 28.800 & 1.250 \\ 1 & \text { CS134 } & 13.900 & 1.140 \\ 1 & \text { CS137 } & 6.100 & 0.950 \\ 1 & \text { MN 54 } & 3.250 & 0.620 \\ 1 & \text { SB125 } & 9.860 & 0.59\end{array}$

$\begin{array}{lrll}3.530 & 33.300 & 3.300 & 0.870 \\ 0.750 & 8.900 & 0.900 & 0.970 \\ 1.250 & 29.500 & 2.900 & 0.980 \\ 1.140 & 14.700 & 1.460 & 0.950 \\ 0.950 & 6.640 & 0.700 & 0.920 \\ 0.620 & 3.440 & 0.380 & 0.950 \\ 0.590 & 9.780 & 1.030 & 1.010\end{array}$

0.14

0.13

0.11

0.12

0.17

0.21

0.12

A
A
A
A
A
A
A

Matrix: WA

$\begin{array}{lllllllll}1 & \text { C0 60 } & 35.500 & 2.720 & 32.800 & 0.580 & 1.080 & 0.09 & \text { A } \\ 1 & \text { CS137 } & 44.300 & 6.230 & 38.300 & 0.881 & 1.160 & 0.17 & W \\ 1 & \text { MN 54 } & 44.300 & 5.440 & 38.400 & 1.160 & 1.150 & 0.15 & W\end{array}$

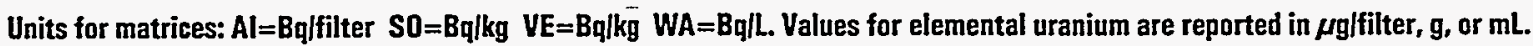

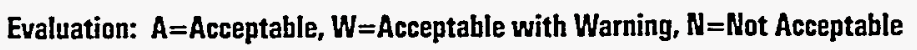
$\mathrm{pCi}=\mathrm{Bq} \times 27$ 


\section{OAP44 Results by Laboratory}

Lab: WN WINCO, Idaho Chemical Processing Plant, Scoville

\begin{tabular}{lllllllll} 
No. & $\begin{array}{l}\text { Radio- } \\
\text { nuclide }\end{array}$ & $\begin{array}{l}\text { Reported } \\
\text { Value }\end{array}$ & $\begin{array}{l}\text { Reported } \\
\text { Error }\end{array}$ & $\begin{array}{c}\text { EML } \\
\text { Value }\end{array}$ & $\begin{array}{l}\text { EML } \\
\text { Error }\end{array}$ & $\frac{\text { Reported }}{\text { EML }}$ & $\begin{array}{l}\text { Ratio } \\
\text { Error }\end{array}$ & Evaluation \\
\hline
\end{tabular}

Matrix: AI

$\begin{array}{llrlrllll}1 & \text { CE144 } & 23.000 & 0.600 & 33.300 & 3.300 & 0.690 & 0.07 & \text { A } \\ 2 & & 23.200 & 0.600 & 33.300 & 3.300 & 0.700 & 0.07 & \text { A } \\ 1 & \text { CO 57 } & 7.110 & 0.120 & 8.900 & 0.900 & 0.800 & 0.08 & \text { A } \\ 1 & \text { CO 60 } & 26.100 & 0.200 & 29.500 & 2.900 & 0.890 & 0.09 & \text { A } \\ 1 & \text { CS134 } & 13.700 & 0.200 & 14.700 & 1.460 & 0.930 & 0.09 & \text { A } \\ 1 & \text { CS137 } & 5.820 & 0.180 & 6.640 & 0.700 & 0.880 & 0.10 & \text { A } \\ 1 & \text { GA 1 } & 1.500 & 0.200 & 1.620 & 0.150 & 0.930 & 0.15 & \text { A } \\ 1 & \text { GB 2 } & 1.680 & 0.090 & 1.770 & 0.150 & 0.950 & 0.10 & \text { A } \\ 1 & \text { MN 54 } & 3.000 & 0.120 & 3.440 & 0.380 & 0.870 & 0.10 & \text { A } \\ 1 & \text { RU106 } & 10.600 & 0.800 & 11.600 & 1.440 & 0.910 & 0.13 & \text { A } \\ 1 & \text { SB125 } & 9.200 & 0.200 & 9.780 & 1.030 & 0.940 & 0.10 & \text { A }\end{array}$

Matrix: So

$\begin{array}{lllllllll}1 & C S 137 & 403.000 & 10.000 & 359.000 & 10.000 & 1.120 & 0.04 & \text { A } \\ 1 & K ~ 40 & 500.000 & 40.000 & 465.000 & 30.000 & 1.080 & 0.11 & \text { A }\end{array}$

Matrix: VE

$\begin{array}{rrrrrrrrr}1 & \text { CO } 60 & 60.000 & 3.000 & 59.700 & 0.963 & 1.010 & 0.05 & A \\ 1 & C S 137 & 1050.000 & 20.000 & 944.000 & 16.200 & 1.110 & 0.03 & A \\ 2 & & 1050.000 & 20.000 & 944.000 & 16.200 & 1.110 & 0.03 & A \\ 1 & K 40 & 1120.000 & 80.000 & 1030.000 & 33.000 & 1.090 & 0.09 & A\end{array}$

Matrix: WA

$\begin{array}{llrrrrrrr}1 & \text { AM241 } & 0.770 & 0.090 & 0.766 & 0.013 & 1.010 & 0.12 & \text { A } \\ 1 & \text { CO 60 } & 30.400 & 0.300 & 32.800 & 0.580 & 0.930 & 0.02 & \text { W } \\ 1 & \text { CS137 } & 41.200 & 0.300 & 38.300 & 0.881 & 1.080 & 0.03 & \text { A } \\ 1 & \text { GA 1 } & 1830.000 & 500.000 & 1850.000 & 185.000 & 0.990 & 0.29 & \text { A } \\ 1 & \text { GB 2 } & 960.000 & 100.000 & 744.000 & 74.000 & 1.290 & 0.19 & \text { A } \\ 1 & \text { H } 3 & 243.000 & 21.000 & 251.000 & 11.400 & 0.970 & 0.09 & \text { A } \\ 1 & \text { MN 54 } & 36.500 & 0.400 & 38.400 & 1.160 & 0.950 & 0.03 & \text { A } \\ 1 & \text { PU238 } & 0.910 & 0.100 & 0.982 & 0.074 & 0.930 & 0.12 & \text { A } \\ 1 & \text { PU239 } & 0.720 & 0.080 & 0.772 & 0.056 & 0.930 & 0.12 & \text { A } \\ 1 & \text { SR 90 } & 2.200 & 0.400 & 1.450 & 0.034 & 1.520 & 0.28 & \text { W } \\ 1 & \text { U 238 } & 0.610 & 0.110 & 0.275 & 0.024 & 2.220 & 0.45 & \text { N }\end{array}$

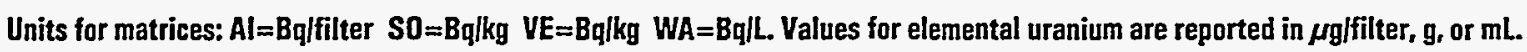

Evaluation: $A=A c c e p t a b l e, W=A c c e p t a b l e$ with Warning, $N=$ Not Acceptable

$\mathrm{pCi}=\mathrm{Bq} \times 27$ 
QAP44 Results by Laboratory

Lab: WP Washington Public Power Supply System, Richland

\begin{tabular}{|c|c|c|c|c|c|c|c|}
\hline $\begin{array}{l}\text { No. } \\
\text { Test }\end{array}$ & $\begin{array}{l}\text { Radio- } \\
\text { nuclide }\end{array}$ & $\begin{array}{l}\text { Reported } \\
\text { Value }\end{array}$ & $\begin{array}{l}\text { Reported } \\
\text { Error }\end{array}$ & $\begin{array}{c}\text { EML } \\
\text { Value }\end{array}$ & $\begin{array}{l}\text { EML } \\
\text { Error }\end{array}$ & $\frac{\text { Reported }}{\text { EML }}$ & $\begin{array}{l}\text { Ratio } \\
\text { Error }\end{array}$ \\
\hline
\end{tabular}

Matrix: Al

$\begin{array}{rrrrrrrrr}1 & \text { CE144 } & 23.900 & 0.790 & 33.300 & 3.300 & 0.720 & 0.08 & \text { A } \\ 1 & \text { CO 57 } & 6.630 & 0.160 & 8.900 & 0.900 & 0.750 & 0.08 & \text { A } \\ 1 & \text { CO 60 } & 25.200 & 0.630 & 29.500 & 2.900 & 0.850 & 0.09 & \text { A } \\ 1 & \text { CS134 } & 12.400 & 0.400 & 14.700 & 1.460 & 0.840 & 0.09 & \text { A } \\ 1 & \text { CS137 } & 6.180 & 0.310 & 6.640 & 0.700 & 0.930 & 0.11 & \text { A } \\ 1 & \text { MN 54 } & 3.100 & 0.280 & 3.440 & 0.380 & 0.900 & 0.13 & \text { A } \\ 1 & \text { RU106 } & 10.800 & 1.900 & 11.600 & 1.440 & 0.930 & 0.20 & \text { A } \\ 1 & \text { SB125 } & 9.560 & 0.630 & 9.780 & 1.030 & 0.980 & 0.12 & \text { A } \\ 1 & \text { SR 90 } & 1.100 & 0.110 & 1.060 & 0.037 & 1.040 & 0.11 & \text { A }\end{array}$

Matrix: So

$\begin{array}{llrrrrrrr}1 & \text { CS137 } & 427.000 & 7.900 & 359.000 & 10.000 & 1.190 & 0.04 & \text { A } \\ 1 & \text { K 40 } & 547.000 & 35.000 & 465.000 & 30.000 & 1.180 & 0.11 & \text { A } \\ 1 & \text { SR 90 } & 1300.000 & 37.000 & 1340.000 & 113.000 & 0.970 & 0.09 & \text { A }\end{array}$

Matrix: VE

$\begin{array}{rlrrrrrrr}1 & \text { CO 60 } & 72.200 & 3.300 & 59.700 & 0.963 & 1.210 & 0.06 & \text { A } \\ 1 & \text { CS137 } & 1250.000 & 10.000 & 944.000 & 16.200 & 1.320 & 0.03 & \text { W } \\ 1 & \text { K 40 } & 1270.000 & 41.000 & 1030.000 & 33.000 & 1.230 & 0.06 & \text { W } \\ 1 & \text { SR 90 } & 1400.000 & 37.000 & 1300.000 & 52.400 & 1.080 & 0.05 & \text { A }\end{array}$

Matrix: WA

$\begin{array}{rlrrrrrrr}1 & \text { CO 60 } & 32.800 & 1.400 & 32.800 & 0.580 & 1.000 & 0.05 & \text { A } \\ 1 & \text { CS137 } & 41.500 & 1.400 & 38.300 & 0.881 & 1.080 & 0.04 & \text { A } \\ 1 & H 3 & 220.000 & 11.000 & 251.000 & 11.400 & 0.880 & 0.06 & \text { A } \\ 1 & \text { MN 54 } & 40.600 & 1.400 & 38.400 & 1.160 & 1.060 & 0.05 & \text { A } \\ 1 & \text { SR 90 } & 1.600 & 0.300 & 1.450 & 0.034 & 1.100 & 0.21 & \text { A }\end{array}$

Units for matrices: $\mathrm{Al}=\mathrm{Bq} / \mathrm{filter} \mathrm{SO}=\mathrm{Bq} / \mathrm{kg}$ VE=Bq/kg $W \mathrm{~A}=\mathrm{Bq} / \mathrm{L}$. Values for elemental uranium are reported in $\mu \mathrm{g} / \mathrm{filter}, \mathrm{g}$, or $\mathrm{mL}$.

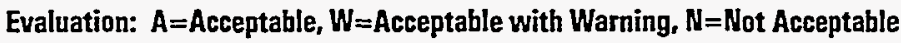

$\mathrm{pCi}=\mathrm{Bq} \times 27$ 


\section{OAP44 Results by Laboratory}

Lab: WS Weldon Springs Site, St Charles, MO

\begin{tabular}{lllllllll} 
No. & Radio- & Reported & Reported & - & EML & EML & Reported & Ratio \\
Test & nuclide & Value & Error & Value & Error & $\frac{\text { EML }}{\text { Error }}$ & Evaluation \\
\hline
\end{tabular}

Matrix: $\quad$ So

$\begin{array}{rlrrrrrrr}1 & \text { AM241 } & 4.300 & 0.900 & 3.690 & 0.454 & 1.170 & 0.28 & \text { A } \\ 1 & \text { CS137 } & 385.000 & 13.700 & 359.000 & 10.000 & 1.070 & 0.05 & \text { A } \\ 1 & \text { K 40 } & 492.000 & 18.100 & 465.000 & 30.000 & 1.060 & 0.08 & \text { A } \\ 1 & \text { U 238 } & 27.800 & 5.600 & 35.900 & 3.630 & 0.770 & 0.18 & \text { A }\end{array}$

Units for matrices: $\mathrm{Al}=\mathrm{Bq} / \mathrm{filter} \mathrm{SO}=\mathrm{Bq} / \mathrm{kg}$ VE=Bq/kg $W A=B q / \mathrm{L}$. Values for elemental uranium are reported in $\mu \mathrm{g} / f i l t e r, \mathrm{~g}$, or $\mathrm{mL}$. 
QAP44 Results by Laboratory

Lab: WV West Valley Nuclear Services Co, Inc, NY

\begin{tabular}{lllllllll} 
No. & Radio- & Reported & Reported & EML & EML & Reported & Ratio & Error \\
Test & nuclide & Value & Error & Value & Error & EML & Evaluation \\
\hline
\end{tabular}

Matrix: Al

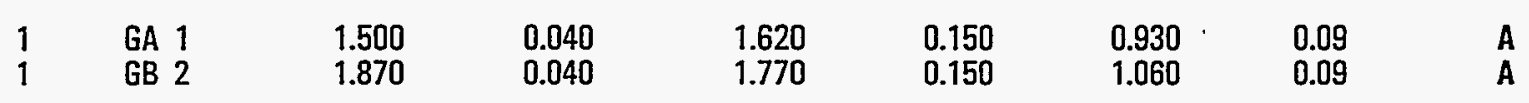

Matrix: WA

$\begin{array}{llrrrrrrr}1 & \text { CO 60 } & 32.900 & 0.880 & 32.800 & 0.580 & 1.000 & 0.03 & \text { A } \\ 1 & \text { CS137 } & 40.900 & 0.850 & 38.300 & 0.881 & 1.070 & 0.03 & \text { A } \\ 1 & \text { GA 1 } & 1800.000 & 26.000 & 1850.000 & 185.000 & 0.970 & 0.10 & \text { A } \\ 1 & \text { GB 2 } & 703.000 & 14.000 & 744.000 & 74.000 & 0.950 & 0.10 & \text { A } \\ 1 & \text { H 3 } & 218.000 & 8.300 & 251.000 & 11.400 & 0.870 & 0.05 & \text { A } \\ 1 & \text { MN 54 } & 42.700 & 0.960 & 38.400 & 1.160 & 1.110 & 0.04 & \text { A } \\ 1 & \text { SR 90 } & 1.900 & 0.360 & 1.450 & 0.034 & 1.310 & 0.25 & \text { W }\end{array}$

Units for matrices: $A I=B q / f i l t e r ~ S O=B q / k g ~ V E=B q / k g ~ W A=B q / L$. Values for elemental uranium are reported in $\mu \mathrm{g} /$ filter, $\mathrm{g}$, or $\mathrm{mL}$. 


\section{QAP44 Results by Laboratory}

Lab: YA Yankee Atomic Electric Company, Westboro, MA

\begin{tabular}{|c|c|c|c|c|c|c|c|c|}
\hline $\begin{array}{l}\text { No. } \\
\text { Test }\end{array}$ & $\begin{array}{l}\text { Radio- } \\
\text { nuclide }\end{array}$ & $\begin{array}{l}\text { Reported } \\
\text { Value }\end{array}$ & $\begin{array}{l}\text { Reported } \\
\text { Error }\end{array}$ & $\begin{array}{l}\text { EML } \\
\text { Value }\end{array}$ & $\begin{array}{l}\text { EML } \\
\text { Error }\end{array}$ & $\frac{\text { Reported }}{\text { EML }}$ & $\begin{array}{l}\text { Ratio } \\
\text { Error }\end{array}$ & Evaluation \\
\hline
\end{tabular}

Matrix: Al

$\begin{array}{lllllllll}1 & \text { AM241 } & 0.180 & 0.004 & 0.189 & 0.007 & 0.950 & 0.04 & \text { A } \\ 1 & \text { GA 1 } & 1.560 & 0.016 & 1.620 & 0.150 & 0.960 & 0.09 & \text { A } \\ 1 & \text { GB 2 } & 1.700 & 0.022 & 1.770 & 0.150 & 0.960 & 0.08 & \text { A } \\ 1 & \text { PU238 } & 0.100 & 0.002 & 0.096 & 0.002 & 1.040 & 0.03 & \text { A } \\ 1 & \text { PU239 } & 0.098 & 0.003 & 0.093 & 0.003 & 1.050 & 0.04 & \text { A }\end{array}$

Matrix: SO

$\begin{array}{rlrlrrrrr}1 & \text { AM241 } & 4.360 & 0.190 & 3.690 & 0.454 & 1.180 & 0.15 & \text { A } \\ =1 & \text { CS137 } & 373.000 & 2.700 & 359.000 & 10.000 & 1.040 & 0.03 & \text { A } \\ 1 & \text { K 40 } & 481.000 & 4.600 & 465.000 & 30.000 & 1.030 & 0.07 & \text { A } \\ 1 & \text { PU238 } & 43.000 & 0.450 & 43.000 & 2.440 & 1.000 & 0.06 & \text { A } \\ 1 & \text { PU239 } & 9.370 & 0.250 & 9.230 & 0.346 & 1.020 & 0.05 & \text { A }\end{array}$

Matrix: VE

$\begin{array}{llr}1 & \text { AM241 } & 5.750 \\ 1 & \text { CM244 } & 4.590 \\ 1 & \text { CO } 60 & 59.600 \\ 1 & \text { CS137 } & 1030.000 \\ 1 & \text { K } 40 & 1120.000 \\ 1 & \text { PU239 } & 9.760\end{array}$

0.300
0.110
1.600
14.000
30.000
0.300

5.600
4.440
59.700
944.000
1030.000
9.820

0.184
0.202
0.963
16.200
33.000
1.220

1.030
1.030
1.000
1.090
1.090
0.990

0.06
0.05
0.03
0.02
0.05
0.13

A
A
A
A
A
A

Matrix: WA

$\begin{array}{llrrrrrrr}1 & \text { AM241 } & 0.756 & 0.021 & 0.766 & 0.013 & 0.990 & 0.03 & \text { A } \\ 1 & \text { CO 60 } & 32.000 & 0.450 & 32.800 & 0.580 & 0.980 & 0.02 & \text { A } \\ 1 & \text { CS137 } & 38.100 & 0.430 & 38.300 & 0.881 & 1.000 & 0.03 & \text { A } \\ 1 & \text { GA 1 } & 1740.000 & 86.000 & 1850.000 & 185.000 & 0.940 & 0.11 & \text { A } \\ 1 & \text { GB 2 } & 588.000 & 12.000 & 744.000 & 74.000 & 0.790 & 0.08 & \text { W } \\ 1 & \text { H 3 } & 237.000 & 12.000 & 251.000 & 11.400 & 0.940 & 0.06 & \text { A } \\ 1 & \text { MN 54 } & 39.000 & 0.750 & 38.400 & 1.160 & 1.020 & 0.04 & \text { A } \\ 1 & \text { PU238 } & 1.000 & 0.028 & 0.982 & 0.074 & 1.020 & 0.08 & \text { A } \\ 1 & \text { PU239 } & 0.763 & 0.010 & 0.772 & 0.056 & 0.990 & 0.07 & \text { A }\end{array}$

Units for matrices: $A|=B q| f i l t e r ~ S O=B q / k g$ VE=Bq/kg $W A=B q / L$. Values for elemental uranium are reported in $\mu \mathrm{g} / f i l t e r, g$, or $\mathrm{mL}$. 


\section{OAP44 Results by Laboratory}

Lab: YP US Army Proving Ground, Yuma, AZ

\begin{tabular}{lllllllll} 
No. & $\begin{array}{l}\text { Radio- } \\
\text { nuclide }\end{array}$ & $\begin{array}{l}\text { Reported } \\
\text { Value }\end{array}$ & $\begin{array}{c}\text { Reported } \\
\text { Error }\end{array}$ & $\begin{array}{c}\text { EML } \\
\text { Value }\end{array}$ & $\begin{array}{c}\text { EML } \\
\text { Error }\end{array}$ & $\begin{array}{c}\text { Reported } \\
\text { EML }\end{array}$ & $\begin{array}{l}\text { Ratio } \\
\text { Error }\end{array}$ & Evaluation \\
\hline
\end{tabular}

Matrix: Al

$1 \quad$ UUG

3.850

0.650

4.310

0.100

0.890

0.15

A

Matrix: So

$1 \quad$ U UG

3.220

0.027

2.900

0.290

1.110

0.11

w

Matrix: WA

$1 \quad U U G$

0.020

0.002

0.022

0.003

0.910

0.14

A

Units for matrices: $\mathrm{Al}=\mathrm{Bq}$ /filter $\mathrm{SO}=\mathrm{Bq} / \mathrm{kg}$ VE=Bqlkg $W \mathrm{~A}=\mathrm{Bq} / \mathrm{L}$. Values for elemental uranium are reported in $\mu \mathrm{g} / \mathrm{filter}, \mathrm{g}$, or $\mathrm{mL}$.

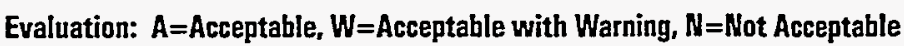
$\mathrm{pCi}=\mathrm{Bq} \times 27$ 
Matrix: Al

Radionuclide: AM241

EML Value: 0.189

EML Error: 0.007

\begin{tabular}{|c|c|c|c|c|c|c|}
\hline Lahconde. & Test\# & $\begin{array}{c}\text { Reported } \\
\text { Value. }\end{array}$ & $\begin{array}{c}\text { Reported } \\
\text { Error }\end{array}$ & $\frac{\text { Reported }}{\text { EML }}$ & $\begin{array}{l}\text { Ratio } \\
\text { Frror }\end{array}$ & Evaluation \\
\hline 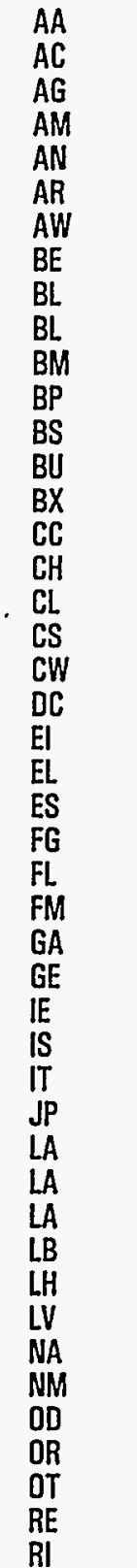 & 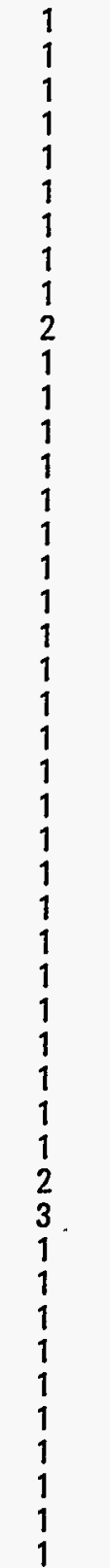 & $\begin{array}{l}0.189 \\
0.174 \\
0.169 \\
0.270 \\
0.192 \\
0.176 \\
0.224 \\
0.180 \\
0.240 \\
0.240 \\
0.260 \\
0.201 \\
0.280 \\
0.580 \\
0.204 \\
0.180 \\
0.197 \\
0.220 \\
0.180 \\
0.196 \\
0.220 \\
0.180 \\
0.245 \\
0.159 \\
0.170 \\
0.234 \\
0.210 \\
0.210 \\
0.206 \\
0.178 \\
0.156 \\
0.173 \\
0.180 \\
0.212 \\
0.200 \\
0.192 \\
0.159 \\
0.140 \\
0.249 \\
0.182 \\
0.186 \\
0.353 \\
0.190 \\
0.280 \\
0.139 \\
0.175\end{array}$ & $\begin{array}{l}0.007 \\
0.005 \\
0.022 \\
0.050 \\
0.006 \\
0.013 \\
0.100 \\
0.010 \\
0.020 \\
0.010 \\
0.100 \\
0.012 \\
0.040 \\
0.130 \\
0.010 \\
0.000 \\
0.034 \\
0.040 \\
0.010 \\
0.007 \\
0.051 \\
0.012 \\
0.020 \\
0.032 \\
0.040 \\
0.054 \\
0.040 \\
0.000 \\
0.030 \\
0.025 \\
0.029 \\
0.006 \\
0.005 \\
0.023 \\
0.022 \\
0.021 \\
0.012 \\
0.020 \\
0.050 \\
0.015 \\
0.012 \\
0.057 \\
0.020 \\
0.080 \\
0.011 \\
0.016\end{array}$ & $\begin{array}{l}1.00 \\
0.92 \\
0.89 \\
1.43 \\
1.02 \\
0.93 \\
1.18 \\
0.95 \\
1.27 \\
1.27 \\
1.38 \\
1.06 \\
1.48 \\
3.07 \\
1.08 \\
0.95 \\
1.04 \\
1.16 \\
0.95 \\
1.04 \\
1.16 \\
0.95 \\
1.30 \\
0.84 \\
0.90 \\
1.24 \\
1.11 \\
1.11 \\
1.09 \\
0.94 \\
0.83 \\
0.92 \\
0.92 \\
0.95 \\
1.12 \\
1.06 \\
1.02 \\
0.84 \\
0.74 \\
1.32 \\
0.96 \\
0.98 \\
1.87 \\
1.01 \\
1.48 \\
0.74 \\
0.93\end{array}$ & $\begin{array}{l}0.05 \\
0.04 \\
0.12 \\
0.27 \\
0.05 \\
0.08 \\
0.53 \\
0.06 \\
0.11 \\
0.07 \\
0.53 \\
0.07 \\
0.22 \\
0.70 \\
0.07 \\
0.03 \\
0.19 \\
0.22 \\
0.06 \\
0.05 \\
0.27 \\
0.07 \\
0.12 \\
0.17 \\
0.21 \\
0.29 \\
0.22 \\
0.04 \\
0.16 \\
0.14 \\
0.16 \\
0.04 \\
0.04 \\
0.13 \\
0.12 \\
0.12 \\
0.07 \\
0.11 \\
0.27 \\
0.09 \\
0.07 \\
0.31 \\
0.11 \\
0.43 \\
0.06 \\
0.09\end{array}$ & $\begin{array}{l}A \\
A \\
A \\
W \\
\mathbf{A} \\
A \\
A \\
A \\
A \\
W \\
W \\
W \\
A \\
W \\
\mathbf{N} \\
A \\
A \\
A \\
A \\
A \\
A \\
A \\
A \\
A \\
W \\
A \\
A \\
A \\
A \\
A \\
A \\
A \\
A \\
A \\
A \\
A \\
A \\
A \\
A \\
A \\
A \\
W \\
W \\
A \\
A \\
W \\
A \\
W \\
W \\
A\end{array}$ \\
\hline
\end{tabular}

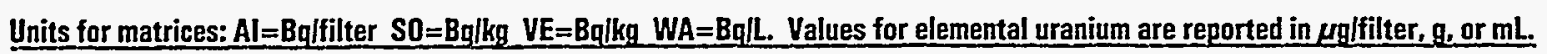
Evaluation: $A=A c c e p t a b l e, W=A c c e p t a b l e$ with Warning, $N=$ Not Acceptable.

$\mathrm{pCi}=\mathrm{Bq} \times 27$ 


\section{OAP44 Results by Nuclide}

Matrix: Al

Radionuclide: AM241

EML Value: 0.189

EML Error: 0.007

\begin{tabular}{|c|c|c|c|c|c|c|}
\hline Lahonde & Test \# & $\begin{array}{c}\text { Reported } \\
\text { Value }\end{array}$ & $\begin{array}{c}\text { Reported } \\
\text { Frror }\end{array}$ & $\frac{\text { Reported }}{\text { FMI }}$ & $\begin{array}{l}\text { Ratio } \\
\text { Error }\end{array}$ & Evaluation \\
\hline SE & 1 & 0.280 & 0.020 & 1.48 & 0.12 & $W$ \\
\hline SR & 1 & 0.158 & 0.022 & 0.84 & 0.12 & A \\
\hline SW & 1 & 0.570 & 0.010 & 3.02 & 0.12 & $\mathbf{N}$ \\
\hline TE & 1 & 0.270 & 0.040 & 1.43 & 0.22 & $W$ \\
\hline TI & 1 & 0.210 & 0.040 & 1.11 & 0.22 & A \\
\hline TM & 1 & 0.214 & 0.009 & 1.13 & 0.06 & $\ddot{A}$ \\
\hline TN & 1 & 0.181 & 0.012 & 0.96 & 0.07 & A \\
\hline TO & $i$ & 0.215 & 0.126 & 1.14 & 0.67 & A \\
\hline TW & 1 & 0.175 & 0.009 & 0.93 & 0.06 & A \\
\hline$T X$ & 1 & 0.194 & 0.014 & 1.03 & 0.08 & A \\
\hline UK & 1 & 0.208 & 0.030 & 1.10 & 0.16 & A \\
\hline UP & 1 & 0.252 & 0.056 & 1.33 & 0.30 & $\mathbf{W}$ \\
\hline UY & 1 & 0.270 & 0.060 & 1.43 & 0.32 & $W$ \\
\hline WA & 1 & 0.162 & 0.016 & 0.86 & 0.09 & A \\
\hline WC & 1 & 0.226 & 0.049 & 1.20 & 0.26 & A \\
\hline YA & 1 & 0.180 & 0.004 & 0.95 & 0.04 & $A$ \\
\hline
\end{tabular}

Total Number Reported: 62

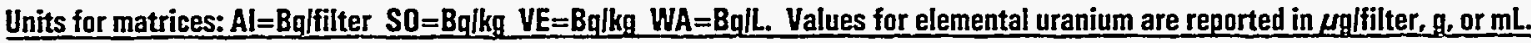


Matrix: Al

Radionuclide: CE144

EML Value: 33.300

EML Error: 3.300

$\begin{array}{cccccc}\text { Lahcade } & \text { Test \# } & \begin{array}{c}\text { Reported } \\ \text { Value }\end{array} & \begin{array}{c}\text { Reported } \\ \text { Error }\end{array} & \frac{\text { Reported }}{\text { EMI }} & \text { Ratio } \\ \text { Frror }\end{array}$

\begin{tabular}{|c|c|c|c|c|c|c|}
\hline$A A$ & 1 & 33.300 & 3.300 & 1.00 & 0.14 & \\
\hline$A C$ & 1 & 23.100 & 2.300 & 0.69 & 0.10 & \\
\hline AG & 1 & 25.300 & 1.800 & 0.76 & 0.09 & \\
\hline Al & 1 & 6.910 & 0.360 & 0.21 & 0.02 & \\
\hline $\mathrm{AL}$ & 1 & 44.400 & 1.060 & 1.33 & 0.14 & \\
\hline$A M$ & 1 & 25.600 & 0.450 & 0.77 & 0.08 & \\
\hline AN & 1 & 23.100 & 0.700 & 0.69 & 0.07 & \\
\hline AR & 1 & 32.200 & 2.100 & 0.97 & 0.12 & \\
\hline$A W$ & 1 & 33.400 & 5.010 & 1.00 & 0.18 & \\
\hline BA & 1 & 30.400 & 2.600 & 0.91 & 0.12 & \\
\hline$B C$ & 1 & 23.100 & 0.670 & 0.69 & 0.07 & \\
\hline $\mathrm{BE}$ & 1 & 21.300 & 2.200 & 0.64 & 0.09 & \\
\hline$B L$ & 1 & 26.300 & 1.300 & 0.79 & 0.09 & \\
\hline $\mathrm{BL}$ & 2 & 26.300 & 1.300 & 0.79 & 0.09 & \\
\hline $\mathrm{BL}$ & 3 & 25.700 & 1.200 & 0.77 & 0.08 & \\
\hline$B M$ & 1 & 28.000 & 2.000 & 0.84 & 0.10 & \\
\hline $\mathrm{BN}$ & 1 & 32.000 & 2.260 & 0.96 & 0.12 & . \\
\hline $\mathrm{BP}$ & 1 & 26.200 & 7.000 & 0.79 & 0.22 & . \\
\hline $\mathrm{BQ}$ & $i$ & 30.000 & 0.900 & 0.90 & 0.09 & \\
\hline BR & $i$ & 29.700 & 2.940 & 0.89 & 0.13 & \\
\hline BS & 1 & 23.300 & 0.240 & 0.70 & 0.07 & \\
\hline BU & 1 & 33.000 & 6.000 & 0.99 & 0.21 & \\
\hline$B X$ & 1 & 23.300 & 0.676 & 0.70 & 0.07 & \\
\hline CA & 1 & 20.300 & 0.900 & 0.61 & 0.07 & \\
\hline CC & 1 & 20.400 & 0.000 & 0.61 & 0.06 & \\
\hline $\mathrm{CH}$ & 1 & 21.100 & 1.710 & 0.63 & 0.08 & \\
\hline CL. & 1 & 20.000 & 1.750 & 0.60 & 0.08 & \\
\hline$C P$ & 1 & 30.600 & 4.300 & 0.92 & 0.16 & \\
\hline CS & 1 & 16.000 & 0.690 & 0.48 & 0.05 & \\
\hline DC & 1 & 21.300 & 5.720 & 0.64 & 0.18 & \\
\hline EG & 1 & 23.400 & 0.800 & 0.70 & 0.07 & \\
\hline EL & 1 & 21.600 & 1.700 & 0.65 & 0.08 & \\
\hline EP & 1 & 26.900 & 0.472 & 0.81 & 0.08 & \\
\hline ES & 1 & 20.600 & 2.970 & 0.62 & 0.11 & \\
\hline$F G$ & 1 & 33.900 & 3.300 & 1.02 & 0.14 & \\
\hline $\mathrm{FL}$ & 1 & 26.700 & 1.100 & 0.80 & 0.09 & \\
\hline $\mathrm{FM}$ & 1 & 25.100 & 0.560 & 0.75 & 0.08 & \\
\hline FN & 1 & 41.500 & 9.800 & 1.25 & 0.32 & \\
\hline GA & 1 & 21.300 & 0.000 & 0.64 & 0.06 & \\
\hline $\mathrm{GE}$ & 1 & 23.700 & 2.270 & 0.71 & 0.10 & \\
\hline 10 & 1 & 33.700 & 2.100 & 1.01 & 0.12 & \\
\hline IE & 1 & 22.000 & 1.210 & D.66 & 0.07 & \\
\hline IN & $i$ & 21.500 & 0.630 & 0.65 & 0.07 & \\
\hline IS & 1 & 22.600 & 3.700 & 0.68 & 0.13 & \\
\hline IT & 1 & 20.000 & 0.530 & 0.60 & 0.06 & \\
\hline
\end{tabular}

Units for matrices: $A l=B q$ lfilter $S O=B q l k g$ VE=Bq/kg $W A=B q l L$. Values for elemental uranium are reported in $\mu g$ lfilter, g, or $\mathrm{mL}$. Evaluation: $A=A c c e p t a b l e, W=A c c e p t a b l e$ with Warning, $N=$ Not Acceptable. $\mathrm{pCi}=\mathrm{Bq} \times 27$ 
QAP44 Results by Nuclide

Matrix: Al

Radionuclide: CE144

EML Value: 33.300

EML Error: 3.300

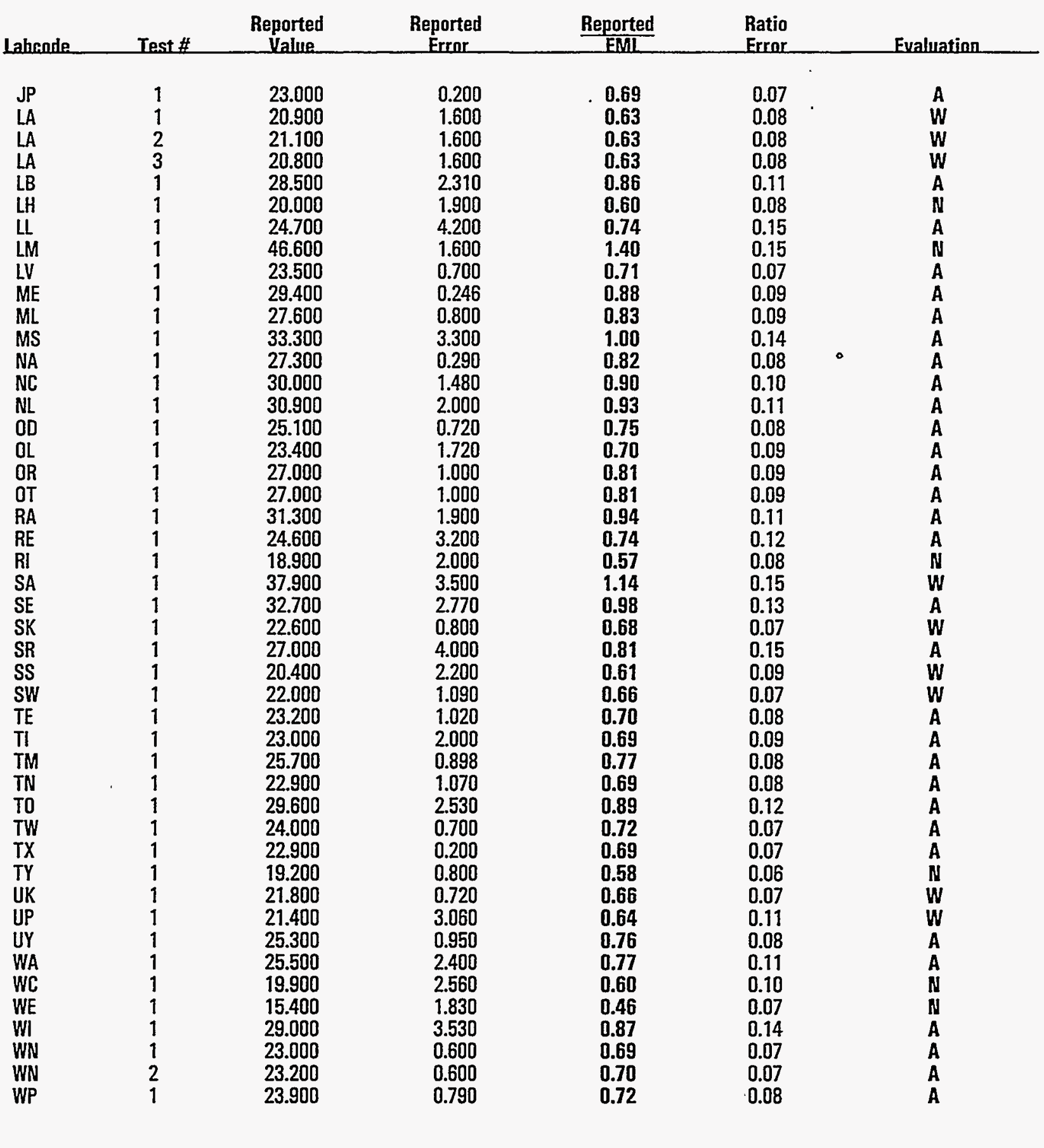

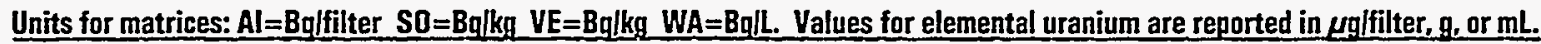

Evaluation: $A=A$ cceptable, $W=$ Acceptable with Warning, $N=$ Hot Acceptable.

$\mathrm{pCi}=\mathrm{Bq} \times 27$ 


\section{QAP44 Results by Nuclide}

Matrix: Al

Radionuclide: CE144

EML Value: 33.300

EML. Error: 3.300

\begin{tabular}{ccccc} 
Reported & Reported & Reported & Ratio \\
Lahande & Test \# & Ealue & Error & $\frac{\text { FMI }}{\text { Evaluation }}$ \\
\hline
\end{tabular}

Total Number Reported: 91

Units for matrices: $\mathrm{Al}=\mathrm{Bq} / \mathrm{filter} \mathrm{SO}=\mathrm{Bq} / \mathrm{kg} \quad \mathrm{VE}=\mathrm{Bq} / \mathrm{kg}$ WA=BglL. Values for elemental uranium are reported in $\mu \mathrm{g} /$ filter, $\mathrm{g}$, or $\mathrm{mL}$. Evaluation: $A=A c c e p t a b l e, W=A c c e p t a b l e$ with Warning, $N=$ Not Acceptable. $\mathrm{pCi}=\mathrm{Bq} \times 27$ 


\section{QAP44 Results by Nuclide}

Matrix: Al

Radionuclide: CO 57

EML Value: 8.900

EML Error: 0.900

\begin{tabular}{|c|c|c|c|c|c|}
\hline laheade & Test \# & Reported & Reported & $\frac{\text { Reported }}{\text { FMI }}$ & Ratio \\
\hline
\end{tabular}

\begin{tabular}{|c|c|c|c|c|c|}
\hline$A A$ & 1 & 8.900 & 0.900 & 1.00 & 0.14 \\
\hline$A C$ & 1 & 6.240 & 0.110 & 0.70 & 0.07 \\
\hline$A G$ & 1 & 7.440 & 0.490 & 0.84 & 0.10 \\
\hline $\mathrm{Al}$ & 1 & 5.850 & 0.055 & 0.66 & 0.07 \\
\hline AM & 1 & 6.220 & 0.070 & 0.70 & 0.07 \\
\hline AN & 1 & 6.620 & 0.200 & 0.74 & 0.08 \\
\hline AR & 1 & 7.110 & 0.300 & 0.80 & 0.09 \\
\hline$A W$ & 1 & 9.390 & 1.410 & 1.05 & 0.19 \\
\hline BA & 1 & 8.460 & 0.620 & 0.95 & 0.12 \\
\hline$B C$ & 1 & 6.770 & 0.196 & 0.76 & 0.08 \\
\hline $\mathrm{BE}$ & 1 & 6.160 & 0.420 & 0.69 & 0.08 \\
\hline$B L$ & 1 & 7.400 & 0.350 & 0.83 & 0.09 \\
\hline BL. & 2 & 7.380 & 0.350 & 0.83 & $0: 09$ \\
\hline $\mathrm{BL}$ & 3 & 7.230 & 0.340 & 0.81 & 0.09 \\
\hline BM & 1 & 8.010 & 0.560 & 0.90 & 0.11 \\
\hline BN & 1 & 9.120 & 0.600 & 1.03 & 0.12 \\
\hline BP & 1 & 5.800 & 1.200 & 0.65 & 0.15 \\
\hline $\mathrm{BQ}$ & 1 & 7.500 & 0.100 & 0.84 & 0.09 \\
\hline $\mathrm{BR}$ & 1 & 7.980 & 0.480 & 0.90 & 0.11 \\
\hline BS & 1 & 6.440 & 0.050 & 0.72 & 0.07 \\
\hline BU & 1 & 8.900 & 0.900 & 1.00 & 0.14 \\
\hline BX & 1 & 6.660 & 0.200 & 0.75 & 0.08 \\
\hline CA & 1 & 6.030 & 0.090 & 0.68 & 0.07 \\
\hline CC & 1 & 6.150 & 0.000 & 0.69 & 0.07 \\
\hline $\mathrm{CH}$ & 1 & 6.370 & 1.700 & 0.72 & 0.20 \\
\hline CL & 1 & 6.500 & 0.230 & 0.73 & 0.08 \\
\hline CP & 1 & 8.770 & 1.190 & 0.99 & 0.17 \\
\hline CS & 1 & 5.040 & 0.160 & 0.57 & 0.06 \\
\hline$D C$ & 1 & 5.500 & 0.931 & 0.62 & 0.12 \\
\hline EG & 1 & 7.100 & 0.200 & 0.80 & 0.08 \\
\hline EL & 1 & 6.800 & 0,600 & 0.76 & 0.10 \\
\hline $\mathrm{EP}$ & 1 & 7.710 & 0.609 & 0.87 & 0.11 \\
\hline ES & 1 & 6.800 & 0.830 & 0.76 & 0.12 \\
\hline$F G$ & 1 & 9.370 & 1.060 & 1.05 & 0.16 \\
\hline FL & 1 & 7.810 & 0.020 & 0.88 & 0.09 \\
\hline FM & 1 & 7.390 & 0.130 & 0.83 & 0.09 \\
\hline FN & 1 & 7.000 & 0.750 & 0.79 & 0.12 \\
\hline GA & 1 & 6.170 & 0.000 & 0.69 & 0.07 \\
\hline GE & 1 & 7.110 & 0.460 & 0.80 & 0.10 \\
\hline ID & 1 & 9.270 & 0.480 & 1.04 & 0.12 \\
\hline IE & 1 & 6.410 & 1.060 & 0.72 & 0.14 \\
\hline IN & 1 & 6.360 & 0.160 & 0.72 & 0.07 \\
\hline IS & 1 & 6.350 & 0.530 & 0.71 & 0.09 \\
\hline IT & 1 & 5.840 & 0.340 & 0.66 & 0.08 \\
\hline JP & 1 & 7.100 & 0.040 & 0.80 & 0.08 \\
\hline
\end{tabular}

Units for matrices: $\mathrm{Al}=\mathrm{Bq}$ /filter $\mathrm{SO}=\mathrm{Bq} / \mathrm{kg}, \mathrm{VE}=\mathrm{Bq} / \mathrm{kg}, W \mathrm{~W}=\mathrm{Bq} / \mathrm{L}$. Values for elemental uranium are reported in $\mu \mathrm{g} /$ filter, $\mathrm{g}$, or $\mathrm{mL}$.

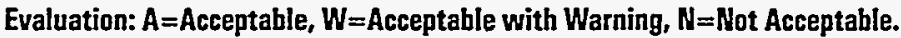

$\mathrm{pCi}=\mathrm{Bq} \times 27$ 
QAP44 Results by Nuclide

Matrix: Al

Radionuclide: CO 57

EML Value: 8.900

EML Error: 0.900

\begin{tabular}{|c|c|c|c|c|c|c|}
\hline Lahconde & Test \# & $\begin{array}{c}\text { Reported } \\
\text { Value }\end{array}$ & $\begin{array}{c}\text { Reported } \\
\text { Error }\end{array}$ & $\frac{\text { Reported }}{\text { EML }}$ & $\begin{array}{l}\text { Ratio } \\
\text { Error }\end{array}$ & Evaluation \\
\hline LA & 1 & 5.800 & 0.400 & 0.65 & 0.08 & $W$ \\
\hline LA & 2 & 5.900 & 0.400 & 0.66 & 0.08 & $W$ \\
\hline LA & 3 & 5.900 & 0.400 & 0.66 & 0.08 & $\mathbf{W}$ \\
\hline LB & 1 & 6.630 & 0.186 & 0.75 & 0.08 & $A$ \\
\hline LH & 1 & 5.900 & 0.600 & 0.66 & 0.10 & W \\
\hline LL. & 1 & 7.120 & 3.600 & 0.80 & 0.41 & $A$ \\
\hline$L M$ & 1 & 11.400 & 0.300 & 1.28 & 0.13 & $\ddot{w}$ \\
\hline LV & 1 & 6.600 & 0.100 & 0.74 & 0.08 & $\ddot{A}$ \\
\hline ME & $i$ & 6.700 & 0.035 & 0.75 & 0.08 & A \\
\hline MI & 1 & 5.820 & 0.181 & 0.65 & 0.07 & $W$ \\
\hline ML & 1 & 6.700 & 0.200 & 0.75 & 0.08 & A \\
\hline MS & 1 & 9.900 & 0.990 & 1.11 & 0.16 & $W$ \\
\hline NA & 1 & 7.930 & 0.050 & 0.89 & 0.09 & A \\
\hline NC & $i$ & 9.090 & 0.178 & 1.02 & 0.11 & A \\
\hline NL. & $i$ & 8.640 & 0.680 & 0.97 & 0.12 & $\ddot{A}$ \\
\hline 00 & 1 & 7.490 & 0.181 & 0.84 & 0.09 & A \\
\hline OL & $i$ & 6.890 & 0.190 & 0.77 & 0.08 & $\ddot{A}$ \\
\hline OR & 1 & 7.800 & 0.200 & 0.88 & 0.09 & $A$ \\
\hline OT & 1 & 7.200 & 0.100 & 0.81 & 0.08 & A \\
\hline $\mathrm{RA}$ & 1 & 9.250 & 0.390 & 1.04 & 0.11 & A \\
\hline $\mathrm{RE}$ & 1 & 6.700 & 0.740 & 0.75 & 0.11 & A \\
\hline RI & 1 & 5.020 & 0.340 & 0.56 & 0.07 & N \\
\hline SA & 1 & 11.000 & 1.100 & 1.24 & 0.18 & 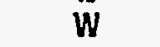 \\
\hline $\mathrm{SE}$ & 1 & 8.470 & 0.160 & 0.95 & 0.10 & A \\
\hline SK & 1 & 6.230 & 0.090 & 0.70 & 0.07 & $\ddot{W}$ \\
\hline SR & $i$ & 7.600 & 0.600 & 0.85 & 0.11 & $A$ \\
\hline SS & 1 & 5.740 & 0.940 & 0.65 & 0.12 & $\ddot{W}$ \\
\hline SW & 1 & 6.510 & 0.220 & 0.73 & 0.08 & $A$ \\
\hline TE & 1 & 6.050 & 0.130 & 0.68 & 0.07 & $\ddot{W}$ \\
\hline $\mathrm{TI}$ & 1 & 6.700 & 0.700 & 0.75 & 0.11 & A \\
\hline $\mathrm{TM}$ & 1 & 7.340 & 0.237 & 0.83 & 0.09 & A \\
\hline TN & 1 & 6.800 & 0.286 & 0.76 & 0.08 & A \\
\hline TO & $i$ & 7.990 & 0.461 & 0.90 & 0.11 & $\ddot{A}$ \\
\hline TW & 1 & 7.400 & 0.100 & 0.83 & 0.08 & $A$ \\
\hline TX & 1 & 6.120 & 0.040 & 0.69 & 0.07 & W \\
\hline TY & 1 & 4.300 & 0.400 & 0.48 & 0.07 & $\mathbb{N}$ \\
\hline UK & 1 & 6.380 & 0.130 & 0.72 & 0.07 & $\ddot{A}$ \\
\hline UP & 1 & 6.310 & 0.344 & 0.71 & 0.08 & $\ddot{w}$ \\
\hline UY & 1 & 7.200 & 0.200 & 0.81 & 0.08 & A \\
\hline WA & 1 & 7.300 & 0.370 & 0.82 & 0.09 & A \\
\hline WC & 1 & 5.820 & 0.588 & 0.65 & 0.09 & $\mathbf{W}$ \\
\hline WE & 1 & 4.440 & 0.296 & 0.50 & 0.06 & $\mathbf{N}$ \\
\hline$W I$ & 1 & 8.630 & 0.750 & 0.97 & 0.13 & A \\
\hline WN & 1 & 7.110 & 0.120 & 0.80 & 0.08 & A \\
\hline WP & $i$ & 6.630 & 0.160 & 0.75 & 0.08 & A \\
\hline
\end{tabular}

Units for matrices: $\mathrm{Al}=\mathrm{Bq}$ lfilter $\mathrm{SO}=\mathrm{Bq} \mathrm{kg}, \mathrm{VE}=\mathrm{Bq} / \mathrm{kg}, W A=\mathrm{Bq} / \mathrm{L}$. Values for elemental uranium are reported in $\mu \mathrm{glfilter}, \mathrm{g}$, or $\mathrm{mL}$. Evaluation: $A=$ Acceptahle, $W=$ Acceptable with Warning, $N=$ Not Acceptable. $\mathrm{pCi}=\mathrm{Bq} \times 27$ 


\section{QAP44 Results by Nuclide}

Matrix: Al

Radionuclide: CO 57

EML Value: 8.900

EML Error: 0.900

Reported

Reported

Reported

Ratio

Value

Errar

EMI

Errar

Evaluation

Total Number Reported: 90

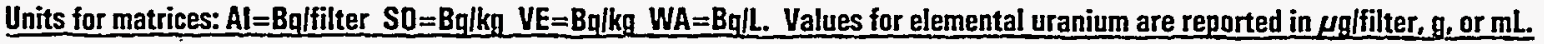

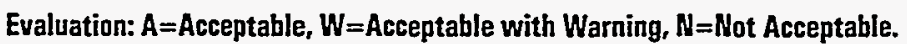

$\mathrm{pCi}=\mathrm{Bq} \times 27$ 
Matrix: Al

Radionuclide: CO 60

EML Value: 29.500

EML. Error: 2.900

\begin{tabular}{ccccc} 
Lahconde & Reported & Reported & Reported & Ratio \\
Value & Error & FML & Frror & Evaluation \\
\hline
\end{tabular}

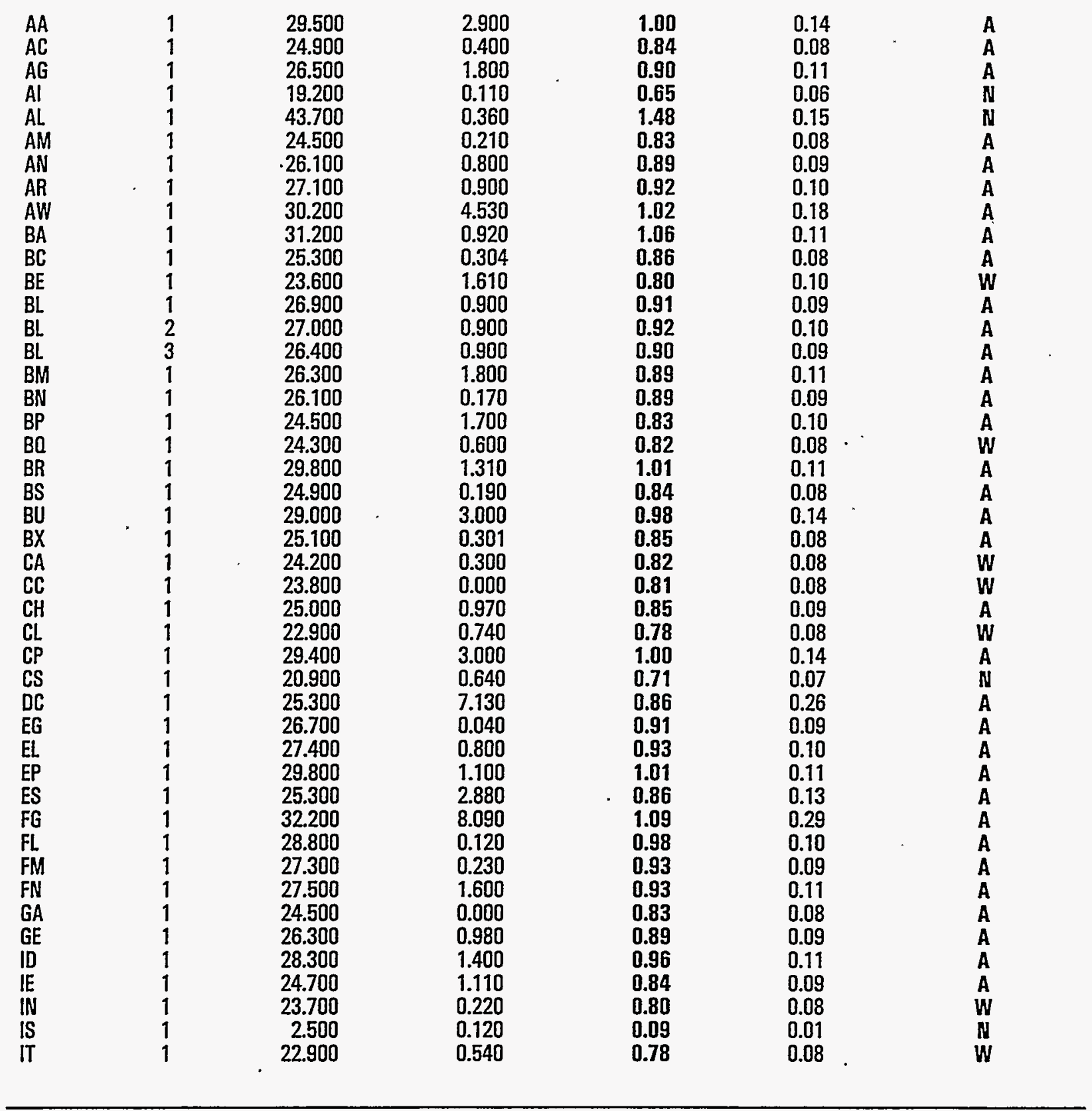

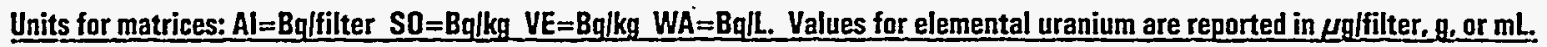
Evaluation: $A=A c c e p t a b l e, W=A c c e p t a b l e$ with Warning, $N=$ Not Acceptable.

$\mathrm{pCi}=\mathrm{Bq} \times 27$ 
QAP44 Results by Nuclide

Matrix: AI

Radionuclide: 6060

EML Value: 29.500

EML Error: 2.900

\begin{tabular}{|c|c|c|c|c|c|c|}
\hline Lahonde & Iest \# & $\begin{array}{c}\text { Reported } \\
\text { Value }\end{array}$ & $\begin{array}{c}\text { Reported } \\
\text { Error }\end{array}$ & $\frac{\text { Reported }}{\text { EMI }}$ & $\begin{array}{l}\text { Ratio } \\
\text { Errar }\end{array}$ & Evaluation \\
\hline$J P$ & 1 & 24.000 & 0.200 & 0.81 & 0.08 & $\mathbf{W}$ \\
\hline LA & 1 & 23.300 & 1.600 & 0.79 & 0.09 & $\mathbf{W}$ \\
\hline LA & 2 & 23.400 & 1.600 & 0.79 & 0.10 & $W$ \\
\hline LA & 3 & 23.400 & 1.600 & 0.79 & 0.10 & $W$ \\
\hline $\mathrm{LB}$ & 1 & 25.700 & 6.160 & 0.87 & 0.23 & A \\
\hline $\mathrm{LH}$ & 1 & 21.800 & 1.600 & 0.74 & 0.09 & W \\
\hline LL & 1 & 28.700 & 1.800 & 0.97 & 0.11 & A \\
\hline$L M$ & $i$ & 40.900 & 0.900 & 1.39 & 0.14 & $\mathbf{N}$ \\
\hline LV & 1 & 25.100 & 0.200 & 0.85 & 0.08 & A \\
\hline ME & 1 & 28.500 & 0.154 & 0.97 & 0.10 & A \\
\hline MI & 1 & 26.800 & 0.865 & 0.91 & 0.09 & A \\
\hline ML & 1 & 25.800 & 0.400 & 0.88 & 0.09 & A \\
\hline MS & 1 & 30.400 & 3.000 & 1.03 & 0.14 & $\ddot{A}$ \\
\hline NA & $i$ & 27.600 & 0.130 & 0.94 & 0.09 & A \\
\hline NC & 1 & 28.700 & 0.470 & 0.97 & 0.10 & A \\
\hline NL. & 1 & 28.400 & 1.100 & 0.96 & 0.10 & A \\
\hline NM & 1 & 20.400 & 0.600 & 0.69 & 0.07 & $\mathbf{N}$ \\
\hline 00 & 1 & 27.100 & 0.290 & 0.92 & 0.09 & A \\
\hline $\mathrm{OL}$ & 1 & 25.600 & 0.400 & 0.87 & 0.09 & A \\
\hline OR & 1 & 29.000 & 1.000 & 0.98 & 0.10 & A \\
\hline OT & 1 & 26.000 & 1.000 & 0.88 & 0.09 & A \\
\hline RA & $i$ & 22.900 & 0.800 & 0.78 & 0.08 & $W$ \\
\hline $\mathrm{RE}$ & $i$ & 24.900 & 2.370 & 0.84 & 0.12 & $A$ \\
\hline $\mathrm{Rl}$ & 1 & 2.230 & 0.700 & 0.08 & 0.02 & $\mathbf{N}$ \\
\hline SA & 1 & 33.900 & 1.800 & 1.15 & 0.13 & $\ddot{W}$ \\
\hline SE & 1 & 29.200 & 1.640 & 0.99 & 0.11 & $A$ \\
\hline SK & 1 & 27.700 & 0.900 & 0.94 & 0.10 & A \\
\hline SR & 1 & 27.000 & 1.000 & 0.92 & 0.10 & A \\
\hline SS & 1 & 24.600 & 0.550 & 0.83 & 0.08 & A \\
\hline SW & 1 & 24.000 & 0.510 & 0.81 & 0.08 & $w$ \\
\hline TE & 1 & 26.500 & 0.430 & 0.90 & 0.09 & $A$ \\
\hline $\mathrm{TI}$ & $i$ & 25.000 & 3.000 & 0.85 & 0.13 & $\ddot{A}$ \\
\hline TM & 1 & 26.600 & 0.830 & 0.90 & 0.09 & $\ddot{A}$ \\
\hline TN & 1 & 25.400 & 0.864 & 0.86 & 0.09 & A \\
\hline TO & 1 & 27.800 & 1.270 & 0.94 & 0.10 . & A \\
\hline TW & 1 & 27.000 & 0.500 & 0.92 & 0.09 & A \\
\hline $\mathrm{TX}$ & 1 & 24.000 & 0.200 & 0.81 & 0.08 & $w$ \\
\hline TY & $i$ & 18.500 & 0.600 & 0.63 & 0.06 & $N$ \\
\hline UC & 1 & 25.100 & 0.130 & 0.85 & 0.08 & A \\
\hline UK & 1 & 24.900 & 0.370 & 0.84 & 0.08 & $\ddot{A}$ \\
\hline UP & 1 & 24.800 & 0.629 & 0.84 & 0.09 & $A$ \\
\hline UY & 1 & 27.000 & 1.700 & 0.92 & 0.11 & $A$ \\
\hline WA & 1 & 27.400 & 0.700 & 0.93 & 0.09 & $A$ \\
\hline WC & $i$ & 23.400 & 1.820 & 0.79 & 0.10 & $W$ \\
\hline WE & 1 & 47.100 & 1.570 & 1.60 & 0.17 & $N$ \\
\hline WI & $i$ & 28.800 & 1.250 & 0.98 & 0.11 & A \\
\hline
\end{tabular}

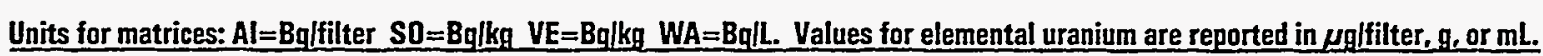

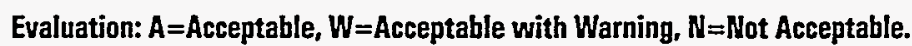

$p C i=B q \times 27$ 


\section{QAP44 Results by Nuclide}

Matrix: Al

Radionuclide: 6060

EML Value: 29.500

EML Error: 2.900

\begin{tabular}{|c|c|c|c|c|c|c|}
\hline Lahande & Test \#\# & $\begin{array}{c}\text { Reported } \\
\text { Value }\end{array}$ & $\begin{array}{c}\text { Reported } \\
\text { Errar }\end{array}$ & $\frac{\text { Reported }}{\text { EMLL }}$ & $\begin{array}{l}\text { Ratio } \\
\text { Frror }\end{array}$ & Fvaluation \\
\hline $\begin{array}{l}\text { WN } \\
\text { WP }\end{array}$ & $\begin{array}{l}1 \\
1\end{array}$ & $\begin{array}{l}26.100 \\
25.200\end{array}$ & $\begin{array}{l}0.200 \\
0.630\end{array}$ & $\begin{array}{l}0.89 \\
0.85\end{array}$ & $\begin{array}{l}0.09 \\
0.09\end{array}$ & $\begin{array}{l}A \\
A\end{array}$ \\
\hline
\end{tabular}

Total Number Reported: 93

Units for matrices: $\mathrm{Al}=\mathrm{Bg} / \mathrm{filter} \mathrm{SO}=\mathrm{Bq} / \mathrm{kg}$ VE $=\mathrm{Bq} / \mathrm{kg}, W \mathrm{~A}=\mathrm{Bq} / \mathrm{L}$. Values for elemental uranium are reported in $\mu \mathrm{g} / \mathrm{filter}, \mathrm{g}$, or $\mathrm{mL}$. 
QAP44 Results by Nuclide

Matrix: Al

Radionuclide: CS134

EML Value: 14.700

EML Error: 1.460

\begin{tabular}{cccccc} 
Lahrode & $\begin{array}{c}\text { Reported } \\
\text { Value }\end{array}$ & $\begin{array}{c}\text { Reported } \\
\text { Frror }\end{array}$ & $\frac{\text { Reported }}{\text { EMll }}$ & $\begin{array}{l}\text { Ratio } \\
\text { Error }\end{array}$ \\
\hline
\end{tabular}

\begin{tabular}{|c|c|c|c|c|c|c|}
\hline$A A$ & 1 & 14.700 & 1.460 & 1.00 & 0.14 & A \\
\hline$A C$ & 1 & 13.500 & 0.300 & 0.92 & 0.09 & A \\
\hline AG & 1 & 14.500 & 0.940 & 0.99 & 0.12 & A \\
\hline Al & 1 & 10.600 & 0.820 & 0.72 & 0.09 & $W$ \\
\hline $\mathrm{AL}$ & 1 & 13.500 & 0.210 & 0.92 & 0.09 & A \\
\hline AM & 1 & 9.810 & 0.130 & 0.67 & 0.07 & N \\
\hline AN & 1 & 13.100 & 0.400 & 0.89 & 0.09 & A \\
\hline AR & 1 & 13.000 & 0.600 & 0.88 & 0.10 & A \\
\hline AW & 1 & 14.500 & 2.180 & 0.99 & 0.18 & $\ddot{A}$ \\
\hline BA & 1 & 15.300 & 0.850 & 1.84 & 0.12 & $\ddot{A}$ \\
\hline $\mathrm{BC}$ & 1 & 13.300 & 0.613 & 0.91 & 0.10 & $\ddot{A}$ \\
\hline $\mathrm{BE}$ & 1 & 11.600 & 0.820 & 0.79 & 0.10 & 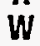 \\
\hline BL & 1 & 13.400 & 0.500 & 0.91 & 0.10 & A \\
\hline BL & 2 & 13.300 & 0.500 & 0.91 & 0.10 & A \\
\hline BL & 3 & 12.800 & 0.500 & 0.87 & 0.09 & A \\
\hline BM & 1 & 14.600 & 0.700 & 0.99 & 0.11 & A \\
\hline$B N$ & 1 & 13.000 & 0.020 & 0.88 & 0.09 & A \\
\hline BP & 1 & 14.100 & 1.100 & 0.96 & 0.12 & $\ddot{A}$ \\
\hline $\mathrm{BO}$ & $i$ & 14.500 & 0.500 & 0.99 & 0.10 & A \\
\hline BR & 1 & 14.300 & 0.880 & 0.97 & 0.11 & A \\
\hline BS & 1 & 13.100 & 0.150 & 0.89 & 0.09 & A \\
\hline$B U$ & 1 & 14.000 & 1.000 & 0.95 & 0.12 & A \\
\hline$B X$ & 1 & 13.300 & 0.611 & 0.91 & 0.10 & A \\
\hline CA & 1 & 13.200 & 0.100 & 0.90 & 0.09 & A \\
\hline CC & 1 & 12.700 & 0.000 & 0.86 & 0.09 & $\ddot{A}$ \\
\hline $\mathrm{CH}$ & 1 & 12.600 & 0.552 & 0.86 & 0.09 & A \\
\hline$C L$ & 1 & 13.100 & 0.450 & 0.89 & 0.09 & $\ddot{A}$ \\
\hline$C P$ & 1 & 13.800 & 2.000 & 0.94 & 0.17 & A \\
\hline CS & 1 & 8.960 & 0.190 & 0.61 & 0.06 & $\mathbb{N}$ \\
\hline DC & 1 & 16.000 & 1.910 & 1.09 & 0.17 & $W$ \\
\hline EG & 1 & 13.800 & 0.300 & 0.94 & 0.10 & A \\
\hline EL & 1 & 12.800 & 0.500 & 0.87 & 0.09 & A \\
\hline EP & 1 & 14.400 & 1.170 & 0.98 & 0.13 & $\ddot{A}$ \\
\hline ES & 1 & 12.500 & 1.440 & 0.85 & 0.13 & $\ddot{A}$ \\
\hline FG & 1 & 14.700 & 2.740 & 1.00 & 0.21 & $\ddot{A}$ \\
\hline $\mathrm{FL}$ & 1 & 14.100 & 0.150 & 0.96 & 0.10 & A \\
\hline$F M$ & 1 & 13.700 & 0.230 & 0.93 & 0.09 & A \\
\hline FN & 1 & 14.400 & 0.700 & 0.98 & 0.11 & A \\
\hline GA & 1 & 11.900 & 0.000 & 0.81 & 0.08 & $\ddot{A}$ \\
\hline GE & 1 & 13.600 & 0.600 & 0.93 & 0.10 & A \\
\hline ID & 1 & 15.900 & 0.800 & 1.08 & 0.12 & $\ddot{A}$ \\
\hline IE & 1 & 12.300 & 0.180 & 0.84 & 0.08 & A \\
\hline IN & 1 & 12.700 & 0.180 & 0.86 & 0.09 & $\ddot{A}$ \\
\hline IS & 1 & 12.300 & 0.800 & 0.84 & 0.10 & A \\
\hline IT & 1 & 11.400 & 0.380 & 0.78 & 0.08 & $\mathbf{W}$ \\
\hline
\end{tabular}

Units for matrices: $\mathrm{Al}=\mathrm{Bg}$ /filter $\mathrm{SO}=\mathrm{Bq} / \mathrm{kg}$ VE=Bqlkg WA=BqlL . Values for elemental uranium are reported in $\mu q$ lfilter, $g$, or $\mathrm{mL}$. Evaluation: A=Acceptable, $W=$ Acceptable with Warning, $N=$ Not Acceptable.

$\mathbf{p C i}=\mathrm{Bq} \times 27$ 
Matrix: Al

Radionuclide: CS134

EML Value: 14.700

EML Error: 1.460

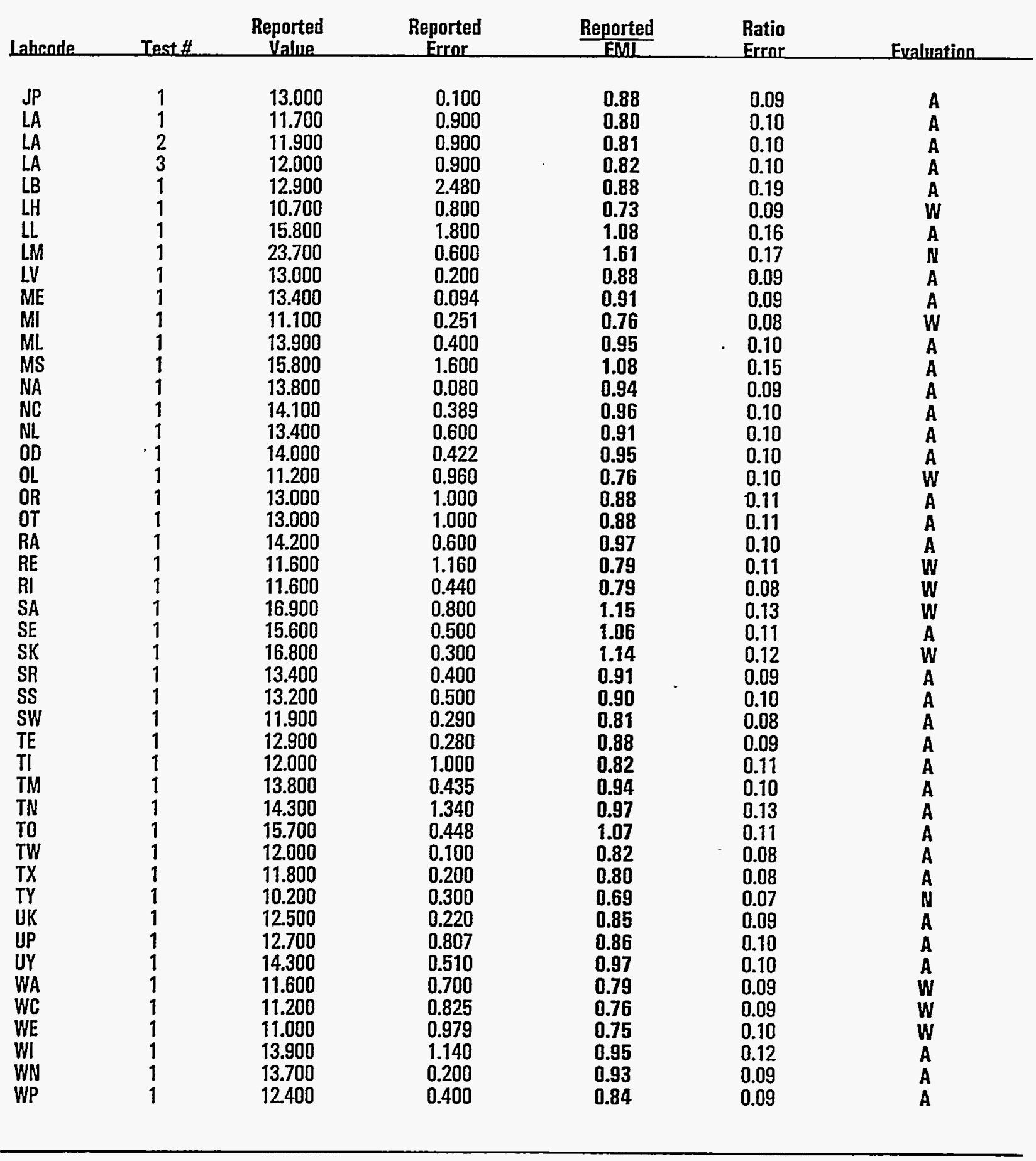

Units for matrices: $A I=B q$ lfilter $S O=B q / k g ~ V E=B q / k g$ WA=BqlL. Values for elemental uranium are reported in $\mu q$ ifilter, $g$, or $\mathrm{mL}$. 


\section{QAP44 Results by Nuelide}

Matrix: Al

Radionuclide: CS134

EML Value: 14.700

EML Error: 1.460

Reported

Value

Reported

Frror

Reported

Ratio

Lahcoide Test \#

Total Number Reported: 91

Units for matrices: $A l=B q$ filter $S O=B q l k g$ VE $=B q / k g ~ W A=B q l L$. Values for elemental uranium are reported in $\mu g$ lfilter, $q$, or $\mathrm{mL}$. Evaluation: $A=A c c e p t a b l e, W=A c c e p t a b l e$ with Warning, $N=$ Not Acceptable.

$\mathrm{pCi}=\mathrm{Bq} \times 27$ 


\section{QAP44 Results by Nuclide}

Matrix: Al

Radionuclide: CS137

EML Value: 6.640

EML Error: 0.700

\begin{tabular}{cccccc} 
Lahoode & Test\# & $\begin{array}{c}\text { Reported } \\
\text { Value }\end{array}$ & $\begin{array}{c}\text { Reported } \\
\text { Frror }\end{array}$ & $\frac{\text { Reported }}{\text { FMII }}$ & $\begin{array}{c}\text { Ratio } \\
\text { Frror }\end{array}$ Evaluation \\
\hline
\end{tabular}

\begin{tabular}{|c|c|c|c|c|c|c|}
\hline $\begin{array}{l}A A \\
A C \\
A G \\
A I \\
A L \\
A M \\
A N \\
A R \\
A W \\
B A \\
B C . \\
B E \\
B L \\
B L \\
B L \\
B M \\
B N \\
B P \\
B D \\
B R \\
B S \\
B S \\
B U \\
B X \\
C A \\
C C \\
C H \\
C L \\
C P \\
C S \\
D C \\
E G \\
E L \\
E P \\
E S \\
F G \\
F L \\
F M \\
F N \\
G A \\
G E \\
I D \\
I E \\
I N \\
I S \\
I T\end{array}$ & $\begin{array}{l}1 \\
1 \\
1 \\
1 \\
1 \\
1 \\
1 \\
1 \\
1 \\
1 \\
1 \\
1 \\
1 \\
2 \\
3 \\
1 \\
1 \\
1 \\
1 \\
1 \\
1 \\
1 \\
1 \\
1 \\
1 \\
1 \\
1 \\
1 \\
1 \\
1 \\
1 \\
1 \\
1 \\
1 \\
1 \\
1 \\
1 \\
1 \\
1 \\
1 \\
1 \\
1 \\
1 \\
1 \\
1\end{array}$ & $\begin{array}{r}6.640 \\
5.530 \\
6.090 \\
4.830 \\
10.500 \\
5.630 \\
5.850 \\
6.000 \\
6.950 \\
7.120 \\
5.850 \\
5.480 \\
5.870 \\
5.870 \\
5.760 \\
6.390 \\
7.940 \\
7.400 \\
6.800 \\
6.640 \\
5.370 \\
6.900 \\
5.920 \\
5.200 \\
5.570 \\
5.560 \\
5.840 \\
7.100 \\
4.470 \\
7.290 \\
5.800 \\
6.900 \\
6.190 \\
5.140 \\
7.420 \\
6.720 \\
6.300 \\
5.870 \\
5.150 \\
5.900 \\
7.400 \\
5.380 \\
4.950 \\
5.510 \\
5.050\end{array}$ & $\begin{array}{l}0.700 \\
0.210 \\
0.440 \\
0.085 \\
0.230 \\
0.150 \\
0.180 \\
0.300 \\
1.040 \\
0.680 \\
0.322 \\
0.390 \\
0.240 \\
0.240 \\
0.230 \\
0.380 \\
0.400 \\
1.400 \\
0.300 \\
0.930 \\
0.090 \\
0.700 \\
0.320 \\
0.500 \\
0.000 \\
0.552 \\
0.370 \\
0.830 \\
0.190 \\
3.670 \\
0.200 \\
0.600 \\
0.515 \\
0.760 \\
1.410 \\
0.110 \\
0.140 \\
0.480 \\
0.000 \\
0.556 \\
0.380 \\
0.320 \\
0.250 \\
0.770 \\
0.180\end{array}$ & $\begin{array}{l}1.00 \\
0.83 \\
0.92 \\
0.73 \\
1.58 \\
0.85 \\
0.88 \\
0.90 \\
1.05 \\
1.07 \\
0.88 \\
0.83 \\
0.88 \\
0.88 \\
0.87 \\
0.96 \\
1.20 \\
1.11 \\
1.02 \\
1.00 \\
0.81 \\
1.04 \\
0.89 \\
0.78 \\
0.84 \\
0.84 \\
0.88 \\
1.07 \\
0.67 \\
1.10 \\
0.87 \\
1.04 \\
0.93 \\
0.77 \\
1.12 \\
1.01 \\
0.95 \\
0.88 \\
0.78 \\
0.89 \\
1.11 \\
0.81 \\
0.75 \\
0.83 \\
0.76\end{array}$ & \begin{tabular}{l}
0.15 \\
0.09 \\
0.12 \\
0.08 \\
0.17 \\
0.09 \\
0.10 \\
0.11 \\
0.19 \\
0.15 \\
0.11 \\
0.11 \\
0.10 \\
0.10 \\
0.10 \\
0.12 \\
0.14 \\
0.24 \\
0.12 \\
0.18 \\
0.09 \\
0.15 \\
0.11 \\
0.11 \\
0.09 \\
0.12 \\
0.11 \\
0.17 \\
0.08 \\
0.56 \\
\hdashline
\end{tabular} & $\begin{array}{l}A \\
W \\
A \\
W \\
\text { N } \\
A \\
A \\
A \\
A \\
A \\
A \\
W \\
A \\
A \\
A \\
A \\
W \\
A \\
A \\
A \\
W \\
A \\
A \\
W \\
A \\
A \\
A \\
A \\
\text { W } \\
\text { A } \\
\text { A } \\
A \\
A \\
W \\
A \\
A \\
A \\
A \\
W \\
A \\
A \\
W \\
W \\
W \\
W\end{array}$ \\
\hline
\end{tabular}

Units for matrices: $\mathrm{Al}=\mathrm{Bq}$ /filiter $\mathrm{SD}=\mathrm{Bq} / \mathrm{kg}$ VE=Bglkg $W A=\mathrm{Bq} / \mathrm{L}$. Values for elemental uranium are reported in $\mu \mathrm{g} /$ filter, $\mathrm{g}$, or $\mathrm{mL}$. 
Matrix: Al

Radionuclide: CS137

EML Value: 6.640

EML Error: 0.700

\begin{tabular}{|c|c|c|c|c|c|c|}
\hline Lahende & Test \# & $\begin{array}{c}\text { Reported } \\
\text { Value }\end{array}$ & $\begin{array}{c}\text { Reported } \\
\text { Errar }\end{array}$ & $\frac{\text { Reported }}{\text { FML }}$ & $\begin{array}{l}\text { Ratio } \\
\text { Frror }\end{array}$ & Evaluatinn \\
\hline$J P$ & 1 & 5.700 & 0.070 & 0.86 & 0.09 & $A$ \\
\hline LA & 1 & 5.600 & 0.400 & 0.84 & 0.11 & A \\
\hline LA & 2 & 5.400 & 0.400 & 0.81 & 0.11 & $\ddot{W}$ \\
\hline LA & 3 & 5.500 & 0.400 & 0.83 & 0.11 & $W$ \\
\hline LB & 1 & 5.280 & 0.940 & 0.80 & 0.17 & $W$ \\
\hline LH & 1 & 5.200 & 0.500 & 0.78 & 0.11 & $w$ \\
\hline LL & 1 & 6.320 & 4.000 & 0.95 & 0.61 & A \\
\hline$L M$ & $i$ & 9.100 & 0.500 & 1.37 & 0.16 & $\mathbf{N}$ \\
\hline LV & 1 & 5.630 & 0.070 & 0.85 & 0.09 & $A$ \\
\hline ME & 1 & 5.980 & 0.062 & 0.90 & 0.10 & $A$ \\
\hline MI & 1 & 5.210 & 0.249 & 0.79 & 0.09 & $W$ \\
\hline ML & 1 & 5.600 & 0.200 & 0.84 & 0.09 & A \\
\hline MS & 1 & 6.700 & 0.670 & 1.01 & 0.15 & $\ddot{A}$ \\
\hline NA & 1 & 6.630 & 0.090 & 1.00 & 0.11 & $\ddot{A}$ \\
\hline NC & 1 & 6.650 & 0.251 & 1.00 & 0.11 & A \\
\hline NL & 1 & 6.430 & 0.390 & 0.97 & 0.12 & A \\
\hline NM & 1 & 4.500 & 0.260 & 0.68 & 0.08 & $\mathbf{N}$ \\
\hline oD & 1 & 6.410 & 0.379 & 0.97 & 0.12 & A \\
\hline $\mathrm{OL}$ & 1 & 6.060 & 0.190 & 0.91 & 0.10 & $\ddot{A}$ \\
\hline $\mathrm{OR}$ & 1 & 6.700 & 0.400 & 1.01 & 0.12 & A \\
\hline OT & 1 & 6.200 & 0.200 & 0.93 & 0.10 & $\ddot{A}$ \\
\hline $\mathrm{RA}$ & 1 & 6.210 & 0.320 & 0.94 & 0.11 & $\ddot{A}$ \\
\hline $\mathrm{RE}$ & 1 & 5.470 & 0.710 & 0.82 & 0.14 & $w$ \\
\hline $\mathrm{RI}$ & 1 & 5.210 & 0.710 & 0.79 & 0.14 & $W$ \\
\hline SA & 1 & 8.400 & 0.400 & 1.27 & 0.15 & $w$ \\
\hline SE & $i$ & 6.750 & 0.680 & 1.02 & 0.15 & $A$ \\
\hline SK & $i$ & 5.070 & 0.040 & 0.76 & 0.08 & $w$ \\
\hline SR & $i$ & 6.000 & 2.000 & 0.90 & 0.32 & A \\
\hline SS & $j$ & 5.400 & 0.400 & 0.81 & 0.11 & $w$ \\
\hline SW & 1 & 5.320 & 0.350 & 0.80 & 0.10 & $W$ \\
\hline TE & $i$ & 6.200 & 0.900 & 0.93 & 0.17 & A \\
\hline $\mathrm{TI}$ & $i$ & 6.100 & 0.600 & 0.92 & 0.13 & A \\
\hline TM & 1 & 6.020 & 0.360 & 0.91 & 0.11 & $\ddot{A}$ \\
\hline TN & $i$ & 5.530 & 0.498 & 0.83 & 0.12 & $\ddot{w}$ \\
\hline TO & $i$ & 6.030 & 0.490 & 0.91 & 0.12 & $A$ \\
\hline TW & 1 & 6.200 & 0.200 & 0.93 & 0.10 & $A$ \\
\hline $\mathrm{TX}$ & $i$ & 5.350 & 0.080 & 0.81 & 0.09 & $w$ \\
\hline TY & 1 & 5.300 & 0.300 & 0.80 & 0.10 & $w$ \\
\hline UC & 1 & 6.960 & 0.310 & 1.05 & 0.12 & $A$ \\
\hline UK & 1 & 5.190 & 0.260 & 0.78 & 0.09 & $\mathbf{W}$ \\
\hline UP & 1 & 5.420 & 0.457 & 0.82 & 0.11 & $W$ \\
\hline UY & 1 & 5.800 & 0.410 & 0.87 & 0.11 & $A$ \\
\hline WA & 1 & 6.200 & 1.000 & 0.93 & 0.18 & $\hat{A}$ \\
\hline WC & 1 & 5.190 & 0.741 & 0.78 & 0.14 & $w$ \\
\hline WE & 1 & 4.510 & 0.663 & 0.68 & 0.12 & $N$ \\
\hline WI & 1 & 6.100 & 0.950 & 0.92 & 0.17 & A \\
\hline
\end{tabular}

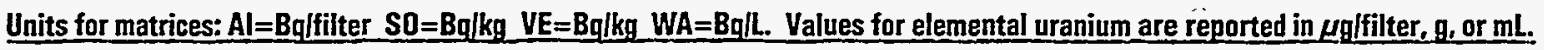
Evaluation: $A=A c c e p t a b l e, ~ W=A c c e p t a b l e$ with Warning, $N=$ Not Acceptable. $\mathrm{pCi}=\mathrm{Bq} \times 27$ 


\section{QAP44 Results by Nuclide}

Matrix: Al

Radionuclide: CS137

EML Value: 6.640

EML Error: 0.700

\begin{tabular}{|c|c|c|c|c|c|c|}
\hline Lahcode & Lest\# & $\begin{array}{c}\text { Reported } \\
\text { Value }\end{array}$ & $\begin{array}{c}\text { Reported } \\
\text { Error }\end{array}$ & $\frac{\text { Reported }}{\text { FMI }}$ & $\begin{array}{l}\text { Ratio } \\
\text { Frror }\end{array}$ & Evaluation \\
\hline $\begin{array}{l}\text { WN } \\
W P\end{array}$ & $\begin{array}{l}1 \\
1\end{array}$ & $\begin{array}{l}5.820 \\
6.180\end{array}$ & $\begin{array}{l}0.180 \\
0.310\end{array}$ & $\begin{array}{l}0.88 \\
0.93\end{array}$ & $\begin{array}{l}0.10 \\
0.11\end{array}$ & $\begin{array}{l}A \\
A\end{array}$ \\
\hline
\end{tabular}

Total Number Reported: 93

Units for matrices: $\mathrm{Al}=\mathrm{Bq}$ lfilter $\mathrm{SO}=\mathrm{Bq} / \mathrm{kg}, \mathrm{VE}=\mathrm{Bq} / \mathrm{kg}$ WA=BqlL. Values for elemental uranium are reported in $\mu \mathrm{glfilter}$. $\mathrm{g}$. or $\mathrm{mL}$. Evaluation: $A=A c c e p t a b l e, W=A c c e p t a b l e$ with Warning, $N=$ Not Acceptable. $\mathrm{pCi}=\mathrm{Bq} \times 27$ 


\section{QAP44 Results by Nuclide}

Matrix: Al

Radionuclide: GA 1

EML Value: 1.620

EML Error: 0.150

$\begin{array}{cccc}\text { Lahonde } & \text { Reported } & \text { Reported } & \text { Reported } \\ \text { Value } & \text { Frror } & \text { Rati } \\ \text { Erro }\end{array}$

Error

Evaluation

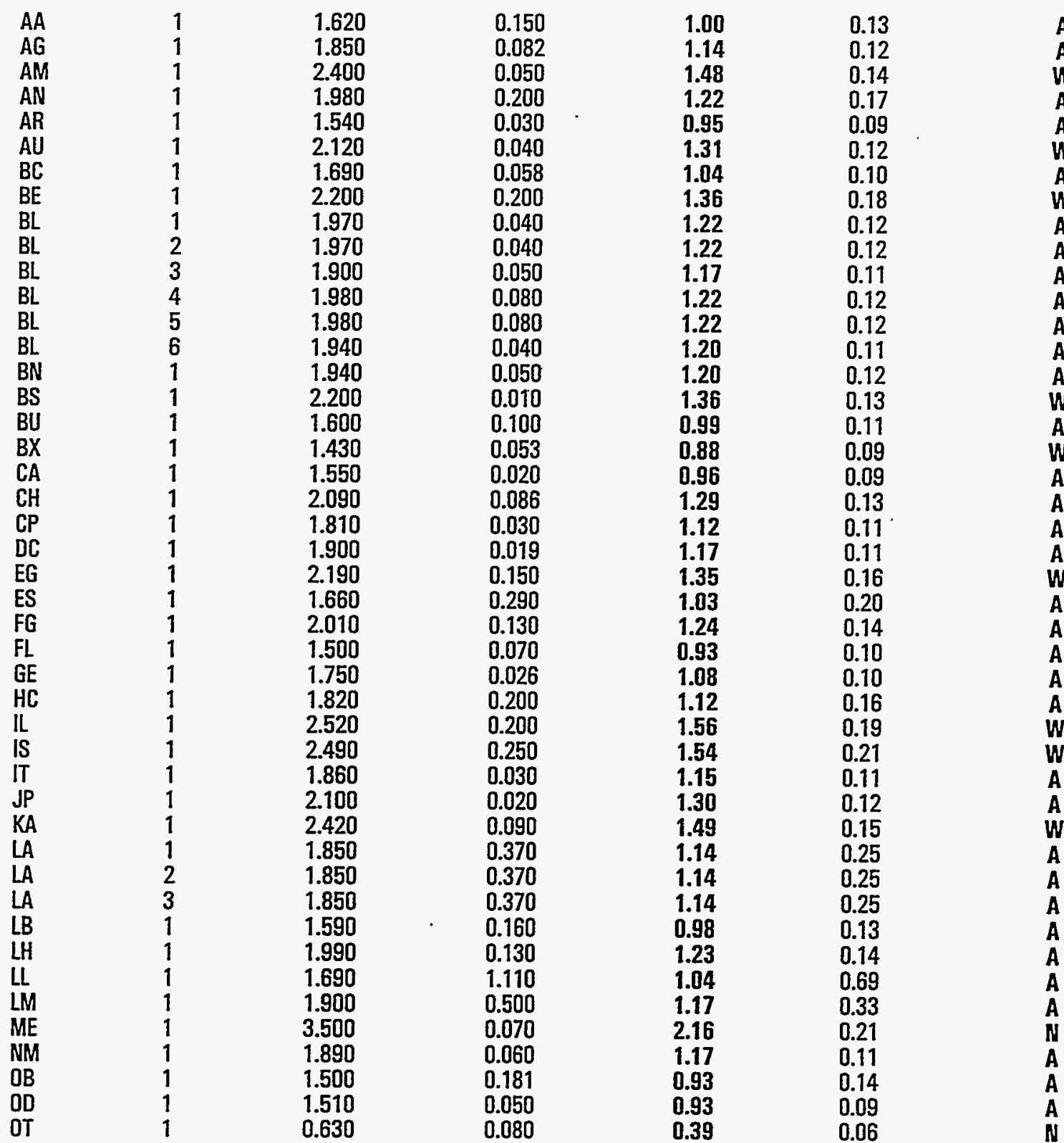

$A$
$A$
$W$
$A$
$A$
W
$A$
$W$
A
$A$
$A$
$A$
$A$
$A$
$A$
$A$
$W$
$A$
$W$
$A$
$A$
$A$
$A$
$W$
$A$
$A$
$A$
$A$
$A$
$W$
$W$
$A$
$A$
$W$
$A$
$A$
$A$
$A$
$A$
$A$
$A$
$N$
$A$
$A$
$A$
$N$

Units for matrices: $\mathrm{Al}=\mathrm{Bq}$ /filter $\mathrm{SO}=\mathrm{Bq} / \mathrm{kg}$ VE=Bqlkg $W_{A}=\mathrm{Bq} / \mathrm{L}$. Values for elemental uranium are reported in $\mu \mathrm{g} / \mathrm{filter}, \mathrm{g}$, or mL. Evaluation: $A=A c c e p t a b l e, W=A c c e p t a b l e$ with Warning, $N=$ Not Acceptable.

$\mathrm{pCi}=\mathrm{Bq} \times 27$ 


\section{QAP44 Results by Nuclide}

Matrix: Al

Radionuclide: GA 1

EML Value: 1.620

EML. Error: 0.150

\begin{tabular}{|c|c|c|c|c|c|c|}
\hline Lahconde. & Test\# & $\begin{array}{c}\text { Reported } \\
\text { Value }\end{array}$ & $\begin{array}{c}\text { Reported } \\
\text { Error }\end{array}$ & $\frac{\text { Reported }}{\text { FMI }}$ & $\begin{array}{l}\text { Ratio } \\
\text { Frror }\end{array}$ & Evaluation \\
\hline $\begin{array}{l}P A \\
R E \\
R F \\
S C \\
S R \\
T E \\
T I \\
T M \\
T N \\
T D \\
T W \\
\text { TC } \\
\text { UP } \\
\text { UP } \\
\text { UY } \\
W A \\
W C \\
W N \\
W V \\
\text { YA }\end{array}$ & $\begin{array}{l}1 \\
1 \\
1 \\
1 \\
1 \\
1 \\
1 \\
1 \\
1 \\
1 \\
1 \\
1 \\
1 \\
1 \\
1 \\
1 \\
1 \\
1 \\
1\end{array}$ & $\begin{array}{l}1.490 \\
2.640 \\
0.926 \\
1.620 \\
1.800 \\
2.210 \\
2.400 \\
2.700 \\
2.010 \\
2.090 \\
2.230 \\
1.690 \\
1.600 \\
1.950 \\
2.200 \\
1.530 \\
1.500 \\
1.500 \\
1.560\end{array}$ & $\begin{array}{l}0.040 \\
0.550 \\
0.140 \\
0.020 \\
0.130 \\
0.050 \\
0.100 \\
0.300 \\
0.033 \\
0.060 \\
0.060 \\
0.090 \\
0.000 \\
0.000 \\
0.080 \\
0.031 \\
0.200 \\
0.040 \\
0.016\end{array}$ & $\begin{array}{l}0.92 \\
1.63 \\
0.57 \\
1.00 \\
1.11 \\
1.36 \\
1.48 \\
1.67 \\
1.24 \\
1.29 \\
1.38 \\
1.04 \\
0.99 \\
1.20 \\
1.36 \\
0.94 \\
0.93 \\
0.93 \\
0.96\end{array}$ & $\begin{array}{l}0.09 \\
0.37 \\
0.10 \\
0.09 \\
0.13 \\
0.13 \\
0.15 \\
0.24 \\
0.12 \\
0.13 \\
0.13 \\
0.11 \\
0.09 \\
0.11 \\
0.14 \\
0.09 \\
0.15 \\
0.09 \\
0.09\end{array}$ & $\begin{array}{l}A \\
N \\
N \\
A \\
A \\
W \\
W \\
W \\
N \\
A \\
A \\
W \\
A \\
A \\
A \\
W \\
A \\
A \\
A \\
A\end{array}$ \\
\hline
\end{tabular}

Total Number Reported: 64

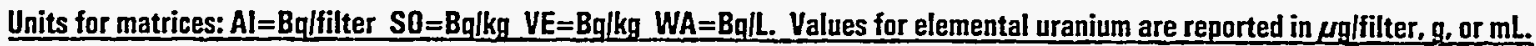


Matrix: Al

Radionuclide: GB 2

EML Value: 1.770

EML Error: 0.150

$\begin{array}{cccccc}\text { Lahronle } & \text { Reported } & \text { Reported } & \text { Reported } & \text { Ratio } \\ \text { Value. } & \text { Frror } & \text { EMI } & \text { Error } & \text { Evaluation }\end{array}$

\begin{tabular}{|c|c|c|c|c|c|}
\hline $\begin{array}{l}A A \\
A G \\
A M \\
A N \\
A R \\
A U \\
B C \\
B E \\
B L \\
B L \\
B L \\
B L \\
B L \\
B L \\
B N \\
B S \\
B X \\
C A \\
C H \\
C P \\
D C \\
E G \\
E S \\
F G \\
F L \\
G E \\
H C \\
I L \\
I S \\
I T \\
K A \\
L A \\
L A \\
L A \\
L B \\
L H \\
L L \\
L M \\
M E \\
N M \\
O B \\
O D \\
O T \\
P A \\
R E\end{array}$ & $\begin{array}{l}1 \\
1 \\
1 \\
1 \\
1 \\
1 \\
1 \\
1 \\
1 \\
2 \\
3 \\
4 \\
5 \\
6 \\
1 \\
1 \\
1 \\
1 \\
1 \\
1 \\
1 \\
1 \\
1 \\
1 \\
1 \\
1 \\
1 \\
1 \\
1 \\
1 \\
1 \\
1 \\
2 \\
3 \\
1 \\
1 \\
1 \\
1 \\
1 \\
1 \\
1 \\
1 \\
1 \\
1 \\
1\end{array}$ & $\begin{array}{l}1.770 \\
1.730 \\
2.540 \\
2.250 \\
1.500 \\
2.090 \\
3.050 \\
1.800 \\
1.700 \\
1.710 \\
1.670 \\
1.660 \\
1.700 \\
2.240 \\
2.290 \\
1.870 \\
2.690 \\
1.460 \\
1.840 \\
1.170 \\
2.000 \\
2.190 \\
1.260 \\
1.480 \\
2.160 \\
1.640 \\
1.710 \\
1.620 \\
1.580 \\
2.990 \\
1.970 \\
1.590 \\
1.630 \\
1.630 \\
1.480 \\
1.740 \\
2.310 \\
2.000 \\
2.490 \\
1.870 \\
1.870 \\
2.180 \\
0.970 \\
1.760 \\
5.350\end{array}$ & $\begin{array}{l}0.150 \\
0.066 \\
0.040 \\
0.230 \\
0.030 \\
0.040 \\
0.083 \\
0.100 \\
0.050 \\
0.050 \\
0.060 \\
0.100 \\
0.100 \\
0.050 \\
0.050 \\
0.010 \\
0.077 \\
0.030 \\
0.126 \\
0.010 \\
0.018 \\
0.140 \\
0.220 \\
0.130 \\
0.070 \\
0.022 \\
0.200 \\
0.160 \\
0.160 \\
0.073 \\
0.060 \\
0.220 \\
0.220 \\
0.220 \\
0.150 \\
0.100 \\
0.860 \\
0.500 \\
0.046 \\
0.090 \\
0.207 \\
0.060 \\
0.140 \\
0.030 \\
0.840\end{array}$ & $\begin{array}{l}1.00 \\
0.98 \\
1.44 \\
1.27 \\
0.85 \\
1.18 \\
1.72 \\
1.02 \\
0.96 \\
0.97 \\
0.94 \\
0.94 \\
0.96 \\
1.27 \\
1.29 \\
1.06 \\
1.52 \\
0.83 \\
1.04 \\
0.66 \\
1.13 \\
1.24 \\
0.71 \\
0.84 \\
1.22 \\
0.93 \\
0.97 \\
0.92 \\
0.89 \\
1.69 \\
1.11 \\
0.90 \\
0.92 \\
0.92 \\
.0 .84 \\
0.98 \\
1.31 \\
1.13 \\
1.41 \\
1.06 \\
1.06 \\
1.23 \\
0.55 \\
0.99 \\
3.02\end{array}$ & \begin{tabular}{l}
0.12 \\
0.09 \\
0.12 \\
0.17 \\
0.07 \\
0.10 \\
0.15 \\
0.10 \\
0.09 \\
0.09 \\
0.09 \\
0.10 \\
0.10 \\
0.11 \\
0.11 \\
0.09 \\
0.14 \\
0.07 \\
0.11 \\
0.06 \\
0.10 \\
0.13 \\
0.14 \\
0.10 \\
0.11 \\
0.08 \\
0.14 \\
0.12 \\
\hdashline
\end{tabular} \\
\hline
\end{tabular}

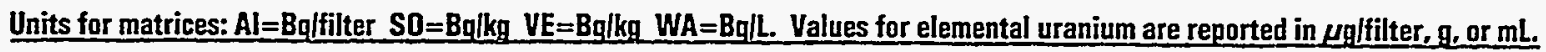


QAP44 Results by Nuclide

Matrix: Al

Radionuclide: GB 2

EML Value: 1.770

EML Error: 0.150

\begin{tabular}{|c|c|c|c|c|c|c|}
\hline Lahende & Test\# & $\begin{array}{c}\text { Reported } \\
\text { Value }\end{array}$ & $\begin{array}{c}\text { Reported } \\
\text { Error }\end{array}$ & $\frac{\text { Reported }}{\text { EML }}$ & $\begin{array}{l}\text { Ratio } \\
\text { Frrar }\end{array}$ & Evaluation \\
\hline RF & 1 & 1.180 & 0.210 & 0.67 & 0.13 & $\mathbf{N}$ \\
\hline SC & $i$ & 1.920 & 0.020 & 1.09 & 0.09 & A \\
\hline SR. & 1 & 1.780 & 0.110 & 1.01 & 0.11 & A \\
\hline TE & $i$ & 1.950 & 0.040 & 1.10 & 0.10 & A \\
\hline $\mathrm{TI}$ & $i$ & 2.200 & 0.100 & 1.24 & 0.12 & $\ddot{A}$ \\
\hline $\mathrm{TM}$ & 1 & 1.540 & 0.118 & 0.87 & 0.10 & $\mathbf{W}$ \\
\hline TN & 1 & 1.920 & 0.032 & 1.09 & 0.09 & A \\
\hline TO & 1 & 1.560 & 0.050 & 0.88 & 0.08 & $w$ \\
\hline TW & 1 & 1.790 & 0.040 & 1.01 & 0.09 & A \\
\hline UC & 1 & 1.640 & 0.060 & 0.93 & 0.09 & A \\
\hline UP & 1 & 1.820 & 0.000 & 1.03 & 0.09 & A \\
\hline UY & 1 & 2.150 & 0.000 & 1.22 & 0.10 & A \\
\hline WA & $i$ & 2.170 & 0.060 & 1.23 & 0.11 & $\ddot{A}$ \\
\hline WC & 1 & 1.880 & 0.038 & 1.06 & 0.09 & A \\
\hline WN & 1 & 1.680 & 0.090 & 0.95 & 0.10 & $\ddot{A}$ \\
\hline WV & 1 & 1.870 & 0.040 & 1.06 & 0.09 & A \\
\hline YA & 1 & 1.700 & 0.022 & 0.96 & 0.08 & A \\
\hline
\end{tabular}

Total Number Reported: 62

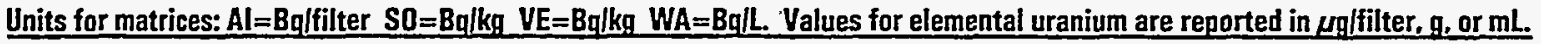
Evaluation: $A=A c c e p t a b l e, W=A c c e p t a b l e$ with Warning, $N=N o t$ Acceptable.

$\mathrm{pCi}=\mathrm{Bq} \times 27$ 
Matrix: Al

Radionuclide: MN 54

EML Value: 3.440

EML Error: 0.380

\begin{tabular}{|c|c|c|c|c|c|c|}
\hline Lahcode & Test\# & $\begin{array}{c}\text { Reported } \\
\text { Value }\end{array}$ & $\begin{array}{c}\text { Reported } \\
\text { Errnr }\end{array}$ & $\frac{\text { Reported }}{\text { FML }}$ & $\begin{array}{l}\text { Ratio } \\
\text { Error }\end{array}$ & Evaluation \\
\hline
\end{tabular}

\begin{tabular}{|c|c|c|c|c|c|}
\hline 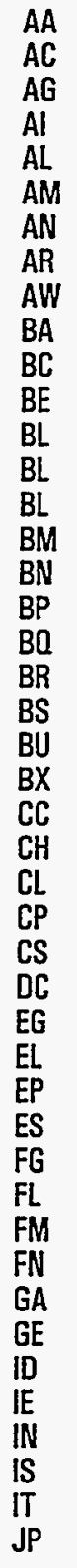 & $\begin{array}{l}1 \\
1 \\
1 \\
1 \\
1 \\
1 \\
1 \\
1 \\
1 \\
1 \\
1 \\
1 \\
1 \\
2 \\
3 \\
1 \\
1 \\
1 \\
1 \\
1 \\
1 \\
1 \\
1 \\
1 \\
1 \\
1 \\
1 \\
1 \\
1 \\
1 \\
1 \\
1 \\
1 \\
1 \\
1 \\
1 \\
1 \\
1 \\
1 \\
1 \\
1 \\
1 \\
1 \\
1 \\
1\end{array}$ & $\begin{array}{l}3.440 \\
2.660 \\
2.890 \\
2.300 \\
3.200 \\
2.850 \\
2.890 \\
3.000 \\
3.500 \\
4.170 \\
2.990 \\
2.900 \\
3.330 \\
3.260 \\
3.140 \\
3.260 \\
3.860 \\
3.100 \\
2.900 \\
4.450 \\
2.860 \\
3.400 \\
3.040 \\
2.750 \\
2.880 \\
2.890 \\
3.710 \\
2.580 \\
3.470 \\
3.100 \\
4.360 \\
3.240 \\
2.450 \\
3.210 \\
3.610 \\
3.340 \\
3.070 \\
2.940 \\
3.420 \\
3.630 \\
2.750 \\
2.580 \\
2.470 \\
2.600 \\
3.100\end{array}$ & $\begin{array}{l}0.380 \\
0.140 \\
0.200 \\
0.089 \\
0.120 \\
0.240 \\
0.140 \\
0.180 \\
0.530 \\
0.600 \\
0.156 \\
0.230 \\
0.160 \\
0.160 \\
0.150 \\
0.230 \\
0.160 \\
1.400 \\
0.300 \\
1.200 \\
0.100 \\
0.300 \\
0.164 \\
0.000 \\
0.504 \\
0.400 \\
0.840 \\
0.130 \\
0.966 \\
0.200 \\
0.300 \\
0.437 \\
0.560 \\
0.790 \\
0.250 \\
0.120 \\
0.250 \\
0.000 \\
0.570 \\
0.220 \\
0.340 \\
0.010 \\
0.620 \\
0.230 \\
0.110\end{array}$ & \begin{tabular}{l}
1.00 \\
0.77 \\
0.84 \\
0.67 \\
0.93 \\
0.83 \\
0.84 \\
0.87 \\
1.02 \\
1.21 \\
0.87 \\
0.84 \\
0.97 \\
0.95 \\
0.91 \\
0.95 \\
1.12 \\
0.90 \\
0.84 \\
1.29 \\
0.83 \\
0.99 \\
0.88 \\
0.80 \\
0.84 \\
0.84 \\
1.08 \\
0.75 \\
1.01 \\
0.90 \\
1.27 \\
0.94 \\
0.71 \\
0.93 \\
1.05 \\
0.97 \\
0.89 \\
0.86 \\
\hdashline
\end{tabular} & \begin{tabular}{l}
0.16 \\
0.09 \\
0.11 \\
0.08 \\
0.11 \\
0.12 \\
0.10 \\
0.11 \\
0.19 \\
0.22 \\
0.11 \\
0.12 \\
0.12 \\
0.12 \\
0.11 \\
0.12 \\
0.13 \\
0.42 \\
0.13 \\
0.38 \\
0.10 \\
0.14 \\
0.11 \\
0.09 \\
0.17 \\
0.15 \\
\hdashline
\end{tabular} \\
\hline
\end{tabular}

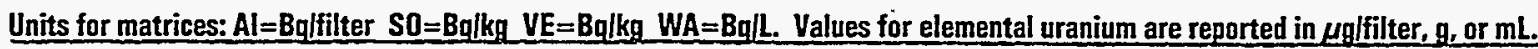
Evaluation: $A=$ Acceptable, $W=$ Acceptable with Warning, $N=$ Not Acceptable. 
QAP44 Results by Nuclide

Matrix: Al

Radionuclide: MN 54

EML Value: 3.440

EML Error: 0.380

\begin{tabular}{|c|c|c|c|c|c|c|}
\hline Lahcade. & Test \# & $\begin{array}{c}\text { Reported } \\
\text { Value }\end{array}$ & $\begin{array}{c}\text { Reported } \\
\text { Error }\end{array}$ & $\frac{\text { Reported }}{\text { EMML }}$ & $\begin{array}{l}\text { Ratio } \\
\text { Frror }\end{array}$ & Evaluation \\
\hline $\begin{array}{l}L A \\
L A \\
L A \\
L B \\
L H \\
L H \\
L L \\
L M \\
L V \\
M E \\
M I \\
M L \\
M S \\
N A \\
N C \\
N L \\
O D \\
O L \\
O R \\
O T \\
R A \\
R E \\
R I \\
\text { SA } \\
S E \\
S K \\
S R \\
S S \\
S W \\
T E \\
T E \\
T I \\
T M \\
T N \\
T O \\
T W \\
T X \\
T Y \\
U K \\
U P \\
U Y \\
U Y \\
W A \\
W C \\
W E \\
W I \\
W N \\
W P\end{array}$ & 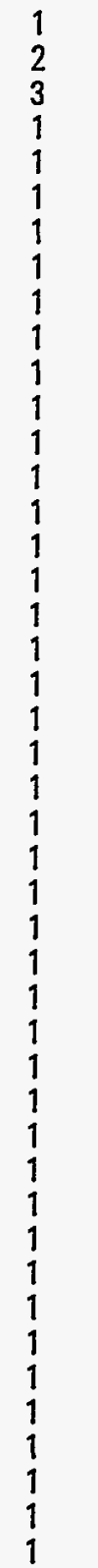 & $\begin{array}{l}2.800 \\
2.900 \\
2.800 \\
2.840 \\
2.700 \\
3.130 \\
5.000 \\
2.760 \\
3.380 \\
2.980 \\
2.800 \\
3.400 \\
3.400 \\
3.700 \\
3.580 \\
3.160 \\
3.140 \\
3.400 \\
3.100 \\
2.900 \\
2.920 \\
2.220 \\
4.300 \\
3.450 \\
2.410 \\
3.000 \\
3.000 \\
2.540 \\
3.270 \\
3.100 \\
2.790 \\
3.020 \\
3.180 \\
3.150 \\
3.170 \\
2.700 \\
2.770 \\
2.760 \\
3.200 \\
3.420 \\
2.790 \\
4.250 \\
3.250 \\
3.000 \\
3.100\end{array}$ & $\begin{array}{l}0.200 \\
0.300 \\
0.300 \\
0.550 \\
0.300 \\
6.400 \\
0.500 \\
0.100 \\
0.064 \\
0.145 \\
0.200 \\
0.340 \\
0.080 \\
0.245 \\
0.240 \\
0.214 \\
0.100 \\
0.400 \\
0.200 \\
0.140 \\
0.470 \\
0.560 \\
0.300 \\
0.170 \\
0.040 \\
1.000 \\
0.400 \\
0.350 \\
0.350 \\
0.300 \\
0.188 \\
0.520 \\
0.593 \\
0.090 \\
0.110 \\
0.200 \\
0.270 \\
0.342 \\
0.320 \\
0.260 \\
0.459 \\
0.799 \\
0.620 \\
0.120 \\
0.280\end{array}$ & $\begin{array}{l}0.81 \\
0.84 \\
0.81 \\
0.83 \\
0.79 \\
0.91 \\
1.45 \\
0.80 \\
0.98 \\
0.87 \\
0.81 \\
0.99 \\
0.99 \\
1.08 \\
1.04 \\
0.92 \\
0.91 \\
0.99 \\
0.90 \\
0.84 \\
0.85 \\
0.65 \\
1.25 \\
1.00 \\
0.70 \\
0.87 \\
0.87 \\
0.74 \\
0.95 \\
0.90 \\
0.81 \\
0.88 \\
0.92 \\
0.92 \\
0.92 \\
0.79 \\
0.81 \\
0.80 \\
0.93 \\
0.99 \\
0.81 \\
1.24 \\
0.95 \\
0.87 \\
0.90\end{array}$ & 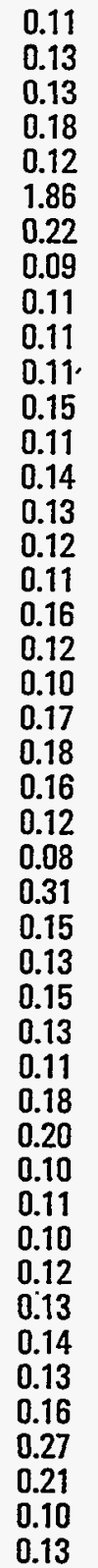 & $\begin{array}{l}W \\
W \\
W \\
W \\
W \\
W \\
A \\
N \\
W \\
A \\
A \\
W \\
A \\
A \\
A \\
A \\
A \\
A \\
A \\
A \\
W \\
A \\
N \\
W \\
A \\
N \\
A \\
A \\
N \\
A \\
A \\
W \\
A \\
A \\
A \\
A \\
W \\
W \\
W \\
A \\
A \\
W \\
W \\
A \\
A \\
A\end{array}$ \\
\hline
\end{tabular}

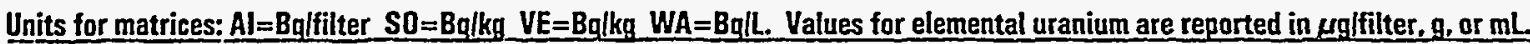




\section{OAP44 Results by Nuclide}

Matrix: Al

Radionuclide: MN 54

EML Value: 3.440

EML Error: 0.380
Reported Reported $\quad$ Ratio Error
Error

Evaluation

Total Number Reported: 90

Units for matrices: $\mathrm{Al}=\mathrm{Bq}$ lfilter $\mathrm{SO}=\mathrm{Bq} / \mathrm{kg}$ VE=Bqlkg WA=BqlL. Values for elemental uranium are reported in $\mu \mathrm{g} / \mathrm{filter}, \mathrm{g}$, or $\mathrm{mL}$.

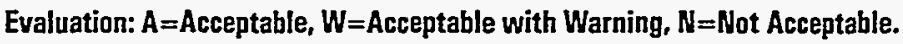
$\mathrm{pCi}=\mathrm{Bq} \times 27$ 
OAP44 Results by Nuclide

Matrix: AI

Radionuclide: PU238

EML Value: 0.096

EML. Error: 0.002

\begin{tabular}{|c|c|c|c|c|c|c|}
\hline Lahende & Test \# & $\begin{array}{c}\text { Reported } \\
\text { Yalue }\end{array}$ & $\begin{array}{c}\text { Reported } \\
\text { Error }\end{array}$ & $\frac{\text { Reported }}{\text { FMI }}$ & $\begin{array}{l}\text { Ratio } \\
\text { Frror }\end{array}$ & Evaluation \\
\hline
\end{tabular}

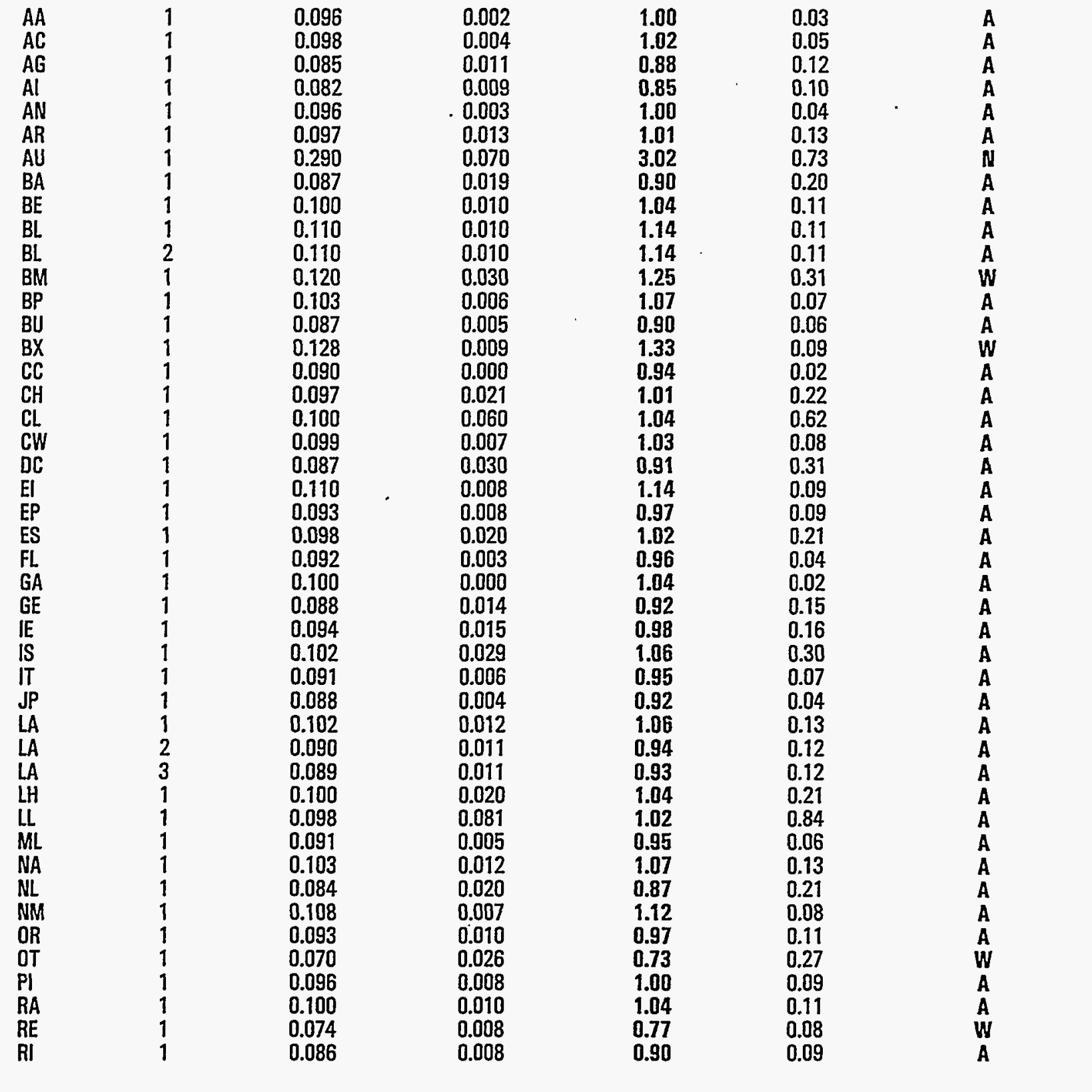

Units for matrices: $\mathrm{Al}=\mathrm{Bq} q$ filter $\mathrm{SO}=\mathrm{Bq} / \mathrm{kg}$ VE $=\mathrm{Bq} \mid \mathrm{kg}, W \mathrm{~A}=\mathrm{Bq} / \mathrm{L}$. Values for elemental uranium are reported in $\mu \mathrm{q} / \mathrm{filter}, \mathrm{g}$, or $\mathrm{mL}$.

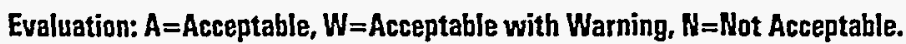

$\mathrm{pCi}=\mathrm{Bq} \times 27$ 


\section{QAP44 Results by Nuclide}

Matrix: Al

Radionuclide: PU238

EML Value: 0.096

EML Error: 0.002

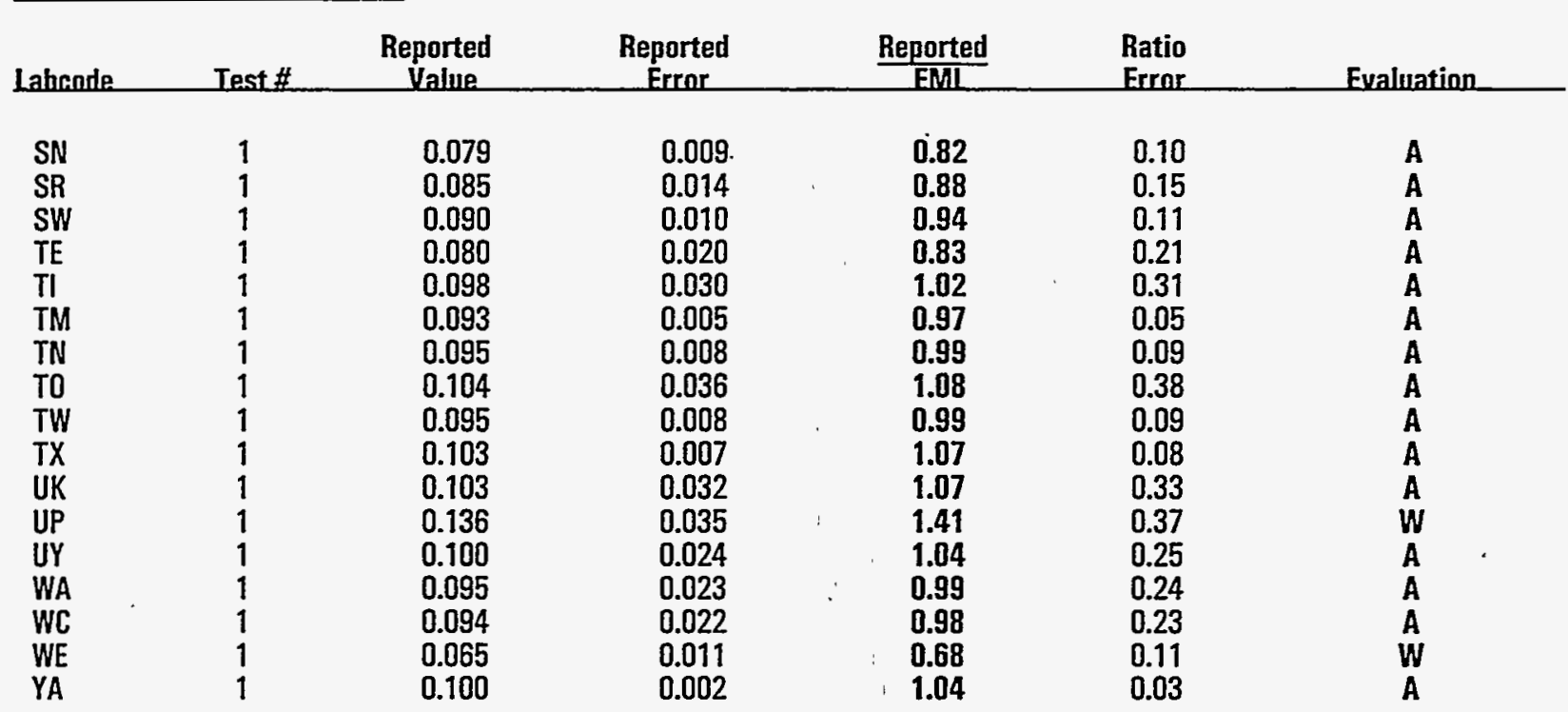

Total Number Reported: $\mathbf{6 2}$

Units for matrices: $\mathrm{Al}=\mathrm{Bq}$ /filter $\mathrm{SD}=\mathrm{Bq} / \mathrm{kg}$ VE=Bq/kg $W \mathrm{~A}=\mathrm{Bq} / \mathrm{L}$. Values for elemental uranium are reported in $\mu \mathrm{g} / \mathrm{filter}, \mathrm{g}$, or $\mathrm{mL}$. $\mathrm{pCi}=\mathrm{Bq} \times 27$ 
Matrix: Al

Radionuclide: PU239

EML Value: 0.093

EML Error: 0.003

\begin{tabular}{lccccc} 
Lahcade & Reported & Reported & Reported & Ratio \\
Value & Frror & $\frac{\text { Frror }}{\text { EMI }}$ & Evaluation \\
\hline
\end{tabular}

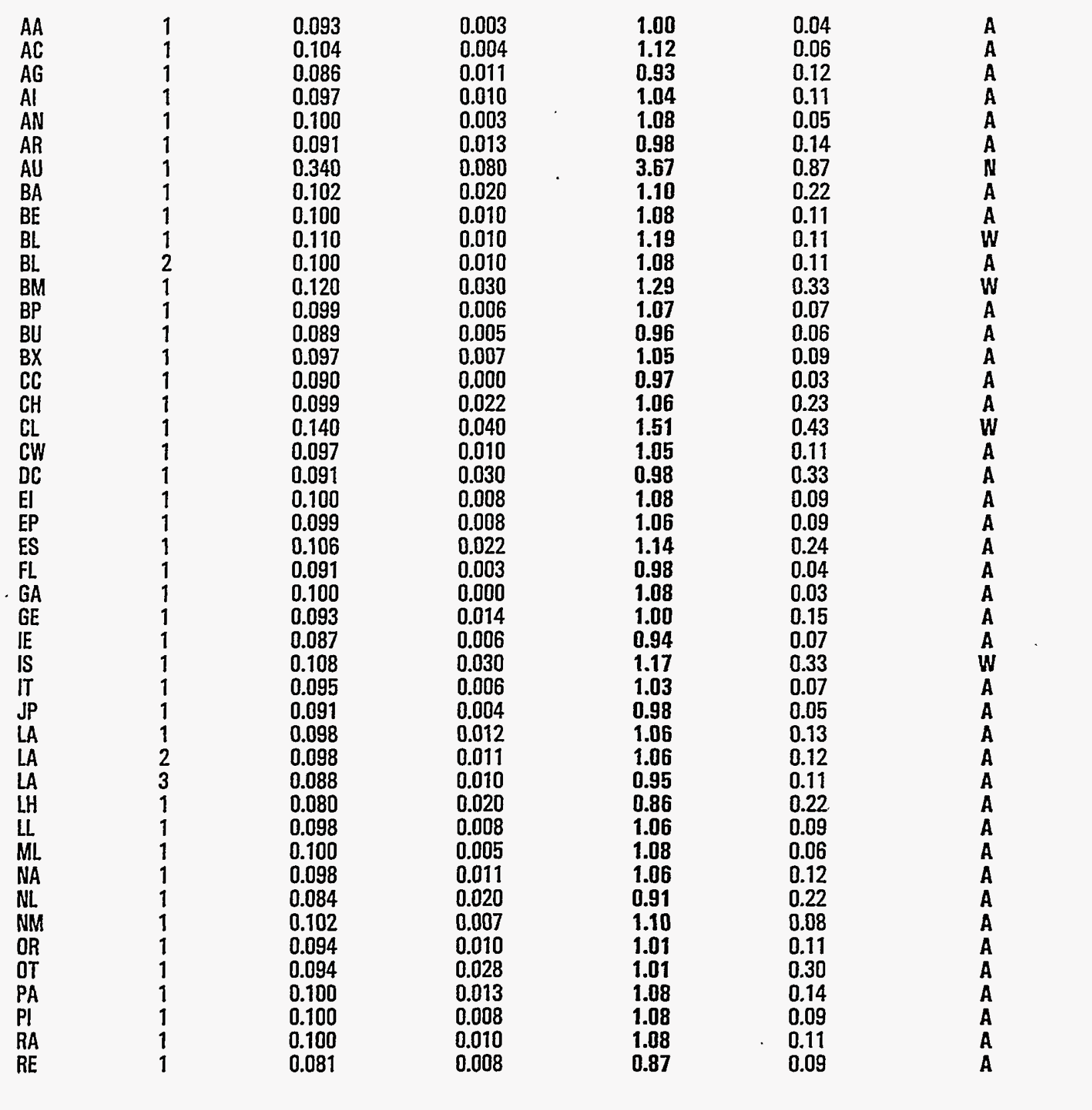

Units for matrices: $\mathrm{Al}=\mathrm{Bq} / \mathrm{filter} \mathrm{SO}=\mathrm{Bq} / \mathrm{kg}$ VE $=\mathrm{Bq} / \mathrm{kg}$ WA=BqlL . Yalues for elemental uranium are reported in $\mu \mathrm{g} / \mathrm{filter}, \mathrm{g}$, or $\mathrm{mL}$. Evaluation: $A=$ Acceptable, $W=$ Acceptable with Warning, $N=$ Not Acceptable. $\mathrm{pCi}=\mathrm{Bq} \times 27$ 
DAP44 Results by Nuclide

Matrix: Al

Radionuclide: PU239

EML Value: 0.093

EML Error: 0.003

\begin{tabular}{|c|c|c|c|c|c|c|}
\hline Lahconde & Test \# & $\begin{array}{c}\text { Reported } \\
\text { Value }\end{array}$ & $\begin{array}{c}\text { Reported } \\
\text { Error }\end{array}$ & $\frac{\text { Reported }}{\text { EML }}$ & $\begin{array}{l}\text { Ratio } \\
\text { Error }\end{array}$ & Evaluation \\
\hline RI & 1 & 0.077 & 0.007 & 0.83 & 0.08 & $W$ \\
\hline SN & 1 & 0.087 & 0.010 & 0.94 & 0.11 & A \\
\hline SR & 1 & 0.094 & 0.016 & 1.01 & 0.18 & A \\
\hline SW & 1 & 0.070 & 0.010 & 0.76 & 0.11 & $w$ \\
\hline $\mathrm{TE}$ & 1 & 0.090 & 0.020 & 0.97 & 0.22 & A \\
\hline $\mathrm{TI}$ & 1 & 0.120 & 0.030 & 1.29 & 0.33 & $\mathbf{W}$ \\
\hline$T M$ & 1 & 0.092 & 0.005 & 0.99 & 0.06 & A \\
\hline TN & 1 & 0.089 & 0.008 & 0.96 & 0.09 & A \\
\hline TO & 1 & 0.110 & 0.037 & 1.19 & 0.40 & $W$ \\
\hline TW & 1 & 0.086 & 0.008 & 0.93 & 0.09 & A \\
\hline $\mathrm{TX}$ & 1 & 0.105 & 0.007 & 1.13 & 0.08 & $A$ \\
\hline UK & 1 & 0.111 & 0.033 & 1.20 & 0.36 & $W$ \\
\hline UP & 1 & 0.085 & 0.026 & 0.92 & 0.29 & A \\
\hline UY & 1 & 0.094 & 0.023 & 1.01 & 0.25 & $A$ \\
\hline WA & 1 & 0.084 & 0.019 & 0.91 & 0.21 & $A$ \\
\hline WC & 1 & 0.097 & 0.023 & 1.05 & 0.25 & A \\
\hline WE & 1 & 0.057 & 0.010 & 0.61 & 0.11 & N \\
\hline YA & 1 & 0.098 & 0.003 & 1.05 & 0.04 & A \\
\hline
\end{tabular}

Total Number Reported: 63

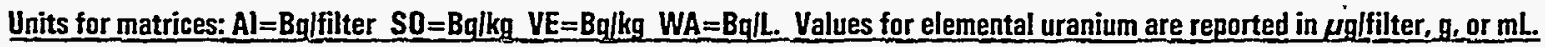

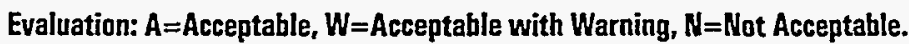

$\mathrm{pCi}=\mathrm{Bq} \times 27$ 


\section{QAP44 Results by Nuclide}

Matrix: Al

Radionuclide: RU106

EML Value: 11.600

EML Error: 1.440

\begin{tabular}{|c|c|c|c|c|c|c|}
\hline Lahmonie & Test\# & $\begin{array}{c}\text { Reported } \\
\text { Value }\end{array}$ & $\begin{array}{c}\text { Reported } \\
\text { Frrar }\end{array}$ & $\frac{\text { Reported }}{\text { EMII }}$ & $\begin{array}{l}\text { Ratio } \\
\text { Errar }\end{array}$ & Evaluation \\
\hline
\end{tabular}

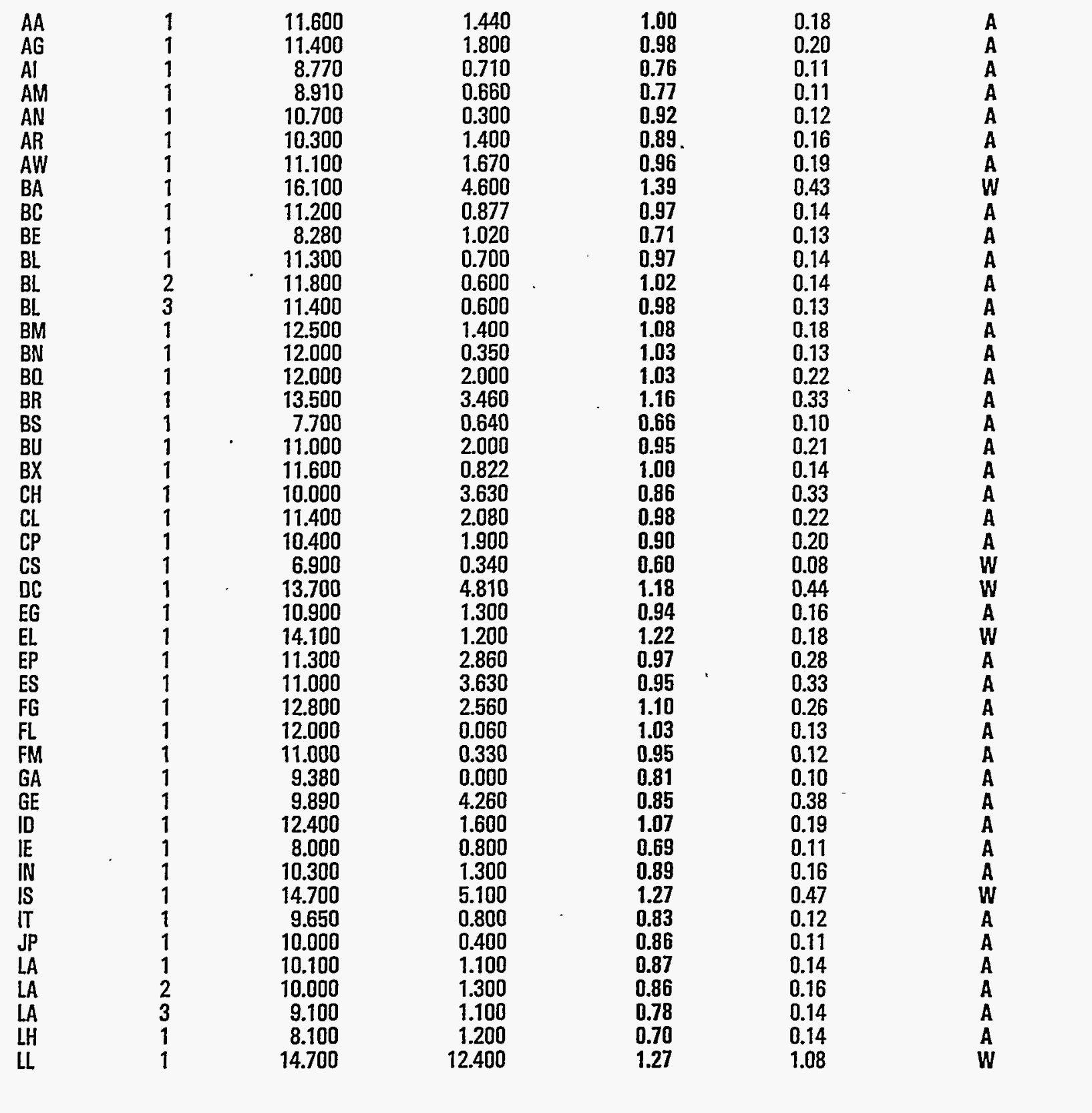

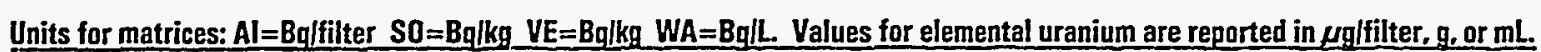
Evaluation: $A=A c c e p t a b l e, W=A c c e p t a b l e$ with Warning, $N=N o t$ Acceptable.

$\mathrm{pCi}=\mathrm{Bq} \times 27$ 


\section{QAP44 Results by Nuclide}

Matrix: Al

Radionuclide: RU106

EML Value: 11.600

EML Error: 1.440

\begin{tabular}{|c|c|c|c|c|c|c|}
\hline Lahcorie & Test\# & $\begin{array}{c}\text { Reported } \\
\text { Value }\end{array}$ & $\begin{array}{c}\text { Reported } \\
\text { Error }\end{array}$ & $\frac{\text { Reported }}{\text { EML }}$ & $\begin{array}{l}\text { Ratio } \\
\text { Frrar }\end{array}$ & Evaluation \\
\hline LM & 1 & 15.100 & 3.100 & 1.30 & 0.31 & $w$ \\
\hline LV & 1 & 10.400 & 0.800 & 0.90 & 0.13 & A \\
\hline ML & 1 & 11.400 & 1.000 & 0.98 & 0.15 & A \\
\hline NA & 1 & 10.300 & 0.560 & 0.89 & 0.12 & $\ddot{A}$ \\
\hline NL & 1 & 10.600 & 1.100 & 0.91 & 0.15 & $\ddot{A}$ \\
\hline OD & 1 & 11.400 & 0.635 & 0.98 & 0.13 & A \\
\hline $\mathrm{OL}$ & 1 & 9.000 & 0.930 & 0.78 & 0.13 & A \\
\hline OR & 1 & 11.000 & 3.000 & 0.95 & 0.28 & A \\
\hline OT & 1 & 9.800 & 1.100 & 0.85 & 0.14 & A \\
\hline $\mathrm{RA}$ & 1 & 12.300 & 1.400 & 1.06 & 0.18 & A \\
\hline $\mathrm{RI}$ & 1 & 8.780 & 3.900 & 0.76 & 0.35 & A \\
\hline SE & $i$ & 11.000 & 1.230 & 0.95 & 0.16 & A \\
\hline SK & $i$ & 12.600 & 0.400 & 1.09 & 0.14 & $\ddot{A}$ \\
\hline SR & 1 & 16.000 & 11.000 & 1.38 & 0.96 & W \\
\hline SS & 1 & 9.500 & 3.400 & 0.82 & 0.31 & A \\
\hline SW & 1 & 10.400 & 2.570 & 0.90 & 0.25 & A \\
\hline TE & 1 & 10.200 & 1.850 & 0.88 & 0.19 & A \\
\hline TI & 1 & 10.000 & 1.000 & 0.86 & 0.14 & A \\
\hline$T M$ & 1 & 11.400 & 1.130 & 0.98 & 0.16 & A \\
\hline TN & 1 & 11.700 & 3.190 & 1.01 & 0.30 & A \\
\hline TO & 1 & 11.400 & 3.570 & 0.98 & 0.33 & A \\
\hline TW & 1 & 10.000 & 0.400 & 0.86 & 0.11 & A \\
\hline TX & 1 & 8.190 & 0.490 & 0.71 & 0.10 & $\ddot{A}$ \\
\hline TY & 1 & 8.600 & 0.700 & 0.74 & 0.11 & A \\
\hline UP & 1 & 6.720 & 1.990 & 0.58 & 0.19 & $\mathbf{W}$ \\
\hline UY & 1 & 11.300 & 1.900 & 0.97 & 0.20 & A \\
\hline WA & 1 & 9.300 & 1.600 & 0.80 & 0.17 & A \\
\hline WC & 1 & 9.820 & 2.150 & 0.85 & 0.21 & A \\
\hline WE & 1 & 12.500 & 5.970 & 1.08 & 0.53 & A \\
\hline WN & 1 & 10.600 & 0.800 & 0.91 & 0.13 & A \\
\hline WP & 1 & 10.800 & 1.900 & 0.93 & 0.20 & A \\
\hline
\end{tabular}

Total Number Reported: 76

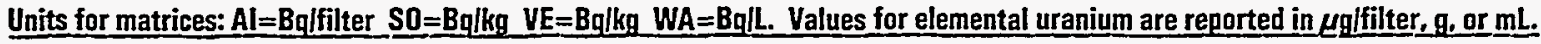
Evaluation: $A=A c c e p t a b l e, W=A c c e p t a b l e$ with Warning, $N=$ Not Acceptable. $\mathrm{pCi}=\mathrm{Bq} \times 27$ 
QAP44 Results by Nuclide

Matrix: Al

Radionuclide: SB125

EML Value: 9.780

EML Error: 1.030

\begin{tabular}{ccccc} 
Lahcode & $\begin{array}{c}\text { Reported } \\
\text { Value }\end{array}$ & $\begin{array}{c}\text { Reported } \\
\text { Frror }\end{array}$ & $\frac{\text { Reported }}{\text { EML }}$ & $\begin{array}{l}\text { Ratio } \\
\text { Frrar }\end{array}$ \\
\hline
\end{tabular}

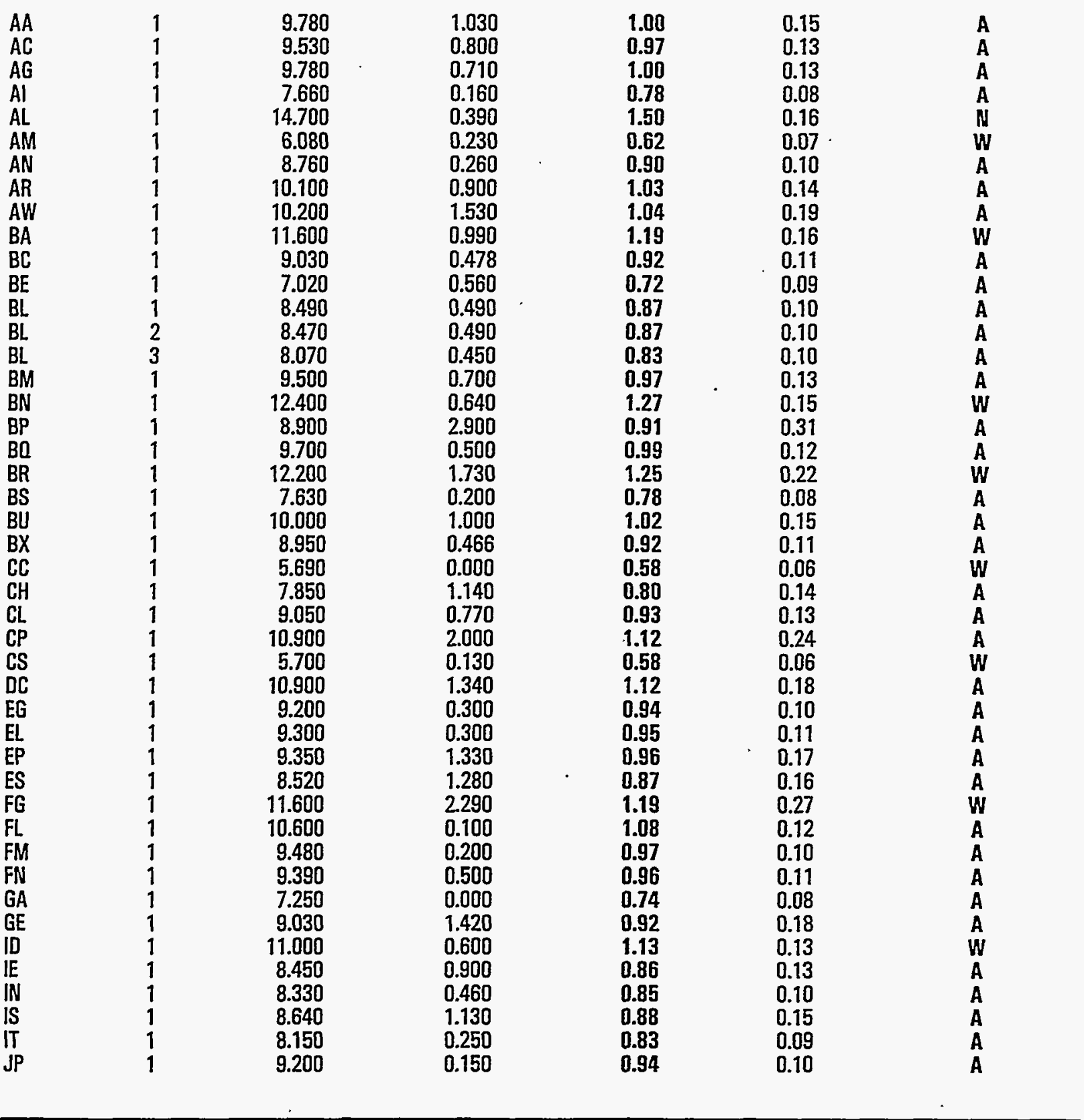

Units for matrices: $A \mathrm{~A}=\mathrm{Bq}$ lfilter $\mathrm{SQ}=\mathrm{Bq} / \mathrm{kg} \mathrm{VE}=\mathrm{Bq} / \mathrm{kg} W \mathrm{WA}=\mathrm{Bq} / \mathrm{L}$. Values for elemental uranium are reported in $\mu \mathrm{g} / \mathrm{filter}, \mathrm{g}$, or $\mathrm{mL}$. Evaluation: $A=A c c e p t a b l e, W=A c c e p t a b l e$ with Warning, $N=$ Not Acceptable. $p C i=B q \times 27$ 


\section{QAP44 Results by Nuclide}

Matrix: Al

Radionuclide: SB125

EML Value: 9.780

EML Error: 1.030

\begin{tabular}{|c|c|c|c|c|c|c|}
\hline Lahrade & Test\# & $\begin{array}{c}\text { Reported } \\
\text { Value }\end{array}$ & $\begin{array}{c}\text { Reported } \\
\text { Error }\end{array}$ & $\frac{\text { Reported }}{\text { EMI }}$ & $\begin{array}{l}\text { Ratio } \\
\text { Frror }\end{array}$ & Evaluation \\
\hline LA & 1 & 9.200 & 0.800 & 0.94 & 0.13 & $A$ \\
\hline LA & 2 & 9.300 & 0.800 & 0.95 & 0.13 & A \\
\hline LA & 3 & 9.000 & 0.700 & 0.92 & 0.12 & A \\
\hline LB & 1 & 8.230 & 0.913 & 0.84 & 0.13 & $\ddot{A}$ \\
\hline LH & 1 & 7.700 & 0.500 & 0.79 & 0.10 & $\ddot{A}$ \\
\hline LL & 1 & 9.810 & 4.200 & 1.00 & 0.44 & $\ddot{A}$ \\
\hline LM & 1 & 13.700 & 1.200 & 1.40 & 0.19 & $\dddot{W}$ \\
\hline LV & 1 & 7.650 & 0.220 & 0.78 & 0.09 & A \\
\hline ML & 1 & 9.300 & 0.400 & 0.95 & 0.11 & A \\
\hline MS & 1 & 10.000 & 1.000 & 1.02 & 0.15 & A \\
\hline NA & $i$ & 10.500 & 0.150 & 1.07 & 0.11 & $\ddot{A}$ \\
\hline $\mathrm{NC}$ & $i$ & 10.000 & 0.512 & 1.02 & 0.12 & A \\
\hline NL & 1 & 10.600 & 0.900 & 1.08 & 0.15 & $\ddot{A}$ \\
\hline OD & 1 & 9.560 & 0.377 & 0.98 & 0.11 & A \\
\hline $\mathrm{OL}$ & 1 & 8.600 & 0.540 & 0.88 & 0.11 & A \\
\hline$O R$ & $i$ & 10.000 & 1.000 & 1.02 & 0.15 & $\hat{A}$ \\
\hline OT & $i$ & 9.200 & 0.400 & 0.94 & 0.11 & $A$ \\
\hline RA & 1 & 11.000 & 0.400 & 1.13 & 0.13 & W \\
\hline $\mathrm{RE}$ & 1 & 7.860 & 1.340 & 0.80 & 0.16 & $A$ \\
\hline RI & $i$ & 8.270 & 1.100 & 0.85 & 0.14 & $\ddot{A}$ \\
\hline$S A$ & 1 & 12.500 & 1.700 & 1.28 & 0.22 & $W$ \\
\hline SE & 1 & 10.100 & 0.420 & 1.03 & 0.12 & $A$ \\
\hline SK & 1 & 8.100 & 0.300 & 0.83 & 0.09 & A \\
\hline SR & 1 & 9.700 & 0.500 & 0.99 & 0.12 & A \\
\hline SS & 1 & 7.900 & 0.700 & 0.81 & 0.11 & $\hat{A}$ \\
\hline SW & 1 & 8.510 & 0.560 & 0.87 & 0.11 & $A$ \\
\hline $\mathrm{TE}$ & 1 & 10.100 & 0.750 & 1.03 & 0.13 & A \\
\hline TI & 1 & 9.100 & 0.900 & 0.93 & 0.13 & A \\
\hline$\ddot{T M}$ & $i$ & 9.660 & 0.475 & 0.99 & 0.12 & $\ddot{A}$ \\
\hline TN & 1 & 8.230 & 1.150 & 0.84 & 0.15 & $\ddot{A}$ \\
\hline TO & $i$ & 9.650 & 1.010 & 0.99 & 0.15 & $\ddot{A}$ \\
\hline TW & $i$ & 9.300 & 0.200 & 0.95 & 0.10 & A \\
\hline TX & $i$ & 7.460 & 0.190 & 0.76 & 0.08 & A \\
\hline TY & $i$ & 8.200 & 0.500 & 0.84 & 0.10 & $A$ \\
\hline UP & $i$ & 4.740 & 0.757 & 0.49 & 0.09 & $W$ \\
\hline UY & 1 & 9.530 & 0.560 & 0.97 & 0.12 & $A$ \\
\hline WA & $i$ & 10.100 & 1.000 & 1.03 & 0.15 & $\ddot{A}$ \\
\hline WC & $i$ & 8.260 & 0.687 & 0.85 & 0.11 & $\ddot{A}$ \\
\hline WE & 1 & 7.050 & 1.310 & 0.72 & 0.15 & A \\
\hline$W I$ & 1 & 9.860 & 0.590 & 1.81 & 0.12 & A \\
\hline WN & 1 & 9.200 & 0.200 & 0.94 & 0.10 & A \\
\hline WP & 1 & 9.560 & 0.630 & 0.98 & 0.12 & A \\
\hline
\end{tabular}

Total Number Reported: 87

Units for matrices: $\mathrm{Al}=\mathrm{Bq}$ lfilter $\mathrm{SO}=\mathrm{Bq} / \mathrm{kg}$ VE=Bq/kq$W A=B q L$. Values for elemental uranium are reported in $\mu \mathrm{glfilter}, \mathrm{g}$, or $\mathrm{mL}$. Evaluation: $A=$ Acceptable, $W=$ Acceptable with Warning, $N=$ Not Acceptable. $p C \mathrm{i}=\mathrm{Bq} \times 27$ 
Matrix: Al

Radionuclide: SR 90

EML Value: 1.860

EML Error: 0.037

\begin{tabular}{lcccc}
\hline Lahonde & Reported & Reported & Reported & Ratio \\
Value & Error & EML & Error
\end{tabular}

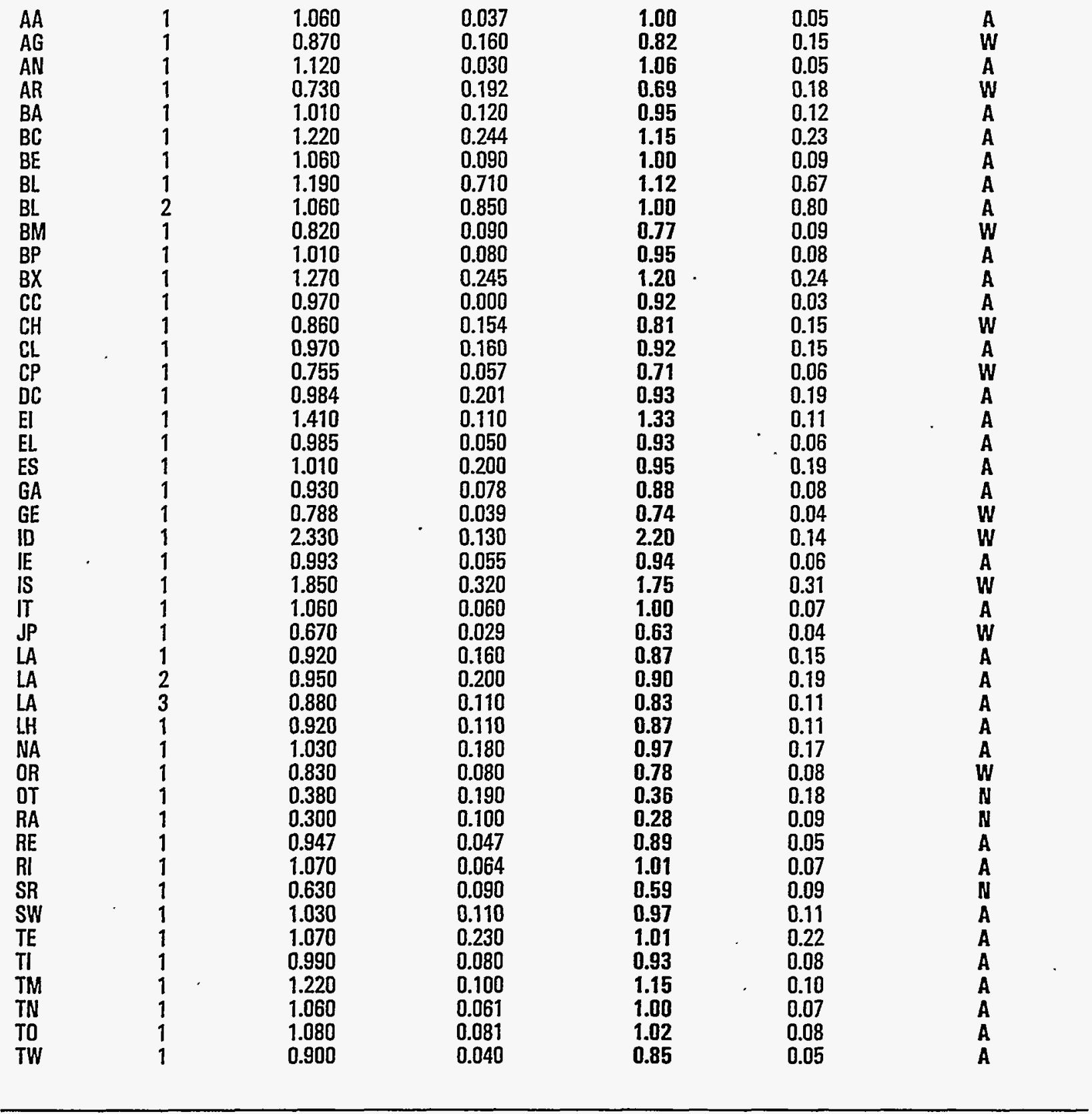

Units for matrices: $\mathrm{Al}=\mathrm{Bq}$ lfilter $\mathrm{SO}=\mathrm{Bq} / \mathrm{kg}$ VE=Bqlkg $W \mathrm{~A}=\mathrm{Bq} / \mathrm{L}$. Values for elemental uranium are reported in $\mu \mathrm{g}$ /filter, $\mathrm{g}$, or $\mathrm{mL}$.

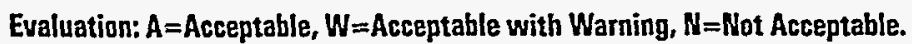
$p C i=B q \times 27$ 


\section{QAP44 Results by Nuclide}

Matrix: Al

Radionuclide: SR 90

EML Value: 1.060

EML Error: 0.037

\begin{tabular}{|c|c|c|c|c|c|c|}
\hline Lahconde & Test \# & $\begin{array}{c}\text { Reported } \\
\text { Value }\end{array}$ & $\begin{array}{c}\text { Reported } \\
\text { Error }\end{array}$ & $\frac{\text { Reported }}{\text { FML }}$ & $\begin{array}{l}\text { Ratio } \\
\text { Errar }\end{array}$ & Evaluation \\
\hline $\begin{array}{l}\text { UP } \\
\text { UY } \\
\text { WA } \\
\text { WC } \\
\text { WE } \\
\text { WP }\end{array}$ & $\begin{array}{l}1 \\
1 \\
1 \\
1 \\
1 \\
1\end{array}$ & $\begin{array}{l}0.881 \\
1.120 \\
1.180 \\
0.768 \\
0.002 \\
1.100\end{array}$ & $\begin{array}{l}0.252 \\
0.200 \\
0.150 \\
0.174 \\
0.001 \\
0.110\end{array}$ & $\begin{array}{l}0.83 \\
1.06 \\
1.11 \\
0.73 \\
0.00 \\
1.04\end{array}$ & $\begin{array}{l}0.24 \\
0.19 \\
0.15 \\
0.17 \\
0.00 \\
0.11\end{array}$ & $\begin{array}{l}A \\
A \\
A \\
W \\
N \\
A\end{array}$ \\
\hline
\end{tabular}

Total Number Reported: 51

Units for matrices: $A l=B q$ lfilter $S D=B q / k g$ VE $=B q / k g ~ W A=B q l L$. Values for elemental uranium are reported in $\mu g$ lfilter, $g$, or $\mathrm{mL}$.

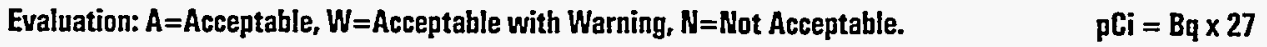


QAP44 Results by Nuclide

Matrix: Al

Radionuclide: U 234

EML Value: 0.052

EML Error: 0.002

\begin{tabular}{|c|c|c|c|c|c|c|}
\hline Laheorie & Test \# & $\begin{array}{c}\text { Reported } \\
\text { Value }\end{array}$ & $\begin{array}{c}\text { Reported } \\
\text { Errar }\end{array}$ & $\frac{\text { Reported }}{\text { FML }}$ & $\begin{array}{l}\text { Ratio } \\
\text { Errar }\end{array}$ & Evaluatian \\
\hline
\end{tabular}

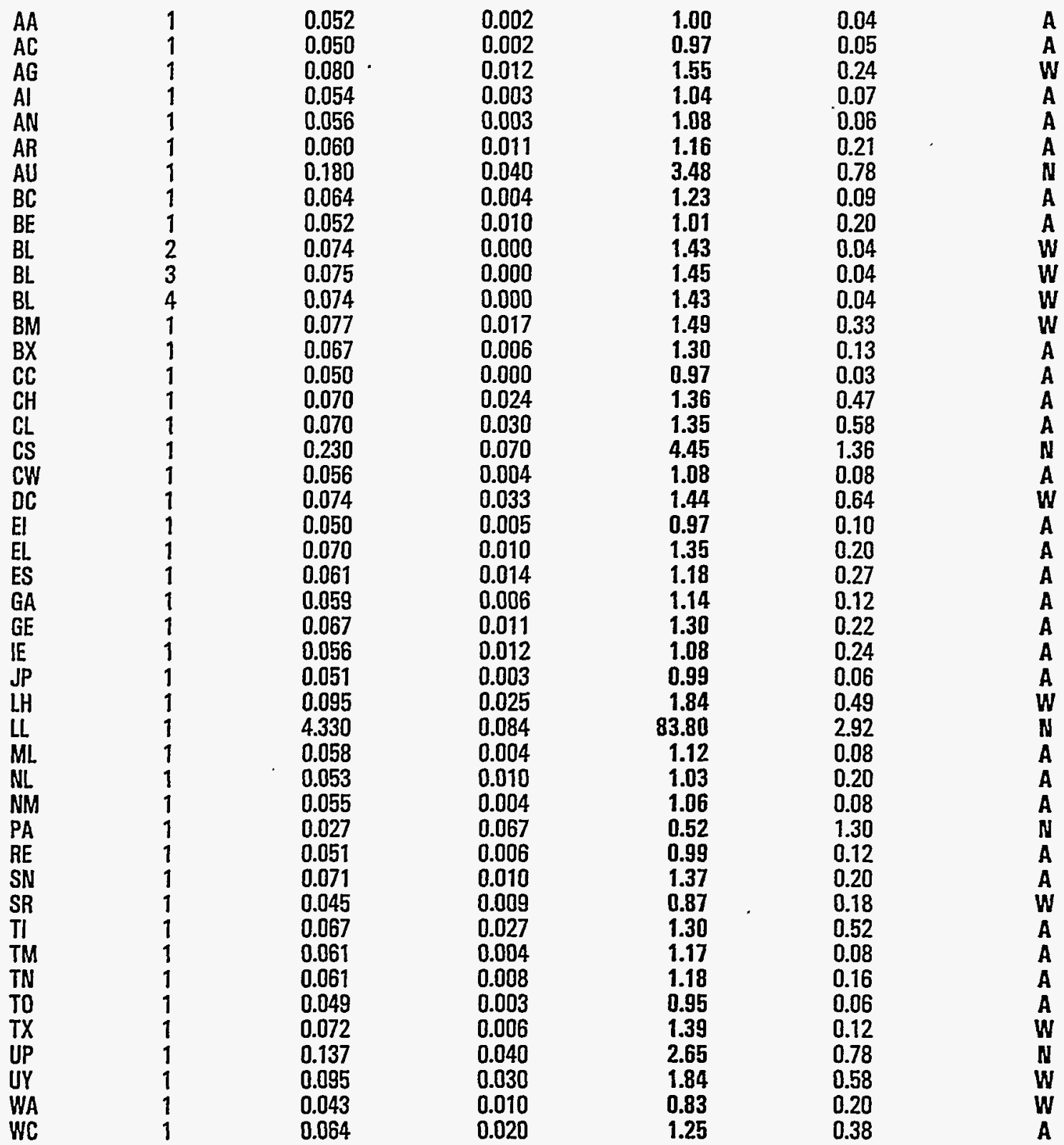

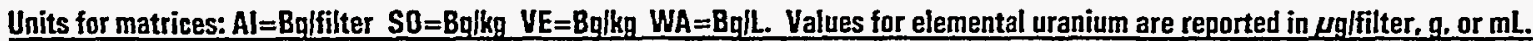
Evaluation: $A=$ Acceptable, $W=$ Acceptable with Warning, $N=$ Not Acceptable. $\mathrm{pCi}=\mathrm{Bq} \times 27$ 


\section{QAP44 Results by Nuclide}

Matrix: AI

Radionuclide: U 234

EML Value: 0.052

EML Error: 0.002

\begin{tabular}{|c|c|c|c|c|c|c|}
\hline Lahonde & Test\# & $\begin{array}{c}\text { Reported } \\
\text { Value }\end{array}$ & $\begin{array}{c}\text { Reported } \\
\text { Errar }\end{array}$ & $\frac{\text { Reported }}{\text { FML }}$ & $\begin{array}{l}\text { Ratio } \\
\text { Errar }\end{array}$ & Evaluatinn \\
\hline WE & 1 & 0.047 & 0.010 & 0.91 & 0.19 & A \\
\hline
\end{tabular}

Total Number Reported: 46

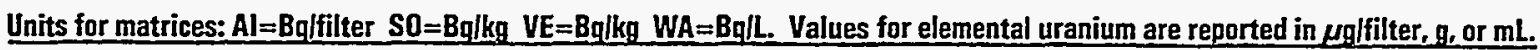

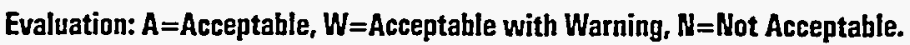
$\mathrm{pCi}=\mathrm{Bq} \times 27$ 
QAP44 Results by Nuclide

Matrix: Al

Radionuclide: U 238

EML Value: 0.053

EML Error: 0.002

\begin{tabular}{|c|c|c|c|c|c|c|}
\hline Lahonde & Test \# & $\begin{array}{c}\text { Reported } \\
\text { Value }\end{array}$ & $\begin{array}{c}\text { Reported } \\
\text { Error }\end{array}$ & $\frac{\text { Reported }}{\text { EMLL }}$ & $\begin{array}{l}\text { Ratio } \\
\text { Error }\end{array}$ & Evaluation \\
\hline
\end{tabular}

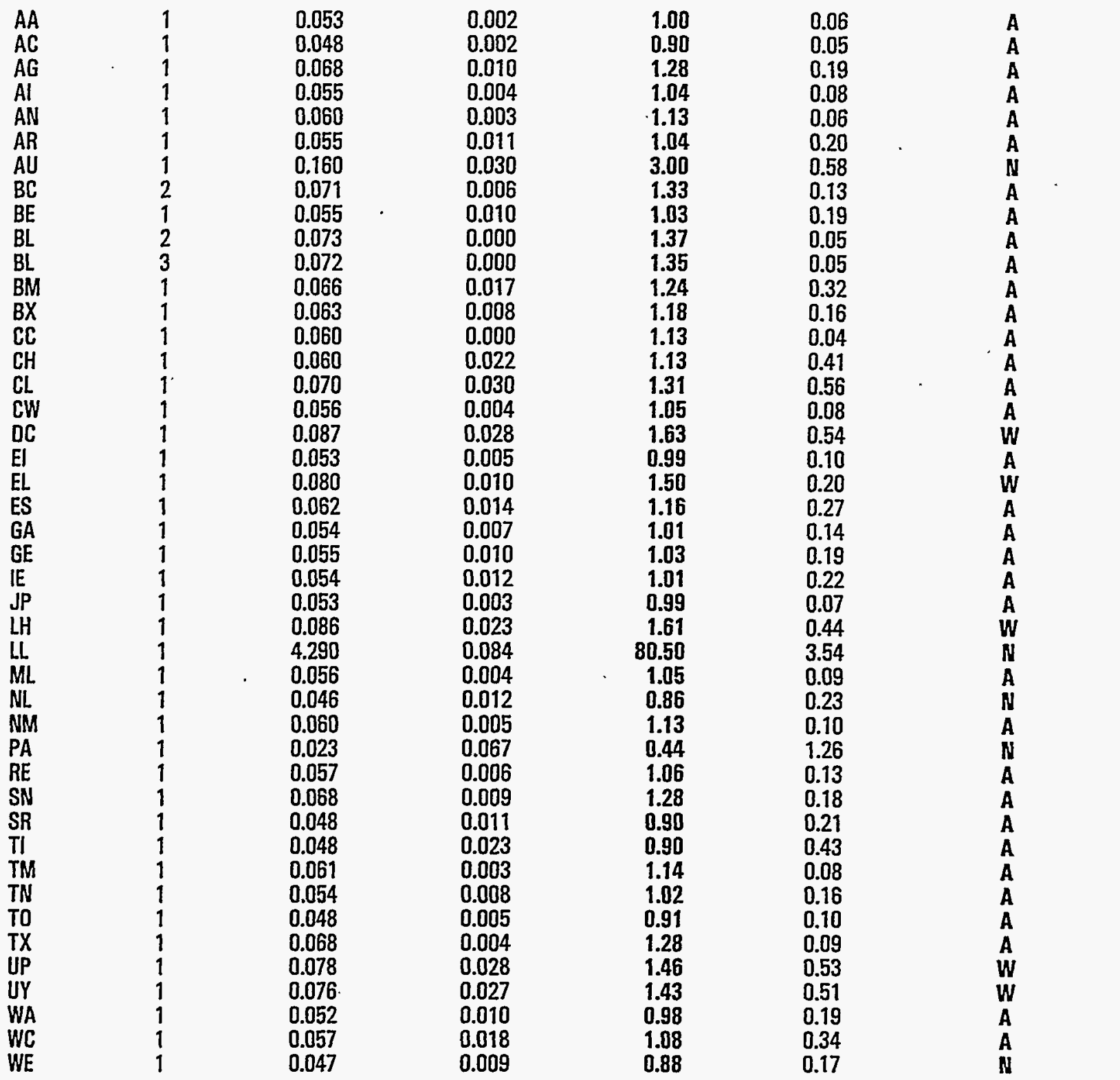

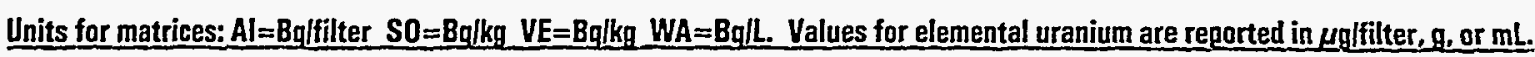
Evaluation: $A=A c c e p t a b l e, W=A c c e p t a b l e$ with Warning, $N=$ Not Acceptable.

$\mathrm{pCi}=\mathrm{Bq} \times 27$ 


\section{QAP44 Results by Nuclide}

Matrix: Al

Radionuclide: U 238

EML Value: 0.053

EML Error: 0.002

Reported

Lahronde Test \# Value

Reported

Error
Reported

EMIL
Ratio

Frrnr
Evaluation

Total Number Reported: 44

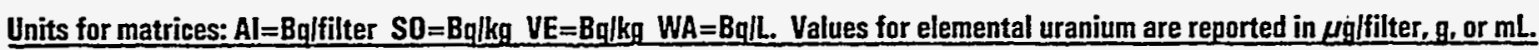
Evaluation: $A=A c c e p t a b l e, W=A c c e p t a b l e$ with Warning, $N=$ Not Acceptable. $\mathrm{pCi}=\mathrm{Bq} \times 27$ 


\section{QAP44 Results by Nuclide}

Matrix: Al

Radionuclide: U BO

EML Value: 0.107

EML Error: 0.003

\begin{tabular}{|c|c|c|c|c|c|c|}
\hline Lahande & Iest \#\# & $\begin{array}{c}\text { Reported } \\
\text { Value }\end{array}$ & $\begin{array}{c}\text { Reported } \\
\text { Error }\end{array}$ & $\frac{\text { Reported }}{\text { EML }}$ & $\begin{array}{l}\text { Ratio } \\
\text { Error }\end{array}$ & Eyaluation \\
\hline$A A$ & 1 & 0.107 & 0.003 & 1.00 & 0.03 & $A$ \\
\hline AR & $i$ & 0.115 & 0.015 & 1.08 & 0.14 & A \\
\hline BU & 1 & 5.800 & 0.400 & 54.20 & 3.96 & N \\
\hline $\mathrm{CH}$ & $i$ & 0.131 & 0.033 & 1.22 & 0.31 & $A$ \\
\hline CL & 1 & 0.120 & 0.020 & 1.12 & 0.19 & A \\
\hline EL & 1 & 0.148 & 0.050 & 1.38 & 0.47 & $\ddot{A}$ \\
\hline ES & 1 & 0.126 & 0.029 & 1.18 & 0.27 & A \\
\hline ID & 1 & 0.115 & 0.006 & 1.08 & 0.06 & A \\
\hline OR & $i$ & 0.133 & 0.020 & 1.24 & 0.19 & $\hat{A}$ \\
\hline OT & 1 & 0.680 & 0.070 & 6.36 & 0.67 & $\mathbb{N}$ \\
\hline $\mathrm{RE}$ & $i$ & 0.113 & 0.006 & 1.06 & 0.06 & A \\
\hline TE & i & 0.118 & 0.020 & 1.10 & 0.19 & $\hat{A}$ \\
\hline TW & $i$ & 0.430 & 0.020 & 4.02 & 0.21 & $\mathbf{N}$ \\
\hline UK & $i$ & 0.184 & 0.039 & 1.72 & 0.37 & $W$ \\
\hline UP & $i$ & 0.215 & 0.049 & 2.01 & 0.46 & $w$ \\
\hline WA & 1 & 0.106 & 0.015 & 0.99 & 0.14 & A \\
\hline
\end{tabular}

Total Number Reported: 16

Units for matrices: $\mathrm{Al}=\mathrm{Bq}$ qfilter $\mathrm{SO}=\mathrm{Bq} / \mathrm{kg}$ VE=Bglkg $W \mathrm{~A}=\mathrm{Bg} / \mathrm{L}$. Values for elemental uranium are reported in $\mu \mathrm{g} /$ filter,, $\mathrm{g}$, or $\mathrm{mL}$

$\mathrm{pCi}=\mathrm{Bq} \times 27$ 


\section{OAP44 Results by Nuclide}

Matrix: Al

Radionuclide: U UG

EML Value: 4.310

EML Error: 0.100

\begin{tabular}{|c|c|c|c|c|c|c|}
\hline Lahmofle & Test \# & $\begin{array}{c}\text { Reported } \\
\text { Value }\end{array}$ & $\begin{array}{c}\text { Reported } \\
\text { Frrnr }\end{array}$ & $\frac{\text { Reported }}{\text { EMI }}$ & $\begin{array}{l}\text { Ratio } \\
\text { Error }\end{array}$ & Evalua \\
\hline$A A$ & 1 & 4.310 & 0.100 & 1.00 & 0.03 & A \\
\hline$A R$ & 1 & 4.400 & 0.060 & 1.02 & 0.03 & $\ddot{A}$ \\
\hline $\mathrm{BE}$ & 1 & 4.500 & 0.000 & 1.04 & 0.02 & A \\
\hline BL & 1 & 6.080 & 0.110 & 1.41 & 0.04 & $W$ \\
\hline$B L$ & 2 & 6.110 & 0.120 & 1.42 & 0.04 & $W$ \\
\hline$B L$ & 3 & 6.080 & 0.110 & 1.41 & 0.04 & $W$ \\
\hline $\mathrm{BO}$ & 1 & 4.300 & 0.200 & 1.00 & 0.05 & $\begin{array}{l}A^{\prime} \\
\end{array}$ \\
\hline CC & 1 & 4.560 & 0.000 & 1.06 & 0.02 & A \\
\hline $\mathrm{CH}$ & 1 & 4.150 & 0.420 & 0.96 & 0.10 & A \\
\hline DC & 1 & 7.000 & 0.700 & 1.62 & 0.17 & $\ddot{W}$ \\
\hline ES & $i$ & 4.990 & 0.490 & 1.16 & 0.12 & A \\
\hline GA & 1 & 4.310 & 0.000 & 1.00 & 0.02 & A \\
\hline $\mathrm{GE}$ & $i$ & 4.360 & 0.060 & 1.01 & 0.03 & A \\
\hline IE & $i$ & 4.400 & 0.937 & 1.02 & 0.22 & A \\
\hline IR & 1 & 4.270 & 0.470 & 0.99 & 0.11 & $\ddot{A}$ \\
\hline IT & $i$ & 4.690 & 0.640 & 1.09 & 0.15 & $\ddot{A}$ \\
\hline LA & 1 & 4.100 & 0.400 & 0.95 & 0.10 & A \\
\hline LA & 2 & 4.100 & 0.400 & 0.95 & 0.10 & $\cdot$ \\
\hline LA & 3 & 4.100 & 0.400 & 0.95 & 0.10 & A \\
\hline NL & 1 & 4.300 & 0.500 & 1.00 & 0.12 & $\ddot{A}$ \\
\hline SW & 1 & 5.450 & 0.960 & 1.27 & 0.23 & $\ddot{W}$ \\
\hline TI & 1 & 8.800 & 1.300 & 2.04 & 0.31 & N \\
\hline $\mathrm{TM}$ & 1 & 4.920 & 0.059 & 1.14 & 0.03 & A \\
\hline TN & 1 & 4.560 & 1.190 & 1.06 & 0.28 & A \\
\hline TO & $i$ & 3.980 & 0.251 & 0.92 & 0.06 & A \\
\hline$T W$ & 1 & 17.400 & 0.900 & 4.04 & 0.23 & $\boldsymbol{N}$ \\
\hline$Y P$ & 1 & 3.850 & 0.650 & 0.89 & 0.15 & $\mathbf{A}$ \\
\hline
\end{tabular}

Total Number Reported: 27

Units for matrices: $\mathrm{Al}=\mathrm{Bq}$ lfilter $\mathrm{SO}=\mathrm{Bq} / \mathrm{kg}$ VE=Bqlkg $\mathrm{WA}=\mathrm{Bq} / \mathrm{L}$. Values for elemental uranium are reported in eglfilter, $\mathrm{g}$, or $\mathrm{mL}$. 
QAP44 Results by Nuclide

Matrix: So

Radionuclide: AM241

EML. Value: 3.690

EML. Error: 0.454

\begin{tabular}{cccccc} 
Lahande & Rest & $\begin{array}{c}\text { Reported } \\
\text { Value }\end{array}$ & $\begin{array}{c}\text { Reported } \\
\text { Frror }\end{array}$ & $\frac{\text { Reported }}{\text { FML }}$ & $\begin{array}{l}\text { Ratio } \\
\text { Frrar }\end{array}$ \\
\hline
\end{tabular}

\begin{tabular}{|c|c|c|c|c|c|}
\hline $\begin{array}{l}A A \\
A C \\
A E \\
A G \\
A M \\
A N \\
A R \\
A U \\
B E \\
B L \\
B P \\
B R \\
B S \\
B U \\
B X \\
C C \\
C H \\
C L \\
C W \\
D C \\
D C \\
D C \\
E G \\
E L \\
E S \\
F G \\
F L \\
F S \\
G A \\
G E \\
H L \\
I D \\
I E \\
I N \\
I S \\
I T \\
J P \\
L A \\
L A \\
L A \\
L B \\
L H \\
L V \\
L W \\
M A\end{array}$ & $\begin{array}{l}1 \\
1 \\
1 \\
1 \\
1 \\
1 \\
1 \\
1 \\
1 \\
1 \\
1 \\
1 \\
1 \\
1 \\
1 \\
1 \\
1 \\
1 \\
1 \\
1 \\
2 \\
3 \\
1 \\
1 \\
1 \\
1 \\
1 \\
1 \\
1 \\
1 \\
1 \\
1 \\
1 \\
1 \\
1 \\
1 \\
1 \\
1 \\
2 \\
3 \\
1 \\
1 \\
1 \\
1 \\
1\end{array}$ & $\begin{array}{l}3.690 \\
4.550 \\
2.660 \\
3.330 \\
3.290 \\
3.460 \\
2.320 \\
2.960 \\
3.530 \\
2.630 \\
3.440 \\
6.000 \\
4.600 \\
2.900 \\
2.020 \\
2.510 \\
4.670 \\
5.640 \\
3.520 \\
5.590 \\
5.590 \\
5.590 \\
4.000 \\
4.330 \\
3.310 \\
2.500 \\
2.800 \\
3.770 \\
3.300 \\
4.370 \\
5.500 \\
4.330 \\
2.860 \\
2.400 \\
2.860 \\
3.430 \\
3.700 \\
3.940 \\
3.490 \\
3.810 \\
1.940 \\
3.400 \\
5.960 \\
3.800 \\
5.200\end{array}$ & $\begin{array}{c}0.454 \\
0.440 \\
0.510 \\
0.440 \\
1.660 \\
0.120 \\
0.310 \\
1.480 \\
0.320 \\
0.180 \\
0.320 \\
3.800 \\
0.100 \\
0.700 \\
0.051 \\
0.000 \\
0.852 \\
3.700 \\
0.170 \\
0.180 \\
0.180 \\
0.180 \\
0.600 \\
0.300 \\
0.600 \\
0.320 \\
0.500 \\
0.260 \\
0.000 \\
0.850 \\
1.900 \\
0.620 \\
0.199 \\
0.290 \\
0.890 \\
0.830 \\
0.230 \\
0.400 \\
0.400 \\
0.400 \\
0.868 \\
0.500 \\
0.670 \\
53.800 \\
2.200\end{array}$ & $\begin{array}{l}1.00 \\
1.23 \\
0.72 \\
0.90 \\
0.89 \\
0.94 \\
0.63 \\
0.80 \\
0.96 \\
0.71 \\
0.93 \\
1.63 \\
1.25 \\
0.79 \\
0.55 \\
0.68 \\
1.27 \\
1.53 \\
0.95 \\
1.52 \\
1.52 \\
1.52 \\
1.08 \\
1.17 \\
0.90 \\
0.68 \\
0.76 \\
1.02 \\
0.89 \\
1.18 \\
1.49 \\
1.17 \\
0.78 \\
0.65 \\
0.78 \\
0.93 \\
1.00 \\
1.07 \\
0.95 \\
1.03 \\
0.53 \\
0.92 \\
1.62 \\
1.03 \\
1.41\end{array}$ & $\begin{array}{r}0.17 \\
0.19 \\
0.16 \\
0.16 \\
0.46 \\
0.12 \\
0.11 \\
0.41 \\
0.15 \\
0.10 \\
0.14 \\
1.05 \\
0.16 \\
0.21 \\
0.07 \\
0.08 \\
0.28 \\
1.02 \\
0.13 \\
0.19 \\
0.19 \\
0.19 \\
0.21 \\
0.17 \\
0.20 \\
0.12 \\
0.17 \\
0.14 \\
0.11 \\
0.27 \\
0.55 \\
0.22 \\
0.11 \\
0.11 \\
0.26 \\
0.25 \\
0.14 \\
0.17 \\
0.16 \\
0.17 \\
0.24 \\
0.18 \\
0.27 \\
14.60 \\
0.62\end{array}$ \\
\hline
\end{tabular}

Units for matrices: $\mathrm{Al}=\mathrm{Bq} /$ filter $\mathrm{SO}=\mathrm{Bq} / \mathrm{kg}$ VE=Bq/kg $W A=B q \mathrm{~L}$. Values for elemental uranium are reported in $\mu \mathrm{glfiiter}, \mathrm{g}$, or $\mathrm{mL}$. Evaluation: $A=A c c e p t a b l e, W=A c c e p t a b l e$ with Warning, $N=$ Not Acceptable. $\mathrm{pCi}=\mathrm{Bq} \times 27$ 


\section{QAP44 Results by Nuclide}

Matrix: SO

Radionuclide: AM241

EML Value: 3.690

EML Error: 0.454

\begin{tabular}{|c|c|c|c|c|c|c|}
\hline Lahronde & Iest \# & $\begin{array}{c}\text { Reported } \\
\text { Value }\end{array}$ & $\begin{array}{c}\text { Reported } \\
\text { Frrar }\end{array}$ & $\frac{\text { Reported }}{\text { FMI }}$ & $\begin{array}{l}\text { Ratio } \\
\text { Errar }\end{array}$ & Evaluation \\
\hline NM & 1 & 4.900 & 0.800 & 1.33 & 0.27 & A \\
\hline OR & 1 & 1.800 & 0.900 & 0.49 & 0.25 & $\mathrm{~N}$ \\
\hline $\mathrm{RF}$ & $i$ & 4.240 & 0.460 & 1.15 & 0.19 & $\mathbf{A}$ \\
\hline RG & 1 & 3.890 & 0.420 & 1.05 & 0.17 & A \\
\hline SE & 1 & 7.830 & 1.930 & 2.12 & 0.59 & $w$ \\
\hline SW & 1 & 5.700 & 1.850 & 1.55 & 0.54 & $w$ \\
\hline TE & $i$ & 6.230 & 2.880 & 1.69 & 0.81 & $W$ \\
\hline $\mathrm{TI}$ & 1 & 5.000 & 2.100 & 1.36 & 0.59 & $A$ \\
\hline TM & 1 & 3.480 & 0.259 & 0.94 & 0.14 & A \\
\hline TN & 1 & 3.060 & 0.358 & 0.83 & 0.14 & A \\
\hline TO & $i$ & 3.230 & 2.210 & 0.88 & 0.61 & A \\
\hline TW & 1 & 3.300 & 0.300 & 0.89 & 0.14 & A \\
\hline$T X$ & 1 & 5.920 & 0.930 & 1.60 & 0.32 & $w$ \\
\hline UK & 1 & 10.100 & 0.810 & 2.74 & 0.40 & $\mathbf{N}$ \\
\hline WA & $i$ & 2.850 & 0.320 & 0.77 & 0.13 & $\bar{A}$ \\
\hline WS & 1 & 4.300 & 0.900 & 1.17 & 0.28 & $\hat{A}$ \\
\hline YA & 1 & 4.360 & 0.190 & 1.18 & 0.15 & $\hat{A}$ \\
\hline
\end{tabular}

Total Number Reported: 62

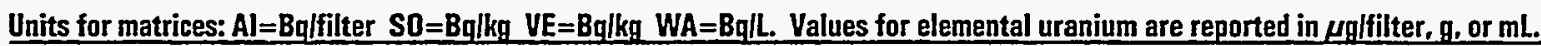
Evaluation: $A=A c c e p t a b l e, W=A c c e p t a b l e$ with Warning, $N=N$ ot Acceptable. 
QAP44 Results by Nuelide

Matrix: So

Radionuclide: CS137

EML Value: 359.000

EML Error: 10.000

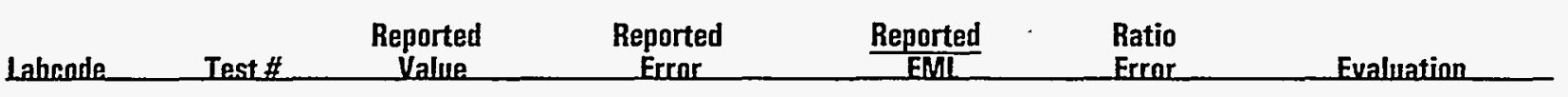

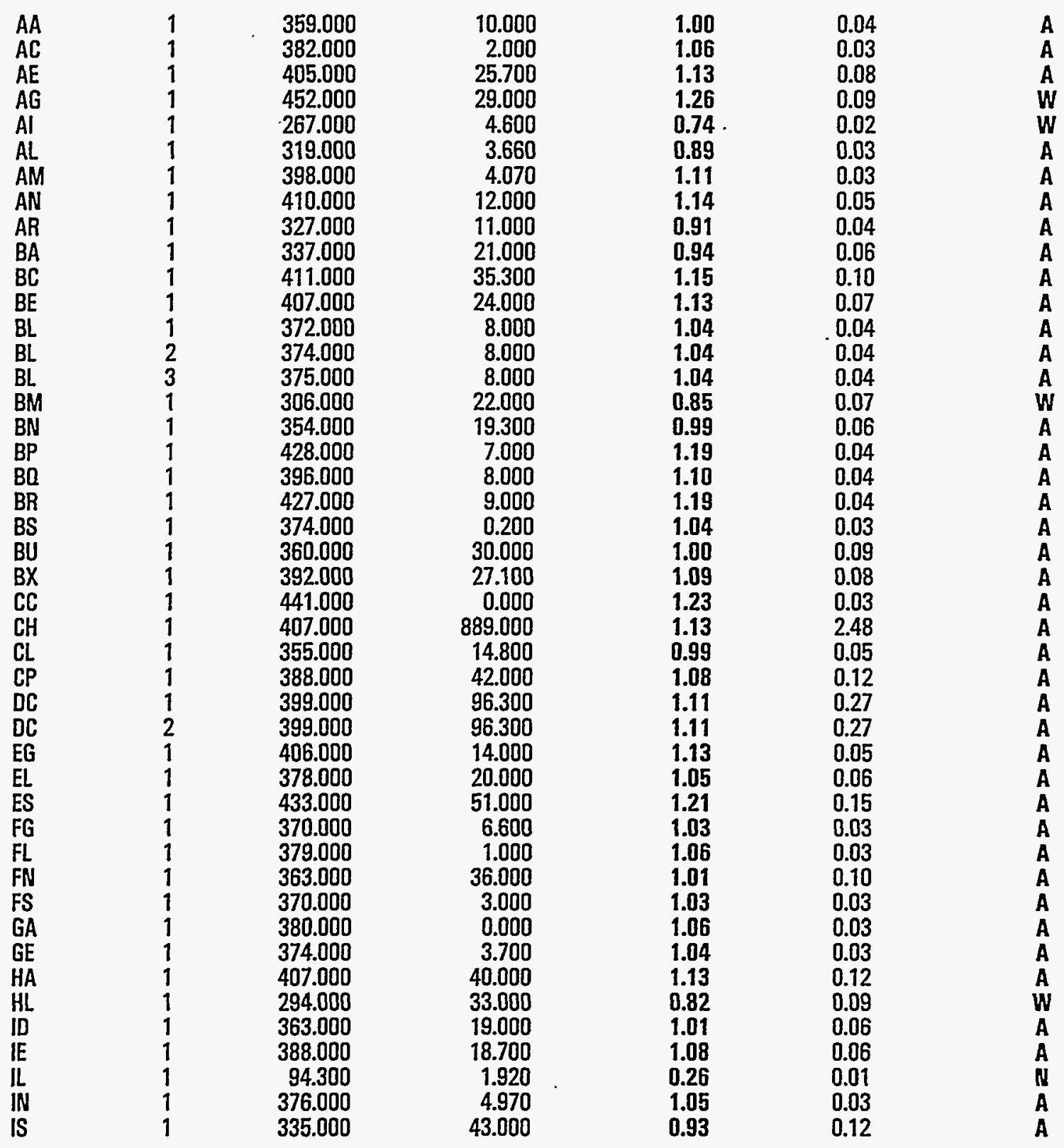

Units for matrices: $\mathrm{Al}=\mathrm{Bq}$ lfilter $S \mathrm{SD}=\mathrm{Bq} / \mathrm{kg}$ VE=Bqlkg $W \mathrm{WA}=\mathrm{Bq} / \mathrm{L}$. Values for elemental uranium are reported in $\mu$ glfilter, $\mathrm{g}$, or $\mathrm{mL}$. 
Matrix: So

Radionuclide: CS137

EML Value: 359.000

EML Error: 10.000

\begin{tabular}{|c|c|c|c|c|c|c|}
\hline Lahende & Test\# & $\begin{array}{c}\text { Reported } \\
\text { Value }\end{array}$ & $\begin{array}{c}\text { Reported } \\
\text { Errar }\end{array}$ & $\frac{\text { Reported }}{\text { FMl }}$ & $\begin{array}{l}\text { Ratio } \\
\text { Frror }\end{array}$ & Fvaluation \\
\hline IT & 1 & 432.000 & 12000 & 120 & 0.05 & A \\
\hline$J P$ & 1 & 390.000 & 3.000 & 1.09 & 0.03 & A \\
\hline KA & $i$ & 434.000 & 20.000 & 1.21 & 0.07 & $\ddot{A}$ \\
\hline LA & 1 & 316.000 & 24.000 & 0.88 & 0.07 & $\ddot{A}$ \\
\hline $\mathrm{LA}$ & 2 & 320.000 & 25.000 & 0.89 & 0.07 & A \\
\hline LA & 3 & 329.000 & 26.000 & 0.92 & 0.08 & $\ddot{A}$ \\
\hline LB & 1 & 332.000 & 13.700 & 0.93 & 0.05 & $\ddot{A}$ \\
\hline LH & 1 & 419.000 & .60 .000 & 1.17 & 0.17 & A \\
\hline LL & $i$ & 400.000 & 1.800 & 1.11 & 0.03 & $\hat{A}$ \\
\hline LM & 1 & 850.000 & 13.000 & 2.37 & 0.08 & N \\
\hline LV & 1 & 370.000 & 2.000 & 1.03 & 0.03 & A \\
\hline$L W$ & 1 & 395.000 & 1.800 & 1.10 & 0.03 & $\ddot{A}$ \\
\hline MA & 1 & 444.000 & 33.000 & 1.24 & 0.10 & W \\
\hline ME & 1 & 370.000 & 12.100 & 1.03 & 0.04 & $\ddot{A}$ \\
\hline$M L$ & 1 & 356.000 & 36.000 & 0.99 & 0.10 & A \\
\hline MS & 1 & 362.000 & 36.000 & 1.01 & 0.10 & A \\
\hline NA & $i$ & 382.000 & 1.940 & 1.06 & 0.03 & $\ddot{A}$ \\
\hline NC & $i$ & 366.000 & 2.230 & 1.02 & 0.03 & A \\
\hline NL & 1 & 399.000 & 8.000 & 1.11 & 0.04 & $\ddot{A}$ \\
\hline NR & 1 & 312.000 & 62.000 & 0.87 & 0.17 & w \\
\hline $\mathrm{OL}$ & 1 & 391.000 & 11.300 & 1.09 & 0.04 & $\ddot{A}$ \\
\hline $\mathrm{OR}$ & 1 & 380.000 & 10.000 & 1.06 & 0.04 & A \\
\hline OS & 1 & 384.000 & 4.300 & 1.07 & 0.03 & $\ddot{A}$ \\
\hline OT & 1 & 380.000 & 10.000 & 1.06 & 0.04 & A \\
\hline RA & 1 & 368.000 & 11.000 & 1.03 & 0.04 & $\ddot{A}$ \\
\hline $\mathrm{RE}$ & 1 & 309.000 & 26.600 & 0.86 & 0.08 & $\hat{w}$ \\
\hline Ri & 1 & 185.000 & 5.000 & 0.52 & 0.02 & N \\
\hline SA & 1 & 372.000 & 20.000 & 1.04 & 0.06 & A \\
\hline SC & 1 & 256.000 & 1.000 & 0.71 & 0.02 & N \\
\hline SE & 1 & 423.000 & 63.600 & 1.18 & 0.18 & $\ddot{A}$ \\
\hline SK & $i$ & 443.000 & 13.000 & 1.23 & 0.05 & A \\
\hline SN & 1 & 399.000 & 40.000 & 1.11 & 0.12 & $\ddot{A}$ \\
\hline SR & $i$ & 395.000 & 23.000 & 1.10 & 0.07 & A \\
\hline SS & 1 & 445.000 & 23.000 & 1.24 & 0.07 & $\mathbf{W}$ \\
\hline SW & $i$ & 483.000 & 14.300 & 1.35 & 0.05 & $w$ \\
\hline TE & $i$ & 405.000 & 4.150 & 1.13 & 0.03 & A \\
\hline $\mathrm{TI}$ & $i$ & 430.000 & 40.000 & 1.20 & 0.12 & A \\
\hline TM & 1 & 413.000 & 13.300 & 1.15 & 0.05 & $\ddot{A}$ \\
\hline TN & 1 & 314.000 & 44.000 & 0.88 & 0.13 & $\ddot{A}$ \\
\hline TO & 1 & 405.000 & 7.590 & 1.13 & 0.04 & $\ddot{A}$ \\
\hline TW & 1 & 360.000 & 11.000 & 1.00 & 0.04 & $\ddot{A}$ \\
\hline TX & 1 & 393.000 & 4.000 & 1.10 & 0.03 & A \\
\hline$T Y$ & 1 & 359.000 & 4.000 & 1.00 & 0.03 & $\ddot{A}$ \\
\hline UC & 1 & 404.000 & 17.900 & 1.13 & 0.06 & $\ddot{A}$ \\
\hline UK & 1 & 375.000 & 2.600 & 1.04 & 0.03 & $\ddot{A}$ \\
\hline UP & 1 & 325.000 & 34.900 & 0.91 & 0.10 & $\ddot{A}$ \\
\hline
\end{tabular}

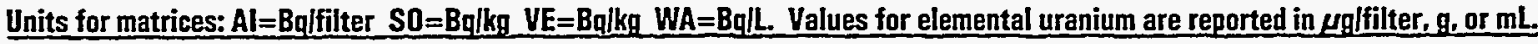




\section{QAP44 Results by Nuclide}

Matrix: SO

Radionuclide: CS137

EML Value: 359.000

EML Error: 10.000

\begin{tabular}{|c|c|c|c|c|c|c|}
\hline Lahoade & Iest\# \# & $\begin{array}{c}\text { Reported } \\
\text { Value }\end{array}$ & $\begin{array}{c}\text { Reported } \\
\text { Error }\end{array}$ & $\frac{\text { Reported }}{\text { EML }}$ & $\begin{array}{l}\text { Ratio } \\
\text { Error }\end{array}$ & Fvaluation \\
\hline $\begin{array}{l}\text { UY } \\
\text { WA } \\
\text { WC } \\
\text { WE } \\
\text { WN } \\
\text { WP } \\
\text { WS } \\
\text { YA }\end{array}$ & $\begin{array}{l}1 \\
1 \\
1 \\
1 \\
1 \\
1 \\
1 \\
1\end{array}$ & $\begin{array}{l}377.000 \\
401.000 \\
407.000 \\
372.000 \\
403.000 \\
427.000 \\
385.000 \\
373.000\end{array}$ & $\begin{array}{r}40.000 \\
31.000 \\
44.200 \\
9.500 \\
10.000 \\
7.900 \\
13.700 \\
2.700\end{array}$ & $\begin{array}{l}1.05 \\
1.12 \\
1.13 \\
1.04 \\
1.12 \\
1.19 \\
1.07 \\
1.04\end{array}$ & $\begin{array}{l}0.12 \\
0.09 \\
0.13 \\
0.04 \\
0.04 \\
0.04 \\
0.05 \\
0.03\end{array}$ & $\begin{array}{l}\text { A } \\
\text { A } \\
\text { A } \\
\text { A } \\
\text { A } \\
\text { A } \\
\text { A } \\
\text { A }\end{array}$ \\
\hline
\end{tabular}

Total Number Reported: 99

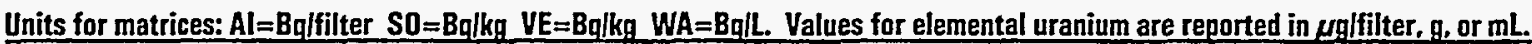


QAP44 Results by Nuclide

Matrix: SO

Radionuclide: K 40

EML Value: 465.000

EML Error: 30.000

\begin{tabular}{cccccc} 
Lahanife & Test\# & $\begin{array}{c}\text { Reported } \\
\text { Value }\end{array}$ & $\begin{array}{c}\text { Reported } \\
\text { Error }\end{array}$ & $\frac{\text { Reported }}{\text { EML }}$ & $\begin{array}{c}\text { Ratio } \\
\text { Frror }\end{array}$ Evaluation \\
\hline
\end{tabular}

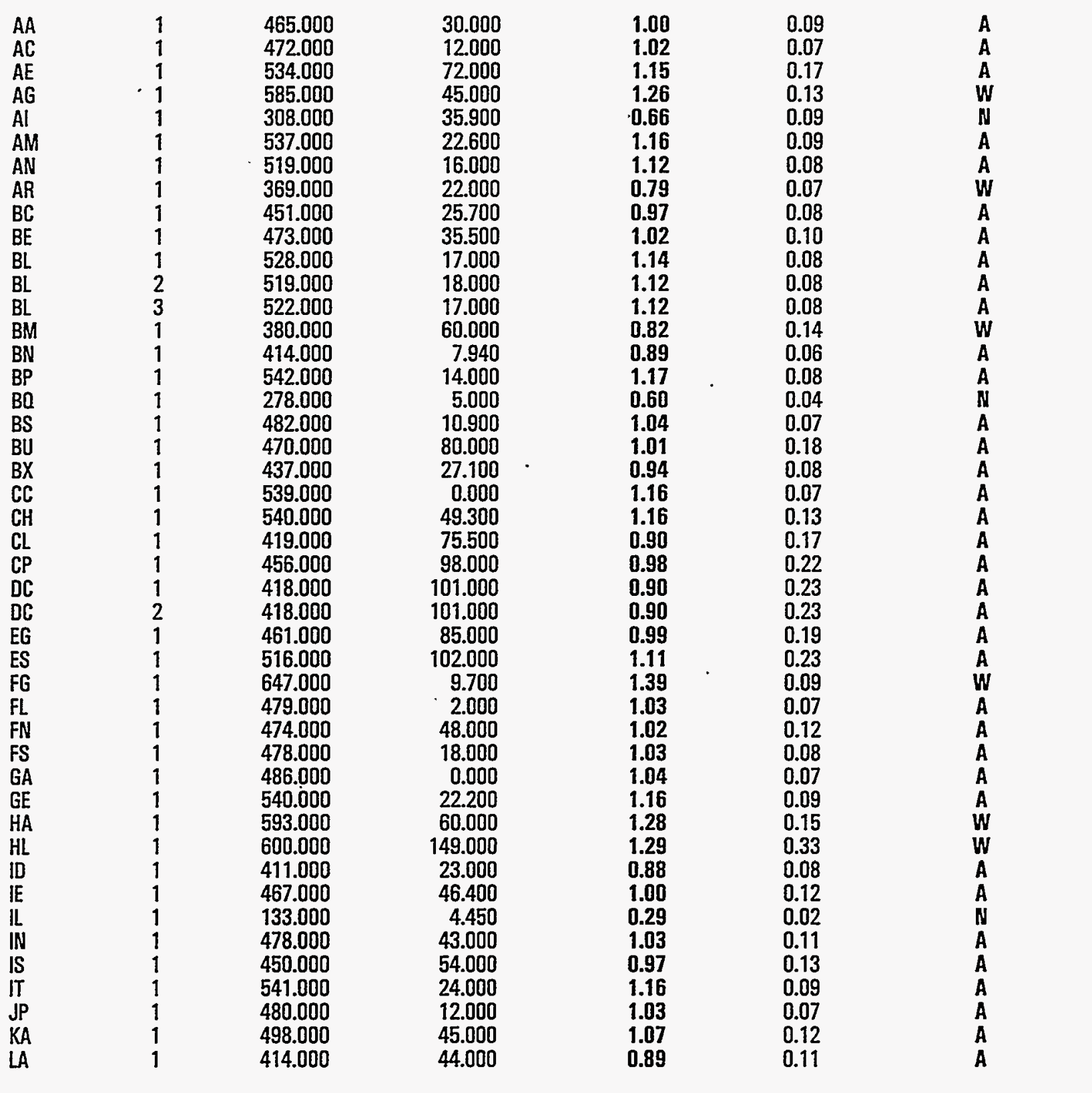

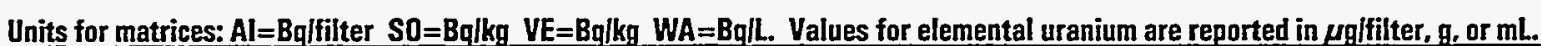
Evaluation: $A=$ Acceptable, $W=$ Acceptable with Warning, $N=$ Not Acceptable.

$\mathrm{pCi}=\mathrm{Bq} \times 27$ 
Matrix: SO

Radionuclide: $K \mathbf{4 0}$

EML Value: 465.000

EML Error: 30.000

\begin{tabular}{|c|c|c|c|c|c|c|}
\hline Laheade & Iest\# \# & $\begin{array}{c}\text { Reported } \\
\text { Value }\end{array}$ & $\begin{array}{c}\text { Reported } \\
\text { Error }\end{array}$ & $\frac{\text { Reported }}{\text { FMII }}$ & $\begin{array}{l}\text { Ratio } \\
\text { Errar }\end{array}$ & Evaluation \\
\hline 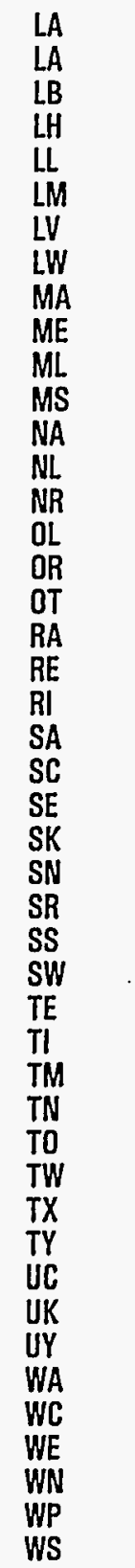 & $\begin{array}{l}2 \\
3 \\
1 \\
1 \\
1 \\
1 \\
1 \\
1 \\
1 \\
1 \\
1 \\
1 \\
1 \\
1 \\
1 \\
1 \\
1 \\
1 \\
1 \\
1 \\
1 \\
1 \\
1 \\
1 \\
1 \\
1 \\
1 \\
1 \\
1 \\
1 \\
1 \\
1 \\
1 \\
1 \\
1 \\
1 \\
1 \\
1 \\
1 \\
1 \\
1 \\
1 \\
1 \\
1 \\
1 \\
1\end{array}$ & $\begin{array}{r}414.000 \\
411.000 \\
496.000 \\
497.000 \\
496.000 \\
1030.000 \\
403.000 \\
468.000 \\
518.000 \\
622.000 \\
473.000 \\
458.000 \\
480.000 \\
584.000 \\
407.000 \\
524.000 \\
520.000 \\
480.000 \\
550.000 \\
392.000 \\
253.000 \\
472.000 \\
387.000 \\
693.000 \\
525.000 \\
528.000 \\
502.000 \\
554.000 \\
848.000 \\
525.000 \\
540.000 \\
439.000 \\
404.000 \\
411.000 \\
470.000 \\
505.000 \\
478.000 \\
552.000 \\
506.000 \\
544.000 \\
549.000 \\
518.000 \\
431.000 \\
500.000 \\
547.000 \\
492.000\end{array}$ & $\begin{array}{r}44.000 \\
44.000 \\
33.400 \\
82.000 \\
6.800 \\
65.000 \\
10.000 \\
7.400 \\
59.000 \\
21.400 \\
52.000 \\
46.000 \\
9.460 \\
80.000 \\
81.000 \\
36.500 \\
80.000 \\
60.000 \\
50.000 \\
50.900 \\
43.000 \\
58.000 \\
4.000 \\
25.300 \\
17.000 \\
50.900 \\
60.000 \\
46.000 \\
121.000 \\
23.300 \\
50.000 \\
47.000 \\
31.600 \\
32.900 \\
19.000 \\
11.000 \\
15.000 \\
24.900 \\
15.000 \\
136.000 \\
31.000 \\
73.600 \\
59.500 \\
40.000 \\
35.000 \\
18.100 \\
\end{array}$ & $\begin{array}{l}0.89 \\
0.88 \\
1.07 \\
1.07 \\
1.07 \\
2.21 \\
0.87 \\
1.01 \\
1.11 \\
1.34 \\
1.02 \\
0.99 \\
1.03 \\
1.26 \\
0.88 \\
1.13 \\
1.12 \\
1.03 \\
1.18 \\
0.84 \\
0.54 \\
1.02 \\
0.83 \\
1.49 \\
1.13 \\
1.14 \\
1.08 \\
1.19 \\
1.82 \\
1.13 \\
1.16 \\
0.94 \\
0.87 \\
0.88 \\
1.01 \\
1.09 \\
1.03 \\
1.19 \\
1.09 \\
1.17 \\
1.18 \\
1.11 \\
0.93 \\
1.08 \\
1.18 \\
1.06\end{array}$ & $\begin{array}{l}0.11 \\
0.11 \\
0.10 \\
0.19 \\
0.07 \\
0.20 \\
0.06 \\
0.07 \\
0.15 \\
0.10 \\
0.13 \\
0.12 \\
0.07 \\
0.19 \\
0.18 \\
0.11 \\
0.19 \\
0.15 \\
0.13 \\
0.12 \\
0.10 \\
0.14 \\
0.05 \\
0.11 \\
0.08 \\
0.13 \\
0.15 \\
0.13 \\
0.29 \\
0.09 \\
0.13 \\
0.12 \\
0.09 \\
0.09 \\
0.08 \\
0.07 \\
0.07 \\
0.09 \\
0.08 \\
0.30 \\
0.10 \\
0.17 \\
0.14 \\
0.11 \\
0.11 \\
0.08\end{array}$ & $\begin{array}{l}A \\
A \\
A \\
A \\
A \\
A \\
A \\
A \\
A \\
A \\
W \\
A \\
A \\
A \\
\mathbf{W} \\
A \\
A \\
A \\
A \\
A \\
A \\
A \\
N \\
A \\
W \\
W \\
A \\
A \\
A \\
A \\
A \\
N \\
A \\
A \\
A \\
A \\
A \\
A \\
A \\
A \\
A \\
A \\
A \\
A \\
A \\
A \\
A \\
A \\
A \\
A \\
A\end{array}$ \\
\hline
\end{tabular}

Units for matrices: $\mathrm{Al}=\mathrm{Bq} /$ filter $\mathrm{SO}=\mathrm{Bq} / \mathrm{kg}$ VE $=\mathrm{Bq} / \mathrm{kg}, W \mathrm{~A}=\mathrm{Bq} / \mathrm{L}$. Values for elemental uranium are reported in $\mu \mathrm{g} /$ filter, $\mathrm{g}$, or $\mathrm{mL}$. Evaluation: $A=A c c e p t a b l e, W=A c c e p t a b l e$ with Warning, $N=$ Not Acceptable. $\mathrm{pCi}=\mathrm{Bq} \times 27$ 
QAP44 Results by Nuclide

Matrix: SO

Radionuclide: K $\mathbf{4 0}$

EML Value: 465.000

EML Error: 30.000

$\begin{array}{ccccccc}\text { Lahconde } & \text { Test \# } & \begin{array}{c}\text { Reported } \\ \text { Value }\end{array} & \begin{array}{c}\text { Reported } \\ \text { Frror }\end{array} & \frac{\text { Reported }}{\text { FMI }} & \begin{array}{c}\text { Ratio } \\ \text { Frror }\end{array} & \text { Fualuation } \\ \text { YA } & 1 & 481.000 & 4.600 & 1.03 & 0.07 & \text { A }\end{array}$

Total Number Reported: 92

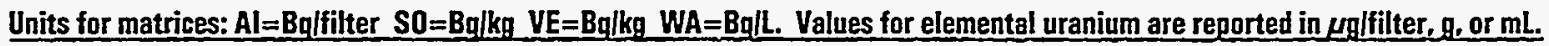
Evaluation: $A=A c c e p t a b l e, W=A c c e p t a b l e$ with Warning, $N=$ Not Acceptable.

$\mathrm{pCi}=\mathrm{Bq} \times 27$ 
QAP44 Results by Nuclide

Matrix: $\mathbf{S O}$

Radionuclide: PU238

EML Value: 43.000

EML Error: 2.440

$\begin{array}{ccccc}\text { Lahende } & \text { Reported } & \text { Reported } & \text { Reported } & \text { Ratio } \\ \text { Emal } & \text { Value } & \text { Error } & \text { Frror } & \text { Evaluation }\end{array}$

\begin{tabular}{|c|c|c|c|c|c|c|}
\hline$A A$ & 1 & 43.000 & 2.440 & 1.00 & 0.08 & A \\
\hline$A C$ & $i$ & 44.800 & 1.500 & 1.04 & 0.07 & $\ddot{A}$ \\
\hline$A E$ & $i$ & 42.800 & 1.620 & 1.00 & 0.07 & A \\
\hline $\mathbf{A G}$ & 1 & 41.000 & 4.800 & 0.95 & 0.12 & $\ddot{A}$ \\
\hline $\mathrm{Al}$ & 1 & 42.300 & 2.500 & 0.98 & 0.08 & A \\
\hline AN & 1 & 46.000 & 1.400 & 1.07 & 0.07 & A \\
\hline AR & 1 & 44.200 & 3.300 & 1.03 & 0.10 & A \\
\hline AU & 1 & 114.000 & 21.000 & 2.65 & 0.51 & $\mathbb{N}$ \\
\hline $\mathrm{BE}$ & 1 & 42.900 & 1.760 & 1.00 & 0.07 & $\mathbf{A}$ \\
\hline $\mathrm{BL}$ & 1 & 45.000 & 0.900 & 1.05 & 0.06 & A \\
\hline BL & 2 & 46.600 & 0.020 & 1.08 & 0.06 & A \\
\hline $\mathrm{BP}$ & 1 & 45.200 & 1.900 & 1.05 & 0.07 & A \\
\hline BU & 1 & 39.100 & 0.900 & 0.91 & 0.06 & A \\
\hline BX & 1 & 36.900 & 0.940 & 0.86 & 0.05 & A \\
\hline CC & 1 & 45.500 & 0.000 & 1.06 & 0.06 & A \\
\hline $\mathrm{CH}$ & 1 & 45.600 & 1.480 & 1.06 & 0.07 & A \\
\hline $\mathrm{Cl}$ & 1 & 38.100 & 6.300 & 0.89 & 0.16 & A \\
\hline CW & 1 & 41.200 & 2.500 & 0.96 & 0.08 & A \\
\hline$D C$ & 1 & 44.300 & 1.800 & 1.03 & 0.07 & A \\
\hline DC & 2 & 44.300 & 1.800 & 1.03 & 0.07 & A \\
\hline EG & 1 & 40.500 & 3.300 & 0.94 & 0.09 & A \\
\hline EP & 1 & 42.200 & 3.660 & 0.98 & 0.10 & A \\
\hline ES & 1 & 44.100 & 7.940 & 1.03 & 0.19 & $\ddot{A}$ \\
\hline ES & 2 & 44.100 & 7.840 & 1.03 & 0.19 & $\ddot{A}$ \\
\hline $\mathrm{FL}$ & 1 & 42.000 & 0.910 & 0.98 & 0.06 & $\ddot{A}$ \\
\hline FS & 1 & 40.700 & 1.400 & 0.95 & 0.06 & A \\
\hline GA & 1 & 44.000 & 0.000 & 1.02 & 0.06 & A \\
\hline GE & 1 & 50.300 & 0.890 & 1.17 & 0.07 & A \\
\hline $\mathrm{HA}$ & 1 & 36.600 & 4.000 & 0.85 & 0.11 & $\ddot{A}$ \\
\hline ID & 1 & 41.700 & 3.700 & 0.97 & 0.10 & $\ddot{A}$ \\
\hline IE & 1 & 40.000 & 0.620 & 0.93 & 0.05 & $\ddot{A}$ \\
\hline IN & 1 & 44.000 & 5.000 & 1.02 & 0.13 & A \\
\hline IS & 1 & 28.500 & 5.000 & 0.66 & 0.12 & $\ddot{A}$ \\
\hline IT & 1 & 30.900 & 3.950 & 0.72 & 0.10 & A \\
\hline JP & 1 & 45.000 & 1.500 & 1.05 & 0.07 & A \\
\hline$K A$ & 1 & 47.000 & 5.000 & 1.09 & 0.13 & A \\
\hline LA & 1 & 41.400 & 1.400 & 0.96 & 0.06 & A \\
\hline LA & 2 & 38.500 & 1.400 & 0.90 & 0.06 & $\ddot{A}$ \\
\hline LA & 3 & 42.700 & 1.400 & 0.99 & 0.07 & A \\
\hline LH & 1 & 42.900 & 3.300 & 1.00 & 0.10 & $\ddot{A}$ \\
\hline LL & 1 & 44.700 & 3.210 & 1.04 & 0.10 & $\ddot{A}$ \\
\hline$M L$ & 1 & 38.000 & 1.070 & 0.88 & 0.06 & A \\
\hline NA & 1 & 40.000 & 3.260 & 0.93 & 0.09 & A \\
\hline NL & 1 & 43.600 & 10.000 & 1.01 & 0.24 & A \\
\hline NM & 1 & 43.000 & 2.000 & 1.00 & 0.07 & A \\
\hline
\end{tabular}

Units for matrices: $\mathrm{Al}=\mathrm{Bq} /$ filter $\mathrm{SO}=\mathrm{Bq} / \mathrm{kg} \quad \mathrm{VE}=\mathrm{Bq} / \mathrm{kg} \mathrm{WA}=\mathrm{Bq} / \mathrm{L}$. Values for elemental uranium are reported in $\mu$ g/filter, $\mathrm{g}$, or $\mathrm{mL}$. 


\section{QAP44 Results by Nuclide}

Matrix: SO

Radionuclide: PU238

EML Value: 43.000

EML Error: 2.440

\begin{tabular}{|c|c|c|c|c|c|c|}
\hline Lahonde. & Test \# & $\begin{array}{c}\text { Reported } \\
\text { Value }\end{array}$ & $\begin{array}{c}\text { Reported } \\
\text { Error }\end{array}$ & $\frac{\text { Reported }}{\text { FML }}$ & $\begin{array}{l}\text { Ratio } \\
\text { Error }\end{array}$ & Evaluatinn \\
\hline $\mathrm{OR}$ & 1 & 44.000 & 1.000 & 1.02 & 0.06 & A \\
\hline $\mathrm{OT}$ & 1 & 50.000 & 6.000 & 1.16 & 0.15 & A \\
\hline $\mathrm{PI}$ & $i$ & 47.000 & 4.500 & 1.09 & 0.12 & $\hat{A}$ \\
\hline RA & 1 & 47.000 & 2.000 & 1.09 & 0.08 & A \\
\hline $\mathrm{RA}$ & 2 & 45.000 & 2.000 & 1.05 & 0.08 & $\hat{A}$ \\
\hline $\mathrm{RE}$ & 1 & 34.800 & 4.100 & 0.81 & 0.11 & $\ddot{A}$ \\
\hline $\mathrm{RG}$ & 1 & 42.300 & 1.700 & 0.98 & 0.07 & A \\
\hline SC & 1 & 50.000 & 1.000 & 1.16 & 0.07 & $\ddot{A}$ \\
\hline SE & 1 & 56.000 & 2.590 & 1.30 & 0.10 & N \\
\hline SN & 1 & 41.100 & 8.160 & 0.96 & 0.20 & A \\
\hline SR & $i$ & 42.600 & 7.200 & 0.99 & 0.18 & $\ddot{A}$ \\
\hline TE & 1 & 42.300 & 1.590 & 0.98 & 0.07 & A \\
\hline TI & $i$ & 44.000 & 6.000 & 1.02 & 0.15 & $A$ \\
\hline TM & 1 & 42.800 & 2.360 & 1.00 & 0.08 & $A$ \\
\hline TN & 1 & 43.200 & 2.500 & 1.01 & 0.08 & $\ddot{A}$ \\
\hline TO & 1 & 43.800 & 13.700 & 1.02 & 0.32 & $\ddot{A}$ \\
\hline TW & 1 & 39.000 & 2.000 & 0.91 & 0.07 & A \\
\hline$T X$ & $i$ & 42.900 & 2.600 & 1.00 & 0.08 & $\ddot{A}$ \\
\hline UK & 1 & 36.900 & 2.200 & 0.86 & 0.07 & $\ddot{A}$ \\
\hline UP & 1 & 41.400 & 6.020 & 0.96 & 0.15 & $\ddot{A}$ \\
\hline UY & 1 & 49.600 & 10.000 & 1.15 & 0.24 & $\ddot{A}$ \\
\hline WA & 1 & 43.300 & 1.200 & 1.01 & 0.06 & A \\
\hline WE & 1 & 17.200 & 0.926 & 0.40 & 0.03 & $W$ \\
\hline YA & $i$ & 43.000 & 0.450 & 1.00 & 0.06 & $A$ \\
\hline
\end{tabular}

Total Number Reported: 69

Units for matrices: $A l=B q$ ffilter $S O=B q / k g . V E=B q l k g$ WA $=B q \mid L$. Values for elemental uranium are reported in $\mu q$ lfilter, $\mathrm{g}$, or $\mathrm{mL}$. Evaluation: $A=$ Acceptable, $W=$ Acceptable with Warning, $N=$ Not Acceptable.

$\mathbf{p C i}=\mathrm{Bq} \times 27$ 
QAP44 Results by Nuclide

Matrix: SO

Radionuclide: PU239

EML. Value: 9.230

EML Error: 0.346

\begin{tabular}{|c|c|c|c|c|}
\hline Lahoode & Test $t$ & $\begin{array}{c}\text { Reported } \\
\text { Value }\end{array}$ & $\begin{array}{c}\text { Reported } \\
\text { Frrne } \\
\end{array}$ & $\frac{\text { Reported }}{\text { FML }}$ \\
\hline
\end{tabular}

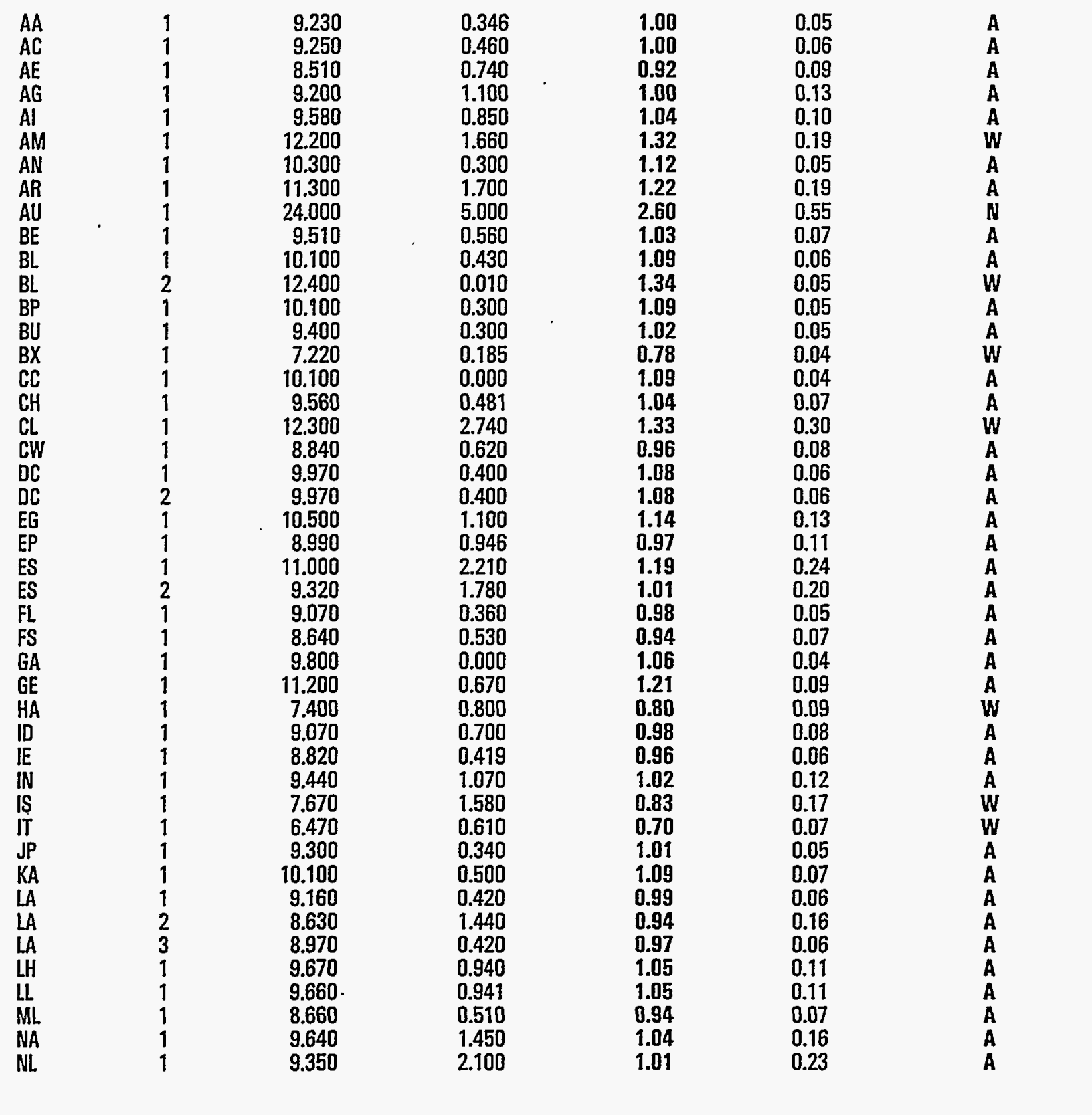

Units for matrices: $\mathrm{Al}=\mathrm{Bq} q$ ffilter $\mathrm{SO}=\mathrm{Bq} / \mathrm{kg}$ VE=Bqlkg $W \mathrm{WA}=\mathrm{Bq} / \mathrm{LL}$. Values for elemental uranium are reported in $\mu \mathrm{g}$ flilter, $\mathrm{g}$, or $\mathrm{mL}$

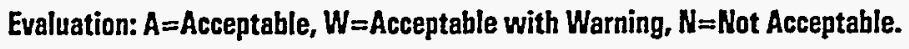
$\mathrm{pCi}=\mathrm{Bq} \times 27$ 
QAP44 Results by Nuclide

Matrix: $\mathbf{S O}$

Radionuclide: PU239

EML Value: 9.230

EML Error: 0.346

\begin{tabular}{|c|c|c|c|c|c|c|}
\hline Lahconde. & Test:\# & $\begin{array}{c}\text { Reported } \\
\text { Value. }\end{array}$ & $\begin{array}{c}\text { Reported } \\
\text { Error }\end{array}$ & $\frac{\text { Reported }}{\text { EML }}$ & $\begin{array}{l}\text { Ratio } \\
\text { Errnr }\end{array}$ & Evaluation \\
\hline NM & 1 & 22.000 & 1.000 & 2.38 & 0.14 & $\mathbf{N}$ \\
\hline OR & 1 & 8.800 & 0.400 & 0.95 & 0.06 & A \\
\hline OT & 1 & 13.000 & 3.000 & 1.41 & 0.33 & $\ddot{W}$ \\
\hline PA & 1 & 10.700 & 1.480 & 1.16 & 0.17 & A \\
\hline PB & 1 & 8.890 & 1.480 & 0.96 & 0.16 & $\hat{A}$ \\
\hline $\mathrm{PI}$ & 1 & 10.000 & 1.300 & 1.08 & 0.15 & $\ddot{A}$ \\
\hline RA & $i$ & 9.000 & 1.000 & 0.98 & 0.11 & $\ddot{A}$ \\
\hline RA & 2 & 9.000 & 1.000 & 0.98 & 0.11 & A \\
\hline RE & 1 & 8.470 & 1.140 & 0.92 & 0.13 & $\ddot{A}$ \\
\hline $\mathrm{RF}$ & 1 & 9.830 & 0.970 & 1.07 & 0.11 & $\ddot{A}$ \\
\hline RG & $i$ & 8.340 & 0.570 & 0.90 & 0.07 & A \\
\hline SC & $i$ & 8.150 & 0.500 & 0.88 & 0.06 & $\hat{A}$ \\
\hline SE & $i$ & 12.400 & 1.620 & 1.34 & 0.18 & $w$ \\
\hline SN & $i$ & 8.460 & 3.290 & 0.92 & 0.36 & A \\
\hline SR & $i$ & 9.290 & 1.930 & 1.01 & 0.21 & $\hat{A}$ \\
\hline TE & $i$ & 9.000 & 0.700 & 0.98 & 0.08 & $\ddot{A}$ \\
\hline TI & 1 & 11.000 & 2.000 & 1.19 & 0.22 & $\ddot{A}$ \\
\hline TM & 1 & 8.530 & 0.888 & 0.92 & 0.10 & A \\
\hline TN & 1 & 9.230 & 0.762 & 1.00 & 0.09 & $\ddot{A}$ \\
\hline TO & 1 & 9.220 & 3.590 & 1.00 & 0.39 & $\ddot{A}$ \\
\hline TW & $i$ & 8.500 & 0.700 & 0.92 & 0.08 & $\hat{A}$ \\
\hline$T X$ & 1 & 10.300 & 0.900 & 1.12 & 0.11 & $\hat{A}$ \\
\hline UK & 1 & 7.110 & 0.980 & 0.77 & 0.11 & $\ddot{w}$ \\
\hline UP & 1 & 8.450 & 1.590 & 0.92 & 0.18 & $A$ \\
\hline UY & 1 & 11.000 & 3.500 & 1.19 & 0.38 & $\ddot{A}$ \\
\hline WA & $i$ & 8.990 & 0.600 & 0.97 & 0.07 & $\ddot{A}$ \\
\hline WE & $i$ & 3.460 & 0.421 & 0.38 & 0.05 & $\mathrm{~N}$ \\
\hline$Y A$ & $i$ & 9.370 & 0.250 & 1.02 & 0.05 & A \\
\hline
\end{tabular}

Total Number Reported: 73

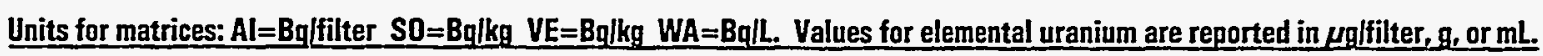
$\mathrm{pCi}=\mathrm{Bq} \times 27$ 


\section{QAP44 Results by Nuclide}

Matrix: SO

Radionuclide: SR 90

EML Value: 1340.000

EML Error: 113.000

\begin{tabular}{cccccc} 
Lahonde & Reported & Reported & Reported & Ratio \\
Value & Frror & Frror & Evaluation \\
\hline
\end{tabular}

\begin{tabular}{|c|c|c|c|c|c|c|}
\hline $\begin{array}{l}A A \\
A G \\
A N \\
A R \\
A D \\
A U \\
B C \\
B E \\
B L \\
B L \\
B M \\
B P \\
B X \\
C C \\
C H \\
C L \\
D C \\
D C \\
E G \\
E I \\
E L \\
E P \\
E S \\
G E \\
H A \\
H L \\
I D \\
I E \\
\text { IN } \\
I S \\
I T \\
J P \\
K A \\
L A \\
L A \\
L A \\
L H \\
N A \\
O R \\
O T \\
R A \\
R A \\
R E \\
\text { SR } \\
T E \\
T I\end{array}$ & $\begin{array}{l}1 \\
1 \\
1 \\
1 \\
1 \\
1 \\
1 \\
1 \\
2 \\
1 \\
1 \\
1 \\
1 \\
1 \\
1 \\
1 \\
2 \\
1 \\
1 \\
1 \\
1 \\
1 \\
1 \\
1 \\
1 \\
1 \\
1 \\
1 \\
1 \\
1 \\
1 \\
1 \\
1 \\
2 \\
3 \\
1 \\
1 \\
1 \\
1 \\
1 \\
2 \\
1 \\
1 \\
1 \\
1\end{array}$ & $\begin{array}{r}1340.000 \\
1040.000 \\
1310.000 \\
1340.000 \\
1280.000 \\
936.000 \\
1210.000 \\
958.000 \\
958.000 \\
506.000 \\
1210.000 \\
618.000 \\
994.000 \\
1040.000 \\
467.000 \\
.1190 .000 \\
1190.000 \\
9900.000 \\
1580.000 \\
595.000 \\
1.220 \\
1010.000 \\
977.000 \\
937.000 \\
1320.000 \\
1050.000 \\
1140.000 \\
1010.000 \\
660.000 \\
1100.000 \\
1200.000 \\
1450.000 \\
1300.000 \\
1400.000 \\
1210.000 \\
1140.000 \\
1450.000 \\
1100.000 \\
1200.000 \\
700.000 \\
650.000 \\
1130.000 \\
695.000 \\
1200.000 \\
1300.000\end{array}$ & $\begin{array}{r}113.000 \\
140.000 \\
39.000 \\
60.000 \\
25.000 \\
71.400 \\
74.000 \\
45.000 \\
34.000 \\
60.000 \\
75.000 \\
42.200 \\
0.000 \\
59.300 \\
11.100 \\
144.000 \\
144.000 \\
30.000 \\
216.000 \\
45.000 \\
0.023 \\
181.000 \\
25.900 \\
100.000 \\
42.200 \\
124.000 \\
248.000 \\
50.500 \\
66.000 \\
111.000 \\
10.000 \\
147.000 \\
87.000 \\
92.000 \\
81.000 \\
86.000 \\
78.000 \\
100.000 \\
100.000 \\
100.000 \\
100.000 \\
210.000 \\
12.000 \\
32.300 \\
100.000\end{array}$ & $\begin{array}{l}1.00 \\
0.78 \\
0.98 \\
1.00 \\
0.96 \\
0.70 \\
0.90 \\
0.72 \\
0.72 \\
0.38 \\
0.90 \\
0.46 \\
0.74 \\
0.78 \\
0.35 \\
0.89 \\
0.89 \\
7.39 \\
1.18 \\
0.44 \\
0.00 \\
0.75 \\
0.73 \\
0.70 \\
0.99 \\
0.78 \\
0.85 \\
0.75 \\
0.49 \\
0.82 \\
0.90 \\
1.08 \\
0.97 \\
1.04 \\
0.90 \\
0.85 \\
1.08 \\
0.82 \\
0.90 \\
0.52 \\
0.49 \\
0.84 \\
0.52 \\
0.90 \\
0.97\end{array}$ & $\begin{array}{l}0.12 \\
0.12 \\
0.09 \\
0.10 \\
0.08 \\
0.08 \\
0.09 \\
0.07 \\
0.07 \\
0.05 \\
0.09 \\
0.05 \\
0.06 \\
0.08 \\
0.03 \\
0.13 \\
0.13 \\
0.62 \\
0.19 \\
0.05 \\
0.00 \\
0.15 \\
0.06 \\
0.10 \\
0.09 \\
0.11 \\
0.20 \\
0.07 \\
0.06 \\
0.11 \\
0.08 \\
0.14 \\
0.10 \\
0.11 \\
0.10 \\
0.10 \\
0.11 \\
0.10 \\
0.11 \\
0.09 \\
0.09 \\
0.17 \\
0.04 \\
0.08 \\
0.11\end{array}$ & $\begin{array}{l}A \\
A \\
A \\
A \\
A \\
W \\
A \\
W \\
W \\
N \\
A \\
\text { N } \\
W \\
A \\
N \\
A \\
A \\
N \\
A \\
N \\
N \\
W \\
W \\
W \\
A \\
A \\
A \\
W \\
N \\
A \\
A \\
A \\
A \\
A \\
A \\
A \\
A \\
A \\
A \\
N \\
N \\
A \\
N \\
A \\
A\end{array}$ \\
\hline
\end{tabular}

Units for matrices: $\mathrm{Al}=\mathrm{Bq}$ (filter $\mathrm{SO}=\mathrm{Bq} / \mathrm{kg}$ VE=Bqlkg $W \mathrm{~A}=\mathrm{Bq} / \mathrm{L}$. Values for elemental uranium are reported in $\mu q$ /filter, $\mathrm{g}$, or $\mathrm{mL}$.

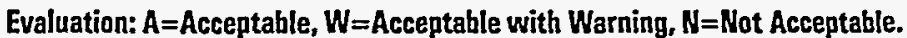

$\mathrm{pCi}=\mathrm{Bq} \times 27$ 


\section{QAP44 Results by Nuclide}

Matrix: SO

Radionuclide: SR 90

EML Value: 1340.000

EML Error: 113.000

\begin{tabular}{|c|c|c|c|c|c|c|}
\hline Lahcode & Test \# & $\begin{array}{c}\text { Reported } \\
\text { Value }\end{array}$ & $\begin{array}{c}\text { Reported } \\
\text { Error }\end{array}$ & $\frac{\text { Reported }}{\text { EML }}$ & $\begin{array}{l}\text { Ratio } \\
\text { Errnor }\end{array}$ & Evaluation \\
\hline$T M$ & 1 & 887.000 & 136.000 & 0.66 & 0.12 & $W$ \\
\hline TN & 1 & 1360.000 & 46.600 & 1.02 & 0.09 & A \\
\hline TO & 1 & 983.000 & 41.700 & 0.73 & 0.07 & $W$ \\
\hline TW & 1 & 909.000 & 9.000 & 0.68 & 0.06 & $w$ \\
\hline $\mathrm{TX}$ & 1 & 1340.000 & 52.000 & 1.00 & 0.09 & A \\
\hline UP & 1 & 1100.000 & 149.000 & 0.82 & 0.13 & $A$ \\
\hline UY & 1 & 1140.000 & 300.000 & 0.85 & 0.24 & $\ddot{A}$ \\
\hline WA & 1 & 1540.000 & 20.000 & 1.15 & 0.10 & A \\
\hline WE & 1 & 2.640 & 0.139 & 0.00 & 0.00 & $\mathbf{N}$ \\
\hline WP & 1 & 1300.000 & 37.000 & 0.97 & 0.09 & A \\
\hline
\end{tabular}

Total Number Reported: 55

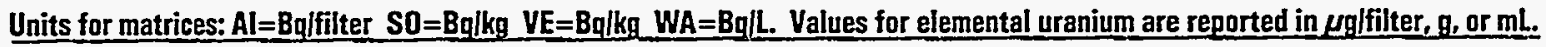

$\mathrm{pCi}=\mathrm{Bq} \times 27$ 
QAP44 Results by Nuclide

Matrix: SO

Radionuclide: U 234

EML. Value: 34.200

EML Error: 3.520

$\begin{array}{ccccc}\text { Lahcode } & \begin{array}{c}\text { Reported } \\ \text { Value }\end{array} & \text { Reported } & \text { Reported } & \text { Ratio } \\ \text { Error } & \frac{\text { FMI }}{\text { Error }} & \text { Evaluation }\end{array}$

\begin{tabular}{|c|c|c|c|c|c|c|}
\hline $\begin{array}{l}A A \\
A E \\
A G \\
A I \\
A N \\
A R \\
A U \\
B C \\
B E \\
B L \\
B L \\
B L \\
B X \\
C C \\
C H \\
C L \\
C W \\
D C \\
D C \\
E G \\
E I \\
E L \\
E S \\
F S \\
G A \\
G E \\
H A \\
H L \\
I E \\
I S \\
J P \\
L H \\
M L \\
N A \\
N L \\
N M \\
O B \\
P A \\
P B \\
R E \\
R F \\
R G \\
S C \\
S N \\
T I\end{array}$ & $\begin{array}{l}1 \\
1 \\
1 \\
1 \\
1 \\
1 \\
1 \\
1 \\
1 \\
1 \\
2 \\
3 \\
1 \\
1 \\
1 \\
1 \\
1 \\
1 \\
2 \\
1 \\
1 \\
1 \\
1 \\
1 \\
1 \\
1 \\
1 \\
1 \\
1 \\
1 \\
1 \\
1 \\
1 \\
1 \\
1 \\
1 \\
1 \\
1 \\
1 \\
1 \\
1 \\
1 \\
1 \\
1 \\
1\end{array}$ & $\begin{array}{l}34.200 \\
32.300 \\
31.400 \\
40.500 \\
37.900 \\
37.800 \\
82.000 \\
42.200 \\
37.700 \\
48.500 \\
49.700 \\
48.500 \\
36.700 \\
35.800 \\
35.900 \\
40.200 \\
32.100 \\
36.900 \\
36.900 \\
14.800 \\
44.200 \\
40.000 \\
31.300 \\
38.800 \\
37.000 \\
35.200 \\
30.700 \\
37.100 \\
35.200 \\
33.100 \\
33.000 \\
33.800 \\
34.400 \\
35.900 \\
34.400 \\
36.000 \\
14.100 \\
29.300 \\
34.400 \\
30.900 \\
37.800 \\
35.500 \\
36.400 \\
57.400 \\
28.000\end{array}$ & $\begin{array}{r}3.520 \\
2.180 \\
3.700 \\
11.000 \\
1.100 \\
3.300 \\
13.000 \\
2.460 \\
4.160 \\
0.000 \\
0.000 \\
0.000 \\
2.500 \\
0.000 \\
2.040 \\
5.200 \\
1.500 \\
3.300 \\
3.300 \\
1.800 \\
3.300 \\
4.000 \\
5.590 \\
1.930 \\
2.000 \\
4.000 \\
4.000 \\
8.000 \\
2.240 \\
13.100 \\
1.400 \\
5.400 \\
1.520 \\
2.830 \\
5.600 \\
1.000 \\
0.000 \\
2.960 \\
2.590 \\
2.900 \\
3.100 \\
1.900 \\
1.400 \\
10.400 \\
8.000\end{array}$ & $\begin{array}{l}1.00 \\
0.94 \\
0.92 \\
1.18 \\
1.11 \\
1.11 \\
2.40 \\
1.23 \\
1.10 \\
1.42 \\
1.45 \\
1.42 \\
1.07 \\
1.05 \\
1.05 \\
1.17 \\
0.94 \\
1.08 \\
1.08 \\
0.43 \\
1.29 \\
1.17 \\
0.92 \\
1.14 \\
1.08 \\
1.03 \\
0.90 \\
1.09 \\
1.03 \\
0.97 \\
0.97 \\
0.99 \\
1.01 \\
1.05 \\
1.01 \\
1.05 \\
0.41 \\
0.86 \\
1.01 \\
0.90 \\
1.11 \\
1.04 \\
1.06 \\
1.68 \\
0.82\end{array}$ & $\begin{array}{l}0.15 \\
0.12 \\
0.14 \\
0.34 \\
0.12 \\
0.15 \\
0.45 \\
0.15 \\
0.17 \\
0.15 \\
0.15 \\
0.15 \\
0.13 \\
0.11 \\
0.12 \\
0.19 \\
0.11 \\
0.15 \\
0.15 \\
0.07 \\
0.16 \\
0.17 \\
0.19 \\
0.13 \\
0.13 \\
0.16 \\
0.15 \\
0.26 \\
0.13 \\
0.40 \\
0.11 \\
0\end{array}$ & $\begin{array}{l}A \\
A \\
A \\
W \\
W \\
W \\
W \\
W \\
W \\
N \\
N \\
N \\
W \\
A \\
A \\
W \\
A \\
W \\
W \\
W \\
N \\
W \\
A \\
W \\
W \\
A \\
A \\
W \\
A \\
A \\
A \\
A \\
A \\
A \\
A \\
A \\
A \\
W \\
A \\
A \\
A \\
W \\
A \\
A \\
N \\
A\end{array}$ \\
\hline
\end{tabular}

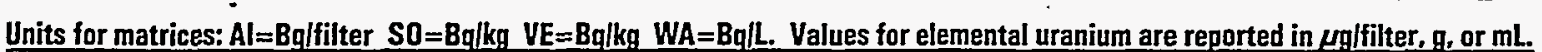

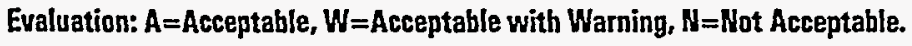

$\mathrm{pCi}=\mathrm{Bq} \times 27$ 


\section{QAP44 Results by Nuclide}

Matrix: So

Radionuclide: U 234

EML Value: 34.200

EML Error: 3.520

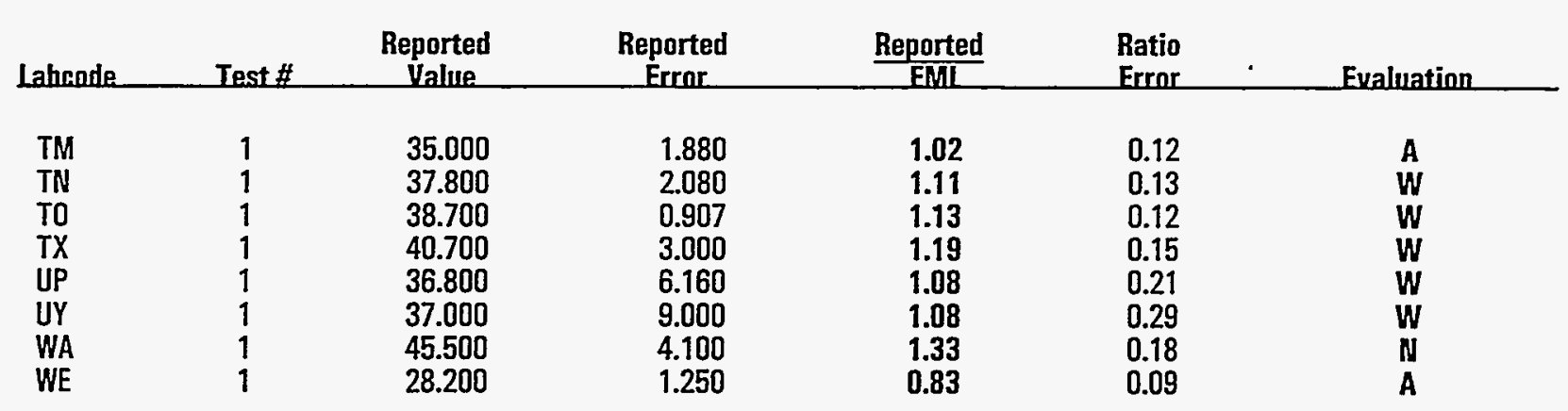

Total Number Reported: 53

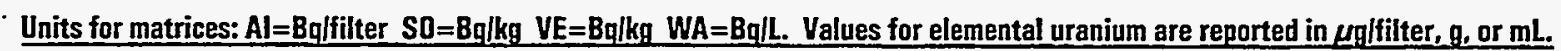
Evaluation: $A=A c c e p t a b l e, W=A c c e p t a b l e$ with Warning, $N=$ Not Acceptable. $\mathrm{pCC}=\mathrm{Bq} \times 27$ 
QAP44 Results by Nuclide

Matrix: SO

Radionuclide: U 238

EML Value: 35.900

EML Errar: 3.630

\begin{tabular}{ccccc} 
Lahoode & Reported & Reported & Reported & Ratio \\
Value & Frror & $\frac{\text { Ful }}{\text { EMror }}$ & Evaluation \\
\hline
\end{tabular}

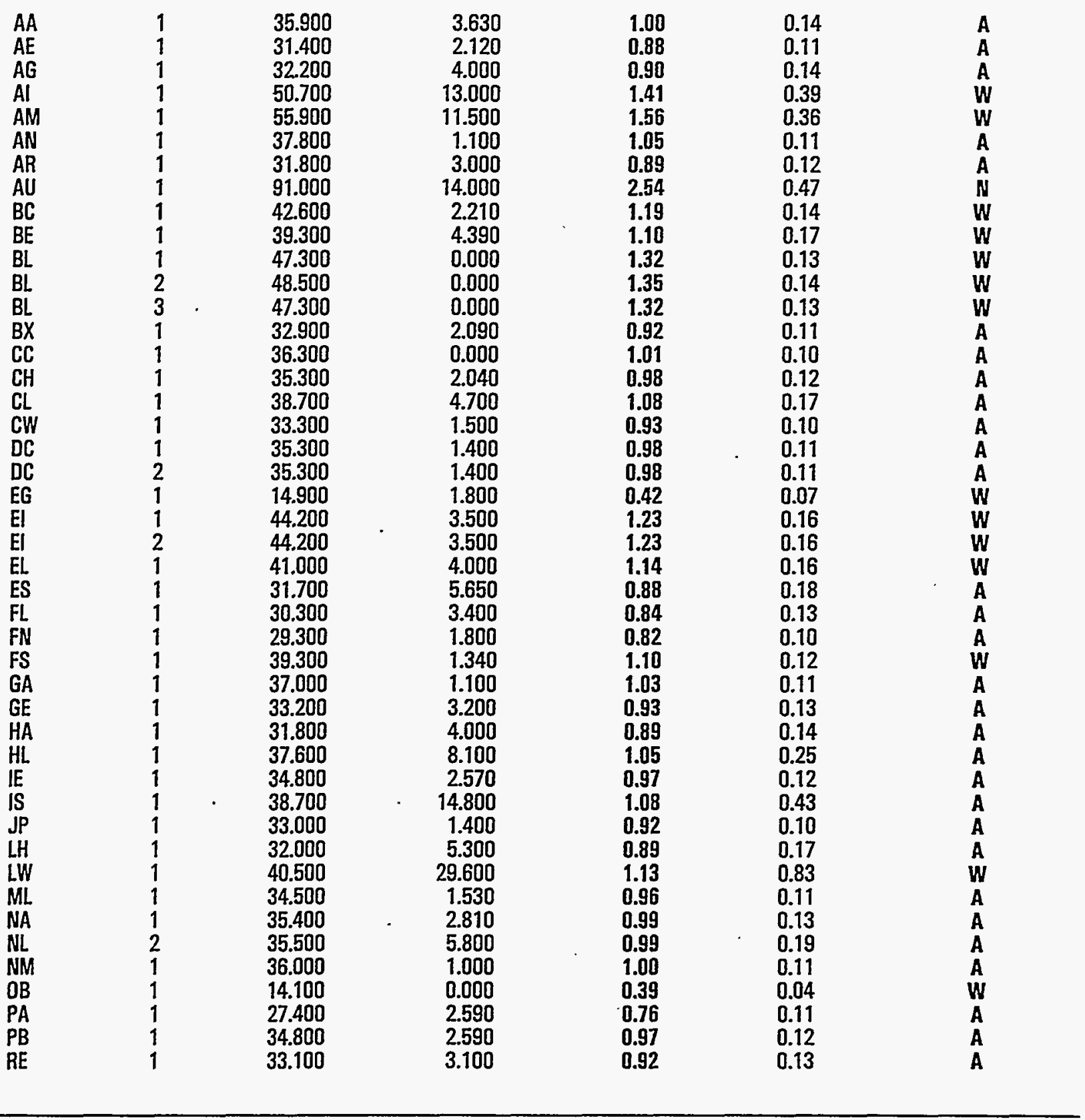

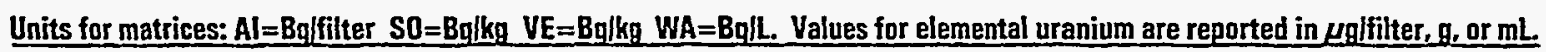

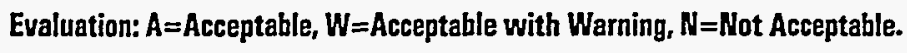

$p C i=B q \times 27$ 


\section{QAP44 Results by Nuclide}

Matrix: SO

Radionuclide: U 238

EML Value: 35.900

EML Error: 3.630

\begin{tabular}{|c|c|c|c|c|c|c|}
\hline Lahonde & Test \# & $\begin{array}{c}\text { Reported } \\
\text { Value }\end{array}$ & $\begin{array}{c}\text { Reported } \\
\text { Error }\end{array}$ & $\frac{\text { Reported }}{\text { EML }}$ & $\begin{array}{l}\text { Ratio } \\
\text { Errnr }\end{array}$ & Evaluatinn \\
\hline $\mathrm{RF}$ & 1 & 37.400 & 3.100 & 1.04 & 0.14 & A \\
\hline RG & 1 & 37.000 & 2.000 & 1.03 & 0.12 & A \\
\hline SC & $i$ & 37.300 & 1.400 & 1.04 & 0.11 & $A$ \\
\hline SN & 1 & 48.200 & 9.270 & 1.34 & 0.29 & $\ddot{W}$ \\
\hline TI & 1 & 35.000 & 9.000 & 0.98 & 0.27 & $A$ \\
\hline TM & 1 & 35.100 & 1.880 & 0.98 & 0.11 & A \\
\hline TN & 1 & 38.800 & 2.030 & 1.08 & 0.12 & $\ddot{A}$ \\
\hline TO & 1 & 38.300 & 0.889 & 1.07 & 0.11 & A \\
\hline TX & 1 & 41.600 & 3.000 & 1.16 & 0.14 & $W$ \\
\hline TY & 1 & 106.000 & 20.000 & 2.95 & 0.63 & $\mathbf{N}$ \\
\hline UP & 1 & 37.100 & 6.220 & 1.03 & 0.20 & $\ddot{A}$ \\
\hline UY & 1 & 36.000 & 9.100 & 1.00 & 0.27 & A \\
\hline WA & $i$ & 45.900 & 4.000 & 1.28 & 0.17 & $W$ \\
\hline WE & $i$ & 28.800 & 1.260 & 0.80 & 0.09 & A \\
\hline WS & 1 & 27.800 & 5.600 & 0.77 & 0.18 & A \\
\hline
\end{tabular}

Total Number Reported: 60

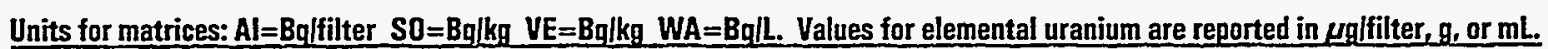

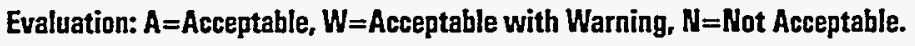

$\mathrm{pCi}=\mathrm{Bq} \times 27$ 
QAP44 Results by Nuclide

Matrix: SO

Radionuclide: U BQ

EML Value: 71.700

EML. Error: 4.150

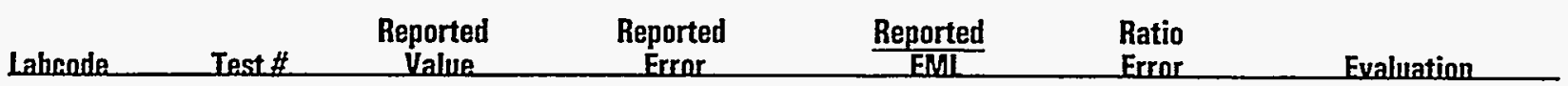

\begin{tabular}{|c|c|c|c|c|c|}
\hline $\begin{array}{l}\mathrm{AA} \\
\mathrm{AR} \\
\mathrm{BS} \\
\mathrm{BU} \\
\mathrm{CH}\end{array}$ & $\begin{array}{l}1 \\
1 \\
1 \\
1 \\
1 \\
1 \\
1 \\
1 \\
1 \\
1 \\
1 \\
1 \\
1 \\
1 \\
1 \\
1 \\
1 \\
1 \\
1\end{array}$ & $\begin{array}{r}71.700 \\
69.600 \\
141.000 \\
7.900 \\
72.900 \\
79.400 \\
50.100 \\
80.400 \\
64.500 \\
42.500 \\
74.800 \\
69.100 \\
70.000 \\
73.900 \\
73.700 \\
68.200 \\
52.600 \\
48.000 \\
93.200\end{array}$ & $\begin{array}{r}4.150 \\
4.500 \\
18.500 \\
0.800 \\
2.900 \\
8.000 \\
5.700 \\
5.500 \\
11.600 \\
5.200 \\
16.100 \\
4.600 \\
2.000 \\
4.200 \\
2.800 \\
2.400 \\
0.900 \\
4.100 \\
5.800\end{array}$ & $\begin{array}{l}1.00 \\
0.97 \\
1.97 \\
0.11 \\
1.02 \\
1.11 \\
0.70 \\
1.12 \\
0.90 \\
0.59 \\
1.04 \\
0.96 \\
0.98 \\
1.03 \\
1.03 \\
0.95 \\
0.73 \\
0.67 \\
1.30\end{array}$ & $\begin{array}{l}0.08 \\
0.08 \\
0.28 \\
0.01 \\
0.07 \\
0.13 \\
0.09 \\
0.10 \\
0.17 \\
0.08 \\
0.23 \\
0.09 \\
0.06 \\
0.08 \\
0.07 \\
0.06 \\
0.04 \\
0.07 \\
0.11\end{array}$ \\
\hline
\end{tabular}

Total Number Reported: 19

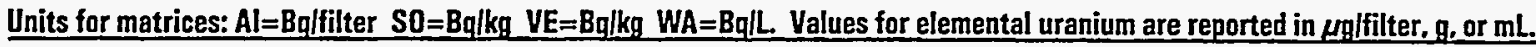

$p C i=B q \times 27$ 


\section{OAP44 Results by Nuclide}

Matrix: SO

Radionuclide: U UG

EML. Value: 2.900

EML Error: 0.290

\begin{tabular}{ccccc} 
Lahrode & Reported & Reported & Reported & Ratio \\
EMIL & Value & Frror & $\frac{\text { Frror }}{\text { Evaluation }}$ \\
\hline
\end{tabular}

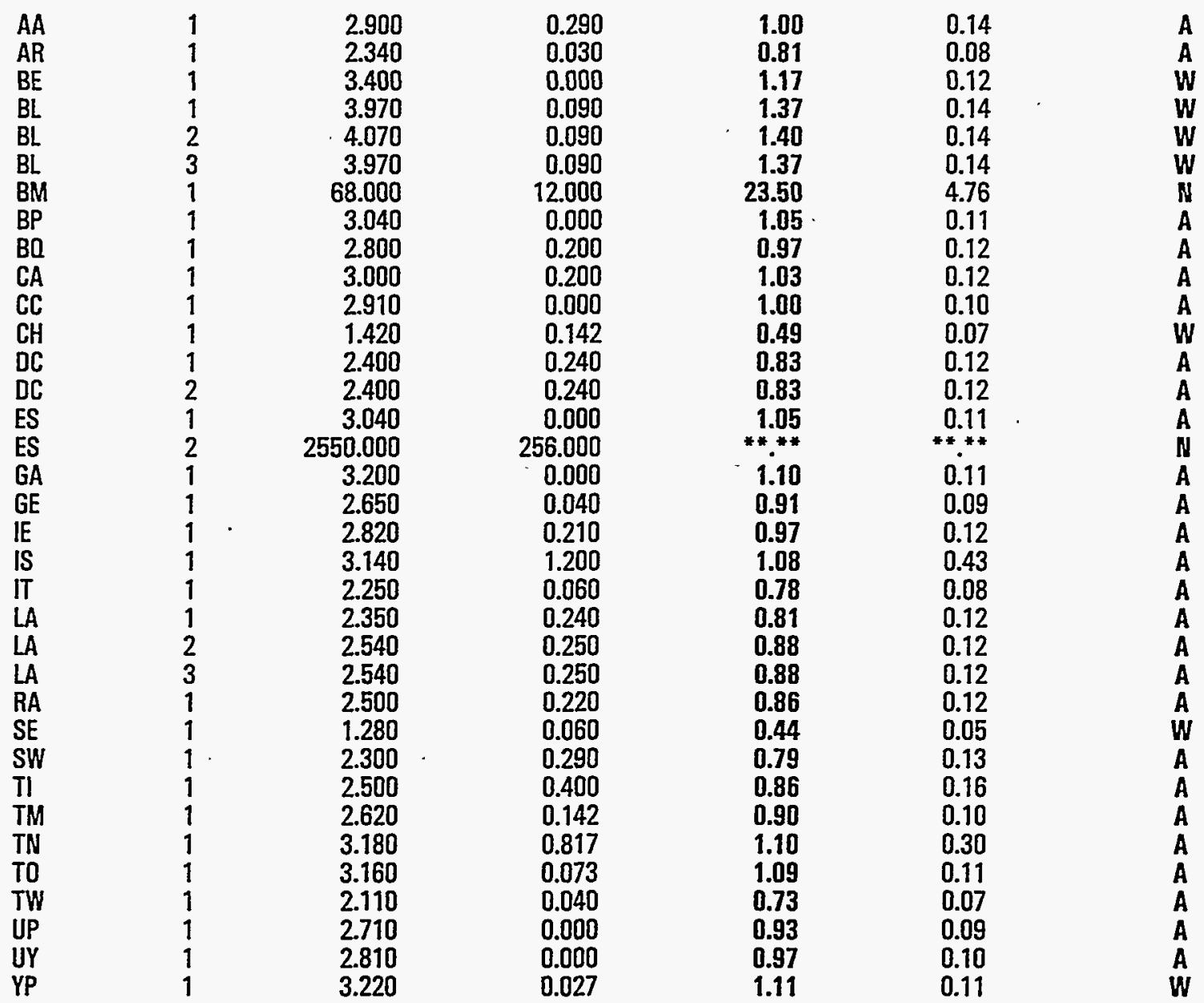

Total Number Reported: 35

Units for matrices: $\mathrm{Al}=\mathrm{Bq}$ lfilter $\mathrm{SB}=\mathrm{Bq} / \mathrm{kg}$ VE=Bqlkg WA=BqlL . Values for elemental uranium are reported in $\mu \mathrm{q} /$ filter, $\mathrm{g}$, or $\mathrm{mL}$. 
OAP44 Results by Nuclide

Matrix: VE

Radionuclide: AM241

EML Value: 5.600

EML Error: 0.184

\begin{tabular}{ccccc} 
Lahrade & $\begin{array}{c}\text { Reported } \\
\text { Value }\end{array}$ & $\begin{array}{c}\text { Reported } \\
\text { Error }\end{array}$ & $\frac{\text { Reported }}{\text { FMl }}$ & $\begin{array}{c}\text { Ratio } \\
\text { Frror }\end{array}$ \\
\hline
\end{tabular}

\begin{tabular}{|c|c|c|c|c|c|c|}
\hline $\begin{array}{l}A A \\
A G \\
A N \\
A R \\
A U \\
B E \\
B L \\
B L \\
B L \\
B L \\
B L \\
B M \\
B S \\
B U \\
B X \\
C C \\
C H \\
C L \\
C W \\
D C \\
E I \\
E L \\
E S \\
F L \\
G A \\
G E \\
I D \\
I E \\
I S \\
I T \\
J P \\
L A \\
L A \\
L A \\
L B \\
L H \\
L V \\
L W \\
M A \\
N A \\
N A \\
N A \\
O R \\
R E \\
R I \\
\text { SE } \\
S W\end{array}$ & $\begin{array}{l}1 \\
1 \\
1 \\
1 \\
1 \\
1 \\
1 \\
2 \\
2 \\
3 \\
1 \\
1 \\
1 \\
1 \\
1 \\
1 \\
1 \\
1 \\
1 \\
1 \\
1 \\
1 \\
1 \\
1 \\
1 \\
1 \\
1 \\
1 \\
1 \\
1 \\
1 \\
2 \\
3 \\
1 \\
1 \\
1 \\
1 \\
1 \\
1 \\
2 \\
1 \\
1 \\
1 \\
1 \\
1\end{array}$ & $\begin{array}{r}5.600 \\
6.170 \\
5.850 \\
5.580 \\
5.700 \\
6.170 \\
7.310 \\
10.900 \\
8.260 \\
8.540 \\
9.100 \\
9.300 \\
7.400 \\
4.700 \\
6.080 \\
6.370 \\
6.270 \\
6.160 \\
6.360 \\
6.100 \\
7.150 \\
6.390 \\
6.300 \\
6.000 \\
6.440 \\
7.000 \\
5.670 \\
7.090 \\
6.160 \\
6.400 \\
5.670 \\
5.710 \\
5.570 \\
4.720 \\
5.230 \\
12.000 \\
5.300 \\
5.200 \\
5.810 \\
5.810 \\
5.600 \\
7.150 \\
5.440 \\
6.280 \\
10.300\end{array}$ & $\begin{array}{l}0.184 \\
0.740 \\
0.180 \\
0.260 \\
1.000 \\
0.580 \\
0.260 \\
0.790 \\
0.290 \\
0.900 \\
4.500 \\
0.100 \\
1.800 \\
0.119 \\
0.000 \\
0.444 \\
1.500 \\
0.310 \\
0.870 \\
0.360 \\
0.700 \\
1.430 \\
1.300 \\
0.000 \\
0.770 \\
1.060 \\
0.530 \\
1.290 \\
0.225 \\
0.150 \\
0.220 \\
0.220 \\
0.220 \\
1.880 \\
0.440 \\
2.000 \\
57.400 \\
1.500 \\
0.570 \\
0.570 \\
1.100 \\
0.650 \\
0.510 \\
0.340 \\
3.340\end{array}$ & $\begin{array}{l}1.00 \\
1.10 \\
1.04 \\
1.00 \\
1.02 \\
1.10 \\
1.31 \\
1.95 \\
1.48 \\
1.53 \\
1.63 \\
1.66 \\
1.32 \\
0.84 \\
1.09 \\
1.14 \\
1.12 \\
1.10 \\
1.14 \\
1.09 \\
1.28 \\
1.14 \\
1.13 \\
1.07 \\
1.15 \\
1.25 \\
1.01 \\
1.27 \\
1.10 \\
1.14 \\
1.01 \\
1.02 \\
1.00 \\
0.84 \\
0.93 \\
2.14 \\
0.95 \\
0.93 \\
1.04 \\
1.04 \\
1.00 \\
1.28 \\
0.97 \\
1.12 \\
1.84\end{array}$ & $\begin{array}{c}0.05 \\
0.14 \\
0.05 \\
0.06 \\
0.18 \\
0.11 \\
0.06 \\
0.16 \\
0.07 \\
0.17 \\
0.81 \\
0.06 \\
0.32 \\
0.03 \\
0.04 \\
0.09 \\
0.27 \\
0.07 \\
0.16 \\
0.07 \\
0.13 \\
0.26 \\
0.24 \\
0.04 \\
0.14 \\
0.19 \\
0.10 \\
0.23 \\
0.05 \\
0.05 \\
0.05 \\
0.05 \\
0.05 \\
0.34 \\
0.08 \\
0.36 \\
10.30 \\
0.27 \\
0.11 \\
0.11 \\
0.20 \\
0.12 \\
0.10 \\
0.07 \\
0.60\end{array}$ & $\begin{array}{l}A \\
A \\
A \\
A \\
A \\
A \\
A \\
W \\
A \\
A \\
W \\
W \\
A \\
A \\
A \\
A \\
A \\
A \\
A \\
A \\
A \\
A \\
A \\
A \\
A \\
A \\
A \\
A \\
A \\
A \\
A \\
A \\
A \\
A \\
A \\
W \\
A \\
A \\
A \\
A \\
A \\
A \\
A \\
A \\
W\end{array}$ \\
\hline
\end{tabular}

Units for matrices: $\mathrm{Al}=\mathrm{Bq}$ lfilter $\mathrm{SO}=\mathrm{Bq} / \mathrm{kg}$ VE=Bqlkg $W \mathrm{~A}=\mathrm{Bq} / \mathrm{L}$. Values for elemental uranium are reported in $\mu \mathrm{g} / \mathrm{filter}$, g, or $\mathrm{mL}$. 


\section{OAP44 Results by Nuclide}

Matrix: VE

Radionuclide: AM241

EML Value: $\mathbf{5 . 6 0 0}$

EML Error: 0.184

\begin{tabular}{|c|c|c|c|c|c|c|}
\hline Lahsonde & Test \# & $\begin{array}{c}\text { Reported } \\
\text { Value }\end{array}$ & $\begin{array}{c}\text { Reported } \\
\text { Error }\end{array}$ & $\frac{\text { Reported }}{\text { EMIL }}$ & $\begin{array}{l}\text { Ratio } \\
\text { Error }\end{array}$ & Eyaluation \\
\hline $\begin{array}{l}\text { TE } \\
\text { TI } \\
\text { TM } \\
\text { TN } \\
\text { TO } \\
\text { TW } \\
\text { TX } \\
W A \\
Y A\end{array}$ & $\begin{array}{l}1 \\
1 \\
1 \\
1 \\
1 \\
1 \\
1 \\
1 \\
1\end{array}$ & $\begin{array}{l}6.100 \\
3.300 \\
6.700 \\
5.930 \\
5.970 \\
5.800 \\
6.660 \\
5.620 \\
5.750\end{array}$ & $\begin{array}{l}1.300 \\
1.000 \\
0.506 \\
0.479 \\
0.288 \\
0.200 \\
0.370 \\
0.630 \\
0.300\end{array}$ & $\begin{array}{l}1.09 \\
0.59 \\
1.20 \\
1.06 \\
1.07 \\
1.04 \\
1.19 \\
1.00 \\
1.03\end{array}$ & $\begin{array}{l}0.24 \\
0.18 \\
0.10 \\
0.09 \\
0.06 \\
0.05 \\
0.08 \\
0.12 \\
0.06\end{array}$ & $\begin{array}{l}A \\
W \\
A \\
A \\
A \\
A \\
A \\
A \\
A\end{array}$ \\
\hline
\end{tabular}

Total Number Reported: 54

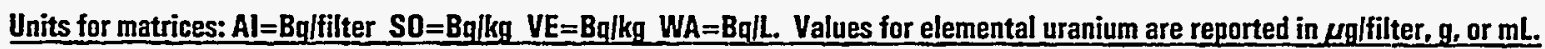
Evaluation: $A=A c c e p t a b l e, W=A c c e p t a b l e$ with Warning, $N=$ Not Acceptable. 


\section{QAP44 Results by Nuclide}

Matrix: VE

Radionuclide: CM244

EML Value: 4.440

EML Error: 0.202

Laheade $\quad$ Test\# $\quad$\begin{tabular}{cccc} 
Reported & Reported & Reported & Ratio \\
EMI & Error & Error & Evaluation \\
\hline
\end{tabular}

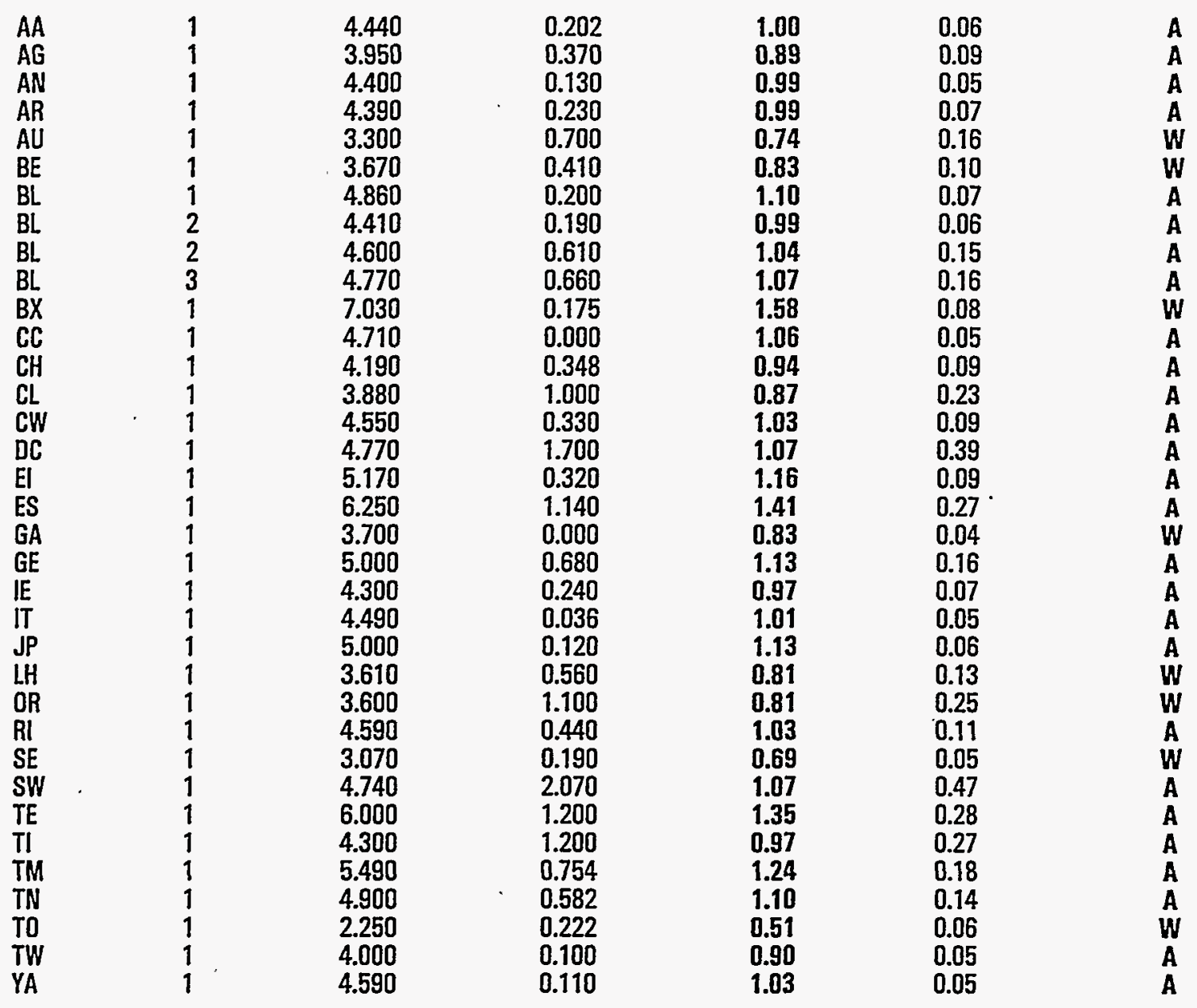

Total Number Reported: 35

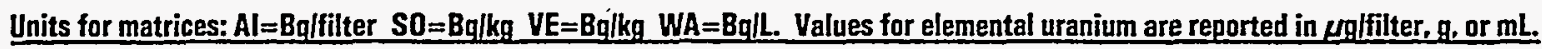
Evaluation: $A=$ Acceptable, $W=$ Acceptable with Warning, $N=$ Not Acceptable.

$\mathrm{pCi}=\mathrm{Bq} \times 27$ 
OAP44 Results by Nuclide

Matrix: VE

Radionuclide: 6060

EML Value: 59.700

EML Error: 0.963

\begin{tabular}{|c|c|c|c|c|c|c|}
\hline Lahende & Test \# & $\begin{array}{c}\text { Reported } \\
\text { Value }\end{array}$ & $\begin{array}{c}\text { Reported } \\
\text { Error }\end{array}$ & $\frac{\text { Reported }}{\text { EML }}$ & $\begin{array}{l}\text { Ratio } \\
\text { Errar }\end{array}$ & Evaluation \\
\hline
\end{tabular}

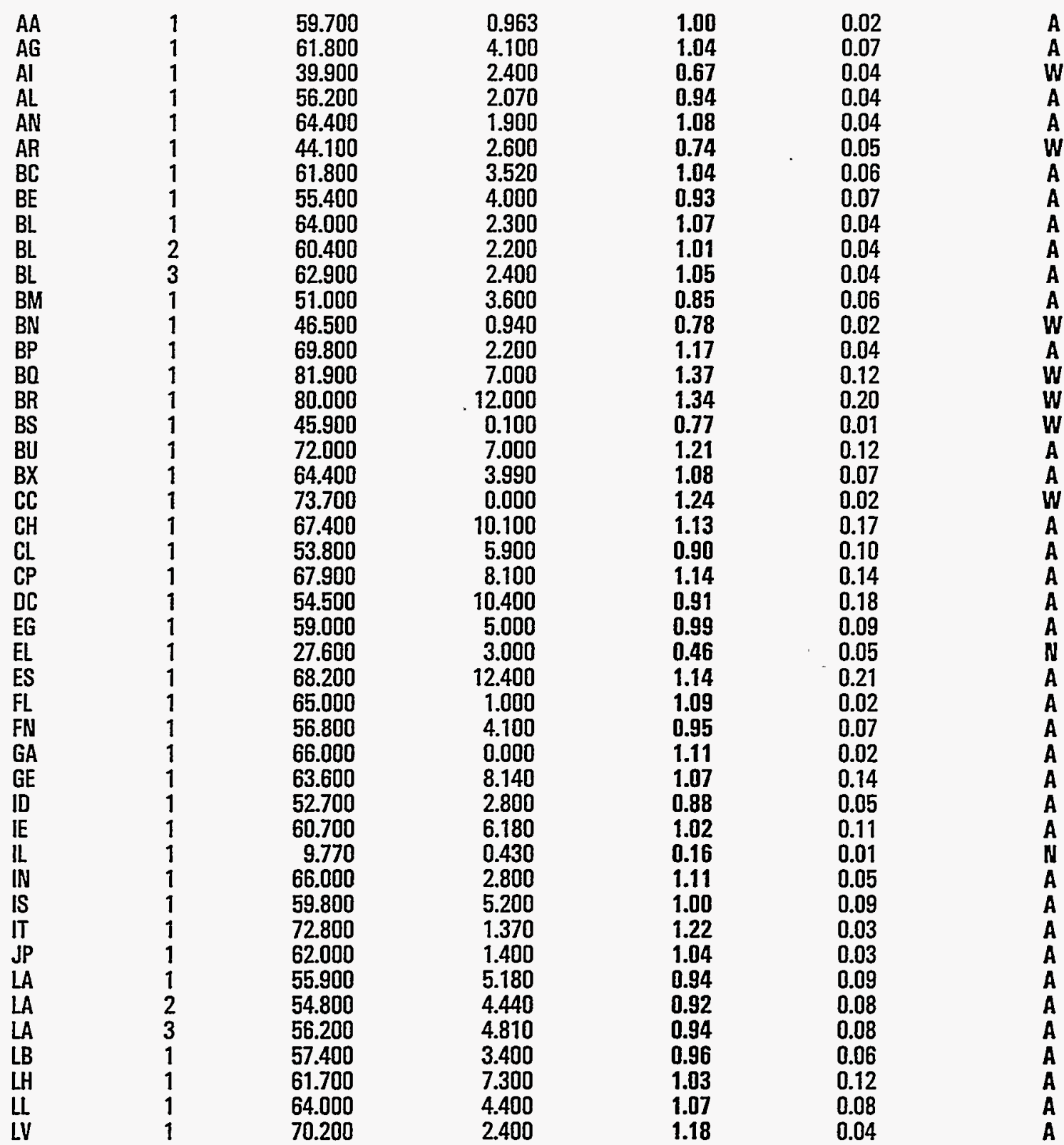

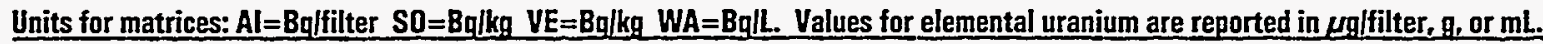
Evaluation: $A=A c c e p t a b l e, W=A c c e p t a b l e$ with Warning, $N=$ Not Acceptable. $\mathrm{pCi}=\mathrm{Bq} \times 27$ 
QAP44 Results by Nuclide

Matrix: VE

Radionuclide: 6060

EML Value: 59.700

EML Error: 0.963

\begin{tabular}{|c|c|c|c|c|c|c|}
\hline Lahcade & Test \# & $\begin{array}{c}\text { Reported } \\
\text { Value }\end{array}$ & $\begin{array}{c}\text { Reported } \\
\text { Frror }\end{array}$ & $\frac{\text { Reported }}{\text { FMI }}$ & $\begin{array}{l}\text { Ratio } \\
\text { Error }\end{array}$ & Evaluatinn \\
\hline$L W$ & 1 & 59.600 & 5.600 & 1.00 & 0.10 & $\mathbf{A}$ \\
\hline$M A$ & $i$ & 67.000 & 7.400 & 1.12 & 0.13 & A \\
\hline ME & 1 & 64.300 & 1.660 & 1.08 & 0.03 & A \\
\hline$M L$ & 1 & 61.500 & 12.800 & 1.03 & 0.22 & A \\
\hline NA & $i$ & 63.000 & 1.100 & 1.05 & 0.03 & $\ddot{A}$ \\
\hline NC & 1 & 58.300 & 1.870 & 0.98 & 0.04 & $A$ \\
\hline NR & 1 & 47.700 & 9.500 & 0.80 & 0.16 & $\mathbf{W}$ \\
\hline $\mathrm{OL}$ & 1 & 64.100 & 2.620 & 1.07 & 0.05 & A \\
\hline OR & 1 & 60.000 & 10.000 & 1.01 & 0.17 & A \\
\hline OT & 1 & 60.000 & 8.000 & 1.01 & 0.14 & A \\
\hline RA & 1 & 55.400 & 2.200 & 0.93 & 0.04 & A \\
\hline $\mathrm{RE}$ & 1 & 62.200 & 9.330 & 1.04 & 0.16 & A \\
\hline RI & $i$ & 30.200 & 2.600 & 0.51 & 0.04 & $\ddot{N}$ \\
\hline SE & $i$ & 71.100 & 2.410 & 1.19 & 0.04 & $\ddot{A}$ \\
\hline SK & 1 & 66.500 & 5.100 & 1.11 & 0.09 & $\ddot{A}$ \\
\hline $\mathrm{SN}$ & 1 & 68.500 & 6.320 & 1.15 & 0.11 & $\ddot{A}$ \\
\hline SR & 1 & 66.000 & 6.000 & 1.11 & 0.10 & $\ddot{A}$ \\
\hline SS & 1 & 64.900 & 3.800 & 1.09 & 0.07 & A \\
\hline SW & 1 & 58.100 & 4.750 & 0.97 & 0.08 & $A$ \\
\hline $\mathrm{TE}$ & 1 & 65.600 & 4.000 & 1.10 & 0.07 & A \\
\hline TI & 1 & 68.000 & 7.000 & 1.14 & 0.12 & A \\
\hline$T M$ & 1 & 66.500 & 6.060 & 1.11 & 0.10 & $\hat{A}$ \\
\hline TN & 1 & 48.700 & 3.990 & 0.82 & 0.07 & $\ddot{A}$ \\
\hline TO & 1 & 66.600 & 3.900 & 1.12 & 0.07 & A \\
\hline TW & 1 & 62.000 & 1.000 & 1.04 & 0.02 & $\ddot{A}$ \\
\hline$T X$ & 1 & 73.300 & 2.200 & 1.23 & 0.04 & $\ddot{A}$ \\
\hline TY & 1 & 56.000 & 3.000 & 0.94 & 0.05 & $\hat{A}$ \\
\hline UC & 1 & 66.200 & 2.370 & 1.11 & 0.04 & A \\
\hline WA & 1 & 59.900 & 3.000 & 1.00 & 0.05 & A \\
\hline WC & $i$ & 65.300 & 6.190 & 1.09 & 0.11 & $\hat{A}$ \\
\hline WE & 1 & 61.300 & 8.320 & 1.03 & 0.14 & $A$ \\
\hline WN & $i$ & 60.000 & 3.000 & 1.01 & 0.05 & A \\
\hline WP & 1 & 72.200 & 3.300 & 1.21 & 0.06 & $\hat{A}$ \\
\hline YA & 1 & 59.600 & 1.600 & 1.00 & 0.03 & $\ddot{A}$ \\
\hline
\end{tabular}

Total Number Reparted: 79 .

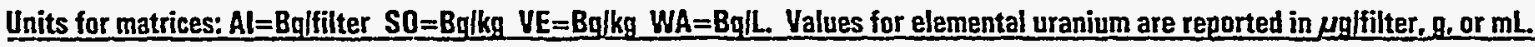
Evaluation: $A=$ Acceptable, $W=A c c e p t a b l e$ with Warning, $N=$ Not Acceptable.

$p C j=B q \times 27$ 
QAP44 Results by Nuclide

Matrix: VE

Radionuclide: CS137

EML Value: 944.000

EML Error: 16.200

\begin{tabular}{cccccc} 
& Reported & Reported & Reported & Ratio & \\
Lahronde & Test\# & Value & Error & $\frac{\text { RML }}{\text { Emror }}$ & Evaluation \\
\hline
\end{tabular}

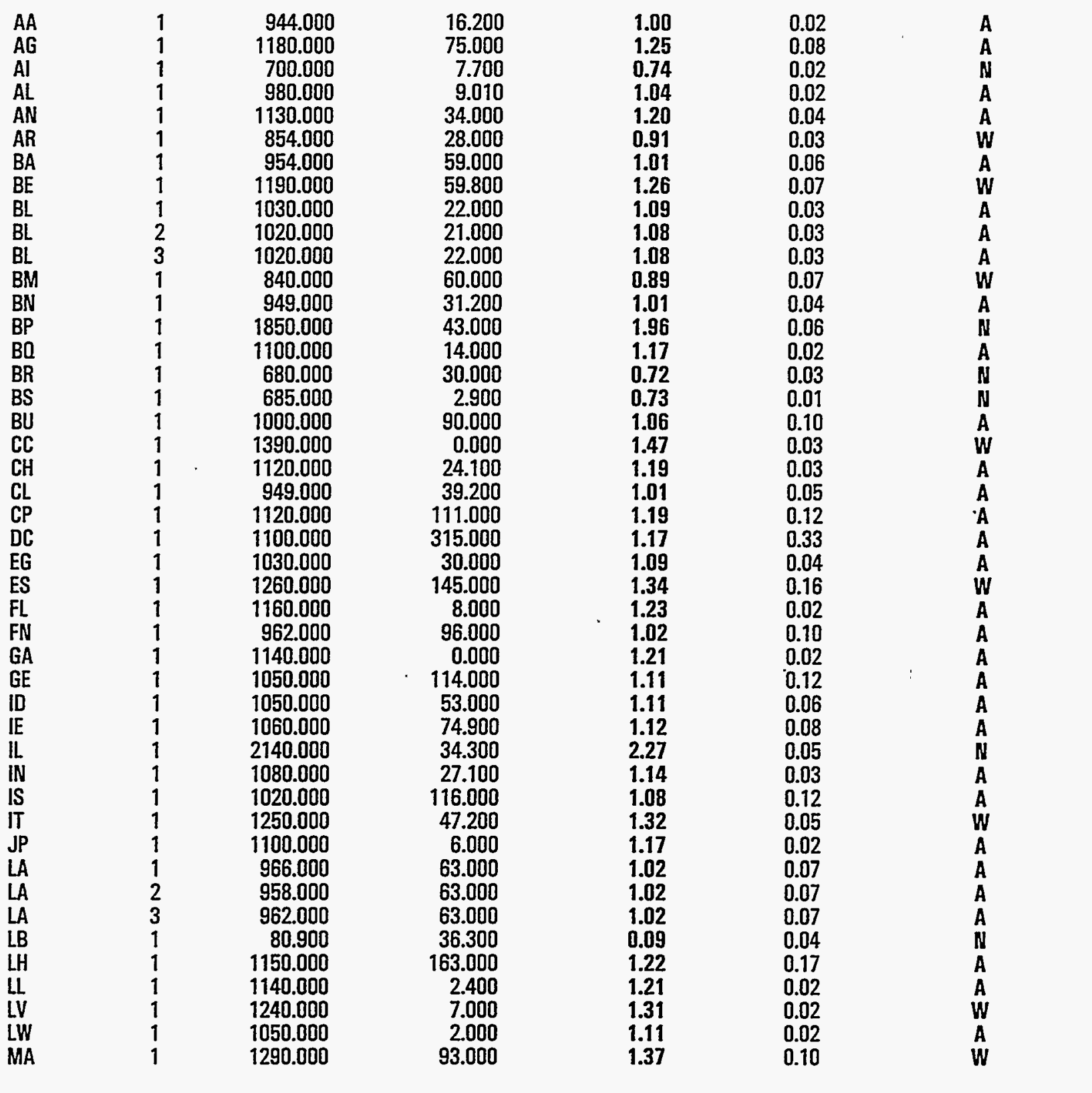

Units for matrices: $\mathrm{Al}=\mathrm{Bq} /$ filter $\mathrm{SO}=\mathrm{Bq} / \mathrm{kg} \quad \mathrm{VE}=\mathrm{Bq} / \mathrm{kg} \quad \mathrm{WA}=\mathrm{Bq} / \mathrm{L}$. Values for elemental uranium are reported in $\mu \mathrm{g} / \mathrm{filter}, \mathrm{g}$, or $\mathrm{mL}$. Evaluation: $A=A c c e p t a b l e, W=A c c e p t a b l e$ with Warning, $N=$ Not Acceptable.

$\mathrm{pCi}=\mathrm{Bq} \times 27$ 


\section{OAP44 Results by Nuclide}

Matrix: VE

Radionuclide: CS137

EML Value: 944.000

EML Error: 16.200

\begin{tabular}{|c|c|c|c|c|c|c|}
\hline Lahanile & Test \# & $\begin{array}{c}\text { Reported } \\
\text { Value. }\end{array}$ & $\begin{array}{c}\text { Reported } \\
\text { Frror }\end{array}$ & $\frac{\text { Reported }}{\text { EMI }}$ & $\begin{array}{l}\text { Ratio } \\
\text { Frror }\end{array}$ & Evaluation \\
\hline$M E$ & 1 & 1170.000 & 37.600 & 1.24 & 0.05 & $A$ \\
\hline$M L$ & $i$ & 1020.000 & 20.000 & 1.08 & 0.03 & A \\
\hline NA & $i$ & 1130.000 & 4.500 & 1.20 & 0.02 & $\ddot{A}$ \\
\hline NC & 1 & 1060.000 & 4.960 & 1.12 & 0.02 & A \\
\hline NR & 1 & 799.000 & 160.000 & 0.85 & 0.17 & $W$ \\
\hline $\mathrm{OL}$ & 1 & 1110.000 & 33.000 & 1.18 & 0.04 & A \\
\hline $\mathrm{OR}$ & $i$ & 1100.000 & 100.000 & 1.17 & 0.11 & $\ddot{A}$ \\
\hline OT & 1 & 1000.000 & 100.000 & 1.06 & 0.11 & A \\
\hline $\mathrm{RA}$ & 1 & 1000.000 & 50.000 & 1.06 & 0.06 & $\ddot{A}$ \\
\hline $\mathrm{RE}$ & 1 & 1080.000 & 90.000 & 1.14 & 0.10 & $\ddot{A}$ \\
\hline $\mathrm{RI}$ & 1 & 532.000 & 8.000 & 0.56 & 0.01 & N \\
\hline SE & 1 & 1130.000 & 84.400 & 1.20 & 0.09 & A \\
\hline SK. & 1 & 1140.000 & 47.000 & 1.21 & 0.05 & A \\
\hline SN & 1 & 1110.000 & 111.000 & 1.18 & 0.12 & A \\
\hline SR & 1 & 85.000 & 44.000 & 0.09 & 0.05 & N \\
\hline SS & 1 & 1100.000 & 48.000 & 1.17 & 0.05 & A \\
\hline SW & $i$ & 868.000 & 14.700 & 0.92 & 0.02 & $\ddot{A}$ \\
\hline TE & 1 & 1100.000 & 12.600 & 1.17 & 0.02 & A \\
\hline$\overline{T I}$ & $i$ & 1200.000 & 100.000 & 1.27 & 0.11 & w \\
\hline TM & 1 & . $\quad 1190.000$ & 36.200 & 1.26 & 0.04 & $w$ \\
\hline TN & 1 & 847.000 & 10.200 & 0.90 & 0.02 & $w$ \\
\hline TO & 1 & 1090.000 & 16.100 & 1.16 & 0.03 & A \\
\hline TW & 1 & 1010.000 & 30.000 & 1.07 & 0.04 & A \\
\hline $\mathrm{TX}$ & 1 & 1260.000 & 8.000 & 1.34 & 0.02 & $\mathbf{W}$ \\
\hline TY & $i$ & 978.000 & 10.000 & 1.04 & 0.02 & A \\
\hline UC & $i$ & 1160.000 & 77.200 & 1.23 & 0.08 & $\ddot{A}$ \\
\hline WA & 1 & 99.200 & 7.400 & 0.11 & 0.01 & $\mathbb{N}$ \\
\hline WC & $i$ & 1140.000 & 124.000 & 1.21 & 0.13 & $A$ \\
\hline WE & $i$ & 1040.000 & 15.100 & 1.10 & 0.02 & $\ddot{A}$ \\
\hline WN & 1 & 1050.000 & 20.000 & 1.11 & 0.03 & A \\
\hline WN & 2 & 1050.000 & 20.000 & 1.11 & 0.03 & A \\
\hline WP & $\overline{1}$ & 1250.000 & 10.000 & 1.32 & 0.03 & $\ddot{W}$ \\
\hline YA & 1 & 1030.000 & 14.000 & 1.09 & 0.02 & A \\
\hline
\end{tabular}

Total Number Reported: 78

Units for matrices: $\mathrm{Al}=\mathrm{Bq} /$ filter $\mathrm{SO}=\mathrm{Bq} / \mathrm{kg}$ VE=Bqlkg $W \mathrm{~A}=\mathrm{Bq} / \mathrm{L}$. Values for elemental uranium are reported in $\mu \mathrm{glfilter}, \mathrm{g}$, or $\mathrm{mL}$. 
QAP44 Results by Nuclide

Matrix: VE

Radionuclide: $K \mathbf{4 0}$

EML Value: 1030.000

EML Error: 33.000

\begin{tabular}{cccccc} 
Lahcade & Repst\# & Reported & Reported & Reported & Ratio \\
EMlue & Error & Frror & Evaluatinn \\
\hline
\end{tabular}

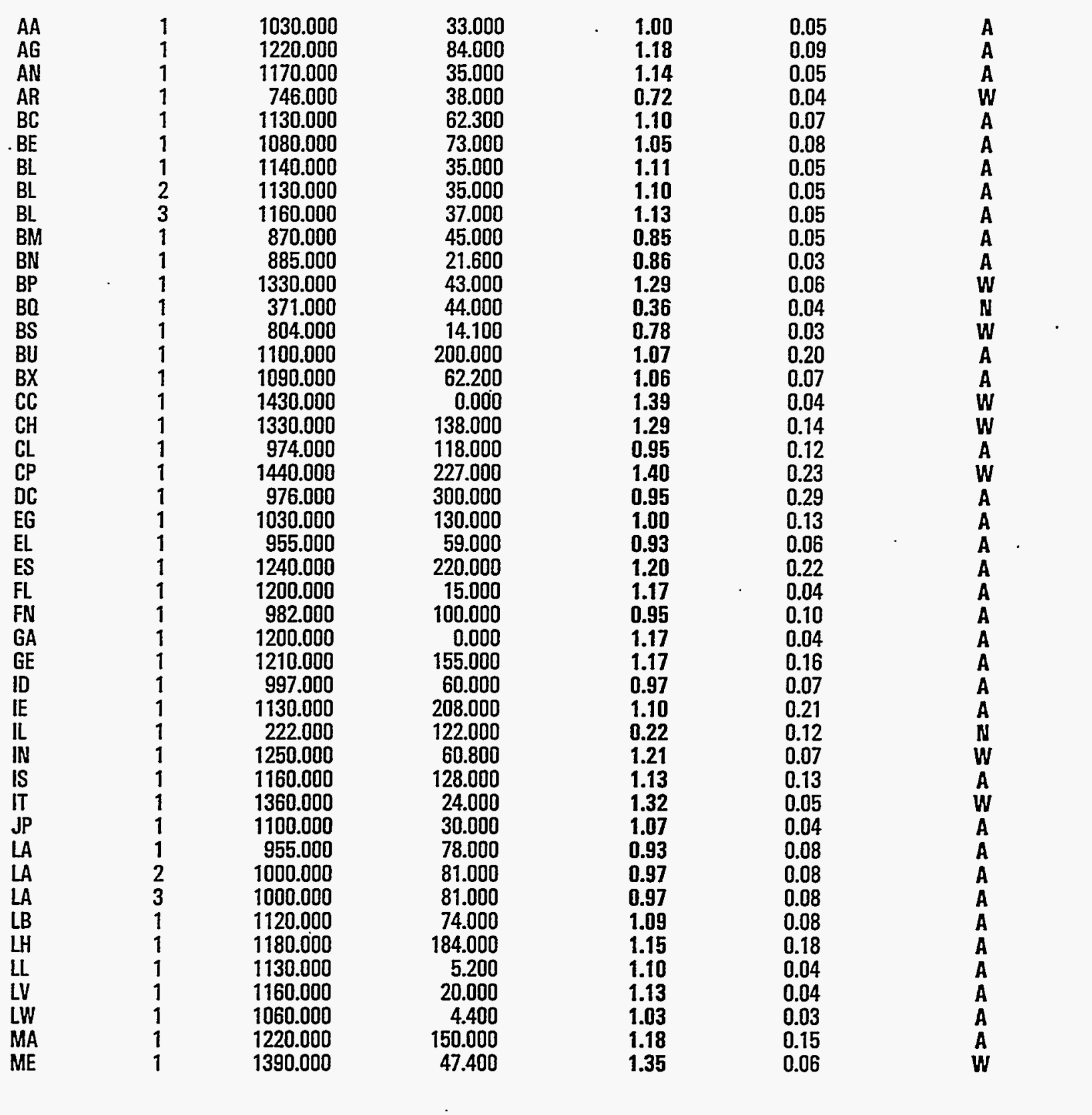

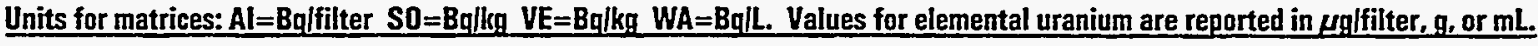
Evaluation: $A=A$ Acceptable, $W=$ Acceptable with Warning, $N=$ Not Acceptable.

$\mathrm{pCi}=\mathrm{Bq} \times 27$ 
QAP44 Results by Nuclide

Matrix: VE

Radionuclide: K $\mathbf{4 0}$

EML Value: 1030.000

EML Error: 33.000

\begin{tabular}{|c|c|c|c|c|c|c|}
\hline Lahcade & Iest\# & $\begin{array}{c}\text { Reported } \\
\text { Value }\end{array}$ & $\begin{array}{c}\text { Reported } \\
\text { Error }\end{array}$ & $\frac{\text { Reported }}{\text { EMML }}$ & $\begin{array}{l}\text { Ratio } \\
\text { Error }\end{array}$ & Evaluatinn \\
\hline ML & 1 & 1190.000 & 244.000 & 1.16 & 0.24 & A \\
\hline NA & 1 & 1180.000 & 20.000 & 1.15 & 0.04 & A \\
\hline NR & 1 & 918.000 & 184.000 & 0.89 & 0.18 & A \\
\hline $\mathrm{OL}$ & 1 & 1200.000 & 75.400 & 1.17 & 0.08 & A \\
\hline $\mathrm{OR}$ & $i$ & 1200.000 & 100.000 & 1.17 & 0.10 & $\ddot{A}$ \\
\hline OT & 1 & 1100.000 & 100.000 & 1.07 & 0.10 & A \\
\hline $\mathrm{RA}$ & 1 & 1050.000 & 70.000 & 1.02 & 0.08 & A \\
\hline RE & 1 & 1120.000 & 146.000 & 1.09 & 0.15 & A \\
\hline RI & 1 & 517.000 & 66.000 & 0.50 & 0.07 & $w$ \\
\hline SE & 1 & 1430.000 & 49.400 & 1.39 & 0.07 & $w$ \\
\hline SK & 1 & 1120.000 & 25.000 & 1.09 & 0.04 & A \\
\hline SN & 1 & 1260.000 & 117.000 & 1.22 & 0.12 & w \\
\hline SR & 1 & 166.000 & 24.000 & 0.16 & 0.02 & $\mathbf{N}$ \\
\hline SS & 1 & 1150.000 & 99.000 & 1.12 & 0.10 & A \\
\hline SW & $i$ & 1030.000 & 129.000 & 1.00 & 0.13 & A \\
\hline TE & 1 & 1190.000 & 61.600 & 1.16 & 0.07 & $\ddot{A}$ \\
\hline $\mathrm{TI}$ & $i$ & 1200.000 & 100.000 & 1.17 & 0.10 & $\ddot{A}$ \\
\hline TM & 1 & 1180.000 & 77.300 & 1.15 & 0.08 & $\ddot{A}$ \\
\hline TN & $i$ & 884.000 & 44.200 & 0.86 & 0.05 & A \\
\hline TO & $i$ & 1050.000 & 59.700 & 1.02 & 0.07 & A \\
\hline TW & $i$ & 1070.000 & 43.000 & 1.04 & 0.05 & $\ddot{A}$ \\
\hline TX & $j$ & 1350.000 & 26.000 & 1.31 & 0.05 & w \\
\hline TY & $i$ & 1120.000 & 50.000 & 1.09 & 0.06 & $\ddot{A}$ \\
\hline Uc & $j$ & 1210.000 & 60.200 & 1.17 & 0.07 & A \\
\hline WA & 1 & 1090.000 & 60.000 & 1.06 & 0.07 & $\ddot{A}$ \\
\hline WC & 1 & 1230.000 & 173.000 & 1.19 & 0.17 & $\ddot{A}$ \\
\hline WE & 1 & 1000.000 & 89.900 & 0.97 & 0.09 & $\ddot{A}$ \\
\hline WN & $i$ & 1120.000 & 80.000 & 1.09 & 0.09 & $\ddot{A}$ \\
\hline WP & 1 & 1270.000 & 41.000 & 1.23 & 0.06 & $\ddot{w}$ \\
\hline YA & 1 & 1120.000 & 30.000 & 1.09 & 0.05 & $\ddot{A}$ \\
\hline
\end{tabular}

Total Number Reported: 75

Units for matrices: $A \mathrm{l}=\mathrm{Bq}$ lfilter $\mathrm{SO}=\mathrm{Bq} / \mathrm{kg}$ VE=Bqlkg $W \mathrm{~A}=\mathrm{Bq} / \mathrm{L}$. Values for elemental uranium are reported in $\mu \mathrm{glfilter}, \mathrm{g}$, or $\mathrm{mL}$. 
QAP44 Results by Nuclide

Matrix: VE

Radionuclide: PU238

EML Value: 0.820

EML Error: 0.104

\begin{tabular}{|c|c|c|c|c|c|c|}
\hline Lahande & Test \# & $\begin{array}{c}\text { Reported } \\
\text { Value }\end{array}$ & $\begin{array}{c}\text { Reported } \\
\text { Errar }\end{array}$ & $\frac{\text { Reported }}{\text { EMIL }}$ & $\begin{array}{l}\text { Ratio } \\
\text { Frror }\end{array}$ & Fualua \\
\hline $\begin{array}{l}A A \\
A N \\
A R \\
A U \\
B A \\
B L \\
B L \\
\text { SE }\end{array}$ & $\begin{array}{l}1 \\
1 \\
1 \\
1 \\
1 \\
1 \\
2 \\
1\end{array}$ & $\begin{array}{l}0.820 \\
0.701 \\
0.604 \\
9.000 \\
1.030 \\
0.940 \\
0.990 \\
1.160\end{array}$ & $\begin{array}{l}0.104 \\
0.028 \\
0.069 \\
2.000 \\
0.210 \\
0.100 \\
0.340 \\
8.370\end{array}$ & $\begin{array}{r}1.00 \\
0.86 \\
0.74 \\
11.00 \\
1.26 \\
1.15 \\
1.21 \\
1.42\end{array}$ & $\begin{array}{r}0.18 \\
0.11 \\
0.13 \\
2.81 \\
0.30 \\
0.19 \\
0.44 \\
10.20\end{array}$ & $\begin{array}{l}A \\
A \\
W \\
N \\
A \\
A \\
A \\
A\end{array}$ \\
\hline
\end{tabular}

Total Number Reported: 8

Units for matrices: $A I=B q]$ filter $S O=B q / k g$ VE $=B q / k g, W A=B q L$. Values for elemental uranium are reported in $\mu g l$ filter, $g$, or $m L$. Evaluation: $A=A c c e p t a b l e, W=A c c e p t a b l e$ with Warning, $N=$ Not Acceptable: $\mathrm{pCi}=\mathrm{Bq} \times 27$ 


\section{QAP44 Results by Nuclide}

Matrix: VE

Radionuclide: PU239

EML Value: 9.820

EML Error: 1.220

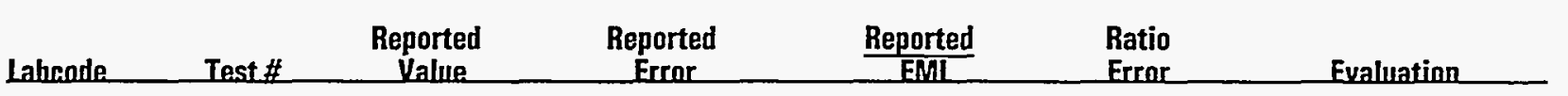

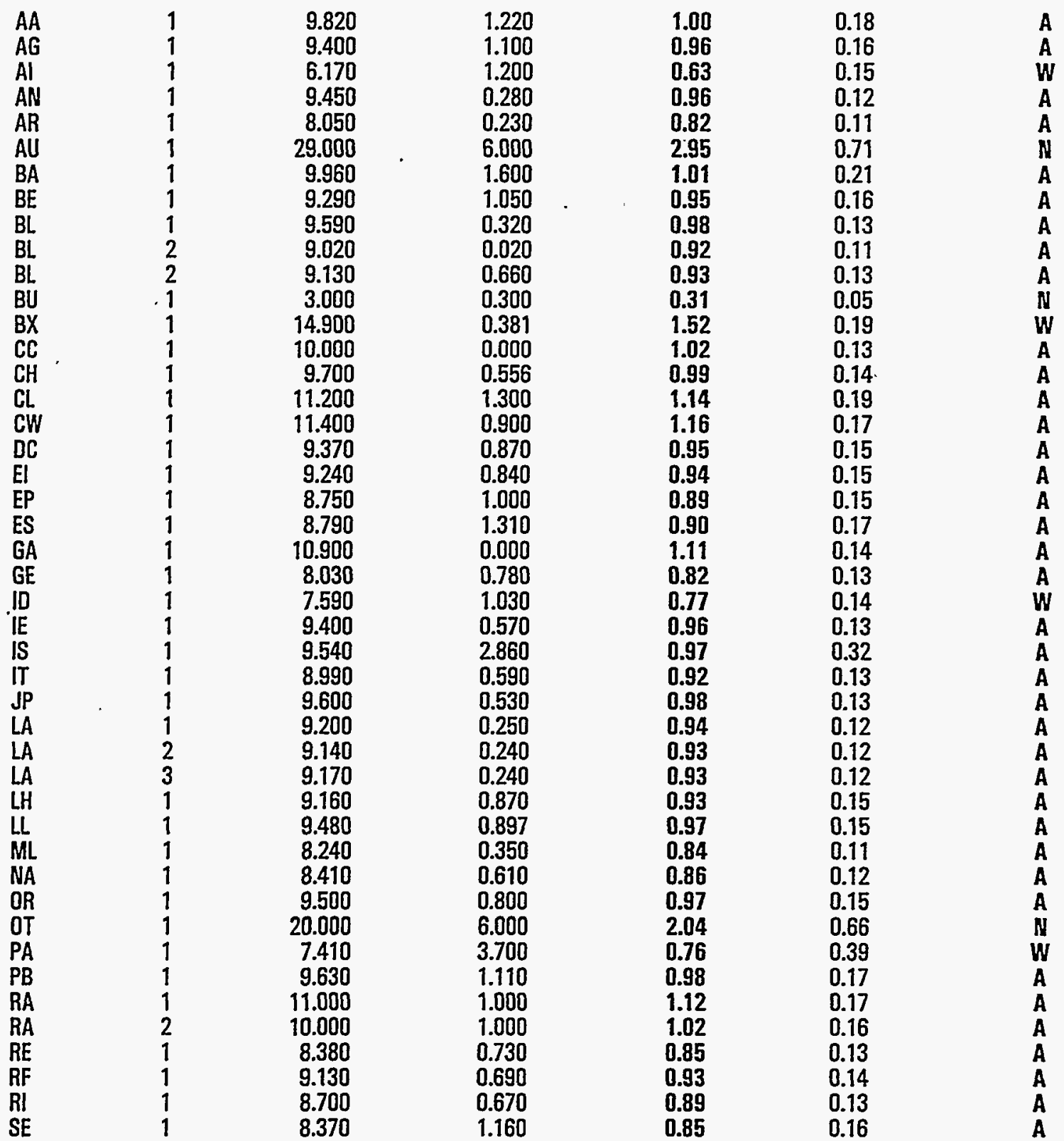

Units for matrices: $\mathrm{Al}=\mathrm{Bq}$ lfilter $\mathrm{SO}=\mathrm{Bq} / \mathrm{kg} \mathrm{VE}=\mathrm{Bq} / \mathrm{kg} \quad W \mathrm{~A}=\mathrm{Bq} \mathrm{LL}$. Values for elemental uranium are reported in $\mu \mathrm{g} /$ filter, $\mathrm{g}$, or $\mathrm{mL}$. 


\section{QAP44 Results by Nuclide}

Matrix: VE

Radionuclide: PU239

EML Value: $\mathbf{9 . 8 2 0}$

EML Error: 1.220

\begin{tabular}{|c|c|c|c|c|c|c|}
\hline Lahoade & Test \# & $\begin{array}{c}\text { Reported } \\
\text { Value }\end{array}$ & $\begin{array}{c}\text { Reported } \\
\text { Frror }\end{array}$ & $\frac{\text { Reported }}{\text { FMl }}$ & $\begin{array}{l}\text { Ratio } \\
\text { Error }\end{array}$ & Evaluation \\
\hline $\begin{array}{l}\text { SN } \\
\text { SR } \\
\text { SW } \\
\text { TE } \\
T I \\
T M \\
T M \\
T N \\
T O \\
T W \\
T X \\
T Y \\
\text { TY } \\
\text { WA } \\
\text { WE } \\
\text { YA }\end{array}$ & $\begin{array}{l}1 \\
1 \\
1 \\
1 \\
1 \\
1 \\
1 \\
1 \\
1 \\
1 \\
1 \\
1 \\
1 \\
1\end{array}$ & $\begin{array}{r}8.150 \\
9.450 \\
16.900 \\
9.200 \\
6.700 \\
8.810 \\
9.580 \\
9.830 \\
8.400 \\
10.300 \\
8.700 \\
9.950 \\
0.529 \\
9.760\end{array}$ & $\begin{array}{l}2.420 \\
1.370 \\
1.840 \\
1.300 \\
1.100 \\
0.638 \\
0.700 \\
3.480 \\
0.400 \\
0.600 \\
1.400 \\
0.520 \\
0.262 \\
0.300\end{array}$ & $\begin{array}{l}0.83 \\
0.96 \\
1.72 \\
0.94 \\
0.68 \\
0.90 \\
0.98 \\
1.00 \\
0.86 \\
1.05 \\
0.89 \\
1.01 \\
0.05 \\
0.99\end{array}$ & $\begin{array}{l}0.27 \\
0.18 \\
0.28 \\
0.18 \\
0.14 \\
0.13 \\
0.14 \\
0.38 \\
0.11 \\
0.14 \\
0.18 \\
0.14 \\
0.03 \\
0.13\end{array}$ & $\begin{array}{l}\mathbf{A} \\
\mathbf{A} \\
\mathbf{W} \\
\mathbf{A} \\
\mathbf{W} \\
\mathbf{A} \\
\mathbf{A} \\
\mathbf{A} \\
\mathbf{A} \\
\mathbf{A} \\
\mathbf{A} \\
\mathbf{A} \\
\mathbf{N} \\
\mathbf{A}\end{array}$ \\
\hline
\end{tabular}

Total Number Reported: 59

Units for matrices: $\mathrm{Al}=\mathrm{Bq}$ ffilter $\mathrm{SO}=\mathrm{Bq} / \mathrm{kg}$ VE $=\mathrm{Bg}$ lkg $W \mathrm{WA}=\mathrm{Bg} / \mathrm{L}$. Values for elemental uranium are reported in $\mu \mathrm{g} / \mathrm{filter}, \mathrm{g}$, or $\mathrm{mL}$. 


\section{QAP44 Results by Nuclide}

Matrix: VE

Radionuclide: SR 90

EML Value: 1300.000

EML Error: 52.400

\begin{tabular}{|c|c|c|c|c|c|c|}
\hline Lahcoide & Test \# & Reported & Reported & $\frac{\text { Reported }}{\text { FMI }}$ & Ratio & Fualuatinn \\
\hline
\end{tabular}

\begin{tabular}{|c|c|c|c|c|c|c|}
\hline 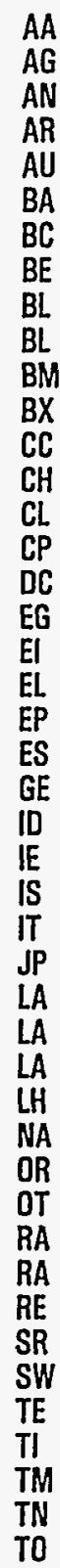 & $\begin{array}{l}1 \\
1 \\
1 \\
1 \\
1 \\
1 \\
1 \\
1 \\
1 \\
2 \\
1 \\
1 \\
1 \\
1 \\
1 \\
1 \\
1 \\
1 \\
1 \\
1 \\
1 \\
1 \\
1 \\
1 \\
1 \\
1 \\
1 \\
1 \\
1 \\
2 \\
3 \\
1 \\
1 \\
1 \\
1 \\
1 \\
2 \\
1 \\
1 \\
1 \\
1 \\
1 \\
1 \\
1 \\
1 \\
1 \\
\end{array}$ & $\begin{array}{r}1300.000 \\
1220.000 \\
1450.000 \\
202.000 \\
1200.000 \\
1130.000 \\
707.000 \\
1120.000 \\
1390.000 \\
1320.000 \\
209.000 \\
677.000 \\
1320.000 \\
1310.000 \\
437.000 \\
1040.000 \\
826.000 \\
1250.000 \\
2060.000 \\
873.000 \\
1.150 \\
1170.000 \\
977.000 \\
1180.000 \\
1220.000 \\
1130.000 \\
1550.000 \\
1400.000 \\
1390.000 \\
1480.000 \\
1220.000 \\
1220.000 \\
1230.000 \\
1060.000 \\
1400.000 \\
1700.000 \\
1900.000 \\
1240.000 \\
806.000 \\
1320.000 \\
1210.000 \\
1400.000 \\
1370.000 \\
1430.000 \\
1370.000\end{array}$ & $\begin{array}{r}52.400 \\
220.000 \\
44.000 \\
6.000 \\
18.000 \\
12.000 \\
42.200 \\
62.000 \\
38.000 \\
34.000 \\
20.000 \\
42.900 \\
0.000 \\
74.100 \\
11.100 \\
13.000 \\
149.000 \\
30.000 \\
193.000 \\
67.000 \\
0.025 \\
164.000 \\
11.500 \\
90.000 \\
18.500 \\
113.000 \\
18.800 \\
10.000 \\
85.100 \\
92.500 \\
77.700 \\
97.000 \\
27.000 \\
100.000 \\
100.000 \\
250.000 \\
250.000 \\
62.000 \\
35.000 \\
15.300 \\
32.200 \\
100.000 \\
167.000 \\
47.400 \\
45.900\end{array}$ & $\begin{array}{l}1.00 \\
0.94 \\
1.12 \\
0.16 \\
0.92 \\
0.87 \\
0.54 \\
0.86 \\
1.07 \\
1.02 \\
0.16 \\
0.52 \\
1.02 \\
1.01 \\
0.34 \\
0.80 \\
0.64 \\
0.96 \\
1.59 \\
0.67 \\
0.00 \\
0.90 \\
0.75 \\
0.91 \\
0.94 \\
0.87 \\
1.19 \\
1.08 \\
1.07 \\
1.14 \\
0.94 \\
0.94 \\
0.95 \\
0.82 \\
1.08 \\
1.31 \\
1.46 \\
0.95 \\
0.62 \\
1.02 \\
0.93 \\
1.08 \\
1.05 \\
1.10 \\
1.05\end{array}$ & $\begin{array}{l}0.06 \\
0.17 \\
0.06 \\
0.01 \\
0.04 \\
0.04 \\
0.04 \\
0.06 \\
0.05 \\
0.05 \\
0.02 \\
0.04 \\
0.04 \\
0.07 \\
0.02 \\
0.03 \\
0.12 \\
0.05 \\
0.16 \\
0.06 \\
0.00 \\
0.13 \\
0.03 \\
0.08 \\
0.04 \\
0.09 \\
0.05 \\
0.04 \\
0.08 \\
0.08 \\
0.07 \\
0.08 \\
0.04 \\
0.08 \\
0.09 \\
0.20 \\
0.20 \\
0.06 \\
0.04 \\
0.04 \\
0.05 \\
0.09 \\
0.14 \\
0.06 \\
0.06\end{array}$ & $\begin{array}{l}A \\
A \\
W \\
N \\
A \\
A \\
W \\
A \\
A \\
A \\
N \\
W \\
A \\
A \\
N \\
A \\
W \\
A \\
N \\
W \\
N \\
A \\
A \\
A \\
A \\
A \\
W \\
A \\
A \\
W \\
A \\
A \\
A \\
A \\
A \\
W \\
N \\
A \\
W \\
A \\
A \\
A \\
A \\
A \\
A\end{array}$ \\
\hline
\end{tabular}

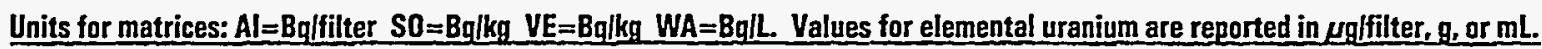
Evaluation: $A=$ Acceptable, $W=$ Acceptable with Warning, $N=$ Not Acceptable. $\mathrm{pCi}=\mathrm{Bq} \times 27$ 


\section{QAP44 Results by Nuclide}

Matrix: VE

Radionuclide: SR 90

EML. Value: 1300.000

EML Error: 52.400

\begin{tabular}{|c|c|c|c|c|c|c|}
\hline Lahande & Test \# & $\begin{array}{c}\text { Reported } \\
\text { Value }\end{array}$ & $\begin{array}{c}\text { Reported } \\
\text { Errar }\end{array}$ & $\frac{\text { Reported }}{\text { EMI }}$ & $\begin{array}{l}\text { Ratio } \\
\text { Error }\end{array}$ & Evaluation \\
\hline $\begin{array}{l}\text { TW } \\
\text { TX } \\
\text { TY } \\
\text { WA } \\
\text { WE } \\
\text { WP }\end{array}$ & $\begin{array}{l}1 \\
1 \\
1 \\
1 \\
1 \\
1\end{array}$ & $\begin{array}{r}930.000 \\
1310.000 \\
1500.000 \\
1470.000 \\
2.810 \\
1400.000\end{array}$ & $\begin{array}{r}10.000 \\
60.000 \\
320.000 \\
26.000 \\
0.198 \\
37.000\end{array}$ & $\begin{array}{l}0.72 \\
1.01 \\
1.15 \\
1.13 \\
0.00 \\
1.08\end{array}$ & $\begin{array}{l}0.03 \\
0.06 \\
0.25 \\
0.05 \\
0.00 \\
0.05\end{array}$ & $\begin{array}{l}\mathbf{A} \\
\mathbf{A} \\
\mathbf{W} \\
\mathbf{W} \\
\mathbf{N} \\
\mathbf{A}\end{array}$ \\
\hline
\end{tabular}

Total Number Reported: 51

Units for matrices: Al=Bqlfilter $S O=B q$ lkg VE=Bqlkg $W A=B q l L$. Values for elemental uranium are reported in $\mu$ glfilter, $q$, or $\mathrm{mL}$. 
QAP44 Results by Nuclide

Matrix: WA

Radionuclide: AM241

EML Value: 0.766

EML Error: 0.013

\begin{tabular}{|c|c|c|c|}
\hline Test\# & Reported & Reported & $\frac{\text { Reported }}{\text { FMI }}$ \\
\hline
\end{tabular}

Evaluation

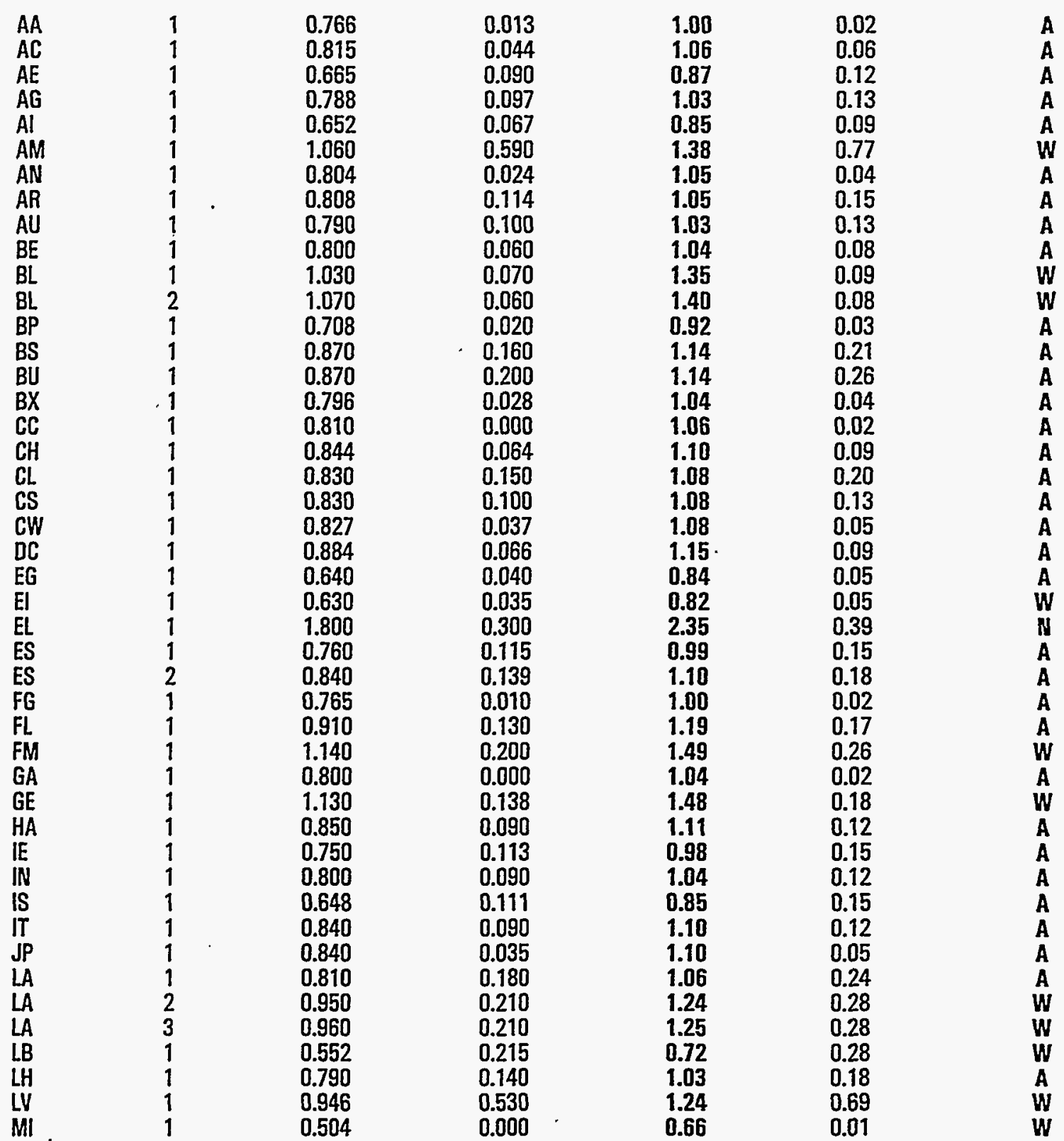

Units for matrices: $\mathrm{Al}=\mathrm{Bq} / \mathrm{filter} \mathrm{SO}=\mathrm{Bg} / \mathrm{kg}$ VE $=\mathrm{Bq} / \mathrm{kg} W \mathrm{WA}=\mathrm{Bq} \mathrm{LL}$. Values for elemental uranium are reported in $\mu \mathrm{g} / \mathrm{filter}, \mathrm{g}$, or $\mathrm{mL}$. $\mathrm{pCi}=\mathrm{Bq} \times 27$ 


\section{QAP44 Results by Nuclide}

Matrix: WA

Radionuclide: AM241

EML Value: 0.766

EML Error: 0.013

\begin{tabular}{|c|c|c|c|c|c|c|}
\hline Lahconde & Test\# & $\begin{array}{c}\text { Reported } \\
\text { Value }\end{array}$ & $\begin{array}{c}\text { Reported } \\
\text { Error }\end{array}$ & $\frac{\text { Reported }}{\text { FMI }}$ & $\begin{array}{l}\text { Ratio } \\
\text { Error }\end{array}$ & Fvaluation \\
\hline MI & 2 & 0.475 & 0.000 & 0.62 & 0.01 & N \\
\hline MS & 1 & 0.980 & 0.100 & 1.28 & 0.13 & $\mathbf{W}$ \\
\hline NA & 1 & 0.732 & 0.045 & 0.96 & 0.06 & A \\
\hline NM & 1 & 0.735 & 0.040 & 0.96 & 0.05 & A \\
\hline OD & 1 & 0.679 & 0.072 & 0.89 & 0.10 & A \\
\hline $\mathrm{OR}$ & 1 & 0.780 & 0.080 & 1.02 & 0.11 & A \\
\hline OT & 1 & 1.100 & 0.300 & 1.44 & 0.39 & $\mathbf{W}$ \\
\hline RE & $i$ & 0.750 & 0.077 & 0.98 & 0.10 & A \\
\hline RF & 1 & 0.784 & 0.042 & 1.02 & 0.06 & A \\
\hline RG & 1 & 0.890 & 0.027 & 1.16 & 0.04 & A \\
\hline RI & 1 & 0.821 & 0.075 & 1.07 & 0.10 & A \\
\hline SC & 1 & 0.600 & 0.050 & 0.78 & 0.07 & $W$ \\
\hline SE & 1 & 0.830 & 0.040 & 1.08 & 0.06 & A \\
\hline SR & 1 & 0.832 & 0.116 & 1.09 & 0.15 & A \\
\hline SW & 1 & 2.950 & 0.270 & 3.85 & 0.36 & N \\
\hline TE & 1 & 0.800 & 0.100 & 1.04 & 0.13 & A \\
\hline $\mathrm{TI}$ & 1 & 0.770 & 0.160 & 1.01 & 0.21 & A \\
\hline TM & 1 & 0.855 & 0.420 & 1.12 & 0.55 & A \\
\hline TN & 1 & 0.731 & 0.037 & 0.95 & 0.05 & A \\
\hline TO & 1 & 1.710 & 0.343 & 2.23 & 0.45 & N \\
\hline TW & 1 & 0.740 & 0.020 & 0.97 & 0.03 & A \\
\hline$T X$ & 1 & 1.000 & 0.093 & 1.31 & 0.12 & $\mathbf{W}$ \\
\hline UK & 1 & 0.835 & 0.091 & 1.09 & 0.12 & A \\
\hline UP & 1 & 0.908 & 0.178 & 1.18 & 0.23 & A \\
\hline UY & 1 & 1.300 & 0.230 & 1.70 & 0.30 & N \\
\hline WA & 1 & 0.730 & 0.110 & 0.95 & 0.14 & A \\
\hline WC & 1 & 0.952 & 0.185 & 1.24 & 0.24 & W \\
\hline WN & 1 & 0.770 & 0.090 & 1.01 & 0.12 & A \\
\hline YA & 1 & 0.756 & 0.021 & 0.99 & 0.03 & A \\
\hline
\end{tabular}

Total Number Reported: 74

Units for matrices: $\mathrm{Al}=\mathrm{Bq}$ /filter $\mathrm{SO}=\mathrm{Bq} / \mathrm{kg}$ VE=Bq/kg $W A=\mathrm{Bq} / \mathrm{L}$. Values for elemental uranium are reported in $\mu$ glfilter, g, or mL. 
QAP44 Results by Nuclide

Matrix: WA

Radionuclide: 6060

EML Value: 32.800

EML Error: 0.580

\begin{tabular}{|c|c|c|c|c|c|c|}
\hline Lahrode & Test\# & $\begin{array}{c}\text { Reported } \\
\text { Value }\end{array}$ & $\begin{array}{c}\text { Reported } \\
\text { Errar }\end{array}$ & $\frac{\text { Reported }}{\text { EMI }}$ & $\begin{array}{l}\text { Ratio } \\
\text { Frror }\end{array}$ & Evaluation \\
\hline
\end{tabular}

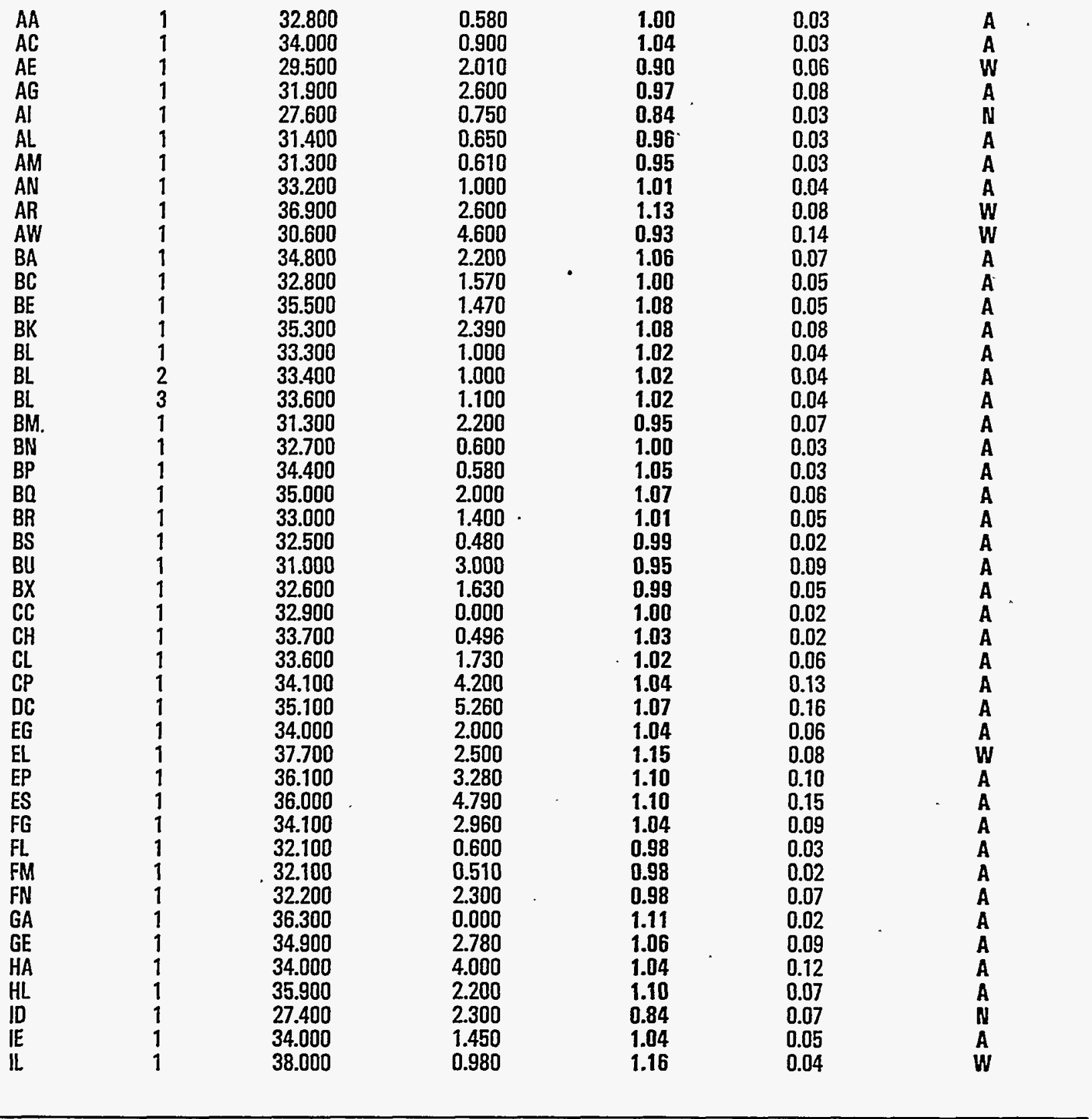

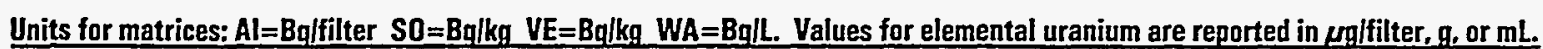


QAP44 Results by Nuclide

Matrix: WA

Radionuclide: 6060

EML Value: 32.800

EML Error: 0.580

\begin{tabular}{|c|c|c|c|c|c|c|}
\hline Lahcorle & Iest\# & $\begin{array}{c}\text { Reported } \\
\text { Value }\end{array}$ & $\begin{array}{c}\text { Reported } \\
\text { Error }\end{array}$ & $\frac{\text { Reported }}{\text { EMML }}$ & $\begin{array}{l}\text { Ratio } \\
\text { Errar }\end{array}$ & Evaluation \\
\hline 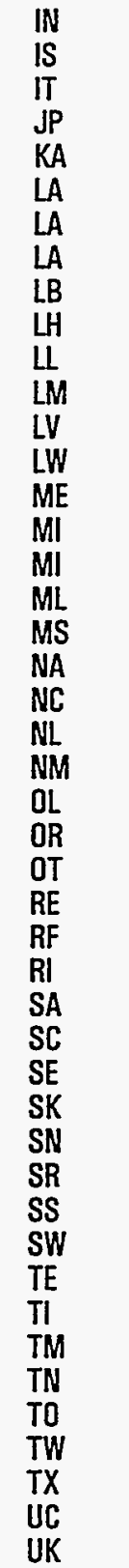 & 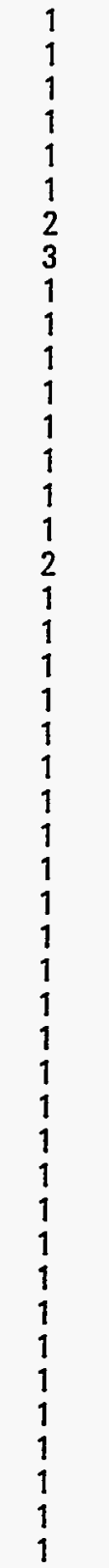 & $\begin{array}{r}35.600 \\
33.800 \\
37.000 \\
33.000 \\
33.000 \\
35.800 \\
37.300 \\
37.100 \\
36.300 \\
31.600 \\
30.800 \\
45.200 \\
32.300 \\
30.200 \\
34.700 \\
31.200 \\
36.300 \\
32.200 \\
34.000 \\
32.900 \\
33.700 \\
34.600 \\
34.500 \\
32.800 \\
32.000 \\
34.000 \\
33.200 \\
33.900 \\
3.360 \\
32.000 \\
34.000 \\
34.200 \\
34.300 \\
32.400 \\
34.000 \\
33.600 \\
31.800 \\
33.600 \\
31.000 \\
34.300 \\
35.600 \\
30.700 \\
32.000 \\
37.600 \\
35.100 \\
36.100\end{array}$ & $\begin{array}{r}0.500 \\
1.600 \\
3.000 \\
0.800 \\
1.000 \\
3.600 \\
4.000 \\
4.000 \\
23.100 \\
3.500 \\
4.000 \\
2.600 \\
0.700 \\
3.200 \\
0.912 \\
0.001 \\
0.001 \\
6.600 \\
3.400 \\
0.410 \\
1.300 \\
0.900 \\
0.300 \\
1.160 \\
1.000 \\
1.000 \\
3.980 \\
1.100 \\
0.160 \\
2.000 \\
1.000 \\
1.120 \\
0.700 \\
2.950 \\
1.000 \\
1.900 \\
0.570 \\
1.000 \\
3.000 \\
1.250 \\
1.990 \\
0.693 \\
0.600 \\
0.700 \\
0.700 \\
0.760\end{array}$ & $\begin{array}{l}1.09 \\
1.03 \\
1.13 \\
1.01 \\
1.01 \\
1.09 \\
1.14 \\
1.13 \\
1.11 \\
0.96 \\
0.94 \\
1.38 \\
0.99 \\
0.92 \\
1.06 \\
0.95 \\
1.11 \\
0.98 \\
1.04 \\
1.00 \\
1.03 \\
1.05 \\
1.05 \\
1.00 \\
0.98 \\
1.04 \\
1.01 \\
1.03 \\
0.10 \\
0.98 \\
1.04 \\
1.04 \\
1.05 \\
0.99 \\
1.04 \\
1.02 \\
0.97 \\
1.02 \\
0.95 \\
1.05 \\
1.09 \\
0.94 \\
0.98 \\
1.15 \\
1.07 \\
1.10\end{array}$ & \begin{tabular}{l}
0.02 \\
0.05 \\
0.09 \\
0.03 \\
0.04 \\
0.11 \\
0.12 \\
0.12 \\
0.71 \\
0.11 \\
0.12 \\
0.08 \\
0.03 \\
0.10 \\
0.03 \\
0.02 \\
0.02 \\
0.20 \\
0.11 \\
0.02 \\
0.04 \\
0.03 \\
0.02 \\
0.04 \\
0.04 \\
0.04 \\
0.12 \\
0.04 \\
0.01 \\
0.06 \\
0.04 \\
0.04 \\
0.03 \\
0.09 \\
0.04 \\
0.06 \\
0.02 \\
0.04 \\
\hdashline
\end{tabular} & $\begin{array}{l}A \\
A \\
W \\
A \\
A \\
A \\
A \\
W \\
W \\
A \\
A \\
W \\
N \\
A \\
W \\
A \\
A \\
A \\
A \\
A \\
A \\
A \\
A \\
A \\
A \\
A \\
A \\
A \\
A \\
N \\
A \\
A \\
A \\
A \\
A \\
A \\
A \\
A \\
A \\
A \\
A \\
A \\
A \\
W \\
A \\
W \\
A \\
A\end{array}$ \\
\hline
\end{tabular}

Units for matrices: $\mathrm{Al}=\mathrm{Bq} / \mathrm{filter} \mathrm{SO}=\mathrm{Bq} / \mathrm{kg} \mathrm{VE}=\mathrm{Bg} / \mathrm{kg}$ WA=Bq/L. Values for elemental uranium are reported in $\mu \mathrm{g} /$ filter, $\mathrm{g}$. or $\mathrm{mL}$. Evaluation: $A=A c c e p t a b l e, W=A c c e p t a b l e$ with Warning, $N=$ Not Acceptable. $\mathrm{pCi}=\mathrm{Bq} \times 27$ 


\section{QAP44 Results by Nuclide}

Matrix: WA

Radionuclide: $\mathbf{C O} 60$

EML Value: 32.800

EML Error: 0.580

\begin{tabular}{|c|c|c|c|c|c|c|}
\hline Lahende & Iest\# & $\begin{array}{c}\text { Reported } \\
\text { Value }\end{array}$ & $\begin{array}{c}\text { Reported } \\
\text { Error }\end{array}$ & $\frac{\text { Reported }}{\text { EML }}$ & $\begin{array}{l}\text { Ratio } \\
\text { Frror }\end{array}$ & Evaluation \\
\hline UP & 1 & 33.600 & 1.180 & 1.02 & 0.04 & A \\
\hline UY & 1 & 32.000 & 2.500 & 0.98 & 0.08 & A \\
\hline WA & 1 & 33.800 & 1.400 & 1.03 & 0.05 & A \\
\hline WC & 1 & 34.300 & 2.660 & 1.05 & 0.08 & A \\
\hline WE & 1 & 30.900 & 1.170 & 0.94 & 0.04 & $W$ \\
\hline Wl & 1 & 35.500 & 2.720 & 1.08 & 0.09 & A \\
\hline WN & 1 & 30.400 & 0.300 & 0.93 & 0.02 & $W$ \\
\hline WP & 1 & 32.800 & 1.400 & 1.00 & 0.05 & A \\
\hline WV & $i$ & 32.900 & 0.880 & 1.00 & 0.03 & A \\
\hline YA & 1 & 32.000 & 0.450 & 0.98 & 0.02 & A \\
\hline
\end{tabular}

Total Number Reported: 101

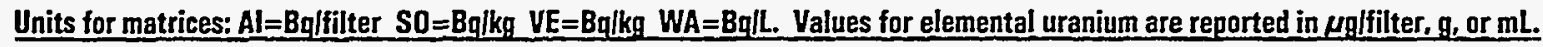




\section{QAP44 Results by Nuclide}

Matrix: WA

Radionuclide: CS137

EML Value: 38.300

EML Error: 0.881

\begin{tabular}{|c|c|c|c|c|c|}
\hline Lahconde. & Test\# & $\begin{array}{c}\text { Reported } \\
\text { Value }\end{array}$ & $\begin{array}{l}\text { Reported } \\
\text { Error }\end{array}$ & $\frac{\text { Reported }}{\text { EML }}$ & $\begin{array}{l}\text { Ratio } \\
\text { Frrar }\end{array}$ \\
\hline
\end{tabular}

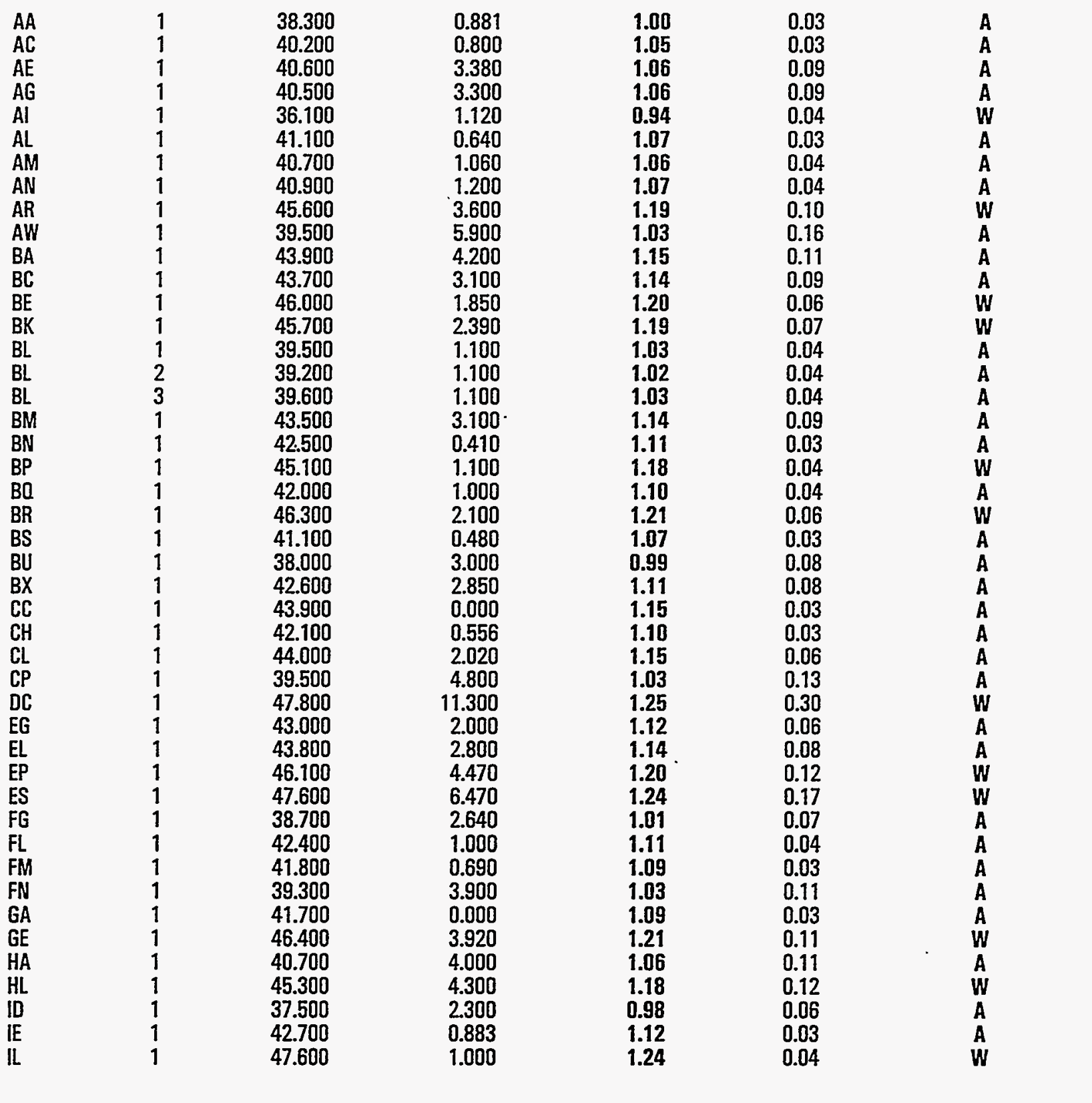

Units for matrices: $\mathrm{Al}=\mathrm{Bq}$ lfilter $\mathrm{SO}=\mathrm{Bq} / \mathrm{kg}$ VE=Bqlkg $\mathrm{WA}=\mathrm{Bq} / \mathrm{L}$. Values for elemental uranium are reported in $\mu \mathrm{g} /$ filter, $\mathrm{g}$, or $\mathrm{mL}$.

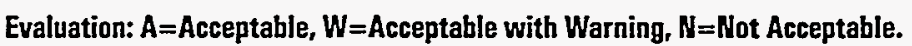
$\mathrm{pCi}=\mathrm{Bq} \times 27$ 
Matrix: WA

Radionuclide: CS137

EML Value: 38.300

EML Error: 0.881

\begin{tabular}{|c|c|c|c|c|c|c|}
\hline Lahonde & Test\# & $\begin{array}{c}\text { Reported } \\
\text { Value }\end{array}$ & $\begin{array}{c}\text { Reported } \\
\text { Error }\end{array}$ & $\frac{\text { Reported }}{\text { FML }}$ & $\begin{array}{l}\text { Ratio } \\
\text { Error }\end{array}$ & Evaluation \\
\hline 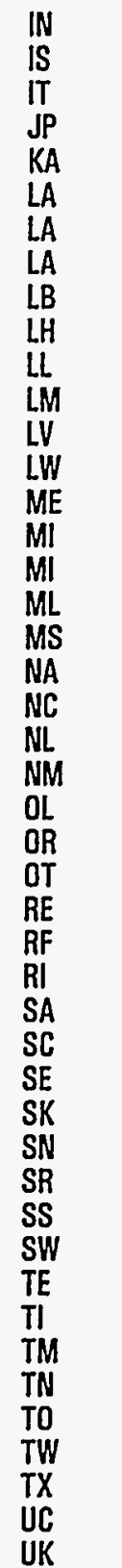 & 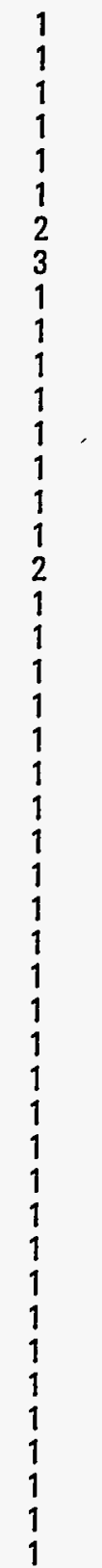 & $\begin{array}{r}42.800 \\
46.100 \\
46.200 \\
43.000 \\
42.000 \\
50.200 \\
49.400 \\
50.000 \\
46.200 \\
41.800 \\
40.200 \\
58.000 \\
43.300 \\
40.800 \\
44.500 \\
0.337 \\
40.900 \\
40.000 \\
40.900 \\
41.000 \\
39.100 \\
42.900 \\
46.900 \\
42.000 \\
40.000 \\
43.000 \\
41.800 \\
42.200 \\
42.300 \\
42.000 \\
40.000 \\
43.600 \\
42.000 \\
38.000 \\
44.000 \\
41.800 \\
39.500 \\
42.800 \\
42.000 \\
44.800 \\
44.300 \\
39.500 \\
40.000 \\
47.400 \\
41.500 \\
43.200\end{array}$ & $\begin{array}{r}1.200 \\
3.100 \\
4.100 \\
1.200 \\
2.000 \\
5.100 \\
5.000 \\
5.100 \\
22.200 \\
6.400 \\
3.870 \\
2.800 \\
0.700 \\
3.200 \\
1.750 \\
0.002 \\
0.002 \\
8.200 \\
4.100 \\
0.560 \\
1.020 \\
0.800 \\
0.500 \\
1.370 \\
1.000 \\
1.000 \\
5.010 \\
1.600 \\
2.200 \\
3.000 \\
1.000 \\
2.140 \\
1.000 \\
3.420 \\
1.000 \\
1.800 \\
0.750 \\
1.300 \\
4.000 \\
1.820 \\
2.040 \\
0.953 \\
2.000 \\
0.700 \\
1.690 \\
0.990\end{array}$ & $\begin{array}{l}1.12 \\
1.20 \\
1.21 \\
1.12 \\
1.10 \\
1.31 \\
1.29 \\
1.31 \\
1.21 \\
1.09 \\
1.05 \\
1.51 \\
1.13 \\
1.07 \\
1.16 \\
0.01 \\
1.07 \\
1.04 \\
1.07 \\
1.07 \\
1.02 \\
1.12 \\
1.23 \\
1.10 \\
1.04 \\
1.12 \\
1.09 \\
1.10 \\
1.10 \\
1.10 \\
1.04 \\
1.14 \\
1.10 \\
0.99 \\
1.15 \\
1.09 \\
1.03 \\
1.12 \\
1.10 \\
1.17 \\
1.16 \\
1.03 \\
1.04 \\
1.24 \\
1.08 \\
1.13\end{array}$ & \begin{tabular}{l}
0.04 \\
0.09 \\
0.11 \\
0.04 \\
0.06 \\
0.14 \\
0.13 \\
0.14 \\
0.58 \\
0.17 \\
0.10 \\
0.08 \\
0.03 \\
0.09 \\
0.05 \\
0.00 \\
0.02 \\
0.22 \\
0.11 \\
0.03 \\
0.04 \\
0.03 \\
0.03 \\
0.04 \\
0.04 \\
0.04 \\
0.13 \\
0.05 \\
0.06 \\
0.08 \\
0.04 \\
0.06 \\
\hdashline
\end{tabular} & $\begin{array}{l}A \\
W \\
W \\
A \\
A \\
A \\
N \\
N \\
W \\
W \\
A \\
A \\
N \\
A \\
A \\
W \\
N \\
A \\
A \\
A \\
A \\
A \\
A \\
W \\
A \\
A \\
A \\
A \\
A \\
A \\
A \\
A \\
A \\
A \\
A \\
A \\
A \\
A \\
A \\
A \\
W \\
W \\
A \\
A \\
W \\
A \\
A\end{array}$ \\
\hline
\end{tabular}

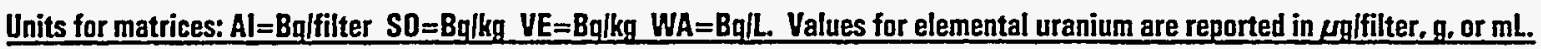
Evaluation: $A=A$ cceptable, $W=$ Acceptable with Warning, $N=$ Not Acceptable.

$\mathrm{pCi}=\mathrm{Bq} \times 27$ 


\section{QAP44 Results by Nuclide}

Matrix: WA

Radionuclide: CS137

EML Value: 38.300

EML Error: 0.881

\begin{tabular}{|c|c|c|c|c|c|c|}
\hline Lahonde & Test \# & $\begin{array}{c}\text { Repported } \\
\text { Yalue }\end{array}$ & $\begin{array}{c}\text { Reported } \\
\text { Errar }\end{array}$ & $\frac{\text { Reported }}{\text { EMI }}$ & $\begin{array}{l}\text { Ratio } \\
\text { Frrar }\end{array}$ & Evaluation \\
\hline $\begin{array}{l}\text { UP } \\
\text { UY } \\
\text { WA } \\
W C \\
W E \\
W I \\
W N \\
W P \\
W V \\
Y A\end{array}$ & $\begin{array}{l}1 \\
1 \\
1 \\
1 \\
1 \\
1 \\
1 \\
1 \\
1 \\
1\end{array}$ & $\begin{array}{l}43.600 \\
37.700 \\
43.100 \\
44.900 \\
39.000 \\
44.300 \\
41.200 \\
41.500 \\
40.900 \\
38.100\end{array}$ & $\begin{array}{l}3.020 \\
2.900 \\
3.700 \\
6.140 \\
1.080 \\
6.230 \\
0.300 \\
1.400 \\
0.850 \\
0.430\end{array}$ & $\begin{array}{l}1.14 \\
0.98 \\
1.13 \\
1.17 \\
1.02 \\
1.16 \\
1.08 \\
1.08 \\
1.07 \\
1.00\end{array}$ & $\begin{array}{l}0.08 \\
0.08 \\
0.10 \\
0.16 \\
0.04 \\
0.17 \\
0.03 \\
0.04 \\
0.03 \\
0.03\end{array}$ & $\begin{array}{l}A \\
A \\
A \\
W \\
A \\
W \\
A \\
A \\
A \\
A\end{array}$ \\
\hline
\end{tabular}

Total Number Reported: 101

Units for matrices: $\mathrm{Al}=\mathrm{Bq}$ lfilter $\mathrm{SO}=\mathrm{Bq} / \mathrm{kg}$ VE $=\mathrm{Bq} / \mathrm{kg}, W \mathrm{~A}=\mathrm{Bq} q \mathrm{~L}$. Values for elemental uranium are reported in $\mu \mathrm{glfilter}$, g, or $\mathrm{mL}$.

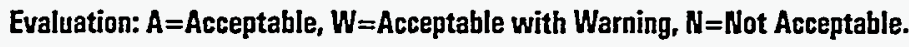
$\mathrm{pCi}=\mathrm{Bq} \times 27$ 


\section{QAP44 Results by Nuclide}

Matrix: WA

Radionuclide: FE 55

EML Value: 83.000

EML Error: 3.440

\begin{tabular}{|c|c|c|c|c|c|c|}
\hline Lahcode & Test\# & $\begin{array}{c}\text { Reported } \\
\text { Value }\end{array}$ & $\begin{array}{c}\text { Reported } \\
\text { Frror }\end{array}$ & $\frac{\text { Reported }}{\text { FMI }}$ & $\begin{array}{l}\text { Ratio } \\
\text { Frror }\end{array}$ & Evaluatinn \\
\hline
\end{tabular}

$\begin{array}{llrrrrr}\text { AA } & 1 & 83.000 & 3.440 & 1.00 & 0.06 & \text { A } \\ \text { BL } & 1 & 77.000 & 4.000 & 0.93 & 0.06 & \text { A } \\ \text { BL } & 2 & 76.000 & 5.000 & 0.92 & 0.07 & \text { A } \\ \text { BL } & 3 & 75.000 & 4.000 & 0.90 & 0.06 & \text { A } \\ \text { BX } & 1 & 57.000 & 4.660 & 0.69 & 0.06 & \text { W } \\ \text { EG } & 1 & 62.000 & 17.000 & 0.75 & 0.21 & \text { W } \\ \text { GE } & 1 & 112.000 & 21.900 & 1.35 & 0.27 & \text { W } \\ \text { HA } & 1 & 81.400 & 0.900 & 0.98 & 0.04 & \text { A } \\ \text { KA } & 1 & 108.000 & 6.000 & 1.30 & 0.09 & \text { W } \\ \text { LH } & 1 & 68.900 & 12.400 & 0.83 & 0.15 & \text { A } \\ \text { TE } & 1 & 109.000 & 21.700 & 1.31 & 0.27 & \text { W } \\ \text { TI } & 1 & 110.000 & 20.000 & 1.33 & 0.25 & \text { W } \\ \text { TN } & 1 & 94.700 & 3.440 & 1.14 & 0.06 & \text { A }\end{array}$

Total Number Reported: 13

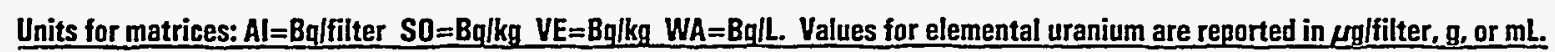
Evaluation: $A=$ Acceptable, $W=$ Acceptable with Warning, $N=$ Not Acceptable. 
QAP44 Results by Nuclide

Matrix: WA

Radionuclide: GA 1

EML Value: 1850.000

EML Error: 185.000

\begin{tabular}{|c|c|c|c|c|c|c|}
\hline Lahende & Test \# & $\begin{array}{c}\text { Reported } \\
\text { Value }\end{array}$ & $\begin{array}{c}\text { Reported } \\
\text { Error }\end{array}$ & $\frac{\text { Reported }}{\text { FMI }}$ & $\begin{array}{l}\text { Ratio } \\
\text { Error }\end{array}$ & Fualuation \\
\hline
\end{tabular}

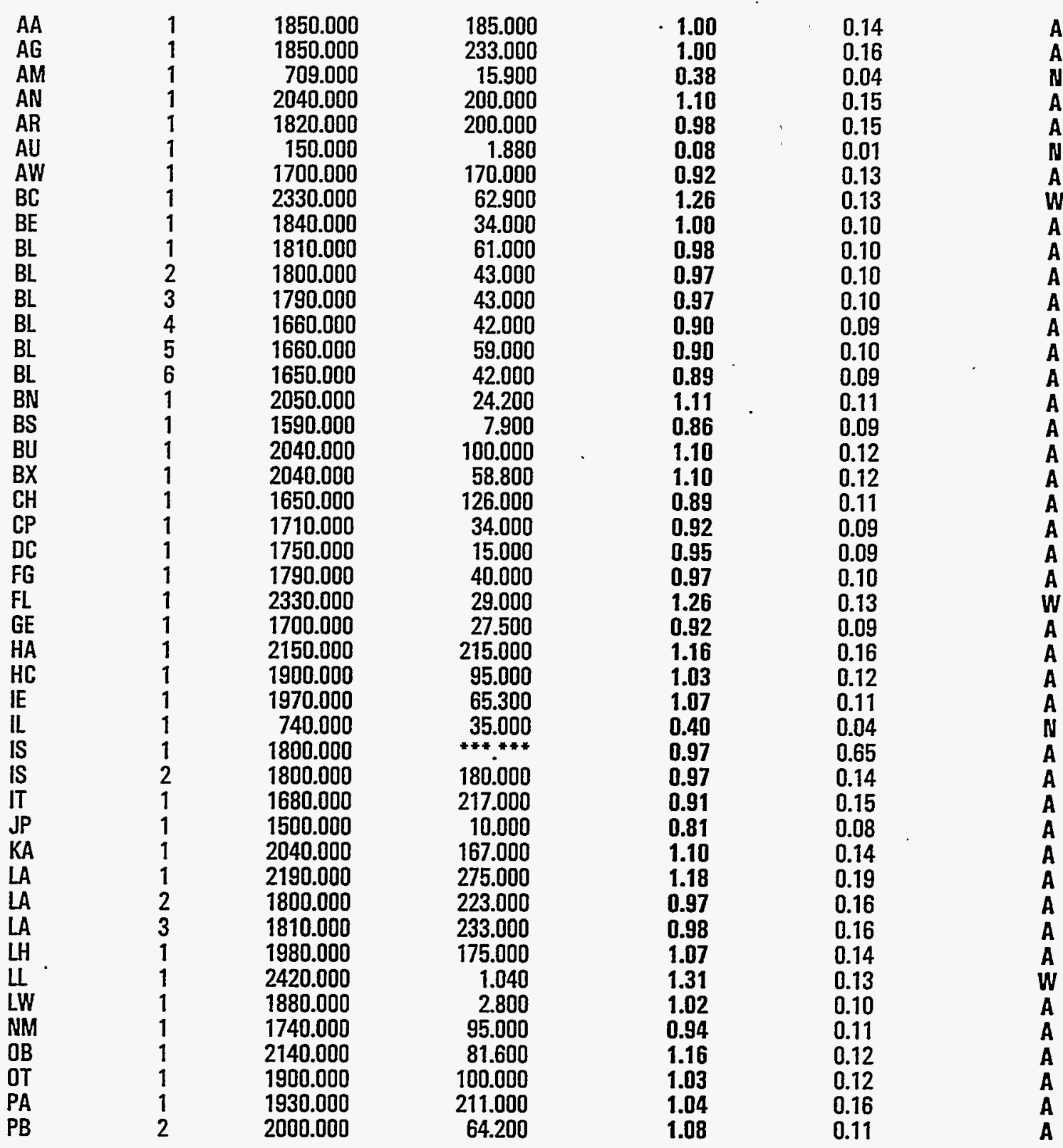

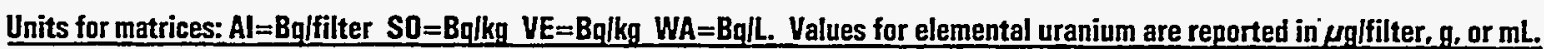
Evaluation: $A=A c c e p t a b l e, ~ W=A c c e p t a b l e$ with Warning, $N=$ Not Acceptable.

$\mathrm{pCi}=\mathrm{Bq} \times 27$ 


\section{OAP44 Results by Nuclide}

Matrix: WA

Radionuclide: GA 1

EML Value: 1850.000

EML Error: 185.000

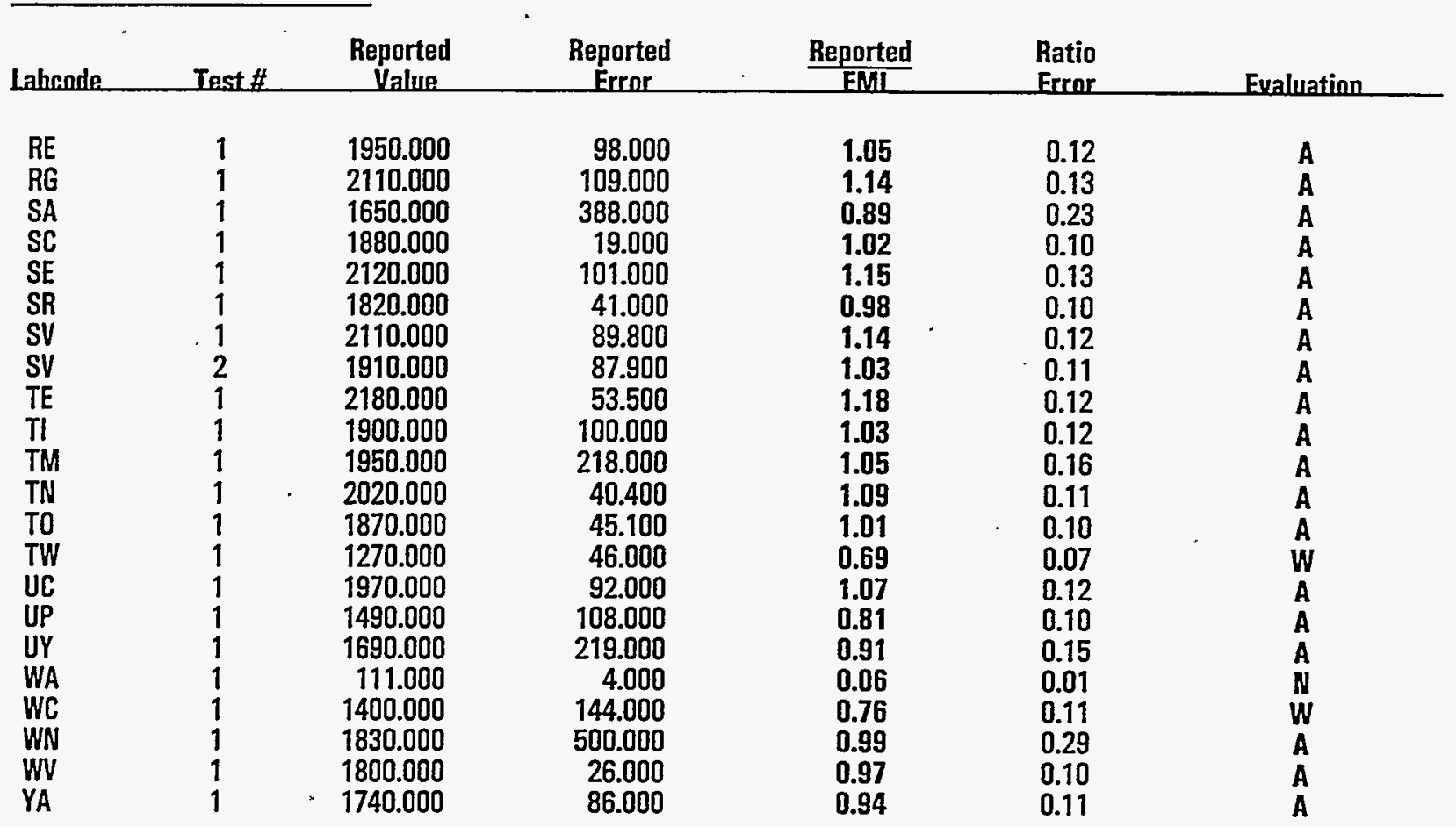

Total Number Reported: 67

Units for matrices: $A l=B q$ ffilter $S O=B q l k g V E=B q l k g, W A=B q l L$. Values for elemental uranium are reported in $\mu \mathrm{g} /$ filter, $g$, or $\mathrm{mL}$.

$\mathrm{pCi}=\mathrm{Bq} \times 27$ 
QAP44 Results by Nuclide

Matrix: WA

Radionuclide: GB 2

EML Value: 744.000

EML Error: 74.000

\begin{tabular}{cccccc} 
Lahoode & Reported & Reported & Reported & Ratio \\
Value & Error & $\frac{\text { FML }}{\text { Error }}$ & Evaluation \\
\hline
\end{tabular}

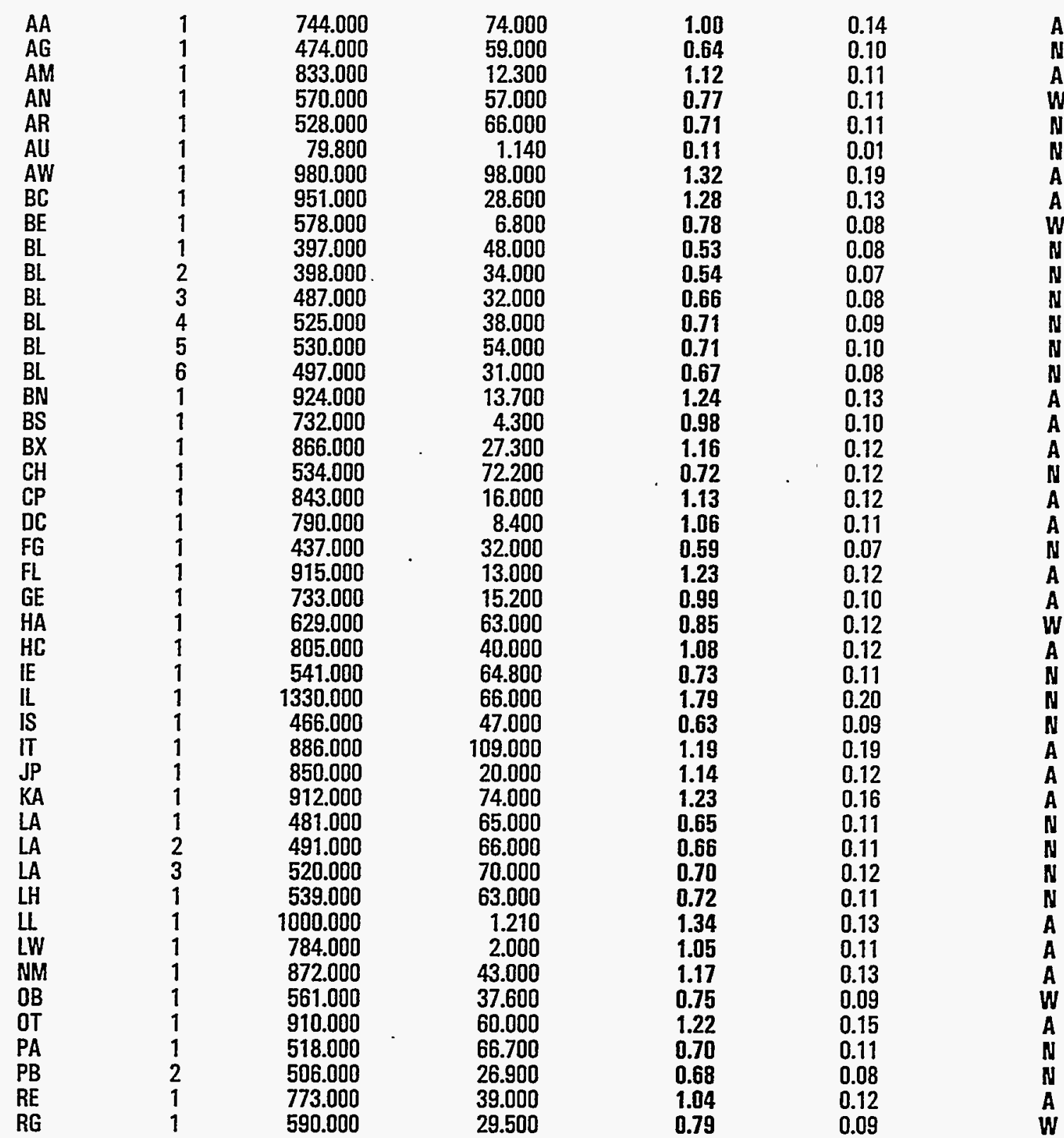

Units for matrices: $\mathrm{Al}=\mathrm{Bq}$ ffilter $\mathrm{SO}=\mathrm{Bq} / \mathrm{kg}$ VE=Bqlkg $\mathrm{WA}=\mathrm{Bq} / \mathrm{L}$. Values for elemental uranium are reported in $\mu \mathrm{g} \mid$ filter, $\mathrm{g}$, or $\mathrm{mL}$. Evaluation: $A=A c c e p t a b l e, W=A c c e p t a b l e$ with Warning, $N=$ Not Acceptable.

$\mathrm{pCi}=\mathrm{Bq} \times 27$ 


\section{QAP44 Results by Nuclide}

Matrix: WA

Radionuclide: GB 2

EML Value: 744.000

EML Error: 74.000

\begin{tabular}{|c|c|c|c|c|c|c|}
\hline Lahende & Iest\# & $\begin{array}{c}\text { Reported } \\
\text { Value }\end{array}$ & $\begin{array}{c}\text { Reported } \\
\text { Errar }\end{array}$ & $\frac{\text { Reported }}{\text { EMI }}$ & $\begin{array}{l}\text { Ratio } \\
\text { Error }\end{array}$ & Evaluation \\
\hline SA & 1 & 1090.000 & 200.000 & 1.47 & 0.31 & A \\
\hline SC & 1 & 495.000 & 10.000 & 0.67 & 0.07 & N \\
\hline SE & 1 & 881.000 & 40.900 & 1.18 & 0.13 & A \\
\hline SR & 1 & 600.000 & 18.000 & 0.81 & 0.08 & $w$ \\
\hline SV & 1 & 874.000 & 41.800 & 1.17 & 0.13 & A. \\
\hline SV & 2 & 930.000 & 43.100 & 1.25 & 0.14 & A \\
\hline TE & 1 & 872.000 & 27.000 & 1.17 & 0.12 & A \\
\hline TI & 1 & 530.000 & 50.000 & 0.71 & 0.10 & N \\
\hline TM & 1 & 877.000 & 69.200 & 1.18 & 0.15 & A \\
\hline TN & 1 & 590.000 & 15.200 & 0.79 & 0.08 & $\mathbf{W}$ \\
\hline TN & 2 & 590.000 & 15.200 & 0.79 & 0.08 & $\mathbf{W}$ \\
\hline TO & 1 & 903.000 & 25.200 & 1.21 & 0.13 & A \\
\hline TW & 1 & 610.000 & 25.000 & 0.82 & 0.09 & $\mathbf{w}$ \\
\hline UC & 1 & 634.000 & 28.000 & 0.85 & 0.09 & $\mathbf{W}$ \\
\hline UP & 1 & 700.000 & 67.200 & 0.94 & 0.13 & $\ddot{A}$ \\
\hline UY & 1 & 794.000 & 112.000 & 1.07 & 0.18 & A \\
\hline WA & 1 & 44.900 & 2.000 & 0.06 & 0.01 & $\mathbf{N}$ \\
\hline WC & 1 & 655.000 & 67.500 & 0.88 & 0.13 & $W$ \\
\hline WN & 1 & 960.000 & 100.000 & 1.29 & 0.19 & A \\
\hline WV & 1 & 703.000 & 14.000 & 0.95 & 0.10 & A \\
\hline YA & 1 & 588.000 & 12.000 & 0.79 & 0.08 & $\mathbf{W}$ \\
\hline
\end{tabular}

Total Number Reported: 66

Units for matrices: $A l=B q \mid$ filter $S O=B q / k q V E=B q / k g \quad W A=B q / L$. Values for elemental uranium are reported in $\mu \mathrm{g} /$ filter, $g$, or $\mathrm{mL}$. 
QAP44 Results by Nuclide

Matrix: WA

Radionuclide: H 3

EML Value: 251.000

EML Error: 11.400

\begin{tabular}{|c|c|c|c|c|c|c|}
\hline Lahende & Test \# & $\begin{array}{l}\text { Reported } \\
\text { Value }\end{array}$ & $\begin{array}{c}\text { Reported } \\
\text { Errar }\end{array}$ & $\frac{\text { Reported }}{\text { EMIL }}$ & $\begin{array}{l}\text { Ratio } \\
\text { Frror }\end{array}$ & Evaluation \\
\hline
\end{tabular}

\begin{tabular}{|c|c|c|c|c|c|c|}
\hline $\begin{array}{l}A A \\
A E \\
A G \\
A I \\
A N \\
A R \\
A U \\
A W \\
B E \\
B L \\
B L \\
B L \\
B N \\
B P \\
B U \\
B X \\
C C \\
C H \\
C L \\
D C \\
E G \\
E I \\
E L \\
E P \\
E S \\
F G \\
F L \\
F N \\
G E \\
H A \\
H C \\
H L \\
I D \\
I E \\
I S \\
I T \\
J P \\
K A \\
L A \\
L A \\
L A \\
L H \\
L L \\
L M \\
L W\end{array}$ & $\begin{array}{l}1 \\
1 \\
1 \\
1 \\
1 \\
1 \\
1 \\
1 \\
1 \\
1 \\
2 \\
3 \\
1 \\
1 \\
1 \\
1 \\
1 \\
1 \\
1 \\
1 \\
1 \\
1 \\
1 \\
1 \\
1 \\
1 \\
1 \\
1 \\
1 \\
1 \\
1 \\
1 \\
1 \\
1 \\
1 \\
1 \\
1 \\
1 \\
1 \\
2 \\
3 \\
1 \\
1 \\
1 \\
1\end{array}$ & $\begin{array}{l}251.000 \\
212.000 \\
195.000 \\
400.000 \\
220.000 \\
224.000 \\
246.000 \\
220.000 \\
206.000 \\
221.000 \\
233.000 \\
248.000 \\
213.000 \\
187.000 \\
226.000 \\
212.000 \\
156.000 \\
227.000 \\
201.000 \\
244.000 \\
180.000 \\
174.000 \\
330.000 \\
222.000 \\
204.000 \\
284.000 \\
185.000 \\
228.000 \\
197.000 \\
226.000 \\
206.000 \\
214.000 \\
216.000 \\
208.000 \\
309.000 \\
209.000 \\
220.000 \\
239.000 \\
238.000 \\
221.000 \\
212.000 \\
214.000 \\
217.000 \\
244.000 \\
228.000\end{array}$ & $\begin{array}{r}11.400 \\
13.800 \\
29.000 \\
45.000 \\
7.000 \\
9.000 \\
18.000 \\
22.000 \\
20.500 \\
18.000 \\
18.000 \\
18.000 \\
7.970 \\
22.000 \\
5.000 \\
23.200 \\
0.000 \\
2.610 \\
11.500 \\
27.300 \\
30.000 \\
4.000 \\
25.000 \\
7.990 \\
36.600 \\
15.000 \\
2.710 \\
9.000 \\
7.770 \\
30.000 \\
14.400 \\
8.100 \\
13.000 \\
55.200 \\
31.000 \\
6.100 \\
1.000 \\
13.000 \\
11.200 \\
11.000 \\
10.900 \\
17.000 \\
1.600 \\
24.000 \\
6.200\end{array}$ & $\begin{array}{l}1.00 \\
0.85 \\
0.78 \\
1.59 \\
0.88 \\
0.89 \\
0.98 \\
0.88 \\
0.82 \\
0.88 \\
0.93 \\
0.99 \\
0.85 \\
0.75 \\
0.90 \\
0.85 \\
0.62 \\
0.90 \\
0.80 \\
0.97 \\
0.72 \\
0.69 \\
1.32 \\
0.88 \\
0.81 \\
1.13 \\
0.74 \\
0.91 \\
0.79 \\
0.90 \\
0.82 \\
0.85 \\
0.86 \\
0.83 \\
1.23 \\
0.83 \\
0.88 \\
0.95 \\
0.95 \\
0.88 \\
0.85 \\
0.85 \\
0.87 \\
0.97 \\
0.91\end{array}$ & $\begin{array}{l}0.06 \\
0.07 \\
0.12 \\
0.19 \\
0.05 \\
0.05 \\
0.08 \\
0.10 \\
0.09 \\
0.08 \\
0.08 \\
0.08 \\
0.05 \\
0.09 \\
0.05 \\
0.10 \\
0.03 \\
0.04 \\
0.06 \\
0.12 \\
0.12 \\
0.04 \\
0.12 \\
0.05 \\
0.15 \\
0.08 \\
0.04 \\
0.05 \\
0.05 \\
0.13 \\
0.07 \\
0.05 \\
0.06 \\
0.22 \\
0.14 \\
0.05 \\
0.04 \\
0.07 \\
0.06 \\
0.06 \\
0.06 \\
0.08 \\
0.04 \\
0.11 \\
0.05\end{array}$ & $\begin{array}{l}A \\
A \\
W \\
W \\
W \\
A \\
A \\
A \\
A \\
W \\
A \\
A \\
A \\
A \\
W \\
A \\
A \\
W \\
A \\
W \\
A \\
W \\
W \\
W \\
A \\
W \\
A \\
W \\
A \\
W \\
A \\
W \\
A \\
A \\
W \\
W \\
W \\
A \\
A \\
A \\
A \\
A \\
A \\
A \\
A \\
A\end{array}$ \\
\hline
\end{tabular}

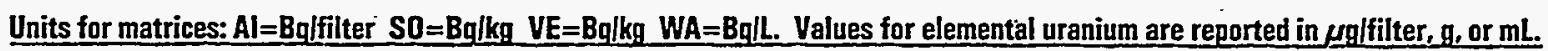
Evaluation: $A=A c c e p t a b l e, W=A c c e p t a b l e$ with Warning, $N=N$ ot Acceptable. $\mathbf{p C i}=\mathbf{B q} \times 27$ 
QAP44 Results by Nuclide

Matrix: WA

Radionuclide: H 3

\section{EML Value: 251.000}

EML. Error: 11.400

\begin{tabular}{|c|c|c|c|c|c|c|}
\hline Lahande & Test\# & $\begin{array}{c}\text { Reported } \\
\text { Value }\end{array}$ & $\begin{array}{c}\text { Reported } \\
\text { Error }\end{array}$ & $\frac{\text { Reported }}{\text { FML }}$ & $\begin{array}{l}\text { Ratio } \\
\text { Error }\end{array}$ & Evaluatinn. \\
\hline MI & 1 & 405.000 & 0.000 & 1.61 & 0.07 & $W$ \\
\hline MI & 2 & 385.000 & 0.000 & 1.53 & 0.07 & $W$ \\
\hline MI & 3 & 385.000 & 0.000 & 1.53 & 0.07 & $W$ \\
\hline MI & 4 & 385.000 & 0.000 & 1.53 & 0.07 & $W$ \\
\hline$M L$ & 1 & 197.000 & 20.700 & 0.79 & 0.09 & W \\
\hline NA & 1 & 199.000 & 4.200 & 0.79 & 0.04 & $W$ \\
\hline OD & 1 & 207.000 & 15.700 & 0.83 & 0.07 & $W$ \\
\hline OR & 1 & 220.000 & 30.000 & 0.88 & 0.13 & A \\
\hline OT & 1 & 370.000 & 30.000 & 1.47 & 0.14 & $W$ \\
\hline PA & 1 & 222.000 & 7.040 & 0.88 & 0.05 & A \\
\hline PB & $i$ & 222.000 & 11.700 & 0.88 & 0.06 & A \\
\hline $\mathrm{PI}$ & 1 & 214,000 & 11.000 & 0.85 & 0.06 & $\ddot{A}$ \\
\hline RE & $i$ & 208.000 & 20.000 & 0.83 & 0.09 & $w$ \\
\hline $\mathrm{RF}$ & 1 & 240.000 & 5.300 & 0.96 & 0.05 & A \\
\hline RG & 1 & 210.000 & 5.500 & 0.84 & 0.04 & $\ddot{A}$ \\
\hline $\mathrm{RI}$ & 1 & 304.000 & 18.000 & 1.21 & 0.09 & $\ddot{W}$ \\
\hline SC & $i$ & 227.000 & 7.000 & 0.90 & 0.05 & A \\
\hline SE & $i$ & 191.000 & 88.500 & 0.76 & 0.35 & $W$ \\
\hline SE & 2 & 191.000 & 5.960 & 0.76 & 0.04 & $\mathbf{W}$ \\
\hline SK & 1 & 223.000 & 3.000 & 0.89 & 0.04 & $A$ \\
\hline SR & $i$ & 214.000 & 5.400 & 0.85 & 0.04 & $\ddot{A}$ \\
\hline SV & 1 & 267.000 & 10.000 & 1.06 & 0.06 & A \\
\hline SW & 1 & 290.000 & 8.640 & 1.16 & 0.06 & A \\
\hline TE & 1 & 434.000 & 34.100 & 1.73 & 0.16 & $w$ \\
\hline $\mathrm{TI}$ & $i$ & 210.000 & 10.000 & 0.84 & 0.06 & $\bar{A}$ \\
\hline$T M$ & 1 & 206.000 & 10.900 & 0.82 & 0.06 & $W$ \\
\hline TN & 1 & 221.000 & 17.000 & 0.88 & 0.08 & A \\
\hline TO & 1 & 219.000 & 42.200 & 0.87 & 0.17 & A \\
\hline TW & 1 & 186.000 & 1.000 & 0.74 & 0.03 & $W$ \\
\hline TX & 1 & 209.000 & 11.000 & 0.83 & 0.06 & $W$ \\
\hline UP & $i$ & 227.000 & 15.400 & 0.90 & 0.07 & A \\
\hline UY & 1 & 161.000 & 23.000 & 0.64 & 0.10 & $\ddot{N}$ \\
\hline WA & 1 & 214.000 & 25.000 & 0.85 & 0.11 & $A$ \\
\hline WC & 1 & 186.000 & 20.500 & 0.74 & 0.09 & $w$ \\
\hline WN & 1 & 243.000 & 21.000 & 0.97 & 0.09 & A \\
\hline WP & 1 & 220.000 & 11.000 & 0.88 & 0.06 & A \\
\hline WV & 1 & 218.000 & 8.300 & 0.87 & 0.05 & $\ddot{A}$ \\
\hline YA & $i$ & 237.000 & 12.000 & 0.94 & 0.06 & A \\
\hline
\end{tabular}

Total Number Reported: 83

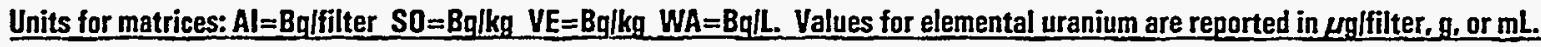


QAP44 Results by Nuclide

Matrix: WA

Radionuclide: MN 54

EML Value: 38.400

EML Error: 1.160

\begin{tabular}{|c|c|c|c|c|}
\hline ahcorde & Test \# & $\begin{array}{c}\text { Reported } \\
\text { Value }\end{array}$ & $\begin{array}{c}\text { Reported } \\
\text { Error }\end{array}$ & $\frac{\text { Reported }}{\text { FMI }}$ \\
\hline
\end{tabular}

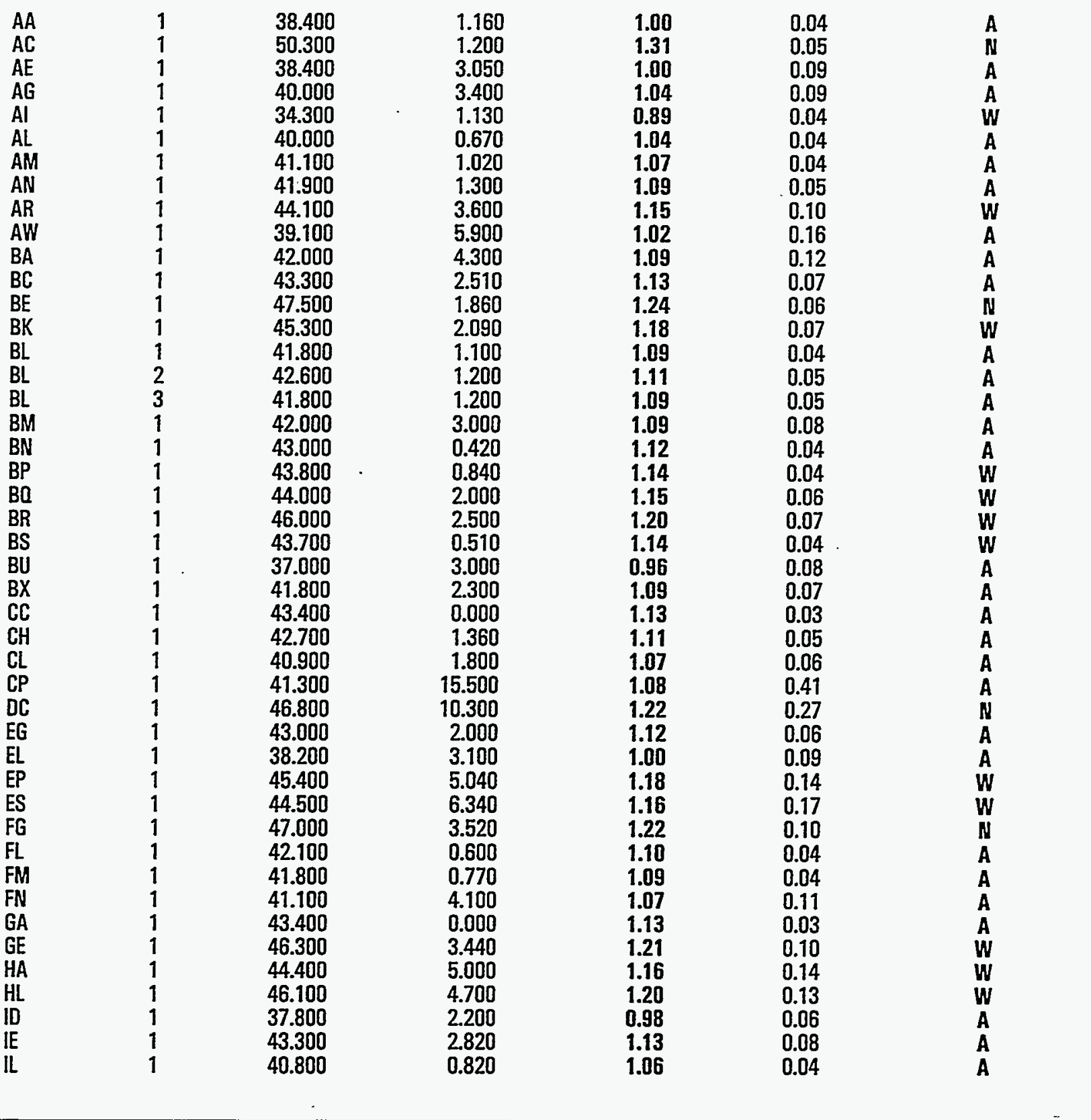

Units for matrices: $\mathrm{Al}=\mathrm{Bq}$ /filter $\mathrm{SO}=\mathrm{Bq} / \mathrm{kg}$ VE=Bqlkg $W \mathrm{~A}=\mathrm{Bq} / \mathrm{L}$. Values for elemental uranium are reported in $\mu \mathrm{g} /$ filter, $\mathrm{g}$, or $\mathrm{mL}$. Evaluation: $A=$ Acceptable, $W=$ Acceptable with Warning, $N=N$ Not Acceptable. $p C i=B q \times 27$ 


\section{QAP44 Results by Nuclide}

Matrix: WA

Radionuclide: MN 54

EML Value: 38.400

EML Error: 1.160

\begin{tabular}{|c|c|c|c|c|c|c|}
\hline Lahconde & Test\# & $\begin{array}{c}\text { Reported } \\
\text { Value }\end{array}$ & $\begin{array}{c}\text { Reported } \\
\text { Frror }\end{array}$ & $\frac{\text { Reported }}{\text { EMI }}$ & $\begin{array}{l}\text { Ratio } \\
\text { Error }\end{array}$ & Evaluation \\
\hline 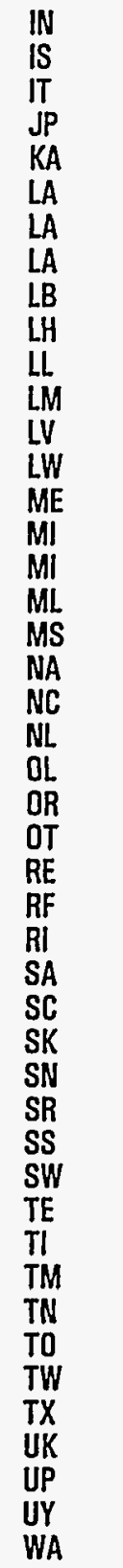 & $\begin{array}{l}1 \\
1 \\
1 \\
1 \\
1 \\
1 \\
1 \\
2 \\
3 \\
1 \\
1 \\
1 \\
1 \\
1 \\
1 \\
1 \\
1 \\
1 \\
1 \\
2 \\
1 \\
1 \\
1 \\
1 \\
1 \\
1 \\
1 \\
1 \\
1 \\
1 \\
1 \\
1 \\
1 \\
1 \\
1 \\
1 \\
1 \\
1 \\
1 \\
1 \\
1 \\
1 \\
1 \\
1 \\
1 \\
1 \\
1 \\
1 \\
1 \\
1 \\
1 \\
1 \\
1 \\
1 \\
1\end{array}$ & $\begin{array}{r}43.700 \\
46.500 \\
46.500 \\
42.000 \\
41.000 \\
49.400 \\
50.800 \\
49.500 \\
47.500 \\
41.500 \\
4.200 \\
58.500 \\
45.500 \\
40.200 \\
46.100 \\
36.100 \\
42.700 \\
41.100 \\
42.500 \\
41.300 \\
40.600 \\
46.200 \\
42.700 \\
41.000 \\
44.000 \\
41.300 \\
42.700 \\
38.700 \\
41.000 \\
41.000 \\
41.300 \\
41.100 \\
44.000 \\
41.700 \\
39.900 \\
41.900 \\
41.000 \\
44.200 \\
45.100 \\
40.100 \\
40.000 \\
48.300 \\
48.100 \\
43.300 \\
40.000 \\
42.700\end{array}$ & $\begin{array}{r}0.800 \\
4.800 \\
5.000 \\
1.600 \\
2.000 \\
5.000 \\
5.100 \\
5.000 \\
25.200 \\
6.400 \\
3.800 \\
3.000 \\
0.900 \\
3.400 \\
1.560 \\
0.002 \\
.0 .002 \\
8.400 \\
4.300 \\
0.580 \\
1.350 \\
0.200 \\
1.250 \\
1.000 \\
1.000 \\
4.540 \\
1.500 \\
1.700 \\
3.000 \\
1.000 \\
1.400 \\
4.340 \\
1.000 \\
1.600 \\
0.760 \\
1.400 \\
4.000 \\
1.490 \\
2.160 \\
1.070 \\
0.800 \\
0.800 \\
1.100 \\
3.290 \\
2.900 \\
3.700\end{array}$ & $\begin{array}{l}1.14 \\
1.21 \\
1.21 \\
1.09 \\
1.07 \\
1.29 \\
1.32 \\
1.29 \\
1.24 \\
1.08 \\
0.11 \\
1.52 \\
1.18 \\
1.05 \\
1.20 \\
0.94 \\
1.11 \\
1.07 \\
1.11 \\
1.08 \\
1.06 \\
1.20 \\
1.11 \\
1.07 \\
1.15 \\
1.08 \\
1.11 \\
1.01 \\
1.07 \\
1.07 \\
1.08 \\
1.07 \\
1.15 \\
1.09 \\
1.04 \\
1.09 \\
1.07 \\
1.15 \\
1.17 \\
1.04 \\
1.04 \\
1.26 \\
1.25 \\
1.13 \\
1.04 \\
1.11\end{array}$ & $\begin{array}{l}0.04 \\
0.13 \\
0.14 \\
0.05 \\
0.06 \\
0.14 \\
0.14 \\
0.14 \\
0.66 \\
0.17 \\
0.10 \\
0.09 \\
0.04 \\
0.09 \\
0.05 \\
0.03 \\
0.03 \\
0.22 \\
0.12 \\
0.04 \\
0.05 \\
0.04 \\
0.05 \\
0.04 \\
0.04 \\
0.12 \\
0.05 \\
0.05 \\
0.08 \\
0.04 \\
0.05 \\
0.12 \\
0.04 \\
0.05 \\
0.04 \\
0.05 \\
0.11 \\
0.05 \\
0.07 \\
0.04 \\
0.04 \\
0.04 \\
0.05 \\
0.09 \\
0.08 \\
0.10\end{array}$ & $\begin{array}{l}W \\
W \\
W \\
W \\
A \\
A \\
N \\
N \\
N \\
N \\
A \\
N \\
N \\
W \\
W \\
A \\
W \\
W \\
A \\
A \\
A \\
A \\
A \\
W \\
A \\
A \\
W \\
A \\
A \\
A \\
A \\
A \\
A \\
A \\
W \\
A \\
A \\
A \\
A \\
W \\
W \\
A \\
A \\
N \\
N \\
A \\
A \\
A\end{array}$ \\
\hline
\end{tabular}

Units for matrices: $\mathrm{Al}=\mathrm{Bq}$ qfilter $\mathrm{SO}=\mathrm{Bq} / \mathrm{kg}$ YE $=\mathrm{Bq} / \mathrm{kg}, \mathrm{WA}=\mathrm{Bq} / \mathrm{L}$. Values for elemental uranium are reported in $\mu \mathrm{q} /$ filter, $\mathrm{g}$, or $\mathrm{mL}$. 


\section{OAP44 Results by Nuclide}

Matrix: WA

Radionuclide: MN 54

EML. Value: 38.400

EML Error: 1.160

\begin{tabular}{|c|c|c|c|c|c|c|}
\hline Lahronde & Test\# & $\begin{array}{c}\text { Reported } \\
\text { Value }\end{array}$ & $\begin{array}{c}\text { Reported } \\
\text { Frror }\end{array}$ & $\frac{\text { Reported }}{\text { EML }}$ & $\begin{array}{l}\text { Ratio } \\
\text { Error }\end{array}$ & Evaluation \\
\hline $\begin{array}{l}\text { WC } \\
\text { WE } \\
\text { WI } \\
\text { WN } \\
\text { WP } \\
W V \\
\text { YA }\end{array}$ & $\begin{array}{l}1 \\
1 \\
1 \\
1 \\
1 \\
1 \\
1\end{array}$ & $\begin{array}{l}44.900 \\
39.700 \\
44.300 \\
36.500 \\
40.600 \\
42.700 \\
39.000\end{array}$ & $\begin{array}{l}6.470 \\
1.190 \\
5.440 \\
0.400 \\
1.400 \\
0.960 \\
0.750\end{array}$ & $\begin{array}{l}1.17 \\
1.03 \\
1.15 \\
0.95 \\
1.06 \\
1.11 \\
1.02\end{array}$ & $\begin{array}{l}0.17 \\
0.04 \\
0.15 \\
0.03 \\
0.05 \\
0.04 \\
0.04\end{array}$ & $\begin{array}{l}\mathbf{W} \\
\mathbf{A} \\
\mathbf{W} \\
\mathbf{A} \\
\mathbf{A} \\
\mathbf{A} \\
\mathbf{A}\end{array}$ \\
\hline
\end{tabular}

Total Number Reported: 98

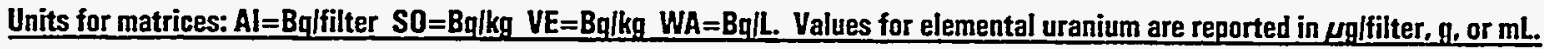
Evaluation: $A=A c c e p t a b l e, W=A c c e p t a b l e$ with Warning, $N=$ Not Acceptable.

$\mathrm{pCi}=\mathrm{Bq} \times 27$ 
QAP44 Results by Nuclide

Matrix: WA

Radionuclide: PU238

EML Value: 0.982

EML Error: 0.074

\begin{tabular}{|c|c|c|c|c|c|c|}
\hline Lahende. & Test \# & $\begin{array}{c}\text { Reported } \\
\text { Value. }\end{array}$ & $\begin{array}{c}\text { Reported } \\
\text { Frror }\end{array}$ & $\frac{\text { Reported }}{\text { EML }}$ & $\begin{array}{l}\text { Ratio } \\
\text { Error }\end{array}$ & Evaluation \\
\hline
\end{tabular}

\begin{tabular}{|c|c|c|c|c|c|c|}
\hline 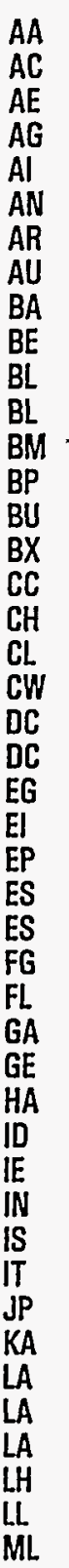 & $\begin{array}{l}1 \\
1 \\
1 \\
1 \\
1 \\
1 \\
1 \\
1 \\
1 \\
1 \\
1 \\
2 \\
1 \\
1 \\
1 \\
1 \\
1 \\
1 \\
1 \\
1 \\
1 \\
2 \\
1 \\
1 \\
1 \\
1 \\
2 \\
1 \\
1 \\
1 \\
1 \\
1 \\
1 \\
1 \\
1 \\
1 \\
1 \\
1 \\
1 \\
1 \\
2 \\
3 \\
1 \\
1 \\
1\end{array}$ & $\begin{array}{l}0.982 \\
1.010 \\
0.950 \\
0.940 \\
1.020 \\
0.920 \\
0.853 \\
1.360 \\
1.040 \\
0.960 \\
1.130 \\
1.050 \\
0.930 \\
0.931 \\
0.740 \\
0.969 \\
1.000 \\
0.948 \\
0.910 \\
1.010 \\
0.897 \\
0.897 \\
0.830 \\
0.880 \\
0.990 \\
0.953 \\
0.883 \\
0.925 \\
0.750 \\
1.120 \\
0.890 \\
0.810 \\
0.870 \\
0.975 \\
0.960 \\
0.936 \\
1.000 \\
1.100 \\
1.010 \\
0.990 \\
0.940 \\
0.930 \\
0.930 \\
1.040 \\
0.980\end{array}$ & $\begin{array}{l}0.074 \\
0.060 \\
0.070 \\
0.120 \\
0.073 \\
0.028 \\
0.122 \\
0.360 \\
0.170 \\
0.090 \\
0.070 \\
0.070 \\
0.160 \\
0.063 \\
0.030 \\
0.039 \\
0.000 \\
0.089 \\
0.220 \\
0.100 \\
0.020 \\
0.020 \\
0.060 \\
0.053 \\
0.087 \\
0.141 \\
0.131 \\
0.010 \\
0.017 \\
0.000 \\
0.090 \\
0.090 \\
0.130 \\
0.110 \\
0.110 \\
0.193 \\
0.020 \\
0.060 \\
0.010 \\
0.140 \\
0.130 \\
0.130 \\
0.120 \\
0.104 \\
0.040\end{array}$ & $\begin{array}{l}1.00 \\
1.03 \\
0.97 \\
0.96 \\
1.04 \\
0.94 \\
0.87 \\
1.39 \\
1.06 \\
0.98 \\
1.15 \\
1.07 \\
0.95 \\
0.95 \\
0.75 \\
0.99 \\
1.02 \\
0.97 \\
0.93 \\
1.03 \\
0.91 \\
0.91 \\
0.85 \\
0.90 \\
1.01 \\
0.97 \\
0.90 \\
0.94 \\
0.76 \\
1.14 \\
0.91 \\
0.83 \\
0.89 \\
0.99 \\
0.98 \\
0.95 \\
1.02 \\
1.12 \\
1.03 \\
1.01 \\
0.96 \\
0.95 \\
0.95 \\
1.06 \\
1.00\end{array}$ & $\begin{array}{l}0.11 \\
0.10 \\
0.10 \\
0.14 \\
0.11 \\
0.08 \\
0.14 \\
0.38 \\
0.19 \\
0.12 \\
0.11 \\
0.11 \\
0.18 \\
0.10 \\
0.06 \\
0.08 \\
0.08 \\
0.12 \\
0.24 \\
0.13 \\
0.07 \\
0.07 \\
0.09 \\
0.09 \\
0.12 \\
0.16 \\
0.15 \\
0.07 \\
0.06 \\
0.09 \\
0.11 \\
0.11 \\
0.15 \\
0.13 \\
0.13 \\
0.21 \\
0.08 \\
0.10 \\
0.08 \\
0.16 \\
0.15 \\
0.15 \\
0.14 \\
0.13 \\
0.09\end{array}$ & $\begin{array}{l}A \\
A \\
A \\
A \\
A \\
A \\
W \\
N \\
A \\
A \\
W \\
A \\
A \\
A \\
W \\
A \\
A \\
A \\
A \\
A \\
A \\
A \\
W \\
W \\
A \\
A \\
W \\
A \\
W \\
A \\
A \\
W \\
W \\
A \\
A \\
A \\
A \\
A \\
A \\
A \\
A \\
A \\
A \\
A \\
A \\
A\end{array}$ \\
\hline
\end{tabular}

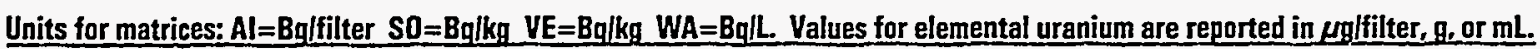

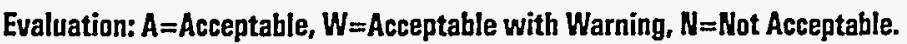
$\mathrm{pCi}=\mathrm{Bq} \times 27$ 
QAP44 Results by Nuclide

Matrix: WA

Radionuclide: PU238

EML Value: 0.982

EML Error: 0.074

\begin{tabular}{|c|c|c|c|c|c|c|}
\hline Lahende & Iest \# & $\begin{array}{c}\text { Reported } \\
\text { Value }\end{array}$ & $\begin{array}{c}\text { Reported } \\
\text { Frror }\end{array}$ & $\frac{\text { Reported }}{\text { FMIL }}$ & $\begin{array}{l}\text { Ratio } \\
\text { Error }\end{array}$ & Evaluation \\
\hline NL & 1 & 0.864 & 0.210 & 0.88 & 0.22 & $\mathbf{w}$ \\
\hline NM & 1 & 0.940 & 0.038 & 0.96 & 0.08 & $A$ \\
\hline OD & 1 & 0.881 & 0.091 & 0.90 & 0.11 & $\ddot{W}$ \\
\hline OR & 1 & 0.970 & 0.050 & 0.99 & 0.09 & A \\
\hline OT & 1 & 1.000 & 0.100 & 1.02 & 0.13 & $\ddot{A}$ \\
\hline $\mathrm{PI}$ & 1 & 0.950 & 0.070 & 0.97 & 0.10 & A \\
\hline RE & 1 & 0.860 & 0.090 & 0.88 & 0.11 & $W$ \\
\hline RG & 1 & 0.941 & 0.027 & 0.96 & 0.08 & $A$ \\
\hline RI & 1 & 0.810 & 0.065 & 0.83 & 0.09 & $W$ \\
\hline SC & 1 & 1.140 & 0.016 & 1.16 & 0.09 & $W$ \\
\hline SE & 1 & 0.740 & 0.060 & 0.75 & 0.08 & $\boldsymbol{W}$ \\
\hline SN & 1 & 0.847 & 0.094 & 0.86 & 0.12 & $W$ \\
\hline SR & 1 & 1.040 & 0.140 & 1.06 & 0.16 & A \\
\hline TE & $i$ & 0.900 & 0.100 & 0.92 & 0.12 & A \\
\hline $\mathrm{TI}$ & 1 & 0.970 & 0.170 & 0.99 & 0.19 & A \\
\hline TM & 1 & 0.984 & 0.043 & 1.00 & 0.09 & A \\
\hline TN & 1 & 0.942 & 0.045 & 0.96 & 0.09 & A \\
\hline TO & 1 & 1.010 & 0.332 & 1.03 & 0.35 & $\ddot{A}$ \\
\hline TW & $i$ & 0.930 & 0.070 & 0.95 & 0.10 & A \\
\hline TX & 1 & 0.970 & 0.071 & 0.99 & 0.10 & $\ddot{A}$ \\
\hline UK & 1 & 0.921 & 0.100 & 0.94 & 0.12 & $\ddot{A}$ \\
\hline UP & 1 & 0.992 & 0.205 & 1.01 & 0.22 & A \\
\hline UY & 1 & 0.970 & 0.170 & 0.99 & 0.19 & A \\
\hline WA & 1 & 0.870 & 0.120 & 0.89 & 0.14 & $\ddot{w}$. \\
\hline WC & 1 & 0.951 & 0.163 & 0.97 & 0.18 & $A$ \\
\hline WE & 1 & 0.842 & 0.079 & 0.86 & 0.10 & $W$ \\
\hline WN & 1 & 0.910 & 0.100 & 0.93 & 0.12 & $A$ \\
\hline YA & 1 & 1.000 & 0.028 & 1.02 & 0.08 & $\ddot{A}$ \\
\hline
\end{tabular}

Total Number Reported: 73

Units for matrices: $A l=B q \mid$ filter $S O=B q / k g$ VE $=B q / \mathrm{kg} W A=B q / L$. Values for elemental uranium are reported in $y$ glfilter, $g$, or $\mathrm{mL}$. 
Matrix: WA

Radionuclide: PU239

EML Value: 0.772

EML Error: 0.056

\begin{tabular}{ccccc} 
Leported & Reported \\
Value & Frror & $\frac{\text { Reported }}{\text { EML }}$ & $\begin{array}{c}\text { Ratio } \\
\text { Error }\end{array}$ & Evaluation \\
\hline
\end{tabular}

\begin{tabular}{|c|c|c|c|c|c|}
\hline $\begin{array}{l}A A \\
A C \\
A E \\
A G \\
A I \\
A N \\
A R \\
A U \\
B A \\
B E \\
B K \\
B L \\
B L \\
B M \\
B P \\
B U \\
B X \\
C C \\
C H \\
C L \\
C W \\
D C \\
D C \\
E G \\
E I \\
E P \\
E S \\
E S \\
F G \\
F L \\
G A \\
G E \\
H A \\
I D \\
I E \\
I N \\
I S \\
I T \\
J P \\
K A \\
L A \\
L A \\
L A \\
L H \\
L L \\
\end{array}$ & $\begin{array}{l}1 \\
1 \\
1 \\
1 \\
1 \\
1 \\
1 \\
1 \\
1 \\
1 \\
1 \\
1 \\
2 \\
1 \\
1 \\
1 \\
1 \\
1 \\
1 \\
1 \\
1 \\
1 \\
2 \\
1 \\
1 \\
1 \\
1 \\
2 \\
1 \\
1 \\
1 \\
1 \\
1 \\
1 \\
1 \\
1 \\
1 \\
1 \\
1 \\
1 \\
1 \\
2 \\
3 \\
1 \\
1\end{array}$ & $\begin{array}{l}0.772 \\
0.813 \\
0.750 \\
0.715 \\
0.824 \\
0.723 \\
0.839 \\
1.060 \\
0.766 \\
0.740 \\
0.550 \\
0.790 \\
0.800 \\
0.710 \\
0.704 \\
0.780 \\
0.703 \\
0.760 \\
0.726 \\
0.880 \\
0.812 \\
0.694 \\
0.694 \\
0.630 \\
0.620 \\
0.778 \\
0.844 \\
0.740 \\
0.763 \\
0.592 \\
0.890 \\
0.688 \\
0.730 \\
0.700 \\
0.730 \\
0.780 \\
0.822 \\
0.945 \\
0.840 \\
0.753 \\
0.900 \\
0.790 \\
0.750 \\
0.800 \\
0.840\end{array}$ & $\begin{array}{l}0.056 \\
0.048 \\
0.060 \\
0.089 \\
0.065 \\
0.022 \\
0.120 \\
0.300 \\
0.120 \\
0.070 \\
0.060 \\
0.060 \\
0.060 \\
0.120 \\
0.023 \\
0.030 \\
0.031 \\
0.000 \\
0.029 \\
0.160 \\
0.060 \\
0.046 \\
0.046 \\
0.050 \\
0.039 \\
0.074 \\
0.125 \\
0.110 \\
0.010 \\
0.014 \\
0.000 \\
0.078 \\
0.080 \\
0.050 \\
0.022 \\
0.090 \\
0.177 \\
0.140 \\
0.045 \\
0.025 \\
0.130 \\
0.110 \\
0.110 \\
0.150 \\
0.090\end{array}$ & $\begin{array}{l}1.00 \\
1.05 \\
0.97 \\
0.93 \\
1.07 \\
0.94 \\
1.09 \\
1.37 \\
0.99 \\
0.96 \\
0.71 \\
1.02 \\
1.04 \\
0.92 \\
0.91 \\
1.01 \\
0.91 \\
0.98 \\
0.94 \\
1.14 \\
1.05 \\
0.90 \\
0.90 \\
0.82 \\
0.80 \\
1.01 \\
1.09 \\
0.96 \\
0.99 \\
0.77 \\
1.15 \\
0.89 \\
0.95 \\
0.91 \\
0.95 \\
1.01 \\
1.07 \\
1.22 \\
1.09 \\
0.98 \\
1.17 \\
1.02 \\
0.97 \\
1.04 \\
1.09\end{array}$ & \begin{tabular}{l}
0.10 \\
0.10 \\
0.11 \\
$D$ \\
0.13 \\
0.07 \\
0.17 \\
0.40 \\
0.17 \\
0.11 \\
0.09 \\
0.11 \\
0.11 \\
0.17 \\
0.07 \\
0.08 \\
0.08 \\
0.07 \\
0.08 \\
0.22 \\
0.11 \\
0.09 \\
\hdashline
\end{tabular} \\
\hline
\end{tabular}

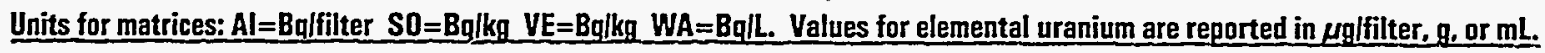
Evaluation: $A=$ Acceptable, $W=$ Acceptable with Warning, $N=$ Not Acceptable.

$\mathrm{pCi}=\mathrm{Bq} \times 27$ 


\section{QAP44 Results by Nuclide}

Matrix: WA

Radionuclide: PU239

EML Value: 0.772

EML Error: 0.056

\begin{tabular}{|c|c|c|c|c|c|c|}
\hline Lahconde & Test \#\# & $\begin{array}{c}\text { Reported } \\
\text { Value }\end{array}$ & $\begin{array}{c}\text { Reported } \\
\text { Frrno } \\
\end{array}$ & $\frac{\text { Reported }}{\text { EMII }}$ & $\begin{array}{l}\text { Ratio } \\
\text { Frror }\end{array}$ & Fualuation \\
\hline ML & 1 & 0.790 & 0.040 & 1.02 & 0.09 & A \\
\hline NL & $i$ & 0.726 & 0.170 & 0.94 & 0.23 & $\ddot{A}$ \\
\hline NM & 1 & 0.711 & 0.031 & 0.92 & 0.08 & $\ddot{A}$ \\
\hline OD & 1 & 0.725 & 0.075 & 0.94 & 0.12 & $\hat{A}$ \\
\hline $\mathrm{OR}$ & 1 & 0.730 & 0.050 & 0.95 & 0.09 & A \\
\hline OT & $i$ & 0.740 & 0.110 & 0.96 & 0.16 & $\ddot{A}$ \\
\hline$P A$ & i & 0.815 & 0.119 & 1.06 & 0.17 & A \\
\hline PB & 1 & 0.731 & 0.082 & 0.95 & 0.13 & A \\
\hline PI & $i$ & 0.740 & 0.060 & 0.96 & 0.10 & $\ddot{A}$ \\
\hline $\mathrm{RE}$ & 1 & 0.696 & 0.075 & 0.90 & 0.12 & $\ddot{A}$ \\
\hline $\mathrm{RF}$ & 1 & 0.763 & 0.043 & 0.99 & 0.09 & $\ddot{A}$ \\
\hline RG & 1 & 0.761 & 0.023 & 0.99 & 0.08 & $\ddot{A}$ \\
\hline $\mathrm{Rl}$ & 1 & 0.633 & 0.057 & 0.82 & 0.09 & A \\
\hline SC & 1 & 0.814 & 0.013 & 1.05 & 0.08 & A \\
\hline SE & 1 & 0.480 & 0.040 & 0.62 & 0.07 & $\ddot{w}$ \\
\hline SN & 1 & 0.653 & 0.075 & 0.85 & 0.12 & $\ddot{A}$ \\
\hline SR & 1 & 0.810 & 0.120 & 1.05 & 0.17 & A \\
\hline TE & 1 & 0.700 & 0.100 & 0.91 & 0.15 & A \\
\hline TI & 1 & 0.810 & 0.150 & 1.05 & 0.21 & A \\
\hline TM & 1 & 0.761 & 0.035 & 0.99 & 0.08 & A \\
\hline TN & 1 & 0.770 & $0.038^{\circ}$ & 1.00 & 0.09 & A \\
\hline TO & $i$ & 0.746 & 0.255 & 0.97 & 0.34 & $\ddot{A}$ \\
\hline$T W$ & 1 & 0.580 & 0.060 & 0.75 & 0.09 & W \\
\hline$T X$ & 1 & 0.840 & 0.069 & 1.09 & 0.12 & $\ddot{A}$ \\
\hline UC & $i$ & 0.780 & 0.110 & 1.01 & 0.16 & A \\
\hline UK & 1 & 0.762 & 0.092 & 0.99 & 0.14 & $\ddot{A}$ \\
\hline UP & $i$ & 0.767 & 0.166 & 0.99 & 0.23 & $\ddot{A}$ \\
\hline UY & $i$ & 0.820 & 0.160 & 1.06 & 0.22 & $\ddot{A}$ \\
\hline WA & $i$ & 0.680 & 0.100 & 0.88 & 0.14 & A \\
\hline WC & 1 & 0.782 & 0.137 & 1.01 & 0.19 & A \\
\hline WE & $i$ & 0.526 & 0.063 & 0.68 & 0.10 & $\ddot{w}$ \\
\hline WN & 1 & 0.720 & 0.080 & 0.93 & 0.12 & A \\
\hline YA & 1 & 0.763 & 0.010 & 0.99 & 0.07 & A \\
\hline
\end{tabular}

Total Number Reported: 78

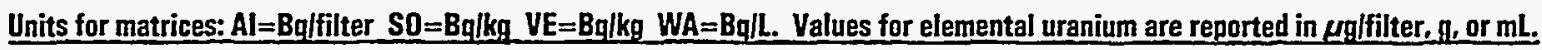
Evaluation: $A=A c c e p t a b l e, ~ W=A c c e p t a b l e$ with Warning, N=Not Acceptable. $\mathrm{pCi}=\mathrm{Bq} \times 27$ 
Matrix: WA

Radionuclide: SR 90

EML Value: 1.450

EML Error: 0.034

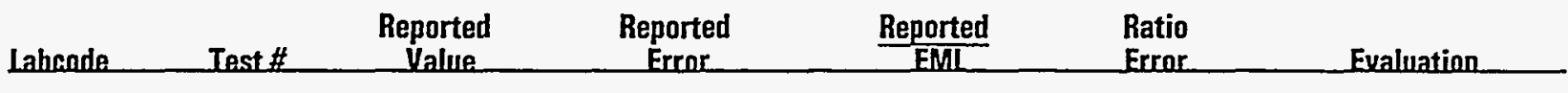

\begin{tabular}{|c|c|c|c|c|c|}
\hline $\begin{array}{l}A A \\
A E \\
A G \\
A N \\
A R \\
A U \\
B C \\
B E \\
B L \\
B L \\
B M \\
B N \\
B P \\
B X \\
C C \\
C H \\
C L \\
C P \\
D C \\
D C \\
E G \\
E I \\
E L \\
E P \\
E S \\
F G \\
G A \\
G E \\
H A \\
H L \\
I D \\
I E \\
I N \\
I S \\
I S \\
I T \\
J P \\
K A \\
L A \\
L A \\
L A \\
L H \\
N A \\
O R \\
O T \\
O R\end{array}$ & $\begin{array}{l}1 \\
1 \\
1 \\
1 \\
1 \\
1 \\
1 \\
1 \\
1 \\
2 \\
1 \\
1 \\
1 \\
1 \\
1 \\
1 \\
1 \\
1 \\
1 \\
2 \\
1 \\
1 \\
1 \\
1 \\
1 \\
1 \\
1 \\
1 \\
1 \\
1 \\
1 \\
1 \\
1 \\
1 \\
2 \\
1 \\
1 \\
1 \\
1 \\
2 \\
3 \\
1 \\
1 \\
1 \\
1\end{array}$ & $\begin{array}{l}1.450 \\
1.500 \\
1.500 \\
1.580 \\
1.520 \\
2.300 \\
2.180 \\
1.860 \\
1.600 \\
1.640 \\
1.170 \\
1.320 \\
1.240 \\
2.000 \\
1.500 \\
1.510 \\
1.700 \\
0.815 \\
1.470 \\
1.470 \\
1.230 \\
2.150 \\
1.550 \\
1.290 \\
1.760 \\
1.680 \\
1.640 \\
1.170 \\
1.240 \\
1.560 \\
1.710 \\
1.330 \\
2.000 \\
1.540 \\
1.540 \\
1.460 \\
1.600 \\
1.460 \\
1.290 \\
1.410 \\
1.540 \\
1.420 \\
1.550 \\
1.370 \\
2.400\end{array}$ & $\begin{array}{l}0.034 \\
0.340 \\
0.300 \\
0.050 \\
0.190 \\
0.370 \\
0.518 \\
0.350 \\
0.650 \\
0.670 \\
0.230 \\
0.000 \\
0.110 \\
0.485 \\
0.000 \\
0.385 \\
0.470 \\
0.211 \\
0.372 \\
0.372 \\
0.060 \\
0.190 \\
0.200 \\
0.343 \\
0.300 \\
0.180 \\
0.310 \\
0.084 \\
0.200 \\
0.280 \\
0.150 \\
0.060 \\
0.200 \\
0.000 \\
0.520 \\
0.160 \\
0.140 \\
0.240 \\
0.140 \\
0.160 \\
0.160 \\
0.360 \\
0.260 \\
0.300 \\
0.600\end{array}$ & $\begin{array}{l}1.00 \\
1.03 \\
1.03 \\
1.09 \\
1.05 \\
1.59 \\
1.50 \\
1.28 \\
1.10 \\
1.13 \\
0.81 \\
0.91 \\
0.86 \\
1.38 \\
1.03 \\
1.04 \\
1.17 \\
0.56 \\
1.01 \\
1.01 \\
0.85 \\
1.48 \\
1.07 \\
0.89 \\
1.21 \\
1.16 \\
1.13 \\
0.81 \\
0.86 \\
1.08 \\
1.18 \\
0.92 \\
1.38 \\
1.06 \\
1.06 \\
1.01 \\
1.10 \\
1.01 \\
0.89 \\
0.97 \\
1.06 \\
0.98 \\
1.07 \\
0.95 \\
1.66\end{array}$ & \begin{tabular}{l}
0.03 \\
0.24 \\
0.21 \\
0.04 \\
0.13 \\
0.26 \\
0.36 \\
0.24 \\
0.45 \\
0.46 \\
0.16 \\
0.02 \\
0.08 \\
0.34 \\
0.02 \\
0.27 \\
0.33 \\
0.15 \\
0.26 \\
0.26 \\
0.05 \\
0.14 \\
0.14 \\
0.24 \\
0.21 \\
0.13 \\
0.22 \\
0.06 \\
0.14 \\
0.20 \\
0.11 \\
\hdashline
\end{tabular} \\
\hline
\end{tabular}

Units for matrices: $\mathrm{Al}=\mathrm{Bq}$ qfilter $\mathrm{SO}=\mathrm{Bq}$ lkg $\mathrm{VE}=\mathrm{Bq} / \mathrm{kg} W \mathrm{WA}=\mathrm{Bq} \mathrm{lL}$. Values for elemental uranium are reported in $\mu \mathrm{g} /$ filter, $\mathrm{g}$. or $\mathrm{mL}$.

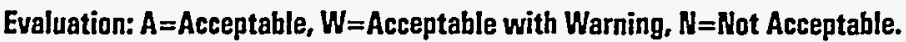

$\mathrm{pCi}=\mathrm{Bq} \times 27$ 
OAP44 Results by Nuclide

Matrix: WA

Radionuclide: SR 90

EML Value: 1.450

EML Error: 0.034

\begin{tabular}{|c|c|c|c|c|c|c|}
\hline Lahconde & Test \# & $\begin{array}{c}\text { Reported } \\
\text { Value }\end{array}$ & $\begin{array}{c}\text { Reported } \\
\text { Frrar }\end{array}$ & $\frac{\text { Reported }}{\text { EMII }}$ & $\begin{array}{l}\text { Ratio } \\
\text { Frror }\end{array}$ & Fyaluatinn \\
\hline RE & 1 & 1.470 & 0.150 & 1.01 & 0.11 & A \\
\hline Rl & 1 & 1.740 & 0.370 & 1.20 & 0.26 & A \\
\hline SC & 1 & 2.570 & 0.060 & 1.77 & 0.06 & $\mathbf{N}$ \\
\hline SR & 1 & 1.630 & 0.240 & 1.12 & 0.17 & A \\
\hline SW & 1 & 1.650 & 0.220 & 1.14 & 0.15 & A \\
\hline TE & 1 & 2.200 & 0.700 & 1.52 & 0.48 & $W$ \\
\hline $\mathrm{TI}$ & $i$ & 1.600 & 0.300 & 1.10 & 0.21 & A \\
\hline$T M$ & 1 & 1.450 & 0.142 & 1.00 & 0.10 & A \\
\hline TN & 1 & 1.620 & 0.093 & 1.12 & 0.07 & A \\
\hline TO & $i$ & 1.450 & 0.247 & 1.00 & 0.17 & $\ddot{A}$ \\
\hline - TW & $i$ & 1.300 & 0.100 & 0.90 & 0.07 & A \\
\hline$T X$ & 1 & 1.920 & 0.620 & 1.32 & 0.43 & $w$ \\
\hline UP & 1 & 1.190 & 0.670 & 0.82 & 0.46 & $W$. \\
\hline UY & 1 & 1.300 & 0.480 & 0.90 & 0.33 & $A$ \\
\hline WA & 1 & 1.630 & 0.240 & 1.12 & 0.17 & $\ddot{A}$ \\
\hline WC & 1 & 1.010 & 0.250 & 0.70 & 0.17 & $\ddot{N}$ \\
\hline WE & 1 & 0.004 & 0.001 & 0.00 & 0.00 & $\mathbf{N}$ \\
\hline WN & 1 & 2.200 & 0.400 & 1.52 & 0.28 & W \\
\hline WP & 1 & 1.600 & 0.300 & 1.10 & 0.21 & $\ddot{A}$ \\
\hline WV & 1 & 1.900 & 0.360 & 1.31 & 0.25 & $w$ \\
\hline
\end{tabular}

Total Number Reported: 65

Units for matrices: $\mathrm{Al}=\mathrm{Bq} /$ filter $\mathrm{SO}=\mathrm{Bq} / \mathrm{kg}$ VE=Bqlkg $W A=B q \mid \mathrm{L}$. Values for elemental uranium are reported in $\mu \mathrm{g} /$ filter, $\mathrm{g}$, or $\mathrm{mL}$. Evaluation: $A=$ Acceptable, $W=$ Acceptable with Warning, $N=$ Not Acceptable.

$\mathrm{pCi}=\mathrm{Bq} \times 27$ 
OAP44 Results by Nuclide

Matrix: WA

Radionuclide: U 234

EML Value: 0.274

EML Error: 0.019

\begin{tabular}{|c|c|c|c|c|c|c|}
\hline Lahonde & Test \# & $\begin{array}{c}\text { Reported } \\
\text { Value. }\end{array}$ & $\begin{array}{l}\text { Reported } \\
\text { Frror }\end{array}$ & $\frac{\text { Reported }}{\text { FMII }}$ & $\begin{array}{l}\text { Ratio } \\
\text { Error }\end{array}$ & Evaluatinn \\
\hline
\end{tabular}

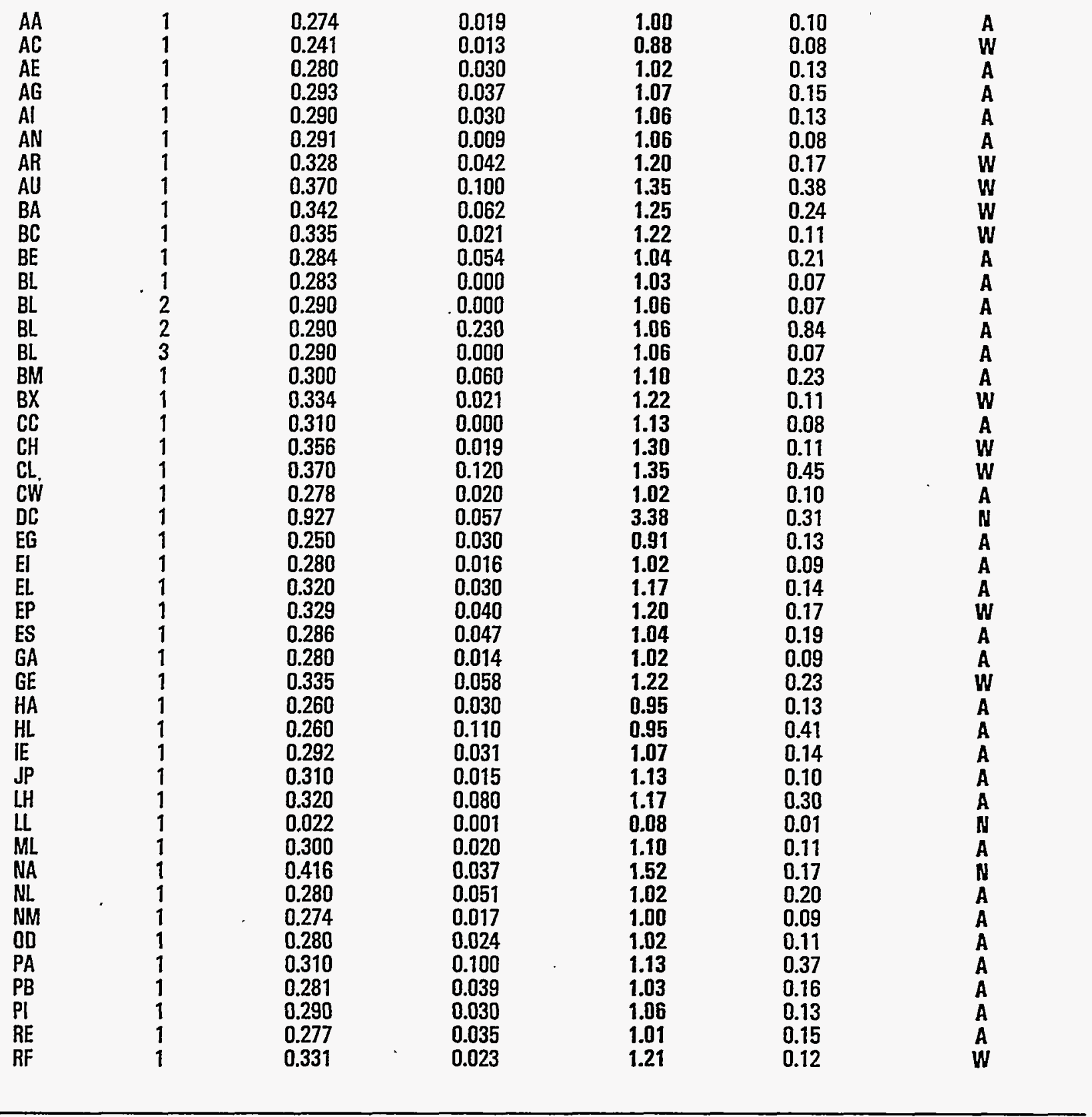

Units for matrices: $\mathrm{Al}=\mathrm{Bq}$ lfilter $\mathrm{SO}=\mathrm{Bq} / \mathrm{kg}$ VE=Bq/kg$W \mathrm{~A}=\mathrm{Bq} / \mathrm{L}$. Values for elemental uranium are reported in $\mu \mathrm{g} / \mathrm{filter}$, $\mathrm{g}$. or $\mathrm{mL}$. Evaluation: $A=$ Acceptable, $W=A c c e p t a b l e$ with Warning, $N=$ Not Acceptable.

$$
\mathrm{pCi}=\mathrm{Bq} \times 27
$$




\section{QAP44 Results by Nuclide}

Matrix: WA

Radionuclide: U 234

EML Value: 0.274

EML Error: 0.019

\begin{tabular}{|c|c|c|c|c|c|c|}
\hline Lahende & Test\# & $\begin{array}{c}\text { Reported } \\
\text { Yalue. }\end{array}$ & $\begin{array}{c}\text { Reported } \\
\text { Frror }\end{array}$ & $\frac{\text { Reported }}{\text { FML }}$ & $\begin{array}{l}\text { Ratio } \\
\text { Frror }\end{array}$ & Evaluation \\
\hline $\begin{array}{l}\text { RG } \\
\text { SC } \\
\text { SN } \\
\text { SR } \\
\text { II } \\
\text { TM } \\
\text { TN } \\
\text { TO } \\
\text { TX } \\
\text { UP } \\
\text { UY } \\
\text { WA } \\
\text { WC } \\
\text { WE }\end{array}$ & $\begin{array}{l}1 \\
1 \\
1 \\
1 \\
1 \\
1 \\
1 \\
1 \\
1 \\
1 \\
1 \\
1 \\
1 \\
1\end{array}$ & $\begin{array}{l}0.328 \\
0.300 \\
0.314 \\
0.289 \\
0.520 \\
0.311 \\
0.289 \\
0.257 \\
0.440 \\
0.349 \\
0.320 \\
0.290 \\
0.350 \\
0.278\end{array}$ & $\begin{array}{l}0.018 \\
0.020 \\
0.043 \\
0.052 \\
0.280 \\
0.026 \\
0.022 \\
0.024 \\
0.026 \\
0.113 \\
0.099 \\
0.040 \\
0.081 \\
0.057\end{array}$ & $\begin{array}{l}1.20 \\
1.10 \\
1.15 \\
1.05 \\
1.90 \\
1.14 \\
1.05 \\
0.94 \\
1.61 \\
1.27 \\
1.17 \\
1.06 \\
1.28 \\
1.02\end{array}$ & $\begin{array}{l}0.11 \\
0.11 \\
0.18 \\
0.20 \\
1.03 \\
0.12 \\
0.11 \\
0.11 \\
0.15 \\
0.42 \\
0.37 \\
0.16 \\
0.31 \\
0.22\end{array}$ & $\begin{array}{l}W \\
A \\
A \\
A \\
N \\
A \\
A \\
A \\
N \\
W \\
A \\
A \\
W \\
A\end{array}$ \\
\hline
\end{tabular}

Total Number Reported: 59

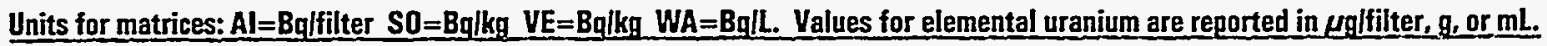

$\mathrm{pCi}=\mathrm{Bq} \times 27$ 


\section{QAP44 Results by Nuclide}

Matrix: WA

Radionuclide: U 238

EML Value: 0.275

EML Error: 0.024

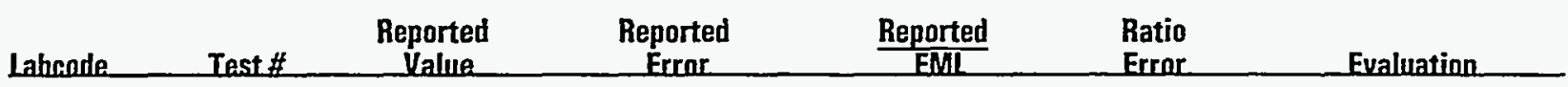

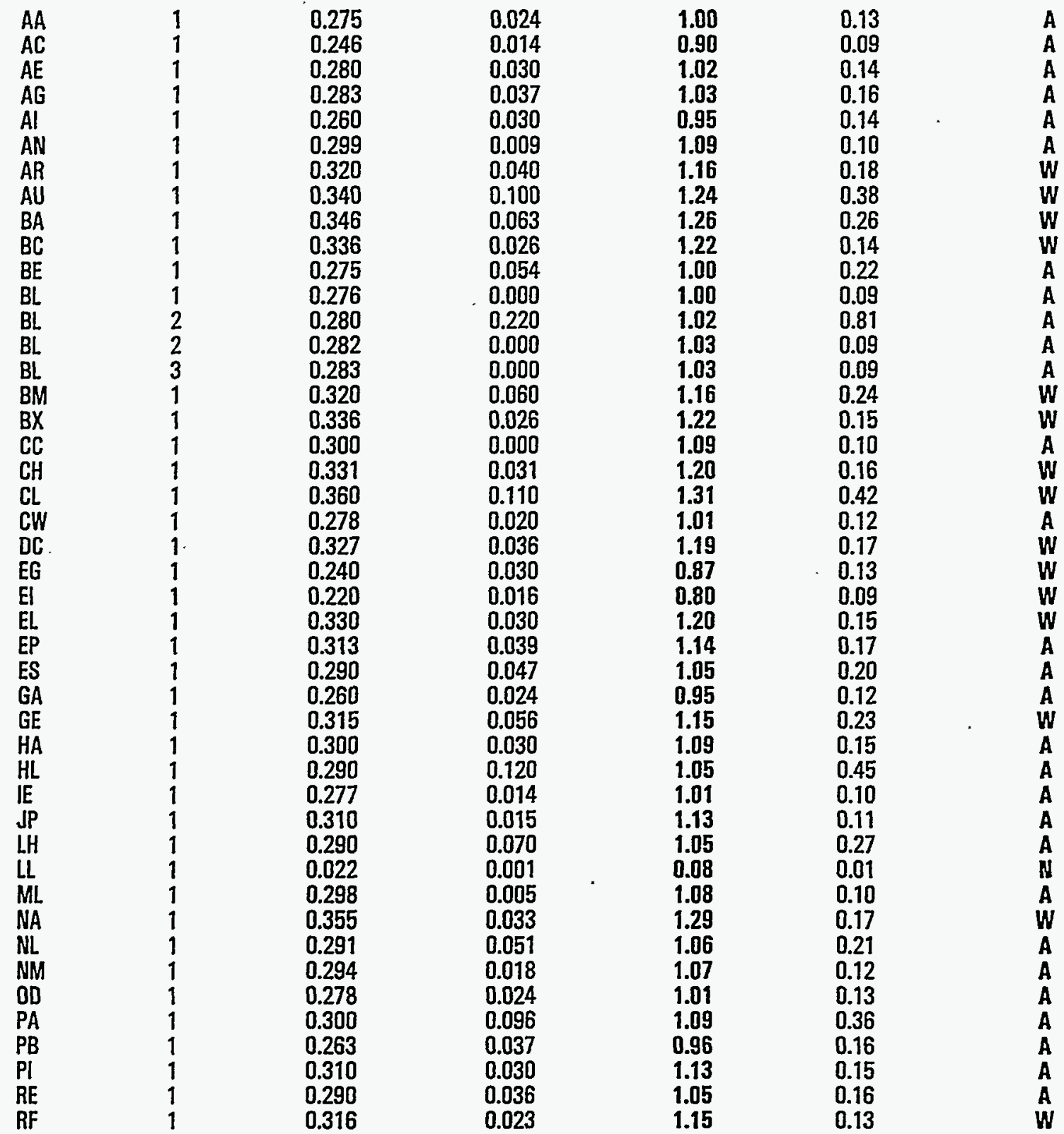

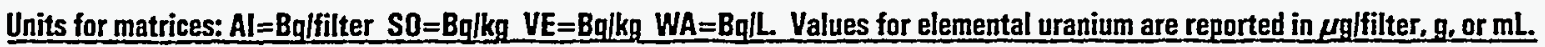


QAP44 Results by Nuclide

Matrix: WA

Radionuclide: U 238

EML Value: 0.275

EML Error: 0.024

\begin{tabular}{|c|c|c|c|c|c|c|}
\hline Lahente & Test \# & $\begin{array}{c}\text { Reported } \\
\text { Value }\end{array}$ & $\begin{array}{c}\text { Reported } \\
\text { Error }\end{array}$ & $\frac{\text { Reported }}{\text { FMIL }}$ & $\begin{array}{l}\text { Ratio } \\
\text { Error }\end{array}$ & Evaluatinn \\
\hline$R G$ & 1 & 0.295 & 0.017 & 1.07 & 0,11 & A \\
\hline SC & $i$ & 0.300 & 0.020 & 1.09 & 0.12 & $\ddot{A}$ \\
\hline SN & 1 & 0.303 & 0.041 & 1.10 & 0.18 & A \\
\hline SR & 1 & 0.285 & 0.051 & 1.04 & 0.21 & $\ddot{A}$ \\
\hline $\mathrm{TI}$ & 1 & 1.300 & 0.500 & 4.73 & 1.87 & N \\
\hline$T M$ & 1 & 0.259 & 0.024 & 0.94 & 0.12 & A \\
\hline TN & 1 & 0.299 & 0.022 & 1.09 & 0.13 & $\ddot{A}$ \\
\hline TO & 1 & 0.262 & 0.024 & 0.95 & 0.12 & $\hat{A}$ \\
\hline $\mathrm{TX}$ & 1 & 0.440 & 0.026 & 1.60 & 0.17 & $\mathbf{N}$ \\
\hline UP & 1 & 0.303 & 0.157 & 1.10 & 0.58 & A \\
\hline UY & $i$ & 0.310 & 0.100 & 1.13 & 0.38 & $A$ \\
\hline WA & 1 & 0.300 & 0.050 & 1.09 & 0.21 & A \\
\hline WC & 1 & 0.300 & 0.072 & 1.09 & 0.28 & $\ddot{A}$ \\
\hline WE & 1 & 0.316 & 0.059 & 1.15 & 0.24 & $\mathbf{W}$ \\
\hline WN & 1 & 0.610 & 0.110 & 2.22 & 0.45 & N \\
\hline
\end{tabular}

Total Number Reported: 60

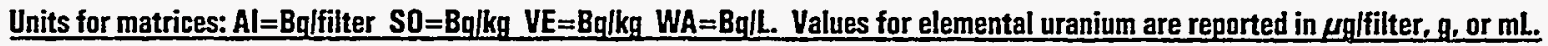
Evaluation: $A=A c c e p t a b l e, W=A c c e p t a b l e$ with Warning, $N=$ Not Acceptable.

$\mathrm{pCi}=\mathrm{Bq} \times 27$ 


\section{QAP44 Results by Nuclide}

Matrix: WA

Radionuclide: U BQ

EML Value: 0.559

EML Error: 0.047

\begin{tabular}{|c|c|c|c|c|c|c|}
\hline Lahende. & Test \# & $\begin{array}{c}\text { Reported } \\
\text { Yalue. }\end{array}$ & $\begin{array}{c}\text { Reported } \\
\text { Errar }\end{array}$ & $\frac{\text { Reported }}{\text { EMIL }}$ & $\begin{array}{l}\text { Ratio } \\
\text { Errno }\end{array}$ & Fyaluation \\
\hline
\end{tabular}

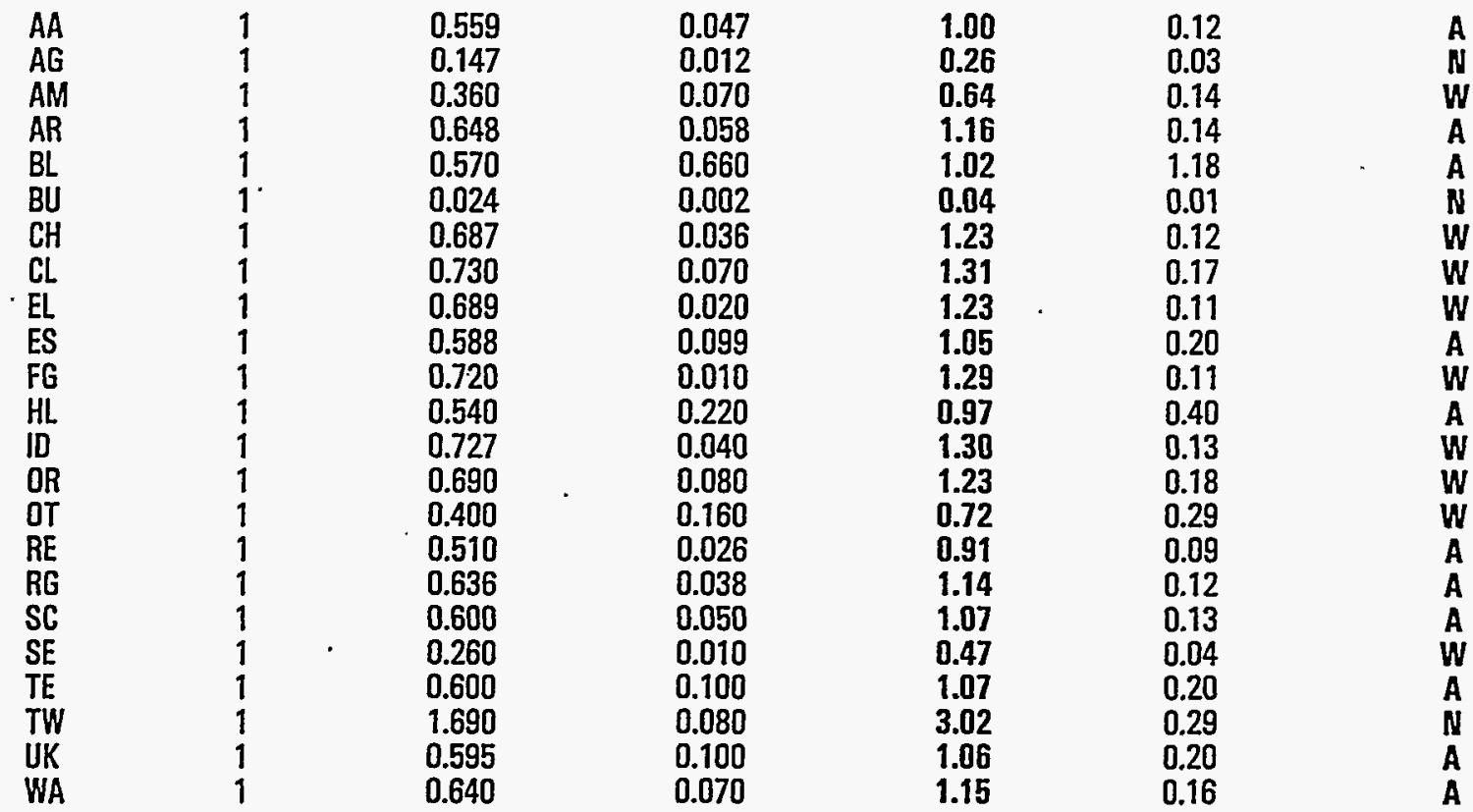

Total Number Reported: 23

Units for matrices: $A l=B q$ lfilter $S O=B q l k g$ VE=Bglkg $W A=B q l L$. Values for elemental uranium are reported in pgifilter, $\mathrm{g}$, or $\mathrm{mL}$. 


\section{QAP44 Results by Nuclide}

Matrix: WA

Radionuclide: U UG

EML Value: 0.022

EML Error: 0.003

\begin{tabular}{|c|c|c|c|c|c|c|}
\hline Lahonde & Test \# & $\begin{array}{l}\text { Reported } \\
\text { Value }\end{array}$ & $\begin{array}{l}\text { Reported } \\
\text { Frrar }\end{array}$ & $\frac{\text { Beported }}{\text { FML }}$ & $\begin{array}{l}\text { Ratio } \\
\text { Error }\end{array}$ & Eyaluatinn \\
\hline
\end{tabular}

\begin{tabular}{|c|c|c|c|c|c|c|}
\hline AA & 1 & 0.022 & 0.003 & 1.00 & 0.17 & A \\
\hline $\mathrm{AR}$ & 1 & 25.700 & 1.100 & $* * * *$ & $* *$ & $\mathbf{N}$ \\
\hline AW & 1 & 0.016 & 0.002 & 0.72 & 0.11 & $\mathbf{N}$ \\
\hline $\mathrm{BE}$ & 1 & 23.900 & 0.000 & $* * * *$ & $* *$ & $\ddot{N}$ \\
\hline BL & 1 & 0.023 & 0.000 & 1.04 & 0.12 & A \\
\hline $\mathrm{BL}$ & 2 & 0.024 & 0.000 & 1.07 & 0.13 & $\ddot{A}$ \\
\hline $\mathrm{BL}$ & 3 & 0.024 & 0.000 & 1.07 & 0.13 & A \\
\hline BP & 1 & 24.300 & 0.000 & $* * * *$ & $* * * *$ & $\mathbf{N}$ \\
\hline $\mathrm{BO}$ & 1 & 0.026 & 0.001 & 1.18 & 0.14 & A \\
\hline CC & 1 & 0.020 & 0.000 & 0.90 & 0.11 & A \\
\hline $\mathrm{CH}$ & 1 & 0.023 & 0.002 & 1.02 & 0.16 & A \\
\hline CZ & 1 & 0.027 & 0.000 & 1.22 & 0.14 & A \\
\hline $\mathrm{DC}$ & 1 & 0.023 & 0.002 & 1.04 & 0.16 & A \\
\hline ES & 1 & 0.023 & 0.000 & 1.05 & 0.12 & A \\
\hline ES & 2 & 23.200 & 2.350 & $* * * *$ & $* *{ }^{* *}$ & $\mathbf{N}$ \\
\hline GA & 1 & 0.021 & 0.000 & 0.95 & 0.11 & A \\
\hline GS & 1 & 0.017 & 0.001 & 0.77 & 0.10 & $\mathbf{W}$ \\
\hline IE & 1 & 22.500 & 1.160 & $* * * * *$ & $* * * *$ & N \\
\hline IR & $i$ & 0.035 & 0.001 & 1.58 & 0.19 & $\mathrm{~N}$ \\
\hline IT & 1 & 22.800 & 0.190 & $* * * *$ & $* * * *$ & $N$ \\
\hline KA & 1 & 0.023 & 0.001 & 1.02 & 0.12 & A \\
\hline LA & 1 & 0.021 & 0.000 & 0.93 & 0.11 & A \\
\hline LA & 2 & 0.021 & 0.000 & 0.96 & 0.11 & $\ddot{A}$ \\
\hline LA & 3 & 0.021 & 0.000 & 0.96 & 0.11 & $\ddot{A}$ \\
\hline NL & 1 & 0.023 & 0.003 & 1.04 & 0.18 & $\ddot{A}$ \\
\hline RI & 1 & 0.006 & 0.000 & 0.27 & 0.03 & $\mathbf{N}$ \\
\hline SE & 1 & 0.000 & 0.000 & 0.00 & 0.00 & N \\
\hline sW & 1 & 2.040 & 0.230 & 91.90 & 14.90 & N \\
\hline $\mathrm{TI}$ & 1 & 0.040 & 0.000 & 1.80 & 0.21 & $\mathbf{N}$ \\
\hline TM & 1 & 0.025 & 0.001 & 1.13 & 0.14 & A \\
\hline TN & $i$ & 0.013 & 0.004 & 0.57 & 0.18 & $\ddot{N}$ \\
\hline TO & 1 & 0.021 & 0.001 & 0.95 & 0.12 & A \\
\hline TW & 1 & 0.068 & 0.003 & 3.06 & 0.38 & $\mathbf{N}$ \\
\hline UC & 1 & 0.024 & 0.002 & 1.08 & 0.17 & A \\
\hline UP & 1 & 0.024 & 0.000 & 1.08 & 0.13 & $\ddot{A}$ \\
\hline UY & 1 & 0.024 & 0.000 & 1.08 & 0.13 & A \\
\hline YP & 1 & 0.020 & 0.002 & 0.91 & 0.14 & 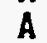 \\
\hline
\end{tabular}

Total Number Reported: 37

Units for matrices: $A|=B q|$ filter $S O=B q \mid k g$ VE=Bqlkg $W A=B q \mid L$. Values for elemental uranium are reported in $\mu g l f i l t e r, g, 0 r \mathrm{~mL}$. Evaluation: $A=A c c e p t a b l e, W=$ Acceptable with Warning, $N=$ Not Acceptable.

$p \mathrm{Ci}=\mathrm{Bq} \times 27$ 


\section{Participating Laboratories in EML QAP44}

\section{Laboratories Reporting Data}

Code Laboratory Name

AC Analytical Chemistry Laboratory Chemical Technology Div., Argonne, IL

AE Analytical Resources, Inc. , Seattle, WA

AG Paragon Analytics, Inc. , Fort Collins, CO

AI Nuclear Technology Services, Inc. Radiochemistry Lab, Roswell, GA

AL Ames Laboratory Safety, Health \& Env. G40TASF, Ames, IA

AM American Radiation Services, Inc. , Baton Rouge, LA

AN Argonne Nat'I Laboratory ESH, Bldg 200, Rm. F109, Argonne, IL

AR Accu-Labs Research Inc. , Golden, CO

AU ORISE EESD/ESSAP PO Box 117, Oak Ridge, TN

AW Argonne National Laboratory, Idaho Falls, ID

BA Westinghouse Electric Corp. Bettis Atomic Power Lab, West Mifflin, PA

BC Babcock \& Wilcox MC \#42 Naval Nuclear Fuel Division, Lynchburg, VA

BE RUST Geotech, Grand Junction, CO

BK Brookhaven Nat'I Laboratory Dept of Adv Tech., Upton, NY

BL Barringer Laboratories Inc. , Golden, CO

BM Battelle Memorial Institute, Columbus, $\mathrm{OH}$

BNI Brookhaven Nat'I Laboratory Bldg. \#535 A, Upton, NY

BP Battelle PNL, BIdg. 325 BRM 8547 c/o Westinghouse Hanford CO., Richland, WA

B0 Becquerel Laboratories Inc. , Mississauga, Ontario, Canada

BR US Army Research Laboratory Attn: AMSRL-OP-AP-RKIRichard M, Aberdeen Proving Ground, MD

BS B\&W Nuclear Envir. Services, Vandergraft, PA

BU Autoridad Regulatoria , Buenos Aires, Argentina

BX B\&W Nuclear Envir. Services Nuclear Envir. Lab, Lynchburg, VA

CA Atomic Energy Control Board, Ottawa, Canada

CC Compuchem Environmental Corp. , Research Triangle Park, NC

CH California State Dept. Health Serv. Sanitation \& Radiation Laborat, Berkeley, CA

CL Core Laboratories, Casper, WY

CP Controls for Envir. Pollution, Santa Fe, NM

CS Rockwell Internat'I Corp. Rad. Protection T100, Canoga Park, CA

CW Carlsbad Environmental Monitoring Res, Carlsbad, NM

CZ ACZ Laboratories, Inc. , Steamboat Springs, CO

DC Datachem Laboratories, Salt Lake City, UT

EG TRA-MTR604 LAB 124 ATTN: Jodie Doherty 208-533.41, Scoville, ID

EI Argonne National Laboratory Building 211, Argonne, IL

EL Energy Laboratories, Inc. Radiochemistry Department, Casper, WY

EP US EPA-LV Mail Stop/ RSD, Las Vegas, NV

ES Environmental Sci. \& Engr., Inc. Inorganic Analyt.Chem., Gainesville, FL

FG FGL Environmental, Santa Paula, CA

FL Dept of Health \& Rehab. Serv. Office of Rad. Control, Orlando, FL

FM Office of Radiation Control Mobile Emergency Radiological, Orlando, FL

FN Shipping and Receiving Department ATTN: Steve Benesch, 21 S Shab, Batavia, IL

FS Florida State University Department of Oceanography, Tallahassee, FL

GA Lockheed Martin Rm. 333 Bldg X710, Pikton, OH

GE General Engineering Labs Environmental Physics, Inc., Charleston, SC

GS USGS/NWQL, Arvada, CO

HA NUS Laboratory, Pittsburgh, PA

HC Lawrence Livermore Laboratory Hazards Control, L383, Livermore, CA

HL Heritage Laboratories, Inc. , Romeoville, IL

ID DPRA - IRD/CNEN Av. Salvador Allende S/N, Jaca, Rio De Janeiro, RJBrasil

IE IEA, Inc. , Morrisville, NC

IL ISU Environmental Monitoring Program Physical Science 103, Pocatello, ID 


\section{Participating Laboratories in EML QAP44}

Laboratories Reporting Data

Code Laboratory Name

IN WINCO MS 5202, Idaho Falls, ID

IR Idaho National Engineering Laboratory, Scoville, ID

IS Quanterra- St. Louis , Earth City, MO

IT Quanterra-Richland Laboratory, Richland, WA

JP Japan Chemical Analysis Center 295-3 Sanno-Cho, Chiba 263, Japan

KA Knolls Atomic Power Lab, Bldg A-3 Rm 2401 River Road, Schenectady, NY

LA Analytical Services, CST-3 MS K484, Los Alamos, NM

LB Lawrence Berkeley Lab UCB Bldg 75, Room 124, Berkeley, CA

LH Lockheed Analytical Laboratory, Las Vegas, NV

LL Lawrence Livermore Nat'I Lab Nuclear Chem. Div., Livermore, CA

LM Los Alamos Nat'I Lab, Mercury, NV

LV UNLV, Dept of Health Physics Bigelow H. S. BLDG, RM 350, Las Vegas, NV

LW Lawrence Livermore Nat'I Lab Nuclear Chem. Div., Livermore, CA

MA ORNL Health Sciences Research Div. BLDG 7710 MS 6379, Oak Ridge, TN

ME Radiation Control Program, Jamaica Plain, MA

MI Massachusetts Inst. of Tech. , Middleton, MA

ML EG\&G Mound Applied Technologies, Miamisburg, $\mathrm{OH}$

MS Manufacturing Sciences Corporation, Oak Ridge, TN

NA USEPA NAREL, Montgomery, AL

NC Head, Nuclear Services North Carolina State Universit, Raleigh, NC

NL FERMCO BLDG 15, room C-11, Cincinnati, OH

NM Environmental'Evaluation Group 505 North Main Street, Carlsbad, NM

NR NRF Chemistry, S1W2, Scoville, ID

OB OBG Laboratories, East Syracuse, NY

OD ORNL, Radiobioassay Lab Bldg 4500-S Rm H-249 MS 6105, Oak Ridge, TN

OL Oak Ridge National Laboratory Environmental Sciences Div., Oak Ridge, TN

OR Oak Ridge National Lab BIdg 4500.N Rm G-5, Oak Ridge, TN

OS Dregon Health Division Radiation Controls Section, Portland, OR

OT ORNLRadioactive Material Analysis Lab BLDG 2026, Room 129, Oak Ridge, TN

PA Mason \& Hanger-Silas Mason Co., Inc. , Amarillo, TX

PB Mason \& Hanger-Silas Mason Co., Inc., Amarillo, TX

PI Lockheed Martin Specialty Components , Largo, FL

RA - V. G. Khlopin Radium Institute , St. Petersburg, Russia

RE Bechtel Nevada Building 650, Warehouse 160, Mercury, NV

RF EG\&G Rocky Flats Plant Bldg 123, Golden, CO

RG EG\&G Rocky Flats Plant, Golden, CO

RI Westinghouse Hanford Co. Analytical Labs, Dept. T6-16, Richland, WA

SA Sandia Labs - Organization 7715 Radioactive Sample Diag. Prog., Albuquerque, NM

SC S.3 Division Maxwell Labs, La Jolla, CA

SE Shealy Environmental Services Inc. Overlook Business Center, Cayce, SC

SK Savannah River Plant Bldg 735-7A Rm 110, Aiken, SC

SN Sanford Cohen Associates, Inc. , Montgomery, AL

SR Savannah River Plant, Aiken, SC

SS Savannah River Tech Center, Aiken, SC

SV Savannah Lab \& Envt Serv., Inc. , Tampa, FL

SW Southwest Research Institute, Div. 01 P.D. Drawer 28510, San Antonio, TX

TE Teledyne Isotopes Midwest Lab, Northbrook, IL

TI Teledyne Brown Engineering Environmental Services, Westwood, NJ

TM TMA/Eberline-Albuquerque Lab, Albuquerque, NM

TN TMAINORCAL, Richmond, CA

TO TMAJEberline Oak Ridge Laboratory, Oak Ridge, TN 


\section{Participating Laboratories in EML OAP44}

Laboratories Reporting Data

Code Laboratory Name

TW Taiwan Radiation Monitoring Center Atomic Energy Council, Executi, Kaohsiung, Taiwan, ROC

TX TDH/Laboratories, Austin, TX

TY Scientific Production Association 82, Lenin Street, Kaluga Region, RUSSIA

UC Lockheed Martin RM 60 BLDG C.710, Paducah, KY

UK K-25 Plant Lockheed Martin Energy Systems, Oak Ridge, TN

UP Y.12 Plant, ASO, OC Laboratory 113C Union Valley Road, Oak Ridge, TN

UY Y.12 Plant, ASO, QC Laboratory 113C Union Valley Road, Oak Ridge, TN

WA Environmental Radiation Lab Off. of Public Health Labs., Seattle, WA

WC Westinghouse Hanford Co. MSIN S3-28, Richland, WA

WE Westinghouse Electric Corp. Chemical \& Materials Tech., Madison, PA

WI Westinghouse Electric Corp. WIPP Site, Carlsbad, NM

WN WINCO CPP-609, Scoville, ID

WP Washington Public Power Supply System, Richland, WA

WS Weldon Springs Site, St. Charles, MO

WV West Valley Nuclear Services Co, Inc MS 307, West Valley, NY

YA Yankee Atomic Electric Company, Westboro, MA

YP US Army Proving Ground ATTN: STEYP-RS-LS-MP, Yuma, AZ

Total Reporting Labs: 119 


\section{Participating Laboratories in EML QAP44}

Laboratories Not Reporting Data

\section{Code Laboratory Name}

ED Maxim Téchnologies , St. Louis, MO

HI Heritage Laboratories, Inc. , Indianapolis, IN

HS RESL - USDOE, Idaho Falls, ID

OI Oak Ridge Research Institute, Inc. , Oak Ridge, TN

PR Princeton Plasma Physics Lab, Princeton, NJ

RD Radiation Detection Company, Sunnyvale, CA

RL Thermo Hanford 2440 Stevens Center MSIN: H6-0, Richland, WA

SL Stanford Linear Accelerator Center Off. of Hlth Physics, MS 84, Menlow Park, CA

Total Non-Reporting Labs: 8 